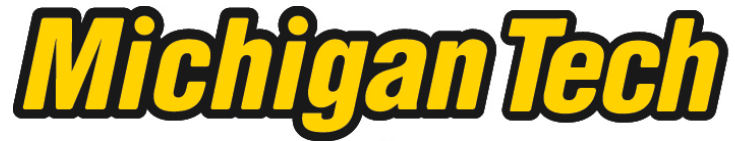 \\ Michigan Technological University Create the Future Digital Commons @ Michigan Tech
}

2013

EXPERIMENTAL INVESTIGATION INTO PARTICULATE MATTER

DISTRIBUTION IN CATALYZED PARTICULATE FILTERS USING A 3D TERAHERTZ WAVE SCANNER

Ryan Kristopher Foley

Michigan Technological University

Follow this and additional works at: https://digitalcommons.mtu.edu/etds

Part of the Mechanical Engineering Commons, and the Power and Energy Commons Copyright 2013 Ryan Kristopher Foley

Recommended Citation

Foley, Ryan Kristopher, "EXPERIMENTAL INVESTIGATION INTO PARTICULATE MATTER DISTRIBUTION IN CATALYZED PARTICULATE FILTERS USING A 3D TERAHERTZ WAVE SCANNER", Master's Thesis, Michigan Technological University, 2013.

https://doi.org/10.37099/mtu.dc.etds/657

Follow this and additional works at: https://digitalcommons.mtu.edu/etds

Part of the Mechanical Engineering Commons, and the Power and Energy Commons 


\title{
EXPERIMENTAL INVESTIGATION INTO PARTICULATE MATTER DISTRIBUTION IN CATALYZED PARTICULATE FILTERS USING A 3D TERAHERTZ WAVE SCANNER
}

\author{
By \\ Ryan Kristopher Foley
}

\begin{abstract}
A THESIS
Submitted in partial fulfillment of the requirements for the degree of MASTER OF SCIENCE

In Mechanical Engineering
\end{abstract}

MICHIGAN TECHNOLOGICAL UNIVERSITY

2013

(C) 2013 Ryan K. Foley 

This thesis has been approved in partial fulfillment of the requirements for the Degree of MASTER OF SCIENCE in Mechanical Engineering.

Department of Mechanical Engineering- Engineering Mechanics

Thesis Co-Advisor: John H. Johnson

Thesis Co-Advisor: Jeffrey D. Naber

Committee Member: $\quad$ Franz X. Tanner

Department Chair: $\quad$ William W. Predebon 



\section{Table of Contents}

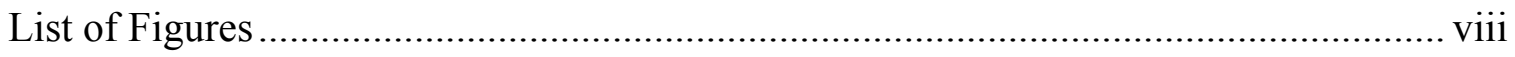

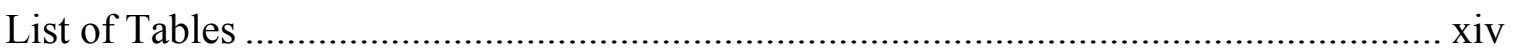

Preface

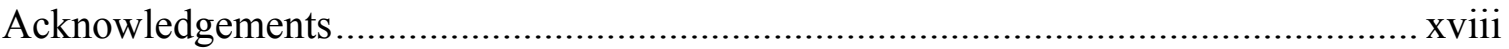

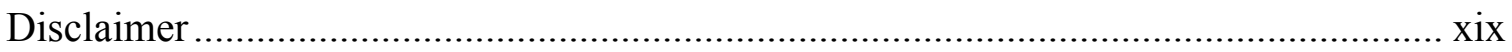

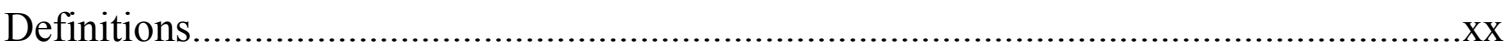

List of Abbreviations ............................................................................................. xxii

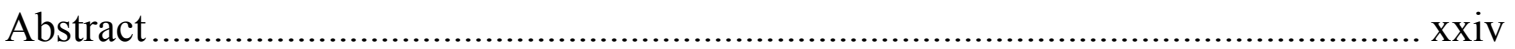

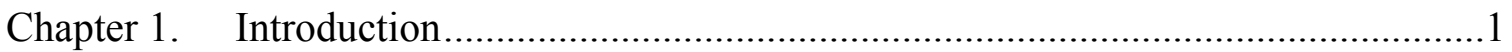

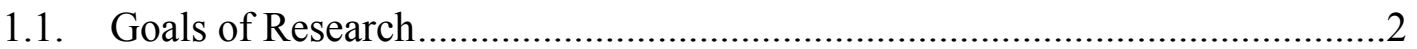

1.1.1. Phase 1: Experimental and Methodology Development.......................3

1.1.2. Phase 2: PM Distribution Trend Analysis ...........................................

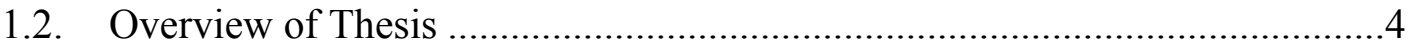

Chapter 2. Literature Review.........................................................................

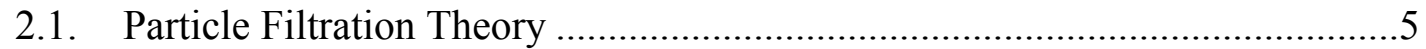

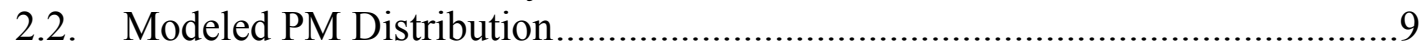

2.3. PM Distribution Measurement Methods ……………...................................14

2.3.1. Restrictive Measurement Methods ....................................................14

2.3.2. Multi-dimensional Imaging Measurement Methods............................15

2.4. Experimental Investigations of PM Distribution............................................17

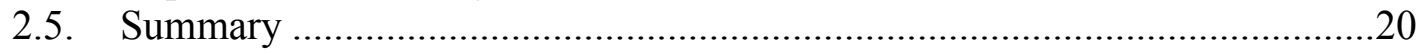

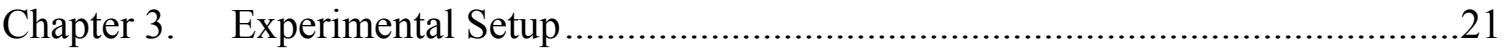

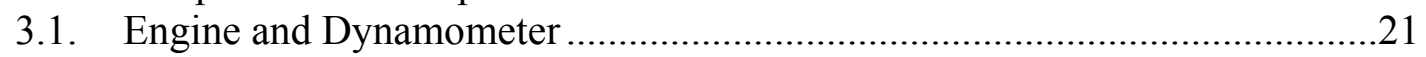

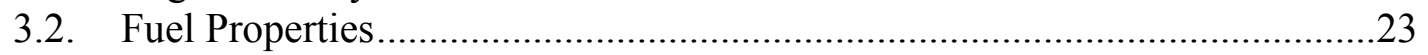

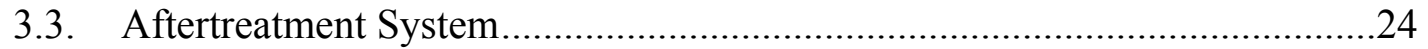

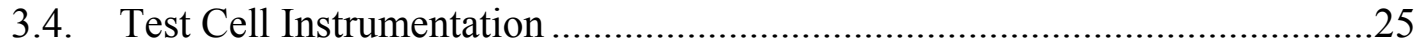

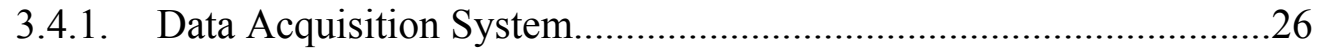

3.4.2. Measurement of Engine Intake Air.................................................26

3.4.3. Fuel Flow Rate Measurements......................................................26

3.4.4. Pressure Transducers ......................................................................27

3.4.5. Temperature Measurements..........................................................28

3.4.6. Gaseous Emissions Measurements ..................................................30

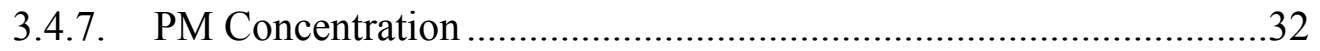

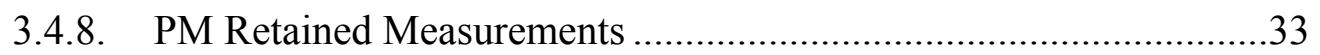

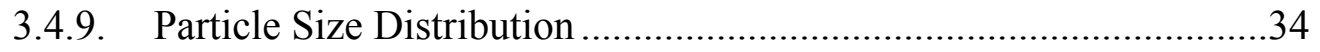

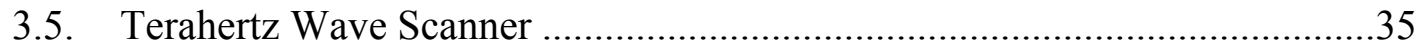




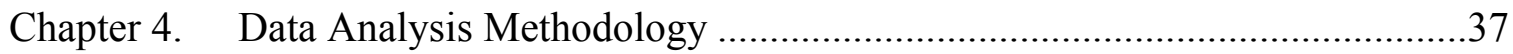

4.1. Conversion of Scan Data to Grams per Liter ..................................................37

4.2. Conversion of Scan Data to Grams per Surface Area ....................................39

4.3. Development of Directionality ..................................................................4

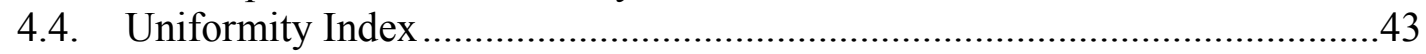

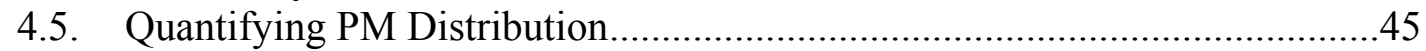

4.6. Requirements for Uniformity ................................................................46

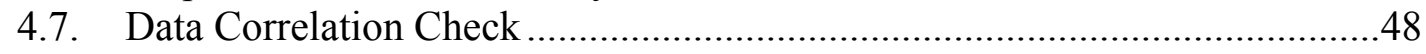

Chapter 5. Experimental Matrix and Procedures.............................................................51

5.1. Phase 1: Experimental and Methodology Development .................................51

5.2. Phase 2: PM Distribution Trend Analysis .......................................................54

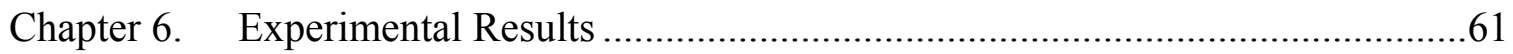

6.1. Developed Data Analysis Method Functionality ..........................................61

6.2. Changes in the PM Density Calculation Process ...........................................81

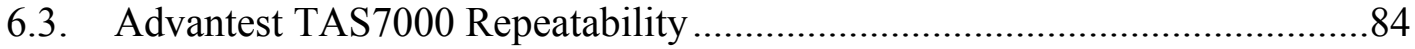

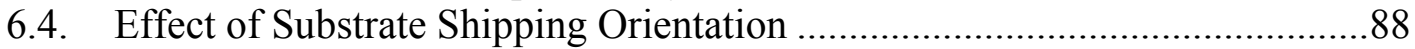

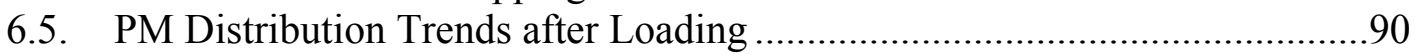

6.6. PM Distribution Trends after Passive Oxidation ...........................................101

6.7. PM Distribution Trends after Active Regeneration .......................................115

6.8. PM Distribution Trends after Post Loading ................................................130

Chapter 7. Summary and Conclusions ………………..............................................138

7.1. Summary of Experiments Conducted......................................................138

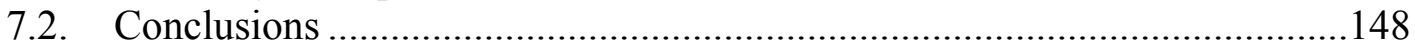

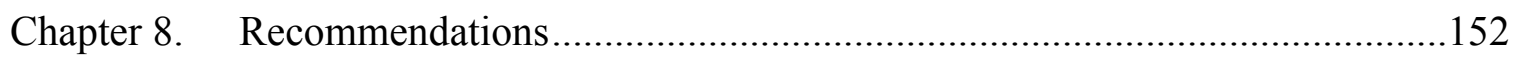

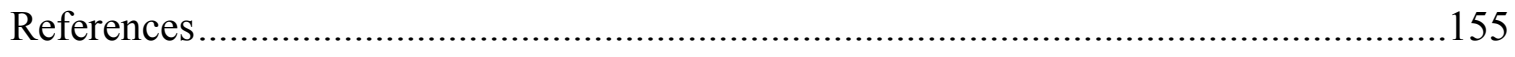

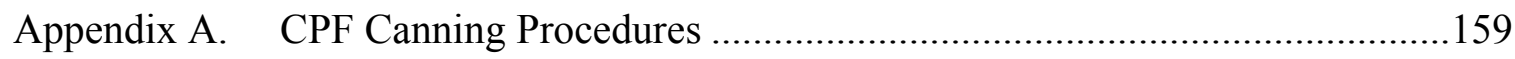

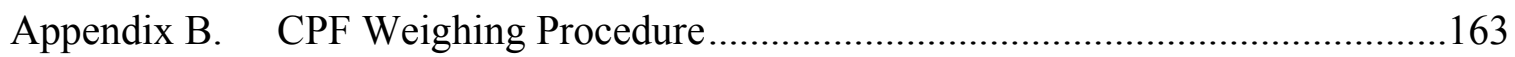

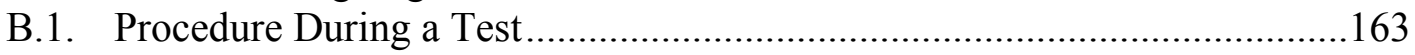

B.2. Substrate Only Measurement Procedure …………......................................165

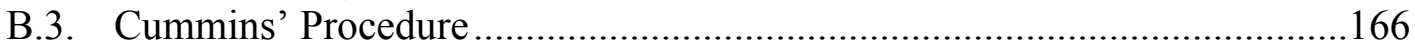

Appendix C. Substrate Shipping Procedure ………………………….......................167

Appendix D. Test Plan Measurements................................................................168

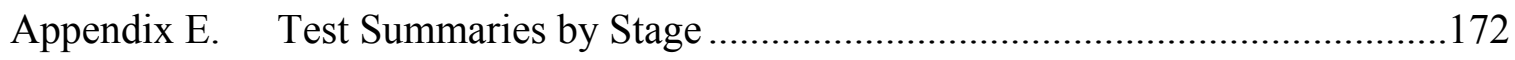

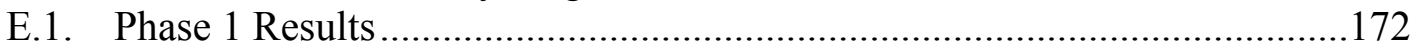

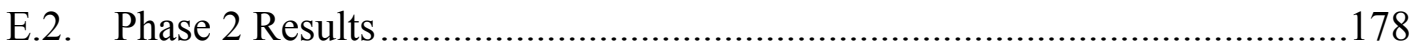

Appendix F. $\quad$ PM Reaction Rate..........................................................................185

Appendix G. Substrate Mass Discrepancies …………….........................................187 
Appendix H. Test Pressure Drop Curves ...............................................................190

Appendix I. Average CPF Temperatures .............................................................197

Appendix J. Emissions Measurements by Stage ……………...................................207

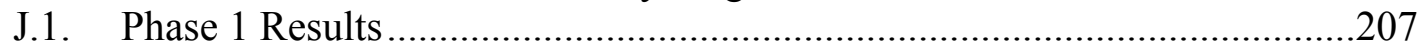

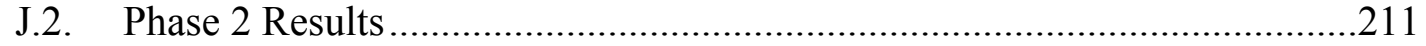

Appendix K. Additional PM Distribution Plots......................................................218

Appendix L. Data Correlation Check Results by Test ..................................................258

Appendix M. Permission to Use Copyrighted Material ...................................................268 


\section{List of Figures}

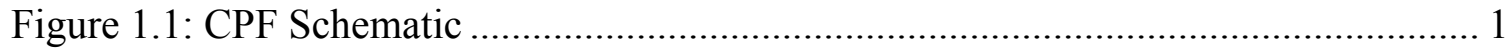

Figure 2.1: Example of Particle Sizes Taken from the Phase 2 Test 1 Loading Stage...... 6

Figure 2.2: Example of Filtration Mechanism Effectiveness v. Particle Size [7].............. 6

Figure 2.3: Change in Overall Permeability as a Function of Loading [8] ........................ 7

Figure 2.4: Axial Through-Wall Flow Distribution as a Function of Permeability [9] ...... 8

Figure 2.5: Effect of Inlet Exhaust Gas Velocity on Wall Flow Distribution [9].............. 8

Figure 2.6 PM Distribution at the Beginning of Cake Formation [13] .......................... 10

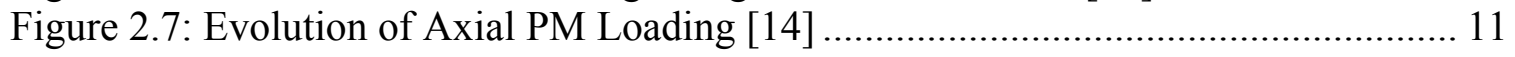

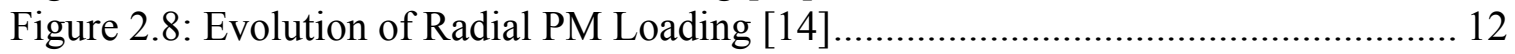

Figure 2.9: Wall Flow Distribution as a Function of PM Loading [15] .......................... 13

Figure 2.10: PM Cake Thickness v. PM Loading [19] .................................................. 19

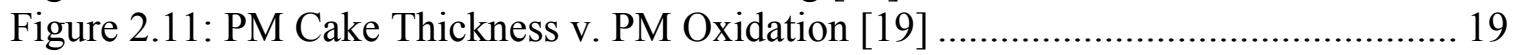

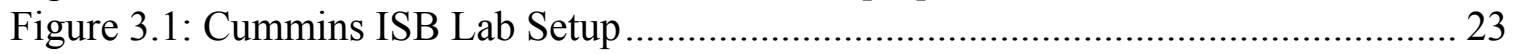

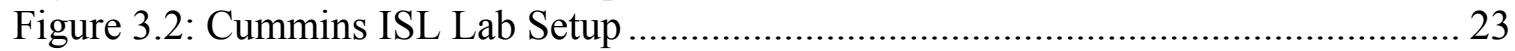

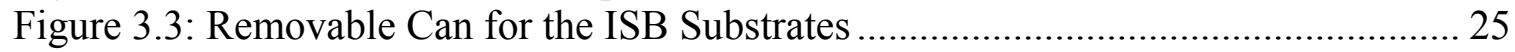

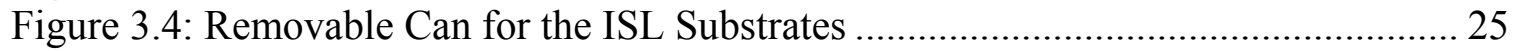

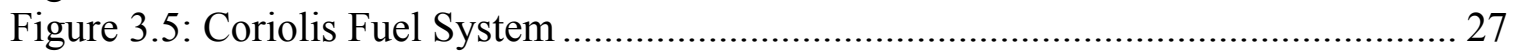

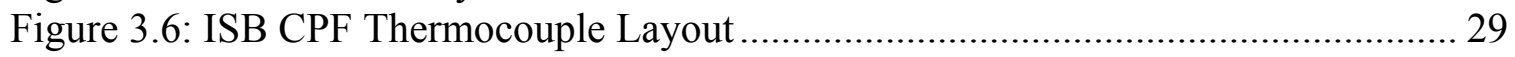

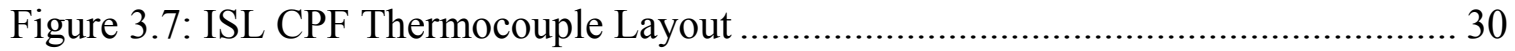

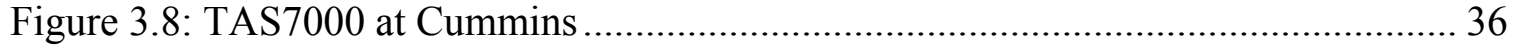

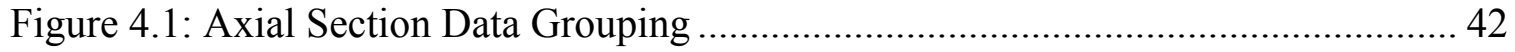

Figure 4.2: R* Values for the Radial Sections............................................................ 43

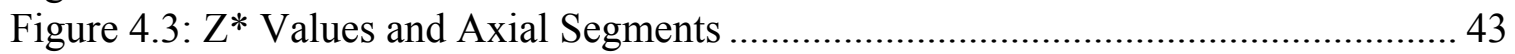

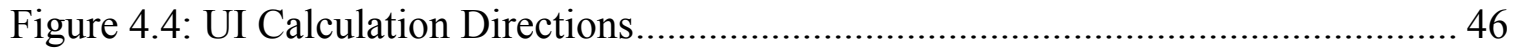

Figure 4.5: Data Correlation: Good Data Set- Taken from Test 1 Phase 2 ................... 50

Figure 4.6: Data Correlation: Shifted Data Set- Taken from Test 1 Phase $1 \ldots \ldots \ldots \ldots \ldots \ldots . . . . . .50$

Figure 5.1: Phase 1 Test Matrix ...................................................................................... 51

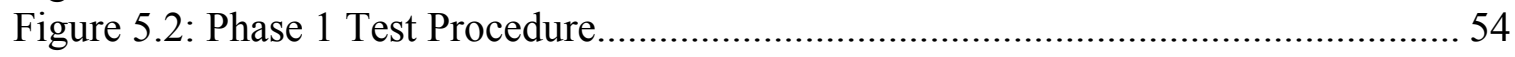

Figure 5.3: Revised Option 3 for Phase 1 Test Procedure ............................................... 54

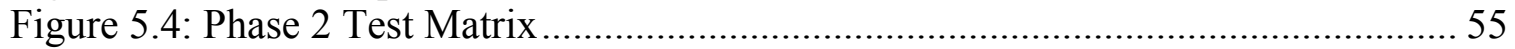

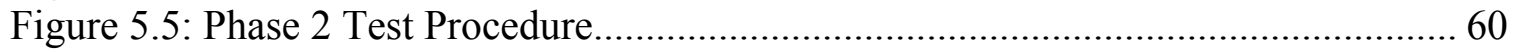

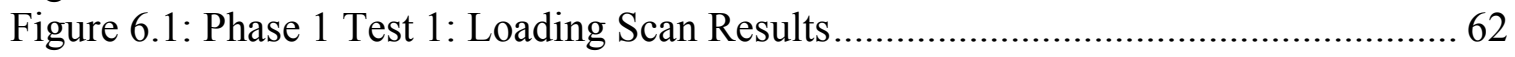

Figure 6.2: Phase 1 Test 1- Loading Axial PM Distribution in Each Radial Section....... 63

Figure 6.3: Phase 1 Test 1- Loading Angular PM Distribution in Each Radial Section .. 65

Figure 6.4: Phase 1 Test 1- Active Regeneration Scan Results ...................................... 67

Figure 6.5: Phase 1 Test 1- Active Regeneration Axial PM Distribution in Each Radial

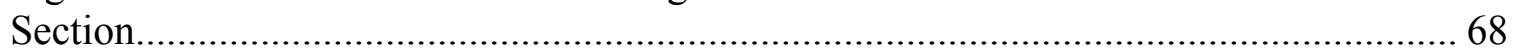

Figure 6.6: Phase 1 Test 1- Active Regeneration Angular PM Distribution in Each Radial

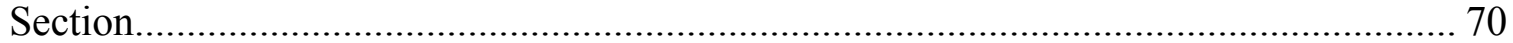

Figure 6.7: Phase 1 Test 2- Active Regeneration Scan Results .................................... 71

Figure 6.8: Phase 1 Test 2- Active Regeneration Axial PM Distribution in Each Radial

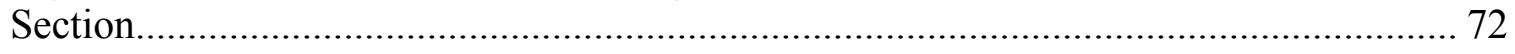


Figure 6.9: Phase 1 Test 2- Active Regeneration Angular PM Distribution in Each Radial

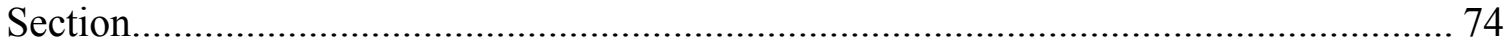

Figure 6.10: Phase 1 Test 3- Passive Oxidation Scan Results ......................................... 75

Figure 6.11: Phase 1 Test 3- Passive Oxidation Axial PM Distribution in Each Radial

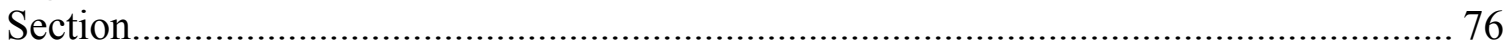

Figure 6.12: Phase 1 Test 3- Passive Oxidation Angular PM Distribution in Each Radial

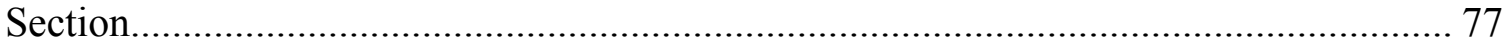

Figure 6.13: Phase 1 Test 4- Active Regeneration Scan Results.................................... 79

Figure 6.14: Phase 1 Test 4- Active Regeneration Axial PM Distribution in Each Radial

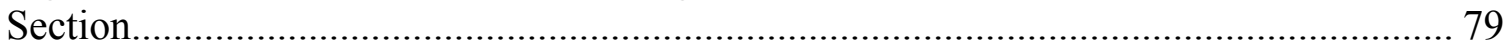

Figure 6.15: Phase 1 Test 4- Active Regeneration Angular PM Distribution in Each

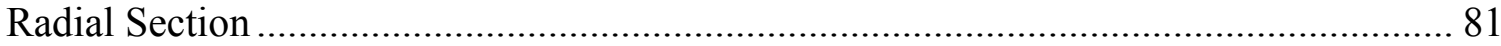

Figure 6.16: Phase 2 Test 1- Loading Difference in Scan Results ................................... 82

Figure 6.17: Phase 2 Test 1- Passive Oxidation Difference in Scan Results.................... 83

Figure 6.18: Repeat Scans of the Phase 1 Test 4 Scan Active Regeneration Scan........... 86

Figure 6.19: Repeat Scans of the Phase 2 Test 1 Passive Oxidation Scan ....................... 87

Figure 6.20: Repeat Scans of the Phase 2 Test 7 Passive Oxidation Scan ....................... 88

Figure 6.21: Phase 2 Test 1- Loading Scan Results..................................................... 91

Figure 6.22: Phase 2 Test 6- Loading Scan Results........................................................ 94

Figure 6.23: Phase 2 Test 6- Second Loading Scan Results............................................ 96

Figure 6.24: Axial PM Distribution for all Loading Tests........................................... 100

Figure 6.25: Axial PM Distribution Comparison Between Experimental and Modeled

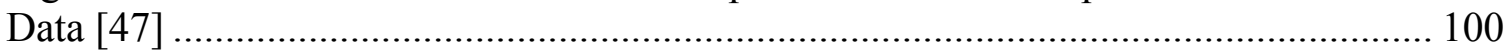

Figure 6.26: Axial UI as a Function of PM Density …………..................................... 101

Figure 6.27: Phase 2 Test 1- Passive Oxidation Scan Results....................................... 103

Figure 6.28: Phase 2 Test 7- Passive Oxidation Scan Results ........................................ 106

Figure 6.29: Phase 2 Test 8- Passive Oxidation Scan Result ....................................... 108

Figure 6.30: Phase 2 Test 9- Balance Point Scan Result ................................................... 111

Figure 6.31: Axial PM Distribution for all Passive Oxidation Tests .............................. 114

Figure 6.32: Axial Uniformity Index as a Function of Percent of PM Oxidized............ 114

Figure 6.33: Phase 2 Test 2- Cleanout Scan Result..................................................... 116

Figure 6.34: Phase 2 Test 3- Active Regeneration Scan Result ..................................... 119

Figure 6.35: Phase 2 Test 4- Active Regeneration Scan Result .................................. 122

Figure 6.36: Phase 2 Test 5- Active Regeneration Scan Result ................................... 124

Figure 6.37: Axial PM Distribution for all Active Regeneration Tests.......................... 128

Figure 6.38: Axial Uniformity Index as a Function of Percent of PM Oxidized............ 129

Figure 6.39: Radial Uniformity Index as a Function of Percent of PM Oxidized .......... 129

Figure 6.40: Angular Uniformity Index as a Function of Percent of PM Oxidized ....... 130

Figure 6.41: Phase 2 Test 4- Post Loading Scan Result ............................................... 131

Figure 6.42: Phase 2 Test 7- Post Loading Scan Results............................................. 134

Figure 6.43: Axial PM Distribution for all Post Loading Tests...................................... 137

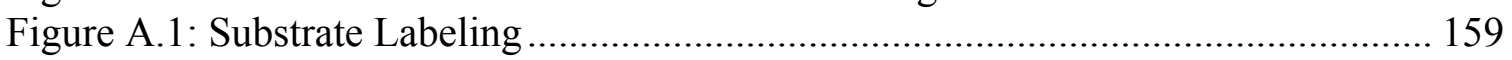

Figure A.2: Substrate Alignment Marks ……………............................................. 160

Figure A.3: Thermocouple Location Markings ……………........................................ 160

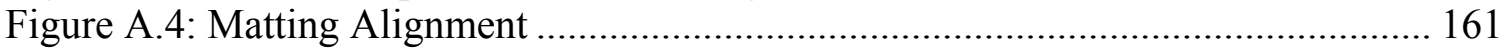


Figure A.5: Substrate and Can Alignment .................................................................. 162

Figure A.6: Bolt Tightening Sequence …………….............................................. 162

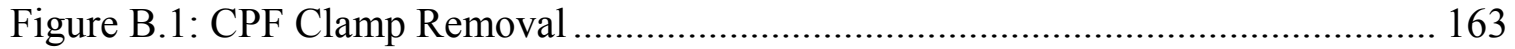

Figure B.2: Temperature Measurement Locations for Substrate Baking ....................... 165

Figure C.1: Substrate in Shipping Crate ............................................................... 167

Figure F.1: PM Reaction Rate Plot ........................................................................ 185

Figure F.2: Experimental Versus Model Predicted Reaction Rates ............................... 186

Figure H.1: Phase 1 Test 1 Pressure Drop Curves........................................................ 190

Figure H.2: Phase 1 Test 2 Pressure Drop Curves...................................................... 191

Figure H.3: Phase 1 Test 3 Pressure Drop Curves.......................................................... 191

Figure H.4: Phase 1 Test 4 Pressure Drop Curves......................................................... 192

Figure H.5: Phase 2 Test 1 Pressure Drop Curves......................................................... 192

Figure H.6: Phase 2 Test 2 Pressure Drop Curves........................................................ 193

Figure H.7: Phase 2 Test 3 Pressure Drop Curves......................................................... 193

Figure H.8: Phase 2 Test 4 Pressure Drop Curves......................................................... 194

Figure H.9: Phase 2 Test 5 Pressure Drop Curves..................................................... 194

Figure H.10: Phase 2 Test 6 Pressure Drop Curves..................................................... 195

Figure H.11: Phase 2 Test 7 Pressure Drop Curves.................................................... 195

Figure H.12: Phase 2 Test 8 Pressure Drop Curves...................................................... 196

Figure H.13: Phase 2 Test 9 Pressure Drop Curves........................................................ 196

Figure I.1: Radial Temperature Distribution for Phase 1 Test 1 Loading ...................... 197

Figure I.2: Radial Temperature Distribution for Phase 1 Test 1 Active Regeneration... 198

Figure I.3: Radial Temperature Distribution for Phase 1 Test 2 Active Regeneration... 198

Figure I.4: Radial Temperature Distribution for Phase 1 Test 3 Passive Oxidation....... 199

Figure I.5: Radial Temperature Distribution for Phase 1 Test 4 Active Regeneration... 199

Figure I.6: Radial Temperature Distribution for Phase 2 Test 1 Loading ...................... 200

Figure I.7: Radial Temperature Distribution for Phase 2 Test 1 Passive Oxidation....... 200

Figure I.8: Radial Temperature Distribution for Phase 2 Test 2 Cleanout ...................... 201

Figure I.9: Radial Temperature Distribution for Phase 2 Test 3 Active Regeneration... 201

Figure I.10: Radial Temperature Distribution for Phase 2 Test 4 Active Regeneration. 202

Figure I.11: Radial Temperature Distribution for Phase 2 Test 4 Post Loading ............ 202

Figure I.12: Radial Temperature Distribution for Phase 2 Test 5 Active Regeneration. 203

Figure I.13: Radial Temperature Distribution for Phase 2 Test 6 Loading .................... 203

Figure I.14: Radial Temperature Distribution for Phase 2 Test 6 Second Loading ....... 204

Figure I.15: Radial Temperature Distribution for Phase 2 Test 7 Passive Oxidation..... 204

Figure I.16 Radial Temperature Distribution for Phase 2 Test 7 Post Loading ............. 205

Figure I.17: Radial Temperature Distribution for Phase 2 Test 8 Passive Oxidation..... 205

Figure I.18: Radial Temperature Distribution for Phase 2 Test 9 Balance Point ........... 206

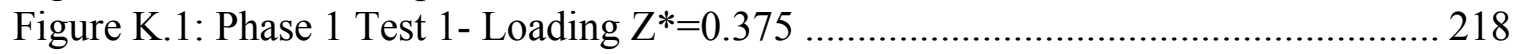

Figure K.2: Phase 1 Test 1- Loading $Z^{*}=0.625$....................................................... 218

Figure K.3: Phase 1 Test 1- Loading $Z^{*}=0.875$ …………...................................... 219

Figure K.4: Phase 1 Test 1- Active Regeneration Z*=0.375 ...................................... 219

Figure K.5: Phase 1 Test 1- Active Regeneration $Z^{*}=0.625$........................................ 220

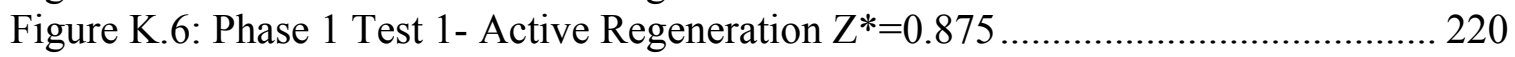

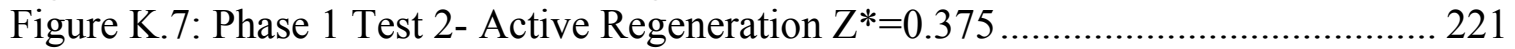




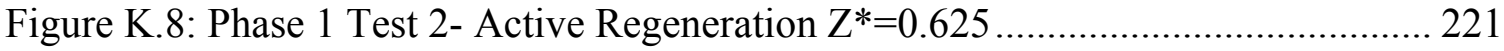

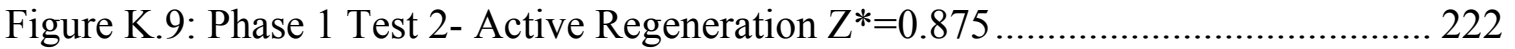

Figure K.10: Phase 1 Test 3- Passive Oxidation $Z^{*}=0.375$....................................... 222

Figure K.11: Phase 1 Test 3- Passive Oxidation $Z^{*}=0.625$........................................ 223

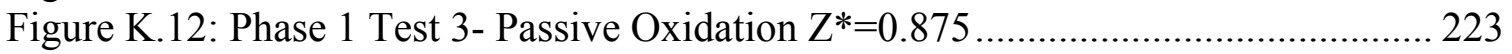

Figure K.13: Phase 1 Test 4- Active Regeneration $Z^{*}=0.375$.................................... 224

Figure K.14: Phase 1 Test 4- Active Regeneration $Z^{*}=0.625$.................................. 224

Figure K.15: Phase 1 Test 4- Active Regeneration $Z^{*}=0.875$................................... 225

Figure K.16: Phase 2 Test 1- Loading Axial Distribution ........................................... 225

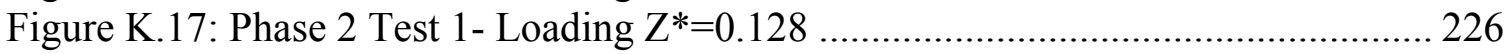

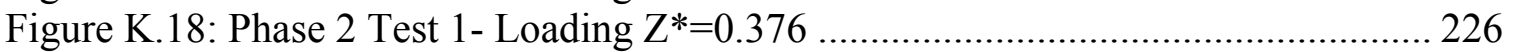

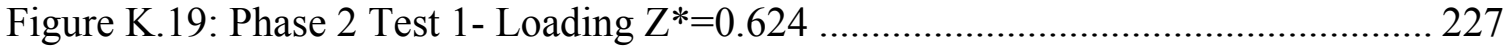

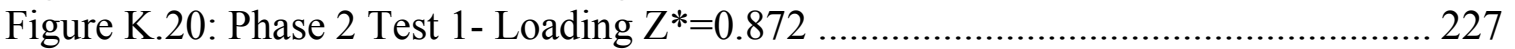

Figure K.21: Phase 2 Test 1- Passive Oxidation Axial Distribution ............................. 228

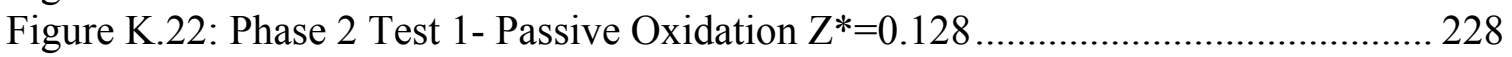

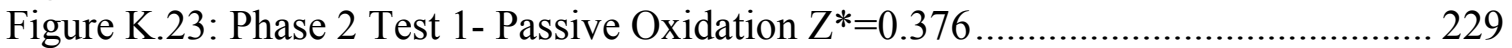

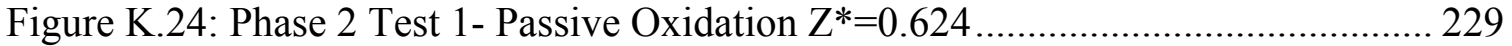

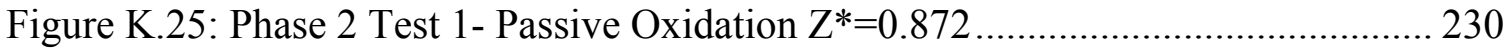

Figure K.26: Phase 2 Test 2- Cleanout Axial Distribution............................................ 230

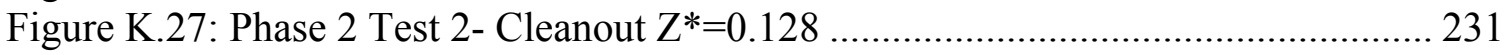

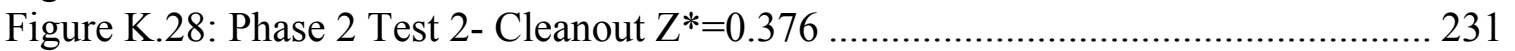

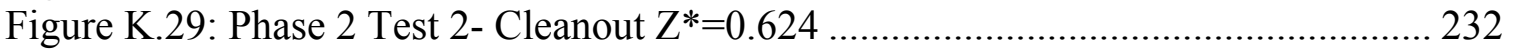

Figure K.30: Phase 2 Test 2- Cleanout $Z^{*}=0.872$................................................... 232

Figure K.31: Phase 2 Test 3- Active Regeneration Axial Distribution ......................... 233

Figure K.32: Phase 2 Test 3- Active Regeneration $Z^{*}=0.128$.................................... 233

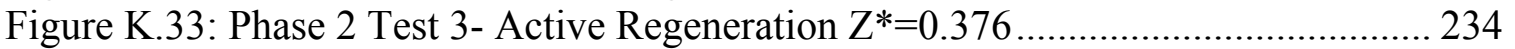

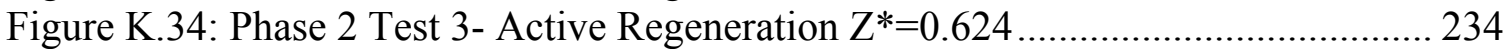

Figure K.35: Phase 2 Test 3- Active Regeneration $Z^{*}=0.872$.................................. 235

Figure K.36: Phase 2 Test 4- Active Regeneration Axial Distribution ......................... 235

Figure K.37: Phase 2 Test 4- Active Regeneration $Z^{*}=0.128$.................................... 236

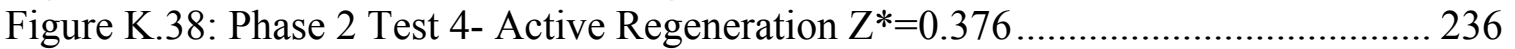

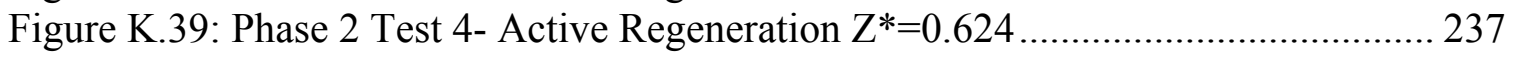

Figure K.40: Phase 2 Test 4- Active Regeneration $Z^{*}=0.872$.................................... 237

Figure K.41: Phase 2 Test 4- Post Loading Axial Distribution ..................................... 238

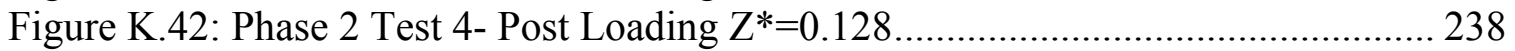

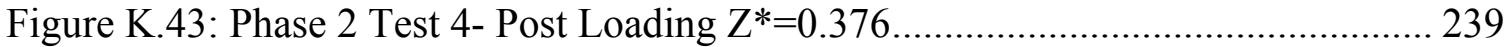

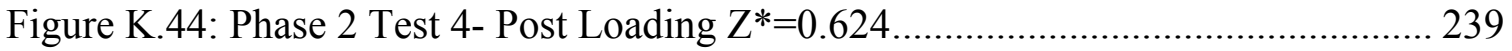

Figure K.45: Phase 2 Test 4- Post Loading $Z^{*}=0.872$................................................ 240

Figure K.46: Phase 2 Test 5- Active Regeneration Axial Distribution ......................... 240

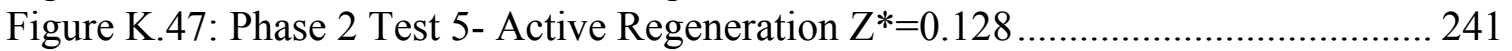

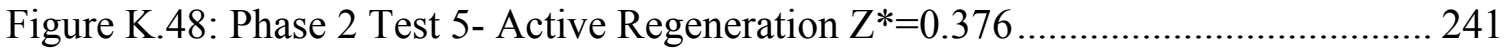

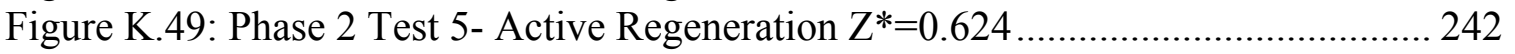

Figure K.50: Phase 2 Test 5- Active Regeneration $Z^{*}=0.872$................................ 242

Figure K.51: Phase 2 Test 6- Loading Axial Distribution ........................................... 243

Figure K.52: Phase 2 Test 6- Loading $Z^{*}=0.128$................................................. 243 


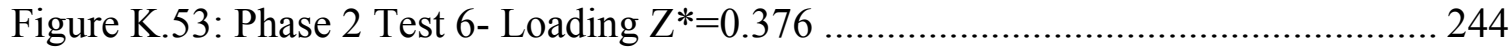

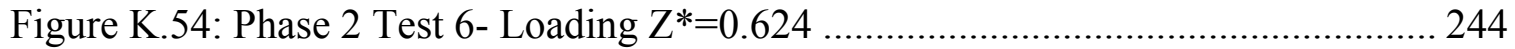

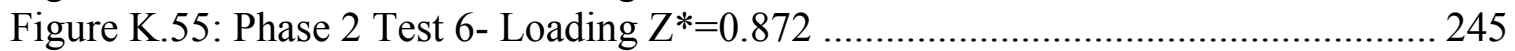

Figure K.56: Phase 2 Test 6- Second Loading Axial Distribution ................................. 245

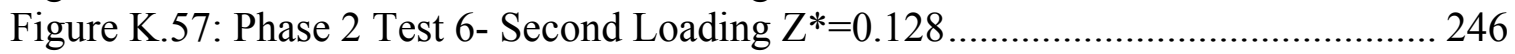

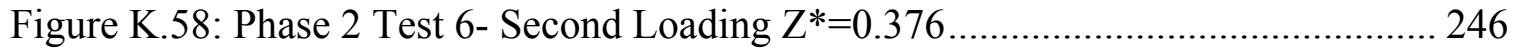

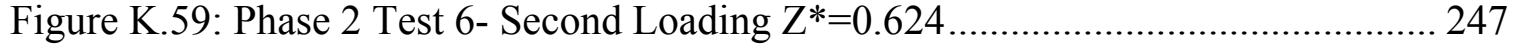

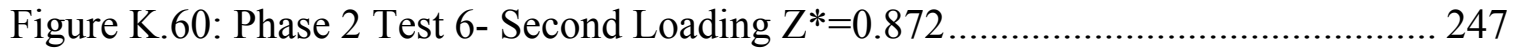

Figure K.61: Phase 2 Test 7- Passive Oxidation Axial Distribution .............................. 248

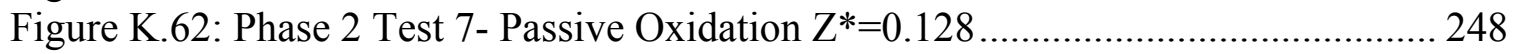

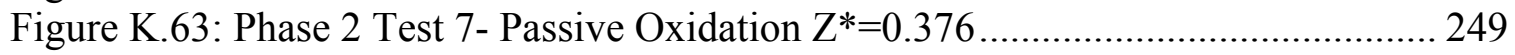

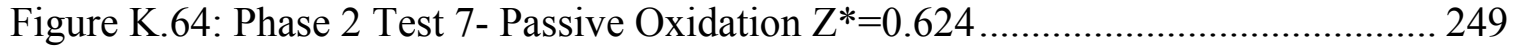

Figure K.65: Phase 2 Test 7- Passive Oxidation $Z^{*}=0.872$........................................ 250

Figure K.66: Phase 2 Test 7- Post Loading Axial Distribution .................................... 250

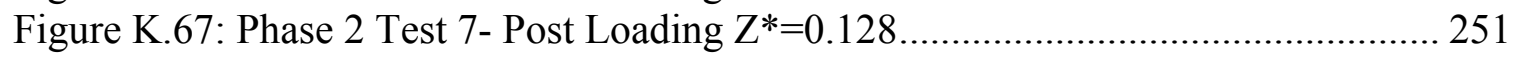

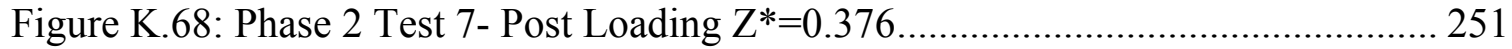

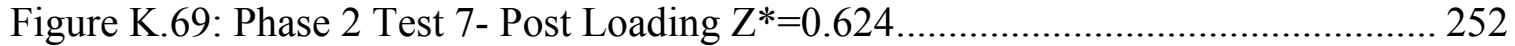

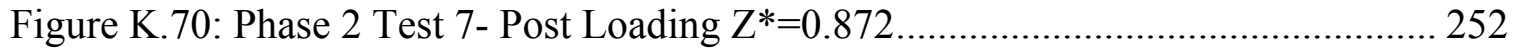

Figure K.71: Phase 2 Test 8- Passive Oxidation Axial Distribution .............................. 253

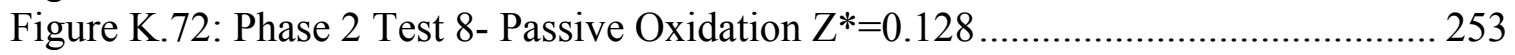

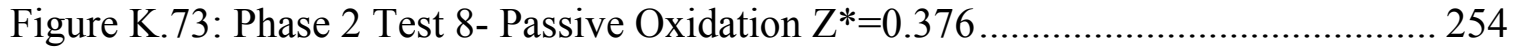

Figure K.74: Phase 2 Test 8- Passive Oxidation $Z *=0.624 \ldots \ldots \ldots \ldots \ldots \ldots \ldots \ldots \ldots \ldots \ldots \ldots \ldots \ldots \ldots \ldots . . .254$

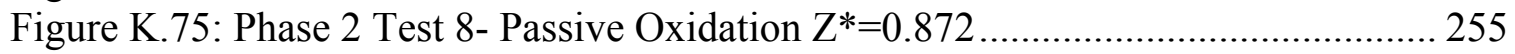

Figure K.76: Phase 2 Test 9- Balance Point Axial Distribution ..................................... 255

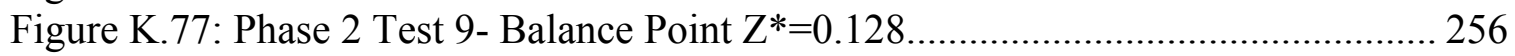

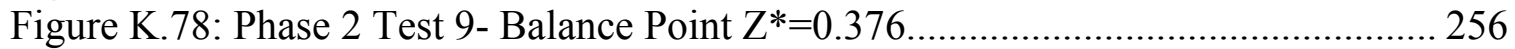

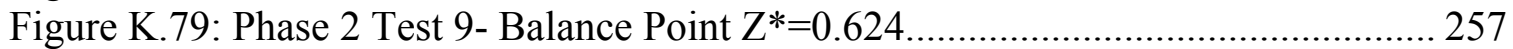

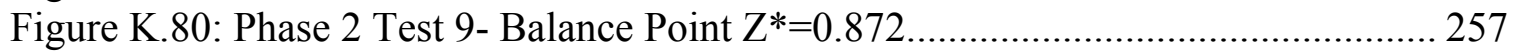

Figure L.1: Data Correlation for Phase 1 Test 1 Loading Scan..................................... 258

Figure L.2: Data Correlation for Phase 1 Test 1 Active Regeneration Scan .................. 259

Figure L.3: Data Correlation for Phase 1 Test 2 Active Regeneration Scan .................. 259

Figure L.4: Data Correlation for Phase 1 Test 3 Passive Oxidation Scan ....................... 260

Figure L.5: Data Correlation for Phase 1 Test 4 Active Regeneration Scan .................. 260

Figure L.6: Data Correlation for Phase 2 Test 1 Loading Scan .................................... 261

Figure L.7: Data Correlation for Phase 2 Test 1 Passive Oxidation Scan ....................... 261

Figure L.8: Data Correlation for Phase 2 Test 2 Cleanout Scan..................................... 262

Figure L.9: Data Correlation for Phase 2 Test 3 Active Regeneration........................... 262

Figure L.10: Data Correlation for Phase 2 Test 4 Active Regeneration Scan ................. 263

Figure L.11: Data Correlation for Phase 2 Test 4 Post Loading Scan............................ 263

Figure L.12: Data Correlation for Phase 2 Test 5 Active Regeneration Scan ................. 264

Figure L.13: Data Correlation for Phase 2 Test 6 Loading Scan.................................... 264

Figure L.14: Data Correlation for Phase 2 Test 6 Second Loading Scan ....................... 265

Figure L.15: Data Correlation for Phase 2 Test 7 Passive Oxidation Scan .................... 265

Figure L.16: Data Correlation for Phase 2 Test 7 Post Loading Scan ............................. 266

Figure L.17: Data Correlation for Phase 2 Test 8 Passive Oxidation Scan ..................... 266 
Figure L.18: Data Correlation for Phase 2 Test 9 Balance Point Scan......................... 267 


\section{List of Tables}

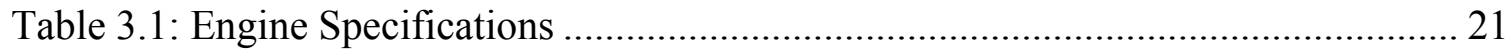

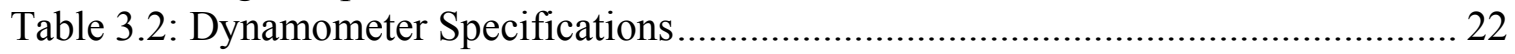

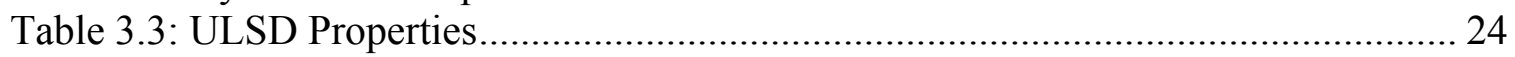

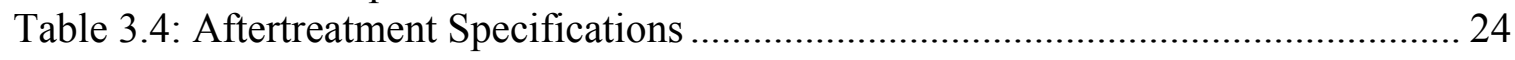

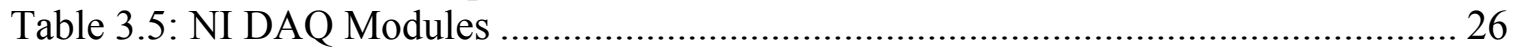

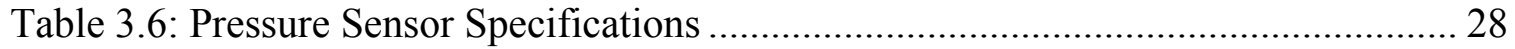

Table 3.7: Thermocouples Used in the MTU Test Cell................................................ 29

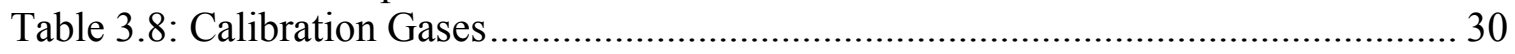

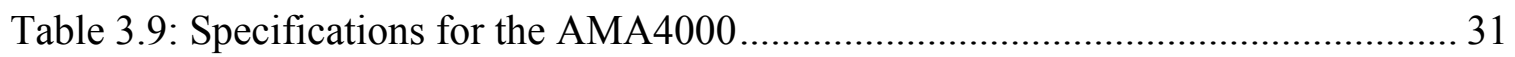

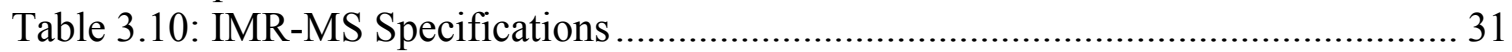

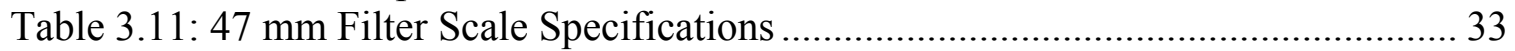

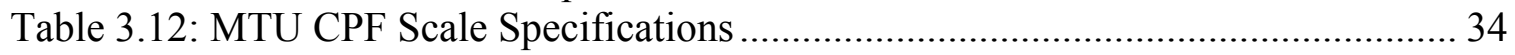

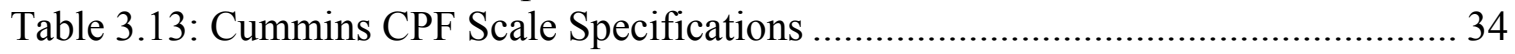

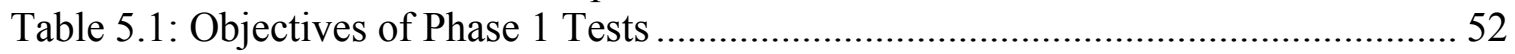

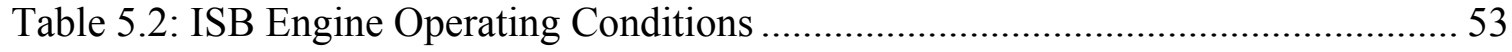

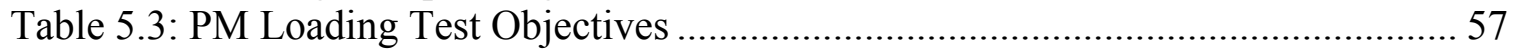

Table 5.4: Multiple Loading Test Cleanout Objectives ............................................... 57

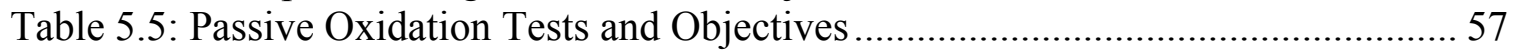

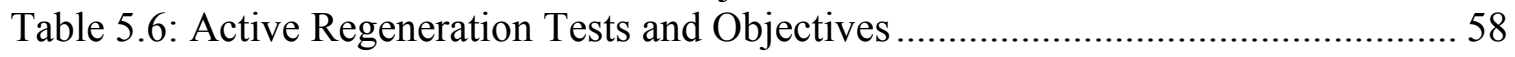

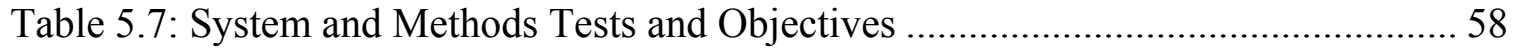

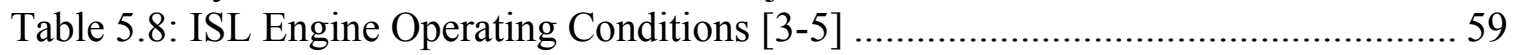

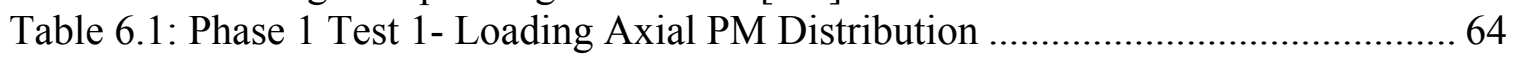

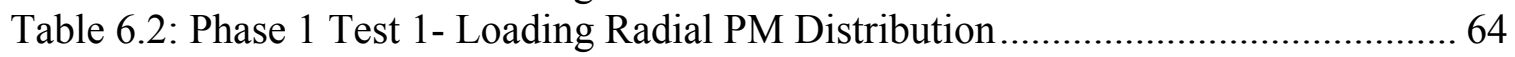

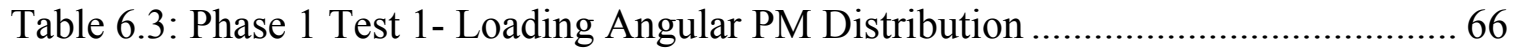

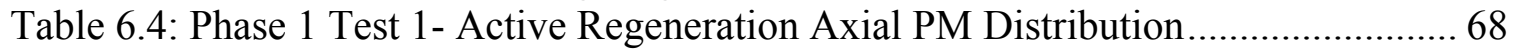

Table 6.5: Phase 1 Test 1- Active Regeneration Radial PM Distribution ......................... 69

Table 6.6: Phase 1 Test 1- Active Regeneration Angular PM Distribution...................... 70

Table 6.7: Phase 1 Test 2- Active Regeneration Axial PM Distribution........................... 72

Table 6.8: Phase 1 Test 2- Active Regeneration Radial PM Distribution ....................... 73

Table 6.9: Phase 1 Test 2- Active Regeneration Angular PM Distribution..................... 74

Table 6.10: Phase 1 Test 3- Passive Oxidation Axial PM Distribution........................... 76

Table 6.11: Phase 1 Test 3- Passive Oxidation Radial PM Distribution .......................... 77

Table 6.12: Phase 1 Test 3- Passive Oxidation Angular PM Distribution......................... 78

Table 6.13: Phase 1 Test 4- Active Regeneration Axial PM Distribution........................ 80

Table 6.14: Phase 1 Test 4- Active Regeneration Radial PM Distribution ..................... 80

Table 6.15: Phase 1 Test 4- Active Regeneration Angular PM Distribution................... 81

Table 6.16: Difference Between Inlet to the Side and Inlet Up Orientations ................... 89

Table 6.17: Difference Between Inlet Down and Inlet to the Side Orientations .............. 89

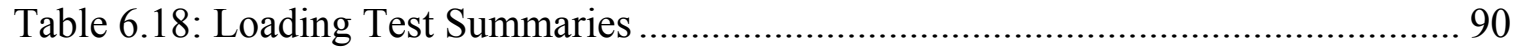

Table 6.19: Phase 2 Test 1- Loading Axial PM Distribution ....................................... 92

Table 6.20: Phase 2 Test 1- Loading Radial PM Distribution ......................................... 92

Table 6.21: Phase 2 Test 1- Loading Angular PM Distribution ..................................... 93

Table 6.22: Phase 2 Test 6- Loading Axial PM Distribution ........................................ 94 
Table 6.23: Phase 2 Test 6- Loading Radial PM Distribution ............................................ 95

Table 6.24: Phase 2 Test 6- Loading Angular PM Distribution ....................................... 95

Table 6.25: Phase 2 Test 6- Second Loading Axial PM Distribution................................ 97

Table 6.26: Phase 2 Test 6- Second Loading Radial PM Distribution .............................. 97

Table 6.27: Phase 2 Test 6- Second Loading Angular PM Distribution ........................... 98

Table 6.28: Passive Oxidation Test Summaries ........................................................... 102

Table 6.29: Phase 2 Test 1- Passive Oxidation Axial PM Distribution............................ 104

Table 6.30: Phase 2 Test 1- Passive Oxidation Radial PM Distribution ......................... 104

Table 6.31: Phase 2 Test 1- Passive Oxidation Angular PM Distribution....................... 105

Table 6.32: Phase 2 Test 7- Passive Oxidation Axial PM Distribution........................... 106

Table 6.33: Phase 2 Test 7- Passive Oxidation Radial PM Distribution ......................... 107

Table 6.34: Phase 2 Test 7- Passive Oxidation Angular PM Distribution....................... 107

Table 6.35: Phase 2 Test 8- Passive Oxidation Axial PM Distribution........................... 109

Table 6.36: Phase 2 Test 8- Passive Oxidation Radial PM Distribution ........................ 109

Table 6.37: Phase 2 Test 8- Passive Oxidation Angular PM Distribution...................... 110

Table 6.38: Phase 2 Test 9- Balance Point Axial PM Distribution ................................. 111

Table 6.39: Phase 2 Test 9- Balance Point Radial PM Distribution................................ 112

Table 6.40: Phase 2 Test 9- Balance Point Angular PM Distribution ............................. 112

Table 6.41: Active Regeneration Test Summaries ...................................................... 115

Table 6.42: Phase 2 Test 2- Cleanout Axial PM Distribution ........................................ 117

Table 6.43: Phase 2 Test 2- Cleanout Radial PM Distribution....................................... 118

Table 6.44: Phase 2 Test 2- Cleanout Angular PM Distribution .................................... 118

Table 6.45: Phase 2 Test 3- Active Regeneration Axial PM Distribution....................... 120

Table 6.46: Phase 2 Test 3- Active Regeneration Radial PM Distribution ..................... 120

Table 6.47: Phase 2 Test 3- Active Regeneration Angular PM Distribution................... 121

Table 6.48: Phase 2 Test 4- Active Regeneration Axial PM Distribution....................... 122

Table 6.49: Phase 2 Test 4- Active Regeneration Radial PM Distribution ..................... 123

Table 6.50: Phase 2 Test 4- Active Regeneration Angular PM Distribution................... 123

Table 6.51: Phase 2 Test 5- Active Regeneration Axial PM Distribution....................... 125

Table 6.52: Phase 2 Test 5- Active Regeneration Radial PM Distribution ..................... 126

Table 6.53: Phase 2 Test 5- Active Regeneration Angular PM Distribution................... 126

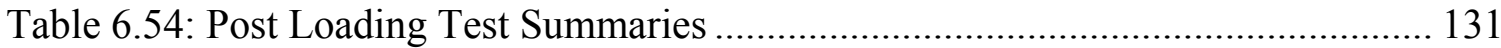

Table 6.55: Phase 2 Test 4- Post Loading Axial PM Distribution................................. 132

Table 6.56: Phase 2 Test 4- Post Loading Radial PM Distribution................................. 133

Table 6.57: Phase 2 Test 4- Post Loading Angular PM Distribution .............................. 133

Table 6.58: Phase 2 Test 7- Post Loading Axial PM Distribution.................................... 135

Table 6.59: Phase 2 Test 7- Post Loading Radial PM Distribution................................ 135

Table 6.60: Phase 2 Test 7- Post Loading Angular PM Distribution ............................. 136

Table 7.1: Loading Tests PM Distribution Summary ..................................................... 141

Table 7.2: Passive Oxidation Tests PM Distribution Summary ....................................... 143

Table 7.3: Active Regeneration Tests PM Distribution Summary ................................. 146

Table 7.4: Post Loading Tests PM Distribution Summary ............................................. 147

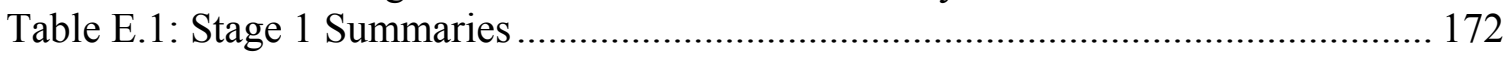

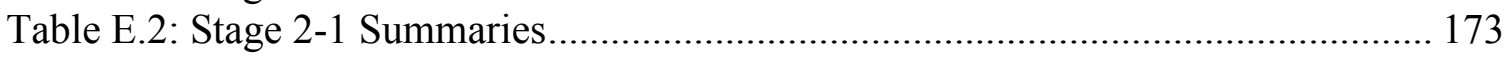

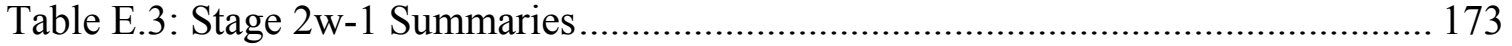




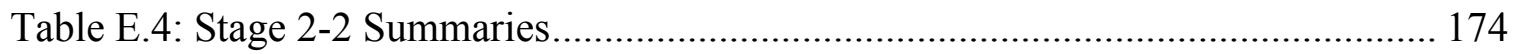

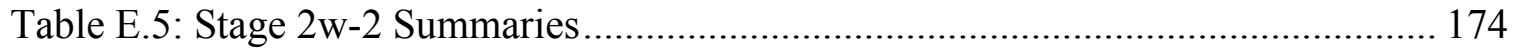

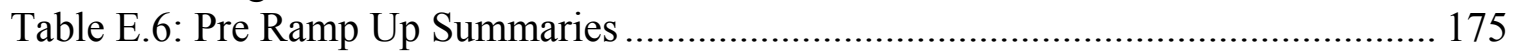

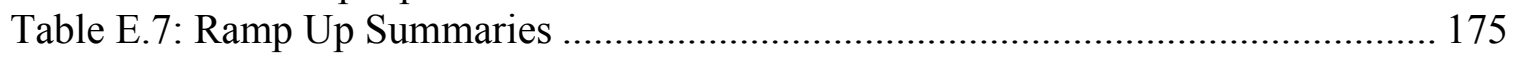

Table E.8: Pre Active Regeneration Ramp Up Summaries ............................................ 176

Table E.9: Oxidation Summaries......................................................................... 176

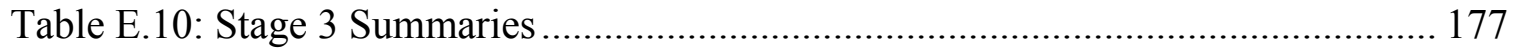

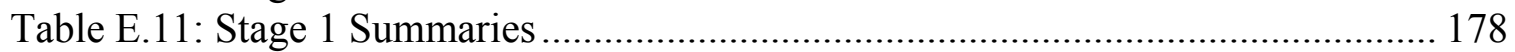

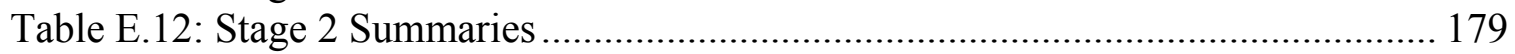

Table E.13: Pre Ramp Up Summaries .................................................................. 179

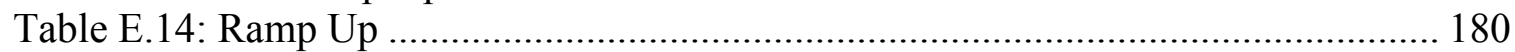

Table E.15: Pre Active Regeneration Ramp Up Summaries ........................................ 180

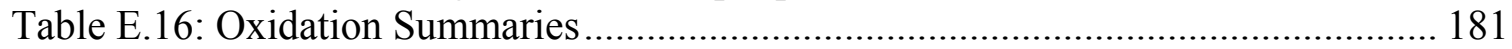

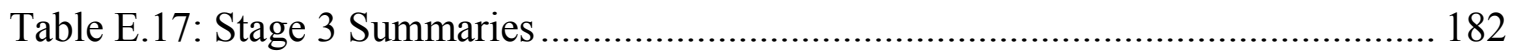

Table E.18: Pre Stage 4 Summaries.......................................................................... 183

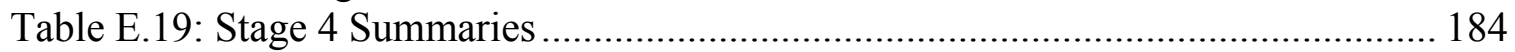

Table G.1: Difference Between In- Test and Substrate Only Mass Measurements ....... 187

Table G.2: Difference Between Initial and Final Mass at Cummins ............................. 188

Table G.3: Corrected Differences Between In-Test and Substrate Only Measurements 189

Table J.1: Stage 1 Emission Summaries ................................................................... 207

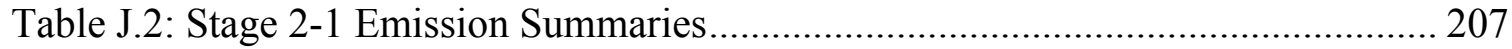

Table J.3: Stage 2w-1 Emission Summaries........................................................... 208

Table J.4: Stage 2-2 Emission Summaries................................................................ 208

Table J.5: Stage 2w-2 Emission Summaries............................................................. 209

Table J.6: Pre Ramp Up Emission Summaries …………......................................... 209

Table J.7: Oxidation Emission Summaries................................................................ 210

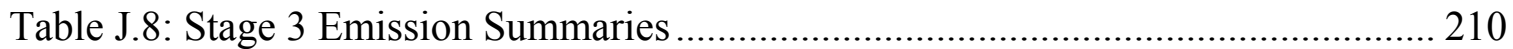

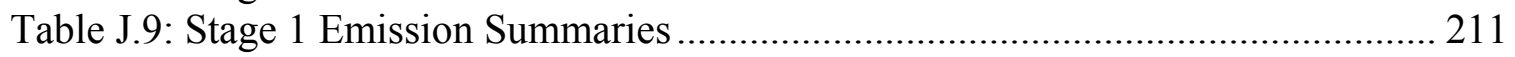

Table J.10: Stage 2 Emission Summaries ………………......................................... 212

Table J.11: Pre Ramp Up Emission Summaries ………………………………....... 213

Table J.12: Oxidation Stage Emission Summaries ………………………………...... 214

Table J.13: Stage 3 Emission Summaries ............................................................... 215

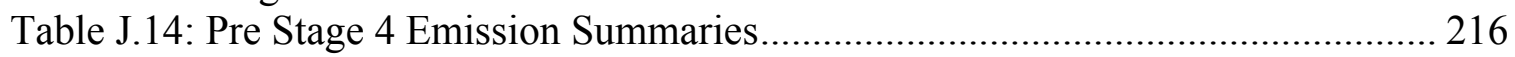

Table J.15: Stage 4 Emission Summaries ………………....................................... 217 


\section{Preface}

The work presented within this thesis is also being prepared for two separate publications. The first publication covers the work referred to Phase 1, and is presented in portions of Chapters 3-7. The publication has been submitted to SAE International for review and is tentatively titled "Development of the Methodology for Using a Terahertz Wave Scanner to Determine the 3D PM Distribution in a Catalyzed Particulate Filter." The authors include Ryan Foley, Jeff Naber, John Johnson, all of Michigan Technological University, and Leigh Rogoski of Cummins Inc. Ryan Foley conducted the on-engine experiments, developed testing practices, developed the analysis method which is the focus of the publication, analyzed the data, and drafted the paper. Jeff Naber assisted in the development of the test matrix, assisted in the development of the analysis method, provided direction to the project, and edited the publication. John Johnson assisted in the development of the test matrix, provided direction and support to the project, and edited the publication. Leigh Rogoski conducted the data collection that was required at Cummins, provided support to the project, and edited the publication.

The second publication covers the work referred to as Phase 2, and is also presented in portions of Chapters 3-7, as both phases are discussed in those chapters. This publication has not been developed yet, but will be drafted in early 2014. The intent is to submit the publication to SAE International. There is no working title at this time. The authors tentatively include Ryan Foley, Jeff Naber, John Johnson, all of Michigan Technological University, and Leigh Rogoski of Cummins Inc. Ryan Foley developed the test matrix, test procedures, conducted the on-engine experiments, analyzed the data, and will draft the publication. Jeff Naber assisted in the development of the test matrix, provided direction and support to the project, and will edit the publication. John Johnson assisted in the development of the test matrix, provided direction and support to the project, and will edit the publication. Leigh Rogoski conducted the data collection that was required at Cummins, provided support to the project, and will edit the final publication.

Finally, a portion of this work was developed collaboratively. Chris Hutton and Ryan Foley worked jointly on Phase 1 of this project. Chris started the work by developing the original test matrix, thermocouple layout, and testing procedure, worked with Cummins to gain an understanding of the Advantest System they were using to collect data, and completed two tests that resulted in substrate scans. Ryan Foley continued the work after Chris Hutton left Michigan Technological University for another job. Ryan added portions to the test matrix that allowed the data processing to be completed, improved the test procedures, completed the other three tests that were used for substrate scans, analyzed all of the data for the tests in Phase 1, continued to work with Cummins to understand the Advantest system, and drafted all of the presentations and the publication that resulted from Phase 1 of the research. 


\section{Acknowledgements}

I would like to first thank my advisers Dr. John Johnson and Dr. Jeff Naber for providing me with the opportunity to perform this research. Additionally, I thank them for the guidance they provided with the development of the test matrix and data analysis methods. They both pushed me to continually find better ways to pursue the research. I also want to thank Dr. Franz Tanner for agreeing to be a part of my thesis committee.

The financial support I received from Cummins and the United States Department of Energy for this research is greatly appreciated. "This material is based upon work supported by the Department of Energy National Energy Technology Laboratory under Award Number(s) DE-EE0000204." Cummins awarded me a fellowship for a majority of my time at Michigan Technological University, for which I am grateful.

Cummins was heavily involved in this research effort. Cummins supported the research by providing the required engines, aftertreatment components, thermocouples, and technical assistance. Joe Brault, Krishna Chilumukuru, and Arvind Suresh all supported the engines and aftertreatment systems that were used and provided technical assistance when issues arose. Leigh Rogoski, Tiffany Morgan, and William Crosby were the main contacts at Cummins that were responsible for the scanning and handling of the substrates. Leigh made sure that substrates were scanned correctly, and that the required testing materials were sent to me in a timely manner. Leigh was also the main contact between the Advantest team and me, ensuring that everyone was communicating and the required work was being completed. The Advantest team consisted of James Powers, Makoto Shinohara, Paul Fabian, and Greg Self. I am thankful for their dedication and support for this project. Cummins and Advantest put in a lot of hours making sure I was getting the correct data, and without them, I would have nothing to discuss.

I want to thank Chris Hutton, Ken Shiel, James Pidgeon, and Xiaobo Song for their work in developing the test facility and procedures at Michigan Technological University and for assisting with my tests. Additionally, I thank Chris Hutton for his work in preparing and executing the initial parts of the Phase 1 test matrix. He did a lot of work in preparation for testing with Cummins and Advantest, and made it easy for me to continue the experimental work when he moved on to a new job, giving me more time to focus on the data analysis. There were three main people that assisted in running tests: Jake Denison, Caleb Bauer, and Krishnan Raghavan. Without the assistance from these people, the testing would not have been able to be completed. Jake and Caleb also assisted in test cell maintenance when I became weighed down with data processing and report writing. Their ability to work with limited instruction made my life easy, and I can't thank them enough for their dedication to the lab. Harsha Surenahalli and Kiran Premchand both helped in my data processing, understanding of the data, and understanding of what PM distribution information was required for the models that were being developed. Marty Toth, Paul Kilpela, and Paul Dice all provided technical assistance with lab problems and worked quickly to resolve the issues, helping me to keep the research moving along. To all of the other Michigan Technological University 
faculty and staff that helped me along the way, I appreciate the effort you put forth on a daily basis and your commitment to us students.

Lastly, I want to thank my friends and family. My friends made it possible to handle the stresses related to graduate school. My family, particularly my parents, supported me in my endeavors and dealt with me being in school for six and a half years. They moved me around the country multiple times, occasionally in the middle of winter, and allowed me to pursue my goals. They provided encouragement during the hard times and helped me to remain focused on the end goal. Their personal investment and support is greatly appreciated.

\section{Disclaimer}

"This report was prepared as an account of work sponsored by an agency of the United States Government. Neither the United States Government nor any agency thereof, nor any of their employees, makes any warranty, express or implied, or assumes any legal liability or responsibility for the accuracy, completeness, or usefulness of any information, apparatus, product, or process disclosed, or represents that its use would not infringe privately owned rights. Reference herein to any specific commercial product, process, or service by trade name, trademark, manufacturer, or otherwise does not necessarily constitute or imply its endorsement, recommendation, or favoring by the United States Government or any agency thereof. The views and opinions of authors expressed herein do not necessarily state or reflect those of the United States Government or any agency thereof." 


\section{Definitions}

Aftertreatment System

Particulate Matter (PM)

Phase 1

Phase 2

Loading

Passive Oxidation

Active Regeneration

Post Loading

Spatial Resolution

Sample Point

Baseline Data Set/Scan

Axial Section

Radial Section

Angular Increment
The combination of the DOC, CPF, and SCR used to reduce the emissions associated with heavy duty diesel engines.

The solid content in the exhaust. This includes carbonaceous and ash content

The first part of the PM distribution study. Focused on developing the test and data analysis methods and seeing the performance of the TAS7000.

The second part of the PM distribution study. Focused on developing an understanding of PM distribution trends after multiple engine conditions.

The portion of a test where PM is loaded into a CPF.

The portion of a test where primarily $\mathrm{NO}_{2}$ assisted oxidation of the PM occurs.

The portion of a test where primarily $\mathrm{O}_{2}$ assisted, thermal oxidation of the PM occurs.

The portion of a test, after oxidation occurs, where PM is loaded into a CPF.

The area (2D) or volume (3D) represented by one sample point.

The data that are generated by a scanning system.

A scan taken of a substrate before any PM loading has occurred.

A division of the substrate along its length, in the data set which is generated by the TAS7000.

A division of the substrate in the radial direction, in the data set and there are 4 equal area radial sections, in each axial section, generated during data processing.

A division of the substrate in the angular direction, in the data set. There are $725^{\circ}$ angular increments, in each axial section, generated during data processing. 
Analysis Point

Quadrant

Axial Segment

Region

Analysis Area

Individual Value

Regional Value

Overall Value

Second Loading
The average of the sample points contained within one axial section, radial section, and angular increment. There are 288 analysis points in each axial section.

A collection of 18 angular increments, used to give general data trends.

A collection of 16 (ISB) or 31-32 (ISL) axial sections used to give general data trends.

A collection of axial sections, radial sections, angular increments, quadrants, or axial segments for which the PM distribution is being analyzed.

A collection of analysis points within an axial section, radial section, angular increment, quadrant, or axial segment used to calculate the PM distribution information. There are multiple analysis areas in one region.

Uniformity index value in one axial section, radial section, or angular increment and they show a high level of detail.

Uniformity index value in one axial segment, radial section, or quadrant and they show general data trends.

One uniformity index value in the axial, radial, or angular direction. These values are used in for comparisons of different test cases.

A PM loading that occurs after the initial loading phase of a test is completed. A passive oxidation or active regeneration stage is not completed in between the original and second loading 


\section{List of Abbreviations}

EPA

Environmental Protection Agency

DOC

Diesel Oxidation Catalyst

CPF

Catalyzed Particulate Filter

SCR

Selective Catalytic Reduction

PM

Particulate Matter

MTU

Michigan Technological University

1D

One Dimensional

3D

Three Dimensional

TAS7000

Advantest TAS7000 3D Imaging Analysis System-

Terahertz Wave Scanner

UDOC

Upstream DOC

DDOC

Downstream DOC

DCPF

Downstream CPF

SEM

Scanning Electron Microscope

$\mathrm{HC}$

Hydrocarbons

FID

Flame Ionization Detector

LDA

Laser Doppler Anemometry

2D

Two Dimensional

ULSD

Ultra-low Sulfur Diesel

PSD

Particle Size Distribution

SMPS

Scanning Mobility Particle Sizer

NI

National Instruments

LFE

Laminar Flow Element

xxii 


\begin{tabular}{ll} 
AMA4000 & Pierburg AMA4000 Five Gas Emissions Analyzer \\
IMR-MS & Ion Molecule Reaction Mass Spectrometer \\
$\mathrm{O}_{2}$ & Oxygen \\
$\mathrm{CO}$ & Carbon Monoxide \\
$\mathrm{CO}_{2}$ & Carbon Dioxide \\
$\mathrm{NO}_{\mathrm{X}}$ & Total Nitrogen Oxides \\
$\mathrm{NO}$ & Nitric Oxide \\
$\mathrm{THC}$ & Total Hydrocarbons \\
$\mathrm{NO}$ & Nitrogen Dioxide \\
$\mathrm{MST}$ & Manual Sampling Train \\
$\mathrm{PR}$ & Percentile Range \\
$\mathrm{UI}$ & Uniformity Index \\
$\mathrm{EOC}$ & Engine Operating Condition \\
$\mathrm{CI}$ & Confidence Interval \\
\hline
\end{tabular}




\begin{abstract}
The particulate matter distribution (PM) trends that exist in catalyzed particulate filters (CPFs) after loading, passive oxidation, active regeneration, and post loading conditions are not clearly understood. These data are required to optimize the operation of CPFs, prevent damage to the CPFs caused by non-uniform distributions, and develop accurate CPF models. To develop an understanding of PM distribution trends, multiple tests were conducted and the PM distribution was measured in three dimensions using a terahertz wave scanner. The results of this work indicate that loading, passive oxidation, active regeneration, and post loading can all cause non-uniform PM distributions. The density of the PM in the substrate after loading and the amount of PM that is oxidized during passive oxidations and active regenerations affect the uniformity of the distribution. Post loading that occurs after active regenerations result in distributions that are less uniform than post loading that occurs after passive oxidations.
\end{abstract}




\section{Chapter 1. Introduction}

The Environmental Protection Agency (EPA) emission standards for 2010 to the present require heavy duty diesel engines to use an aftertreatment system consisting of a diesel oxidation catalyst (DOC), a catalyzed particulate filter (CPF), and a selective catalytic reduction (SCR) system. The DOC, CPF, and SCR consist of ceramic substrates, which are flow through (DOC and SCR) or wall flow (CPF) and are housed within a metal can. The substrate is secured in a can by an interference fit between the can and a mat that is wrapped around the substrate. A wall flow substrate is made by plugging every other inlet and outlet channel, as shown in Figure 1.1. The plugs force the flow through the substrate wall, which will filter the particulate matter (PM) out of the exhaust. The PM is filtered initially in the substrate wall, and eventually it will form a "cake" layer on top of the wall. The cake layer and substrate wall result in a filtration efficiency in excess of $97 \%[1]$.

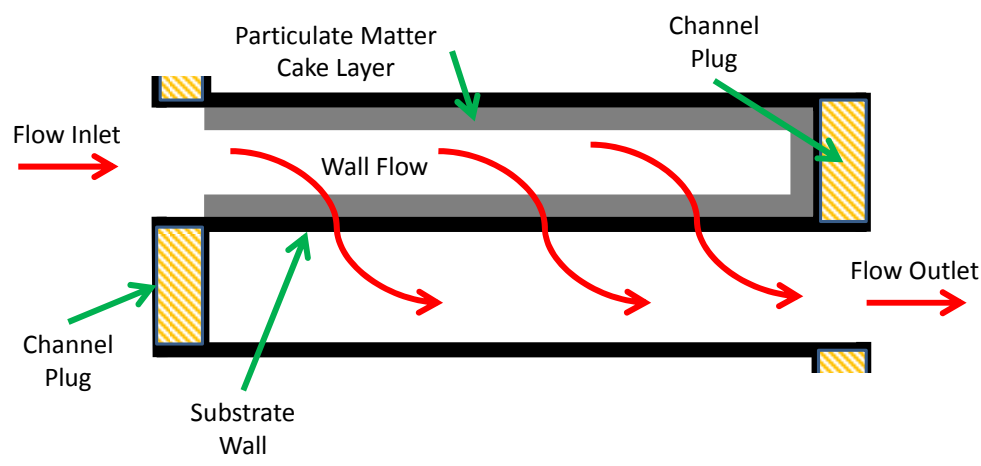

Figure 1.1: CPF Schematic

The current focus of research surrounding the aftertreatment system is on the development of a thorough understanding of the aftertreatment system performance in an effort to optimize and reduce the associated fuel consumption and emissions. Advanced experimental measurements and accurate models that can predict the inlet and outlet conditions, as well as the internal states, of each component in the aftertreatment system are employed to develop a thorough understanding of the aftertreatment system performance. The measurement of the PM distribution in a CPF is one of the advanced experimental measurements that is required to understand the $\mathrm{CPF}$ performance and develop accurate CPF models. The PM distribution needs to be measured after PM loading, passive oxidation, active regeneration, and post loading conditions. Post loading is defined as an additional PM loading stage that takes place after PM is oxidized by a passive oxidation engine condition or an active regeneration test condition.

The measurement of the PM distribution in a CPF is required for three reasons. The first reason is that knowledge of the PM distribution after PM loading, passive oxidation, active regeneration, and post loading events can lead to a better understanding of the effects of those events on the CPF performance, e.g. pressure drop and PM oxidation 
kinetics. The second reason PM distribution measurements are required is that increased knowledge on the effect of PM loading, passive oxidation, active regeneration, and post loading on the PM distribution will help to optimize the operation of CPFs. This includes understanding the maximum allowable PM loading in the substrate and the required frequency of active regenerations to prevent substrate damage caused by non-uniform PM distributions. If the PM distribution in a substrate is not uniform, excessive thermal gradients can be generated during active regenerations, causing damage to the substrate. This was shown by Zhan et al. in reference [2]. The third reason PM distribution measurements are important is to provide data that will lead to the development of CPF models that agree with experimental data.

Accurate models are calibrated and validated using experimental data. Michigan Technological University (MTU) has developed a one dimensional (1D) CPF model, which is described by Premchand, et al., in reference [1]. The developed model is capable of calculating the gaseous emission concentrations, exhaust gas temperatures, filtration efficiency of the PM cake and the substrate wall, total PM retained, PM cake layer thickness, total substrate pressure drop, and various other parameters [1]. The pressure drop, mass retained, exhaust gas temperatures, and gaseous emissions concentration are calibrated using experimental data collected in the MTU test cell. The PM distribution that is predicted by the model, however, is not calibrated and has not been compared to experimental data. This is because experimental measurements of the PM distribution in a CPF are, traditionally, difficult to obtain. The axial PM distribution from the model is a function of the wall flow velocity distribution [1]. The lack of experimental PM distribution data makes it difficult to develop CPF models that agree with experimental data.

Recently, new methods of measuring the PM distribution in a CPF have been developed. These new methods make experimental measurements easier to obtain and can characterize the three dimensional (3D) PM distribution. A research study was developed that used a terahertz wave scanner, which is one of the newly developed methods, to gain an initial understanding of PM distribution trends. The study was focused on analyzing PM distribution trends for loading, passive oxidation, active regeneration, and post loading conditions. In order to analyze the PM distribution data, new methods had to be developed that could handle the 3D data sets. This thesis presents the developed analysis methods, as well as the PM distribution trends that were determined during the study.

\subsection{Goals of Research}

The research that was conducted to investigate PM distribution trends was divided into two phases, with two distinct goals. The first phase, Phase 1, was devoted to developing the methodology that would be used to conduct PM distribution experiments using terahertz wave scanner. The second phase, Phase 2, was devoted to understanding PM distribution trends for loading, passive oxidation, active regeneration, and post loading conditions. Both of these phases had a unique set of objectives tailored for the specific purpose of the phases. 


\subsubsection{Phase 1: Experimental and Methodology Development}

The goal of Phase 1 of the PM distribution research study was to develop an understanding of the system used to measure the PM distribution, as well as to develop the analysis methods that would be required to characterize PM distribution. With that purpose in mind, three objectives were developed:

1. Develop the experimental methods and instrumentation.

2. Evaluate the capabilities of the PM distribution measurement system.

3. Develop an analysis method that can quantify PM distributions.

The experimental methods and instrumentation were based on the research previously conducted in the MTU test cell by Hutton [3], Shiel [4], and Pidgeon [5]. However, modifications had to be made to the methods and instrumentation they developed to accommodate stopping the test and measuring the PM distribution. For this research, an Advantest TAS7000 3D Imaging Analysis System, which uses terahertz waves to measure the PM distribution, was used. However, there was limited data available that discussed the capabilities of the TAS7000 system to measure the PM distribution that resulted from PM loading and oxidation conditions. As a result, the tests that were conducted as part of Phase 1 were designed to investigate PM distribution trends after operating CPFs with PM loading, passive oxidation, and active regeneration conditions. A method had to be developed that would allow PM distributions to be quantified and evaluated; the literature did not present methods for analyzing 3D data sets. During Phase 1, an analysis method was developed that would accomplish that task.

\subsubsection{Phase 2: PM Distribution Trend Analysis}

Phase 2 of PM distribution research study was developed to further explore, and collect data on, PM distribution trends using the engine conditions that were used to calibrate the MTU 1D CPF model in reference [1]. The Phase 2 work was developed with the results of Phase 1 in mind. There were five objectives that were used when developing the experimental plan for the Phase 2 work. The objectives were as follows:

1. Verify and validate the PM distribution trends that were found during Phase 1.

2. Answer questions that arose after analyzing data from Phase 1.

3. Compare the PM distributions that resulted from two different engines and aftertreatment systems.

4. Develop a better understanding of the PM distribution trends that exist for loading, passive oxidation, active regeneration, and post loading conditions.

5. Validate experimental procedures and instruments that are used to measure the PM distribution.

The PM distribution trends that were observed during Phase 1 needed to be validated and resulted in questions that needed to be investigated. Phase 1 and 2 were designed to be conducted on two different engines, with two different aftertreatment systems. As a result, the similarities and differences that may exist in the PM distribution as a result of the different engines and aftertreatment systems needed to be investigated. The first three 
objectives caused the experimental plans for Phase 1 and Phase 2 to be similar in terms of the tests that would be run and the scans that would be taken. However, the main goal of Phase 2 was the fourth objective, which was the deeper investigation into the PM distribution trends that occur in CPFs for loading, passive oxidation, active regeneration, and post loading engine conditions. The majority of tests that were conducted in Phase 2 were designed to provide data sets that would show how the PM distribution in a CPF changes for the different conditions and PM density levels. The data obtained from the tests will be compared to the PM distribution output from the MTU 1D CPF model. To allow for the experimental data and model results to be compared, the tests conducted in Phase 2 were run using similar methods and engine conditions as Hutton [3], Shiel [4], and Pidgeon [4]. The final purpose of the work conducted in Phase 2 was to evaluate the effect of shipping the substrate on the PM distribution and the repeatability of the TAS7000. Data on the effect of shipping will provide an understanding on whether shipping the substrate affects the PM distribution in the substrate. The repeatability of the data from the TAS7000 allows the functionality and accuracy of the system to be understood and accounted for when looking at data trends.

\subsection{Overview of Thesis}

This thesis discusses research that was conducted to investigate PM distribution trends in CPFs. This includes presenting relevant literature, discussing the experimental setup, explaining the analysis methodology, providing the experimental plans, and discussing the results of the experiments that were conducted. Each chapter is focused on one these topics. Additional data that were collected and procedures that were used during the PM distribution work are included in the appendices.

Chapter 2 presents a review of current literature. There are four main focuses of the literature review: PM filtration theory, PM distribution trends as found through modeling, techniques to experimentally measure the PM distribution, and PM distribution trends as

found experimentally. Background on the TAS7000 is presented as well. Chapter 3 explains the experimental setup that was used to conduct the PM distribution research. This includes the engine, aftertreatment system components, fuel, instrumentation and the TAS7000. Chapter 4 discusses the methodology used to process the scan data that were collected with the TAS7000. The calibration procedure, uniformity index development, analysis procedure, uniformity limit, and correlation coefficient are discussed in detail. Chapter 5 provides the experimental plans and procedures that were used to conduct the PM distribution study. Both Phases 1 and 2 are presented and discussed in detail. Chapter 6 presents the results from the experiments that were conducted. These results are discussed and compared to data that was found in the literature review. Chapter 7 summarizes the experimental findings. Chapter 8 provides recommendations for future research on PM distribution trends. 


\section{Chapter 2. Literature Review}

A literature review was conducted with four objectives in mind. The first objective was to understand the mechanisms that affect the deposition of PM in a particulate filter. Having background knowledge on the mechanisms that influence the PM deposition can help to explain trends in the experimental data. The second objective was to determine what current particulate filter models predict for PM distribution trends. The modeled PM distributions are based on fundamental equations and therefore provide information on what trends are expected in the PM distribution. The third objective was to develop an understanding of the methods that are currently available to measure the PM distribution. Comparing the different measurement methods allowed for decisions to be made that determined how experimental data would be collected for this study. The fourth and final objective was to gather and study PM distribution data that was experimentally measured. Although limited, this data would highlight the differences between the modeled PM distribution and the actual PM distribution.

\subsection{Particle Filtration Theory}

To discuss in detail any PM distribution trends, it becomes important to understand what drives the filtration in a particulate filter. The literature review resulted in six possible sources of particle filtration occurring in a particulate filter: 1) diffusion, 2) interception, 3) inertia, 4) gravity, 5) electrostatic forces, and 6) thermophoresis $[6,7]$. The effectiveness of each of those mechanisms is dependent on the size of the particles. Experimental data collected during this study has shown that diesel PM is typically below $1000 \mathrm{~nm}$ in size, as shown in Figure 2.1. As this figure shows, particle size distribution measurements that were taken upstream of the DOC (UDOC), downstream of the DOC (DDOC), and downstream of the CPF (DCPF). The subplot on the left shows the distribution of the particles based on number, and the subplot on the right shows the distribution of the particles based on the volume of the particles. Logarithmic scales are used for both the $\mathrm{x}$ and $\mathrm{y}$ axis. A greater explanation of the equipment and procedures used to collect the data shown in Figure 2.1 is given in Chapter 3. For particles below $1000 \mathrm{~nm}$, the two main causes of particle filtration are diffusion due to Brownian motion and direct interception with the substrate wall [6]. Diffusion has been found to be the primary cause of filtration for particles smaller than $200 \mathrm{~nm}$, while interception is the primary cause above $200 \mathrm{~nm}$, as shown in Figure 2.2 [7]. Diffusion of particles, by definition, is driven by concentration gradients. Interception of particles is related to the manner in which exhaust gases pass through the walls of a substrate, since the particles remain in the streamlines prior to being captured by the substrate wall [7]. The distribution of the flow of the exhaust gas through the substrate wall will therefore have an impact on the PM distribution in the substrate. It is important to look at the wall flow profiles to gain a better understanding of the flow effects on the PM distribution. 

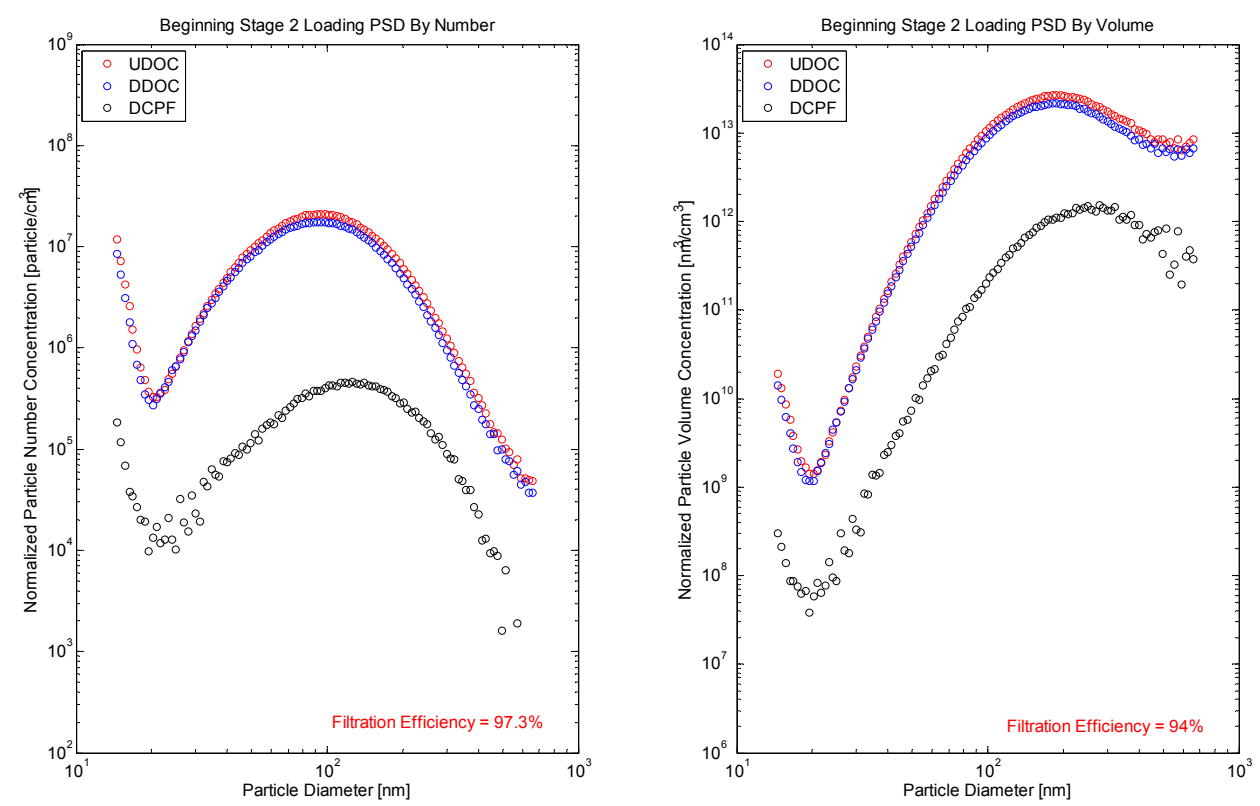

Figure 2.1: Example of Particle Sizes Taken from the Phase 2 Test 1 Loading Stage

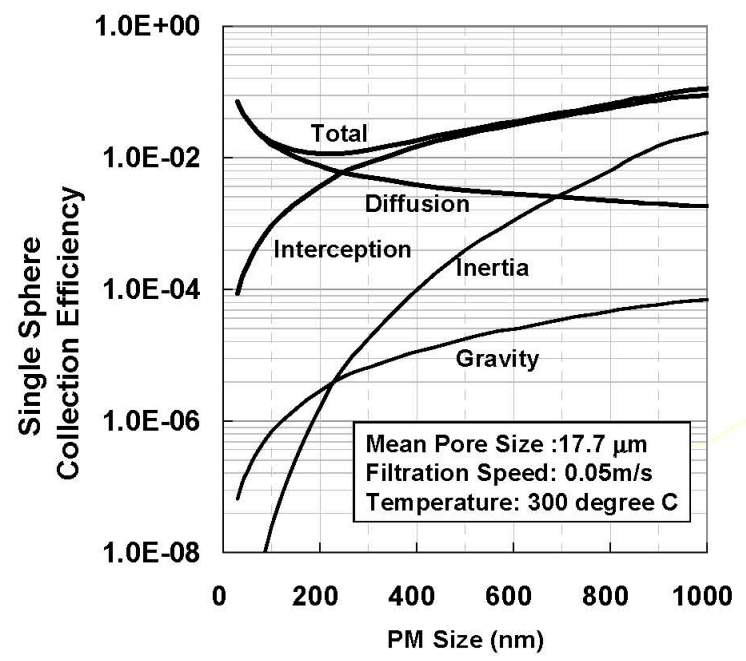

Figure 2.2: Example of Filtration Mechanism Effectiveness v. Particle Size [7]

Reprinted with permission from SAE Paper No. 2007-01-0921 C 2007 SAE International.

The axial through-wall gas flow distribution has been shown to be dependent on the pressure drop through the wall, and therefore the porosity and permeability of the substrate [8]. The PM loading in a substrate will affect the porosity, and therefore permeability, of the substrate since it will take up the open volume in the ceramic material used in the construction of substrates. This was confirmed by Opris, et al. in reference [8], with the results of their work shown in Figure 2.3. The two lines labeled $\mathrm{K}_{\mathrm{e}}, 60 \mathrm{ppm} \mathrm{Cu}$ Additive and $\mathrm{K}_{\mathrm{e}}, 0 \mathrm{ppm} \mathrm{Cu}$ additive show the overall permeability of the substrate as PM is loaded into the substrate. The overall permeability decreased by an order of magnitude as the substrate was loaded with PM. 


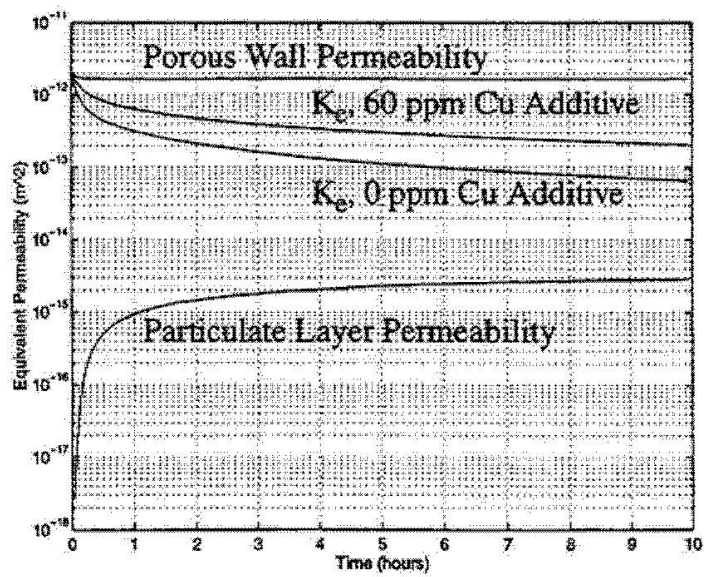

Figure 2.3: Change in Overall Permeability as a Function of Loading [8]

Reprinted with permission from SAE Paper No. 980545 C 1998 SAE International.

Liu, et al. provided an example of the predicted axial distribution in the through-wall gas flow $\left(\mathrm{U}_{\mathrm{w}}\right)$ at three different levels of permeability, as determined through modeling efforts, in reference [9] and the results are shown in Figure 2.4. As the overall permeability of the substrate decreased, the flow distribution was shown to become more uniform. The wall flow distribution is also impacted by the velocity that the exhaust gas enters the channels of the substrate [9]. This is illustrated in Figure 2.5, where the wall flow distribution for three different velocities and two different permeability levels is shown. In Figure 2.5, subplot (a) shows the distribution trends for a permeability of $1.8 \times 10^{-13} \mathrm{~m}^{2}$, and subplot (b) shows the distribution trends for $1.8 \times 10^{-11} \mathrm{~m}^{2}$. As the inlet velocity is increased, the wall flow distribution becomes less uniform. As the permeability increases, the non-uniformity in the flow distribution becomes more significant. The manner in which PM is deposited into the substrate has also been shown to change with respect to exhaust gas velocity. Konstandopoulos, et al. show in reference [10] that as the velocity of the exhaust gas increases, the porosity, and subsequently the permeability, of the deposited PM decreases. 


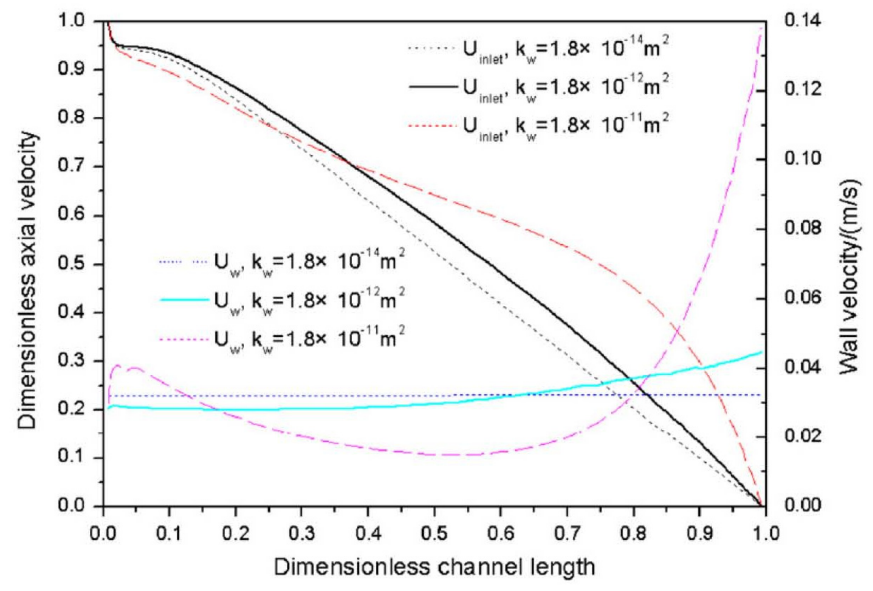

Figure 2.4: Axial Through-Wall Flow Distribution as a Function of Permeability [9]

Reprinted from Journal of Aerosol Science, 40 /4, Liu, Y., Gong, J., Fu, J., Cai, H., Long, G., "Nanoparticle motion trajectories and deposition in an inlet channel of wall-flow diesel particulate filter," Page 316, Copyright 2008, with permission from Elsevier.

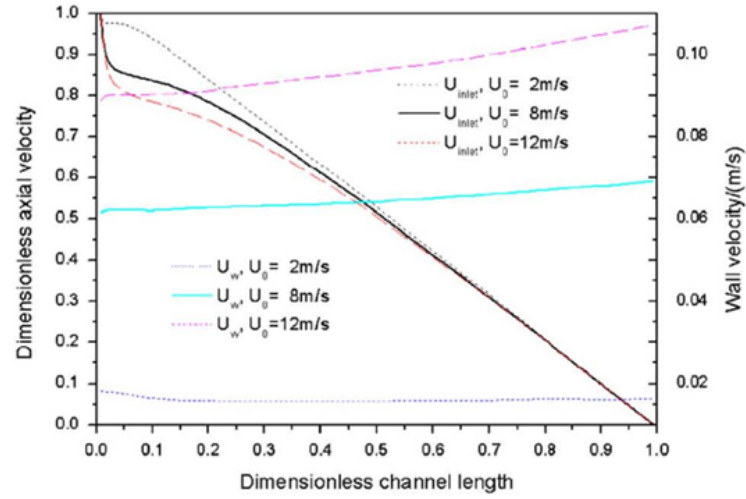

(a)

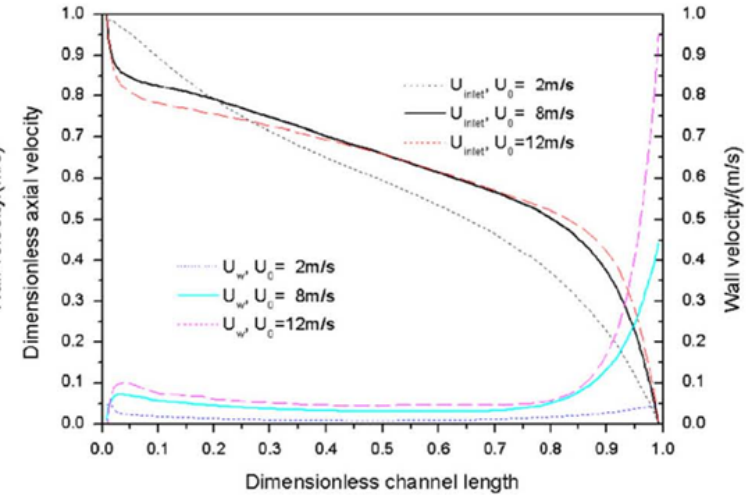

(b)

Figure 2.5: Effect of Inlet Exhaust Gas Velocity on Wall Flow Distribution [9]

Reprinted from Journal of Aerosol Science, 40 /4, Liu, Y., Gong, J., Fu, J., Cai, H., Long, G., "Nanoparticle motion trajectories and deposition in an inlet channel of wall-flow diesel particulate filter," Page 317, Copyright 2008, with permission from Elsevier.

In reference [11], Masoudi comes to the conclusion that the PM distribution should be uniform in the axial direction due to fluid dynamics and the concept of the path of least resistance. The path of least resistance is the idea that the exhaust will flow through the substrate wherever there is the least impedance to flow. Therefore, as an area becomes loaded with PM the flow will be redirected to an area with less PM, resulting in a uniform PM distribution. In general, it has been shown through modeling work that particles will follow the flow streamlines, so the axial through-wall gas flow distribution would have to change significantly for that to be true [9]. However, the lack of empirical data means that the actual PM distribution in the substrate after being exposed to various engine conditions, the effect of permeability and exhaust gas velocity on the PM distribution, 
and the changes in the substrate permeability as the substrate is loaded with PM are not clearly understood.

\subsection{Modeled PM Distribution}

In an effort to better understand how CPFs perform, many researchers have developed models that will predict the exhaust gas flow paths through the substrates. Some of these models will also predict the PM distribution in the channels of the substrates. These models are based on the fundamental equations, a large portion of which can be found in reference [8]. The models are also typically calibrated to experimental data so that the overall pressure drop, mass of PM retained, etc., agree with experimental data. The fundamentals of how PM is loaded into a substrate were discussed in section 2.1, so this section will focus on the results obtained by models that use similar fundamentals.

Liu, et al., in reference [12], discuss the flow of exhaust gas through a clean substrate, since that will impact the initial PM distribution. They developed both an analytical and numerical model, the latter of which allows for multi-dimensional analysis of the gas flow. The results of the numerical model show that a zone exists near the channel plugs where the exhaust gas recirculates, and does not follow the rest of the streamlines. These zones could be part of an explanation as to why the axial through-wall gas flow has a higher velocity near the inlet and the outlet of the channel, as is found in Figure 2.4. The authors state that a high pressure differential and flow inertia may be additional causes for the increased wall flow near the inlet and outlet of the channel, respectively [12].

In reference [13], Piscaglia, et al. developed a multidimensional model that would simulate the deposition of particles within the exhaust stream. Their worked went beyond the clean filters result presented in reference [12], and investigates how PM is deposited into the cake layer. Figure 2.6, which comes from reference [13], shows the PM distribution at the start of the PM cake layer formation for three space velocities and two values of permeability. In subplot (a) of Figure 2.6 , the permeability was $10^{-11} \mathrm{~m}^{2}$ and in subplot (b) the permeability was $10^{-12} \mathrm{~m}^{2}$. In general, the trends that are shown in Figure 2.6 follow the axial wall gas flow distribution shown in Figures 2.4 and 2.5. The developed model shows that the PM deposition profile is dependent on the velocity field at least up to the point where the PM cake is formed [13]. 

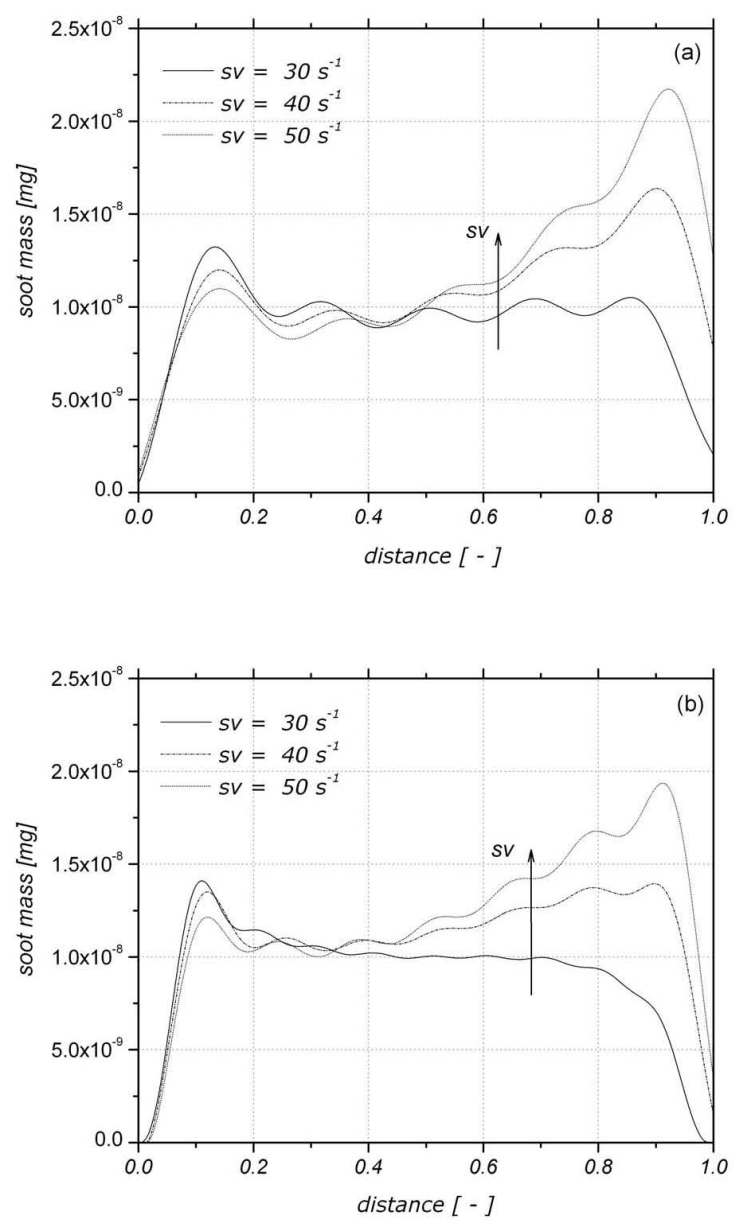

Figure 2.6 PM Distribution at the Beginning of Cake Formation [13]

Reprinted with permission from SAE Paper No. 2005-01-0963 (C) 2005 SAE International.

Yi developed a 3D model in reference [14] and presented results for a simplified representation and a full substrate complete with inlet and outlet connections. It should be mentioned that a DOC was not used in the simulations. The developed model shows the evolution of the PM distribution as a function of time. Subplot (1) in Figure 2.7 shows a clean substrate, and subplot (6) shows a PM loaded substrate. The total amount of PM retained in the substrate in subplot (6) is not known. The subplots in between (1) and (6) show the evolution of the PM distribution as a function of loading. The PM loading increases in subplots (1) - (6) in sequential order. The simulation begins with a PM distribution similar to that found in Figure 2.6, but evolves to a uniform distribution, as shown in Figure 2.7 [14]. This result is in agreement with Masoudi in reference [11] where it was stated that the final PM distribution should be uniform. 


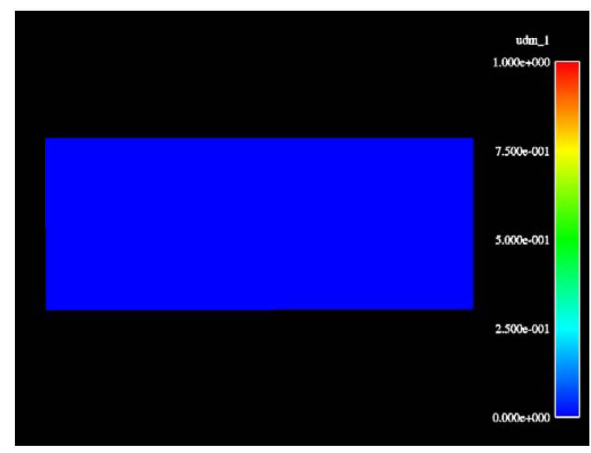

$(1)$

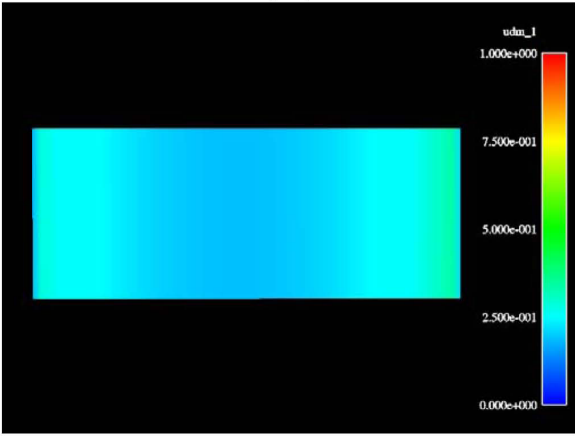

(3)

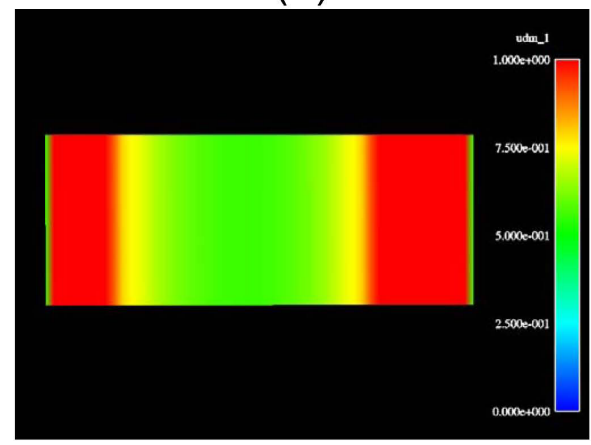

(5)

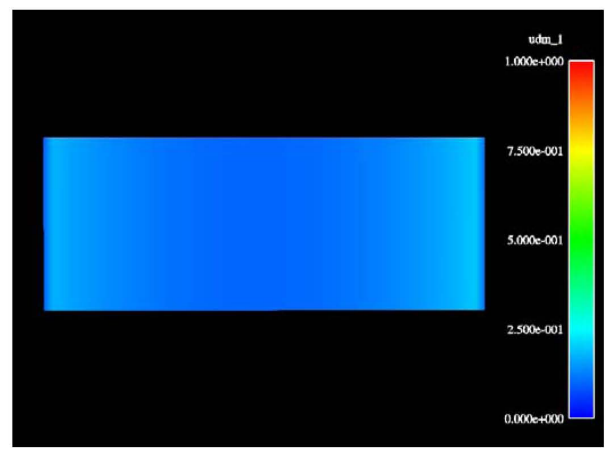

$(2)$

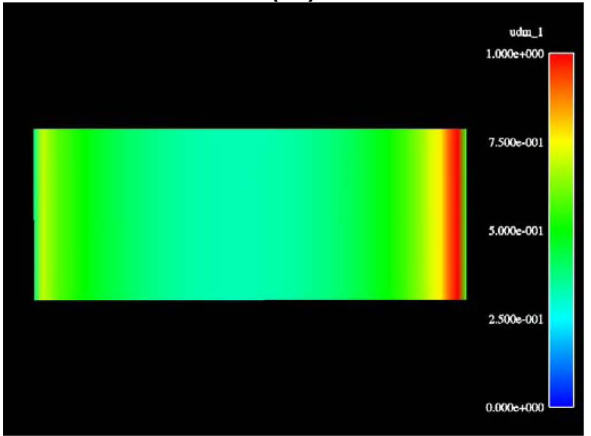

(4)

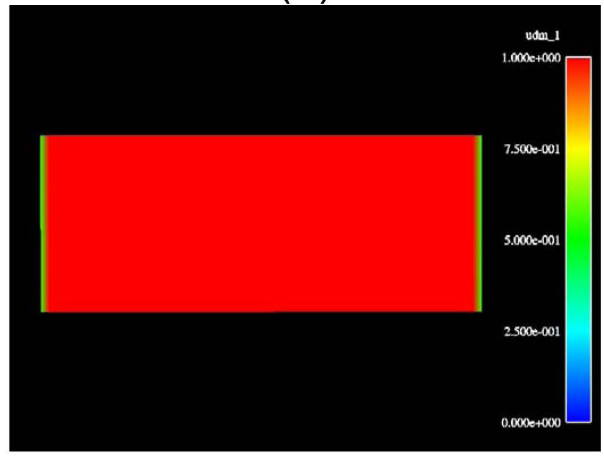

(6)

Figure 2.7: Evolution of Axial PM Loading [14]

Subplot (1) is a clean filter and subplot (6) is a loaded filter. The final PM loading is unknown, and the units used with the color bar are unknown. The results show a uniform axial distribution at the completion of loading.

Reprinted with permission from SAE Paper No. 2006-01-0264 두 2006 SAE International.

The model presented in reference [14] was also run with inlet and outlet connections attached to the substrate. The results are shown in Figure 2.8, which shows the porosity of the substrate as a function of time and a low porosity indicates PM loading [14]. Subplots (1) - (6) show the evolution of the PM distribution as a function of PM loading, with the PM loading increasing in consecutive subplots. Subplot (1) shows a clean substrate and subplot (6) shows a loaded substrate. The amount of PM retained in subplot (6) is not known. The results show that a significant non-uniformity in the radial direction develops as the loading increases. The coloring in the outer edges of the 
substrate indicate that there is little PM loading taking place. Masoudi stated that a nonuniform radial distribution is to be expected [11]. The centerline of the substrate shows a similar axial PM loading distribution as is shown in Figure 2.7. It is unknown if the PM loading in subplot (6) of Figure 2.8 is similar to the PM loading in subplot (6) of Figure 2.7 , but it is noteworthy that the axial PM distribution does not become as uniform in Figure 2.8 as it did in Figure 2.7. Figure 2.8 shows a higher PM loading near the inlet and outlet of the substrate, than in the middle.

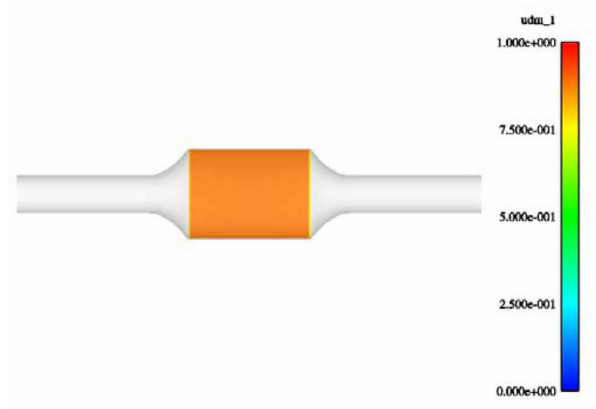

(1)

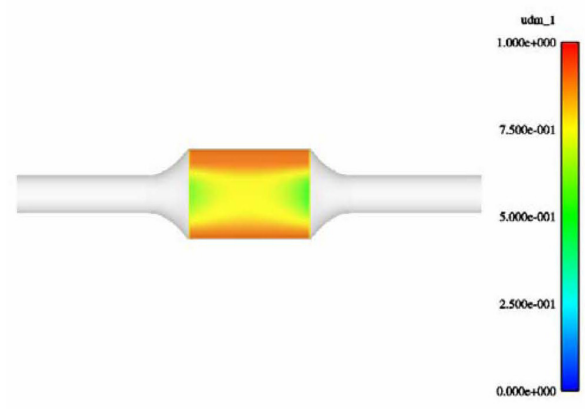

(3)

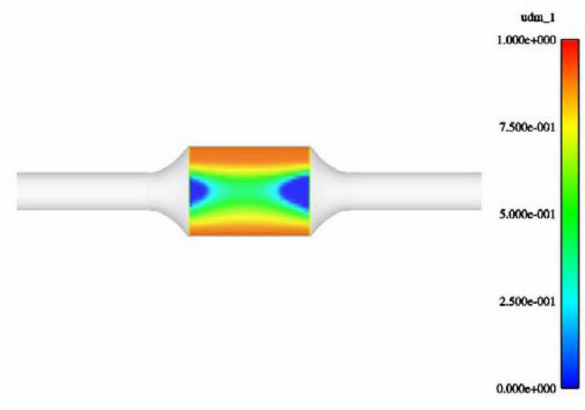

(5)

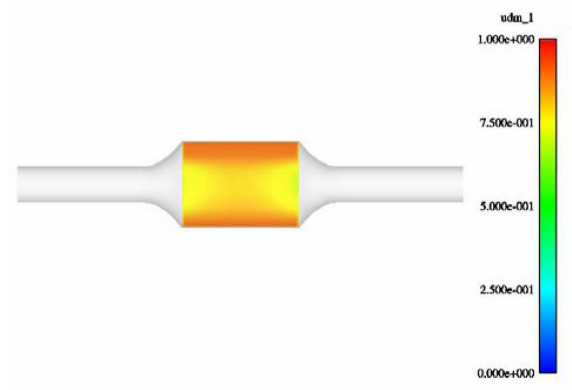

(2)

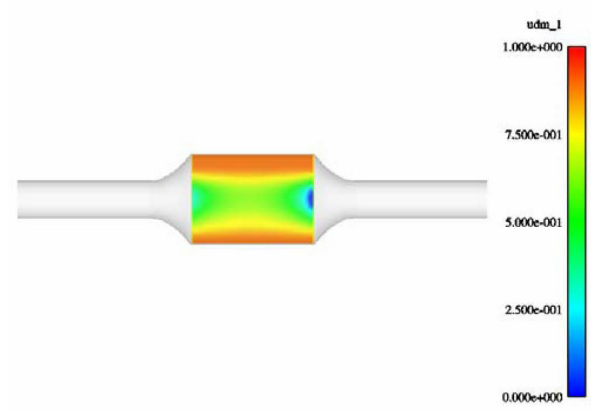

(4)

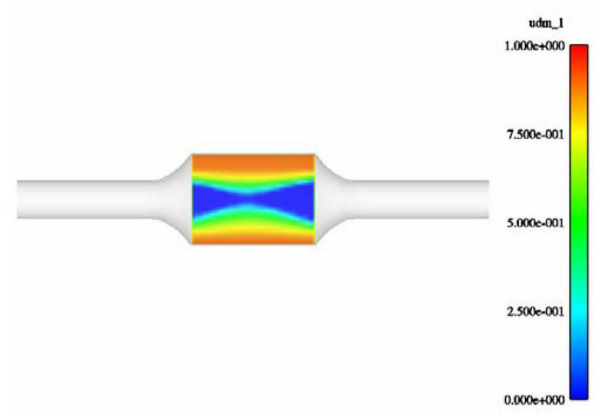

(6)

Figure 2.8: Evolution of Radial PM Loading [14]

Subplot (1) is a clean filter and Subplot (6) is a loaded filter. The plot shows the evolution of the substrate porosity as a function of time. The final PM loading is unknown, and the units used with the color bar are unknown. The results show a significant non-uniform radial distribution.

Reprinted with permission from SAE Paper No. 2006-01-0264 (C) 2006 SAE International. 
Bensaid, et al., in reference [15], developed a 3D model of a particulate filter that would predict PM loading. The results of their work are shown in Figure 2.9, which consists of plots of the axial through-wall gas flow distribution at six different PM loading levels. The PM loading levels are normalized, so they are shown as a fraction of the peak loading [15]. The authors do not provide the peak loading level. The results show that as a substrate is loaded with PM, the area near the inlet and outlet of the substrate experience the greatest amount of change in the flow rates initially. This is in agreement with the work presented previously. The minimum amount of flow is predicted near the middle of the substrate's length. The authors do show a fairly uniform flow distribution at the completion of loading.

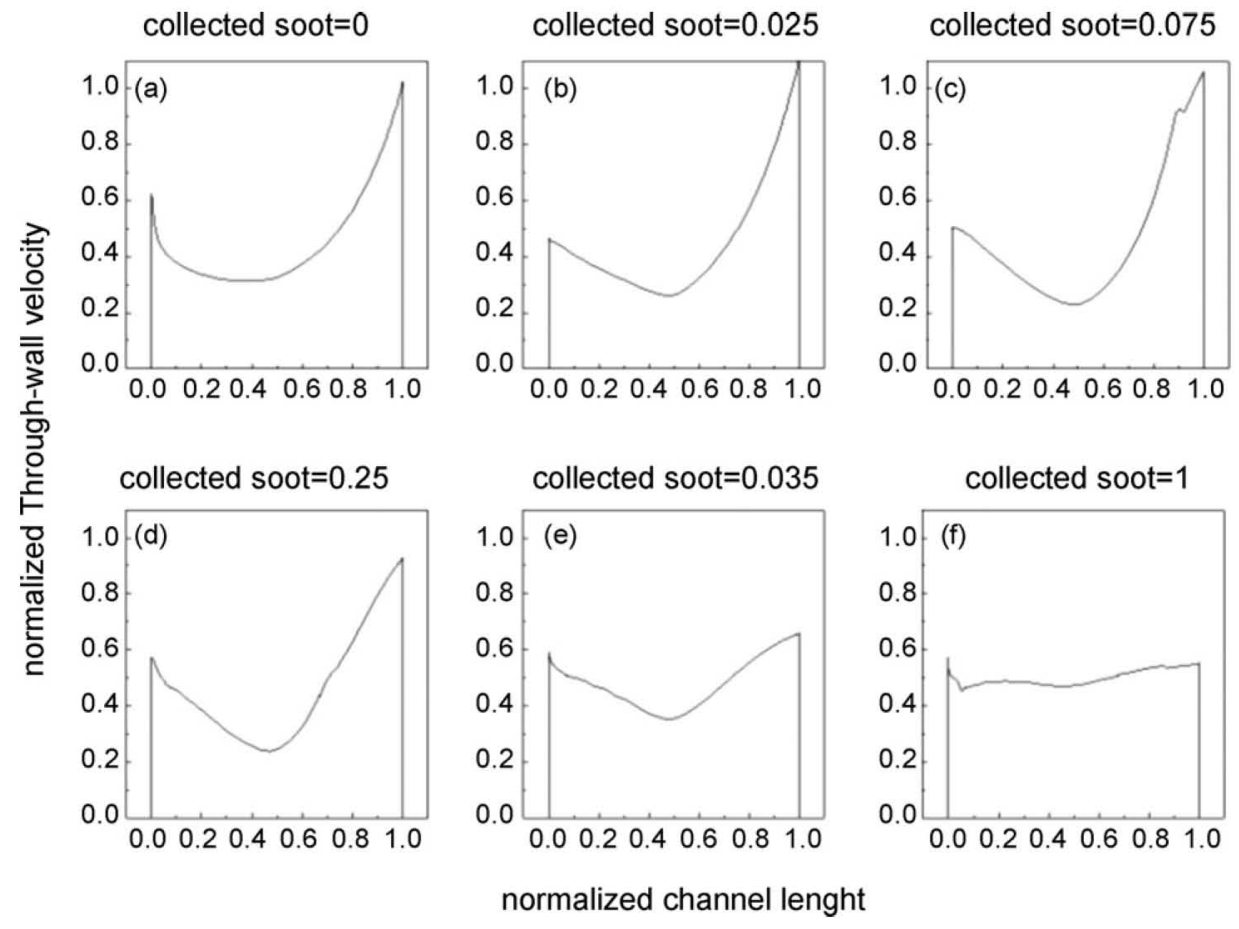

Figure 2.9: Wall Flow Distribution as a Function of PM Loading [15]

The plot shows the evolution of the normalized through wall velocity, as a function of PM loading. The final PM loading is unknown. Subplot (e) is assumed to show the collected soot fraction of 0.35 .

Reprinted from Chemical Engineering Journal, 154 /1-3, Bensaid, S., Marchisio, D., Fino, D., Saracco, G., Specchia, V., "Modelling of diesel particulate filtration in wall-flow traps," Page 216, Copyright 2009, with permission from Elsevier.

The results of the models that were presented in this section provide valuable insight into how PM is possibly distributed in a substrate. These models are accurate in the sense that the total pressure drop across the modeled substrate at a given PM loading is similar to pressure drop data that were experimentally collected [12-14]. The models discussed in this section only looked at PM loading, starting with a clean substrate. In addition to PM loading, the effect of passive oxidation and active regeneration on the PM distribution needs to be studied further. In order to discuss actual PM distribution trends, experimental measurements need to be taken after loading, passive oxidation, active 
regeneration, and post loading conditions in order to fully characterize PM distribution trends. The PM distribution from models can then be compared to the experimental measurements to evaluate their accuracy. One of the main reasons there is little experimental data is that PM distribution measurements are hard to obtain.

\subsection{PM Distribution Measurement Methods}

Taking experimental measurements of PM distribution can be complicated. Many of the measurement methods involve altering the substrate by cutting out sections of the substrate or drilling holes in the channel plugs to introduce additional constituents. Other measurement methods consist of measuring the radial flow distribution. Although these methods are typically non-intrusive, they do not capture the full 3D PM distribution characteristics. Traditionally, both intrusive and non-intrusive measurement methods provide a finite amount of data. Recently, non-intrusive multi-dimensional measurement methods have been developed that use imaging methods to determine the PM distribution. These new methods are still in the validation stage and are not in widespread use. This section will discuss the restrictive measurement methods, both intrusive and non-intrusive, that have been used as well as the multi-dimensional measurement methods.

\subsubsection{Restrictive Measurement Methods}

Measuring the thickness of the PM cake layer is one method used to determine the PM distribution in a substrate. The thickness of the PM cake layer is usually obtained by cutting out sections of the substrate and using an optical microscope [16], digital camera [17], scanning electron microscope (SEM) [15, 18, 19], or stereo microscope [20] to measure the PM cake layer in the multiple sections. Dissecting a substrate and measuring the PM cake thickness allows for measuring axial and radial PM distribution trends. There are, however, two problems associated with this method of measuring PM distribution. The first problem is that cutting the substrate could affect the PM cake thickness near the area of the cut, resulting in less accurate measurements [18]. The second problem is that repeated tests cannot be easily performed on a single substrate due to the cutting. This is important when looking at how the PM distribution changes after multiple loading and oxidation cycles. In reference [19], Pinturaud developed a method to run repeated tests on a single substrate section and take SEM images. A specialized can was designed to hold seven sections of a scaled down substrate and it could be disassembled and reassembled to allow for SEM images [19]. Additionally, by not cutting the substrate each time, there would be little disturbance in the PM cake thickness. This would be difficult, but not impossible, to perform on a full size substrate. The ability to perform successive PM distribution measurements on a single substrate is desired. This would allow for direct changes in the PM distribution between loading and passive oxidation or active regeneration conditions to be measured, as well as the PM distribution that results from multiple loading and passive oxidation or active regeneration experiments.

Another method of measuring PM distribution that involves modifying the substrate is explained by Subbu, et al. in reference [21]. The authors drill a small hole in the channel 
plug placed in the inlet face of the substrate and introduce a hydrocarbon (HC) gas. A second method of injecting $\mathrm{HC}$ gas is proposed and it involves using a small capillary inserted in the outlet channel of the substrate, which would avoid altering the substrate. However, this method was not investigated further. A sample tube, which is connected to a flame ionization detector (FID) used to measure the HC concentration, is inserted in the outlet channel where the $\mathrm{HC}$ gas is injected. Room air is passed through the substrate, and the axial through-wall flow distribution can be determined by measuring the differential dilution ratio of the known $\mathrm{HC}$ gas concentration along the length of the substrate. This method allows for both axial and radial measurements. For radial measurements, multiple HC gas injection sites will be needed. This method allows for repeated measurements if the use of the capillary to inject the $\mathrm{HC}$ gas could be verified, which would avoid altering the substrate. The authors do state that their methods have not been verified, and the effect of the sample tube on wall flow distribution is not known. [21]

Radial flow measurements, which can be correlated to the radial PM distribution, have been completed using a variety of anemometers. Laser Doppler Anemometry (LDA) was used by Ranalli et al. in reference [22]. A problem with LDA is that salt particles have to be introduced to the flow and they can cause interference with the flow through the substrate, affecting the measurement results [19, 22]. In reference [19], Pinturaud, et al. used a $9 \mathrm{~mm}$ propeller anemometer to measure the radial flow distribution in a substrate. The propeller anemometer did not disturb the flow through the substrate [19]. Stratakis, et al. used a hot wire anemometer to measure the radial flow distribution in reference [23]. Similar to the use of an anemometer, Stratakis, et al. used Pitot tubes to measure the radial flow distribution in reference [24]. All of these measurement methods provide a lot of information on the radial PM distribution, but do not provide any axial PM distribution. A second measurement would be required to fully characterize the PM distribution.

The final measurement method that will be discussed in this section is a pressurized air discharge device developed by Stratakis, et al. in reference [25]. This device is capable of measuring the radial PM distribution, similar to an anemometer. It functions by forcing a controlled amount of pressurized air from a pressure vessel through a small group of inlet channels of a substrate. It measures the decay of the pressure inside the pressure vessel, and that can be correlated to the amount of PM loading in those channels. A slower discharge rate would indicate a higher PM loading. By measuring multiple groups of channels, a radial PM distribution trend can be found. Similar to anemometer measurements, no axial PM distribution data is measured so a second measurement method would have to be used to fully understand the PM distribution in the substrate. [25]

\subsubsection{Multi-dimensional Imaging Measurement Methods}

Three multi-dimensional imaging measurement methods were found in the literature review: X-rays, dynamic neutron radiography, and terahertz waves. The effort to use these methods is fairly recent, with the earliest publication being 2009 [26]. These 
methods are, or will soon be, capable of measuring the complete 3D PM distribution. The biggest advantage of these methods is that the substrate itself does not have to be altered in any way for the measurements, allowing for repeated testing on one substrate. Additionally, the full PM distribution can be measured with just one measurement device.

In reference [26], Zandhuis, et al. demonstrate the capability of an X-ray measurement system to measure the PM distribution in a substrate. When possible, substrates were scanned prior to any loading to provide baseline data sets that could be used to separate the substrate from the PM loading. The system was able to measure PM loading at levels as low as 0.1 grams. The system was able to measure ash loading in addition to PM loading, although it can be difficult to distinguish one from the other. Ash has a slightly stronger signal than PM, but no other distinguishing characteristics according to the authors. The authors also demonstrated that the substrate would not have to be removed from the can for measurements, indicating that measurements could be performed on any substrate. Lastly, the X-ray system was able to detect that a substrate had internal damage. Although not currently available, the authors suggest that $3 \mathrm{D}$ imaging would be possible with the addition of rotating stages. The spatial resolution, or the amount of area for a two dimensional (2D) scan or the amount of volume for a 3D scan represented by one sample point, was not discussed in great detail. The spatial resolution is a function of the number of pixels used in the X-ray system. [26]

A measurement system that uses dynamic neutron radiography was presented by Harvel, et al. in reference [27]. Similar to the X-ray system, data acquired from a clean substrate is used as a baseline measurement to enable more accurate calculations of PM loading. The system discussed had the necessary equipment to produce $3 \mathrm{D}$ data sets, but the required software was not available at the time of the publication. For the system to provide accurate results, a calibration curve has to be produced for each test using the PM that was generated in that test. This is because the calibration values for PM are dependent on the amount of hydrogen, carbon, oxygen, etc. it contains, as those materials attenuate neutron beams. The spatial resolution of this system appears to be a function of the camera used to collect the data, but this was not discussed in great detail. The ability to measure PM loading separate from ash loading was not discussed. [27]

A system that uses terahertz waves to measure the PM distribution was discussed by Nishina, et al. in reference [28]. This paper discusses the Advantest TAS7000 3D Imaging Analysis System. The TAS7000 is the only imaging system of the three discussed in this section that is capable of $3 \mathrm{D}$ measurements at this time. It measures the PM distribution in the radial, $r$, angular, $\theta$, and axial directions, $z$. The authors do not provide the exact spatial resolution of the system. The TAS7000 has the ability to measure both the catalyst washcoat and PM distribution. Terahertz waves are also safer to work around since they have a lower energy level than X-rays. A baseline scan is taken of a substrate prior to any loading and the difference in the measurements between the loaded and clean substrate is used to determine the PM distribution, similar to the X-ray and dynamic neutron radiography measurement methods. [28] 
The TAS7000 is the measurement system that was used for this research, which will be discussed in Chapter 6. As such, it is important to provide more background on how the system works. The TAS7000 is able to measure the loading of the washcoat, PM, ash, or other substances due to a unique property of terahertz waves known as the spectral fingerprint. The spectral finger print is the unique motion that molecules exhibit when a terahertz waves passes through them, causing the terahertz wave to be attenuated in a very specific frequency spectrum $[28,29]$. The amount of the substance of interest that is on or in a substrate is determined by setting average attenuation of the terahertz wave equal to the average loading of a substance [28]. Terahertz waves were shown to not penetrate metal, so prior to a substrate being scanned it has to be removed from the metal can [30]. Specific parameters of the TAS7000 used in this study will be discussed in Chapter 3.

\subsection{Experimental Investigations of PM Distribution}

The measurement methods described in section 2.3 have been used to collect experimental data in a few studies. This section will present a summary of the radial and axial PM distribution trends that were found in those studies. The goal of this is to look for general trends. Each study was conducted using different substrates, flow rates, engines, and experimental setups. Some studies used soot generators to load the substrates and other studies did not have a DOC in front of the particulate filter. All of these factors could contribute to different PM distributions, and so it may not be appropriate to compare the results from two different studies.

Ranalli, et al., in reference [22], loaded a substrate at two different exhaust flow rates. The first flow rate was $60 \mathrm{~kg} / \mathrm{hr}$ and it produced a uniform radial PM distribution from 0 to $9.3 \mathrm{~g} / \mathrm{L}$. The second flow rate was $320 \mathrm{~kg} / \mathrm{hr}$ and it produced a non-uniform radial PM distribution after $5 \mathrm{~g} / \mathrm{L}$ of PM was loaded into the substrate. The results show that the periphery of the filter has a lower PM loading than the centerline. The actual difference in PM loading levels is unknown since the PM distribution is measured using LDA techniques, meaning that only the difference in flow rates is available. A result similar to this was published by Pinturaud, et al. in reference [19]. Pinturaud, et al. also showed that the radial PM distribution after an active regeneration remained uniform [19]. In reference [31], Ranalli, et al. show that optimizing the geometry of the piping going into the substrate can produce a uniform radial flow distribution at exhaust flow rates as high as $500 \mathrm{~kg} / \mathrm{hr}$. Stratakis, et al. show the ability of the DOC to redirect and even the exhaust flow prior to it entering the substrate in reference [23]. This indicates that whether or not the system is optimized, or if it has a DOC in front of the particulate filter to assist in flow evening, can have an impact on the radial PM distribution.

In reference [23], Stratakis, et al. used radial flow rate measurements to estimate the radial PM distribution as well. The results show that halfway through the loading cycle, the center of the substrate had a higher PM loading than the edges. However, at the completion of the loading cycle, the PM distribution becomes more uniform. The PM loading was done at an exhaust flow rate of $190 \mathrm{~kg} / \mathrm{hr}$, approximately halfway between 
the two flow rates that Ranalli, et al. used in reference [22], and the authors did not state the total amount of PM loaded.

The thickness of the PM cake layer was measured in both the axial and radial direction by Nagata, et al. in reference [16]. Their substrates were loaded without a DOC upstream and were loaded to an average PM density of 9.5-9.8 g/L. The results show that the inlet of the substrate, nearest the centerline, has the highest PM density, regardless of the exhaust flow rate. Additionally, near the inlet of the substrate, the periphery of the substrate has a lower loading than the centerline by $2 \mathrm{~g} / \mathrm{L}$ for a low flow rate and $3 \mathrm{~g} / \mathrm{L}$ for high flow rates. The results for the other axial and radial locations show a more uniform distribution. Only the results near the inlet of the substrate show significant nonuniformities.

In reference [17], Koltsakis, et al. measured the PM cake thickness in a single channel of a substrate. The results show that the local PM cake thickness near the inlet of the substrate is around $90 \mu \mathrm{m}, 75 \mu \mathrm{m}$ near the middle of the substrate, and $85 \mu \mathrm{m}$ near the outlet of the substrate, when the overall PM density is around $3.4 \mathrm{~g} / \mathrm{L}$. When the overall PM density is increased to $8.2 \mathrm{~g} / \mathrm{L}$, the PM cake layer thickness is between 250 and 300 $\mu \mathrm{m}$ throughout the axial length. [17]

Bensaid, et al., in reference [18], measured PM cake layer thickness in substrates using two different housing configurations. One configuration had the substrate directly behind the inlet cone, and the other housing had a straight section of pipe between the inlet cone and substrate. The straight section would allow the flow to stabilize prior to it entering the substrate. The PM distribution trends that were measured were similar for both housings. The center section of the substrate, near $50 \%$ of the axial length, had a PM cake layer thickness 16-20\% lower than the inlet and outlet PM cake thickness. The housing with the straight section of pipe produced a similar axial PM distribution and a more uniform PM distribution in the radial direction. Bensaid, et al. did collect data at lower PM loading levels as well. Those results show that the PM cake thickness near the outlet of the substrate is higher than near the inlet. The results also show that the minimum cake thickness occurred within $35 \%$ of the axial length. The different PM loading levels used are not known. It is also not known what caused the shift in the axial PM distribution trend. [18]

Pinturaud, et al. measured the PM cake layer thickness using the sectioned substrate, which was discussed in section 2.3, and an SEM [19]. For multiple substrate loading levels, the thickest PM cake was found near the outlet of the substrate, and the thinnest PM cake was found near the inlet. There was no amount of PM loading that showed a uniform PM distribution. These results are shown in Figure 2.10. This trend is different than that reported in references [16-18]. Pinturaud, et al. used this same apparatus to measure the PM cake thickness after an active regeneration was performed, with an initial PM loading of $7 \mathrm{~g} / \mathrm{L}$. The results of this test are shown in Figure 2.11. The PM cake thickness after 15,34 , and $41 \%$ of the PM was oxidized was higher than the PM cake thickness prior to the active regeneration. When $45 \%$ of the PM was oxidized, the PM cake thickness was similar to the PM cake thickness prior to the regeneration. The PM 
distribution trends after 61 and $73 \%$ of the PM was oxidized were similar to the PM distribution trends during loading. The axial PM distribution after $83 \%$ of the PM was oxidized was fairly uniform, with the cake thickness between 10 and $20 \mu \mathrm{m}$ throughout the length of the substrate. [19]

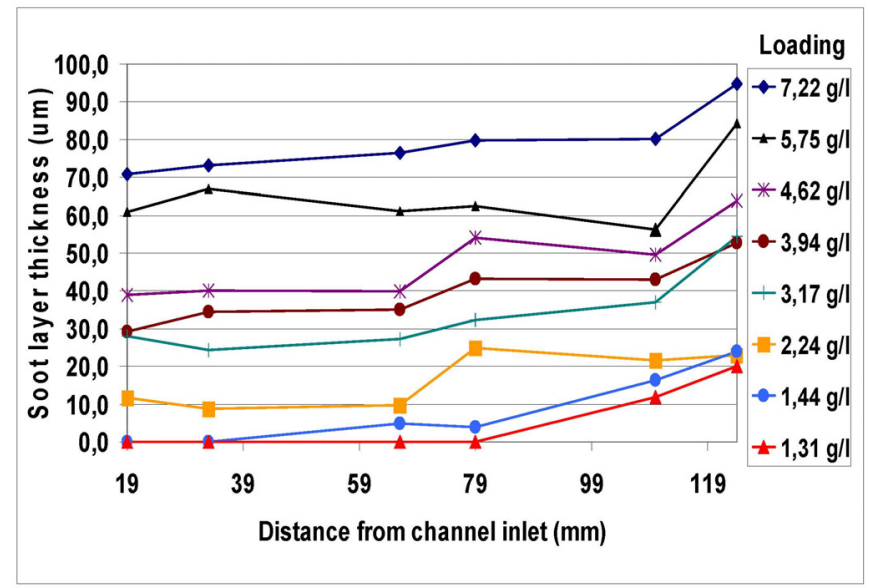

Figure 2.10: PM Cake Thickness v. PM Loading [19]

Reprinted with permission from SAE Paper No. 2007-24-0094 (c) 2007 SAE International.

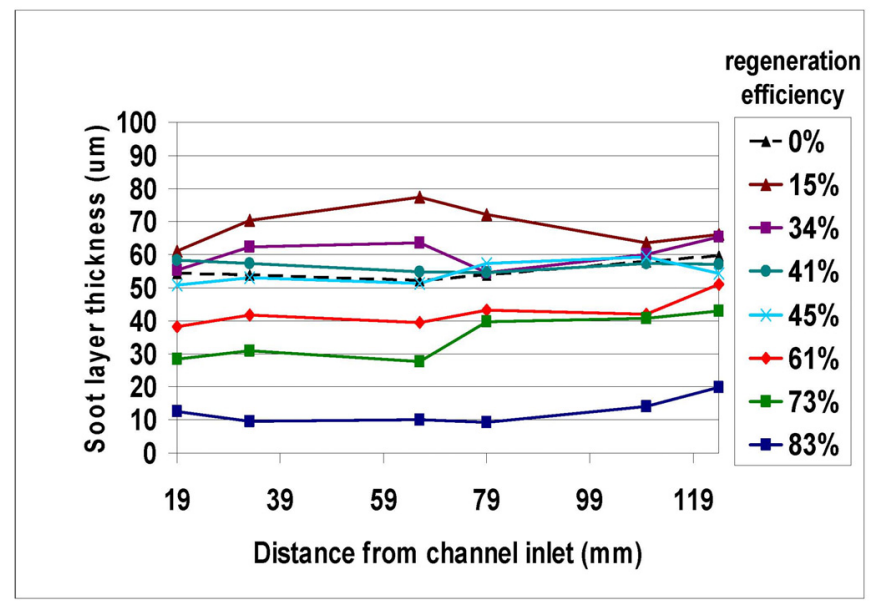

Figure 2.11: PM Cake Thickness v. PM Oxidation [19]

Reprinted with permission from SAE Paper No. 2007-24-0094 (C) 2007 SAE International.

In reference [32], Harvel, et al. loaded a full size substrate to approximately $3 \mathrm{~g} / \mathrm{L}$ and used a dynamic neutron radiography system to analyze the axial PM distribution. The results show a PM loading that increased from the inlet to the outlet of the substrate, with the region near the outlet having the highest PM loading. These results are in agreement with the results shown in reference [19]. These results are also in agreement with the models that were presented in section 2.2. 


\subsection{Summary}

This section provided some of the theoretical background on PM filtration in filters. The primary filtration mechanisms are diffusion and interception of the particles. The interception mechanism is a part of the through-wall gas flow, and therefore the PM distribution is a function of the wall flow distribution. Two factors that can impact the wall flow distribution were discussed, which were permeability and inlet velocity. Models were then investigated that were based on similar fundamental equations. Three models provided predicted PM distributions, based on multi-dimensional substrate models. The models show a non-uniform PM distribution at the start of the filtration process, but two of the models predict the PM distribution will be uniform eventually, although the PM density at that time is not known. An overview of the methods that have been used to measure PM distributions was presented. Three new measurement methods were discussed that are non-intrusive and can provide 3D data. Finally, experimental results were presented. The experimental measurements show that the radial PM distribution is a function of the PM loading level and the exhaust flow rate. The radial distribution was found to be slightly non-uniform, with the periphery of the filter having a lower loading than the centerline. Researchers were able to achieve a uniform radial distribution by straightening the incoming flow. There were four distinct axial PM distribution trends that were found in the experiments:

- Some of the results showed a uniform PM distribution at certain PM loading levels, while other results did not.

- One researcher found that the PM loading was the highest near the inlet of the substrate.

- The axial PM distribution found from two different researchers showed that the area near the inlet and outlet of the substrate would have a higher PM loading than the area near the middle of the axial length.

- Two other researchers had results that showed the PM loading increased from the inlet to the outlet of the substrate.

The amount of variation that exists in the collected data makes it difficult to draw conclusions and make comparisons to models. More data that is collected using consistent methods is required to begin to understand PM distribution trends. The work that is presented within this thesis will show PM distribution trends for loading, passive oxidation, active regeneration, and post loading experimental conditions. This work is designed to develop the ability to measure 3D PM distributions and begin to explain fundamental PM distribution trends found for various test conditions. The next chapters will discuss, in detail, the experimental work that was conducted. 


\section{Chapter 3. Experimental Setup ${ }^{1}$}

This chapter describes the experimental setup that was used for the PM distribution trend research, which will be discussed in Chapter 6. This chapter serves to provide information on the manufacturer of the equipment and the specifications of the equipment. The procedures that accompany some of the equipment listed here are provided in the appendices. The engines and aftertreatment systems used, along with the terahertz wave scanner, are of particular interest to this research. Two different engines and CPFs were used for this research, a 2010 Cummins ISB with a CPF that had a 229 $\mathrm{mm}$ diameter and was $280 \mathrm{~mm}$ long and a 2007 Cummins ISL with a CPF that had a 267 $\mathrm{mm}$ diameter and was $305 \mathrm{~mm}$ long. Both CPFs were 2010 models, so the catalyst washcoat used is similar. The same terahertz wave scanner was used for both substrates, with hardware and software changes made to accommodate the different sizes.

\subsection{Engine and Dynamometer}

Two engines were used in this research. As a result two different lab setups had to be used. The engine that was used for the Phase 1 research was a 2010 Cummins ISB rated at $224 \mathrm{~kW}$. The engine that was used for the Phase 2 research was a 2007 Cummins ISL rated at $272 \mathrm{~kW}$. The specifications for both engines are given in Table 3.1. Cummins provided proprietary software that could be used to control various parameters of the engines as well as enable them for lab use. The throttle command for the engine was controlled using a potentiometer or a command in the software.

Table 3.1: Engine Specifications

\begin{tabular}{|c|c|c|}
\hline Model & $\begin{array}{c}\text { Cummins ISB 224 kW } \\
(300 \mathrm{HP})\end{array}$ & $\begin{array}{c}\text { Cummins ISL 272 kW } \\
(365 \mathrm{HP})\end{array}$ \\
\hline $\begin{array}{c}\text { Year of } \\
\text { Manufacture }\end{array}$ & 2010 & 2007 \\
\hline Cylinders & Inline 6 & Inline 6 \\
\hline Bore \& Stroke & $107 \times 124 \mathrm{~mm}$ & $114 \times 144.5$ \\
\hline Displacement & $6.7 \mathrm{~L}$ & $8.9 \mathrm{~L}$ \\
\hline Aspiration & Turbocharged & Turbocharged \\
\hline Aftercooling & $\begin{array}{c}\text { Cummins Charge Air } \\
\text { Cooler }\end{array}$ & $\begin{array}{c}\text { Cummins Charge Air } \\
\text { Cooler }\end{array}$ \\
\hline Turbocharger & $\begin{array}{c}\text { Holset Variable } \\
\text { Geometry Turbine }\end{array}$ & $\begin{array}{c}\text { Holset Variable } \\
\text { Geometry Turbine }\end{array}$ \\
\hline Rated Power & $224 \mathrm{~kW} @$ 2600 RPM & 272 kW @ 2100 RPM \\
\hline Peak Torque & 896 Nm @ 1600 RPM & 1695 @ 1400 RPM \\
\hline EGR system & $\begin{array}{c}\text { Electronically Controlled } \\
\text { and Cooled }\end{array}$ & $\begin{array}{c}\text { Electronically Controlled } \\
\text { and Cooled }\end{array}$ \\
\hline
\end{tabular}

${ }^{1}$ Parts of the material contained in this chapter have been submitted, or are being considered for submission, to SAE International for publication consideration. 
A wet gap Eddy current dynamometer was used to control the speed and load of the engine. The dynamometer was manufactured by Dynamatic and had a peak power rating of $373 \mathrm{~kW}$. The entire specifications for the dynamometer are given in Table 3.2. The dynamometer was controlled using a Digalog 1022A controller.

Table 3.2: Dynamometer Specifications

\begin{tabular}{|c|c|}
\hline Manufacturer & Dynamatic \\
\hline Model Number & AD8121 \\
\hline Max Power $(\mathrm{kW})$ & $373 @ 1750-7000$ RPM \\
\hline Max Torque $(\mathrm{Nm})$ & $2035 @ 1750$ RPM \\
\hline Inertia $\left(\mathrm{kg}-\mathrm{m}^{2}\right)$ & 1.56 \\
\hline Construction & Wet Gap \\
\hline
\end{tabular}

The two different engines that were used in this study resulted in two different lab setups. The lab setup for the Cummins ISB is given in Figure 3.1, and the lab setup for the Cummins ISL is given in Figure 3.2. Both figures also show two exhaust paths that are controlled through the use of pneumatic valves. The "Trapline" exhaust path consists of the aftertreatment system and is the path that is used when tests are taking place. The "Baseline" exhaust path bypasses the aftertreatment system and allows a test to be started and stopped at specific points in time. When a test needs to be started, the valve for the "Trapline" is opened and the "Baseline" is closed. When a test needs to be stopped, the "Baseline" is opened and the "Trapline" is closed. The "Baseline" also allows the engine to be warmed up without changing the PM loading in the substrate. The exhaust system has a vacuum of approximately $0.5 \mathrm{kPa}$ applied during testing. 


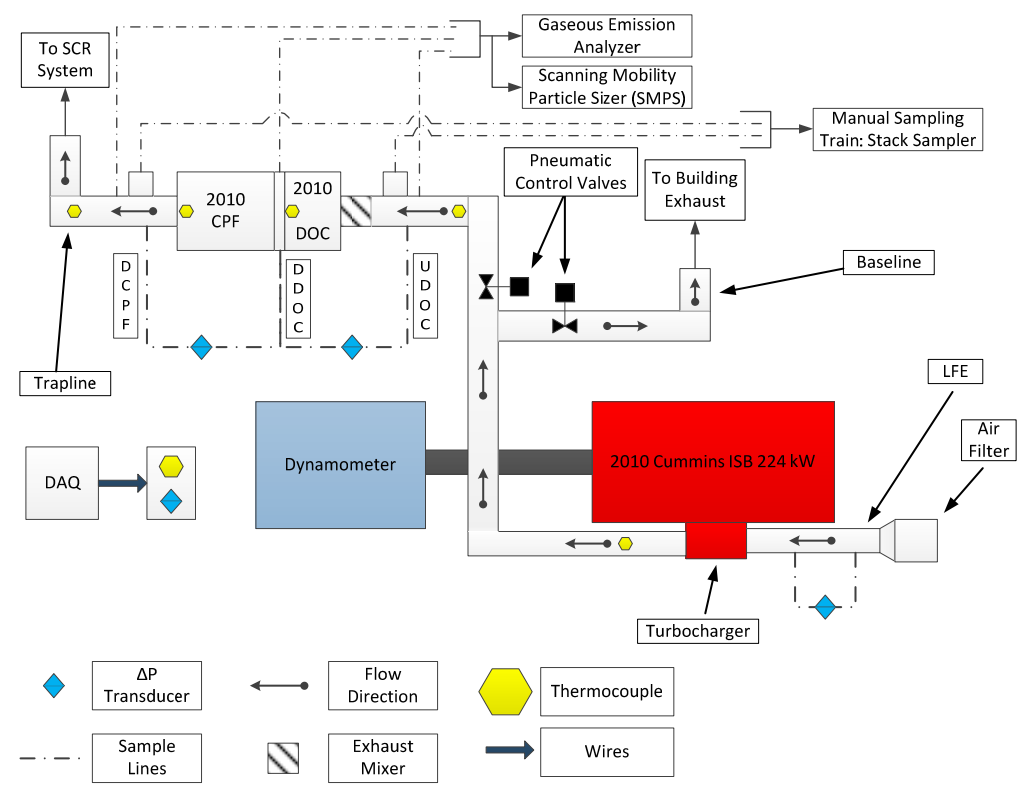

Figure 3.1: Cummins ISB Lab Setup

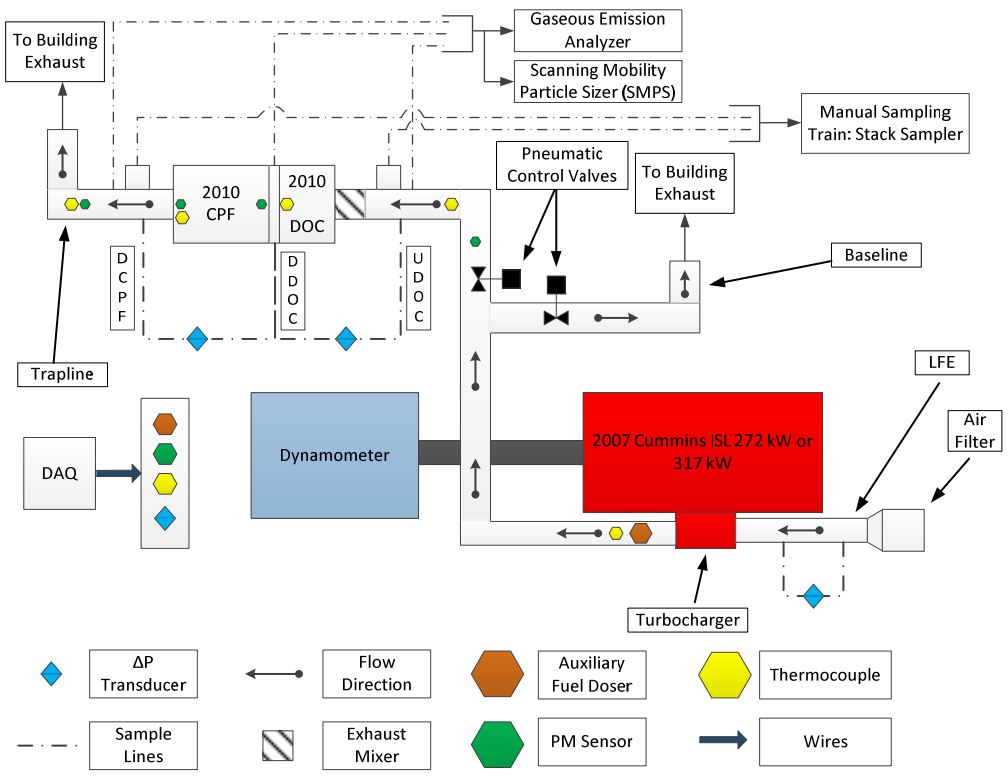

Figure 3.2: Cummins ISL Lab Setup

\subsection{Fuel Properties}

All of the tests that were conducted on the ISL and ISB engines used ultra-low sulfur diesel fuel (ULSD) as the fuel. Table 3.3 summarizes the properties for the USLD used. All metal content in the fuel was found to be less than $1 \mathrm{ppm}$. The fuel testing was completed by Cummins. 
Table 3.3: ULSD Properties

\begin{tabular}{|c|c|}
\hline Higher Heating Value $(\mathrm{MJ} / \mathrm{kg})$ & 45.68 \\
\hline Lower Heating Value $(\mathrm{MJ} / \mathrm{kg})$ & 42.89 \\
\hline Cetane Index & 46.9 \\
\hline Viscosity @ $40{ }^{\circ} \mathrm{C}(\mathrm{cSt})$ & 3.009 \\
\hline API (Gravity) & 35.2 \\
\hline Specific Gravity & 0.85 \\
\hline Sulfur (ppm) & 6 \\
\hline Water $(\mathrm{ppm})$ & 57 \\
\hline Initial Boiling Point $\left({ }^{\circ} \mathrm{C}\right)$ & 181 \\
\hline Final Boiling Point $\left({ }^{\circ} \mathrm{C}\right)$ & 359 \\
\hline
\end{tabular}

\subsection{Aftertreatment System}

The Cummins ISL and ISB engines used two different sized aftertreatment systems. Both systems consisted of 2010 components for this study. The specifications for the aftertreatment system for both engines are provided in Table 3.4. The 2010 ISB engine does have an SCR system after the CPF, but those specifications are not provided here since it is not the focus of this work. The dimensions provided for the diameter and length of the substrates are nominal values, with the actual values varying within $\pm 0.5 \mathrm{~mm}$. Two different CPF substrates were used with the ISB engine and four CPF substrates were used with the ISL engine.

Table 3.4: Aftertreatment Specifications

\begin{tabular}{|c|c|c|c|c|}
\cline { 2 - 5 } \multicolumn{1}{c|}{} & \multicolumn{2}{c|}{ ISB } & \multicolumn{2}{c|}{ ISL } \\
\cline { 2 - 5 } \multicolumn{1}{c|}{} & DOC & CPF & DOC & CPF \\
\hline Model Year & 2010 & 2010 & 2010 & 2010 \\
\hline Material & Cordierite & Cordierite & Cordierite & Cordierite \\
\hline Diameter $(\mathrm{mm})$ & 229 & 229 & 267 & 267 \\
\hline Length $(\mathrm{mm})$ & 102 & 280 & 102 & 305 \\
\hline Cell Geometry & Square & Square & Square & Square \\
\hline Total Volume $(\mathrm{L})$ & 4.2 & 11.5 & 5.7 & 17.0 \\
\hline Cell Density $\left(\mathrm{cells} / \mathrm{mm}^{2}\right)$ & 0.62 & 0.31 & 0.62 & 0.31 \\
\hline Cell Width $(\mathrm{mm})$ & 1.17 & 1.49 & 1.17 & 1.49 \\
\hline Filtration Area $\left(\mathrm{m}^{2}\right)$ & N/A & 10.62 & N/A & 15.72 \\
\hline Open Frontal Area $\left(\mathrm{mm}^{2}\right)$ & 34747 & 14151 & 47520 & 19243 \\
\hline Channel Wall Thickness $(\mathrm{mm})$ & 0.10 & 0.30 & 0.11 & 0.30 \\
\hline Wall density $\left(\mathrm{g} / \mathrm{cm}^{3}\right)$ & 1.2 & 1.2 & 1.2 & 1.2 \\
\hline Porosity & $35 \%$ & $52 \%$ & $35 \%$ & $52 \%$ \\
\hline Mean Pore Size $(\mu \mathrm{m})$ & N/A & 15 & N/A & 13 \\
\hline Number of inlet cells & 25453 & 6363 & 34714 & 8653 \\
\hline
\end{tabular}


In section 2.3 it was mentioned that the terahertz waves would not pass through the can that contains the substrate. Due to this, removable cans were designed that opened like a clamshell. The designed can had a flange along one side that used seven bolts to hold it closed. Inside of the can, a sleeve of sheet metal was used as the sealing element against the matting that is designed to hold and seal the substrate inside the can. The cans that were used for the ISB and ISL substrates are shown in Figures 3.3 and 3.4, respectively. Figures 3.3a and 3.4a show the can in the open position, and Figures $3.3 \mathrm{~b}$ and $3.4 \mathrm{~b}$ show the can as it is during testing. The cans were manufactured by Cole Technologies in Columbus, IN. Both the ISB and ISL aftertreatment systems were designed to allow for emissions, temperature, and pressure drop measurements. The pressure drop measurements were taken across the DOC and CPF. The filtration efficiencies of the $\mathrm{CPFs}$ canned in the removable cans and the production components were compared to verify that the removable cans would produce similar results. The procedure used to can the $\mathrm{CPF}$ is given in Appendix A.
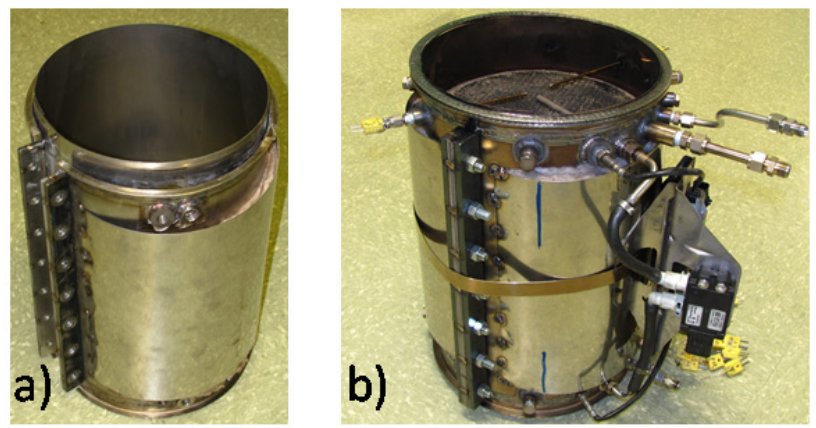

Figure 3.3: Removable Can for the ISB Substrates
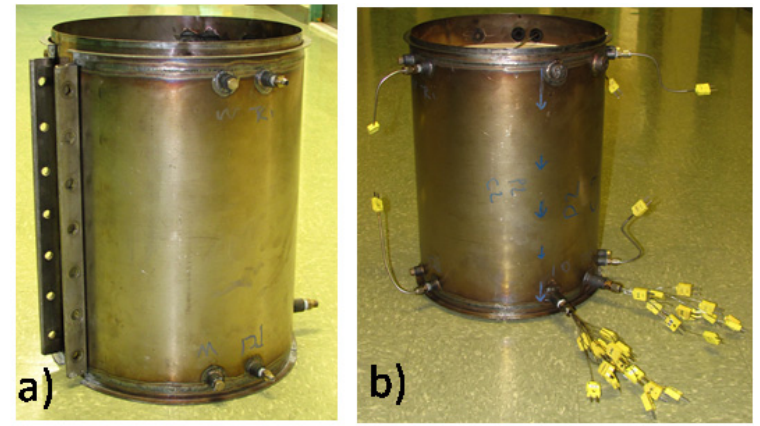

Figure 3.4: Removable Can for the ISL Substrates

\subsection{Test Cell Instrumentation}

This section describes in detail the equipment and instruments used to take experimental measurements. The measurements include engine intake air flow rate and properties, fuel flow rate, pressures, temperatures, gaseous emissions, PM concentration, PM retained, and PM particle size distribution (PSD). Figures 3.1 and 3.2 show the location and use of a majority of the instrumentation. The data that is collected with these instruments is 
used to ensure the repeatability of test conditions, perform data analysis on the tests conducted, and assists in the development and calibration of models.

\subsubsection{Data Acquisition System}

Data acquisition was accomplished with a variety of programs. The proprietary Cummins software had a data logging feature, which was used to record various engine parameters. The emissions measurement instruments, scanning mobility particle sizer (SMPS) instruments, and a few of the additional sensors used in the lab had their own software which was used for data collection. The majority of the data, however, was collected using a LabVIEW program that was specifically designed for the MTU test cell. The LabVIEW program collected data from two National Instruments (NI) cDAQ-9178 chassis, one NI USB CAN-8473, and a specially built RS-485 driver. The two NI chassis used 10 modules for the inputs and output. The details of the 10 modules are listed in Table 3.5.

Table 3.5: NI DAQ Modules

\begin{tabular}{|c|c|c|c|}
\hline Module & No. of Channels & Quantity & Measurement \\
\hline NI 9263 & 4 & 1 & Analog Output \\
\hline NI 9237 & 4 & 1 & Wheatstone Bridge Analog Voltage Input \\
\hline NI 9239 & 4 & 2 & Analog Input Module \\
\hline NI 9472 & 8 & 1 & 24V Logic, Digital Output \\
\hline NI 9213 & 16 & 4 & T/C Module \\
\hline NI 9401 & 8 & 1 & TTL Digital Input/Output Module \\
\hline
\end{tabular}

\subsubsection{Measurement of Engine Intake Air}

The flow rate of air into the engines was measured using a Meriam Instruments laminar flow element (LFE), model number 50MC2-06F. The flow rate through the LFE is calculated based on the pressure drop that occurs through a laminar section of the instrument. The specifications for the pressure sensor used for this measurement will be presented in section 3.4.4. The accuracy of the calibration is $0.72-0.86 \%$ of the reading and the repeatability is $0.10 \%$. The LFE is equipped with temperature, relative humidity, and barometric pressure sensors. The temperature and relative humidity of the air is measured using an Omega Engineering $\mathrm{HX} 94 \mathrm{~V}$. The range of the temperature measurement is $0-100{ }^{\circ} \mathrm{C}$, with an accuracy of $\pm 0.6 \%$ and a repeatability of $\pm 0.3{ }^{\circ} \mathrm{C}$. The range of the relative humidity measurement is $3-95 \%$, with an accuracy of $\pm 2 \%$ and a repeatability of $\pm 1 \%$. More information on the barometric pressure sensor will be presented in section 3.4.4, with the rest of the pressure sensors.

\subsubsection{Fuel Flow Rate Measurements}

Fuel flow rate into the engine was measured with two different systems. The fuel flow rate was measured using an AVL $703 \mathrm{G}$ fuel balance system for all the tests conducted on the ISB engine and one of the tests conducted on the ISL engine. After the first test on the ISL engine was complete, the fuel system was updated and a Coriolis fuel system was installed. The system installed was a Micro-Motion Coriolis flow meter and transmitter 
with part numbers CMFS015M319N2BAECZZ and 2700R12ABAEZCZ, respectively. The transmitter was set to output the measurements, using RS-485 communication, to the developed LabVIEW program. The maximum range on the accuracy was stated to be $\pm 1.667 \%$ of the flow rate, but that decreases to $0.10 \%$ for flow rates above $0.17 \mathrm{~kg} / \mathrm{min}$. The ISL engine typically used more than $0.17 \mathrm{~kg} / \mathrm{min}$ of fuel for the engine conditions tested. Additionally, the Coriolis system measures the density of the fuel within \pm 0.02 $\mathrm{kg} / \mathrm{m}^{3}$. The Coriolis fuel measurement system had to be developed from a variety of components, all of which are shown in the schematic in Figure 3.5. The components were selected based on conversations with Rob Kost of CNH America, LLC. who had recently developed a similar system [33].

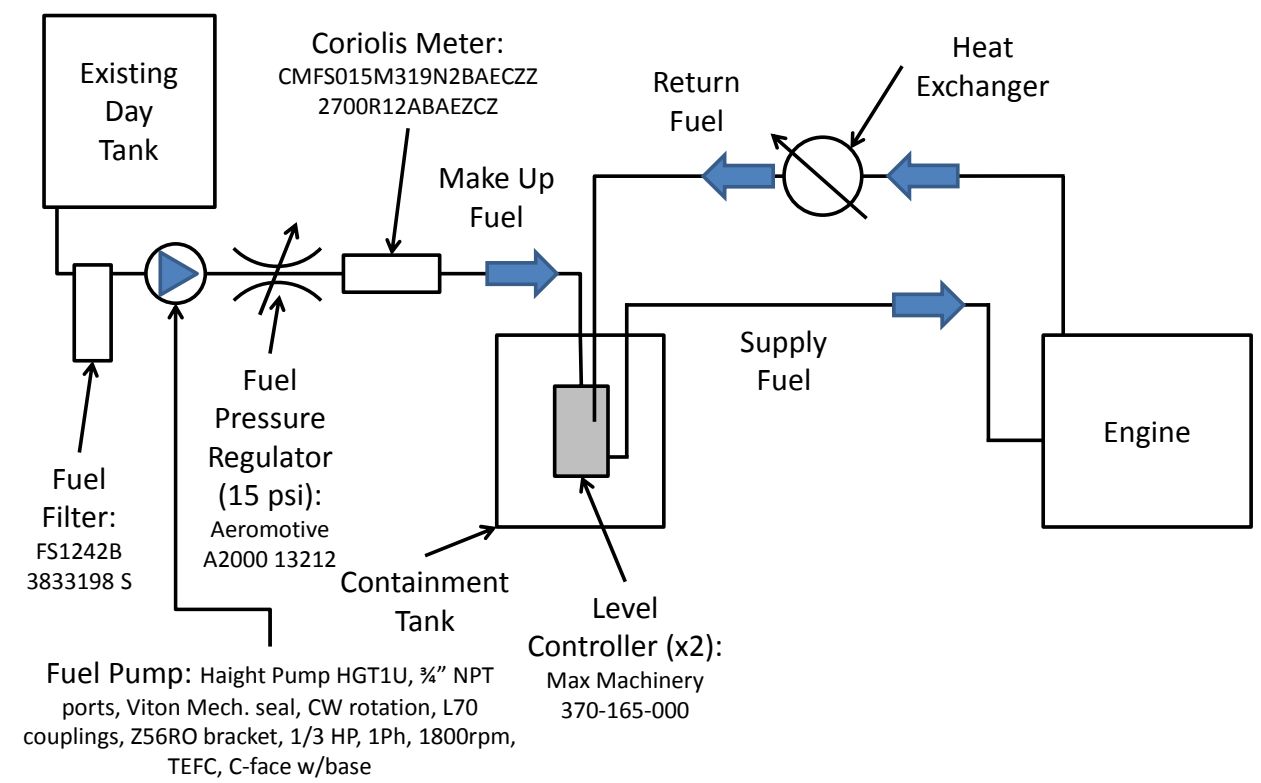

Figure 3.5: Coriolis Fuel System

Based on work discussed in [33].

\subsubsection{Pressure Transducers}

Five pressure sensors were used in this research. The pressure sensors were used to measure the barometric pressure, the pressure drop across the LFE, the pressure drop across the DOC, the pressure drop across the CPF, and the pressure drop across the SCR. The barometric pressure sensor measures the absolute pressure in the test cell during tests, which is used in the air flow rate calculations. The $\triangle \mathrm{P}$ LFE sensor is used to measure the flow rate of air through the LFE. Its value is the main parameter in the air flow rate calculation. The $\triangle \mathrm{P} \mathrm{DOC}$ sensor is mostly used as an indicator of a phenomenon known as face plugging. If the face of the DOC is plugged during testing with HCs and PM, the pressure drop across the DOC will increase, indicating that there is a problem. The $\triangle \mathrm{P} C P F$ is used for two purposes. During testing, the $\triangle \mathrm{P} C P F$ provides an indication of the loading in the CPF. Loading a CPF to a similar $\triangle \mathrm{P}$ provides repeatable mass retained measurements between tests. The $\triangle \mathrm{P} C \mathrm{CPF}$ is also used in the calibration of the MTU 1D CPF model. Although it was not used in this study, the 
pressure drop across the SCR, as measured by the $\triangle \mathrm{P}$ SCR sensor, could be used to calculate the absolute pressure in the exhaust system for the ISB engine, so it will be included in this discussion. The specifications of the pressure sensors used are given in Table 3.6.

Table 3.6: Pressure Sensor Specifications

\begin{tabular}{|c|c|c|c|c|c|}
\hline & $\begin{array}{c}\text { Barometric } \\
\text { Pressure }\end{array}$ & $\triangle \mathrm{P}$ LFE & $\triangle \mathrm{P} D O C$ & $\triangle \mathrm{P} C \mathrm{CPF}$ & $\triangle \mathrm{P}$ SCR \\
\hline $\begin{array}{l}\text { Sensor } \\
\text { Brand }\end{array}$ & $\begin{array}{c}\text { Omega } \\
\text { Engineering }\end{array}$ & Sensotec & Sensotec & Sensotec & $\begin{array}{c}\text { Omega } \\
\text { Engineering }\end{array}$ \\
\hline $\begin{array}{c}\text { Model } \\
\text { Number }\end{array}$ & $\begin{array}{l}\text { PX419- } \\
26 B 5 V\end{array}$ & $\begin{array}{c}\text { 060-0882- } \\
\text { 28ZD }\end{array}$ & $\begin{array}{c}\text { 060-0882- } \\
07 \mathrm{ZD}\end{array}$ & $\begin{array}{c}060-0882- \\
15 Z D\end{array}$ & $\begin{array}{c}\text { PX409C- } \\
2.5 \mathrm{DWU} 5 \mathrm{~V}\end{array}$ \\
\hline Sensor Type & Absolute & Differential & Differential & Differential & Differential \\
\hline Range & $26.00-32.00$ & $0.00-3.44$ & $0.00-13.79$ & $0.00-68.95$ & $0.00-17.24$ \\
\hline Units & in. $\mathrm{Hg}$ & $\mathrm{kPa}$ & $\mathrm{kPa}$ & $\mathrm{kPa}$ & $\mathrm{kPa}$ \\
\hline Accuracy & \multirow{4}{*}{ $\pm 0.08 \%$} & $\pm 0.25 \%$ FS & $\pm 0.25 \% \mathrm{FS}$ & $\pm 0.25 \% \mathrm{FS}$ & $\pm 0.25 \%$ FS \\
\hline Linearity & & $\pm 0.15 \%$ FS & $\pm 0.15 \% \mathrm{FS}$ & $\pm 0.15 \% \mathrm{FS}$ & $\pm 0.15 \%$ FS \\
\hline Hysteresis & & $\pm 0.10 \% \mathrm{FS}$ & $\pm 0.10 \% \mathrm{FS}$ & $\pm 0.10 \% \mathrm{FS}$ & $\pm 0.10 \% \mathrm{FS}$ \\
\hline Repeatability & & $\pm 0.05 \%$ FS & $\pm 0.05 \% \mathrm{FS}$ & $\pm 0.05 \% \mathrm{FS}$ & $\pm 0.05 \%$ FS \\
\hline
\end{tabular}

\subsubsection{Temperature Measurements}

Thermocouples were used throughout the MTU test cell for this research, a majority of which were type K. The ISL engine used type E thermocouples for some of the onengine measurements. The complete list of different thermocouples used is provided in Table 3.7. The $3.175 \mathrm{~mm}$ diameter thermocouples where used to measure temperatures throughout the test cell, including the ISL and ISB engine, the exhaust lines, and the PM sampling system. The $0.813 \mathrm{~mm}$ diameter thermocouples were inserted into the outlet channels of the CPF. These thermocouples measured the gas temperature inside the CPF at seven radial and four axial locations. The thermocouple layouts used for the CPF on the ISB and ISL engine are provided in Figures 3.6 and 3.7, respectively. The dimensions used in Figures 3.6 and 3.7 are millimeters and each thermocouple location is marked by an identifier. In Figure 3.7, the parenthesis after each identifier is the length of the thermocouple used, in inches since that is how the thermocouples were ordered. For reference 12 inches is $304.8 \mathrm{~mm}, 18$ inches is $457.2 \mathrm{~mm}$, and 24 inches is $609.6 \mathrm{~mm}$. The $0.510 \mathrm{~mm}$ diameter thermocouples were inserted into the DOC to measure the internal temperatures at different axial and radial locations. 
Table 3.7: Thermocouples Used in the MTU Test Cell

\begin{tabular}{|c|c|c|c|c|c|}
\hline Manufacturer & $\begin{array}{c}\text { Diameter } \\
(\mathrm{mm})\end{array}$ & $\begin{array}{l}\text { Length } \\
(\mathrm{mm})\end{array}$ & Type & Part Number & Accuracy \\
\hline \multirow{3}{*}{ Watlow } & 0.510 & 304.8 & $\mathrm{~K}$ & G554558 & \multirow{9}{*}{ $\pm 2.2{ }^{\circ} \mathrm{C}$} \\
\hline & 0.510 & 431.8 & $\mathrm{~K}$ & G563304 & \\
\hline & 3.175 & 152.4 & $\mathrm{~K}$ & G563302 & \\
\hline \multirow{8}{*}{$\begin{array}{c}\text { Omega } \\
\text { Engineering }\end{array}$} & 0.510 & 457.2 & K & KMQSS-020U-18 & \\
\hline & 0.813 & 304.8 & $\mathrm{~K}$ & KMQSS-032U-12 & \\
\hline & 0.813 & 457.2 & $\mathrm{~K}$ & KMQSS-032U-18 & \\
\hline & 0.813 & 609.6 & $\mathrm{~K}$ & KMQSS-032U-24 & \\
\hline & 3.175 & 152.4 & $\mathrm{~K}$ & KMQSS-125U-6 & \\
\hline & 3.175 & 304.8 & $\mathrm{~K}$ & KMQSS-125U-12 & \\
\hline & 3.175 & 152.4 & E & $\begin{array}{c}\text { TJ36-CXSS-18U- } \\
\text { 6-CC-XSIB }\end{array}$ & \multirow[t]{2}{*}{ $\pm 1.7^{\circ} \mathrm{C}$} \\
\hline & 3.175 & 304.8 & $\mathrm{E}$ & EMTSS-125U-12 & \\
\hline
\end{tabular}

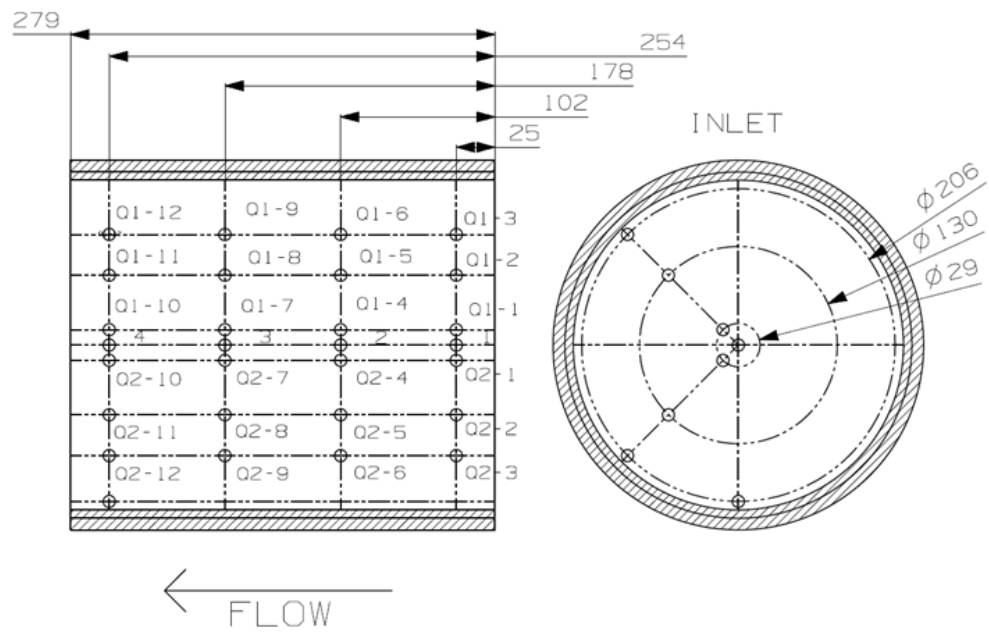

Figure 3.6: ISB CPF Thermocouple Layout

Adapted from CPF Layout Developed by Chris Hutton 


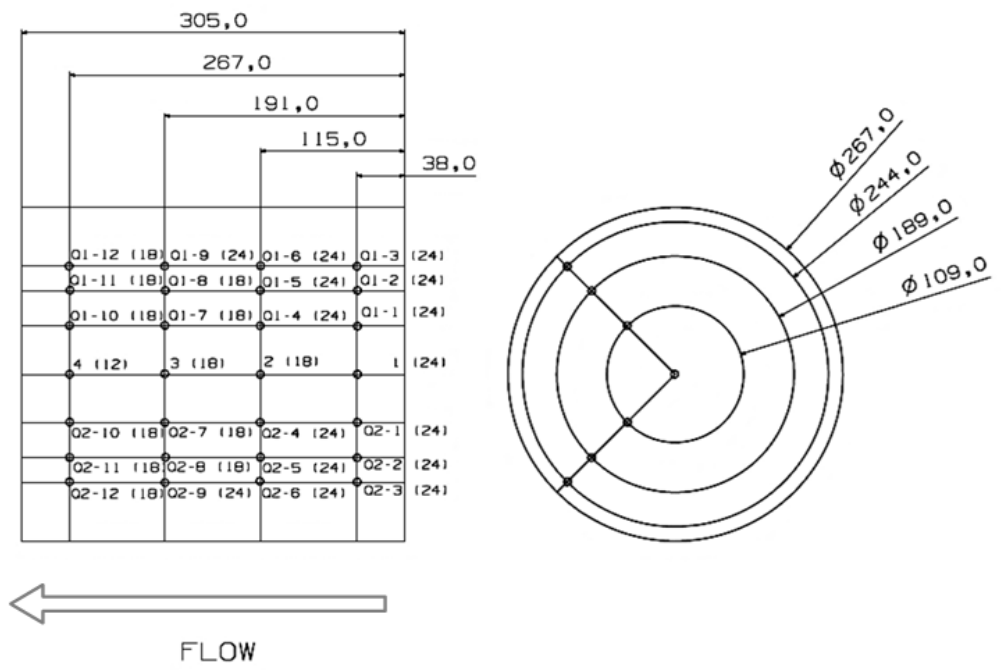

Figure 3.7: ISL CPF Thermocouple Layout

\subsubsection{Gaseous Emissions Measurements}

The gaseous emissions were measured upstream of the DOC (UDOC), downstream of the DOC (DDOC), and downstream of the CPF (DCPF). This is shown by the sample lines in Figures 3.1 and 3.2. Two instruments were used to measure the gaseous emissions: a Pierburg AMA4000 and a V \& F Analyse- und Messtechnik GmbH AirSense Ion Molecule Reaction Mass Spectrometer (IMR-MS). The list of calibration gases used to calibrate the emissions instruments is provided in Table 3.8. Both the AMA4000 and IMR-MS were calibrated each day before use. The AMA4000 was used to measure oxygen $\left(\mathrm{O}_{2}\right)$, carbon monoxide $(\mathrm{CO})$, carbon dioxide $\left(\mathrm{CO}_{2}\right)$, total nitrogen oxides $\left(\mathrm{NO}_{\mathrm{X}}\right)$, nitric oxide (NO), and total hydrocarbons (THC). The specifications for the AMA4000 are given in Table 3.9. The IMR-MS was used to measure nitrogen dioxide $\left(\mathrm{NO}_{2}\right), \mathrm{NO}$, and $\mathrm{O}_{2}$. The $\mathrm{NO}_{\mathrm{X}}$ measurement for the IMR-MS is completed by adding the $\mathrm{NO}_{2}$ and NO measurements. The specifications for the IMR-MS are provided in Table 3.10. The $\mathrm{NO}_{\mathrm{X}}$ measurements from the AMA4000 and IMR-MS were compared to determine how similar the two measurements were. When both instruments were working properly, the IMR-MS $\mathrm{NO}_{\mathrm{X}}$ measurement was 10-20 ppm, or 5-10\%, lower than the AMA4000.

Table 3.8: Calibration Gases

\begin{tabular}{|c|c|}
\hline Gas & Concentration \\
\hline \multirow{2}{*}{$\mathrm{O}_{2}(\%)$} & 12.94 \\
\cline { 2 - 2 } & 20.90 \\
\hline $\mathrm{CO}(\mathrm{ppm})$ & 299.70 \\
\hline $\mathrm{CO}_{2}(\%)$ & 14.11 \\
\hline $\mathrm{NO}(\mathrm{ppm})$ & 500.00 \\
\hline \multirow{2}{*}{$\mathrm{CH}_{3}(\mathrm{ppm})$} & 30.12 \\
\cline { 2 - 2 } $\mathrm{NO}_{2}(\mathrm{ppm})$ & 1399.00 \\
\hline
\end{tabular}


Table 3.9: Specifications for the AMA4000

\begin{tabular}{|c|c|c|c|c|c|c|}
\hline & $\mathrm{O}_{2}$ & $\mathrm{CO}$ & $\mathrm{CO}_{2}$ & $\mathrm{NO}_{\mathrm{x}}$ & NO & $\mathrm{THC}$ \\
\hline Range & $0-25 \%$ & $\begin{array}{c}0-5000 \\
\text { ppm }\end{array}$ & $0-20 \%$ & \multicolumn{2}{|c|}{$\begin{array}{c}0-10,000 \\
\text { ppm }\end{array}$} & $\begin{array}{c}0-20,000 \\
\text { ppm C }_{3}\end{array}$ \\
\hline $\begin{array}{l}\text { Detection } \\
\text { Limit }\end{array}$ & $15 \mathrm{ppm}$ & $125 \mathrm{ppb}$ & 15 ppm & \multicolumn{2}{|c|}{$35 \mathrm{ppb}$} & $30 \mathrm{ppb} \mathrm{C}_{3}$ \\
\hline Accuracy & \multicolumn{6}{|c|}{ Not Available } \\
\hline Repeatability & $\begin{array}{c}\leq 0.5 \% \text { of } \\
\text { the Measured } \\
\text { Value }+2 \mathrm{x} \\
\text { Detection } \\
\text { Limit }\end{array}$ & $\begin{array}{c}\leq 0.5 \% \\
\text { of the } \\
\text { Measured } \\
\text { Value }+ \\
2 x \\
\text { Detection } \\
\text { Limit }\end{array}$ & $\begin{array}{c}\leq 0.5 \% \\
\text { of the } \\
\text { Measured } \\
\text { Value }+ \\
2 \mathrm{x} \\
\text { Detection } \\
\text { Limit } \\
\end{array}$ & \multicolumn{2}{|c|}{$\begin{array}{c}\leq 0.3 \% \text { of } \\
\text { the } \\
\text { Measured } \\
\text { Value }+2 \mathrm{x} \\
\text { Detection } \\
\text { Limit }\end{array}$} & $\begin{array}{c}\leq 0.5 \% \\
\text { of the } \\
\text { Measured } \\
\text { Value }+ \\
2 \mathrm{x} \\
\text { Detection } \\
\text { Limit }\end{array}$ \\
\hline Noise & $\begin{array}{c}\leq 1.0 \% \text { of } \\
\text { the Measured } \\
\text { Value }+2 \mathrm{x} \\
\text { Detection } \\
\text { Limit }\end{array}$ & $\begin{array}{c}\leq 1.0 \% \text { of } \\
\text { the } \\
\text { Measured } \\
\text { Value }+ \\
3 x \\
\text { Detection } \\
\text { Limit }\end{array}$ & $\begin{array}{c}\leq 1.0 \% \\
\text { of the } \\
\text { Measured } \\
\text { Value }+ \\
2 \mathrm{x} \\
\text { Detection } \\
\text { Limit } \\
\end{array}$ & \multicolumn{2}{|c|}{$\begin{array}{c}\leq 1.0 \% \text { of } \\
\text { the } \\
\text { Measured } \\
\text { Value }+2 \mathrm{x} \\
\text { Detection } \\
\text { Limit }\end{array}$} & $\begin{array}{c}\leq 1.0 \% \\
\text { of the } \\
\text { Measured } \\
\text { Value }+ \\
2 \mathrm{x} \\
\text { Detection } \\
\text { Limit }\end{array}$ \\
\hline $\begin{array}{c}\text { Analyzer } \\
\text { Type }\end{array}$ & Paramagnetic & IRD & IRD & CLD & CLD & FID \\
\hline $\begin{array}{l}\text { Measurement } \\
\text { Type }\end{array}$ & Dry & Dry & Dry & Wet & Wet & Wet \\
\hline
\end{tabular}

Table 3.10: IMR-MS Specifications

\begin{tabular}{|c|c|}
\hline Mass Range & $0-500 \mathrm{amu}$ \\
\hline Resolution & $<1 \mathrm{amu}$ \\
\hline \multirow{3}{*}{ Lower Detection Limit } & $<1 \mathrm{ppb}$ (Benzene in Air) \\
\cline { 2 - 2 } & $\begin{array}{c}<10 \mathrm{ppb} \text { (Benzene in Exhaust } \\
\text { Gas) }\end{array}$ \\
\hline Drift Concentration & $< \pm 5 \%$ over 12 Hours \\
\hline Reproducibility & $< \pm 3 \%$ \\
\hline Accuracy & $< \pm 2 \%$ \\
\hline Max Humidity & Wet \\
\hline Measurement Type & $10-6500 \mathrm{msec} / \mathrm{amu}$ \\
\hline Analysis Time & T90 $<50 \mathrm{msec}$ \\
\hline Response Time &
\end{tabular}




\subsubsection{PM Concentration}

The PM concentration in the exhaust was measured using the MTU Hot Sampling Method. This method consists of sampling hot, undiluted, exhaust through a $47 \mathrm{~mm}$ glass fiber filter using a stack sampler. The $47 \mathrm{~mm}$ filters used in this work were Pall Corporation 61631 filters. The stack sampler used was an Andersen Instruments Inc. Manual Sampling Train (MST). Details on the design of the sample probe used can be found in reference [34]. After the exhaust sample passes through the $47 \mathrm{~mm}$ filter, it is pulled through two impingers that contain desiccant and are placed in a water bath to help cool and dry the sample. After the impingers, the sample passes through a vacuum pump, a dry gas meter, and a flow orifice. The MST uses a dry gas meter to measure the cumulative volume of exhaust that is sampled. The $47 \mathrm{~mm}$ filters are weighed before and after sampling to determine the amount of PM retained on the filter. The readings of the dry gas meter are recorded before and after sampling to determine the total volume of gas that was sampled. The volume measurement is corrected to standard conditions based on the temperature and pressure of the gas inside the dry gas meter. The concentration of the PM is calculated using Eq. 3.1.

$$
P M_{\text {conc }}=\frac{m_{\text {end }}-m_{\text {start }}}{\Delta V_{\text {std }}}
$$

In Eq. 3.1, $m_{\text {end }}$ is the mass of the filter after testing, $m_{\text {start }}$ is the mass of the filter before testing, $\Delta V_{s t d}$ is the standard volume of the sample that was taken, and is calculated using Eq. 3.2.

$$
\Delta V_{s t d}=\left(V_{\text {end }}-V_{\text {start }}\right)\left(\frac{T_{s t d}}{P_{s t d}}\right)\left(\frac{P_{a c t}}{T_{a v g}}\right)
$$

In Eq. 3.2, $V_{\text {end }}$ is the reading from the dry gas meter at the end of the sample, $V_{\text {start }}$ is the reading from the dry gas meter at the start of the sample, $T_{s t d}$ is the standard temperature, $P_{\text {std }}$ is the standard pressure, $P_{\text {act }}$ is the actual measured pressure, and $T_{\text {avg }}$ is the average measured temperature inside the dry gas meter. For this research a value of $100 \mathrm{kPa}$ was used for $P_{s t d}$, and a value of $298.15 \mathrm{~K}$ was used for $T_{s t d}$.

The mass of the filters was determined using a Mettler Toledo UMT2 scale. The specifications for the UMT2 are given in Table 3.11. Prior to any mass measurements on the filters, they are baked at $316^{\circ} \mathrm{C}$ for 15 minutes to remove any volatile compounds. The filters are then allowed to stabilize in a clean environment for a minimum of 24 hours and then the initial weights are taken. Each filter is weighed three times to get the average weight. After PM sampling has been completed on the filters they are stored in a box containing desiccant to prevent moisture absorption by sulfates that may be in the PM sample. Sulfates are hygroscopic, so an effort needs to be made to limit the amount 
of moisture to which they are exposed [34]. The desiccant in the box provides a "dry" ambient condition to store the filters in until all sampling is done for a particular test. Once all of the PM samples have been collected for a particular test, the samples are treated with ammonium hydroxide for at least one hour to prevent additional moisture absorption by the sulfate content in the PM samples by converting any sulfates on $47 \mathrm{~mm}$ filters to ammonium sulfate. Lakkireddy, in reference [34], states that ammonium sulfate is less hygroscopic and describes the procedure for treating the $47 \mathrm{~mm}$ filters with ammonium hydroxide. The filters are then placed back in the clean environment for a minimum of 24 hours before being weighed again. Each filter is weighed three times again. The filters are stored in Millipore PDMA04700 plastic filter holders when they are not being used for sampling or being weighed. Additional information on the MTU sampling method can be found in references [34, 35].

Table 3.11: 47 mm Filter Scale Specifications

\begin{tabular}{|c|c|}
\hline Manufacturer & Mettler Toledo GmbH \\
\hline Model & UMT2 \\
\hline Capacity & $2100 \mathrm{mg}$ \\
\hline Resolution & $0.1 \mu \mathrm{g}$ \\
\hline Repeatability & $0.25 \mu \mathrm{g}$ \\
\hline Linearity & $\pm 0.5 \mu \mathrm{g}$ \\
\hline
\end{tabular}

The PM concentration in the exhaust is measured UDOC and DCPF. The UDOC samples were collected for five to ten minutes and the DCPF samples were collected for 30 to 60 minutes. The UDOC measurements are used as inputs to the MTU 1D CPF model. The DCPF measurement is used to determine the filtration efficiency of the CPF, which is also an input to the MTU 1D CPF model. The filtration efficiency is determined using Eq. 3.3. In Eq. 3.3, $P M_{U D O C}$ is the PM concentration measured UDOC and $P M_{D C P F}$ is the PM concentration measured DCPF.

$$
\eta_{C P F}=\left(\frac{P M_{U D O C}-P M_{D C P F}}{P M_{U D O C}}\right) \times 100
$$

\subsubsection{PM Retained Measurements}

The mass of the PM retained in the CPF is determined by weighing the CPF before, during, and after testing. The weight of the CPF is measured after a cleanout and the loading, oxidation, and post loading stages that occur during testing. The procedure used to weigh the substrate at the different stages in the testing can be found in Appendix B. All of the procedures involve weighing the CPF when it is hot. Austin, in reference [36], found that the temperature the CPF was weighed at had a significant impact on the measurement. Due to this, Hutton, in reference [3], recommended that the internal 
temperature of the $\mathrm{CPF}$ does not vary more than $\pm 15{ }^{\circ} \mathrm{C}$ for $\mathrm{CPF}$ weights taken at different points in the test procedure. The mass of the substrate was measured at MTU using an Ohaus Ranger scale. The specifications for the scale are in Table 3.12. The mass of the substrate was measured at Cummins using an A\&D Company, LTD GP-30K scale. The specifications for that scale are given in Table 3.13.

Table 3.12: MTU CPF Scale Specifications

\begin{tabular}{|c|c|}
\hline Manufacturer & Ohaus \\
\hline Model & Ranger RD35LM \\
\hline Capacity & $35,000 \mathrm{~g}$ \\
\hline Resolution & $0.1 \mathrm{~g}$ \\
\hline Repeatability & $\pm 0.1 \mathrm{~g}$ \\
\hline Linearity & $\pm 0.3 \mathrm{~g}$ \\
\hline Overall Accuracy & $\pm 0.4 \mathrm{~g}$ \\
\hline Certified Accuracy & $\pm 1.0 \mathrm{~g}$ \\
\hline
\end{tabular}

Table 3.13: Cummins CPF Scale Specifications

\begin{tabular}{|c|c|}
\hline Manufacturer & A\&D Company, LTD \\
\hline Model & GP-30K \\
\hline Capacity & $31,000 \mathrm{~g}$ \\
\hline Resolution & $0.1 \mathrm{~g}$ \\
\hline Repeatability & $\pm 0.1 \mathrm{~g}$ \\
\hline Linearity & $\pm 0.2 \mathrm{~g}$ \\
\hline Stated Accuracy & $\pm 1.5 \mathrm{~g}$ \\
\hline
\end{tabular}

\subsubsection{Particle Size Distribution}

The PSD was measured UDOC, DDOC, and DCPF, as shown in Figures 3.1 and 3.2, using the SMPS instruments. The SMPS consisted of a TSI 3065 thermodenuder, TSI 3080 electrostatic classifier, and a TSI 3025A ultra-fine condensation particle counter. Prior to the exhaust gas going through the SMPS, it was diluted at a ratio of 10:1, using a diluter manufactured by MTU, with cleaned, dried, and heated compressed air. The diluter used an Air-Vac TD110H single stage vacuum generator as the source of dilution. The dilution ratio was found to be a function of the pressure of the dilution air, the exhaust pressure, and the exhaust flow rate [3]. To minimize fluctuations in the dilution ratio, the pressure of the compressed air was set to $206.8 \mathrm{kPa}$. The dilution ratio was measured by sampling the gaseous exhaust emissions before and after dilution and using Eq. 3.4. 


$$
D R=\frac{\operatorname{Conc}_{\text {Raw }}}{\text { Conc }_{\text {Diluted }}}
$$

Eq. 3.4

In Eq. 3.4, $D R$ is the dilution ratio, $C_{0 n c_{R a w}}$ is the raw gaseous emission concentration, and Conc $_{\text {Diluted }}$ is the diluted gaseous emission concentration. Either $\mathrm{NO}_{\mathrm{X}}$ or $\mathrm{CO}_{2}$ was used to measure the dilution ratio, with both gases providing similar ratios. Additional information on the setup of the SMPS instruments can be found in reference [3].

\subsection{Terahertz Wave Scanner}

An Advantest TAS7000 3D Imaging Analysis System was used to measure the PM distribution, as mentioned in section 2.3. The TAS7000 used in this study was located in the Catalyst Technology Lab at the Cummins Technical Center in Columbus, IN and is pictured in Figure 3.8. Since the scanner is not located at MTU, the substrates had to be shipped to Cummins for scanning. The procedure to ship the substrates is given in Appendix C. Prior to the substrates being scanned, they were weighed at Cummins to determine the PM loading. The procedure used to weigh the substrate at Cummins is given in Appendix B. The spatial resolution of the TAS7000 was dependent on the size of the substrate being scanned. The spatial resolution is defined as the volume of the sample point for which the PM loading measurement is taken. For the substrates used on with the ISB engine, the spatial resolution was a $4 \times 4 \times 4.4 \mathrm{~mm}$ space. For the substrates used with the ISL engine, the spatial resolution was a $4.5 \times 4.5 \times 2.4 \mathrm{~mm}$ space. The resolutions are different for the two substrates because settings had to be changed within the TAS7000 to account for the different substrate size and provide quality data. The most noticeable change is in the number of $z$ sections, or axial sections, produced by the TAS7000. For the ISB substrates 64 axial sections were used, and for the ISL substrates 125 axial sections were used. The TAS7000 scans a substrate in six to twenty hours [37]. The frequency range utilized by the TAS7000 is $400 \mathrm{GHz}$ to $4 \mathrm{THz}$ [38]. 


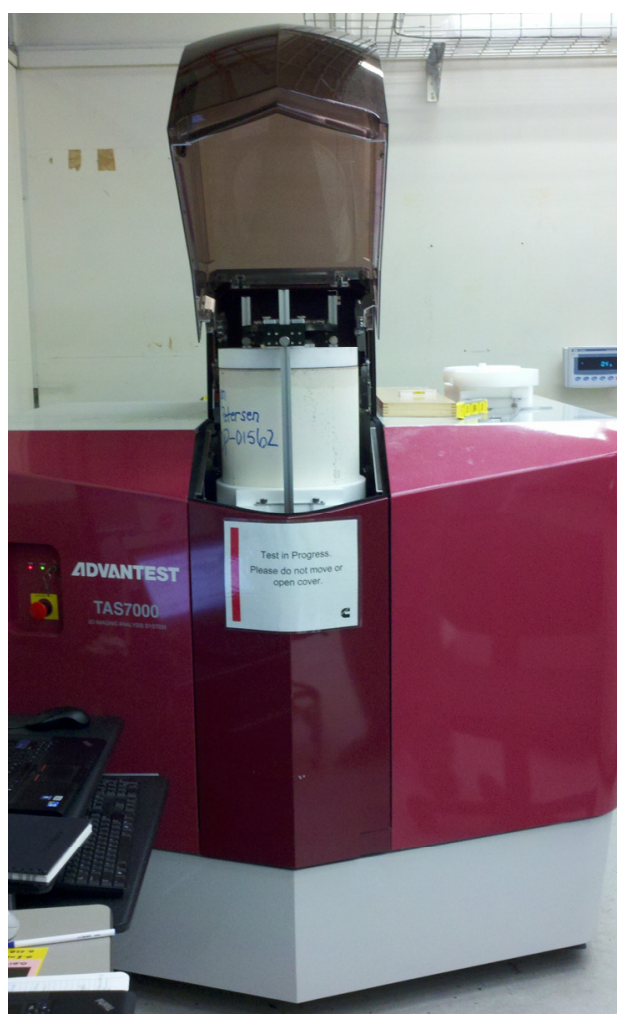

Figure 3.8: TAS7000 at Cummins 


\section{Chapter 4. Data Analysis Methodology ${ }^{2}$}

The data that is produced by the TAS7000 has to be handled in such a way that the final results have units and directionality. In section 2.3 it was mentioned that the TAS7000 scans a substrate in the radial, $r$, angular, $\theta$, and axial, $z$, directions. During the exporting of the data, the TAS7000 converts the $r$ and $\theta$ scan locations to a Cartesian coordinate system and saves the corresponding data in the rows and columns of a matrix. Each $Z$ location, herein referred to as axial sections, has a matrix containing the data for the $r$ and $\theta$ directions. The data handling discussed in this section works with the data in this form. Originally the TAS7000 had software that would convert the data into the appropriate units, but due to a software malfunction, MTU had to develop a new process. A method to quantify the uniformity of the PM distribution had to be developed, which would allow for analysis of the of the PM distribution trends beyond the visually observable trends. Lastly, since the data is collected using a rotating stage, a method to check for shifts in the data was developed.

\subsection{Conversion of Scan Data to Grams per Liter}

The data that were originally produced by the TAS7000 were calibrated to give the PM density in units of grams per liter through software that was developed by Advantest. A summary of the calibration process used is given in reference [28]. The calibration consisted of subtracting the baseline data set from the PM loaded data set and setting the average attenuation value from the TAS7000 equal to the average PM loading in the substrate. However, due to software issues, that procedure was not used during Phase 2 of the research. Instead, a calibration procedure developed at MTU was used to convert the raw TAS7000 data to PM density data in units of grams per liter. That method is discussed in the following paragraphs.

One important difference between the original calibration procedure, which was developed by Advantest and the new calibration procedure, which was developed by MTU, is the constituent of the PM density that was measured. The original Advantest software measured only the carbonaceous PM density. The software had the ability to distinguish between the carbonaceous PM loading and any ash loading that may have taken place in the substrate. The new PM density calculation method that was developed by MTU does not have this capability. As a result, the TAS7000 data for Phase 2 measured both the carbonaceous and ash content, and the PM density calculation method used the total amount of ash and carbonaceous PM retained in the substrate. During the results section, distinctions will be made when describing PM trends between the measurements of just the carbonaceous, or measurements of both the carbonaceous and ash content. There may be differences in the resulting distributions, depending on the amount of ash content. The amount of ash in the substrates used during Phase 2 is discussed in Appendix G, but it was relatively minor $(0.2 \mathrm{~g} / \mathrm{L})$ for three of the four

${ }^{2}$ Parts of the material contained in this chapter have been submitted, or are being considered for submission, to SAE International for publication consideration. 
substrates used in Phase 2. The fourth substrate had an ash loading of $0.8 \mathrm{~g} / \mathrm{L}$ during the Phase 2 tests. Ash loading in the substrate is caused by incombustible material from the engine lube oil, metal fragments from engine wear, corrosion in the engine or exhaust, and metal content or additives in the fuel [39].

The new PM density calculation method, as developed by MTU, first subtracts the baseline data, taken from the clean substrate, from the loaded data, taken from substrates loaded with PM. It then finds the valid data inside the generic matrix generated by the TAS7000. It is worth noting that the TAS7000 up- samples the data by a factor of two prior to the generic matrices being written. This means that the $4 \times 4 \times 4.4 \mathrm{~mm}$ or $4.5 \mathrm{x}$ $4.5 \times 2.4 \mathrm{~mm}$ space discussed in section 3.5 becomes a $2 \times 2 \times 4.4 \mathrm{~mm}$ or $2.25 \times 2.25 \times$ $2.4 \mathrm{~mm}$ space. The data set is then zeroed to minimize the number of negative values in the data set. Negative values have no physical meaning, and are considered noise in the measurement. The zeroing process allows the negative values to become equal to, or near, zero. Zeroing is not applied to data sets where the minimum value is greater than 0 , since values greater than 0 have a physical meaning.

The data set is zeroed by finding the lowest data value that is still within a $95^{\text {th }}$ percentile range (PR) of the mean of the data. The $95^{\text {th }} \mathrm{PR}$ is defined as the range of the data between the values of the 2.5 and 97.5 percentile for the data set. By only using data that is within a $95^{\text {th }} \mathrm{PR}$ of the mean, additional measurement noise can be removed from the calculation. The zeroing is completed using the 2.5 percentile values from each axial section. The minimum 2.5 percentile value out of all of the axial sections becomes the value used to zero the data set. Each sample point in the entire substrate is adjusted by value of the minimum 2.5 percentile out of all the axial sections. This process preserves relative differences between sample points, while eliminating negative sample points. The basic mathematics of this process are shown in Eq. 4.1.

$$
w n_{(i, j, k)}=w o_{(i, j, k)}-w_{\text {min }}
$$

Eq. 4.1

In Eq. 4.1, $w n_{(i, j, k)}$ is the zeroed data value at coordinates $i, j$, and $k, w o_{(i, j)}$ is the original data value at coordinates $i, j$, and $k$, and $w_{\min }$ is the minimum 2.5 percentile value in the entire substrate.

After zeroing, the data is then multiplied by the volume of the sample point $(\Delta v)$. This is the volume of the $2 \times 2 \times 4.4 \mathrm{~mm}$ or $2.25 \times 2.25 \times 2.4 \mathrm{~mm}$ space, $1.76 \times 10^{-5}$ or $1.22 \times 10^{-5} \mathrm{~L}$ respectively. The data is then summed together to get the total attenuation value for the data set. This is shown by Eq. 4.2. 


$$
w_{t}=\sum_{k=1}^{o} \sum_{j=1}^{n} \sum_{i=1}^{m}\left(w n_{(i, j, k)} \times \Delta v\right)
$$

In Eq. 4.2, $w_{t}$ is the total attenuation value for the data set, $w n_{(i, j, k)}$ is the zeroed sample point at coordinates $i, j$, and $k, \Delta v$ is the volume of the sample point, $m$ is the number of rows being analyzed in the matrix, $n$ is the number of columns being analyzed in the matrix, and $o$ is the number of axial sections being analyzed for the substrate. The total attenuation value is proportional to the amount of PM loaded into the substrate, in grams. The gain that is used to scale the zeroed data set to the appropriate units is calculated using Eq. 4.3.

$$
g=\frac{P M_{g}}{w_{t}}
$$

In Eq. 4.3, $g$ is the gain, $P M_{g}$ is the amount of PM loaded in the substrate in grams, and $w_{t}$ is the total attenuation value for the substrate. The gain is then applied to all of the zeroed sample points to obtain a calibrated data set in units of grams per liter. This is shown in Eq. 4.4.

$$
w c=w n \times g
$$

Eq. 4.4

In Eq. 4.4, $w c$ is the calibrated data set, $w n$ is the zeroed data set, and $g$ is the gain used to calibrate the data.

\subsection{Conversion of Scan Data to Grams per Surface Area}

The units of grams per liter are useful for looking at general trends in the PM distribution. The PM loading in a substrate can be described by the PM density, PM cake thickness, or the PM mass per unit surface area. Surface area is commonly used in filtration theory, so a method to represent the PM distribution in terms of grams per square millimeter was developed. The equation used to convert between grams per liter and grams per square millimeter is given as Eq. 4.5.

$$
w_{S A}=\frac{w c \times \Delta v}{S A_{d p}}
$$


In Eq. 4.5, $w_{S A}$ is the converted data set, $w c$ is the calibrated data set with units of grams per liter, $\Delta v$ is the volume of a sample point in liters, and $S A_{d p}$ is the surface area of the sample point, which is given by Eq. 4.6 for the ISB engine and Eq. 4.7 for the ISL engine.

$$
\begin{gathered}
S A_{d p}=\left(z_{s z}\left(4\left(x y_{S z}-w_{t}\right)\right)\right)\left(1-\varepsilon^{\frac{2}{3}}\right) \\
S A_{d p}=\left(z_{S Z}\left(8\left(x y_{s z}-2 w_{t}\right)+8 a\right)\right)\left(1-\varepsilon^{\frac{2}{3}}\right)
\end{gathered}
$$

In Eqs. 4.6 and 4.7, $z_{S Z}$ is the size of the sample point in the $z$ direction (4.4 or $2.4 \mathrm{~mm}$ ), $x y_{s z}$ is the size of the sample point in the $x$ and $y$ direction $(2$ or $2.25 \mathrm{~mm}), w_{t}$ is the channel wall thickness, $a$ is the channel width, and $\varepsilon$ is the porosity of the substrate. Since it is unknown where the sample point would be located within the substrate at the time of the scan, the exact surface area of the sample point is unknown. Therefore, the maximum amount of surface area that could exist in one sample point is calculated using Eqs. 4.6 and 4.7. For the grams per surface area calculations to be accurate, the location of each sample point, with reference to the channels of the substrate, would be needed.

Having the ability to analyze data with units of grams per surface area is advantageous because it allows for the comparison of filters with different properties, since different properties could have an impact on the PM distribution trends. Those properties include channel width, wall thickness, channel density. As cell density increases, for example, the surface area of the substrate will increase and the PM cake thickness will decrease. For reference, the surface area for the entire substrate is given by Eq. 4.8.

$$
\begin{gathered}
S A_{f}=\frac{1}{2} \rho_{C}\left(\frac{\pi D^{2}}{4}\right)\left[\left[4\left(a-2 w_{c a t}\right)\left(L_{S}-\left(L_{P}+w_{c a t}\right)\right)\right.\right. \\
\left.\left.\left.+\left(a-2 w_{c a t}\right)^{2}\right)\right]\left(1-\varepsilon^{\frac{2}{3}}\right)\right]
\end{gathered}
$$

In Eq. 4.8, $S A_{f}$ is the surface area of the substrate, $\rho_{C}$ is the channel density, $D$ is the substrate diameter, $a$ is the channel width, $w_{\text {cat }}$ is the thickness of the washcoat, $L_{S}$ is the length of the substrate, $L_{P}$ is the length of the channel plugs, and $\varepsilon$ is the substrate porosity. The channel density is defined as the number of channels per unit area and is given by Eq. 4.9. 


$$
\rho_{c}=\frac{1}{\left(a+w_{t}\right)^{2}}
$$

Eq. 4.9

In Eq. 4.9, $\rho_{c}$ is the channel density, $a$ is the channel width, and $w_{t}$ is the substrate wall thickness.

\subsection{Development of Directionality}

Regardless of the units used with the data, the data needs to be organized in such a way that it can be processed in specific directions. The directions of interest are the axial, radial, and angular. The data produced by the TAS7000 is in standard matrix form, as discussed in the first paragraph of this chapter, so the only directionality produced by the TAS7000 is in the axial direction through the axial sections. The TAS7000 was set to produce 64 axial sections for the ISB substrates and 125 axial sections for the ISL substrates. To obtain the radial and angular directionality, a method of grouping the data was developed.

The data in each axial section were divided into four radial sections and seventy two $5^{\circ}$ angular increments. The sample points that exist in one angular increment and one radial section are averaged together to form one analysis point. Grouping the data in this form produced 288 analysis points in each axial section. The axial sections allow for data analysis in the $z$ direction, the radial sections allow for analysis in the $r$ direction, and the angular increments allow for analysis in the $\theta$ direction. In order to discuss general data trends, the 72 angular increments were grouped into four quadrants, with each quadrant containing 18 angular increments. The substrate was also divided into 4 axial segments, each one containing 16 axial sections for the ISB substrates or 31-32 axial sections for the ISL substrates. The axial sections, axial segments, radial sections, angular increments, and quadrants are collectively known as regions. The data analysis that is discussed in sections 4.4 and 4.5 is performed within these developed regions.

An example of the data grouping that takes place in each axial section is provided in Figure 4.1. The positive $r$ direction is indicated by the solid blue arrow and the positive $\theta$ direction is indicated by the white arrow with the blue outline. When discussing PM distribution trends in the angular directions, the variable $\Theta$ will be used to identify the angle of rotation. It follows the $\theta$ plane defined by the white arrow with the blue outline. The radial sections are shown by the red lines and the corresponding dimensions are given in the red text. The dimensions used for the ISL and ISB substrates are provided. An example of an angular increment is given as the area between the two solid black lines. The divisions used for the quadrants are given by the green lines, and the corresponding angles are given in the green text. 


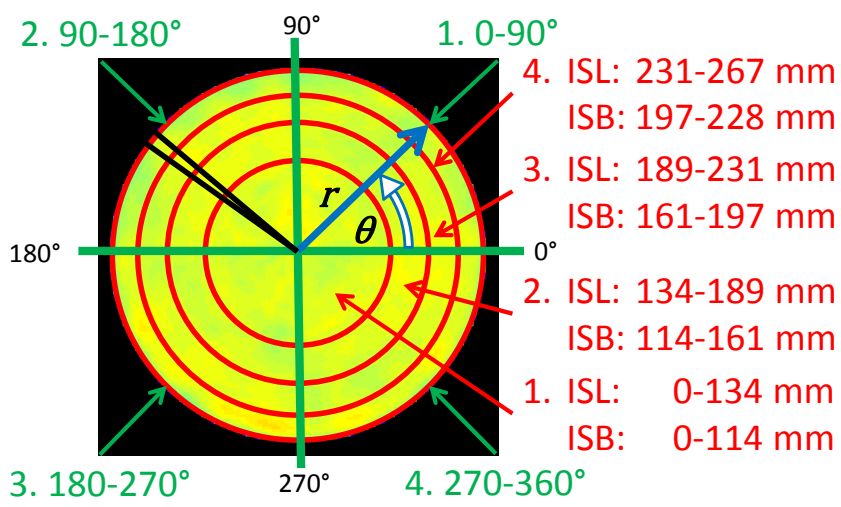

Figure 4.1: Axial Section Data Grouping

Since two different size substrates are used, data can be represented by normalized units for the radius and length, $\mathrm{R}^{*}$ and $\mathrm{Z}^{*}$ respectively. The equation for $\mathrm{R}^{*}$ is given as Eq. 4.10. The equation for $Z^{*}$ is given as Eq. 4.11.

$$
\begin{gathered}
R^{*}=\frac{\text { Radial Distance from Centerline }}{\text { Radius of the Substrate }} \\
Z^{*}=\frac{\text { Distance from Inlet }}{\text { Total Axial Length }}
\end{gathered}
$$

The radial sections and the corresponding $\mathrm{R}^{*}$ values are shown in Figure 4.2. The value of $\mathrm{R}^{*}$ is shown to approach 1 as the distance from the centerline increases. Figure 4.3 shows that $Z^{*}$ approaches 1 as the value of $z$ increases, where $z$ is the distance from the inlet of the substrate. Figure 4.3 also shows the divisions used to generate the axial segments as the vertical solid blue lines. The ranges for the axial segments are provided in the blue text. 


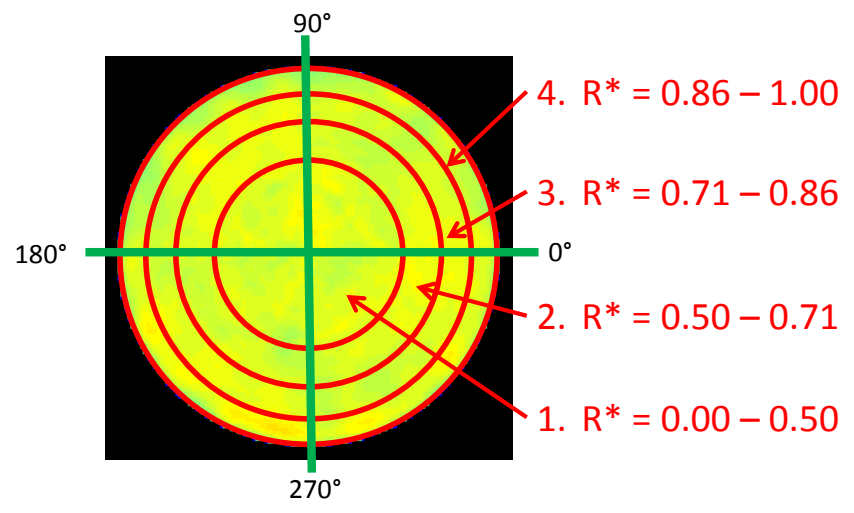

Figure 4.2: R* Values for the Radial Sections

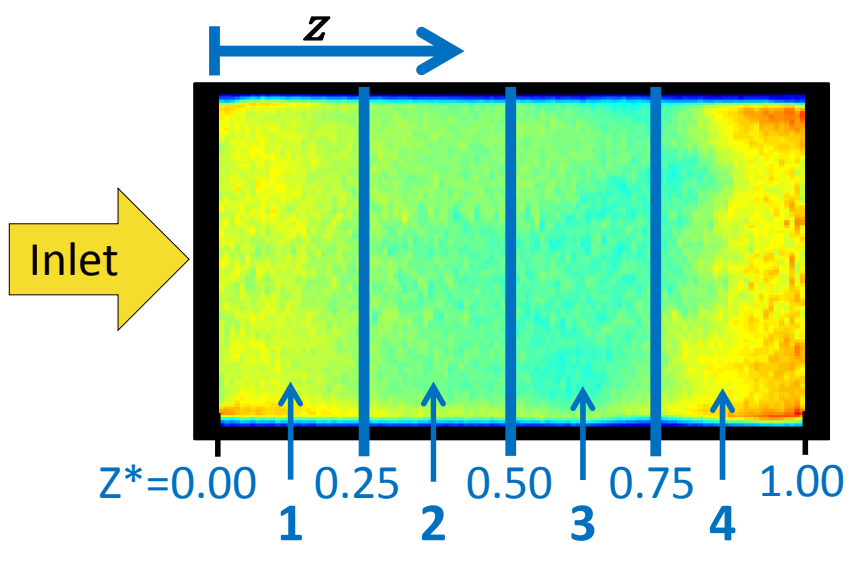

Figure 4.3: $Z^{*}$ Values and Axial Segments

\subsection{Uniformity Index}

An analysis method was developed that quantifies the PM distribution. That method utilizes a parameter known as the uniformity index (UI). The UI is traditionally used in fluid dynamics to describe the uniformity of fluid flow. Tao, et al. present two traditional UI equations, equations 5 and 6 , in reference [40]. Both of these equations were designed to work in $2 \mathrm{D}$ and would not work with the 3D data produced by the TAS7000. A new UI equation was developed, based on equation 6 presented in reference [40]. Equation 6 was selected because it was shown to be more sensitive to variation, both in reference [40] and during the initial development of the new UI equation. Additionally, Stratakis, et al. used a similar equation to describe the distribution uniformity of 2D PM distribution data in reference [23].

The UI quantifies the amount of variation in the average PM density in a specific area in the substrate. An analysis area is defined as a collection of analysis points in one specific direction inside the region being analyzed for the PM distribution. The value is 
normalized so that the valid range is 0 to 1 . A value of 1 indicates a uniform PM distribution. The developed UI equation is given in Eq. 4.12.

$$
U I=1-\left(\frac{\sigma}{\bar{W}}\right)
$$

In Eq. 4.12, $\sigma$ is the standard deviation, and $\bar{w}$ is the PM density in the substrate at the completion of the loading stage of the test. (The different stages of the tests are presented in Chapter 5.) The value of $\bar{w}$ was set to the overall average PM density in the substrate at the completion of the loading stage to allow for loading, passive oxidation, and active regeneration tests to be compared. It was determined through sensitivity analysis that if the value of $\bar{w}$ varied by $\pm 0.8 \mathrm{~g} / \mathrm{L}$, the UI varies by $\pm 4 \%$. The overall PM density in the substrate after passive oxidation or active regeneration tests were completed was $2-4 \mathrm{~g} / \mathrm{L}$ lower than at the completion of loading. Therefore, if the overall PM density in the substrate at the time the substrate was scanned was used, it would be difficult to compare the data from the three different test conditions. The overall PM density at the completion of the loading portion of the tests that were conducted is similar since the tests were designed to load the substrate with the same amount of PM. The equation used to calculate $\sigma$ is given as Eq. 4.13.

$$
\sigma=\sqrt{\frac{\sum_{j=1}^{n_{s}}\left[\sum_{i=1}^{n}\left(w_{i}-\overline{w_{l}}\right)^{2}\right]}{n_{t}}}
$$

In Eq. 4.13, $w_{i}$ is the value of one analysis point inside the analysis area being analyzed, $\overline{w_{l}}$ is the average value of the $w_{i}$ values in the analysis area, $n$ is the number of analysis points in the analysis area, $n_{s}$ is the number of analysis areas in a region, and $n_{t}$ is the total number of analysis points used. The value of $n_{t}$ is calculated using Eq. 4.14.

$$
n_{t}=(n)\left(n_{s}\right)
$$

Eq. 4.14

The developed UI is capable of performing 3D uniformity calculations through the use of the double summation and changing the number of analysis areas used. The double summations also allow directionality to be maintained, since each analysis point is compared to the average value in a specific direction. This also allows for a large area to be analyzed without averaging the data over the large area and decreasing the sensitivity of the UI. The direction that the UI is calculated for is dependent on the direction of the $\overline{w_{l}}$ value, which will be discussed further in section 4.5 . 


\subsection{Quantifying PM Distribution}

The PM distribution is quantified in the axial, radial, and angular directions, using the regions developed in section 4.3. The PM distribution is quantified numerically through the use of the average PM density, $95^{\text {th }}$ PR of the density, and the UI that was developed in section 4.4. The average PM density shows the trends in the PM distribution. The $95^{\text {th }}$ PR shows the amount of variation that exists in the data that is used to calculate the average PM density. The UI is a measure of the consistency in the average PM density. All three parameters are needed to fully understand the PM distribution.

The PM distribution can be quantified using the UI three different ways: an individual value, a regional value, or an overall value. The individual value provides the UI for one analysis point in one axial section, one radial section, and one angular increment. It is most useful when generating plots of the data and analyzing trends. The regional UI value is calculated using the analysis points in the four axial segments, four radial sections, and four quadrants. The regional value is most useful when looking for general data trends that occur for the entire substrate. The overall UI value generates one axial, one radial, and one angular UI for the entire substrate. These values are useful for making comparisons between PM distribution trends that result from loading, passive oxidation, active regeneration, and post loading test conditions. The process for calculating the UI does not change between the individual, regional, and overall values. The number of analysis areas used in the calculations is the only difference. The average PM density and $95^{\text {th }}$ PR can be calculated as individual, regional, or overall values as well by using the same analysis areas as the UI calculations.

The different directions that the UI can be calculated are summarized in Figure 4.4. The four axial segments, four radial sections, and four quadrants are each numbered in a unique color. The arrows in Figure 4.4 indicate the direction that the UI calculations are performed and are color coded to match the regions for which that calculation is performed. The axial UI is calculated using the axial sections, which make up the axial segments. The radial UI is calculated using the angular increments, which make up the quadrants. The angular UI is calculated using the radial sections. More details on how the UI calculations are performed are given in the following paragraphs. 


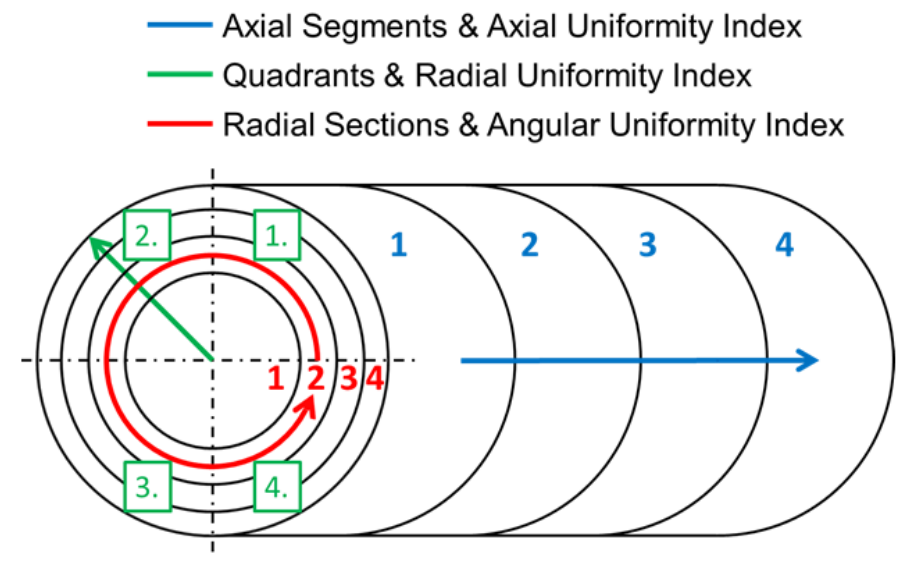

Figure 4.4: UI Calculation Directions

The axial UI is always calculated using the average PM density over the entire substrate, either in one radial section or as a whole, for the value of $\overline{w_{l}}$. The average PM density in the entire substrate and one radial section in the entire substrate are taken as the axial average PM density. The axial sections that are located within the inlet and outlet areas of the substrate that contain channel plugs are excluded from the UI and average PM density calculations. This is because there is an increase in the error in those areas due to the way the substrate is scanned and analyzed for PM loading. For this reason, only the data between $Z^{*}$ values of 0.05 and 0.97 for the ISB substrates and 0.05 and 0.96 for the ISL substrates are used in the axial UI calculation. There are four analysis areas that are used when calculating the axial UI: 1) one radial section in one axial section, 2) one radial section in one axial segment, 3) one radial section over the entire substrate, or 4) the entire substrate.

The radial UI is always calculated using the average PM density in one angular increment for the value of $\overline{w_{l}}$, regardless of the analysis area. An example of an angular increment is provided in Figure 4.1 as the area between the two solid black lines. There are five analysis areas that are used in the radial UI calculation: 1) one angular increment in one axial section, 2) each angular increment in one axial section, 3) each angular increment in one quadrant in one axial segment, 4) each angular increment in one quadrant over the entire substrate, 5) each angular increment in the entire substrate.

The angular UI is always calculated using the average PM density in one radial section as the value of $\overline{w_{l}}$. An example of a radial section is provided in Figure 4.1 as the area between any two red lines. There are four analysis areas that are used when calculating the angular UI: 1) one radial section in one axial section, 2) one radial section in one axial segment, 3) one radial section over the entire substrate, 4) each radial section over the entire substrate.

\subsection{Requirements for Uniformity}

The developed UI quantifies the amount of variation in the average PM density. The goal of the UI is to determine when a PM distribution can be classified as uniform or not 
uniform. To achieve this, the lower limit for the UI that would classify a distribution as uniform was established. The lower limit needed to be connected to a physical phenomenon for it to be logical and appropriate to apply.

The lower limit of the UI that would indicate a uniform PM distribution was established by measuring the absolute difference between the individual values and the corresponding average value. The absolute difference calculations were performed for six data sets and UI values between 0.84 and 0.99 . The absolute difference was used since it is part of the UI calculation, as shown in Eq. 4.13. For each UI value, the average absolute difference between the individual values and the average value was calculated to allow different UI values to be compared. To find the lower limit of the UI that corresponded to a uniform PM distribution, the gradient between the absolute difference calculations for consecutive UI values was calculated. The equation used in this process is given as Eq. 4.15.

$$
\nabla \bar{d}=\frac{\overline{d_{2}}-\overline{d_{1}}}{U I_{2}-U I_{1}}
$$

In Eq. $4.15, \nabla \bar{d}$ is the gradient of the difference between two consecutive points, $\bar{d}$ is the average absolute difference between the individual analysis points and the corresponding average value for one UI value, and $U I$ is the corresponding UI value. The calculation of $\bar{d}$ is completed using Eq. 4.16.

$$
\bar{d}=\frac{\sum_{i=1}^{n}\left|w_{i}-\overline{w_{l}}\right|}{n}
$$

In Eq. $4.16, w_{i}$ is the PM loading value in one analysis point, $\overline{w_{l}}$ is the average of the $w_{i}$ values, and $n$ is the number of $w_{i}$ values. This is similar to Eq. 4.13.

The purpose of the gradient calculations was to identify when the increase in the absolute differences in the data sets between two UI values was the largest. The first gradient value in each of the six data sets that was greater than the gradients calculated for adjacent UI values was considered to be the UI value that represented a non-uniform distribution. The maximum local gradient was used instead of the maximum gradient since the transition between a uniform and non-uniform data set was taken to be gradual. The largest local gradient would indicate when the gradual change had taken place. When the UI values that had the greatest gradient values for the six data sets were averaged together, the result was that the PM distribution at a UI value of 0.93 was the first distribution that was no longer uniform. This indicates that a UI value of 0.94 should be considered the lower limit of the UI for a uniform distribution. The change in the gradient is the physical phenomenon that is currently used to indicate when a 
distribution is no longer uniform. This is a subjective process currently, and more research will be required in the future to validate this conclusion. The future research is discussed in Chapter 8.

\subsection{Data Correlation Check}

The TAS7000 generates 3D scans of a substrate by rotating the substrate. The rotation of the substrate can cause additional errors in the data if the starting point for the rotation of each axial section that is scanned is not identical. This can be caused by the substrate slipping inside the holding fixture, or the motor that rotates the substrate malfunctioning. A method to check the data correlation was developed to ensure that each axial section that is scanned is properly aligned. The value used to judge the alignment of the axial sections is the coefficient of correlation. The valid range of the coefficient of correlation is -1 to 1 [41]. A value of 1 indicates that the two axial sections are in perfect alignment.

The developed method uses one column of data from the data matrix generated by the TAS7000 for each axial section. The column is in a plane that passes through the centerline of the substrate. The method compares one column from one axial section to the same column in the next axial section. The correlation of one axial section to the next is completed in sequential order ( 1 to 2,2 to 3 , etc.) for all of the axial sections in the substrate. If the two columns are not in alignment, the coefficient of correlation will be near 0.90. Experimental data has shown that the coefficient of correlation is between 0.90 and 1 for data sets that are aligned. The coefficient of correlation equation was found in reference [41], as is repeated here as Eq. 4.17.

$$
r=\frac{S S X Y}{\sqrt{S S X} \sqrt{S S Y}}
$$

The equation for $S S X Y$ is given as Eq. 4.18, the equation for $S S X$ is given as Eq. 4.19, and the equation for $S S Y$ is given as Eq. 4.20.

$$
\begin{gathered}
S S X Y=\sum_{i=1}^{n}\left(X_{i}-\bar{X}\right)\left(Y_{i}-\bar{Y}\right) \\
S S X=\sum_{i=1}^{n}\left(X_{i}-\bar{X}\right)^{2}
\end{gathered}
$$




$$
S S Y=\sum_{i=1}^{n}\left(Y_{i}-\bar{Y}\right)^{2}
$$

In Eqs. 4.18, 4.19, and 4.20, $X_{i}$ is one sample point in the column of data from the first axial section being compared, $\bar{X}$ is the average of the sample points in the column of data from the first axial section being compared, $Y_{i}$ is one sample point in the column of data from the second axial section being compared, $\bar{Y}$ is the average of the sample points in the column of data from the second axial section being compared, and $n$ is the number of sample points in the column of data.

Examples of the functionality of the developed method to check the correlation of the data are provided in Figures 4.5 and 4.6. The data in Figure 4.5 is taken from the loading scan in Test 1 of Phase 2 and the data in Figure 4.6 is taken from the loading scan in Test 1 of Phase 1. The top plot in each figure is the data correlation value, with the range of 0 to 1 . This range will show all large and small shifts in data. Showing the whole range of -1 to 1 could make it difficult to see minor shifts in the data. The bottom plot in each figure is the data that was used in the calculation of the coefficient of correlation. It represents the axial PM distribution, with units of grams per liter. The data set in Figure 4.5 shows good correlation between the axial sections. The coefficient of correlation for the data in-between the channel plugs is above 0.90. The data set in Figure 4.6 does not show a good correlation. In the bottom plot of Figure 4.6, around a $Z^{*}$ value of 0.45 , there is a noticeable shift in the data. The coefficient of correlation detected that shift, as indicated by the coefficient of correlation being below 0.90 at a $Z^{*}$ value of 0.45 . The coefficient of correlation showed another shift near a $Z^{*}$ value of 0.80 . The coefficient of correlation at $Z^{*}$ values of $0.21,0.62$, and 0.75 , is slightly lower than the surrounding coefficient of correlations. The data in the bottom plot of Figure 4.6 show slight data shifts at these points as well. The developed data correlation evaluation method functioned correctly for detecting alignment issues in the data. The complete results for the data correlation checks are presented in Appendix L. A majority of the data sets showed evidence of possible shifting. It is unknown if the variation in the coefficient of correlation in those data sets is caused by shifting or experimental noise in the data. It was also found that the coefficient of correlation has more variation in it when the average PM density in the substrate was lower. The data sets that had the least amount of variation were:

- $\quad$ The loading scan from Phase 2 Test 1.

- $\quad$ The active regeneration scan from Phase 2 Test 3.

- $\quad$ The active regeneration scan from Phase 2 Test 4.

- $\quad$ The second loading scan from Phase 2 Test 6. 
- The passive oxidation scan from Phase 2 Test 7.
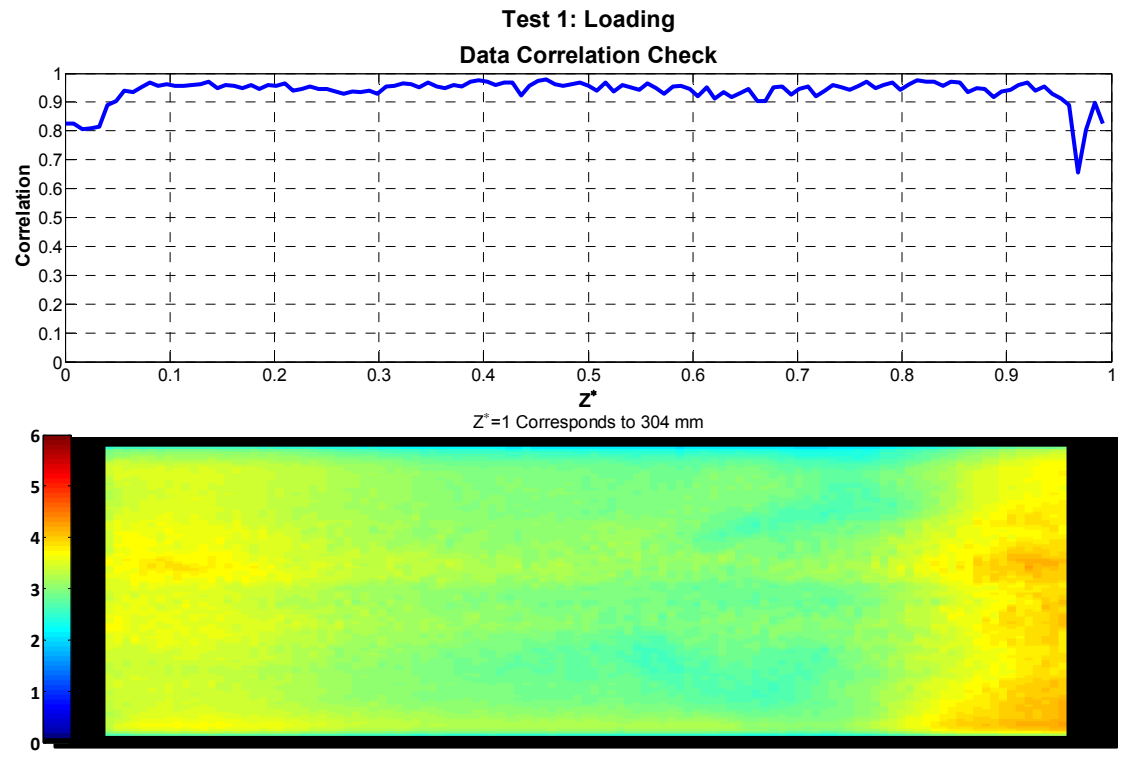

Figure 4.5: Data Correlation: Good Data Set- Taken from Test 1 Phase 2
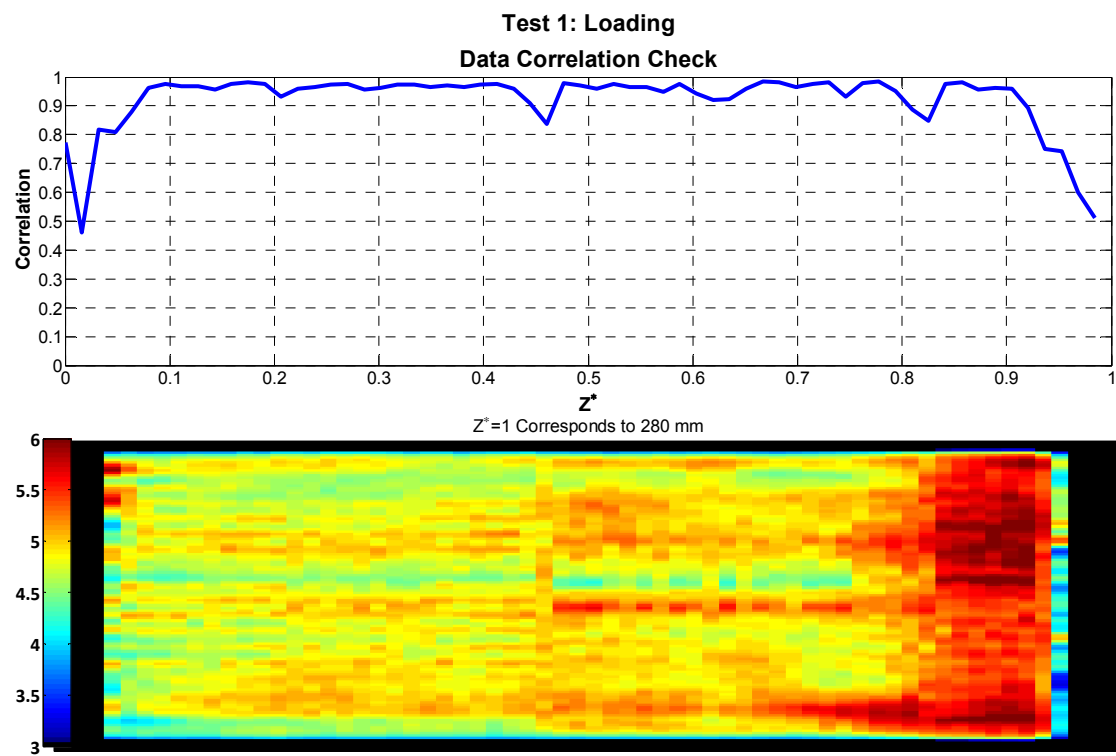

Figure 4.6: Data Correlation: Shifted Data Set- Taken from Test 1 Phase 1 


\section{Chapter 5. Experimental Matrix and Procedures ${ }^{3}$}

This chapter discusses the test matrices and procedures that were used during Phase 1 and 2 of the experimental work conducted. The overall test goals, developed test matrix, engine operating conditions (EOCs), and the procedures used during testing will be discussed. The two phases will be discussed independently since they were designed with different goals in mind. The two phases also used two different engines. Phase 1 work was completed using the 2010 Cummins ISB and Phase 2 work was completed using the 2007 Cummins ISL.

\subsection{Phase 1: Experimental and Methodology Development}

There were three objectives associated with the main goal of Phase 1, which were mentioned in Chapter 1. The main goal was to develop an understanding of the system used to measure the PM distribution, as well as to develop the analysis methods that would be required to characterize PM distribution The first objective was to develop the methods and instrumentation that would be used to conduct PM distribution experiments. The second objective was to evaluate the capability of the TAS7000 to measure the PM distribution. The third objective was to develop a method that can be used to evaluate and quantify the PM distribution. The resulting method was presented in Chapter 4. The test matrix for Phase 1 was developed with these three objectives in mind. The developed test matrix is shown in Figure 5.1 and it consists of four tests and seven substrate scans. Two substrate scans were the baseline scans taken of the substrates before any PM loading occurred. One substrate scan was taken after loading the substrate to $5 \mathrm{~g} / \mathrm{L}$. Three substrate scans were taken after active regenerations were performed on loaded substrates. The active regenerations were performed at different temperatures and resulted in a different amount of available PM being oxidized. One substrate scan was taken after PM was passively oxidized from a loaded substrate. The points in the test where substrate scans were taken are highlighted in green in Figure 5.1.

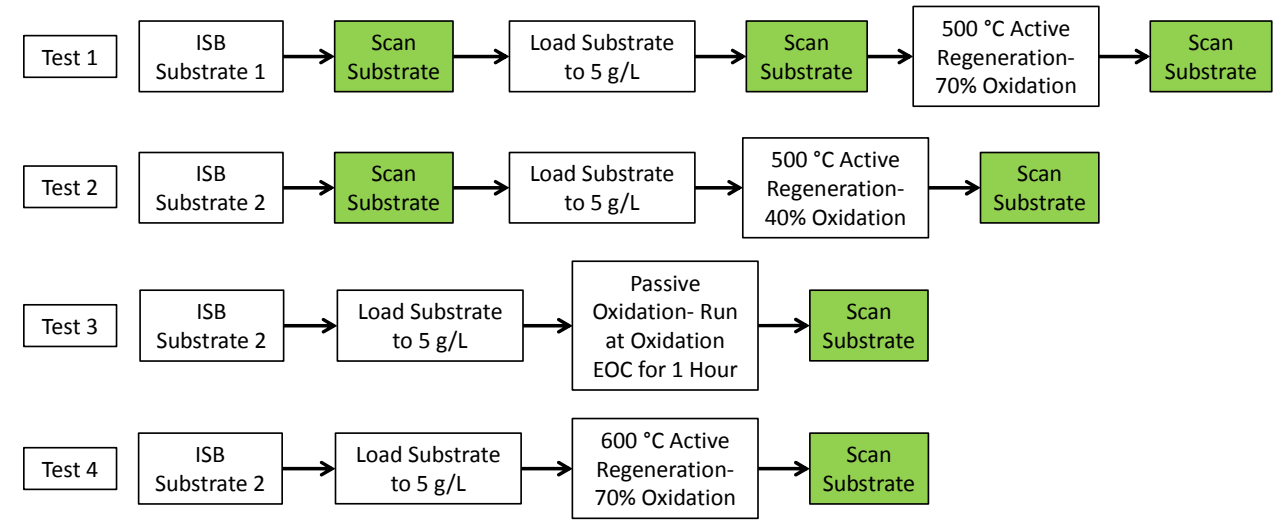

Figure 5.1: Phase 1 Test Matrix

3 Parts of the material contained in this chapter have been submitted, or are being considered for submission, to SAE International for publication consideration. 
The actual tests that were conducted deviated slightly from the ideal test plan shown in Figure 5.1. The PM loadings in the CPF for Tests 1,2 , and 4 were around $5 \mathrm{~g} / \mathrm{L}$, but the PM loading for Test 3 was around $5.8 \mathrm{~g} / \mathrm{L}$. The length of time the CPF was loaded for was similar for tests $1-3$. The engine out PM concentration during the Tests 1 and 2 was around $55 \mathrm{mg} / \mathrm{scm}$, but Test $3 \mathrm{had}$ an engine out PM concentration around $71 \mathrm{mg} / \mathrm{scm}$. This caused the CPF to be loaded quicker. The other deviation was that only $58 \%$ of the available PM was oxidized in Test 1 during the active regeneration, instead of the target of $70 \%$. This was caused by stopping the active regeneration too soon.

In addition to the Phase 1 tests being used to develop the testing methods, evaluate the TAS7000, and develop an analysis method, the individual tests were designed to accomplish certain objectives. The objectives of each test in the Phase 1 test matrix are given in Table 5.1. Overall, the tests were designed develop an initial understanding of PM distribution trends. The trends found for Phase 1 were explored further in Phase 2.

Table 5.1: Objectives of Phase 1 Tests

\begin{tabular}{|c|c|}
\hline Test & Objectives \\
\hline \multirow{3}{*}{1} & Determine the PM Distribution Trends After Loading \\
\hline & $\begin{array}{l}\text { View the PM Distribution Trends After a } 500{ }^{\circ} \mathrm{C} \text { Active Regeneration with } \\
70 \% \text { of the Available PM Oxidized }\end{array}$ \\
\hline & $\begin{array}{l}\text { Compare the PM Distribution After an Active Regeneration to the PM } \\
\text { Distribution After Loading }\end{array}$ \\
\hline \multirow{3}{*}{2} & $\begin{array}{l}\text { Determine the PM Distribution Trends After a } 500{ }^{\circ} \mathrm{C} \text { Active Regeneration } \\
\text { with } 40 \% \text { of the Available PM Oxidized }\end{array}$ \\
\hline & $\begin{array}{l}\text { Compare the PM Distribution After an Active Regeneration to the PM } \\
\text { Distribution After Loading }\end{array}$ \\
\hline & $\begin{array}{l}\text { Compare the PM Distribution Resulting from } 40 \text { and } 70 \% \text { of the Available } \\
\text { PM being Oxidized During an Active Regeneration }\end{array}$ \\
\hline \multirow{3}{*}{3} & Determine the PM Distribution Trends After a Passive Oxidation \\
\hline & $\begin{array}{l}\text { Compare the PM Distribution After a Passive Oxidation to the PM } \\
\text { Distribution After Loading }\end{array}$ \\
\hline & $\begin{array}{l}\text { Compare the PM Distribution Resulting from Passive Oxidation and Active } \\
\text { Regeneration Conditions }\end{array}$ \\
\hline \multirow{3}{*}{4} & $\begin{array}{l}\text { Determine the PM Distribution Trends After a } 600{ }^{\circ} \mathrm{C} \text { Active Regeneration } \\
\text { with } 70 \% \text { of the Available PM Oxidized }\end{array}$ \\
\hline & $\begin{array}{l}\text { Compare the PM Distribution After an Active Regeneration to the PM } \\
\text { Distribution After Loading }\end{array}$ \\
\hline & Compare the PM Distribution After a 500 and $600{ }^{\circ} \mathrm{C}$ Active Regeneration \\
\hline
\end{tabular}

The EOCs used with the 2010 Cummins ISB to achieve the test plan are given in Table 5.2. The "Weighing" EOC was used to stabilize the temperature of the CPF prior to weighing it during tests. The "Loading" EOC was used to load the substrate with PM. The engine calibration was modified during the "Loading" EOC to increase the engine 
out PM concentration, reducing the amount of time it would take to load a CPF to $5 \mathrm{~g} / \mathrm{L}$. The production engine calibration for the ISB engine does not produce high engine out PM concentrations since it is tuned to produce a higher concentration of oxides of nitrogen $\left(\mathrm{NO}_{\mathrm{X}}\right)$. To increase the engine out PM concentration, the fuel rail pressure of the engine was lowered, which reduces the combustion efficiency enabling the production of PM. The "Oxidation" EOC was used for all active regenerations and passive oxidations. For active regeneration tests, the CPF temperature was increased by using in-cylinder fuel dosing. In-cylinder fuel dosing consists of injecting fuel on the exhaust stroke of the engine. This extra fuel will produce an exothermic reaction across the DOC, increasing the temperature of the exhaust gas at the inlet to the CPF.

Table 5.2: ISB Engine Operating Conditions

\begin{tabular}{|c|c|c|c|c|c|c|c|c|}
\hline \multirow{2}{*}{ EOC } & Speed & Load & $\begin{array}{c}\text { Percent } \\
\text { of Full } \\
\text { Load }\end{array}$ & $\begin{array}{c}\mathrm{CPF} \\
\text { Space } \\
\text { Velocity }\end{array}$ & $\begin{array}{c}\text { Average } \\
\text { CPF } \\
\text { Temp }\end{array}$ & $\begin{array}{c}\mathrm{O}_{2} \\
\text { Conc. } \\
\text { Into } \\
\text { CPF }\end{array}$ & $\begin{array}{c}\text { Engine } \\
\text { Out } \\
\text { PM } \\
\text { Conc. }\end{array}$ & $\begin{array}{c}\mathrm{NO}_{\mathrm{X}} \\
\text { Conc. } \\
\text { into } \\
\text { CPF }\end{array}$ \\
\cline { 2 - 10 } & $\mathrm{RPM}$ & $\mathrm{Nm}$ & $\%$ & $1 \mathrm{k} / \mathrm{hr}$ & ${ }^{\circ} \mathrm{C}$ & $\begin{array}{c}\% \\
\mathrm{mg} / \mathrm{scm}\end{array}$ & $\mathrm{mg} / \mathrm{scm}$ \\
\hline Weighing & 1200 & 180 & 20 & 97 & 272 & 13.6 & 2 & 905 \\
\hline Loading & 2700 & 155 & 17 & 310 & 315 & 13.4 & 71 & 197 \\
\hline Oxidation & 1400 & 440 & 49 & 177 & 372 & 7.9 & 8 & 901 \\
\hline
\end{tabular}

The full procedure used during Phase 1 testing is shown in Figure 5.2. The developed procedure is based on the work of Hutton et al. [42], Shiel et al. [43], and Pidgeon et al. [44]. There are multiple paths that can be followed with the test procedure, and each path depends on the test that is being performed. Prior to any CPF weights being taken, the "Weighing" EOC is run for 30 minutes to stabilize the CPF Temperature. Austin, in reference [36], found that the temperature of the CPF significantly impacted the CPF mass measurement. The "Weighing" EOC is used because the "Loading" EOC would have loaded $1.5 \mathrm{~g} / \mathrm{L}$ of $\mathrm{PM}$ into the $\mathrm{CPF}$ during the temperature stabilization time, possibly hiding or altering the PM distribution trends that resulted from active regeneration and passive oxidation. Prior to shipping the substrate to Cummins for scanning, and after receiving a shipped substrate from Cummins, the substrate was baked and weighed with the instrumentation and can removed. The baking procedure is described in Appendix B.

The passive oxidation test, Test 3, did not follow the exact procedure for passive oxidation tests, shown as option 3, given in Figure 5.2. This is because the original goal of Test 3 was to determine the oxidation rates for the "Weighing" and "Oxidation" EOC. The revised option 3 is shown in Figure 5.3. After the ramp up stage shown for option 3, the "Weighing" EOC was run for one hour. Then the CPF was weighed. A second ramp up was performed, and then the "Oxidation" EOC was run for one hour. The rest of the revised procedure follows the original option 3 procedure. Test 3 was used as a passive oxidation test because it was found that the "Weighing" EOC would not oxidize any PM, and the "Oxidation" EOC oxidized over $40 \%$ of the available PM. Since the "Weighing" 
EOC did not oxidize any PM, the added steps would not impact the ability to discuss the passive oxidation that resulted from the "Oxidation" EOC.

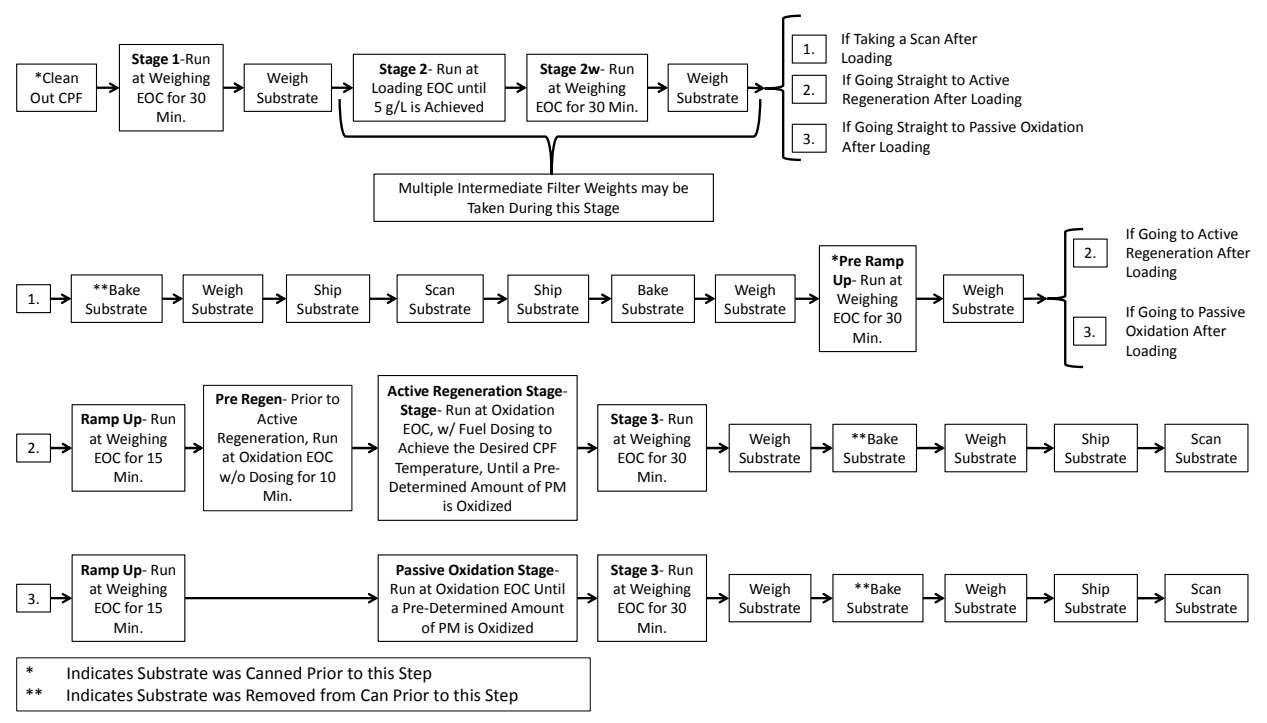

Figure 5.2: Phase 1 Test Procedure

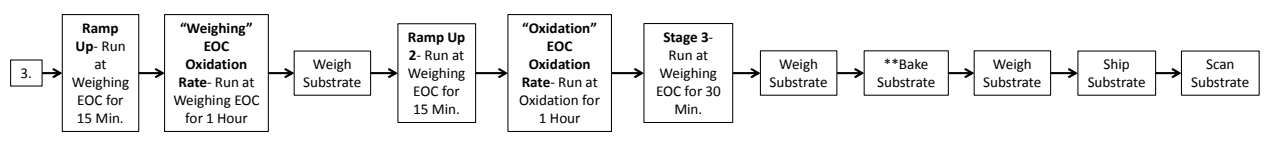

Figure 5.3: Revised Option 3 for Phase 1 Test Procedure

\subsection{Phase 2: PM Distribution Trend Analysis}

The tests developed for Phase 2 were primarily designed based on the results found during Phase 1. The general results from Phase 1 include: non-uniform PM distributions in the axial direction, similar PM distributions for active regeneration and passive oxidation when $40-45 \%$ of the available PM was oxidized, more uniform PM distribution near the centerline of the substrate, a possible percentage of PM oxidation that would result in a uniform distribution, and a PM plug possibly forming near the end of channels after multiple tests. The results from Phase 1 will be discussed in greater detail in Chapters 6 and 7. Additionally, there was interest in investigating trends found in previous data collected at MTU and in understanding the PM distribution trends for passive oxidation and active regeneration engine test conditions being modeled at MTU. The trends of interest include: a change in the slope of the pressure drop curve during passive oxidation events when approximately $30 \%$ of the PM is oxidized, at similar levels of PM retained in the CPF the pressure drop across the CPF is lower after PM is oxidized than before PM is oxidized, and the impact of using accelerated loading during Phase 1. Lastly, there was interest in analyzing the repeatability of the substrate scans and the effect of shipping the substrate. Using this information, five objectives were developed for Phase 2. The first objective was to verify and validate the PM distribution trends that were found during Phase 1. The second objective was to answer questions that existed after the Phase 1 testing was completed. The third objective was to compare the 
distributions of the ISL and ISB substrates. The fourth objective was to gain a better and more thorough understanding of the PM distribution trends that result from loading, passive oxidation, active regeneration, and post loading conditions. This objective was accomplished by running tests similar to those completed by Hutton [42], Shiel [43], and Pidgeon [44], since that data has been modeled at MTU [45]. The PM distribution trends that are experimentally measured can then be compared to the trends produced by the MTU 1D model. The fifth and final objective was to evaluate the experimental procedures and instruments that were used to measure the PM distribution. The shipping process and repeatability of the TAS7000 were the focus of the fifth objective.

A total of 11 tests were developed for Phase 2, nine of which were designed to accomplish objectives 1-4 listed in the previous paragraph. Those nine tests are shown in Figure 5.4. The places where substrate scans were performed are highlighted in green. There were a total of 17 substrate scans taken. Four baseline scans were completed, one for each of the four substrates. Three substrate scans were taken after loading conditions. Four scans were taken after passive oxidation conditions. Four scans were taken after active regeneration conditions. Two scans were taken after post loading conditions.

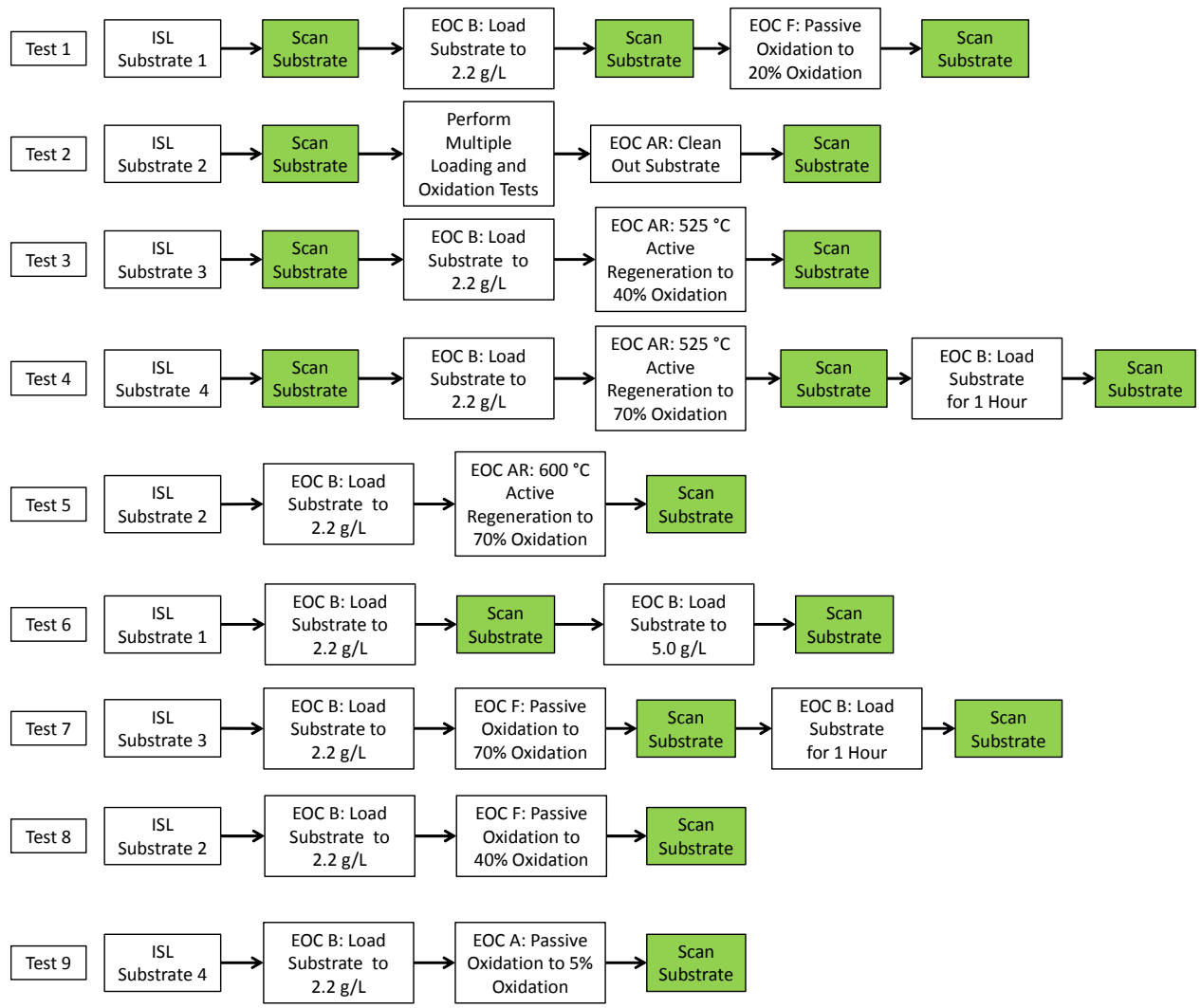

Figure 5.4: Phase 2 Test Matrix

Similar to Phase 1, the actual tests conducted during Phase 2 deviated from the test plan design slightly. The PM loading goal for each test, except Test 2 which did not have a goal, was $2.2 \mathrm{~g} / \mathrm{L}$. This was not achieved for any test. Test 1 was loaded to $3 \mathrm{~g} / \mathrm{L}$ due to 
it taking less time than predicted to load the substrate and the pressure drop across the CPF being lower than previous experiments had shown. The substrate loading rate was constant for the rest of the tests, and the amount of time the CPF was loaded was shortened as much as possible to try and hit $2.2 \mathrm{~g} / \mathrm{L}$ as the peak loading. However, due to the amount of measurements taken during the loading portion of the test, the time could not be shorted far enough to hit $2.2 \mathrm{~g} / \mathrm{L}$. The list of measurements, and the order in which they are taken, are given in Appendix D. The additional loading is not a problem. The CPF loading was also affected by residual PM and ash that was not cleaned out at the start of the test. This is discussed further in Appendix G. The actual PM loading in the substrate at the completion of the loading phase of the test was between 2.8 and $3.9 \mathrm{~g} / \mathrm{L}$. The amount of PM oxidized for Tests 2-5 was lower than planned. This is because the cleanout or active regeneration portion of test was not run for a long enough period of time. Test 2 was supposed to be a cleanout, but only $81 \%$ of available PM was oxidized. This resulted in approximately $1 \mathrm{~g} / \mathrm{L}$ of PM being left in the substrate. Tests 3,4 , and 5 were supposed to achieve 40, 70, and 70\% PM oxidation respectively. Instead, Tests 3, 4 , and 5 achieved 26, 45, and 52\% respectively, which will be enough of a difference to see how the PM distribution changes as the percentage of PM oxidized through active regeneration increases.

The other two tests conducted during Phase 2 were designed to accomplish the fifth objective and validate portions of the experimental methods developed during Phase 1 . The first test was a scanning repeatability test. This test consisted of scanning ISB Substrate 2, from Test 4 of Phase 1, a total of five times. This would allow the repeatability of the TAS7000 to be measured. The second test, which also used ISB Substrate 2 from Test 4 of Phase 1, was a shipping orientation test. This test consisted of shipping the substrate in three different orientations, using a pallet to ensure and maintain its directionality. The three directions were: inlet pointed up, inlet pointed to the side, and inlet pointed down. A substrate scan was performed after each shipping orientation. The results from this test indicated if the shipping orientation had any effect on the PM distribution. Neither of these tests consisted of running a test in the MTU test cell. All of the scans were conducted at Cummins with shipping of the substrates between MTU and Cummins.

Similar to Phase 1, each test had individual objectives that were used to accomplish the objectives for Phase 2. There are five groups of tests that were performed for Phase 2: a PM Loading test, a Multiple Loading Cleanout test, Passive Oxidation tests, Active Regeneration tests, and System and Methods tests. The objectives for the PM Loading test are shown in Table 5.3 and the objectives for the Multiple Loading Cleanout test are shown in Table 5.4. The Passive Oxidation tests and objectives are shown in Table 5.5 and the Active Regeneration tests and objectives are shown in Table 5.6. The System and Methods tests and objectives are shown in Table 5.7. 
Table 5.3: PM Loading Test Objectives

\begin{tabular}{|c|l|}
\hline Test & \multicolumn{1}{c|}{ Objectives } \\
\hline \multirow{4}{*}{6} & Determine the PM Distribution Trends at 2 Different Loadings, 2.2 and $5 \mathrm{~g} / \mathrm{L}$ \\
\cline { 2 - 3 } & Compare the $5 \mathrm{~g} / \mathrm{L}$ Loading from the Phase 1 and Phase 2 \\
\cline { 2 - 3 } & Determine How the PM Distribution Changes as a Function of Loading \\
\cline { 2 - 2 } & $\begin{array}{l}\text { Determine if the Accelerated Loading Used During Phase 1 Produced Results } \\
\text { Similar to a Typical Engine Condition Loading }\end{array}$ \\
\hline
\end{tabular}

Table 5.4: Multiple Loading Test Cleanout Objectives

\begin{tabular}{|c|c|}
\hline Test & Objectives \\
\hline \multirow{3}{*}{2} & Determine the PM Distribution After a Substrate Cleanout \\
\hline & $\begin{array}{l}\text { Determine if Running Multiple Tests on One Substrate Results in a PM Plug } \\
\text { Forming Near the Outlet of the Substrate }\end{array}$ \\
\hline & Determine Effectiveness of CPF Cleanout \\
\hline
\end{tabular}

Table 5.5: Passive Oxidation Tests and Objectives

\begin{tabular}{|c|c|}
\hline Tests & Objectives \\
\hline \multirow{3}{*}{1} & Determine the PM Distribution Trends After Loading \\
\hline & $\begin{array}{l}\text { Determine the PM Distribution Trends After a Passive Oxidation with } 20 \% \\
\text { of the PM Oxidized }\end{array}$ \\
\hline & Compare the PM Distribution After a Passive Oxidation and Loading \\
\hline \multirow{5}{*}{7} & $\begin{array}{l}\text { Determine the PM Distribution Trends After a Passive Oxidation with } 70 \% \\
\text { of the PM Oxidized }\end{array}$ \\
\hline & $\begin{array}{l}\text { Determine the PM Distribution Trends After Performing } 1 \text { Hour of Post } \\
\text { Loading }\end{array}$ \\
\hline & $\begin{array}{l}\text { Compare the PM Distribution Trends from Active Regenerations and Passive } \\
\text { Oxidations Performed with the Same Amount of Available PM Oxidized }\end{array}$ \\
\hline & $\begin{array}{l}\text { Compare the PM Distribution Resulting from Passive Oxidations with } 20 \text { and } \\
70 \% \text { of the Available PM being Oxidized }\end{array}$ \\
\hline & $\begin{array}{l}\text { Compare the PM Distribution Trends After Post Loading to the Passive } \\
\text { Oxidation and the Loading Distribution Trends }\end{array}$ \\
\hline \multirow{4}{*}{8} & $\begin{array}{l}\text { Determine the PM Distribution Trends After a Passive Oxidation with } 40 \% \\
\text { of the PM Oxidized }\end{array}$ \\
\hline & $\begin{array}{l}\text { Compare the PM Distribution Trends from Active Regenerations and Passive } \\
\text { Oxidations Performed with the Same Amount of Available PM Oxidized }\end{array}$ \\
\hline & $\begin{array}{l}\text { Compare the PM Distribution Resulting from Passive Oxidations with 20, } \\
40 \text {, and } 70 \% \text { of the Available PM being Oxidized }\end{array}$ \\
\hline & $\begin{array}{l}\text { Compare the Passive Oxidation Performed During Phase } 1 \text { the Passive } \\
\text { Oxidation from Phase } 2 \text { with } 40 \% \text { of the Available PM being Oxidized }\end{array}$ \\
\hline \multirow{3}{*}{9} & $\begin{array}{l}\text { Determine the PM Distribution Trends After a Passive Oxidation with } 5 \% \text { of } \\
\text { the PM Oxidized }\end{array}$ \\
\hline & Determine How a Balance Point Affects the PM Distribution \\
\hline & $\begin{array}{l}\text { Compare the PM Distributions after a } 5 \% \text { Passive Oxidation to a } 20,40 \text {, and } \\
70 \% \text { Passive Oxidation }\end{array}$ \\
\hline
\end{tabular}


Table 5.6: Active Regeneration Tests and Objectives

\begin{tabular}{|c|c|}
\hline Tests & Objectives \\
\hline \multirow{4}{*}{3} & $\begin{array}{l}\text { Determine the PM Distribution Trends After a } 525{ }^{\circ} \mathrm{C} \text { Active Regeneration } \\
\text { with } 40 \% \text { of the Available PM Oxidized }\end{array}$ \\
\hline & $\begin{array}{l}\text { Compare the PM Distribution After an Active Regeneration to the PM } \\
\text { Distribution After Loading }\end{array}$ \\
\hline & $\begin{array}{l}\text { Compare the PM Distribution Trends from Active Regenerations and Passive } \\
\text { Oxidations Performed with the Same Amount of Available PM Oxidized }\end{array}$ \\
\hline & $\begin{array}{l}\text { Compare the } 500{ }^{\circ} \mathrm{C} \text { Active Regeneration Performed During Phase } 1 \text { to the } \\
525{ }^{\circ} \mathrm{C} \text { Active Regeneration from Phase } 2\end{array}$ \\
\hline \multirow{7}{*}{4} & $\begin{array}{l}\text { Determine the PM Distribution Trends After a } 525{ }^{\circ} \mathrm{C} \text { Active Regeneration } \\
\text { with } 70 \% \text { of the Available PM Oxidized }\end{array}$ \\
\hline & $\begin{array}{l}\text { Determine the PM Distribution Trends After Performing } 1 \text { Hour of Post } \\
\text { Loading }\end{array}$ \\
\hline & $\begin{array}{l}\text { Compare the PM Distribution After an Active Regeneration to the PM } \\
\text { Distribution After Loading }\end{array}$ \\
\hline & $\begin{array}{l}\text { Compare the PM Distribution Trends from Active Regenerations and Passive } \\
\text { Oxidations Performed with the Same Amount of Available PM Oxidized }\end{array}$ \\
\hline & $\begin{array}{l}\text { Compare the PM Distribution Resulting from Active Regenerations with } 40 \\
\text { and } 70 \% \text { of the Available PM being Oxidized }\end{array}$ \\
\hline & $\begin{array}{l}\text { Compare the PM Distribution Trends After Post Loading to the Active } \\
\text { Regeneration and Loading Distribution Trends }\end{array}$ \\
\hline & $\begin{array}{l}\text { Compare the } 500{ }^{\circ} \mathrm{C} \text { Active Regeneration Performed During Phase } 1 \text { to the } \\
525^{\circ} \mathrm{C} \text { Active Regeneration from Phase } 2\end{array}$ \\
\hline \multirow{5}{*}{5} & $\begin{array}{l}\text { Determine the PM Distribution Trends After a } 600{ }^{\circ} \mathrm{C} \text { Active Regeneration } \\
\text { with } 70 \% \text { of the Available PM Oxidized }\end{array}$ \\
\hline & $\begin{array}{l}\text { Compare the PM Distribution After an Active Regeneration to the PM } \\
\text { Distribution After Loading }\end{array}$ \\
\hline & $\begin{array}{l}\text { Compare the PM Distribution Trends from Active Regenerations and Passive } \\
\text { Oxidations Performed with the Same Amount of Available PM Oxidized }\end{array}$ \\
\hline & Compare the PM Distribution After a 525 and $600^{\circ} \mathrm{C}$ Active Regeneration \\
\hline & $\begin{array}{l}\text { Compare the } 600{ }^{\circ} \mathrm{C} \text { Active Regeneration Performed During Phase } 1 \text { to the } \\
600{ }^{\circ} \mathrm{C} \text { Active Regeneration from Phase } 2\end{array}$ \\
\hline
\end{tabular}

Table 5.7: System and Methods Tests and Objectives

\begin{tabular}{|c|l|}
\hline Test & \multicolumn{1}{c|}{ Objective } \\
\hline Repeatability & Determine the Repeatability of the Advantest TAS7000 \\
\hline Shipping & Determine the Effects of Substrate Orientation During Shipping \\
\hline
\end{tabular}

The EOCs used with the 2007 Cummins ISL engine to achieve the test matrix given in Figure 5.4 are given in Table 5.8. These engine points were originally established by Hutton [3], Shiel [4], and Pidgeon [5]. All of the PM loading is accomplished with EOC B. Passive Oxidation tests used EOC A and F. The balance point test, that is part of the 
Passive Oxidation tests, was completed using EOC A. A balance point is defined as a test condition where the PM rate into the CPF is equal to the PM oxidation rate in the $\mathrm{CPF}$, and therefore no PM loading occurs. Active Regeneration tests used EOC AR, and then in-cylinder fuel dosing was used to increase the average CPF temperature. No modifications were made to the engine calibration of the ISL during Phase 2 work.

Table 5.8: ISL Engine Operating Conditions [3-5]

\begin{tabular}{|c|c|c|c|c|c|c|c|c|}
\hline \multirow{2}{*}{ EOC } & Speed & Load & $\begin{array}{c}\text { Percent } \\
\text { of Full } \\
\text { Load }\end{array}$ & $\begin{array}{c}\mathrm{CPF} \\
\text { Space } \\
\text { Velocity }\end{array}$ & $\begin{array}{c}\text { Average } \\
\mathrm{CPF} \\
\text { Temp }\end{array}$ & $\begin{array}{c}\mathrm{O}_{2} \\
\text { Conc. } \\
\text { Into } \\
\mathrm{CPF}\end{array}$ & $\begin{array}{c}\text { Engine } \\
\text { Out } \\
\mathrm{PM} \\
\text { Conc. }\end{array}$ & $\begin{array}{c}\mathrm{NO}_{\mathrm{X}} \\
\text { Conc. } \\
\text { into } \\
\mathrm{CPF}\end{array}$ \\
\hline RPM & $\mathrm{Nm}$ & $\%$ & $1 \mathrm{k} / \mathrm{hr}$ & ${ }^{\circ} \mathrm{C}$ & $\%$ & $\mathrm{mg} / \mathrm{scm}$ & $\mathrm{mg} / \mathrm{scm}$ \\
\hline $\mathrm{B}$ & 2100 & 200 & 12 & 212 & 266 & 13.7 & 21.5 & 175 \\
\hline $\mathrm{F}$ & 1200 & 280 & 17 & 89 & 256 & 12.5 & 5.8 & 576 \\
\hline $\mathrm{AR}$ & 1400 & 460 & 32 & 151 & 350 & 8.7 & 8.1 & 332 \\
\hline
\end{tabular}

The test procedure that was followed during Phase 2 testing is shown in Figure 5.5. This test procedure is based on the work of Shiel et al. [43] and Pidgeon et al. [44], similar to the Phase 1 procedure. The biggest difference between the Phase 1 and Phase 2 procedures, is that Phase 2 does not include a "Weighing" EOC. The loading condition, EOC B, is used to stabilize the CPF temperature prior to taking mass measurements. This was done to match the tests performed previously in the MTU test cell. The Phase 2 test procedure also includes options for post loading on the substrate, which was not a part of the Phase 1 test procedure. The test procedure allows for multiple types of tests to be run, depending on the options chosen. No deviations from this test plan were made. 


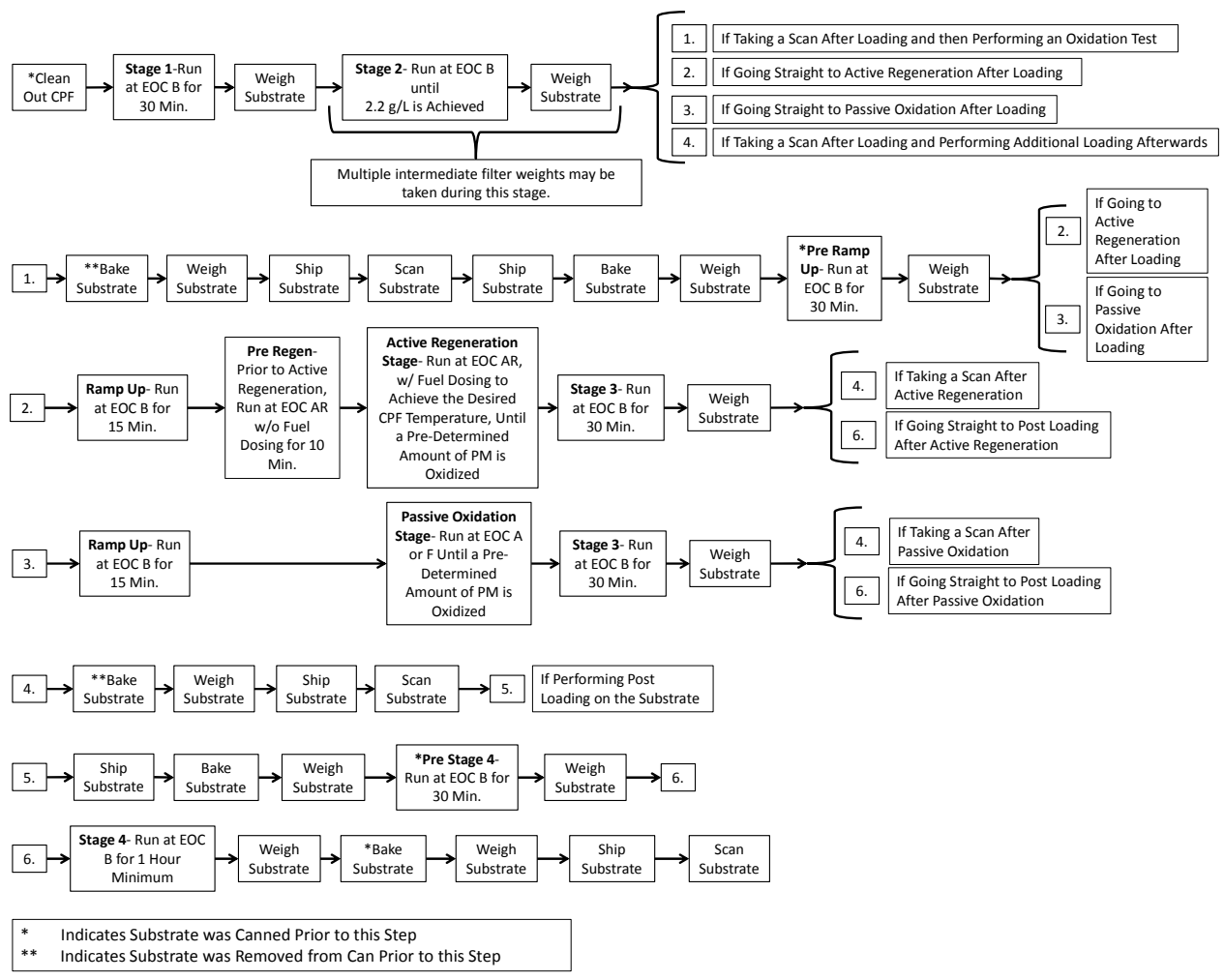

Figure 5.5: Phase 2 Test Procedure 


\section{Chapter 6. Experimental Results ${ }^{4}$}

This chapter presents and discusses the results from experiments that were conducted as part of the investigation into PM distribution trends in a CPF. There are eight groups of results that will be discussed. The first group (6.1) was used to ensure that the developed data analysis method functioned as intended. The second group (6.2) focused on the change in the results due to changes in the PM density calculation process. This group compares the results of the original calculation process to the developed calculation process that was discussed in section 4.1. The third group (6.3) was used to analyze the repeatability of the TAS7000. The fourth group (6.4) was used to determine if the orientation of the substrate during shipping had any effect on the PM distribution. The fifth (6.5), sixth (6.6), seventh (6.7), and eighth (6.8) groups were used to identify PM distribution trends after PM loading, passive oxidation, active regeneration, and post loading, respectively. The PM distribution trends are the main focus of the experimental work that was conducted. Additional data on the tests conducted are available in the appendices. Appendix E presents a summary of the data collected during each test. Appendix F discusses the PM reaction rates found for the PM distribution experiments. Appendix $\mathrm{G}$ discusses discrepancies in the mass measurements of the substrates. Appendix $H$ provides the pressure drop curves that were generated during the experiments. Appendix I gives the average CPF temperatures during the last 5 minutes of the stage that the substrates were scanned. Appendix $\mathrm{J}$ gives a summary of the emissions data that were collected during each experiment. Appendix $\mathrm{K}$ provides additional PM distribution plots, which are not presented in the main body of the thesis. Appendix L presents the results of the data correlation tests that were performed.

\subsection{Developed Data Analysis Method Functionality}

The data that were collected during Phase 1 of the experimental study discussed in this thesis were used to better understand the capabilities of the TAS7000 system and develop an analysis method for the data that were produced by the TAS7000, as mentioned in section 1.1.1. The experimental matrix and procedures that were used to study the capabilities and develop the analysis method are given in section 5.1. This section shows the result of applying the developed analysis method to five data sets. The ability of the analysis method to identify and quantify PM distribution trends is discussed. The data shown in this section is representative of PM only, as the ash content in the substrate was not measured.

\section{Phase 1 Test 1: Loading}

There were three substrate scans taken during Test 1 . The first scan was of a clean substrate, which was used as the baseline scan for substrate 1. The second scan was taken after loading the substrate to $4.96 \mathrm{~g} / \mathrm{L}$. The third scan was taken after an active

\footnotetext{
${ }^{4}$ Parts of the material contained in this chapter have been submitted, or are being considered for submission, to SAE International for publication consideration.
} 
regeneration was performed on the loaded substrate. The results of the second scan are shown in Figure 6.1. The top four subplots in Figure 6.1 show the PM distribution, in grams per liter, at four different $Z^{*}$ values, $0.125,0.375,0.625$, and 0.875 . The amount of color variation in the top four plots gives an indication of the uniformity of the PM distribution in the radial and angular direction. The bottom plot in Figure 6.1 shows the PM distribution in the axial direction in grams per liter. The data used to generate this plot were taken from a single radial plane, which passed through the centerline of the substrate. The amount of color variation in the bottom plot gives an indication of the axial PM distribution uniformity.

The results in the bottom plot show that for the first $85 \%$ of the axial length, the PM distribution was $4.8 \mathrm{~g} / \mathrm{L}$. In the last $15 \%$ of the axial length the PM density increased to $5.6 \mathrm{~g} / \mathrm{L}$, which is $12 \%$ higher than the substrate average, indicating that more PM was loaded near the outlet of the substrate. This axial PM distribution resulted in an overall axial UI of 0.90 . The results presented in the top four plots show an increase in the PM loading, as indicated by the red regions, in radial sections 3 and 4 between $\Theta$ values of 135 and $225^{\circ}$ for $Z^{*}$ values of $0.125,0.375$, and 0.625 . For a $Z^{*}$ value of 0.875 a region of dark red exists in radial sections 3 and 4 between $\Theta$ values of 180 and $270^{\circ}$, indicating a higher PM loading than the surrounding areas. Even with the regions of higher loading, the overall radial and angular UIs were both 0.96 .

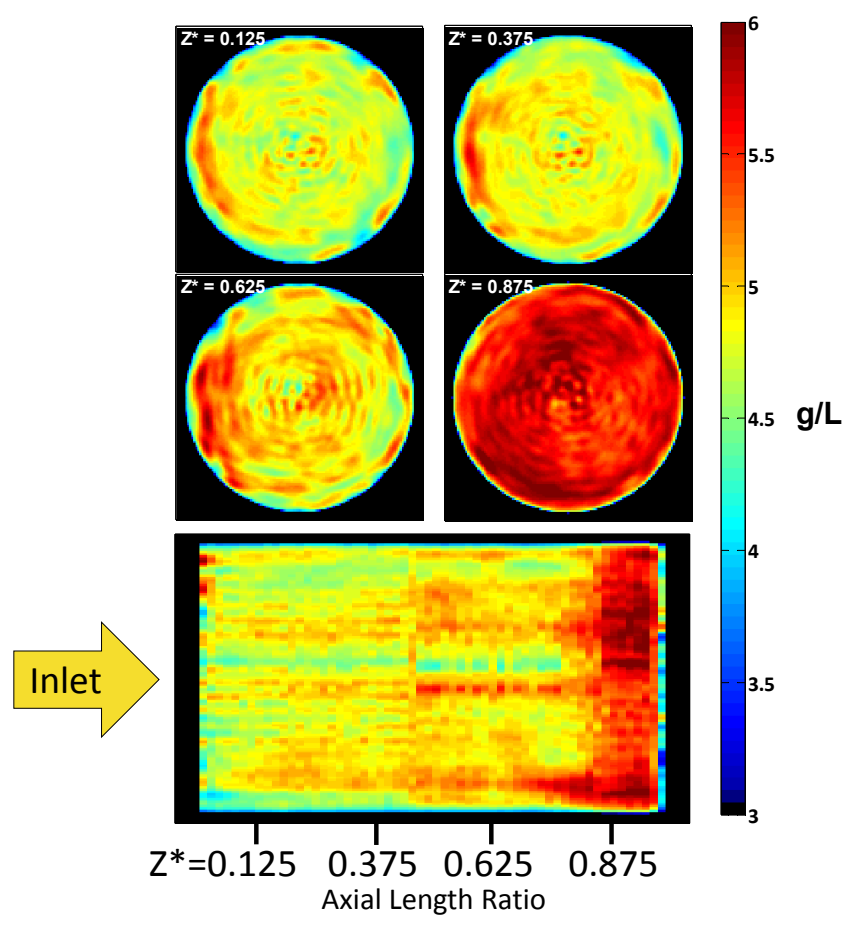

Figure 6.1: Phase 1 Test 1: Loading Scan Results

To further analyze the PM distribution, the axial PM distribution in each radial section was plotted, and the result is shown in Figure 6.2. The y-axis represents the PM density in grams per liter, and the x-axis gives the normalized axial length, $Z^{*}$. The radial 
section number and corresponding dimensions are shown in the top left corner of each plot and the axial uniformity index is given in the top right corner of each plot. The solid blue line in each plot is the average PM density for each axial section and the dashed red line is the $95^{\text {th }} \mathrm{PR}$ for the data. The UI is a measure of the consistency in the blue line and the $95^{\text {th }} \mathrm{PR}$ is a measure of the amount of spread in the data used to generate the blue line. Each plot contains a box containing the average PM density and $95^{\text {th }}$ PR for that radial section. Above radial section 1, the overall radial and angular UIs are given.

The results in Figure 6.2 show that all four radial sections had a similar amount of variation in the axial PM distribution since the axial UI values were all similar. Radial section 4 had a PM density that was 7\% lower than the other three radial sections, which had an average PM density around 4.91- $4.92 \mathrm{~g} / \mathrm{L}$. Although Figure 6.1 shows that the PM density was higher in portions of radial section 3 and 4, radial section 4 also had sample points near the edge of the substrate that had a lower PM density. These sample points caused the average for radial section 4 to be lower than the other radial sections. Figure 6.2 also shows that the $95^{\text {th }} \mathrm{PR}$ increased in each radial section, from $0.38 \mathrm{~g} / \mathrm{L}$ for radial section 1 to $0.99 \mathrm{~g} / \mathrm{L}$ for radial section 4 . This indicates that there is more variation in the PM distribution near the periphery of the substrate than near the center.

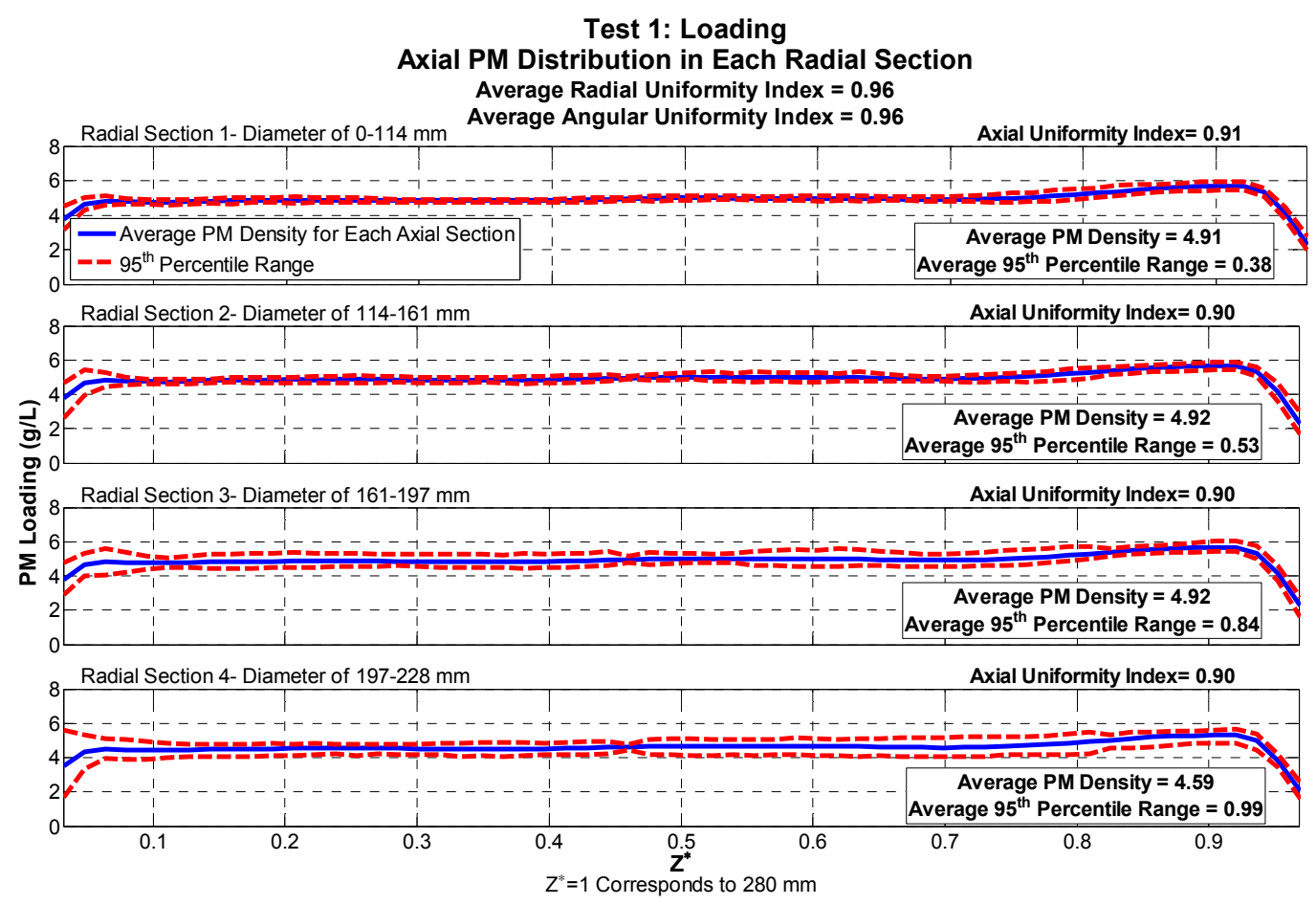

Figure 6.2: Phase 1 Test 1- Loading Axial PM Distribution in Each Radial Section

Table 6.1 gives the regional axial UI, average PM density in grams per liter, and $95^{\text {th }} \mathrm{PR}$ for the average PM density in grams per liter for the four radial sections and four axial segments. The average PM density was found to increase from $4.72 \mathrm{~g} / \mathrm{L}$ in axial segment 1 to $5.10 \mathrm{~g} / \mathrm{L}$ in axial segment 4 , for radial section 1 . The axial segments capture the increase in density shown in Figure 6.1, but the peak value of $5.6 \mathrm{~g} / \mathrm{L}$ is not shown due to 
the size of the axial segment. Due to the consistent increase in PM density from axial segment 1 to 4, axial segments 2 and 3 were found to have a uniform axial UI (above 0.94), indicating that the average PM densities in axial segments 2 and 3 are similar to the average PM density in the entire substrate. The data in Table 6.1 show that the $95^{\text {th }} \mathrm{PR}$ was the lowest for radial section 1 for all four axial segments, which agrees with Figure 6.2 .

Table 6.1: Phase 1 Test 1- Loading Axial PM Distribution

\begin{tabular}{|c|c|c|c|c|c|c|c|c|c|c|c|c|c|}
\hline & \multicolumn{12}{|c|}{ Axial Segment } \\
\hline & & \multicolumn{3}{|c|}{1} & \multicolumn{3}{|c|}{2} & \multicolumn{3}{|c|}{3} & \multicolumn{3}{|c|}{4} \\
\hline & & UI & $\begin{array}{c}\text { Average } \\
\text { PM } \\
\text { Density } \\
(\mathrm{g} / \mathrm{L})\end{array}$ & $\begin{array}{l}95^{\text {th }} \\
\text { PR } \\
(\mathrm{g} / \mathrm{L})\end{array}$ & UI & $\begin{array}{c}\text { Average } \\
\text { PM } \\
\text { Density } \\
(\mathrm{g} / \mathrm{L})\end{array}$ & $\begin{array}{l}95^{\text {th }} \\
\text { PR } \\
(\mathrm{g} / \mathrm{L})\end{array}$ & UI & $\begin{array}{l}\text { Average } \\
\text { PM } \\
\text { Density } \\
(\mathrm{g} / \mathrm{L})\end{array}$ & $\begin{array}{l}95^{\text {th }} \\
\text { PR } \\
(\mathrm{g} / \mathrm{L})\end{array}$ & UI & $\begin{array}{l}\text { Average } \\
\text { PM } \\
\text { Density } \\
(\mathrm{g} / \mathrm{L})\end{array}$ & $\begin{array}{l}95^{\text {th }} \\
\text { PR } \\
(\mathrm{g} / \mathrm{L})\end{array}$ \\
\hline \multirow{4}{*}{ 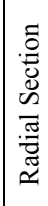 } & 1 & 0.93 & 4.72 & 0.42 & 0.98 & 4.88 & 0.20 & 0.98 & 4.96 & 0.29 & 0.82 & 5.10 & 0.56 \\
\hline & 2 & 0.92 & 4.72 & 0.55 & 0.98 & 4.88 & 0.35 & 0.97 & 4.96 & 0.45 & 0.82 & 5.10 & 0.59 \\
\hline & 3 & 0.91 & 4.73 & 0.98 & 0.96 & 4.88 & 0.73 & 0.95 & 4.97 & 0.80 & 0.82 & 5.11 & 0.69 \\
\hline & 4 & 0.91 & 4.41 & 1.11 & 0.96 & 4.55 & 0.71 & 0.95 & 4.63 & 1.02 & 0.83 & 4.77 & 0.94 \\
\hline
\end{tabular}

Table 6.2 shows the regional radial UI, average PM density in grams per liter, and the $95^{\text {th }}$ PR for the average PM density in grams per liter for the four quadrants and four axial segments. The regions of increased PM loading that are shown in Figure 6.1 are located in quadrants 2 and 3 . For axial segment 1 , which corresponds to the $Z^{*}=0.125$ plot in Figure 6.1, the radial UI was 0.93 in quadrant 2, indicating a non-uniform distribution. This was the only quadrant to have a non-uniform distribution. For axial segments 2 and 3 , which correspond to the $Z^{*}=0.375$ and 0.625 plots in Figure 6.1, the increased PM loading resulted in the $95^{\text {th }}$ PR for quadrant 2 being $38-56 \%$ higher than the adjacent quadrants.

Table 6.2: Phase 1 Test 1- Loading Radial PM Distribution

\begin{tabular}{|c|c|c|c|c|c|c|c|c|c|c|c|c|c|}
\hline & \multicolumn{12}{|c|}{ Axial Segment } \\
\hline & & \multicolumn{3}{|c|}{1} & \multicolumn{3}{|c|}{2} & \multicolumn{3}{|c|}{3} & \multicolumn{3}{|c|}{4} \\
\hline & & UI & $\begin{array}{c}\text { Average } \\
\text { PM } \\
\text { Density } \\
\text { (g/L) }\end{array}$ & $\begin{array}{l}95^{\text {th }} \\
\text { PR } \\
(\mathrm{g} / \mathrm{L})\end{array}$ & UI & $\begin{array}{l}\text { Average } \\
\text { PM } \\
\text { Density } \\
(\mathrm{g} / \mathrm{L})\end{array}$ & $\begin{array}{l}95^{\text {th }} \\
\text { PR } \\
(\mathrm{g} / \mathrm{L})\end{array}$ & UI & $\begin{array}{l}\text { Average } \\
\text { PM } \\
\text { Density } \\
(\mathrm{g} / \mathrm{L})\end{array}$ & $\begin{array}{l}95^{\text {th }} \\
\text { PR } \\
(\mathrm{g} / \mathrm{L})\end{array}$ & UI & $\begin{array}{l}\text { Average } \\
\text { PM } \\
\text { Density } \\
(\mathrm{g} / \mathrm{L})\end{array}$ & $\begin{array}{l}95^{\text {th }} \\
\text { PR } \\
(\mathrm{g} / \mathrm{L})\end{array}$ \\
\hline \multirow{4}{*}{ 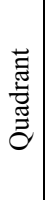 } & 1 & 0.95 & 4.27 & 0.17 & 0.96 & 4.74 & 0.16 & 0.96 & 4.85 & 0.24 & 0.96 & 4.45 & 0.19 \\
\hline & 2 & 0.93 & 4.21 & 0.20 & 0.96 & 4.78 & 0.25 & 0.95 & 4.86 & 0.40 & 0.95 & 4.43 & 0.16 \\
\hline & 3 & 0.94 & 4.26 & 0.13 & 0.96 & 4.85 & 0.16 & 0.96 & 4.94 & 0.25 & 0.96 & 4.48 & 0.17 \\
\hline & 4 & 0.95 & 4.22 & 0.15 & 0.97 & 4.82 & 0.09 & 0.97 & 4.87 & 0.10 & 0.97 & 4.40 & 0.14 \\
\hline
\end{tabular}

The angular PM distribution in each radial section can be plotted for each axial section to further analyze the angular PM distribution, as shown in Figure 6.3. The y-axis represents that PM density in grams per liter, and the $\mathrm{x}$-axis is the angular measurement, 
$\Theta$. The radial section number and corresponding dimensions are shown in the top left corner of each plot, and the angular UI is given in the top right corner of each plot. The solid blue line gives the average PM density for each angular increment, and the dashed red line gives the $95^{\text {th }}$ PR for the average PM density. A box in each plot gives the average PM density and $95^{\text {th }}$ PR for the average density for the radial section. Above radial section 1 , the radial UI for the axial section is given.

Figure 6.3 gives the angular PM distribution for each radial section for a $Z^{*}$ value of 0.125 . The other axial sections show similar results. All of the radial sections had a uniform angular PM distribution, but radial sections 3 and 4 had a lower UI. This indicates that there is more variation in the angular PM distribution near the outer edge of the substrate than near the centerline. The average PM density in radial section 4 was $7 \%$ lower than the other radial sections, which had an average density around $4.74 \mathrm{~g} / \mathrm{L}$. This finding is similar to the results shown in Figure 6.2. The $95^{\text {th }} \mathrm{PR}$ was $1.95 \mathrm{~g} / \mathrm{L}$ in radial section 4 , which is $260-650 \%$ higher than the other radial sections. The higher $95^{\text {th }} \mathrm{PR}$ for radial section 4 may be the result of how the terahertz wave enters the substrate, which can cause additional error. This phenomenon is not fully understood yet.

Test 1: Loading

Angular PM Distribution in Each Radial Section at $Z^{*}=0.125$

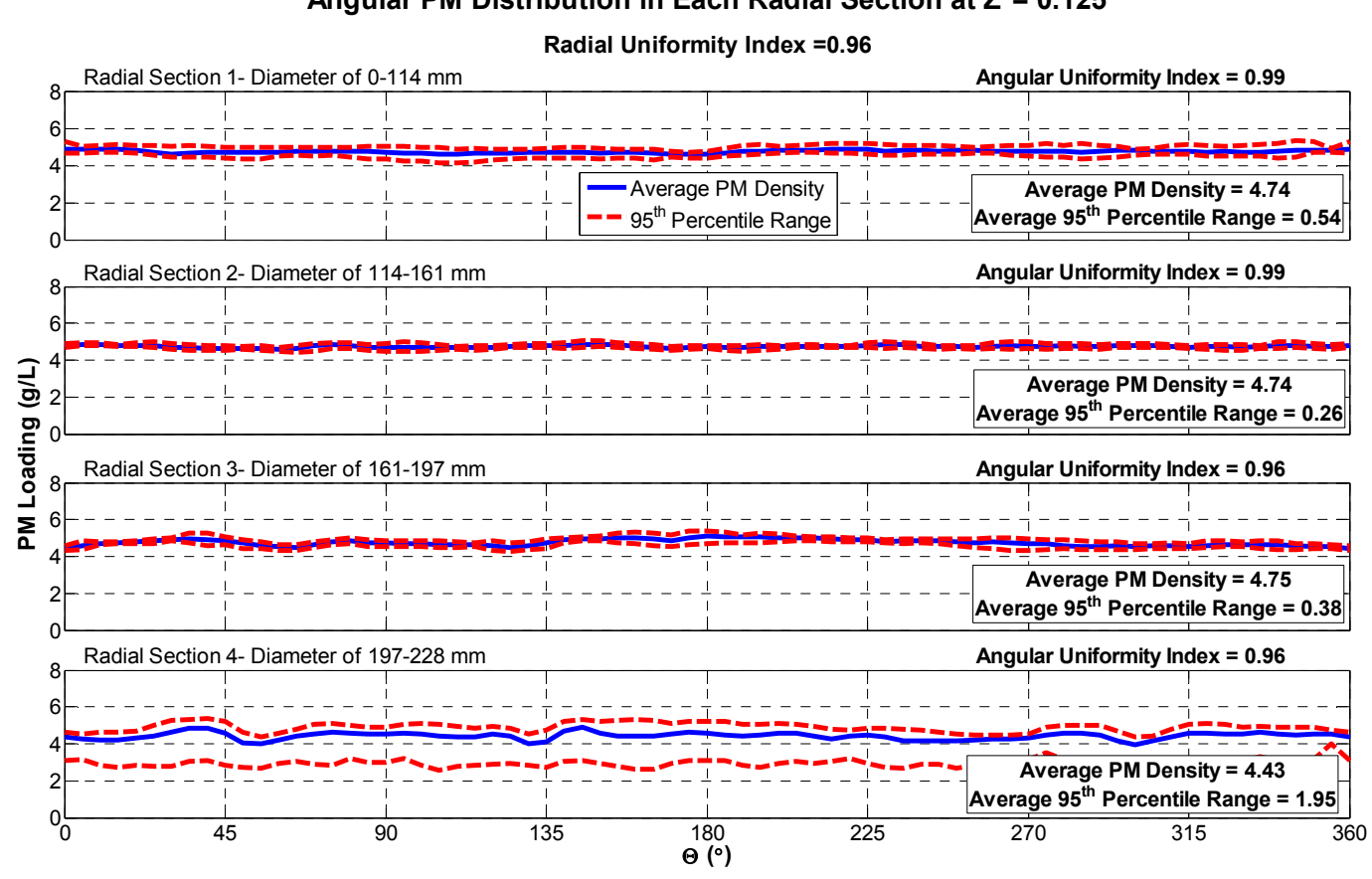

Figure 6.3: Phase 1 Test 1- Loading Angular PM Distribution in Each Radial Section

Table 6.3 gives the regional angular UI, average PM density in grams per liter, and $95^{\text {th }}$ PR for the average PM density in grams per liter for each of the radial sections and all four axial segments. The angular UI was less than 0.94 for radial sections 3 and 4 in axial segment 1 , indicating a non-uniform distribution. This conclusion is different than the results that are shown in Figure 6.3, indicating that there are distribution trends that not represented in a single plot. The $95^{\text {th }}$ PR for radial section 4 was $239-330 \%$ higher 
than radial section 1, which is consistent with Figure 6.3. Radial sections 3 and 4 also had a UI that was lower than radial section 1 and 2 for axial segments 1-3. This indicates that the PM distribution is more uniform near the centerline of the substrate.

Table 6.3: Phase 1 Test 1- Loading Angular PM Distribution

\begin{tabular}{|c|c|c|c|c|c|c|c|c|c|c|c|c|c|}
\hline & \multicolumn{12}{|c|}{ Axial Segment } \\
\hline & & \multicolumn{3}{|c|}{1} & \multicolumn{3}{|c|}{2} & \multicolumn{3}{|c|}{3} & \multicolumn{3}{|c|}{4} \\
\hline & & UI & $\begin{array}{l}\text { Average } \\
\text { PM } \\
\text { Density } \\
(\mathrm{g} / \mathrm{L})\end{array}$ & $\begin{array}{l}95^{\text {th }} \\
\text { PR } \\
(\mathrm{g} / \mathrm{L})\end{array}$ & UI & $\begin{array}{c}\text { Average } \\
\text { PM } \\
\text { Density } \\
(\mathrm{g} / \mathrm{L})\end{array}$ & $\begin{array}{l}95^{\text {th }} \\
\text { PR } \\
(\mathrm{g} / \mathrm{L})\end{array}$ & UI & $\begin{array}{l}\text { Average } \\
\text { PM } \\
\text { Density } \\
(\mathrm{g} / \mathrm{L})\end{array}$ & $\begin{array}{l}95^{\text {th }} \\
\text { PR } \\
(\mathrm{g} / \mathrm{L})\end{array}$ & UI & $\begin{array}{l}\text { Average } \\
\text { PM } \\
\text { Density } \\
(\mathrm{g} / \mathrm{L})\end{array}$ & $\begin{array}{l}95^{\text {th }} \\
\text { PR } \\
(\mathrm{g} / \mathrm{L})\end{array}$ \\
\hline \multirow{4}{*}{ 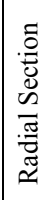 } & 1 & 0.97 & 4.72 & 0.59 & 0.99 & 4.88 & 0.46 & 0.98 & 4.96 & 0.49 & 0.97 & 5.10 & 0.59 \\
\hline & 2 & 0.95 & 4.72 & 0.35 & 0.98 & 4.88 & 0.25 & 0.97 & 4.96 & 0.28 & 0.96 & 5.10 & 0.37 \\
\hline & 3 & 0.93 & 4.73 & 0.50 & 0.96 & 4.88 & 0.37 & 0.95 & 4.97 & 0.42 & 0.96 & 5.11 & 0.40 \\
\hline & 4 & 0.92 & 4.41 & 2.00 & 0.96 & 4.55 & 1.98 & 0.95 & 4.63 & 2.04 & 0.95 & 4.77 & 2.06 \\
\hline
\end{tabular}

\section{Phase 1 Test 1: Active Regeneration}

The third substrate scan from Test 1 was taken after an active regeneration was performed. After loading the substrate to $4.96 \mathrm{~g} / \mathrm{L}$ and taking the loading scan, a $500{ }^{\circ} \mathrm{C}$ active regeneration was performed that oxidized $58 \%$ of the available PM. The PM density in the substrate at the time of the scan was $2.03 \mathrm{~g} / \mathrm{L}$. The result of this scan is shown in Figure 6.4. The bottom plot of Figure 6.4 indicates that there was more PM oxidized near the outlet of the substrate, due to the dark blue color. The first $60 \%$ of the axial length had a PM density around $2.5 \mathrm{~g} / \mathrm{L}$, which is $25 \%$ higher than the substrate average. The last $40 \%$ of the axial length had a PM density of $0.9 \mathrm{~g} / \mathrm{L}$ which is $55 \%$ lower than the substrate average. This distribution resulted in an overall axial UI of 0.84 . For reference, the loading substrate scan had a PM density that was $12 \%$ higher near the outlet of the substrate and an axial UI of 0.90 . The overall radial UI was 0.93 and the overall angular UI was 0.91 after the active regeneration. These results agree with Figure 6.4. In quadrant 1 of $Z^{*}$ values $0.125,0.375$, and 0.625 , the PM distribution fluctuates between 1 and $5 \mathrm{~g} / \mathrm{L}$. This large of a variation should be classified as non-uniform. 


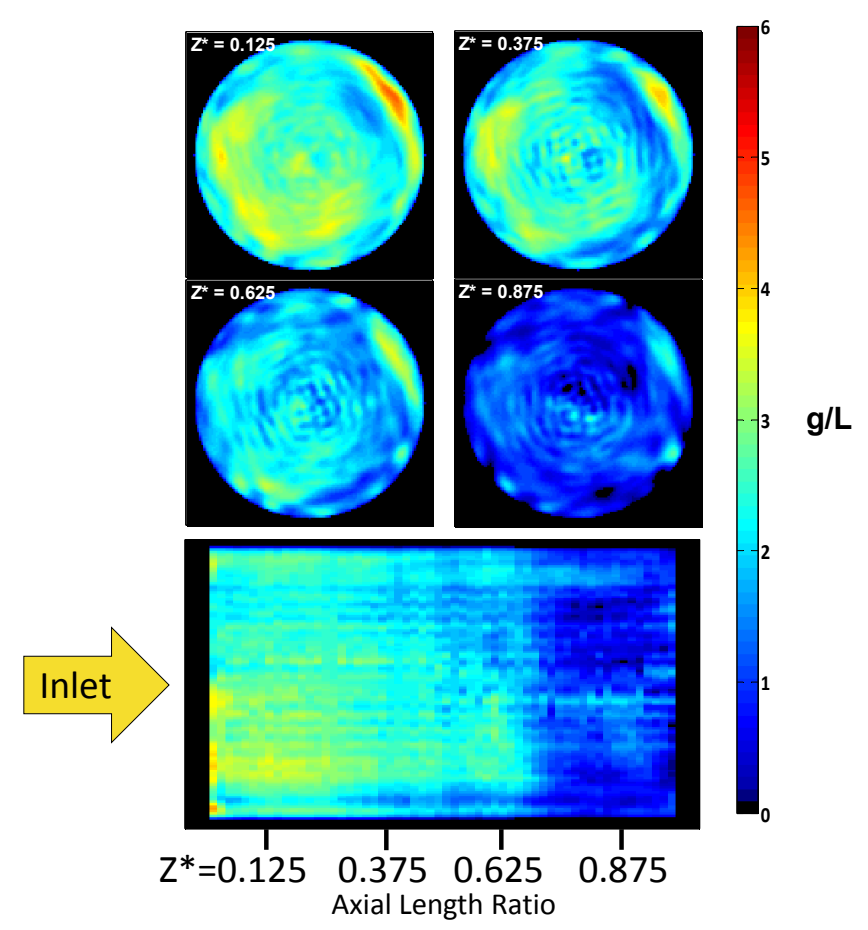

Figure 6.4: Phase 1 Test 1- Active Regeneration Scan Results

The axial PM distribution in each radial section is shown in Figure 6.5. Between $Z^{*}$ values of 0.60 and 0.70 , the PM distribution decreases from $2 \mathrm{~g} / \mathrm{L}$ to $1 \mathrm{~g} / \mathrm{L}$. The axial UIs were similar for all radial sections, indicating that each radial section had a similar amount of variation in the axial PM distribution. Radial section 4 had an average PM density that was $13 \%$ lower than the other three radial sections. The $95^{\text {th }}$ PRs for radial sections 2-4 were two times greater than the $95^{\text {th }}$ PR for radial section 1 , which was $0.80 \mathrm{~g} / \mathrm{L}$, indicating a more consistent PM distribution near the centerline of the substrate. The axial PM distribution data are also presented in Table 6.4. Axial segments 2 and 3 had the highest axial UI, which indicates that the average PM densities in those two segments were closest to the average PM density in the entire substrate. Although the axial PM distribution was not uniform, this trend is similar to the results shown for the substrate scan taken after loading the substrate. Radial sections $2-4$ had a $95^{\text {th }}$ PR that was $50-190 \%$ higher than radial section 1 . Therefore, PM distribution near the centerline of the substrate was more consistent than near the edges of the substrate. This is different, however, than the loading results where radial sections 1 and 2 had a similar $95^{\text {th }} \mathrm{PR}$. 
Test 1: Active Regeneration

Axial PM Distribution in Each Radial Section

Average Radial Uniformity Index $=0.93$

Average Angular Uniformity Index $=0.91$

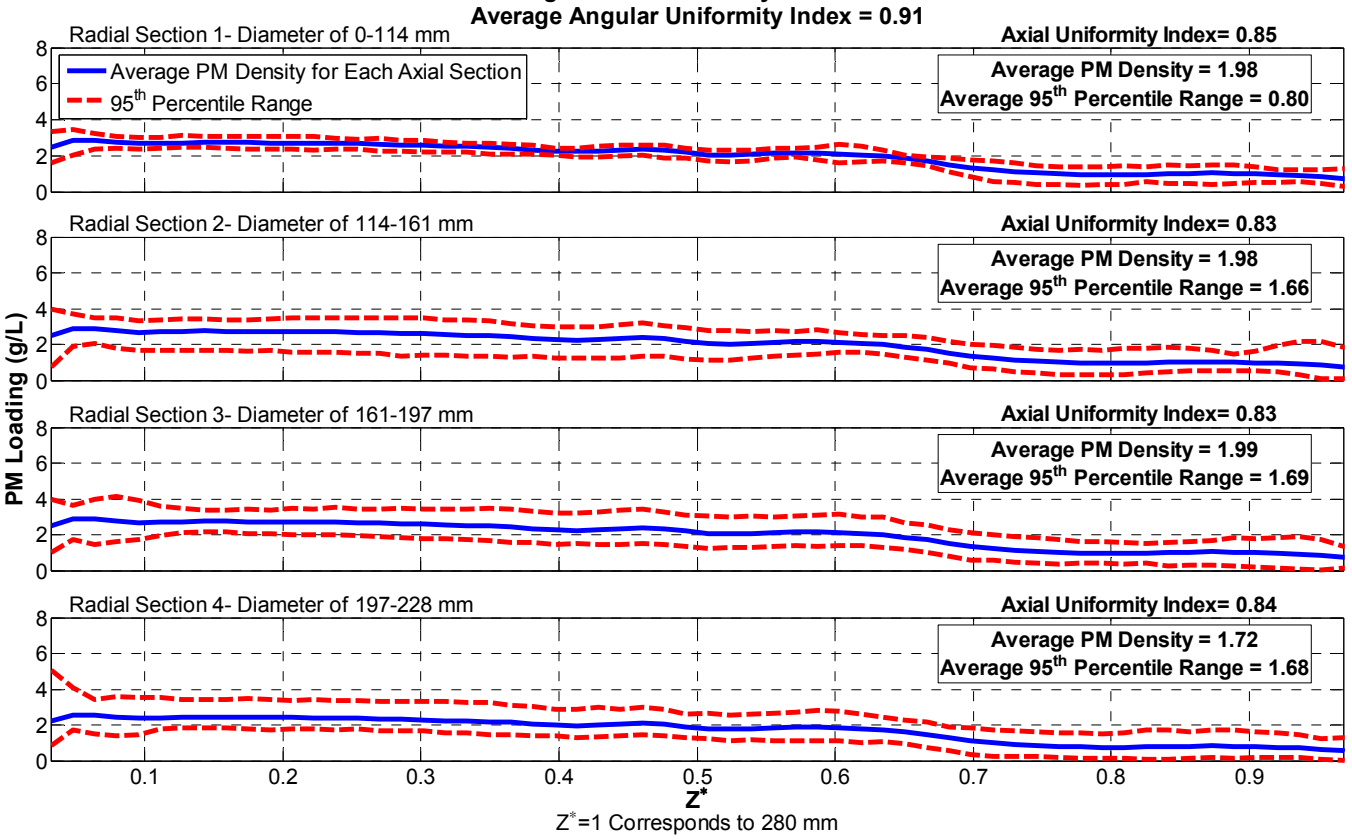

Figure 6.5: Phase 1 Test 1- Active Regeneration Axial PM Distribution in Each Radial Section

Table 6.4: Phase 1 Test 1- Active Regeneration Axial PM Distribution

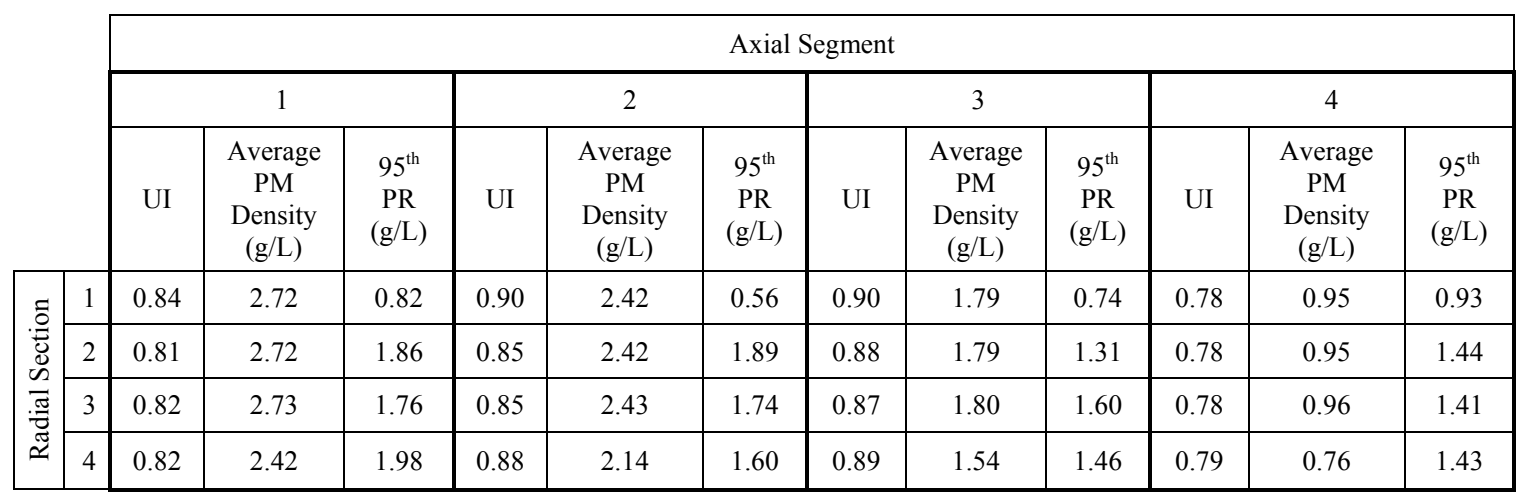

The radial PM distribution data for the four quadrants and four axial segments are shown in Table 6.5. In Figure 6.4, quadrant 1 appears to have the most variation in the data, especially at $Z^{*}$ values of 0.125 (axial segment 1 ) and 0.375 (axial segment 2). In axial segment 2, quadrant 1 was found to have the lowest UI at 0.91 . The other three axial segments had a similar UI for all quadrants. In axial segment 2, quadrant 2 had a UI of 0.92 , which indicates that quadrant 2 was more uniform than quadrant 1 . The $95^{\text {th }} \mathrm{PR}$ for quadrant 2 was $30 \%$ higher than quadrant 1, indicating that the data in quadrant 2 had more variation in it than quadrant 1 . These conflicting statements are possible because the UI and $95^{\text {th }}$ PR measure two different parameters of the PM distribution. The UI indicates the consistency of the analysis points, in an analysis area, with respect to the average value. The $95^{\text {th }} \mathrm{PR}$ indicates the range of the values in an analysis area. For a $Z^{*}$ 
value of 0.375 , the average values for each angular increment in quadrant 2 had a range of $0.62 \mathrm{~g} / \mathrm{L}$ and the values increased between $\Theta$ values of 90 and $180^{\circ}$. In quadrant 1 , the average values in each angular increment had a range that was $62 \%$ lower than quadrant 1. This decrease in the range correlates to a lower $95^{\text {th }} \mathrm{PR}$ for quadrant 1 . The average range of the data used to calculate the average value for each angular increment was $21 \%$ higher in quadrant 1 than quadrant 2. This indicates there is less consistency in the average values for the analysis points in quadrant 1, leading to a lower UI.

Table 6.5: Phase 1 Test 1- Active Regeneration Radial PM Distribution

\begin{tabular}{|c|c|c|c|c|c|c|c|c|c|c|c|c|c|}
\hline & \multicolumn{12}{|c|}{ Axial Segment } \\
\hline & & \multicolumn{3}{|c|}{1} & \multicolumn{3}{|c|}{2} & \multicolumn{3}{|c|}{3} & \multicolumn{3}{|c|}{4} \\
\hline & & UI & $\begin{array}{c}\text { Average } \\
\text { PM } \\
\text { Density } \\
(\mathrm{g} / \mathrm{L}) \\
\end{array}$ & $\begin{array}{l}95^{\text {th }} \\
\text { PR } \\
(\mathrm{g} / \mathrm{L})\end{array}$ & UI & $\begin{array}{l}\text { Average } \\
\text { PM } \\
\text { Density } \\
(\mathrm{g} / \mathrm{L}) \\
\end{array}$ & $\begin{array}{l}95^{\text {th }} \\
\text { PR } \\
(\mathrm{g} / \mathrm{L})\end{array}$ & UI & $\begin{array}{l}\text { Average } \\
\text { PM } \\
\text { Density } \\
(\mathrm{g} / \mathrm{L}) \\
\end{array}$ & $\begin{array}{l}95^{\text {th }} \\
\text { PR } \\
(\mathrm{g} / \mathrm{L})\end{array}$ & UI & $\begin{array}{l}\text { Average } \\
\text { PM } \\
\text { Density } \\
\text { (g/L) } \\
\end{array}$ & $\begin{array}{l}95^{\text {th }} \\
\text { PR } \\
(\mathrm{g} / \mathrm{L})\end{array}$ \\
\hline \multirow{4}{*}{ 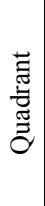 } & 1 & 0.90 & 2.30 & 0.39 & 0.91 & 2.12 & 0.53 & 0.93 & 1.63 & 0.60 & 0.94 & 0.79 & 0.50 \\
\hline & 2 & 0.91 & 2.38 & 0.40 & 0.92 & 2.53 & 0.69 & 0.93 & 1.81 & 0.59 & 0.93 & 0.88 & 0.31 \\
\hline & 3 & 0.91 & 2.64 & 0.30 & 0.93 & 2.61 & 0.30 & 0.94 & 1.93 & 0.36 & 0.94 & 0.90 & 0.26 \\
\hline & 4 & 0.90 & 2.51 & 0.56 & 0.93 & 2.15 & 0.33 & 0.93 & 1.55 & 0.35 & 0.93 & 0.85 & 0.46 \\
\hline
\end{tabular}

The angular PM distribution for a $Z^{*}$ value of 0.125 is shown in Figure 6.6. Radial section 1 was the only radial section that has a uniform angular PM distribution at $Z^{*}=0.125$. This indicates that the $P M$ distribution near the centerline is more uniform than the PM distribution near the edges of the substrate. Radial section 4 had an average PM density that was $11 \%$ lower than radial sections $1-3$. The $95^{\text {th }} \mathrm{PR}$ for radial section 1 is $53 \%$ greater than radial section 2 . This indicates that the data used to calculate the average value for each angular increment were more consistent in radial section 2 , but there is more variation in the resulting average value, than radial section 1 . This trend highlights the need to use both the UI and $95^{\text {th }}$ PR to quantify a PM distribution. Table 6.6 shows that the trend with the $95^{\text {th }}$ PR was consistent for all axial segments. Table 6.6 also shows that radial section 1 was the only radial section to have a uniform distribution. The UI is radial section 1 was $2-10 \%$ higher than the other radial sections, indicating a more uniform PM distribution was found near the centerline of the substrate. 
Test 1: Active Regeneration

Angular PM Distribution in Each Radial Section at $Z^{*}=0.125$

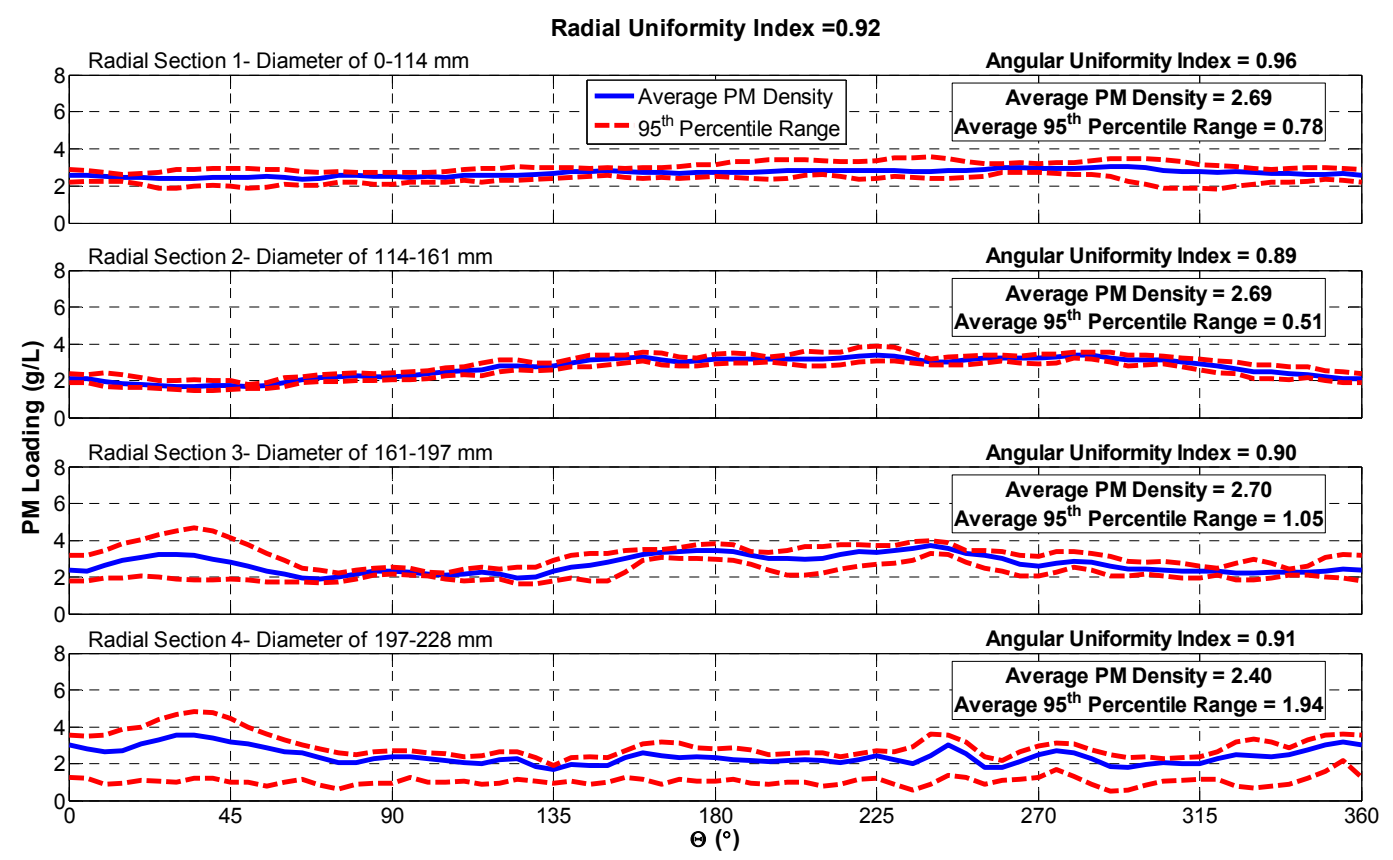

Figure 6.6: Phase 1 Test 1- Active Regeneration Angular PM Distribution in Each Radial Section

Table 6.6: Phase 1 Test 1- Active Regeneration Angular PM Distribution

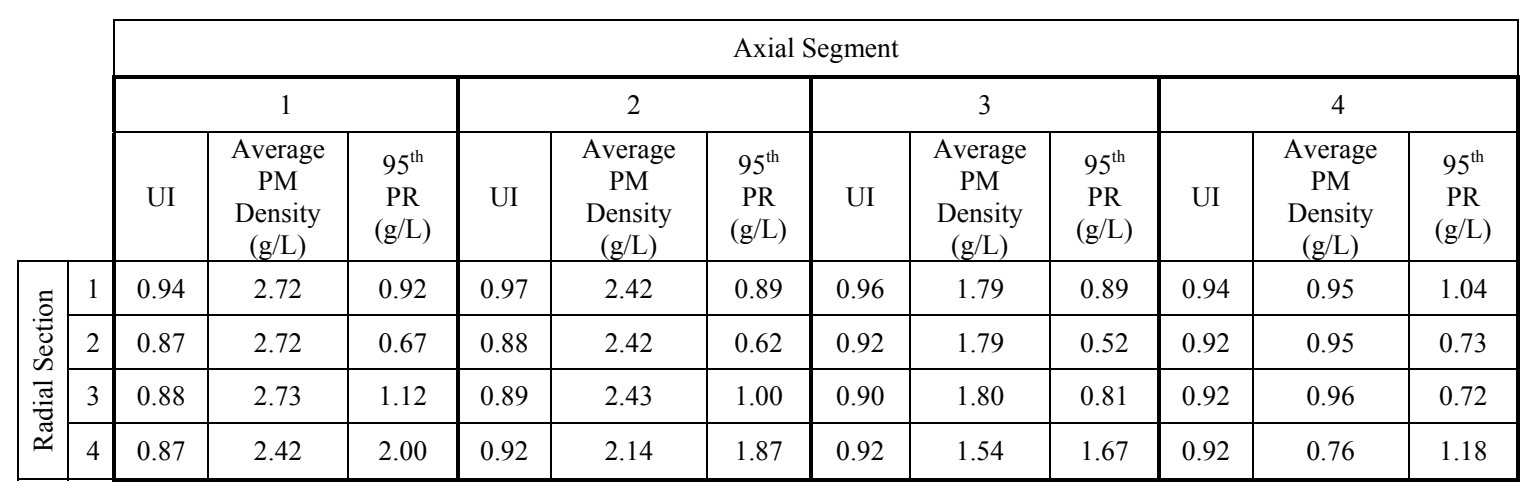

\section{Phase 1 Test 2: Active Regeneration}

There were two substrate scans taken during Test 2. The first scan was the baseline scan, of a clean substrate, for substrate number 2, and the second scan was a scan after the active regeneration. Test 2 consisted of loading a substrate to $5.2 \mathrm{~g} / \mathrm{L}$ and then performing a $500{ }^{\circ} \mathrm{C}$ active regeneration that oxidized $41 \%$ of the available PM. The PM density in the substrate at the time of the scan was $2.99 \mathrm{~g} / \mathrm{L}$. The result of this scan is shown in Figure 6.7. The bottom plot in Figure 6.7 shows that the PM density in the first $40 \%$ of the substrate was $3.4 \mathrm{~g} / \mathrm{L}$, which is $14 \%$ higher than the substrate average. In the last $40 \%$ of the axial length, the PM density was $2.2 \mathrm{~g} / \mathrm{L}$, which is $26 \%$ lower than the substrate average. This PM distribution resulted in an overall axial UI of 0.85 . The UI 
and distribution trend is similar to the result found for the active regeneration scan from Test 1 (axial UI of 0.84). The overall radial and angular UIs were 0.96 and 0.94, respectively, for the active regeneration scan from Test 2, which indicate a uniform distribution. The overall radial and angular UIs from the active regeneration scan of Test 1 , where $58 \%$ of the available PM was oxidized, were not uniform. This indicates that there may be an upper limit to the amount of PM oxidation that will result in a uniform radial and angular distribution.

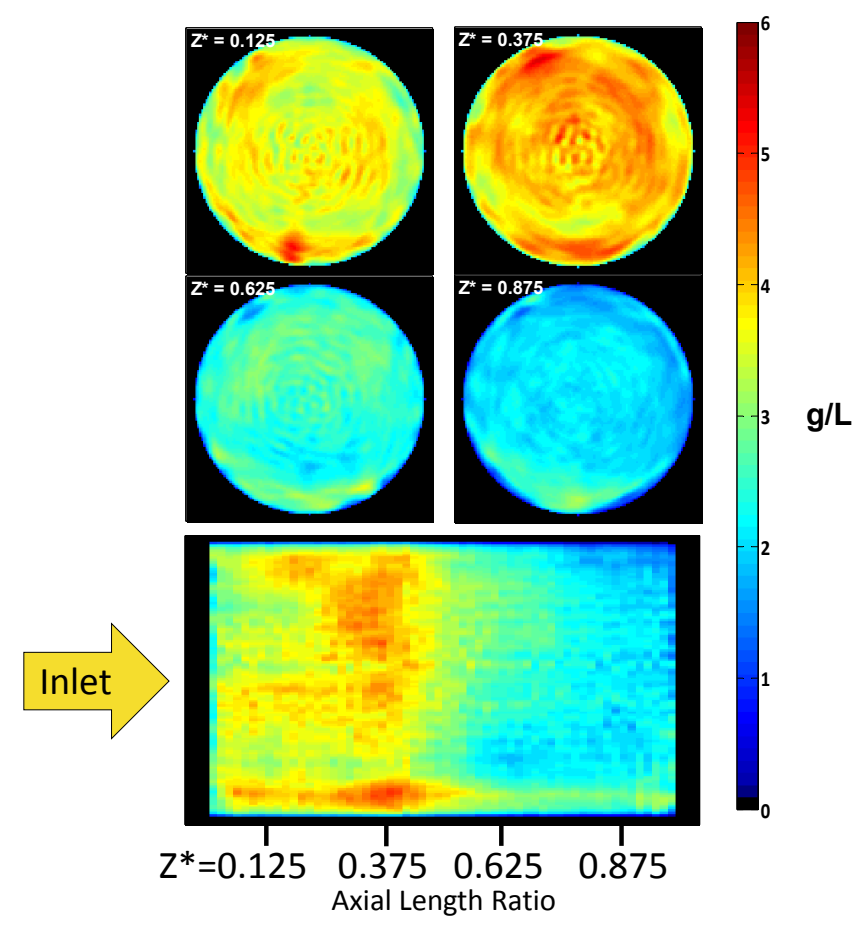

Figure 6.7: Phase 1 Test 2- Active Regeneration Scan Results

The axial PM distribution for each radial section is shown in Figure 6.8. All four radial sections had a similar axial UI, indicating a similar amount of variation in the axial PM distribution. Radial section 4 had an average PM density that was 9\% lower than the substrate average. The $95^{\text {th }}$ PR increased in each radial section, from 0.62 in radial section 1 to 1.20 in radial section 4. These results are similar to the axial PM distribution for both the loading and active regeneration scan from Test 1 . The active regeneration scan from Test 1 showed that a majority of the PM was oxidized after a $Z^{*}$ value of 0.7 . However, Figure 6.8 shows that the PM density starts to decrease at a $Z^{*}$ value of 0.4 . Additionally, the PM density increases until a $Z^{*}$ value of 0.4 . In Test 1 , the PM density was continually decreasing. The data in Table 6.7 confirm these results. Axial segments 1 and 3 had the highest UIs, indicating that the average values in those axial segments are nearest the average value for the substrate. 
Test 2: Active Regeneration

Axial PM Distribution in Each Radial Section

Average Radial Uniformity Index $=0.96$

Average Angular Uniformity Index $=0.94$

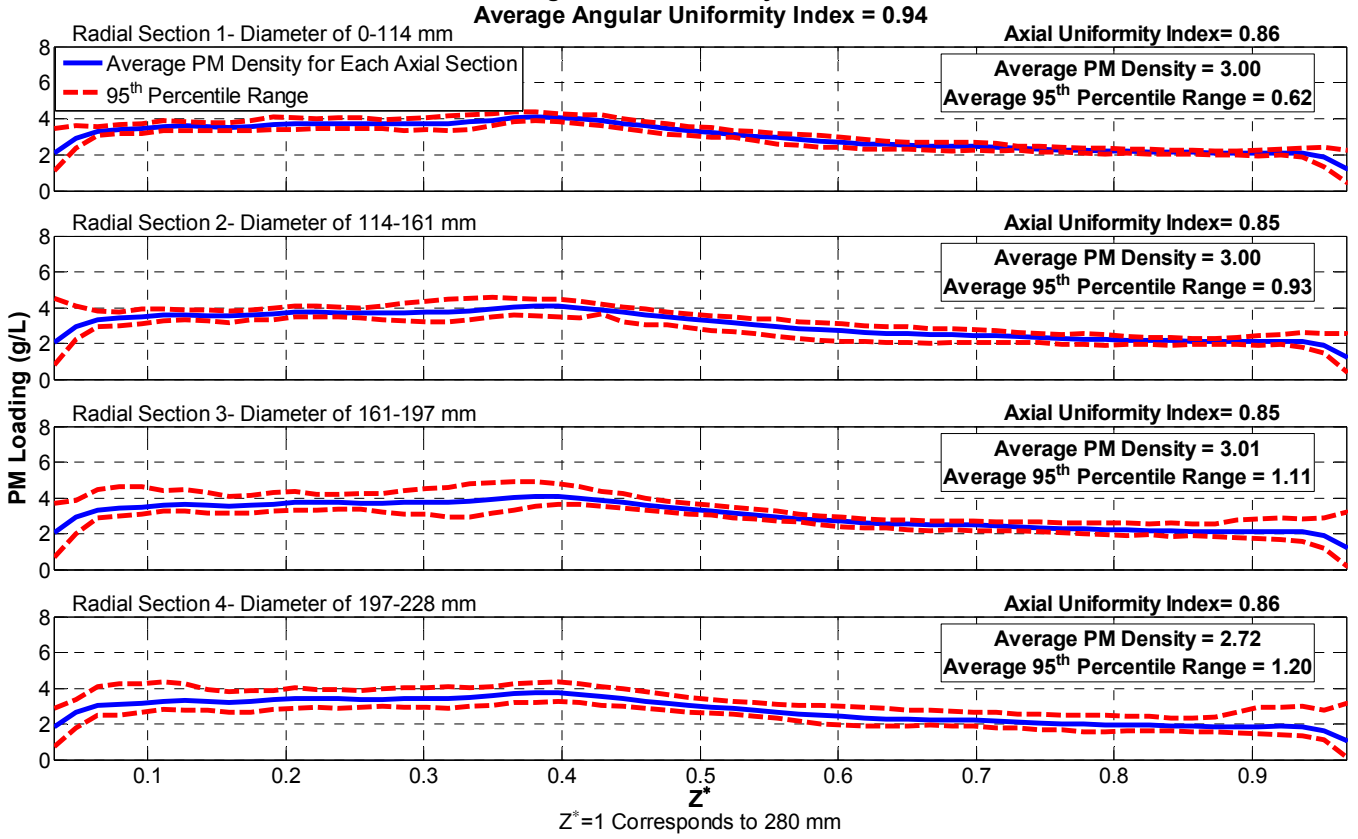

Figure 6.8: Phase 1 Test 2- Active Regeneration Axial PM Distribution in Each Radial Section

Table 6.7: Phase 1 Test 2- Active Regeneration Axial PM Distribution

\begin{tabular}{|c|c|c|c|c|c|c|c|c|c|c|c|c|c|}
\hline & \multicolumn{12}{|c|}{ Axial Segment } \\
\hline & & \multicolumn{3}{|c|}{1} & \multicolumn{3}{|c|}{2} & \multicolumn{3}{|c|}{3} & \multicolumn{3}{|c|}{4} \\
\hline & & UI & $\begin{array}{l}\text { Average } \\
\text { PM } \\
\text { Density } \\
(\mathrm{g} / \mathrm{L})\end{array}$ & $\begin{array}{l}95^{\text {th }} \\
\text { PR } \\
(\mathrm{g} / \mathrm{L})\end{array}$ & UI & $\begin{array}{l}\text { Average } \\
\text { PM } \\
\text { Density } \\
\text { (g/L) }\end{array}$ & $\begin{array}{l}95^{\text {th }} \\
\text { PR } \\
(\mathrm{g} / \mathrm{L})\end{array}$ & UI & $\begin{array}{l}\text { Average } \\
\text { PM } \\
\text { Density } \\
(\mathrm{g} / \mathrm{L})\end{array}$ & $\begin{array}{l}95^{\text {th }} \\
\text { PR } \\
(\mathrm{g} / \mathrm{L})\end{array}$ & UI & $\begin{array}{l}\text { Average } \\
\text { PM } \\
\text { Density } \\
(\mathrm{g} / \mathrm{L})\end{array}$ & $\begin{array}{c}95^{\text {th }} \\
\text { PR } \\
(\mathrm{g} / \mathrm{L})\end{array}$ \\
\hline \multirow{4}{*}{ 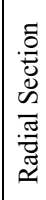 } & 1 & 0.88 & 3.42 & 0.73 & 0.84 & 3.78 & 0.59 & 0.91 & 2.68 & 0.48 & 0.81 & 2.07 & 0.44 \\
\hline & 2 & 0.87 & 3.42 & 0.97 & 0.84 & 3.78 & 0.90 & 0.91 & 2.68 & 0.80 & 0.81 & 2.07 & 0.66 \\
\hline & 3 & 0.87 & 3.43 & 1.35 & 0.84 & 3.79 & 1.16 & 0.91 & 2.69 & 0.51 & 0.80 & 2.07 & 1.05 \\
\hline & 4 & 0.87 & 3.12 & 1.40 & 0.84 & 3.47 & 1.03 & 0.91 & 2.41 & 0.85 & 0.81 & 1.83 & 1.23 \\
\hline
\end{tabular}

Table 6.8 gives the radial PM distribution in the four quadrants and four axial segments. The results show that quadrant 2 had a UI lower than the other 3 quadrants in axial segments 1, 3, and 4. Quadrant 2 was also the only non-uniform quadrant in axial segment 1. Quadrant 2 in the $Z^{*}=0.125$ plot in Figure 6.7 does not visually seem to be non-uniform. The ability of the UI to quantify a distribution, instead of relying on visual observations, is an important characteristic of the developed analysis method. 
Table 6.8: Phase 1 Test 2- Active Regeneration Radial PM Distribution

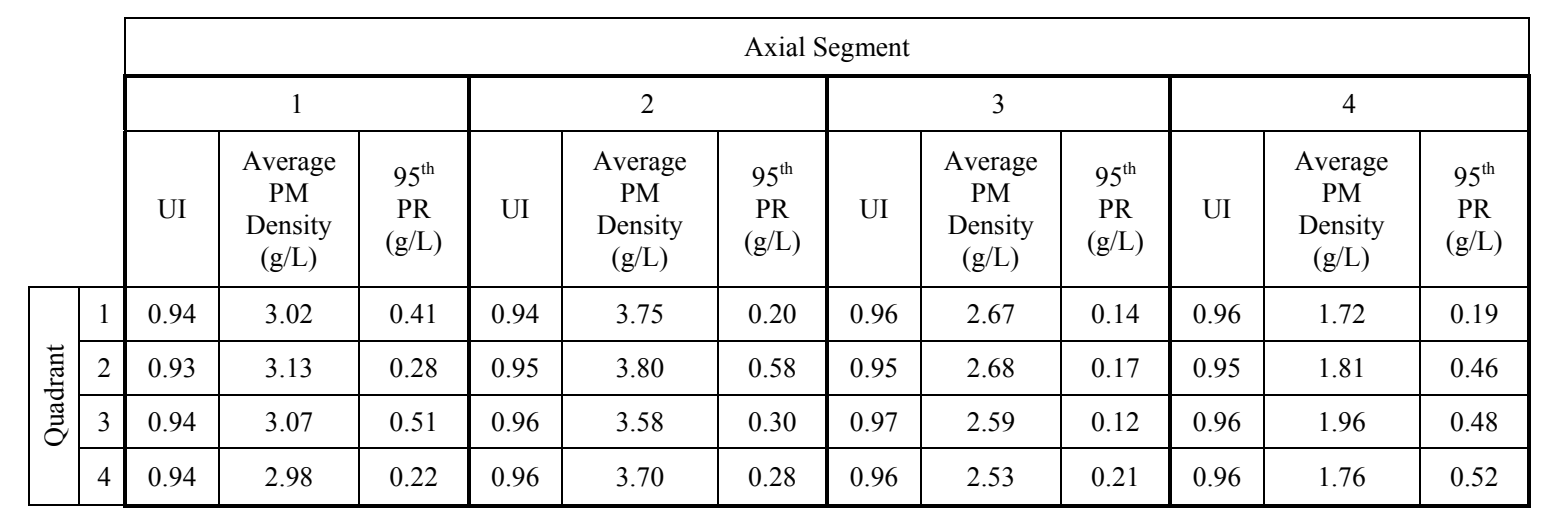

The angular PM distribution in each radial section for a $Z^{*}$ value of 0.125 is shown in Figure 6.9. The angular UI decreased from 0.97 in radial section 1 to 0.94 in radial section 4, which indicates that all radial sections have a uniform angular PM distribution for this particular $Z^{*}$ value. However, the data in Table 6.9 show that axial segment 1 only had a uniform PM distribution for radial section 1 . The only other non-uniform angular PM distributions were in radial sections 3 and 4 in axial segment 4 . Figure 6.9 shows that the $95^{\text {th }} \mathrm{PR}$ in radial section 1 was $77 \%$ higher than radial section 2 and radial sections 1-3 had $95^{\text {th }}$ PRs that were $57-75 \%$ lower than radial section 4 . The data in Table 6.9 confirm this trend, with the $95^{\text {th }} \mathrm{PR}$ in radial section 4 being consistently higher than radial sections 1-3. In all axial segments, except axial segment 3, radial section 1 had a UI that was higher than the other radial sections, indicating there was a more uniform PM distribution near the centerline of the substrate. In axial segment 3 , all of the radial sections were similar. 
Test 2: Active Regeneration

Angular PM Distribution in Each Radial Section at $Z^{*}=0.125$

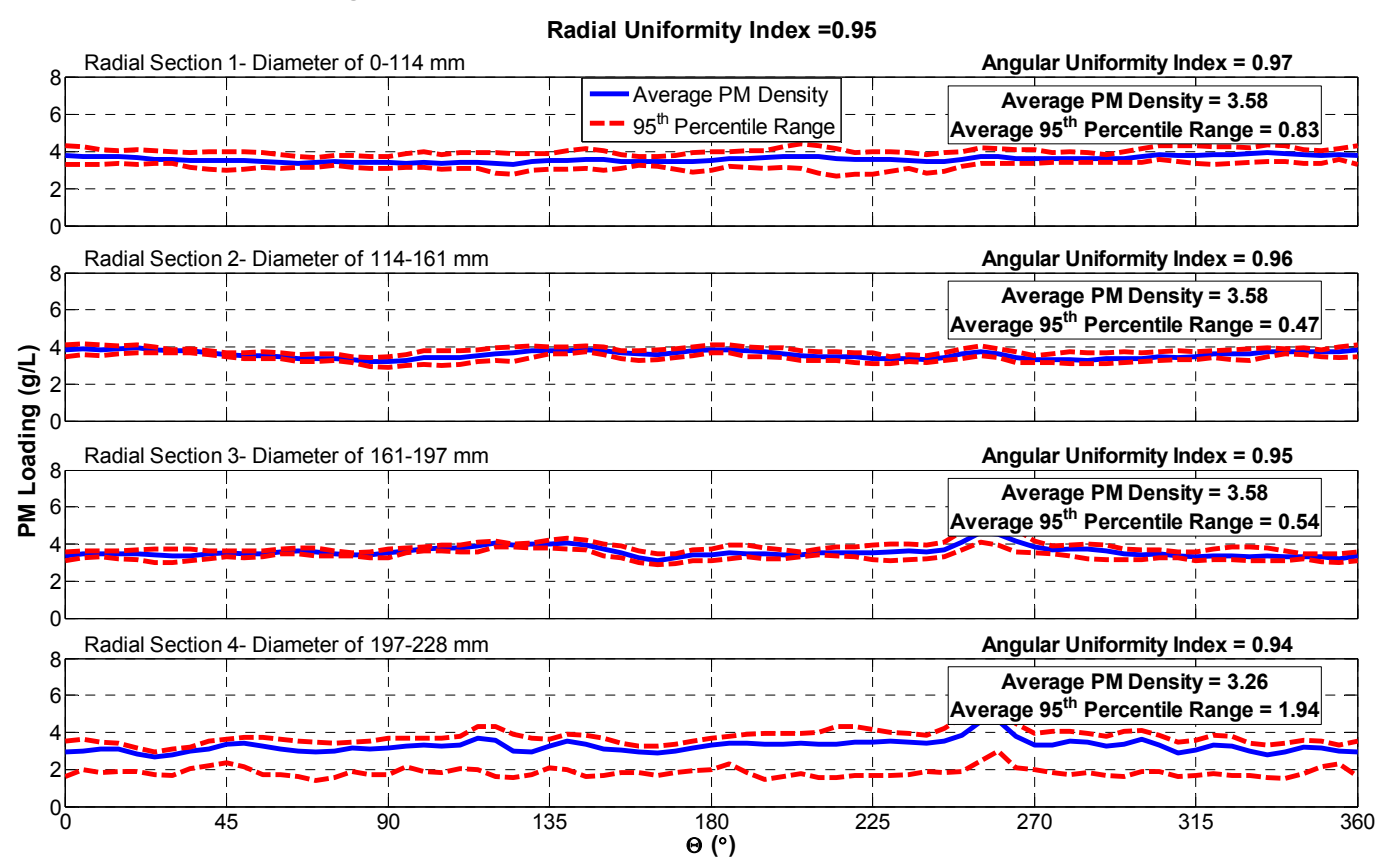

Figure 6.9: Phase 1 Test 2- Active Regeneration Angular PM Distribution in Each Radial Section

Table 6.9: Phase 1 Test 2- Active Regeneration Angular PM Distribution

\begin{tabular}{|c|c|c|c|c|c|c|c|c|c|c|c|c|c|}
\hline & \multicolumn{12}{|c|}{ Axial Segment } \\
\hline & & \multicolumn{3}{|c|}{1} & \multicolumn{3}{|c|}{2} & \multicolumn{3}{|c|}{3} & \multicolumn{3}{|c|}{4} \\
\hline & & UI & $\begin{array}{l}\text { Average } \\
\text { PM } \\
\text { Density } \\
(\mathrm{g} / \mathrm{L})\end{array}$ & $\begin{array}{c}95^{\text {th }} \\
\text { PR } \\
(\mathrm{g} / \mathrm{L})\end{array}$ & UI & $\begin{array}{c}\text { Average } \\
\text { PM } \\
\text { Density } \\
(\mathrm{g} / \mathrm{L})\end{array}$ & $\begin{array}{c}95^{\text {th }} \\
\text { PR } \\
(\mathrm{g} / \mathrm{L})\end{array}$ & UI & $\begin{array}{l}\text { Average } \\
\text { PM } \\
\text { Density } \\
(g / L)\end{array}$ & $\begin{array}{l}95^{\text {th }} \\
\text { PR } \\
(\mathrm{g} / \mathrm{L})\end{array}$ & UI & $\begin{array}{c}\text { Average } \\
\text { PM } \\
\text { Density } \\
(\mathrm{g} / \mathrm{L})\end{array}$ & $\begin{array}{c}95^{\text {th }} \\
\text { PR } \\
(\mathrm{g} / \mathrm{L})\end{array}$ \\
\hline \multirow{4}{*}{ 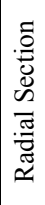 } & 1 & 0.94 & 3.42 & 0.80 & 0.97 & 3.78 & 0.57 & 0.97 & 2.68 & 0.53 & 0.96 & 2.07 & 0.62 \\
\hline & 2 & 0.92 & 3.42 & 0.58 & 0.94 & 3.78 & 0.39 & 0.95 & 2.68 & 0.29 & 0.95 & 2.07 & 0.39 \\
\hline & 3 & 0.91 & 3.43 & 0.61 & 0.95 & 3.79 & 0.50 & 0.97 & 2.69 & 0.44 & 0.93 & 2.07 & 0.42 \\
\hline & 4 & 0.92 & 3.12 & 1.93 & 0.95 & 3.47 & 1.97 & 0.96 & 2.41 & 1.65 & 0.93 & 1.83 & 1.54 \\
\hline
\end{tabular}

\section{Phase 1 Test 3: Passive Oxidation}

Test 3 consisted of loading a substrate to $5.81 \mathrm{~g} / \mathrm{L}$ and then performing a passive oxidation for 52 minutes where $45 \%$ of the available PM was oxidized. The average temperature of the substrate was $372{ }^{\circ} \mathrm{C}$ and the $\mathrm{NO}_{2}$ concentration into the $\mathrm{CPF}$ was 256 $\mathrm{ppm}$. The PM density in the substrate at the end of the passive oxidation was $3.33 \mathrm{~g} / \mathrm{L}$. A substrate scan was performed after the passive oxidation was completed, and the result is shown in Figure 6.10. The bottom plot shows that the first 10 and last $55 \%$ of the axial length had a PM density of $3.2 \mathrm{~g} / \mathrm{L}$, which is $4 \%$ lower than the substrate average. Between 20 and $50 \%$ of the axial length, the PM density was $3.9 \mathrm{~g} / \mathrm{L}$, which is $28 \%$ higher than the substrate average. This indicates that less PM was oxidized near the 
middle of the substrate. The axial distribution, which is similar in trend to the active regeneration scan from Test 2, resulted in an overall axial UI of 0.91 . Test 2 had a lower UI, which was caused by more PM being oxidized near the outlet of the substrate. The overall radial UI was 0.96 and the overall angular UI was 0.94 . These are identical to the overall radial and angular UI for Test 2, and similar to the values calculated for the loading scan of Test 1 . The plots for $Z^{*}$ values of $0.125,0.375$, and 0.875 show an increase in the PM density in quadrants 1 and 2 and radial sections 3 and 4, but those increases did not cause the overall radial and angular UI to be non-uniform.

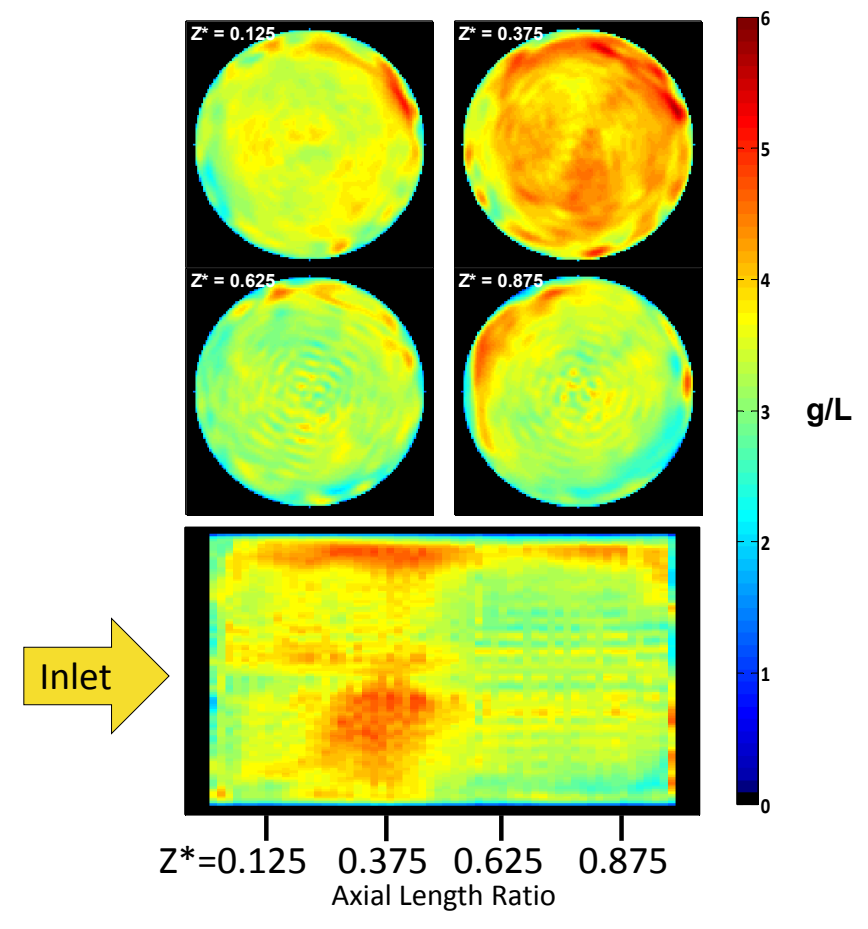

Figure 6.10: Phase 1 Test 3- Passive Oxidation Scan Results

The axial PM distribution in each radial section is shown in Figure 6.11. The average PM density in radial section 4 was $8 \%$ lower than the other three radial sections. This is consistent with the other data from the other scans. Figure 6.11 also shows that at a $Z^{*}$ value of 0.15 the PM density begins to increase and at a $Z^{*}$ value of 0.45 the PM density starts to decrease in all four radial sections. The axial UI decreased from 0.92 in radial section 1 to 0.90 in radial section 4, indicating that there is more variation in the axial PM distribution near the edge of the substrate. The $95^{\text {th }} \mathrm{PR}$ increased in each radial section, from $0.67 \mathrm{~g} / \mathrm{L}$ in radial section 1 to $1.53 \mathrm{~g} / \mathrm{L}$ in radial section 4 . The axial $\mathrm{PM}$ distribution data for each radial section and axial segment are given in Table 6.10. Axial segment 3 is the only axial segment to have a uniform PM distribution. The average PM density trend in Table 6.10, where axial segment 2 has the highest PM density, is similar to the average PM density trend for Test 2 in Table 6.7. The trends for the UI and $95^{\text {th }}$ PR that are shown in Table 6.10 are similar to the trends shown in Figure 6.11. 
Test 3: Passive Oxidation

Axial PM Distribution in Each Radial Section

Average Radial Uniformity Index $=0.96$

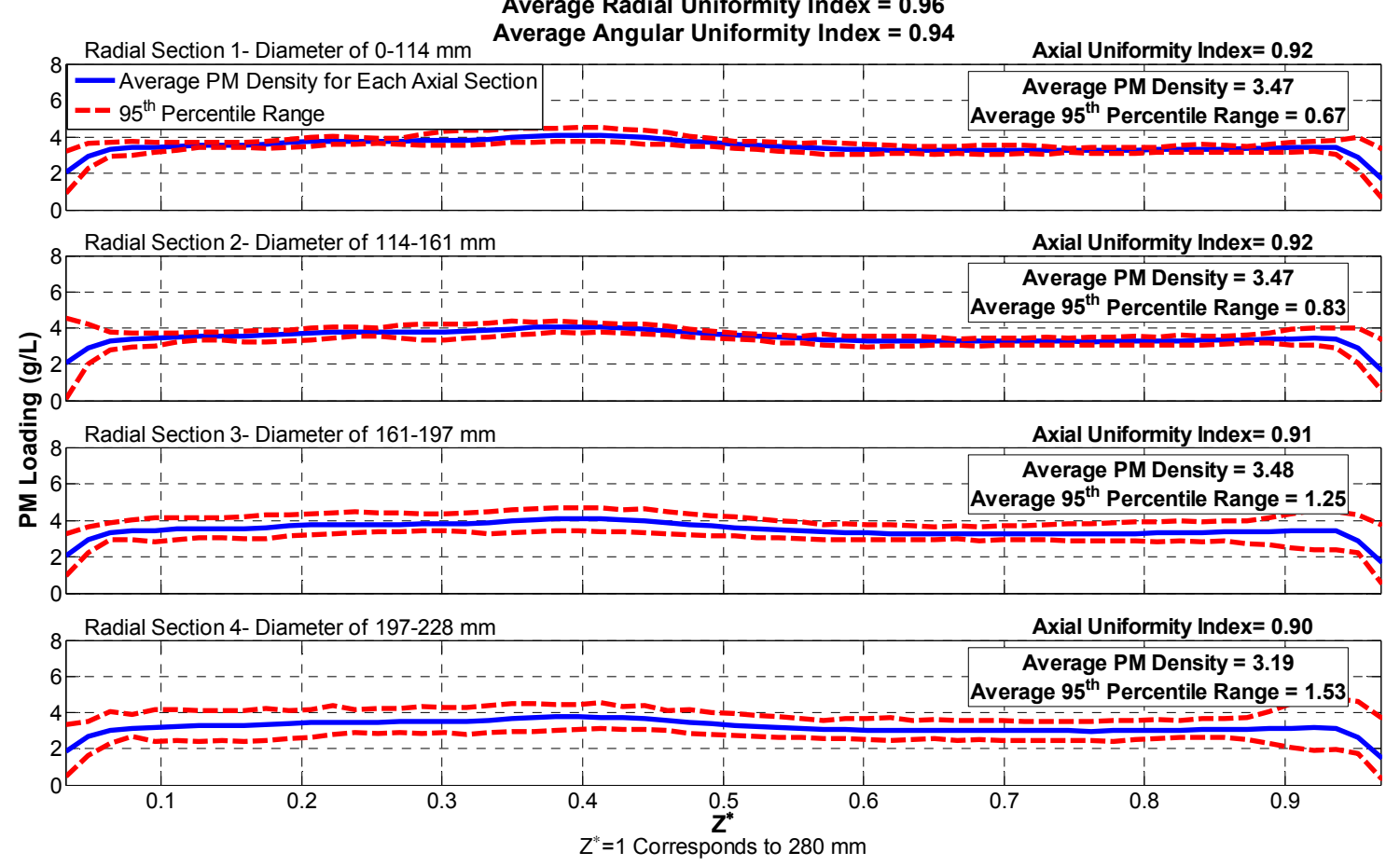

Figure 6.11: Phase 1 Test 3- Passive Oxidation Axial PM Distribution in Each Radial Section

Table 6.10: Phase 1 Test 3- Passive Oxidation Axial PM Distribution

\begin{tabular}{|c|c|c|c|c|c|c|c|c|c|c|c|c|c|}
\hline & \multicolumn{12}{|c|}{ Axial Segment } \\
\hline & & \multicolumn{3}{|c|}{1} & \multicolumn{3}{|c|}{2} & \multicolumn{3}{|c|}{3} & \multicolumn{3}{|c|}{4} \\
\hline & & UI & $\begin{array}{l}\text { Average } \\
\text { PM } \\
\text { Density } \\
(\mathrm{g} / \mathrm{L})\end{array}$ & $\begin{array}{c}95^{\text {th }} \\
\text { PR } \\
(\mathrm{g} / \mathrm{L})\end{array}$ & UI & $\begin{array}{l}\text { Average } \\
\text { PM } \\
\text { Density } \\
(\mathrm{g} / \mathrm{L})\end{array}$ & $\begin{array}{l}95^{\text {th }} \\
\text { PR } \\
(\mathrm{g} / \mathrm{L})\end{array}$ & UI & $\begin{array}{l}\text { Average } \\
\text { PM } \\
\text { Density } \\
(g / L)\end{array}$ & $\begin{array}{l}95^{\text {th }} \\
\text { PR } \\
(\mathrm{g} / \mathrm{L})\end{array}$ & UI & $\begin{array}{c}\text { Average } \\
\text { PM } \\
\text { Density } \\
(\mathrm{g} / \mathrm{L})\end{array}$ & $\begin{array}{c}95^{\text {th }} \\
\text { PR } \\
(\mathrm{g} / \mathrm{L})\end{array}$ \\
\hline \multirow{4}{*}{ 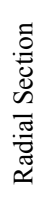 } & 1 & 0.91 & 3.41 & 0.65 & 0.92 & 3.89 & 0.65 & 0.96 & 3.34 & 0.46 & 0.90 & 3.19 & 0.69 \\
\hline & 2 & 0.91 & 3.42 & 1.01 & 0.92 & 3.89 & 0.62 & 0.96 & 3.34 & 0.46 & 0.90 & 3.19 & 0.86 \\
\hline & 3 & 0.90 & 3.42 & 1.26 & 0.91 & 3.90 & 1.16 & 0.95 & 3.35 & 0.85 & 0.88 & 3.19 & 1.53 \\
\hline & 4 & 0.90 & 3.13 & 1.72 & 0.90 & 3.59 & 1.39 & 0.94 & 3.06 & 1.11 & 0.88 & 2.92 & 1.77 \\
\hline
\end{tabular}

The radial PM distribution data for the four quadrants and four axial segments are shown in Table 6.11. All of the quadrants had a radial UI that is greater than 0.94. This indicates that the variation in the PM distribution shown by the red regions in Figure 6.10 was not large enough for the radial distribution to be classified as non-uniform. The variation does cause the $95^{\text {th }} \mathrm{PR}$ for quadrant 1 in axial segment 1 and quadrant 2 in axial segment 4 to be $39-171 \%$ higher than the other quadrants. This shows that the developed analysis method quantifies PM distribution and allows for numerical analysis of the results, instead of relying on visual observations of plots. 
Table 6.11: Phase 1 Test 3- Passive Oxidation Radial PM Distribution

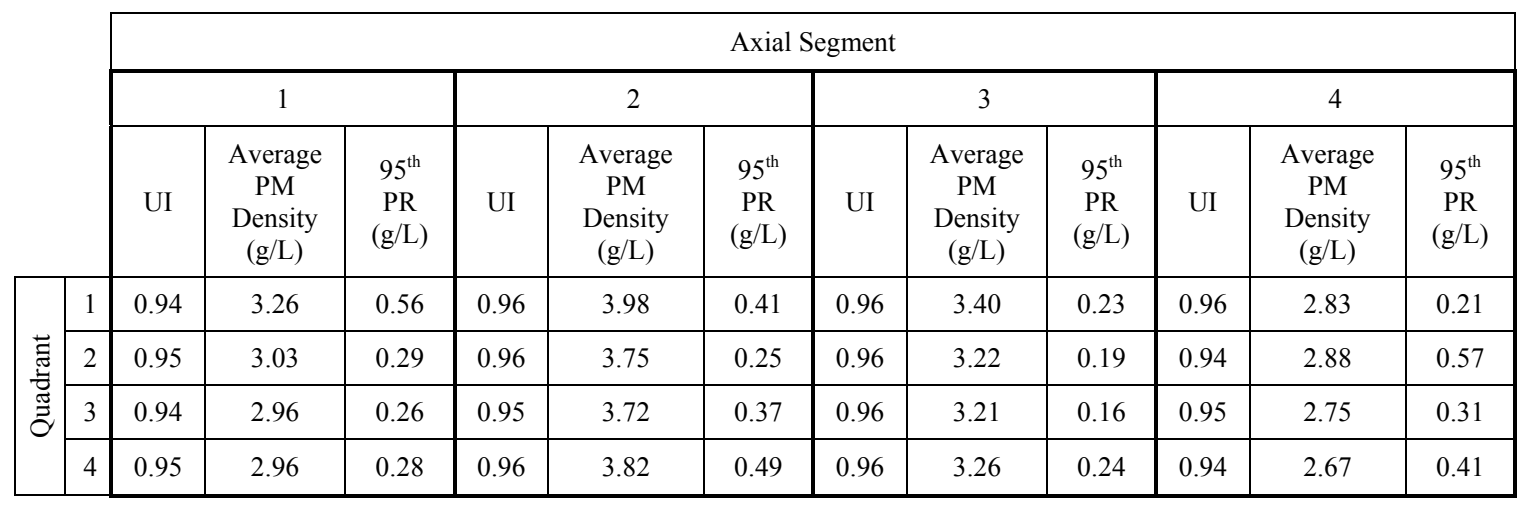

The angular PM distribution in each radial section for a $Z^{*}$ value of 0.125 is shown in Figure 6.12. Similar to the axial PM distribution, radial section 4 had a PM density that was $8 \%$ lower than the other three radial sections. In Figure 6.12, radial section 4 had the only non-uniform PM distribution, with an angular UI of 0.93 . However, the data in Table 6.12 show that radial section 1 had the only uniform PM distribution in axial segment 1 . The only other non-uniform angular PM distributions occur in radial sections 3 and 4 in axial segment 4 . Those radial sections are shown to have a lot of variation in Figure 6.10. The UI for radial sections 1 and 2 were $1-4 \%$ higher than radial sections 3 and 4 , indicating a more uniform PM distribution near the centerline of the substrate.

Test 3: Passive Oxidation

Angular PM Distribution in Each Radial Section at $Z^{*}=0.125$

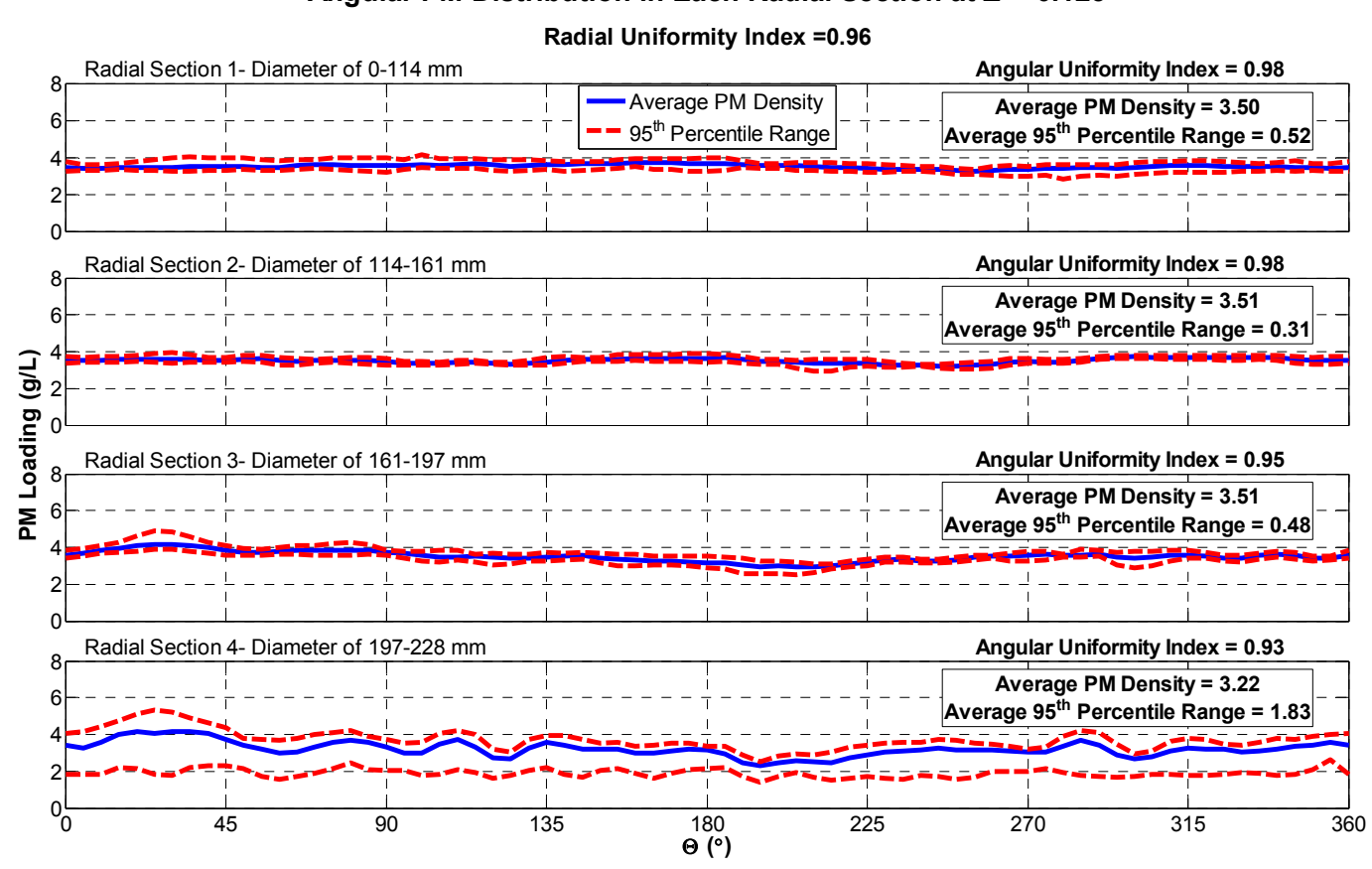

Figure 6.12: Phase 1 Test 3- Passive Oxidation Angular PM Distribution in Each Radial Section 
Table 6.12: Phase 1 Test 3- Passive Oxidation Angular PM Distribution

\begin{tabular}{|c|c|c|c|c|c|c|c|c|c|c|c|c|c|}
\hline & \multicolumn{12}{|c|}{ Axial Segment } \\
\hline & & \multicolumn{3}{|c|}{1} & \multicolumn{3}{|c|}{2} & \multicolumn{3}{|c|}{3} & \multicolumn{3}{|c|}{4} \\
\hline & & UI & $\begin{array}{l}\text { Average } \\
\text { PM } \\
\text { Density } \\
(\mathrm{g} / \mathrm{L})\end{array}$ & $\begin{array}{l}95^{\text {th }} \\
\text { PR } \\
(\mathrm{g} / \mathrm{L})\end{array}$ & UI & $\begin{array}{c}\text { Average } \\
\text { PM } \\
\text { Density } \\
\text { (g/L) }\end{array}$ & $\begin{array}{l}95^{\text {th }} \\
\text { PR } \\
(\mathrm{g} / \mathrm{L})\end{array}$ & UI & $\begin{array}{l}\text { Average } \\
\text { PM } \\
\text { Density } \\
(\mathrm{g} / \mathrm{L})\end{array}$ & $\begin{array}{l}95^{\text {th }} \\
\text { PR } \\
(\mathrm{g} / \mathrm{L})\end{array}$ & UI & $\begin{array}{l}\text { Average } \\
\text { PM } \\
\text { Density } \\
(\mathrm{g} / \mathrm{L})\end{array}$ & $\begin{array}{l}95^{\text {th }} \\
\text { PR } \\
(\mathrm{g} / \mathrm{L})\end{array}$ \\
\hline \multirow{4}{*}{ 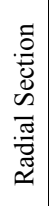 } & 1 & 0.94 & 3.41 & 0.73 & 0.97 & 3.89 & 0.60 & 0.98 & 3.34 & 0.58 & 0.95 & 3.19 & 0.82 \\
\hline & 2 & 0.93 & 3.42 & 0.46 & 0.97 & 3.89 & 0.33 & 0.98 & 3.34 & 0.30 & 0.95 & 3.19 & 0.49 \\
\hline & 3 & 0.93 & 3.42 & 0.56 & 0.95 & 3.90 & 0.56 & 0.96 & 3.35 & 0.49 & 0.91 & 3.19 & 0.61 \\
\hline & 4 & 0.92 & 3.13 & 1.85 & 0.94 & 3.59 & 1.99 & 0.95 & 3.06 & 1.80 & 0.91 & 2.92 & 1.85 \\
\hline
\end{tabular}

\section{Phase 1 Test 4: Active Regeneration}

Test 4 consisted of loading a substrate to $5.12 \mathrm{~g} / \mathrm{L}$ and then performing a $600{ }^{\circ} \mathrm{C}$ active regeneration where $69 \%$ of the available PM was oxidized. A substrate scan was taken at the completion of the active regeneration and the PM density at the time of the scan was $1.54 \mathrm{~g} / \mathrm{L}$. The scan results are shown in Figure 6.13. The axial PM distribution in the bottom plot of Figure 6.13 shows that for the first $40 \%$ and last $10 \%$ of the axial length the PM density was $2.5 \mathrm{~g} / \mathrm{L}$, which is $64 \%$ higher than the substrate average. Between 40 and $90 \%$ of the axial length, the PM density was $0.5 \mathrm{~g} / \mathrm{L}$, which is $67 \%$ lower than the substrate average. This distribution resulted in an overall axial UI of 0.80 , which is the lowest of the five substrate scans analyzed for this work. The overall radial UI was 0.91 and the overall angular UI was 0.89. Figure 6.13 shows a lot of variation in radial sections 3 and 4 in both the radial and angular direction, so the calculated overall UI values agree with the visual representation of the data.

The axial PM distribution for each radial section is shown in Figure 6.14. The axial UI was found to decrease from 0.81 in radial section 1 to 0.78 in radial section 4 . This indicates that radial section 4 had more variation in the axial PM distribution than the other 3 radial sections. Radial section 4 had an average PM density that was $10 \%$ lower than the other radial sections. This is a result similar to the other scan results. The $95^{\text {th }}$ PR increased in each radial section, from 0.76 in radial section 1 to 2.75 in radial section 4. This indicates that the PM distribution is less consistent near the edges of the substrate. This result is similar to previous findings as well. For all radial sections, the PM density started to decrease at a $Z^{*}$ value of 0.40 and then increase again at a $Z^{*}$ value of 0.85. The axial PM distribution data in Table 6.13 show similar results. Radial section 4 had the lowest UI in axial segments 1 and 2. In axial segments 3 and 4 the UIs were similar for all radial sections. In axial segments 1 and 2, 47-58\% of the PM was oxidized (assuming a loading distribution similar to Test 1) and the PM distribution near the centerline was more uniform. In axial segments 3 and 4, the amount of PM oxidized increased to $80-90 \%$ and the PM distribution had a similar uniformity in all radial sections. This indicates that as the amount of PM oxidized increases, the amount of variation in the PM distribution in each radial section becomes similar. 


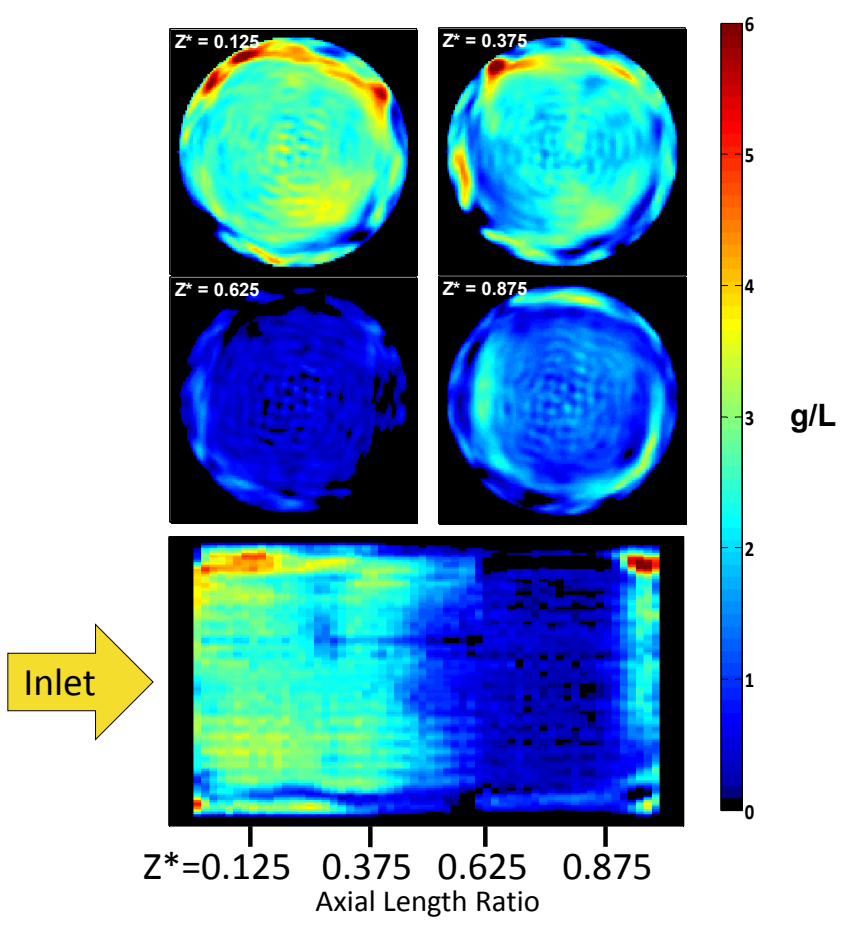

Figure 6.13: Phase 1 Test 4- Active Regeneration Scan Results

Test 4: Active Regeneration

Axial PM Distribution in Each Radial Section

Average Radial Uniformity Index $=0.91$

Average Angular Uniformity Index $=0.89$
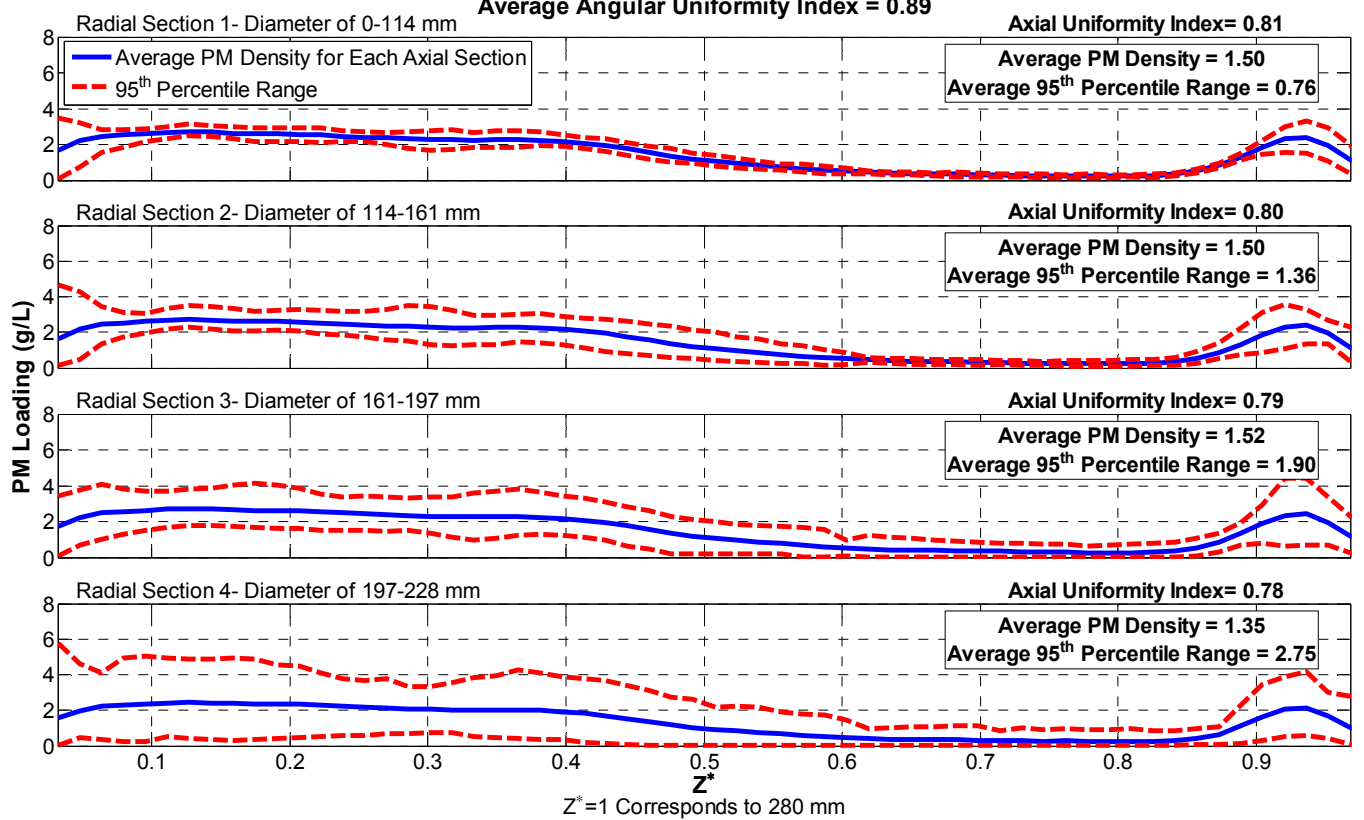

Figure 6.14: Phase 1 Test 4- Active Regeneration Axial PM Distribution in Each Radial Section 
Table 6.13: Phase 1 Test 4- Active Regeneration Axial PM Distribution

\begin{tabular}{|c|c|c|c|c|c|c|c|c|c|c|c|c|c|}
\hline & \multicolumn{12}{|c|}{ Axial Segment } \\
\hline & & \multicolumn{3}{|c|}{1} & \multicolumn{3}{|c|}{2} & \multicolumn{3}{|c|}{3} & \multicolumn{3}{|c|}{4} \\
\hline & & UI & $\begin{array}{c}\text { Average } \\
\text { PM } \\
\text { Density } \\
(\mathrm{g} / \mathrm{L})\end{array}$ & $\begin{array}{c}95^{\text {th }} \\
\text { PR } \\
(\mathrm{g} / \mathrm{L})\end{array}$ & UI & $\begin{array}{c}\text { Average } \\
\text { PM } \\
\text { Density } \\
(\mathrm{g} / \mathrm{L})\end{array}$ & $\begin{array}{l}95^{\text {th }} \\
\text { PR } \\
(\mathrm{g} / \mathrm{L})\end{array}$ & UI & $\begin{array}{c}\text { Average } \\
\text { PM } \\
\text { Density } \\
(\mathrm{g} / \mathrm{L})\end{array}$ & $\begin{array}{c}95^{\text {th }} \\
\text { PR } \\
(\mathrm{g} / \mathrm{L})\end{array}$ & UI & $\begin{array}{c}\text { Average } \\
\text { PM } \\
\text { Density } \\
(\mathrm{g} / \mathrm{L})\end{array}$ & $\begin{array}{c}95^{\text {th }} \\
\text { PR } \\
(\mathrm{g} / \mathrm{L})\end{array}$ \\
\hline \multirow{4}{*}{ 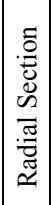 } & 1 & 0.78 & 2.49 & 1.08 & 0.86 & 2.04 & 0.78 & 0.80 & 0.52 & 0.30 & 0.81 & 0.99 & 0.66 \\
\hline & 2 & 0.78 & 2.50 & 1.71 & 0.84 & 2.04 & 1.74 & 0.80 & 0.52 & 0.65 & 0.80 & 0.99 & 1.08 \\
\hline & 3 & 0.76 & 2.51 & 2.41 & 0.83 & 2.05 & 2.18 & 0.79 & 0.55 & 1.17 & 0.79 & 1.02 & 1.56 \\
\hline & 4 & 0.72 & 2.26 & 4.33 & 0.81 & 1.82 & 3.19 & 0.81 & 0.48 & 1.42 & 0.80 & 0.88 & 1.77 \\
\hline
\end{tabular}

The radial PM distribution data for the four quadrants and four axial segments are shown in Table 6.14. Axial segment 3 was the only axial segment to have a uniform radial distribution. This agrees with the PM distribution show in Figure 6.13 for a $Z^{*}$ value of 0.625 .

Table 6.14: Phase 1 Test 4- Active Regeneration Radial PM Distribution

\begin{tabular}{|c|c|c|c|c|c|c|c|c|c|c|c|c|c|}
\hline & \multicolumn{12}{|c|}{ Axial Segment } \\
\hline & & \multicolumn{3}{|c|}{1} & \multicolumn{3}{|c|}{2} & \multicolumn{3}{|c|}{3} & \multicolumn{3}{|c|}{4} \\
\hline & & UI & $\begin{array}{c}\text { Average } \\
\text { PM } \\
\text { Density } \\
(\mathrm{g} / \mathrm{L}) \\
\end{array}$ & $\begin{array}{l}95^{\text {th }} \\
\text { PR } \\
(\mathrm{g} / \mathrm{L})\end{array}$ & UI & $\begin{array}{l}\text { Average } \\
\text { PM } \\
\text { Density } \\
(\mathrm{g} / \mathrm{L}) \\
\end{array}$ & $\begin{array}{l}95^{\text {th }} \\
\text { PR } \\
(\mathrm{g} / \mathrm{L})\end{array}$ & UI & $\begin{array}{l}\text { Average } \\
\text { PM } \\
\text { Density } \\
(\mathrm{g} / \mathrm{L})\end{array}$ & $\begin{array}{l}95^{\text {th }} \\
\text { PR } \\
(\mathrm{g} / \mathrm{L})\end{array}$ & UI & $\begin{array}{l}\text { Average } \\
\text { PM } \\
\text { Density } \\
(\mathrm{g} / \mathrm{L})\end{array}$ & $\begin{array}{l}95^{\text {th }} \\
\text { PR } \\
(\mathrm{g} / \mathrm{L})\end{array}$ \\
\hline \multirow{4}{*}{ 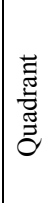 } & 1 & 0.86 & 2.43 & 0.99 & 0.93 & 2.08 & 0.54 & 0.96 & 0.53 & 0.36 & 0.93 & 0.91 & 0.34 \\
\hline & 2 & 0.90 & 2.42 & 0.71 & 0.90 & 2.15 & 0.92 & 0.95 & 0.55 & 0.25 & 0.92 & 0.87 & 0.41 \\
\hline & 3 & 0.88 & 2.21 & 1.17 & 0.92 & 1.81 & 1.05 & 0.96 & 0.57 & 0.22 & 0.92 & 0.92 & 0.35 \\
\hline & 4 & 0.86 & 2.07 & 0.82 & 0.89 & 1.91 & 0.26 & 0.95 & 0.44 & 0.26 & 0.92 & 0.92 & 0.21 \\
\hline
\end{tabular}

The angular PM distribution in each radial section for a $Z^{*}$ value of 0.125 is shown in Figure 6.15. Radial sections 1 and 2 have an angular UI above 0.94, indicating a uniform PM distribution. However, the data in Table 6.15 show that none of the radial sections had a uniform PM distribution in axial segment 1. Radial section 1 did have an angular PM distribution that was uniform in axial segments 2-4, and radial section 2 was uniform in axial segment 3. The other UIs were not uniform, indicating a more uniform PM distribution exists near the center of the substrate. Radial section 4 had a PM density that was $9 \%$ lower than the other three radial sections. Table 6.15 shows that this was true for all axial segments. 
Test 4: Active Regeneration

Angular PM Distribution in Each Radial Section at $Z^{*}=0.125$

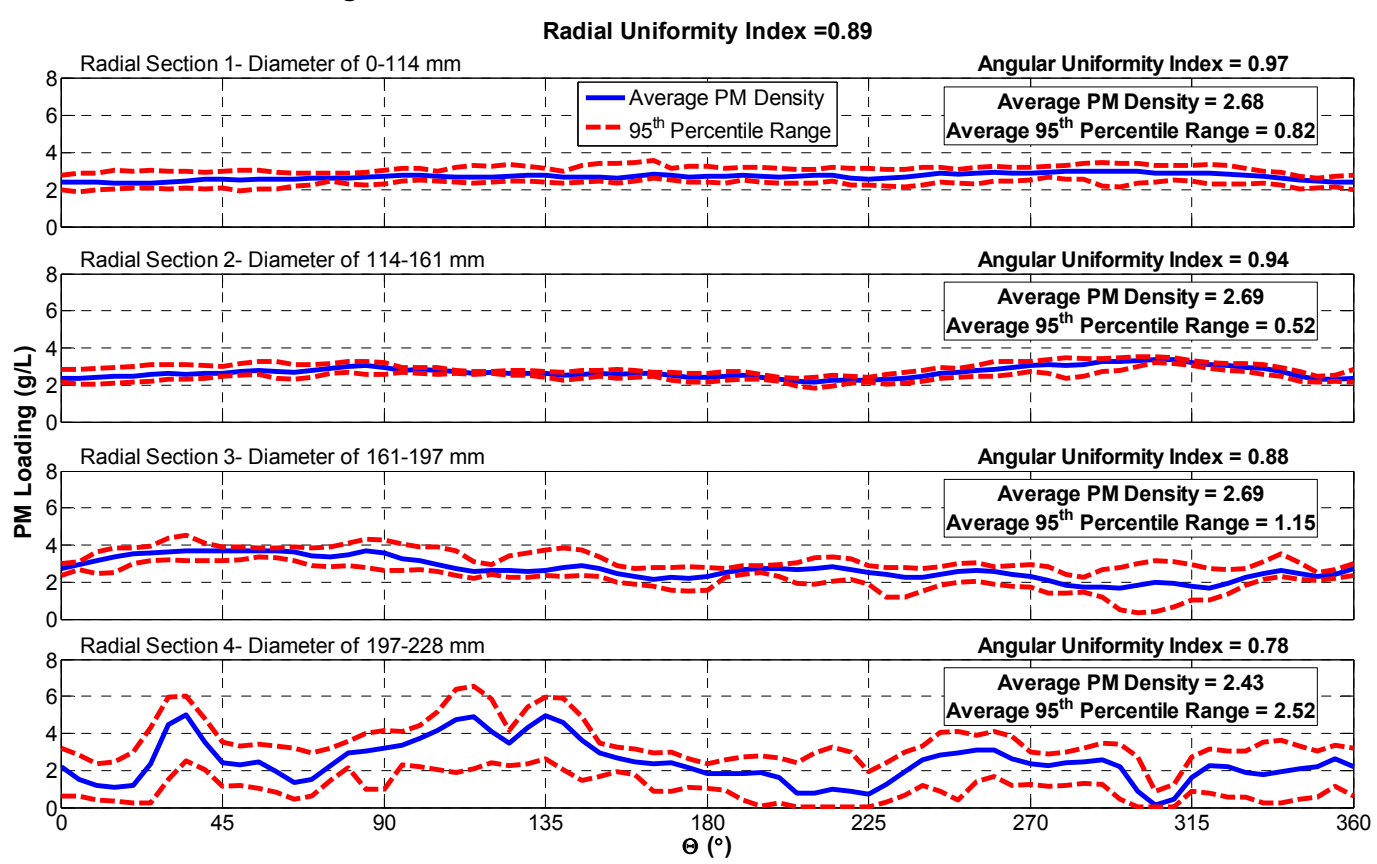

Figure 6.15: Phase 1 Test 4- Active Regeneration Angular PM Distribution in Each Radial Section

Table 6.15: Phase 1 Test 4- Active Regeneration Angular PM Distribution

\begin{tabular}{|c|c|c|c|c|c|c|c|c|c|c|c|c|c|}
\hline & \multicolumn{12}{|c|}{ Axial Segment } \\
\hline & & \multicolumn{3}{|c|}{1} & \multicolumn{3}{|c|}{2} & \multicolumn{3}{|c|}{3} & \multicolumn{3}{|c|}{4} \\
\hline & & UI & $\begin{array}{c}\text { Average } \\
\text { PM } \\
\text { Density } \\
(\mathrm{g} / \mathrm{L}) \\
\end{array}$ & $\begin{array}{l}95^{\text {th }} \\
\text { PR } \\
(\mathrm{g} / \mathrm{L})\end{array}$ & UI & $\begin{array}{l}\text { Average } \\
\text { PM } \\
\text { Density } \\
(\mathrm{g} / \mathrm{L})\end{array}$ & $\begin{array}{c}95^{\text {th }} \\
\text { PR } \\
\text { (g/L) }\end{array}$ & UI & $\begin{array}{l}\text { Average } \\
\text { PM } \\
\text { Density } \\
\text { (g/L) }\end{array}$ & $\begin{array}{l}95^{\text {th }} \\
\text { PR } \\
(\mathrm{g} / \mathrm{L})\end{array}$ & UI & $\begin{array}{l}\text { Average } \\
\text { PM } \\
\text { Density } \\
(\mathrm{g} / \mathrm{L}) \\
\end{array}$ & $\begin{array}{l}95^{\text {th }} \\
\text { PR } \\
(\mathrm{g} / \mathrm{L})\end{array}$ \\
\hline \multirow{4}{*}{ 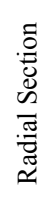 } & 1 & 0.91 & 2.49 & 0.90 & 0.96 & 2.04 & 0.75 & 0.98 & 0.52 & 0.63 & 0.95 & 0.99 & 0.76 \\
\hline & 2 & 0.88 & 2.50 & 0.70 & 0.91 & 2.04 & 0.62 & 0.96 & 0.52 & 0.43 & 0.93 & 0.99 & 0.68 \\
\hline & 3 & 0.84 & 2.51 & 1.41 & 0.88 & 2.05 & 1.51 & 0.93 & 0.55 & 0.72 & 0.90 & 1.02 & 1.18 \\
\hline & 4 & 0.77 & 2.26 & 2.60 & 0.85 & 1.82 & 2.17 & 0.92 & is th0.90 & 0.90 & 0.89 & 0.88 & 1.40 \\
\hline
\end{tabular}

\subsection{Changes in the PM Density Calculation Process}

After Phase 1 of the experimental study discussed in this thesis was completed, changes were made to the way in which the PM density in the substrate was calculated, based on the raw data from the TAS7000. A problem with the software that was originally used with the TAS7000 to calculate the PM density meant that a new method to calculate the PM density had to be developed, which was discussed in section 4.1. The results from the new method were compared to data sets that were processed using the original software to determine if there were any changes in the results of the substrate scan due to the new processing method. The results of the comparison are presented in this section. One of the main differences between the new and old PM density calculation methods is 
that the new method does not have the ability to distinguish between the carbonaceous PM and ash loading. Therefore, the new method measures the density of the PM, including carbonaceous and ash content. The tests that were used for the comparison did not have ash loading above 4 grams, or $0.3 \mathrm{~g} / \mathrm{L}$, so any effect of the ash content is considered minimal.

The first result was taken from the loading scan of Test 1 from the Phase 2 test matrix (section 5.2). The difference between the new data set and the original data set is shown in Figure 6.16. The approach to the plot is identical to the plots shown in section 6.1. The $Z^{*}$ values for the top four subplots are $0.128,0.376,0.624$, and 0.872 . The difference in the PM density is shown in grams per liter. A positive value indicates that the new PM density calculation method produced a PM density that was higher than the old method. A negative value indicates that the new PM density calculation method produced a PM density that was lower than the old method. The results in Figure 6.16 show that the center portion of the substrate had a calculated PM density that was higher with the new method. The maximum difference in the density analysis was found to be $0.50 \mathrm{~g} / \mathrm{L}$, or $18 \%$, in radial section 4 of axial segment 1 . As discussed in section 6.1 , there may be additional error in the data near the edges of the substrate caused by how the terahertz wave enters the substrate. The original PM density calculation process may have accounted for some of those errors. Radial sections 1-3 had differences of 0.06 to $0.15 \mathrm{~g} / \mathrm{L}$, or $2-6 \%$. The average difference for the entire substrate was $0.17 \mathrm{~g} / \mathrm{L}$, or $5.4 \%$.

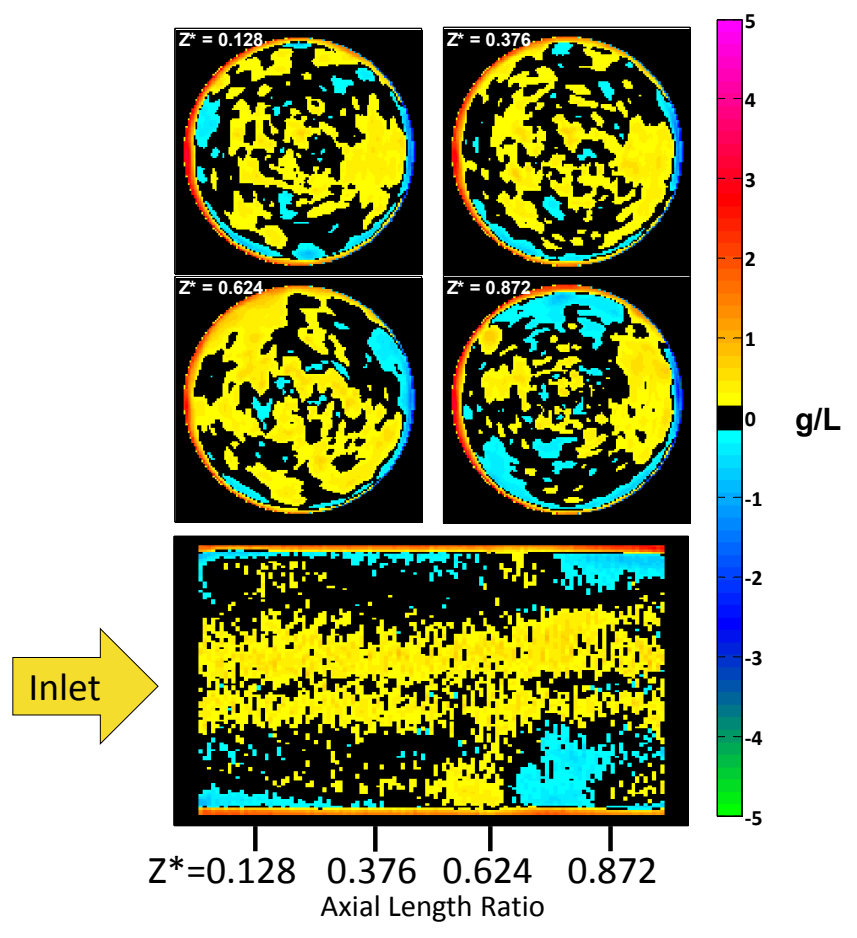

Figure 6.16: Phase 2 Test 1- Loading Difference in Scan Results

The new method of calculating the PM density in the substrate resulted in a more accurate calculation of total amount of PM retained in the substrate. For Test 1 of Phase 
2 , the old method calculated the PM retained to be 2.08 grams lower than the measured value, which is a difference of $4 \%$. The new analysis method calculated the PM retained to be 1.32 grams higher than the measured value, which is a difference of $2.5 \%$.

The second result was taken from the passive oxidation scan in Test 1 of the Phase 2 test matrix. The differences for this test are shown in Figure 6.17. The results are similar to the results in Figure 6.16. The data show that a maximum difference of $0.34 \mathrm{~g} / \mathrm{L}$, or $13 \%$, occurred in radial section 4 of axial segment 2 . Radial sections 1-3 had differences in the range of $0.05-0.15 \mathrm{~g} / \mathrm{L}$, or $1-5 \%$. The overall difference was $0.17 \mathrm{~g} / \mathrm{L}$, or $5.1 \%$. The calculated PM loading went from being 2.8\% lower than the measured value to $2.3 \%$ higher than the measured value. This is not as significant of an improvement in the calculated PM density as what was found for the loading scan from Test 1 of Phase 2.

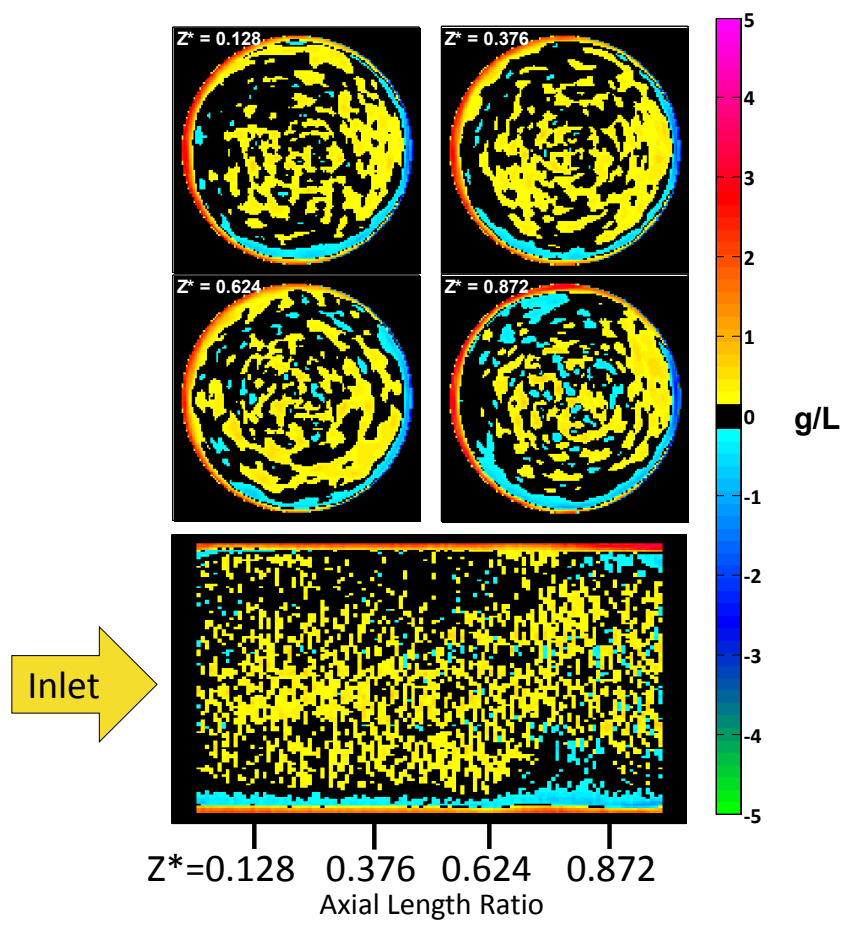

Figure 6.17: Phase 2 Test 1- Passive Oxidation Difference in Scan Results

The difference between the new method of calculating the PM density and the old method was calculated for seven additional data sets. These data sets were from the scanning repeatability and shipping orientation tests mentioned in section 5.2. These tests were completed using the substrate from Test 4 of Phase 1, shown in Figure 6.13. The differences in these tests were more significant than the differences calculated for the two scans from Test 1 . The difference data collected from the repeatability tests had PM densities that were lower in the front half of the substrate and higher in the back half of the substrate, with all radial sections having a similar amount of difference. Axial segments 1 and 2 had PM densities that were -0.81 to $-0.02 \mathrm{~g} / \mathrm{L}$, or -27 to $-1 \%$, different with the new PM density calculation method, than the old PM density calculation method. Axial segments 3 and 4 were $0.30-0.57 \mathrm{~g} / \mathrm{L}$, or $47-147 \%$, higher with the new 
PM density calculation method. Unlike the data sets from Test 1 of Phase 2, the differences for the repeatability tests are significant. One additional difference in the processing of the new PM density calculation method and the original PM density calculation method for the repeatability data sets is the number axial sections that were used. The original PM density calculation method used 64 axial sections, and the new PM density calculation method used 63. It is unknown why the number of axial sections produced by the TAS7000 was different, but that could have an effect on the comparison between the old and new PM density calculation methods.

The difference data from the shipping orientation tests show a trend similar to the difference data from the repeatability tests. Axial segments 1 and 2 had average PM densities that were -1.11 to $-0.19 \mathrm{~g} / \mathrm{L}$, or -34 to $-10 \%$, different with the new PM density calculation method, in all radial sections. Axial segments 3 and 4 had average PM densities that were $0.29-0.52 \mathrm{~g} / \mathrm{L}$, or $38-125 \%$, higher with the new PM density calculation method. The number of axial sections used in the new PM density calculation method for the shipping orientation tests was 63 , similar to the repeatability tests. This may be a source of additional difference in the data set.

The new PM density calculation method produced results that were comparable to the method originally used with the TAS7000 data for the data sets from Test 1 of Phase 2, which had an identical number of axial sections used in the PM density calculation methods. The data from the repeatability and shipping orientation tests were significantly different. The differences between the new and the old calculation method is likely caused in part by the absence of the data blending algorithm used in the old calculation method used with the TAS7000 data [46]. The effect of the blending factor on the PM distribution trends is unknown. The differences between the original and new PM density calculation methods were greater when the PM density in the substrate was lower, so the blending factor may have had more of an impact on the PM distribution at lower average substrate PM densities. The PM density in the substrate was between 3.08 and $3.25 \mathrm{~g} / \mathrm{L}$ for the data sets from Test 1 of Phase 2. The PM density in the substrate for the shipping orientation and repeatability tests was $1.42 \mathrm{~g} / \mathrm{L}$. The small amount of difference that was found in the data sets analyzed from Test 1 of Phase 2 indicates that the new PM density calculation method worked. All of the data from the Phase 1 work was analyzed using the original PM density calculation method. All of the data from the Phase 2 work was analyzed using the new PM density calculation method.

\subsection{Advantest TAS7000 Repeatability}

The TAS7000 that was used to scan the substrates for the PM loading was a new instrument and therefore it was important to understand repeatability of the measurements. The repeatability was measured by scanning one substrate multiple times. Section 5.2 describes the one planned repeatability test that used the substrate from Test 4 of Phase 1 (Figure 6.13). For this test, the substrate was scanned five times. Two other repeatability tests were performed over the duration of the experimental work. One repeat scan was performed on the substrate used for the passive oxidation scan from Test 1 of Phase 2, and one repeat scan was performed on the substrate used for the passive 
oxidation scan from Test 7 of Phase 2. All of the PM density calculations were completed using the new method that was described in section 4.1. As a result, the data shown in this section is the measurement of the carbonaceous and ash content in the substrate.

The repeatability of the TAS7000 was analyzed by averaging the data from the multiple scans together, and calculating the $95 \%$ confidence interval (CI) for the average value. The Central Limit Theorem was used to calculate the $95 \%$ CI. Due to the low number of repeat samples taken, a t-distribution was used to calculate the $95 \%$ CI. The equation used for this was found in reference [41] as equation 8.2. Instead of presenting the $95 \%$ $\mathrm{CI}$ as upper and lower limits, the absolute amount of variation in the data set will be presented. The absolute amount of variation is calculated using Eq. 6.1, which is based on equation 8.2 in reference [41].

$$
C I=t_{n-1} \frac{s}{\sqrt{n}}
$$

Eq. 6.1

In Eq. 6.1, $t_{n-1}$ is the critical value of the t-distribution needed for the desired CI, $s$ is the standard deviation of the sample, and $n$ is the number of samples. The value of $t_{n-1}$ is taken from tables and uses $n-1$ degrees of freedom. The absolute value of the $95 \%$ CI is calculated, instead of the upper and lower limits of the sample mean, since the goal is to evaluate the repeatability of the TAS7000. A lower value for the $95 \% \mathrm{CI}$ indicates a more repeatable system.

The $95 \%$ CI that was calculated for the repeat scans taken on the substrate used for Test 4 of Phase 1 is plotted in Figure 6.18. The largest 95\% CI occurs before a $Z^{*}$ value of 0.25 . There may be a shift in the data around $Z^{*}=0.25$, so data between $Z^{*}$ values of 0 and 0.25 should not be considered accurate. The overall $95 \%$ CI was calculated to be $\pm 0.19 \mathrm{~g} / \mathrm{L}$. The average PM density in the substrate at the time of this scan was $1.42 \mathrm{~g} / \mathrm{L}$, and the $95 \% \mathrm{CI}$ is $14 \%$ of the average PM density in the substrate. 


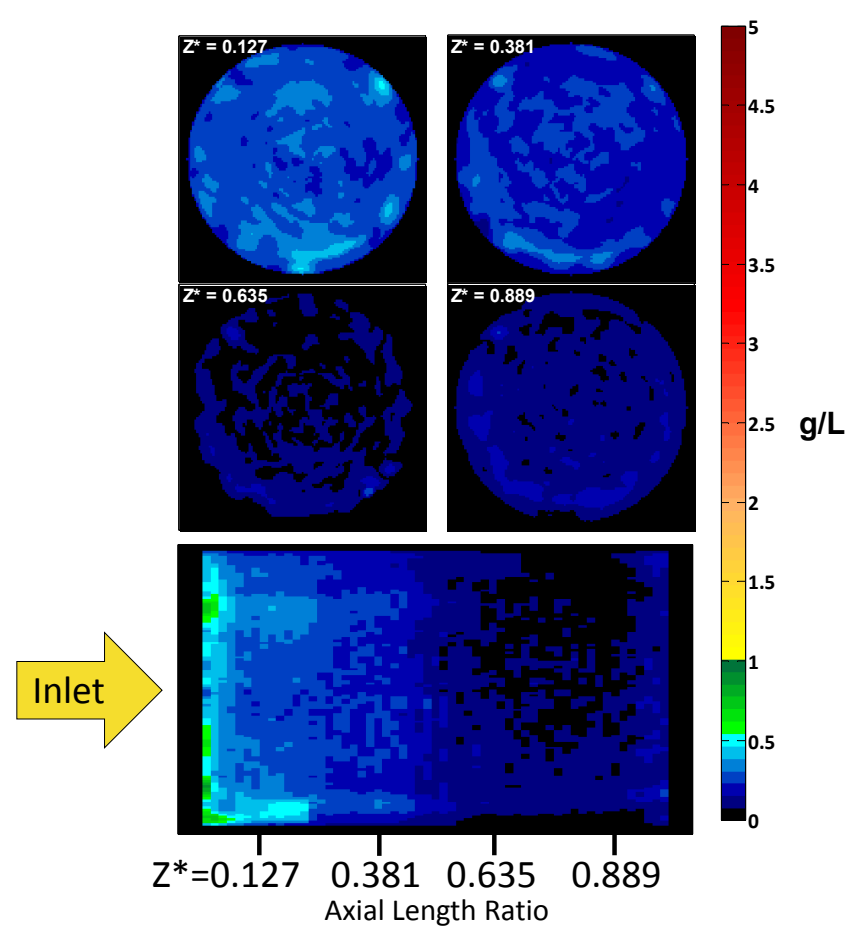

Figure 6.18: Repeat Scans of the Phase 1 Test 4 Scan Active Regeneration Scan

The $95 \%$ CI that was calculated for the repeat scans on the substrate used for the passive oxidation scan from Test 1 of Phase 2 is shown in Figure 6.19. The overall 95\% CI was calculated to be $\pm 0.62 \mathrm{~g} / \mathrm{L}$. This is $223 \%$ higher than the $95 \% \mathrm{CI}$ for the repeat scans from Test 4 of Phase 1. One hypothesis as to why the $95 \%$ CI is larger is that the coefficient of correlation for the data from the passive oxidation scan of Test 1 of Phase 2 had more variation in it than similar data sets. All of the coefficient of correlation plots for the data collected are shown in Appendix L. The increased amount of variation in the coefficient of correlation indicates that there is additional noise, or possible shifting, in the collected data. The notes associated with this substrate scan, and the data associated with this substrate scan, indicate that the TAS7000 did function correctly during the data collection. Therefore, the data set from this substrate scan is representative of normal TAS7000 operation. The data in Figure 6.19 show that all areas of the substrate had an increased $95 \% \mathrm{CI}$. The average PM density in the substrate at the time of the scan was $3.25 \mathrm{~g} / \mathrm{L}$ and the $95 \% \mathrm{CI}$ is $19 \%$ of the average PM density in the substrate. 


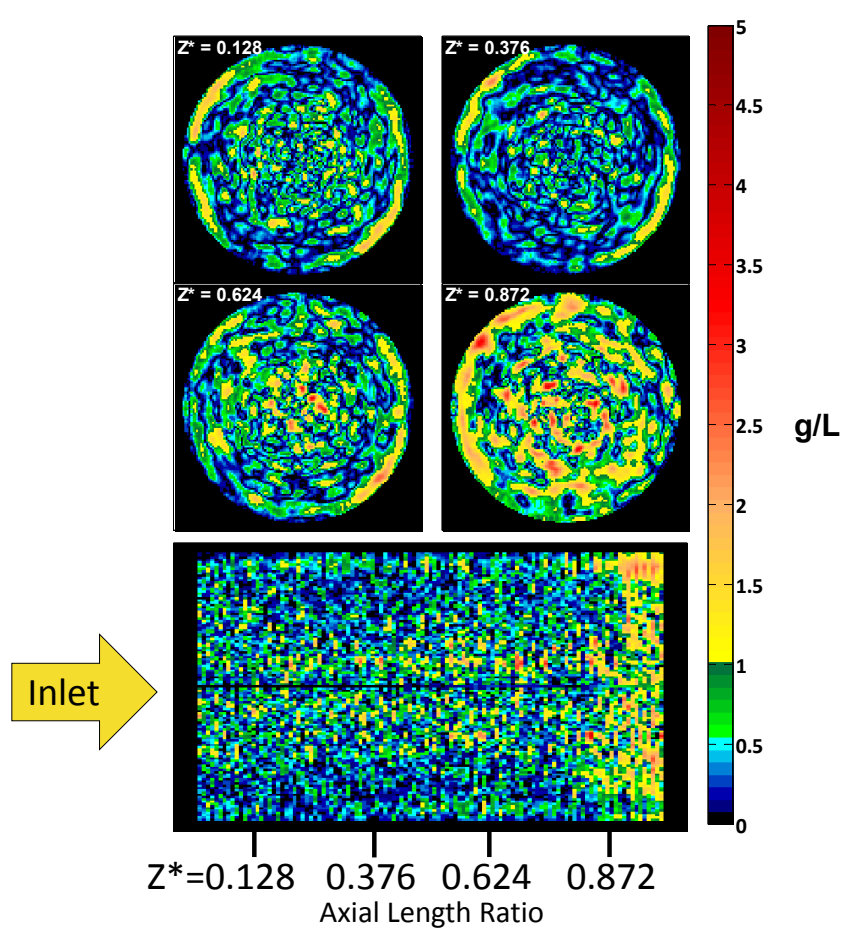

Figure 6.19: Repeat Scans of the Phase 2 Test 1 Passive Oxidation Scan

The $95 \%$ CI for the repeat scans taken from the substrate used for the passive oxidation scan in Test 7 of Phase 2 is shown in Figure 6.20. This result shows a similar amount of variation as Figure 6.18. The overall $95 \%$ CI was calculated to be $\pm 0.11 \mathrm{~g} / \mathrm{L}$. This is $42 \%$ lower than the $95 \%$ CI for the repeat scans of Test 4 from Phase 1 . The data used to calculate this $95 \%$ CI does not show any indicating of shifts. This indicates that the minimum $95 \% \mathrm{CI}$ for the TAS7000 is $\pm 0.11 \mathrm{~g} / \mathrm{L}$. The average PM density in the substrate at the time of the scan was $1.85 \mathrm{~g} / \mathrm{L}$, and the $95 \% \mathrm{CI}$ is $6 \%$ of the average value. 


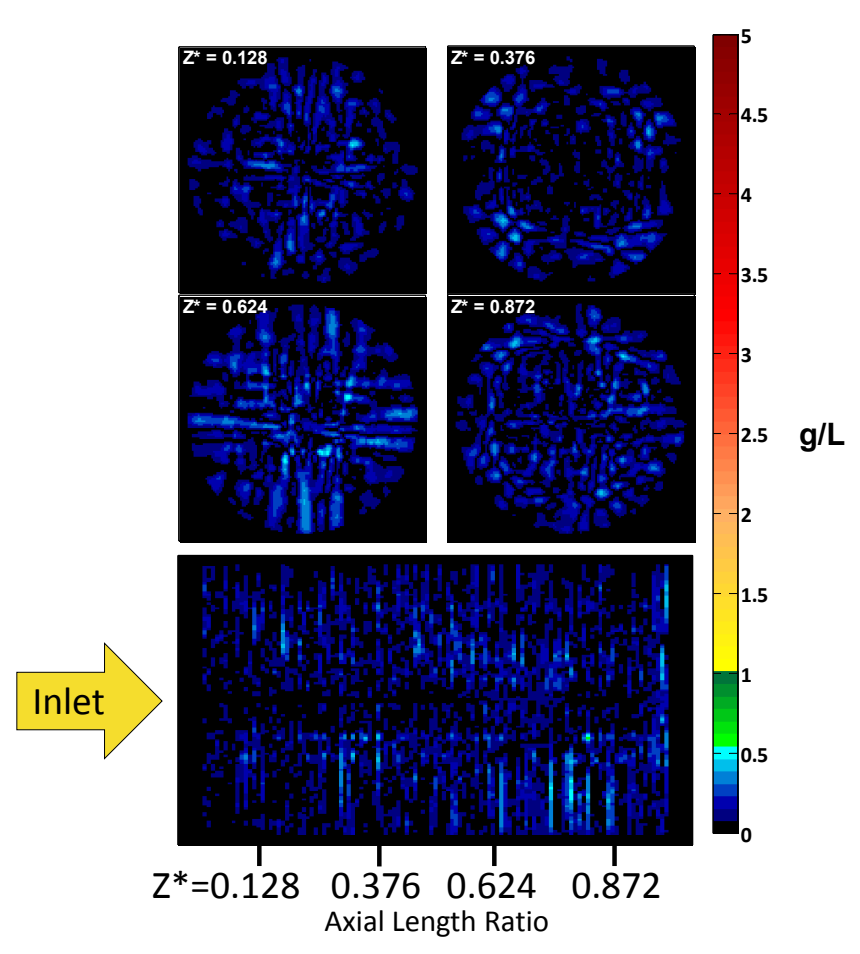

Figure 6.20: Repeat Scans of the Phase 2 Test 7 Passive Oxidation Scan

The average $95 \%$ CI for all three data sets that were used to analyze the repeatability of the TAS7000 was $\pm 0.31 \mathrm{~g} / \mathrm{L}$. Two of those data sets had $95 \%$ CI values between \pm 0.11 and $0.19 \mathrm{~g} / \mathrm{L}$, with the third data set having a $95 \% \mathrm{CI}$ value of $\pm 0.62 \mathrm{~g} / \mathrm{L}$. For the work performed for this thesis, the overall $95 \%$ CI of $\pm 0.31 \mathrm{~g} / \mathrm{L}$ will be considered while analyzing data sets. The higher $95 \%$ CI value of $\pm 0.62 \mathrm{~g} / \mathrm{L}$ is included in the repeatability analysis because the TAS7000 worked as intended, without any problems, during those scans. Therefore, there is no reason to exclude that data from the repeats of the passive oxidation scan for Test 1 of Phase 2. Using the 95\% CI value from the passive oxidation scans from Test 1 of Phase 2 allows the overall 95\% CI to be representative of the repeatability of the TAS7000 under standard operating conditions. The significance of the $95 \% \mathrm{CI}$ is that it indicates the point at which two data values can be said to be different. If the difference between the two data points is within the $95 \%$ $\mathrm{CI}$, the difference is within the measurement repeatability of the system and the difference should not be considered significant.

\subsection{Effect of Substrate Shipping Orientation}

The substrates that were used to study the PM distribution trends had to be shipped to Cummins to be scanned by the TAS7000, as mentioned in section 3.5. All of the substrates were shipped to Cummins with the inlet of the substrate facing upward. To determine if the shipping orientation impacted the PM distribution, a shipping test was conducted. The details of this test are given in section 5.2. The shipping orientation tests were conducted using the substrate that was used for the active regeneration scan in Test 4 of Phase 1. The substrate was shipped with the inlet facing upward, to the side, and 
downward. The shipping test was completed in this order so that the first substrate scan would be similar to the previous substrate scans, since all previous substrates were shipped with the inlet up. The differences between the inlet to the side and inlet up orientations and the inlet down and inlet to the side orientations were calculated to determine the effect of the shipping orientation on the PM distribution. All of the PM density calculations performed for this test used the new PM density calculation method discussed in section 4.1. The measurements shown represent the density of the carbonaceous and ash content in the substrate.

The differences in the axial PM distribution between the inlet to the side shipping orientation and the inlet up shipping orientation are shown in Table 6.16. A negative value indicates that the inlet to the side shipping orientation had a lower PM density in that location. Each axial segment and radial section show that the PM distribution changed. However, the differences are within the $95 \% \mathrm{CI}$ that was established for the TAS7000. Therefore, there are no significant differences in the PM distribution that resulted from shipping the substrate with the inlet to the side and shipping the substrate with the inlet up. The differences in the PM density were between -12 and $4 \%$ of the average PM density in the substrate.

Table 6.16: Difference Between Inlet to the Side and Inlet Up Orientations

\begin{tabular}{|c|c|c|c|c|c|}
\hline & \multicolumn{4}{|c|}{ Average Difference in PM Density in Each Axial Segment $(\mathrm{g} / \mathrm{L})$} \\
\hline & & 1 & 2 & 3 & 4 \\
\hline \multirow{4}{*}{ 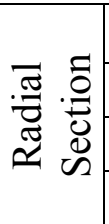 } & 1 & -0.17 & -0.12 & 0.03 & 0.05 \\
\hline & 2 & -0.17 & -0.12 & 0.03 & 0.05 \\
\hline & 3 & -0.17 & -0.12 & 0.03 & 0.05 \\
\hline & 4 & -0.16 & -0.12 & 0.03 & 0.05 \\
\hline
\end{tabular}

The differences in the axial PM distribution between the inlet down and inlet to the side shipping orientations are shown in Table 6.17. A negative value indicates that the inlet down shipping orientation had a lower PM density in that location. Each axial segment and radial section shows differences in the PM distribution. The differences in each axial segment are within the $95 \%$ CI of the TAS7000 data. This indicates that the PM distribution did not change significantly after shipping the substrate with the inlet down. The differences in the PM density were between -15 and $10 \%$ of the average PM density in the substrate.

Table 6.17: Difference Between Inlet Down and Inlet to the Side Orientations

\begin{tabular}{|c|c|c|c|c|c|}
\hline & \multicolumn{4}{|c|}{ Average Difference in PM Density in Each Axial Segment (g/L) } \\
\hline & & 1 & 2 & 3 & 4 \\
\hline \multirow{4}{*}{ 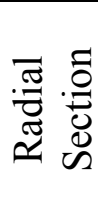 } & 1 & -0.21 & 0.14 & 0.12 & -0.12 \\
\hline & 2 & -0.21 & 0.14 & 0.12 & -0.12 \\
\hline & 3 & -0.21 & 0.14 & 0.12 & -0.12 \\
\hline & 4 & -0.18 & 0.15 & 0.13 & -0.10 \\
\hline
\end{tabular}


These results indicate that the shipping orientation of the substrate should be consistent, since minor changes in the PM distribution may occur, but the shipping orientation is not critical. Shipping the substrate with the inlet up, inlet to the side, and inlet down all produced similar results. It is worth noting that the shipping test was completed nine months after Test 4 of Phase 1 was completed due to problems with the TAS7000. The effect of that time on the PM is not known. The PM distributions that were measured for the tests described in sections 5.1 and 5.2 show that the loaded PM did not all end up at the outlet of the substrate (Figures 6.4, 6.7, 6.10, and 6.13). This indicates that shipping the substrate with the inlet up did not cause all of the PM loaded in the substrate to migrate towards the outlet. The effect of shipping the substrate with the inlet pointed upward cannot be quantified based on the results that were found. Although the shipping orientation did not affect the PM distribution, it is recommended to ship the substrate with the inlet up or to the side to prevent any possible loss of PM.

\subsection{PM Distribution Trends after Loading}

This section presents the results of the scans taken of substrates that were loaded with PM. A total of four scans were taken after substrates were loaded with PM. Two different size CPFs were used, and two different PM density levels were achieved. The first scan of a PM loaded substrate was presented in section 6.1. This was the loading scan from Test 1 of Phase 1 . The results from Phase 2 will be presented in this section and comparisons will be made. The TAS7000 data from Phase 2 was processed using the new PM density calculation method. Therefore, all of the Phase 2 tests results show the PM density, including the carbonaceous and ash content, in the substrate. For reference, the engine conditions used to load the substrates for the tests in Phase 1 and 2 were the "Loading" EOC and EOC B, respectively, and are presented in Tables 5.2 and 5.8, respectively. The important parameters from the loading tests are summarized in Table 6.18. The main parameter of interest during the loading tests was the PM density in the substrate after loading. The full test descriptions are provided in Chapter 5, and the full test summaries are provided in Appendix E. The tables of data that were presented for the tests in section 6.1 provided a better representation of the data for the entire substrate than the plots of the axial and angular PM distribution in each radial section. Therefore, the scan results and tables of data will be given in the main body of this thesis hereafter. Additional PM distribution plots can be found in Appendix K.

Table 6.18: Loading Test Summaries

\begin{tabular}{|c|c|c|c|c|c|c|c|}
\hline \multirow{2}{*}{ Engine } & Test & $\begin{array}{c}\mathrm{CPF} \\
\text { Space } \\
\text { Velocity }\end{array}$ & $\begin{array}{c}\text { Average } \\
\mathrm{CPF} \\
\text { Temp. }\end{array}$ & $\begin{array}{c}\text { Engine } \\
\text { Out PM } \\
\text { Conc. }\end{array}$ & $\begin{array}{c}\mathrm{NO}_{2} \\
\text { Conc. } \\
\text { into the } \\
\mathrm{CPF}\end{array}$ & $\begin{array}{c}\mathrm{O}_{2} \\
\text { Conc. } \\
\text { into the } \\
\mathrm{CPF}\end{array}$ & $\begin{array}{c}\mathrm{PM} \\
\text { Density } \\
\text { after } \\
\text { Loading }\end{array}$ \\
\cline { 3 - 8 } & $1 \mathrm{k} / \mathrm{hr}$ & ${ }^{\circ} \mathrm{C}$ & $\mathrm{mg} / \mathrm{scm}$ & $\mathrm{mg} / \mathrm{scm}$ & $\%$ & $\mathrm{~g} / \mathrm{L}$ \\
\hline ISB & Phase 1 Test 1 & 310 & 311 & 59.5 & 97.1 & 13.2 & 4.96 \\
\hline \multirow{2}{*}{ ISL } & Phase 2 Test 1 & 200 & 263 & 21.4 & 52.2 & 14.0 & 3.08 \\
\cline { 2 - 9 } & Phase 2 Test 6 & 213 & 272 & 21.1 & 60.6 & 13.4 & 2.81 \\
\cline { 2 - 8 } & $\begin{array}{c}\text { Phase 2 Test 6 } \\
\text { Second Loading }\end{array}$ & 222 & 275 & 20.9 & 42.5 & 13.5 & 5.18 \\
\hline
\end{tabular}




\section{Phase 2 Test 1: Loading}

The first loading result presented is from Test 1 of Phase 2. Three substrate scans were taken during Test 1 of Phase 2: one baseline, one after loading, and one after a passive oxidation. The PM density in the substrate at the time of the loading scan was $3.08 \mathrm{~g} / \mathrm{L}$. The result of this scan is shown in Figure 6.21. The overall axial UI was 0.90, which is similar to the overall axial UI for Test 1 of Phase 1 . The PM density distribution is different between the two tests however. The axial PM distribution data are provided in Table 6.19. Test 1 of Phase 1 shows an increasing PM density in all axial segments (Table 6.1). For Test 1 of Phase 1, the PM density in axial segment 1 was $5 \%$ lower than the substrate average and in axial segment 4 it was 3\% higher than the substrate average. For Test 1 of Phase 2, axial segments 1 and 4 had a PM density that was $12-15 \%$ higher than the substrate average and axial segments 2 and 3 had a PM density that was $0-7 \%$ lower than the substrate average. Axial segment 2 was the only axial segment to have a uniform axial PM distribution. The measured distribution for Test 1 of Phase 2 is similar to the predicted distribution shown in Figure 2.6. Radial section 4 has a $95^{\text {th }} \mathrm{PR}$ that is $32-179 \%$ greater than the other radial sections. This increase is likely due to the effect of the terahertz wave entering the edge of the substrate that was discussed in section 6.1. In general, radial section 1 had the lowest $95^{\text {th }} \mathrm{PR}$, which indicates that the PM distribution near the centerline of the substrate is more consistent than near the edges of the substrate. The temperature distribution plot shown in Figure I.6 shows that the temperature of the outer edges of the substrate was $25^{\circ} \mathrm{C}$ lower than the centerline and there was minimal temperature variation in the axial direction.

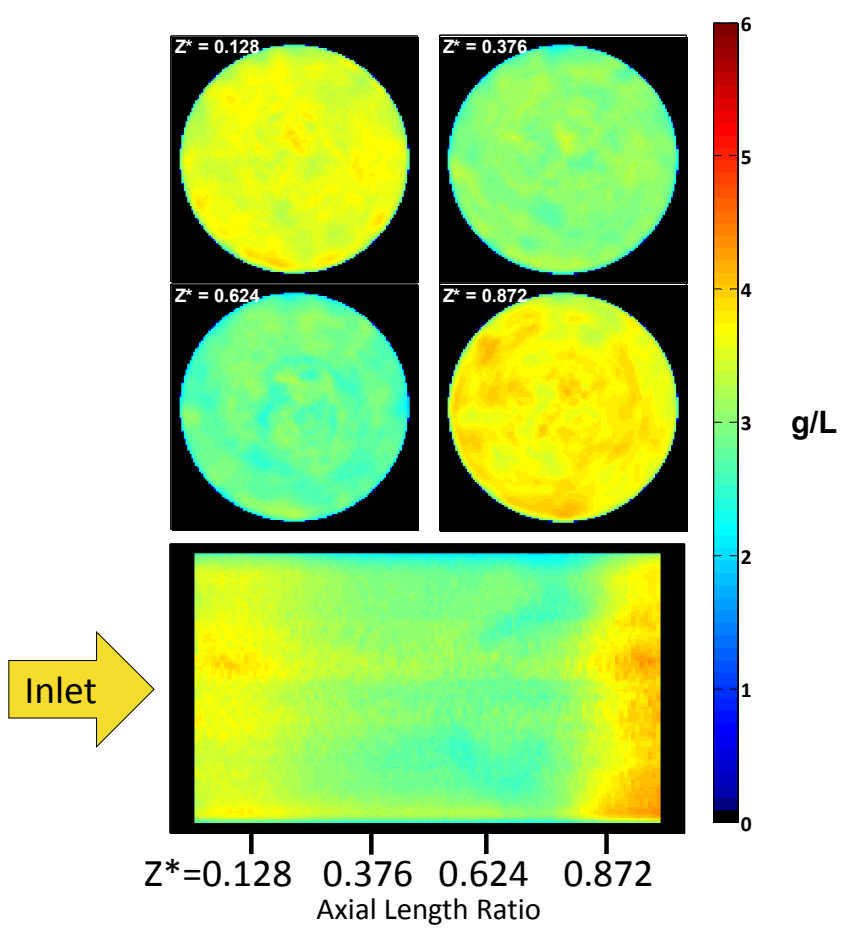

Figure 6.21: Phase 2 Test 1- Loading Scan Results 
Table 6.19: Phase 2 Test 1- Loading Axial PM Distribution

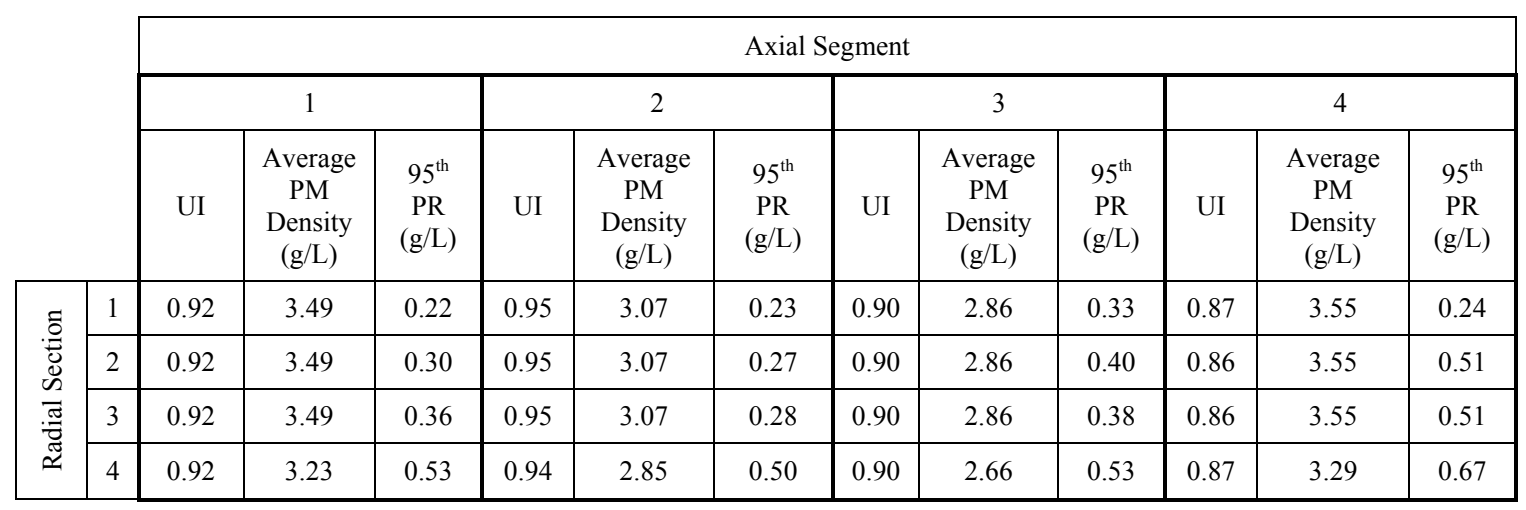

The overall radial UI was 0.96 for the loading scan from Test 1 of Phase 2. This is identical to the overall UI for the loading scan from Test 1 of Phase 1. The radial PM distribution data for the loading scan for Test 1 of Phase 2 are given in Table 6.20. All of the quadrants in all of the axial segments had a uniform distribution. This is consistent with the distributions shown in Figure 6.21, as there was not a large variation in the PM distribution in the radial direction.

Table 6.20: Phase 2 Test 1- Loading Radial PM Distribution

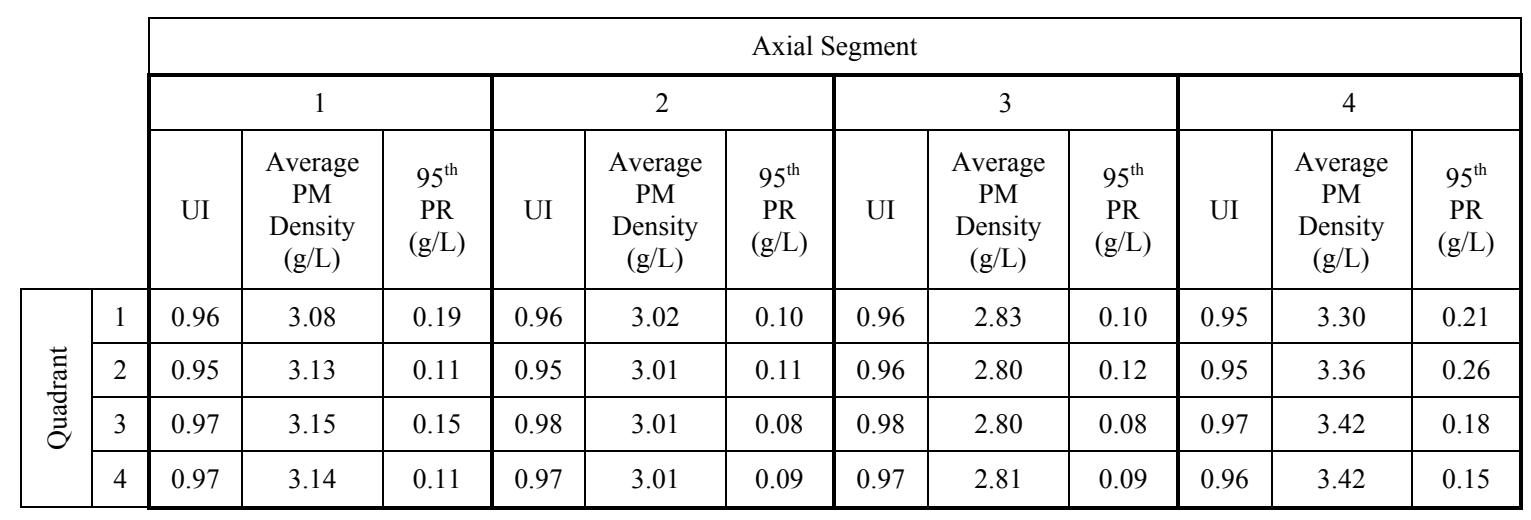

The overall angular UI for the loading scan from Test 1 of Phase 2 was 0.97 . This is similar to the overall angular UI for the loading scan from Test 1 of Phase 1. The angular PM distribution data for the loading scan from Test 1 of Phase 2 are given in Table 6.21. Radial section 4 had an average PM density that was 7\% lower than radial sections 1-3. The $95^{\text {th }} \mathrm{PR}$ is $411-1218 \%$ greater for radial section 4 than the other radial sections. The lower average PM density and higher $95^{\text {th }} \mathrm{PR}$ is caused by sample points on the edge of the substrate having a PM density of $0 \mathrm{~g} / \mathrm{L}$. 
Table 6.21: Phase 2 Test 1- Loading Angular PM Distribution

\begin{tabular}{|c|c|c|c|c|c|c|c|c|c|c|c|c|c|}
\hline & & \multicolumn{12}{|c|}{ Axial Segment } \\
\hline & & \multicolumn{3}{|c|}{1} & \multicolumn{3}{|c|}{2} & \multicolumn{3}{|c|}{3} & \multicolumn{3}{|c|}{4} \\
\hline & & UI & $\begin{array}{l}\text { Average } \\
\text { PM } \\
\text { Density } \\
(g / L)\end{array}$ & $\begin{array}{l}95^{\text {th }} \\
\text { PR } \\
(\mathrm{g} / \mathrm{L})\end{array}$ & UI & $\begin{array}{l}\text { Average } \\
\text { PM } \\
\text { Density } \\
\text { (g/L) }\end{array}$ & $\begin{array}{l}95^{\text {th }} \\
\text { PR } \\
(g / L)\end{array}$ & UI & $\begin{array}{l}\text { Average } \\
\text { PM } \\
\text { Density } \\
(g / L)\end{array}$ & $\begin{array}{l}95^{\text {th }} \\
\text { PR } \\
(\mathrm{g} / \mathrm{L})\end{array}$ & UI & $\begin{array}{c}\text { Average } \\
\text { PM } \\
\text { Density } \\
(\mathrm{g} / \mathrm{L})\end{array}$ & $\begin{array}{c}95^{\text {th }} \\
\text { PR } \\
(\mathrm{g} / \mathrm{L})\end{array}$ \\
\hline \multirow{4}{*}{ 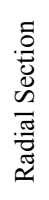 } & 1 & 0.98 & 3.49 & 0.28 & 0.98 & 3.07 & 0.28 & 0.97 & 2.86 & 0.35 & 0.98 & 3.55 & 0.38 \\
\hline & 2 & 0.97 & 3.49 & 0.17 & 0.98 & 3.07 & 0.16 & 0.97 & 2.86 & 0.21 & 0.96 & 3.55 & 0.25 \\
\hline & 3 & 0.97 & 3.49 & 0.17 & 0.98 & 3.07 & 0.16 & 0.97 & 2.86 & 0.18 & 0.96 & 3.55 & 0.19 \\
\hline & 4 & 0.96 & 3.23 & 2.24 & 0.96 & 2.85 & 1.93 & 0.96 & 2.66 & 1.79 & 0.95 & 3.29 & 2.27 \\
\hline
\end{tabular}

\section{Phase 2 Test 6: Loading}

The third loading scan was taken from Test 6 of Phase 2. There were two substrate scans performed during Test 6 , and both of them were for PM loading only. The substrate was loaded to $2.81 \mathrm{~g} / \mathrm{L}$ and then the first substrate scan was performed. The result of this scan is shown in Figure 6.22. The overall axial UI was 0.94, indicating a uniform distribution. This is $4-6 \%$ higher than the two previous loading scans that were analyzed. The axial PM distribution data is presented in Table 6.22. Axial segments 1-3 had a uniform distribution, indicating that the average PM density in those segments was near the average PM density for the substrate. The actual deviation from the substrate average in those axial segments was $0-2 \%$. The actual differences between axial segments 1-3 are within the $95 \%$ CI of the TAS7000 data. Axial segment 4 had a PM density that was 7\% higher than the substrate average. This distribution trend is different than the trends found for the loading scans from Test 1 of Phase 1 and Test 1 of Phase 2 . Test 1 of Phase 1 showed a continually increasing PM density in the axial direction, and Test 1 of Phase 2 had a higher PM density in axial segment 1 than axial segments 2 and 3 . The $95^{\text {th }}$ PR for radial section 1 was $3-78 \%$ lower than the other radial sections, which indicates that the PM distribution near the centerline of the substrate was more consistent than near the edges of the substrate. The temperature distribution plot shown in Figure I.13 shows that the temperature of the outer edges of the substrate was $25^{\circ} \mathrm{C}$ lower than the centerline and there was minimal temperature variation in the axial direction. 


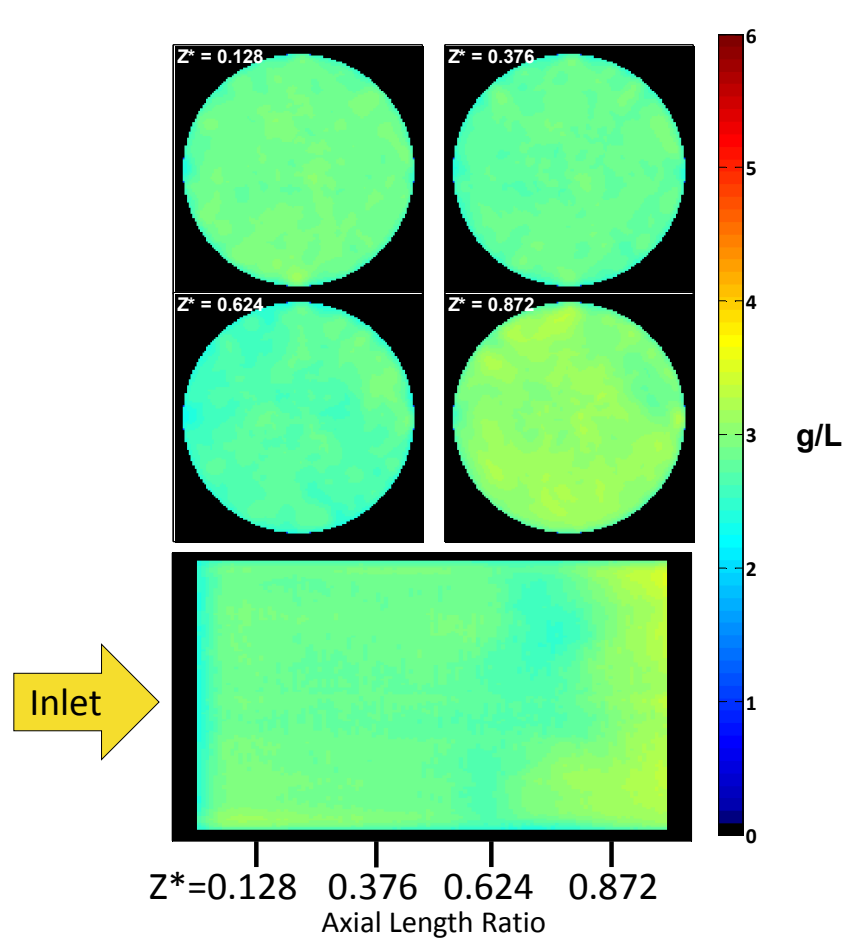

Figure 6.22: Phase 2 Test 6- Loading Scan Results

Table 6.22: Phase 2 Test 6- Loading Axial PM Distribution

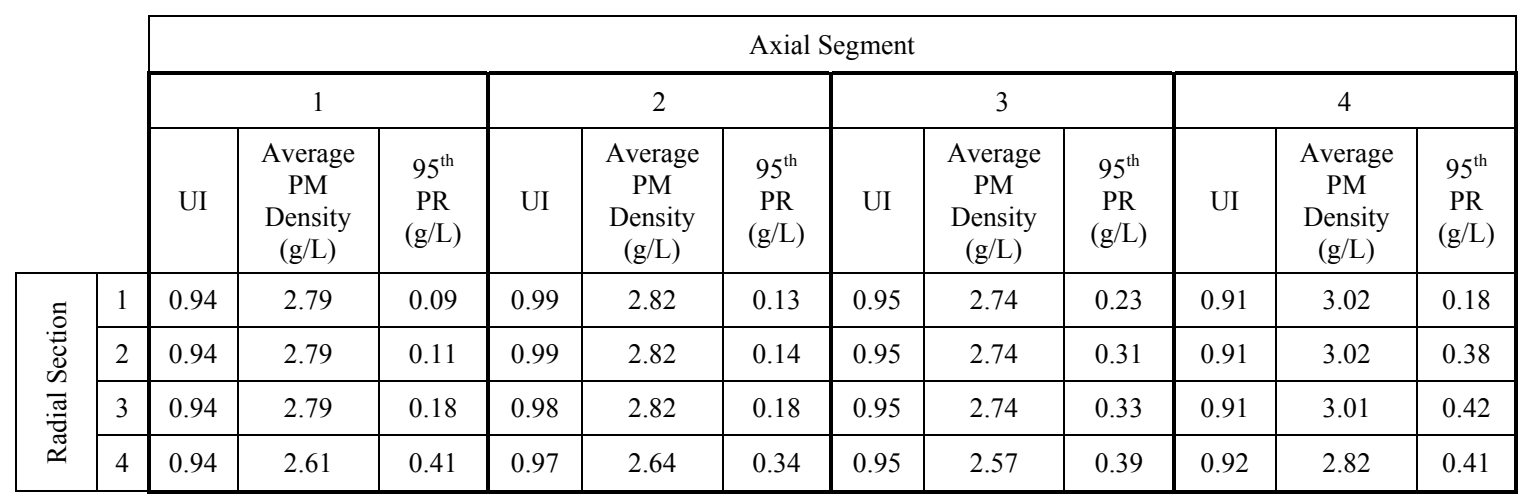

The radial distribution was uniform, with an overall radial UI of 0.97 . The radial distribution data are given in Table 6.23. All of the quadrants in each axial segment had a UI above 0.94, indicating a uniform distribution. This is consistent with the data shown in Figure 6.22. The average PM density in each quadrant was less than $2 \%$ different than the average PM density for the axial segment, indicating each quadrant was similarly loaded. 
Table 6.23: Phase 2 Test 6- Loading Radial PM Distribution

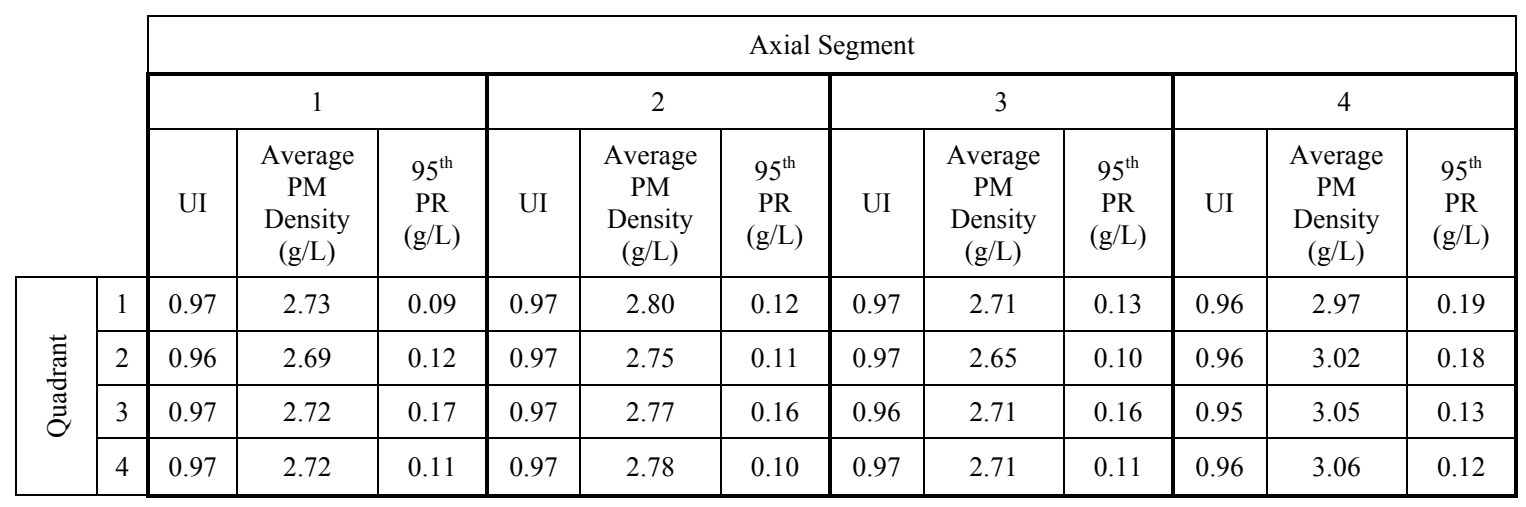

The overall angular UI was 0.97 as well, indicating a uniform distribution. The angular PM distribution data are given in Table 6.24. Each radial section in the axial segments had a UI above 0.94 . Radial section 4 had a PM density that was 6-7\% lower than radial sections 1-3. This is similar to the results from the other loading scans. The $95^{\text {th }} \mathrm{PR}$ for radial section 1 was $28-63 \%$ higher than radial sections 2 and 3 . This is consistent with findings from other tests.

Table 6.24: Phase 2 Test 6- Loading Angular PM Distribution

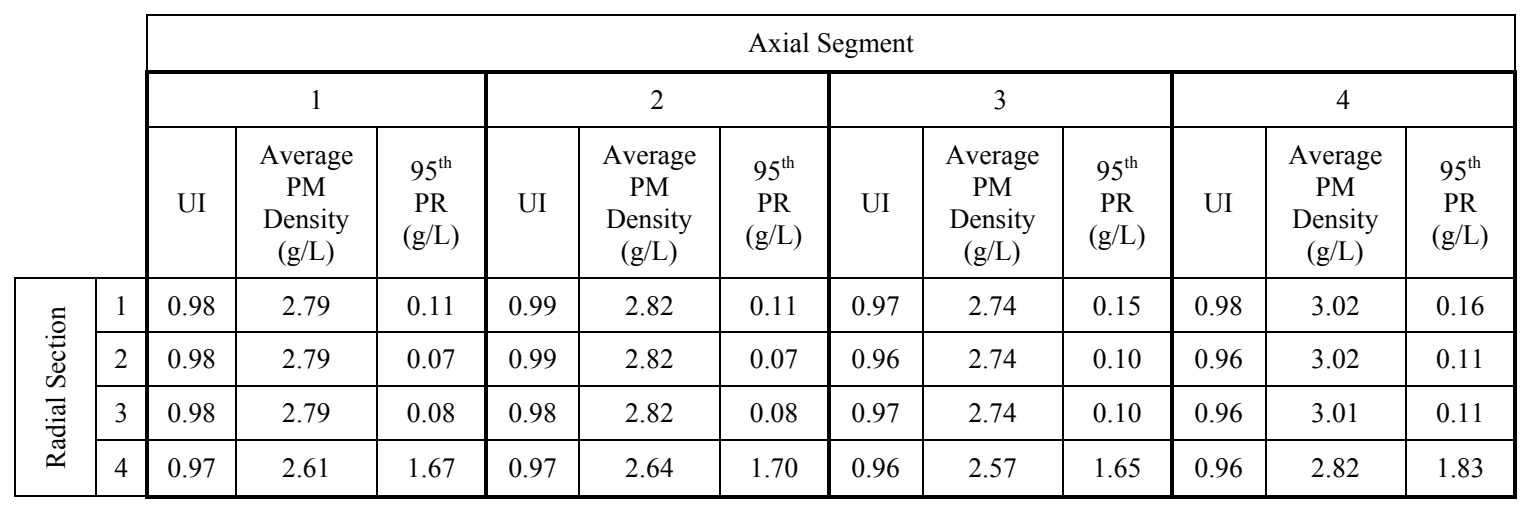

\section{Phase 2 Test 6: Second Loading}

The final loading scan that will be presented is the second scan taken during Test 6 of Phase 2. After the first scan from Test 6 was completed with a PM loading of $2.81 \mathrm{~g} / \mathrm{L}$, the substrate was loaded to $5.18 \mathrm{~g} / \mathrm{L}$. A second scan was taken at this point, which can be compared with the loading scan from Test 1 of Phase 1 . This scan will be referred to as a second loading since it was a continuation of the initial loading, without performing a passive oxidation or active regeneration stage. The scan results for the second loading processing are shown in Figure 6.23. The overall axial UI was 0.89, indicating a nonuniform distribution. This is identical to the overall axial UI for Test 1 of Phase 1 . The axial PM distribution data are given in Table 6.25. For the second loading of Test 6 , the PM density in the axial segment 1 was $1 \%$ lower than the substrate average and the PM 
density in axial segment 4 was 19\% higher than the substrate average. Axial segment 3 does not follow the trend of an increasing PM density in each axial segment, like what was found for the loading scan from Test 1 of Phase 1. Axial segment 3 had a PM density that was $2 \%$ lower than axial segment 2 . Axial segments 1-3 have a range of PM densities that are within the $95 \%$ CI of the TAS7000 data, which means the exact PM distribution trend between those axial segments cannot be precisely understood. Overall the PM distribution trend for the loading scan from Test 1 of Phase 1 and the second loading scan from Test 6 of Phase 2 are similar. The $95^{\text {th }}$ PR for radial section 1 was 21$73 \%$ lower than the other radial sections, indicting a more consistent PM distribution near the centerline of the substrate. The temperature distribution plot shown in Figure I.14 shows that the temperature of the outer edges of the substrate was $25^{\circ} \mathrm{C}$ lower than the centerline and there was minimal temperature variation in the axial direction.

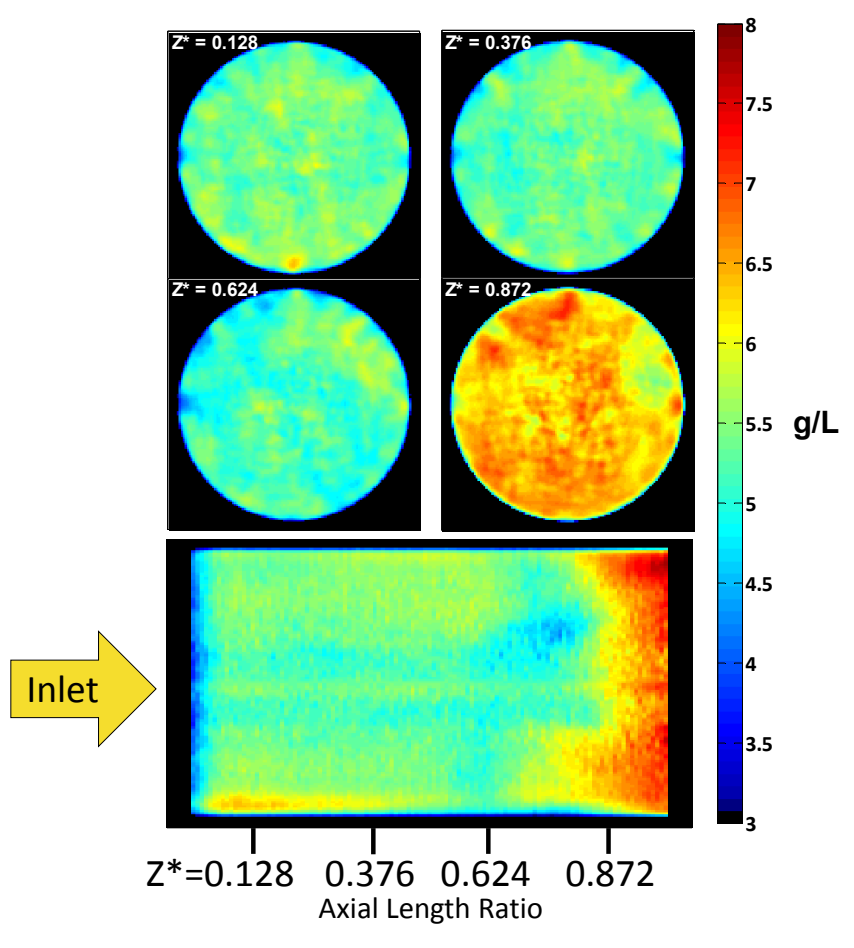

Figure 6.23: Phase 2 Test 6- Second Loading Scan Results 
Table 6.25: Phase 2 Test 6- Second Loading Axial PM Distribution

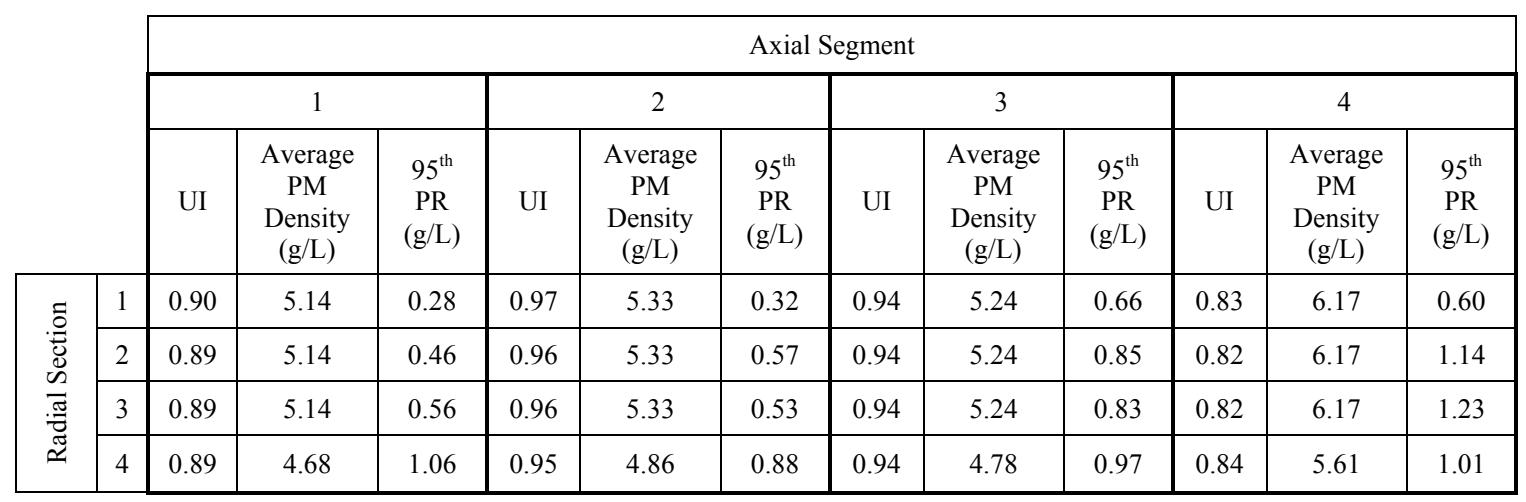

The overall radial UI was 0.95 for the second loading scan from Test 6 of Phase 2. This is similar to the overall radial UI for Test 1 of Phase 1 . The radial distribution data are given in Table 6.26. Every quadrant, except quadrants 1 and 2 in axial segment 4 , had a UI above 0.94, indicating the radial distributions are uniform. In quadrants 1 and 2 in axial segment 4 the UI was 0.93 . In Figure 6.23 , the plot for a $Z^{*}$ value of 0.872 shows variation in the PM density in quadrants 1 and 2 . This agrees with the calculated UI.

Table 6.26: Phase 2 Test 6- Second Loading Radial PM Distribution

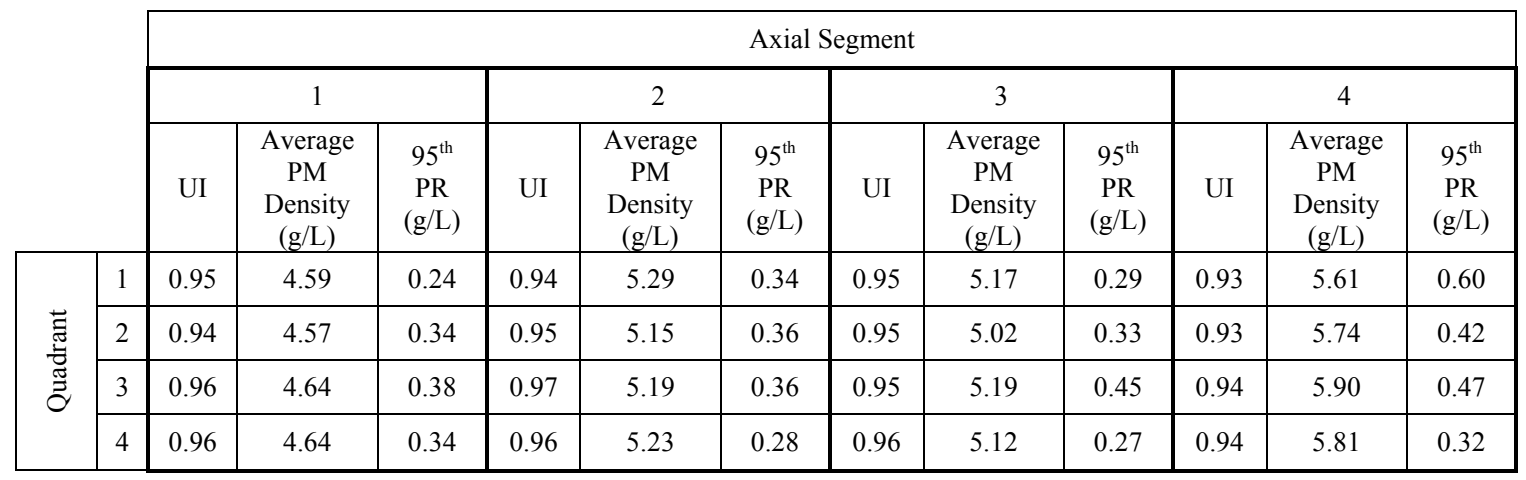

The overall angular UI was 0.95 for the second loading scan from Test 6 of Phase 2, which is similar to the overall angular UI for the loading scan from Test 1 of Phase 1 . The angular PM distribution data are given in Table 6.27. All radial sections, except for radial sections 2 and 3 in axial segment 4 , had a UI above 0.94 . Radial sections 2 and 3 had a UI of 0.93 , indicating a non-uniform distribution. Radial section 4 had a PM density that was $9 \%$ lower than radial sections $1-3$, which is similar to the other tests. 
Table 6.27: Phase 2 Test 6- Second Loading Angular PM Distribution

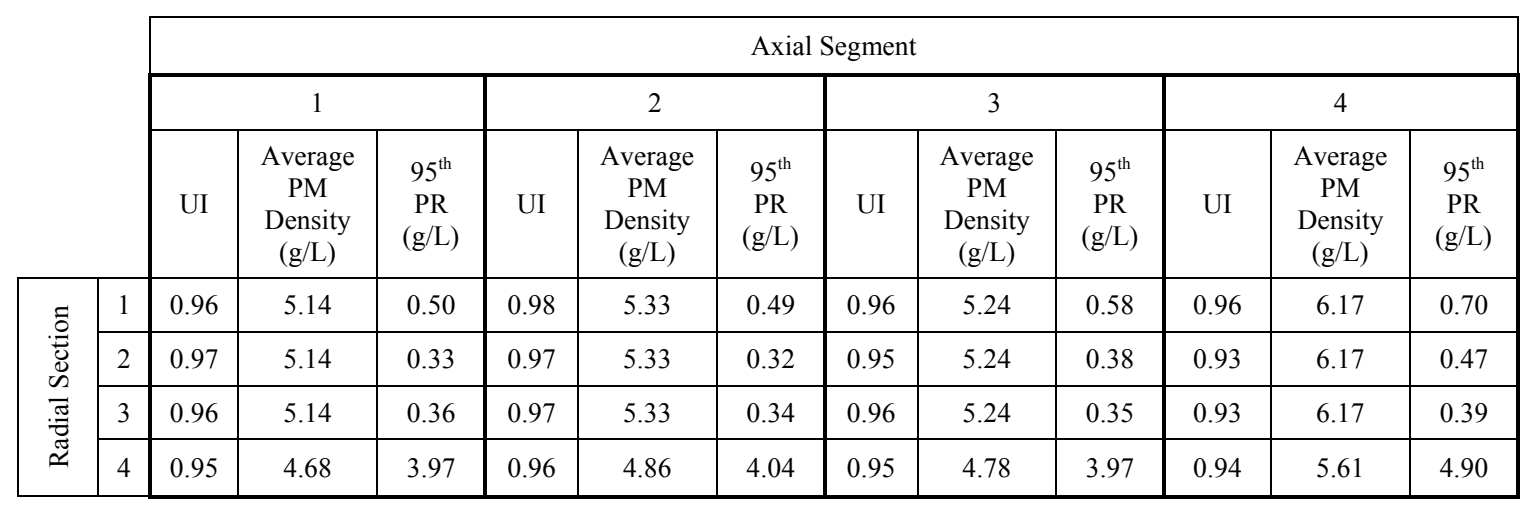

The processing that was done on both data sets from Test 6 of Phase 2 was slightly more complicated than the other data sets. Since there was no oxidation stage in Test 6 , the value of $\bar{w}$ was not as easily defined. The results that are presented in this section use the PM density at the time of the scan for the value of $\bar{w}$, to allow the two data sets to be compared to the other PM loading data sets from Test 1 of Phases 1 and 2 . If the final PM density in the substrate, $5.18 \mathrm{~g} / \mathrm{L}$, was used to process the first loading scan from Test 6 , the UI values increase $1-3 \%$ and the distribution is still classified as uniform. Processing the data in this way is useful when trying to understand how the uniformity of the PM distribution changes as a function of loading. The results of this processing show that the first loading scan from Test 6 of Phase 2 had an overall axial UI of 0.97, and the overall axial UI decreased by $8 \%$ to 0.89 for the second loading scan from Test 6 of Phase 2. The radial and angular overall UIs decreased by $3 \%$ from the first loading scan to the second loading scan, but remained uniform. This indicates that the uniformity of the PM distribution decreases as the PM density increases. If $\bar{w}$ was $2.81 \mathrm{~g} / \mathrm{L}$ when processing the second scan of Test 6 , the UI values would decrease $2-19 \%$. The overall radial and angular PM distributions would also become classified as non-uniform. Processing the results in this way is similar to how a post loading data set is processed. However, since there was no oxidation of the PM during Test 6, it is not appropriate to include those results with the post loading results as the effect of PM oxidation on the loading characteristics of the substrate could not be studied in that data set.

The axial PM distribution and overall axial UI varied for each test. The radial and angular PM distributions were more consistent between tests, and mostly uniform. The uniformity in the radial and angular direction agrees with the research by Ranalli, et al. [22], Pinturaud, et al. [19], Ranalli, et al. [31], and Stratakis, et al. [23] presented in section 2.4. To better understand the axial distribution trends, the PM density for each axial section was plotted versus the $Z^{*}$ values. The result is shown in Figure 6.24 and a summary of each test was provided in Table 6.18. For comparison, Figure 2.10 gives the PM cake thickness under loading conditions, measured for a scaled down particulate filter. Phase 2 Test 6 was the lowest loading, with an average PM density of $2.81 \mathrm{~g} / \mathrm{L}$. Phase 2 Test 1 had a PM loading of $3.08 \mathrm{~g} / \mathrm{L}$. The nearest loading in Figure 2.10 is $3.17 \mathrm{~g} / \mathrm{L}$, and the trend shows a PM loading in the front half of the substrate that is $24 \%$ 
lower than the substrate average and a PM loading in the back half of the substrate that is 18\% higher than the substrate average, which does not match the results in Figure 6.24. The trend shown for a loading of $5.75 \mathrm{~g} / \mathrm{L}$ in Figure 2.10 is similar to the trends shown for the Phase 1 Test 1 and Phase 2 Test 6 Second Loading data. The trend for Phase 2 Test 1 is similar to the trend shown in Figure 2.6b for a space velocity of $40 \mathrm{~s}^{-1}$. The trend for Phase 2 Test 6 is similar to the trend shown in Figure 2.6b for a space velocity of $40 \mathrm{~s}^{-1}$ as well, but the peaks at the inlet and outlet of the substrate are not as pronounced. The loading test from Phase 2 Test 1 was the first test on that particular substrate and Phase 2 Test 6 was the second test on that particular substrate. The difference in the PM distribution between the two tests could be the result of repeated testing on a substrate.

The axial distribution that was found for the second loading from Test 6 of Phase 2 has the same trend as the first loading scan from Test 6 , but the increase near the outlet of the substrate is greater. Axial segment 4 had an average PM density that was $7 \%$ greater than the substrate for the first loading and $19 \%$ greater for the second loading. The greater increase near the outlet of the substrate could be caused by the wall flow velocity distribution changing. In section 2.1, the wall flow velocity distribution was shown to change when the permeability of the substrate and PM cake changed, or when the exhaust gas velocity changed. The exhaust gas velocity increased by $3 \%$ between the first and second scans in Test 6 of Phase 2, which is a minor change. Therefore, any change in wall flow velocity distribution would likely be caused by changes in the permeability of the PM cake and substrate.

The axial PM distribution trend was similar for Test 1 of Phase 1 and the second loading for Test 6 of Phase 2. This indicates that the modified engine calibration used during the Phase 1 loading did not have a significant impact on the PM distribution after loading. The space velocity for Test 1 of Phase 1 was $40 \%$ greater than the space velocity for the second loading of Test 6 of Phase 2, as indicated in Table 6.18. The similarity in the axial PM distribution indicates that the PM distribution was not affected by the space velocity, when the average PM density was near $5 \mathrm{~g} / \mathrm{L}$, for these tests. In general, the axial PM distribution trends in Figure 6.24 follow the wall flow velocity distribution shown in Figures 2.4 and 2.5. The axial PM distribution that was experimentally measured was compared to the axial PM distribution produced by the MTU 1D model by Premchand in reference [47], and the result is shown in Figure 6.25. In general, both the experimental and modeled data show an increase in the PM density near the inlet and outlet of the substrate. The PM density near the outlet is higher than the PM density near the inlet for the three data sets. The modeled data has a minimum density near $40 \%$ of the axial length and the experimental data has a minimum density near $70 \%$ of the axial length. The reason for the difference is unknown. In general, the modeled data and experimental data show similar trends. 


\section{Axial PM Distribution After Loading}

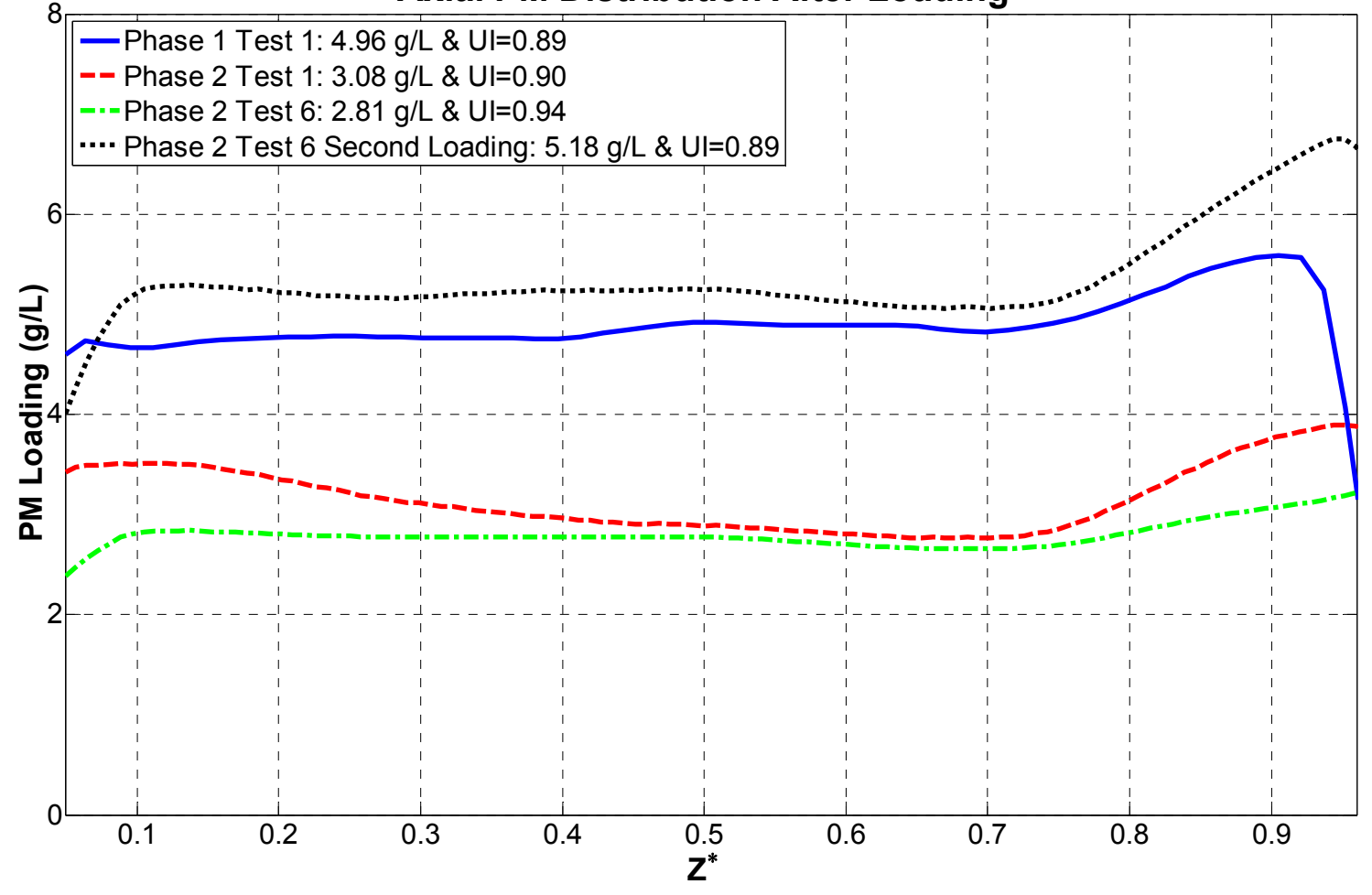

Figure 6.24: Axial PM Distribution for all Loading Tests

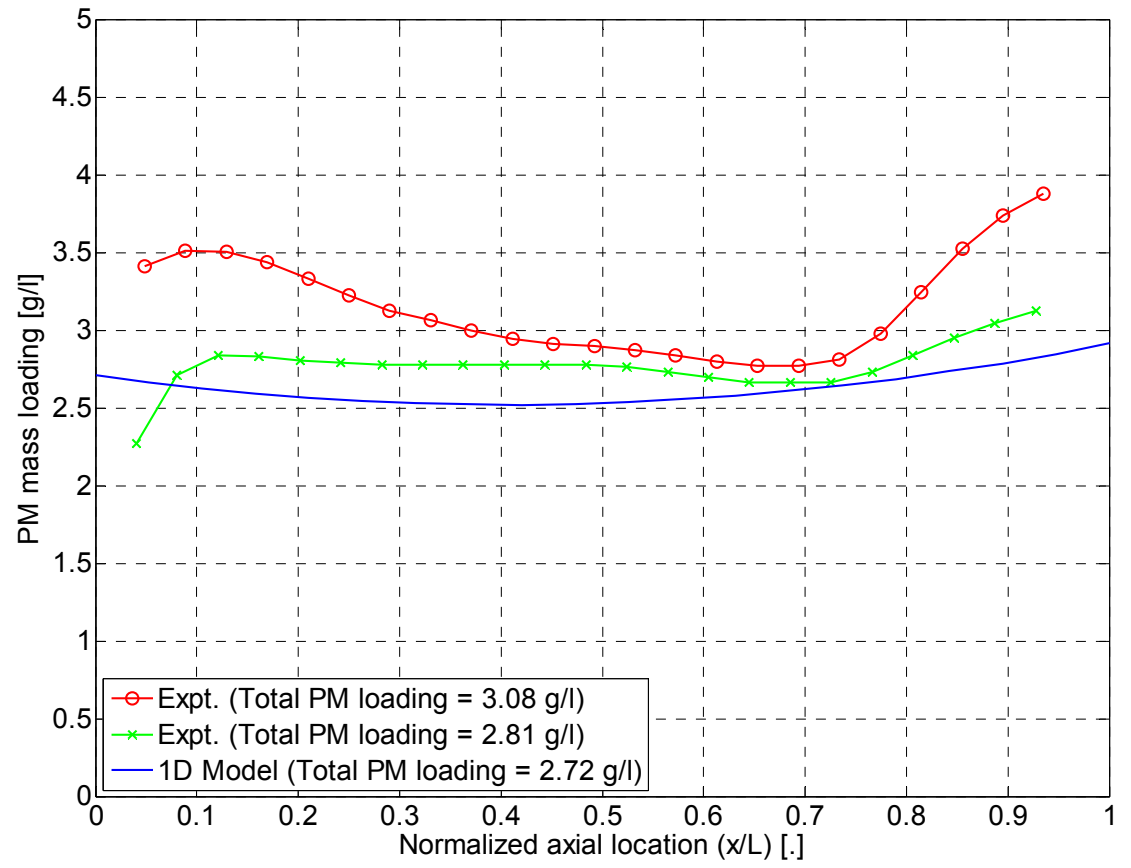

Figure 6.25: Axial PM Distribution Comparison Between Experimental and Modeled Data [47] Reprinted with Permission from Kiran Premchand's Doctoral Dissertation 
The overall axial UI was analyzed as a function of the PM density in the substrate. The result is shown in Figure 6.26. The data show that as the PM density in the substrate increases, the overall axial UI decreases. The axial PM distribution was not uniform after a PM loading of $2.81 \mathrm{~g} / \mathrm{L}$. The overall axial UI decreased by 0.01 between PM densities of 3 and $5.18 \mathrm{~g} / \mathrm{L}$. These data indicate that a PM density of $2.81 \mathrm{~g} / \mathrm{L}$ is the maximum to maintain a uniform distribution. Additionally, the data in Figures 6.24 and 6.26 show that the modified engine calibration used in the loading procedure during Test 1 of Phase 1 resulted in a similar distribution and UI as the standard engine calibration used during all of Phase 2. It can also be said that the two different sized substrates that were used, loaded in a similar fashion.

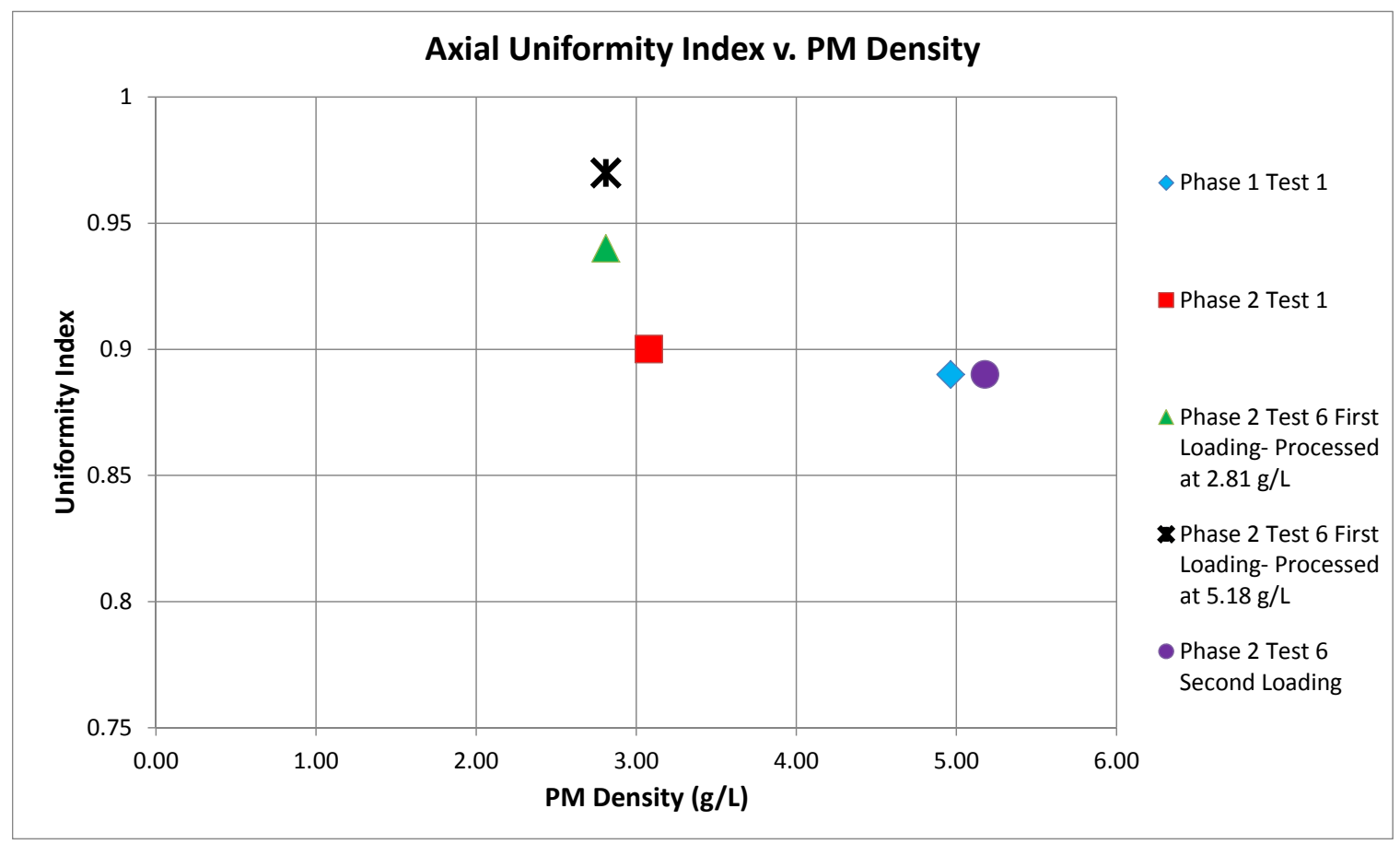

Figure 6.26: Axial UI as a Function of PM Density

\subsection{PM Distribution Trends after Passive Oxidation}

This section presents the results from the five substrate scans that were taken after passive oxidation conditions were run on PM loaded substrates. The substrate scans were completed with two different size substrates and three engine conditions were used for the passive oxidations. The first passive oxidation scan result is shown in section 6.1, in Figure 6.10. That passive oxidation used the "Oxidation" EOC presented in Table 5.2. The four passive oxidation tests that were completed as part of Phase 2 are presented in this section. The Phase 2 tests were processed using the new PM density calculation process, so the data in this section give the density of the PM, including the carbonaceous and ash content, in the substrate. Tests 1, 7, and 8 were all conducted at EOC F, and Test 9 was conducted using EOC A as the passive oxidation engine condition. The parameters of EOCs $\mathrm{F}$ and $\mathrm{A}$ are given in Table 5.8. Table 6.28 provides a summary of the main 
parameters for the five passive oxidation tests. The average $\mathrm{CPF}$ temperature for EOC $\mathrm{F}$ was $348{ }^{\circ} \mathrm{C}$ and the $\mathrm{NO}_{2}$ concentration into the $\mathrm{CPF}$ was $99 \mathrm{ppm}$ or $186 \mathrm{mg} / \mathrm{scm}$. The average CPF temperature for EOC A was $256{ }^{\circ} \mathrm{C}$ and the $\mathrm{NO}_{2}$ concentration into the CPF was $200 \mathrm{ppm}$ or $380 \mathrm{mg} / \mathrm{scm}$. The main parameters that were changed among the passive oxidation tests were the mass of the available PM that was oxidized and the density of the $\mathrm{PM}$ prior to the passive oxidation. The space velocities, $\mathrm{NO}_{2}$ concentrations, and reaction rates varied between tests as well. The full test descriptions are provided in Chapter 5 and the full test summaries are provided in Appendix E. A comparison of the reaction rates listed in Table 6.28 to the reaction rates for other passive oxidation tests can be found in Appendix F.

Table 6.28: Passive Oxidation Test Summaries

\begin{tabular}{|c|c|c|c|c|c|c|c|c|c|c|}
\hline \multirow[t]{2}{*}{ Engine } & \multirow[t]{2}{*}{ Test } & $\begin{array}{c}\text { CPF } \\
\text { Space } \\
\text { Velocity }\end{array}$ & $\begin{array}{c}\text { Average } \\
\text { CPF } \\
\text { Temp. }\end{array}$ & $\begin{array}{c}\text { Engine } \\
\text { Out PM } \\
\text { Conc. }\end{array}$ & $\begin{array}{l}\mathrm{NO}_{2} \\
\text { Conc. } \\
\text { into the } \\
\text { CPF }\end{array}$ & $\begin{array}{c}\mathrm{O}_{2} \\
\text { Conc. } \\
\text { into } \\
\text { the } \\
\mathrm{CPF}\end{array}$ & $\begin{array}{c}\text { PM } \\
\text { Density } \\
\text { before } \\
\text { Passive } \\
\text { Oxidation }\end{array}$ & $\begin{array}{c}\text { Percent } \\
\text { of } \\
\text { Available } \\
\text { PM } \\
\text { Oxidized }\end{array}$ & $\begin{array}{c}\text { PM } \\
\text { Density } \\
\text { at Time } \\
\text { of Scan }\end{array}$ & $\begin{array}{c}\text { Reaction } \\
\text { Rate }\end{array}$ \\
\hline & & $1 \mathrm{k} / \mathrm{hr}$ & ${ }^{\circ} \mathrm{C}$ & $\mathrm{mg} / \mathrm{scm}$ & $\mathrm{mg} / \mathrm{scm}$ & $\%$ & $\mathrm{~g} / \mathrm{L}$ & - & $\mathrm{g} / \mathrm{L}$ & $1 / \mathrm{s}$ \\
\hline ISB & $\begin{array}{c}\text { Phase } 1 \\
\text { Test } 3\end{array}$ & 177 & 372 & 8.3 & 480.7 & 7.9 & 5.81 & $44.9 \%$ & 3.33 & $1.95 \mathrm{E}-04$ \\
\hline \multirow{4}{*}{ ISL } & $\begin{array}{c}\text { Phase } 2 \\
\text { Test } 1\end{array}$ & 153 & 349 & 6.5 & 187.5 & 8.7 & 3.59 & $21.5 \%$ & 3.25 & $0.52 \mathrm{E}-04$ \\
\hline & $\begin{array}{c}\text { Phase } 2 \\
\text { Test } 7 \\
\end{array}$ & 150 & 353 & 10.1 & 175.1 & 8.7 & 3.23 & $64.5 \%$ & 1.85 & $1.14 \mathrm{E}-04$ \\
\hline & $\begin{array}{c}\text { Phase } 2 \\
\text { Test } 8\end{array}$ & 151 & 348 & 7.9 & 195.8 & 8.8 & 3.57 & $28.0 \%$ & 3.25 & $0.74 \mathrm{E}-04$ \\
\hline & $\begin{array}{c}\text { Phase } 2 \\
\text { Test } 9\end{array}$ & 89 & 256 & 5.8 & 379.8 & 12.5 & 3.13 & $5.6 \%$ & 3.51 & $0.10 \mathrm{E}-04$ \\
\hline
\end{tabular}

\section{Phase 2 Test 1: Passive Oxidation}

The third substrate scan that was taken during Test 1 of Phase 2 was taken after a passive oxidation experiment. The substrate was loaded to $3.59 \mathrm{~g} / \mathrm{L}$ and then a passive oxidation was performed using EOC F, from Table 5.8, for 81 minutes. This resulted in $22 \%$ of the available PM being oxidized. The PM density at the time of the scan was $3.25 \mathrm{~g} / \mathrm{L}$. The result of this scan is shown in Figure 6.27. The results of the substrate scan taken after loading the substrate are shown in Figure 6.21. 


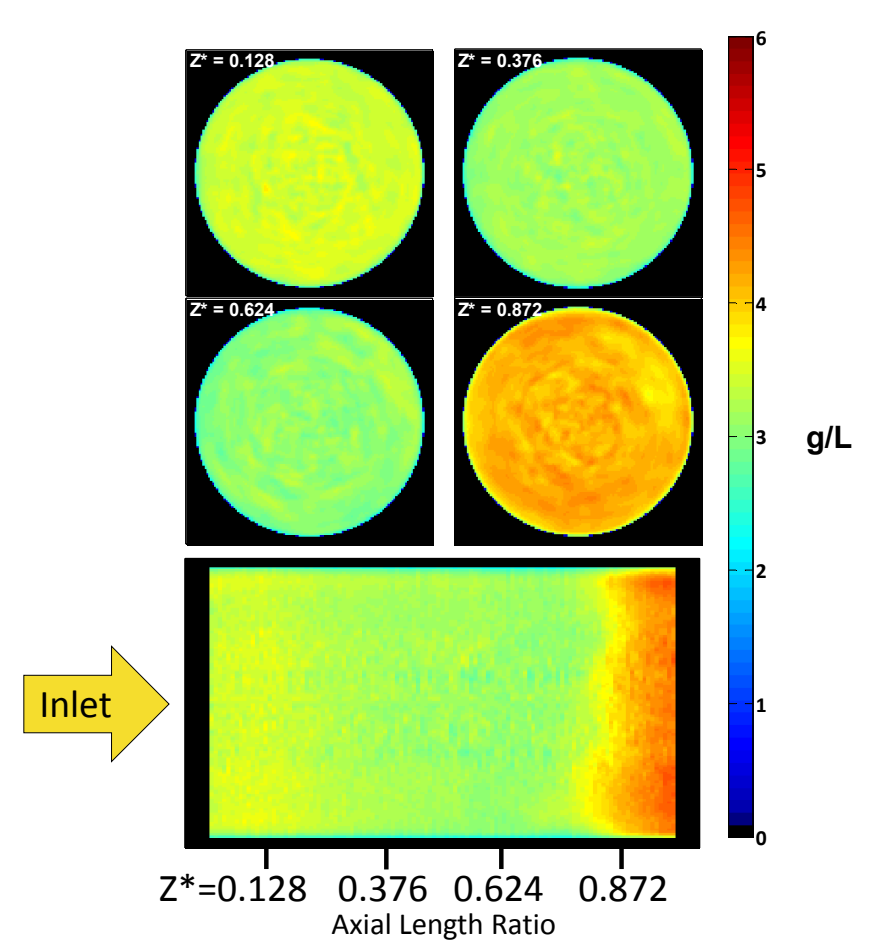

Figure 6.27: Phase 2 Test 1- Passive Oxidation Scan Results

The overall axial UI was 0.89 for the passive oxidation scan from Test 1 of Phase 2. For comparison, the overall axial UI after loading the substrate was 0.90. The axial PM distribution data are provided in Table 6.29. The PM distribution trend after passive oxidation is similar to the trend found after loading, which is given in Table 6.19. The average PM density in axial segment 1 was $4 \%$ higher than the substrate average and in axial segment 4 it was $21 \%$ higher than the substrate average. At the time of the loading scan, axial segments 1 and 4 were 12 and $15 \%$ higher than the substrate average PM density respectively. Axial segments 2 and 3, after passive oxidation, were 2 and $4 \%$ lower than the substrate average, respectively. This is similar to the loading scan data, where axial segments 2 and 3 were 0 and 7\% lower than the substrate average. These data indicate that more PM was oxidized near the inlet of the substrate than near the outlet of the substrate. The PM density near the middle of the axial length of the substrate maintained a similar distribution. It is difficult to make exact conclusions from this data on the differences between the loading and passive oxidation scans because of the Stage 3 loading that takes place after the passive oxidation stage. Stage 3 loaded 5.5 grams of PM, or $0.32 \mathrm{~g} / \mathrm{L}$, into the substrate. The $95^{\text {th }} \mathrm{PR}$ for radial section 1 was $9-56 \%$ lower than radial sections 2 and 3, indicating the data were more consistent near the centerline of the substrate. Axial segments 3 and 4 did not have uniform axial PM distributions, indicating that there was a greater difference between the average PM density for the substrate and the average PM density for those axial segments. The temperature distribution plot shown in Figure I.7 shows that the temperature of the outer edges of the substrate was $25{ }^{\circ} \mathrm{C}$ lower than the centerline and there was minimal temperature variation in the axial direction. 
Table 6.29: Phase 2 Test 1- Passive Oxidation Axial PM Distribution

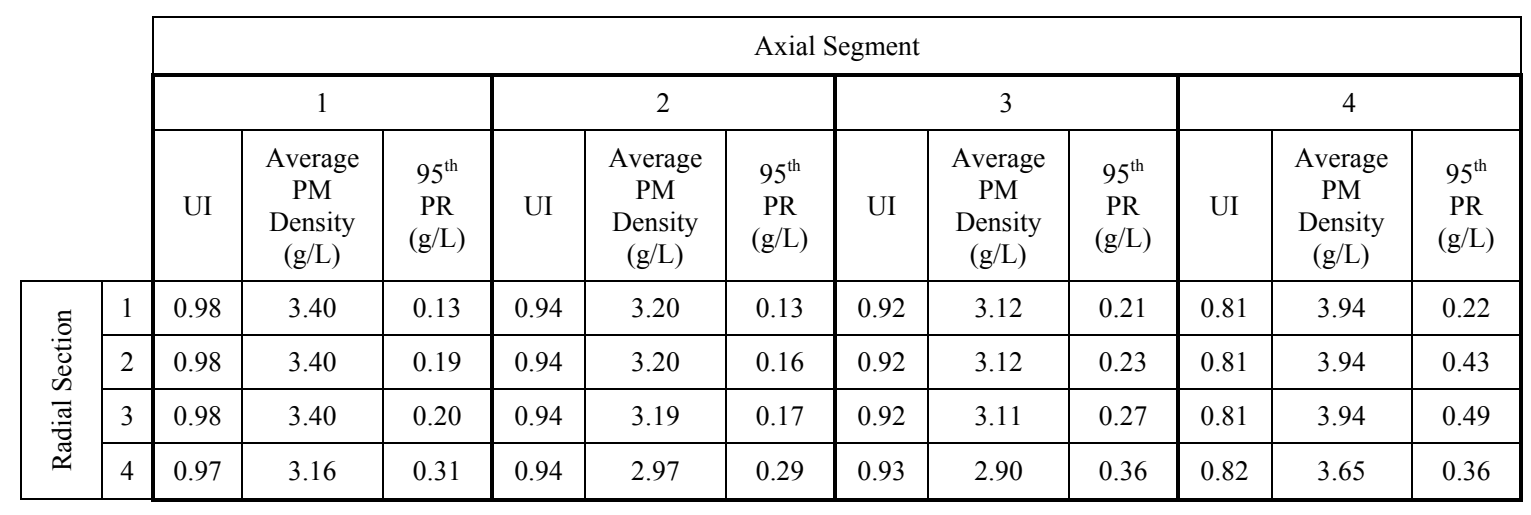

The overall radial UI was 0.97 for the passive oxidation scan from Test 1 of Phase 2, which is similar to the loading scan from Test 1 of Phase 2. This indicates that after a passive oxidation at EOC F where $22 \%$ of the available PM was oxidized, the amount of variation in the radial PM distribution did not change significantly. The radial PM distribution data are given in Table 6.30. All of the quadrants in all of the axial segments had a UI above 0.94 , indicating a uniform distribution. The average PM density and $95^{\text {th }}$ PR were similar for all quadrants. The data in Table 6.30 are in agreement with the PM density plot shown in Figure 6.27.

Table 6.30: Phase 2 Test 1- Passive Oxidation Radial PM Distribution

\begin{tabular}{|c|c|c|c|c|c|c|c|c|c|c|c|c|c|}
\hline & & \multicolumn{12}{|c|}{ Axial Segment } \\
\hline & & \multicolumn{3}{|c|}{1} & \multicolumn{3}{|c|}{2} & \multicolumn{3}{|c|}{3} & \multicolumn{3}{|c|}{4} \\
\hline & & UI & $\begin{array}{c}\text { Average } \\
\text { PM } \\
\text { Density } \\
(\mathrm{g} / \mathrm{L})\end{array}$ & $\begin{array}{c}95^{\text {th }} \\
\mathrm{PR} \\
(\mathrm{g} / \mathrm{L})\end{array}$ & UI & $\begin{array}{c}\text { Average } \\
\text { PM } \\
\text { Density } \\
(\mathrm{g} / \mathrm{L})\end{array}$ & $\begin{array}{c}95^{\text {th }} \\
\text { PR } \\
(\mathrm{g} / \mathrm{L})\end{array}$ & UI & $\begin{array}{c}\text { Average } \\
\text { PM } \\
\text { Density } \\
(\mathrm{g} / \mathrm{L})\end{array}$ & $\begin{array}{c}95^{\text {th }} \\
\text { PR } \\
(\mathrm{g} / \mathrm{L})\end{array}$ & UI & $\begin{array}{c}\text { Average } \\
\text { PM } \\
\text { Density } \\
(\mathrm{g} / \mathrm{L})\end{array}$ & $\begin{array}{c}95^{\text {th }} \\
\text { PR } \\
(\mathrm{g} / \mathrm{L})\end{array}$ \\
\hline \multirow{4}{*}{ 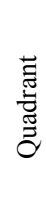 } & 1 & 0.98 & 3.03 & 0.06 & 0.98 & 3.15 & 0.06 & 0.98 & 3.09 & 0.07 & 0.96 & 3.69 & 0.11 \\
\hline & 2 & 0.97 & 3.04 & 0.04 & 0.97 & 3.13 & 0.08 & 0.97 & 3.04 & 0.08 & 0.96 & 3.73 & 0.09 \\
\hline & 3 & 0.96 & 3.05 & 0.10 & 0.97 & 3.13 & 0.10 & 0.96 & 3.06 & 0.11 & 0.95 & 3.82 & 0.18 \\
\hline & 4 & 0.97 & 3.08 & 0.08 & 0.97 & 3.14 & 0.09 & 0.97 & 3.05 & 0.09 & 0.96 & 3.79 & 0.16 \\
\hline
\end{tabular}

The overall angular UI was 0.98 for the passive oxidation scan from Test 1 of Phase 2, which is similar to the loading scan from Test 1 of Phase 2. The angular PM distribution data are given in Table 6.31. All of the radial sections in all of the axial segments had a UI above 0.94 , indicating a uniform distribution. Radial section 4 had an average PM density that was $7 \%$ lower than the other radial sections. This finding is consistent with the distribution data collected after PM loading. The data show that the passive oxidation did not affect the angular PM distribution trends for this experiment where $22 \%$ of the available PM was oxidized. 
Table 6.31: Phase 2 Test 1- Passive Oxidation Angular PM Distribution

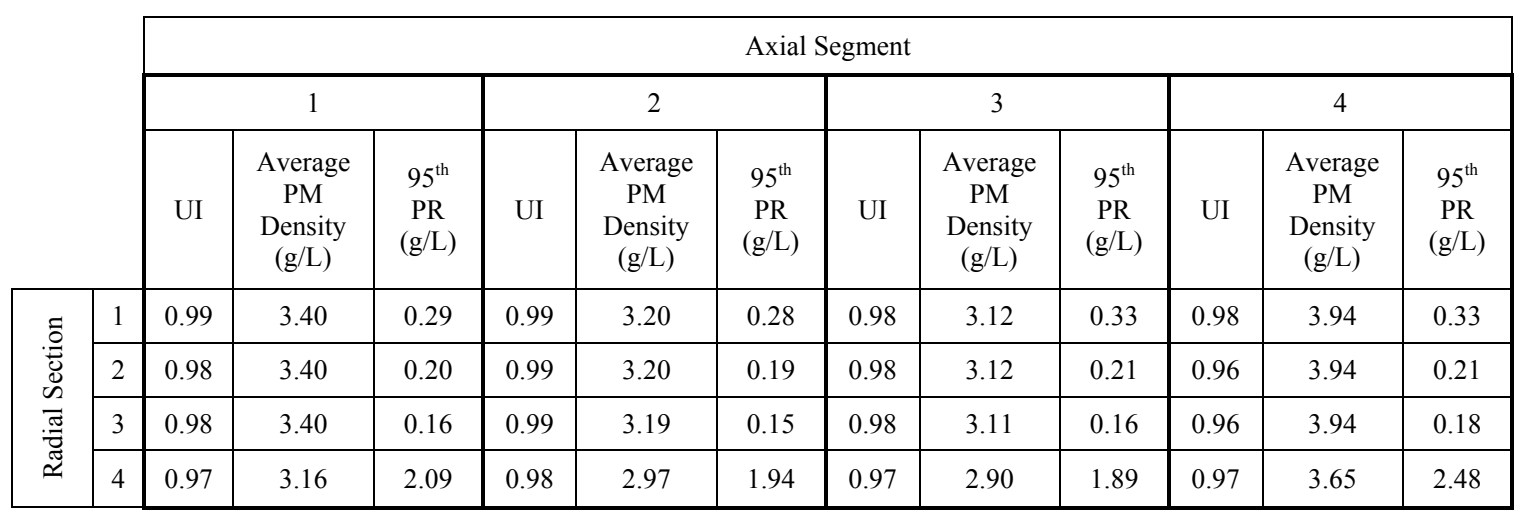

\section{Phase 2 Test 7: Passive Oxidation}

The third passive oxidation experiment that will be discussed is from Test 7 of Phase 2 . There were two substrate scans taken during Test 7 . The first was a passive oxidation scan, and the second was a post loading scan. The results for the post loading scan are given in section 6.8. For Test 7, a substrate was loaded to $3.23 \mathrm{~g} / \mathrm{L}$. Then a passive oxidation was performed with EOC F, from Table 5.8, for 227 minutes. This resulted in $65 \%$ of the available PM being oxidized. The PM density at the time of the passive oxidation substrate scan was $1.85 \mathrm{~g} / \mathrm{L}$. The result of this scan is shown in Figure 6.28. The overall axial UI for the passive oxidation scan was 0.96 , indicating a uniform axial distribution. This overall axial UI is higher than all the overall axial UIs from the loading experiments, which indicates that running a passive oxidation at EOC F until $65 \%$ of the available PM was oxidized improved the uniformity of the PM distribution. The data for the axial PM distribution are given in Table 6.32. Axial segments 1-3 had a similar average PM density around $1.82 \mathrm{~g} / \mathrm{L}$, with a range of $0.04 \mathrm{~g} / \mathrm{L}$, or $2 \%$ of the measured PM density. The average PM densities in axial segments 1-3 were 1-3\% lower than the average PM density in the substrate. Axial segment 4 had an average PM density that was $13 \%$ higher than axial segments $1-3$ and $11 \%$ higher than the substrate average. This trend is similar to the trend that was found for the loading scan from Test 6 of Phase 2. This indicates that a similar amount of PM was oxidized in all axial segments, if the substrate was loaded similar to Test 6 . The UI in radial sections 2 and 3 in axial segment 4 was below 0.94 , but those are the only non-uniform radial sections. The $95^{\text {th }}$ PR was $14-45 \%$ lower in radial section 1 than radial sections 2-3, indicating a more consistent PM distribution near the centerline of the substrate. The temperature distribution plot shown in Figure I.15 shows that the temperature of the outer edges of the substrate was $25^{\circ} \mathrm{C}$ lower than the centerline and there was minimal temperature variation in the axial direction. 


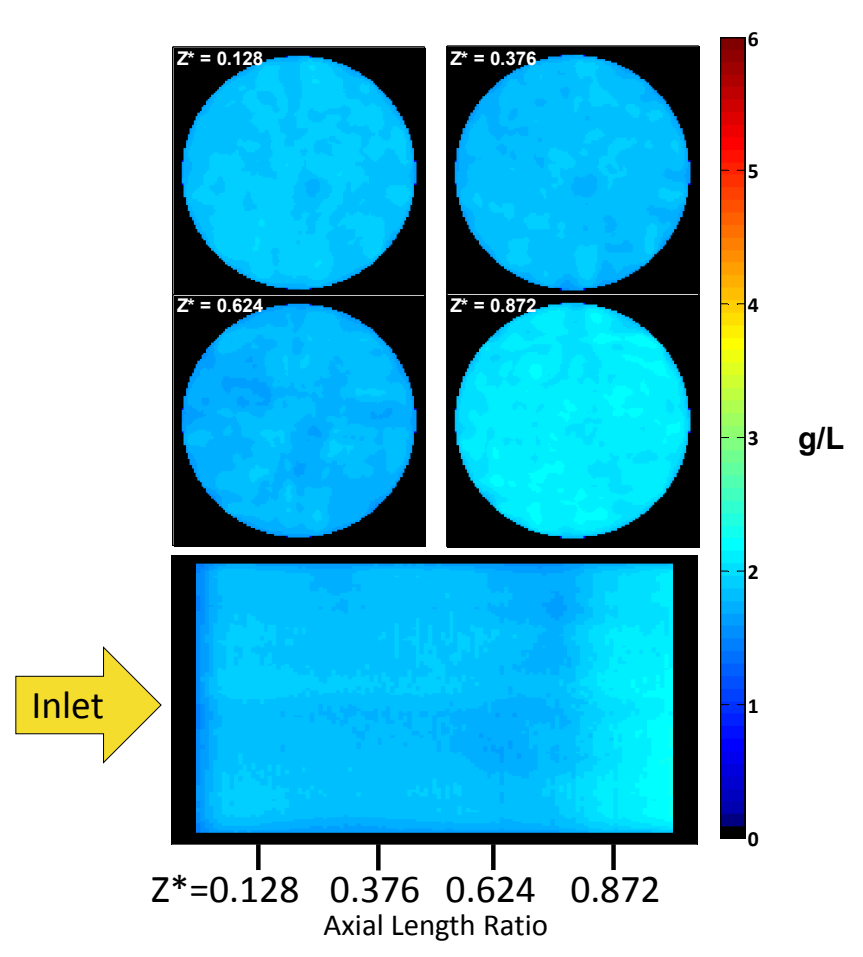

Figure 6.28: Phase 2 Test 7- Passive Oxidation Scan Results

Table 6.32: Phase 2 Test 7- Passive Oxidation Axial PM Distribution

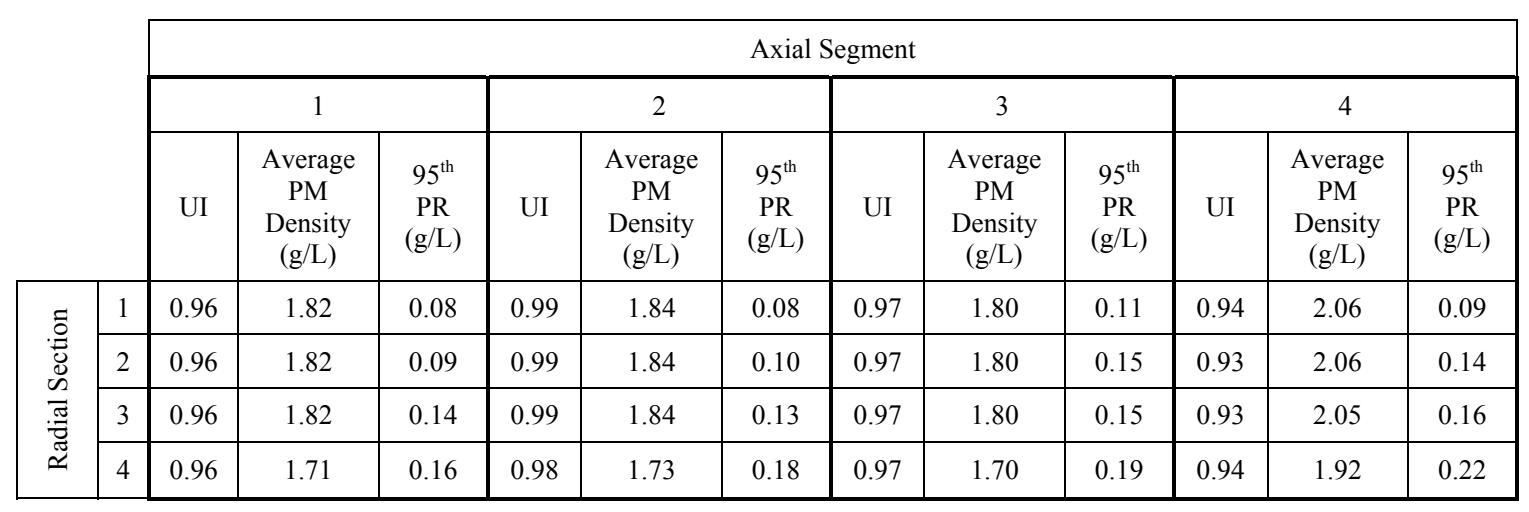

The overall radial UI was 0.98 for the passive oxidation scan from Test 7 of Phase 2. The radial PM distribution data are given in Table 6.33. All of the quadrants in each axial segment had a UI of 0.98 . This indicates a uniform PM distribution, and that the PM distribution in each angular increment had a similar amount of variation. The plots in Figure 6.28 show little variation in the PM density, which agrees with this result. These results indicate that a passive oxidation at EOC $\mathrm{F}$, where $65 \%$ of the available PM was oxidized, did not cause a non-uniform radial distribution. 
Table 6.33: Phase 2 Test 7- Passive Oxidation Radial PM Distribution

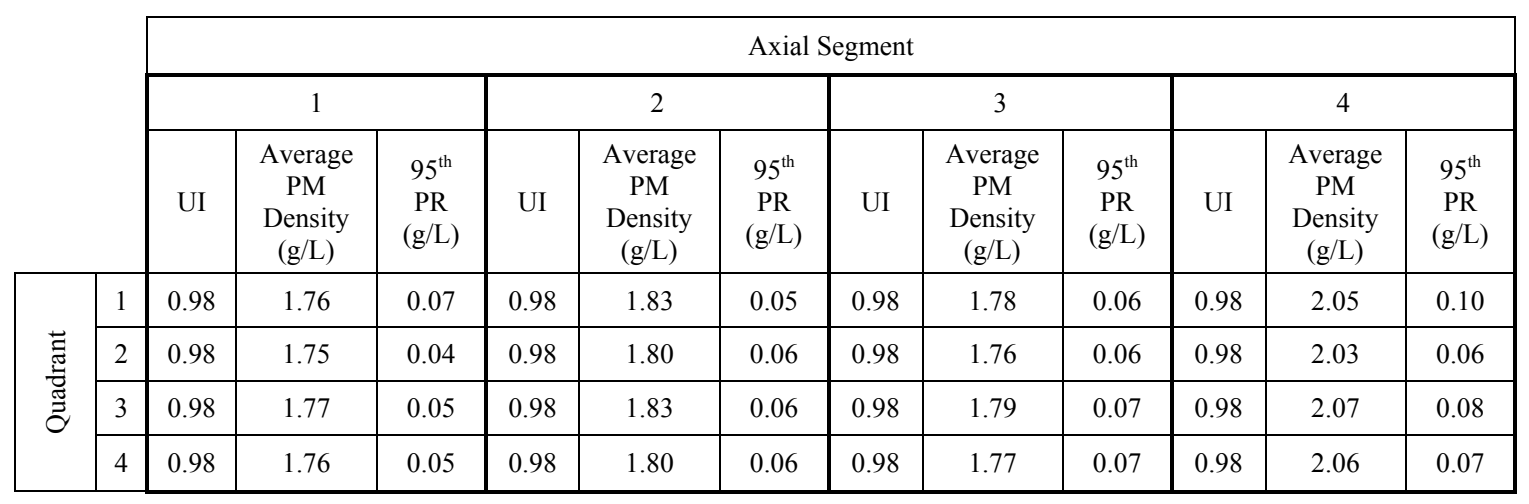

The overall angular UI for the passive oxidation scan from Test 7 of Phase 2 was 0.99 . The angular PM distribution data are given in Table 6.34. The angular UI was between 0.98 and 0.99 for each radial section in each axial segment. This indicates a consistent and uniform angular PM distribution. Radial section 4 had a PM density that was 6\% lower than the substrate average, which is consistent with the findings from other scans. These results indicate that this passive oxidation at EOC F, where $65 \%$ of the available PM was oxidized, did not cause a non-uniform angular distribution.

Table 6.34: Phase 2 Test 7- Passive Oxidation Angular PM Distribution

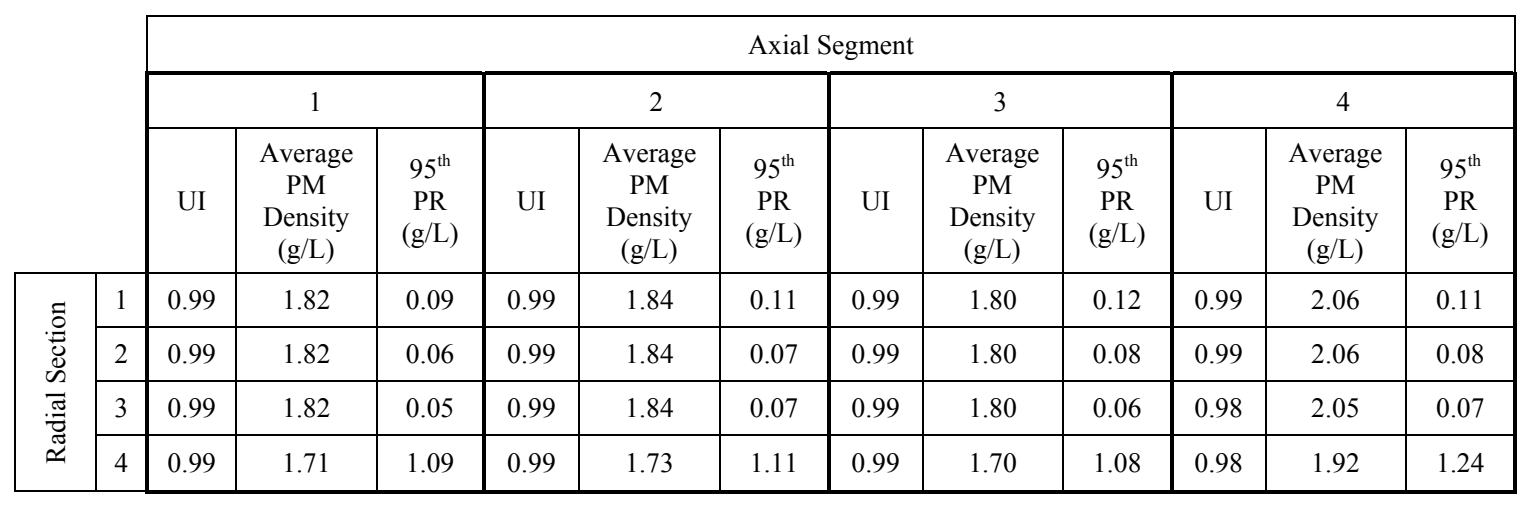

\section{Phase 2 Test 8: Passive Oxidation}

The fourth passive oxidation scan was taken during Test 8 of Phase 2. For this test, a substrate was loaded to $3.57 \mathrm{~g} / \mathrm{L}$ and then a passive oxidation was performed using EOC $\mathrm{F}$, from Table 5.8. The substrate was loaded to a higher PM density for this test because of an ash loading of approximately 13 grams, or $0.8 \mathrm{~g} / \mathrm{L}$. The ash loading was caused by approximately 70 hours of operation prior to Test 2 of Phase 2 being completed, which was the first PM distribution test performed on substrate 2 which was used for Test 8 of Phase 2. The ash loading is discussed further in Appendix G. The test results from Test 2 of Phase 2 are presented in section 6.7. The passive oxidation was run for 111 minutes, and resulted in $28 \%$ of the available PM being oxidized. The PM density in the substrate at the time of scanning was $3.25 \mathrm{~g} / \mathrm{L}$. The result of the passive oxidation scan is shown in 
Figure 6.29. The passive oxidation scan from Test 3 of Phase 1 had a similar amount of PM oxidized, and that result is shown in Figure 6.10 in section 6.1.

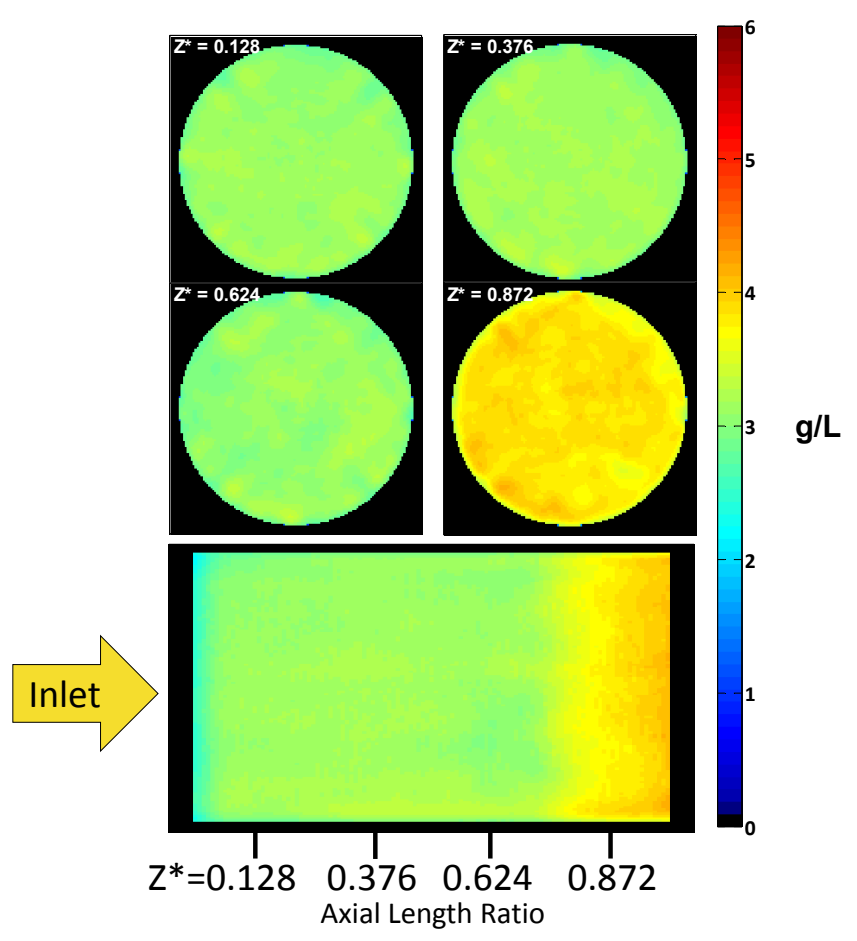

Figure 6.29: Phase 2 Test 8- Passive Oxidation Scan Result

The overall axial UI was 0.91 , indicating a non-uniform distribution. The overall axial UI for Test 8 of Phase 2 was higher than the overall axial UI for the passive oxidation scan from Test 1 of Phase 2 and identical to the overall axial UI from Test 3 of Phase 1. The axial PM distribution data are shown in Table 6.35. The PM density distribution trend is similar to the PM distribution trend from the passive oxidation scans from Tests 1 and 7 of Phase 2 and different than the passive oxidation scan from Test 3 of Phase 1. Axial segment 1 had an average PM density that was 7\% lower than the substrate average. Axial segments 2 and 3 were $2 \%$ lower than the substrate average and axial segment 4 was $17 \%$ higher. This indicates that more PM was oxidized near the inlet of the substrate than near the outlet of the substrate, similar to the results from the passive oxidation scan in Test 1 of Phase 2. In Test 3 of Phase 1, axial segment 2 had an average PM density that was $17 \%$ higher than the substrate average. Axial segments 1,3 , and 4 had average PM densities that were -4 to $2 \%$ different than the average PM density in the substrate. The average PM density in the substrate prior to the passive oxidation in Test 3 of Phase 1 was $62 \%$ higher than the average PM density in Test 8 of Phase 2, which could be a cause of the difference in the distributions. The engine calibration was modified during the loading portion of Test 3 of Phase 1, which may have caused the difference as well. The stage 3 loading that took place during Test 8 of Phase 2 could also be a cause of the difference. The difference could also be caused by Test 3 of Phase 1 having a space velocity that was $17 \%$ greater than Test 8 of Phase 2 . The $95^{\text {th }} \mathrm{PR}$ in radial section 1 is $40-57 \%$ lower than the $95^{\text {th }} \mathrm{PR}$ in radial sections 2 and 3 . This finding 
is consistent with other data sets. The temperature distribution plot shown in Figure I.17 shows that the temperature of the outer edges of the substrate was $25{ }^{\circ} \mathrm{C}$ lower than the centerline and there was minimal temperature variation in the axial direction.

Table 6.35: Phase 2 Test 8- Passive Oxidation Axial PM Distribution

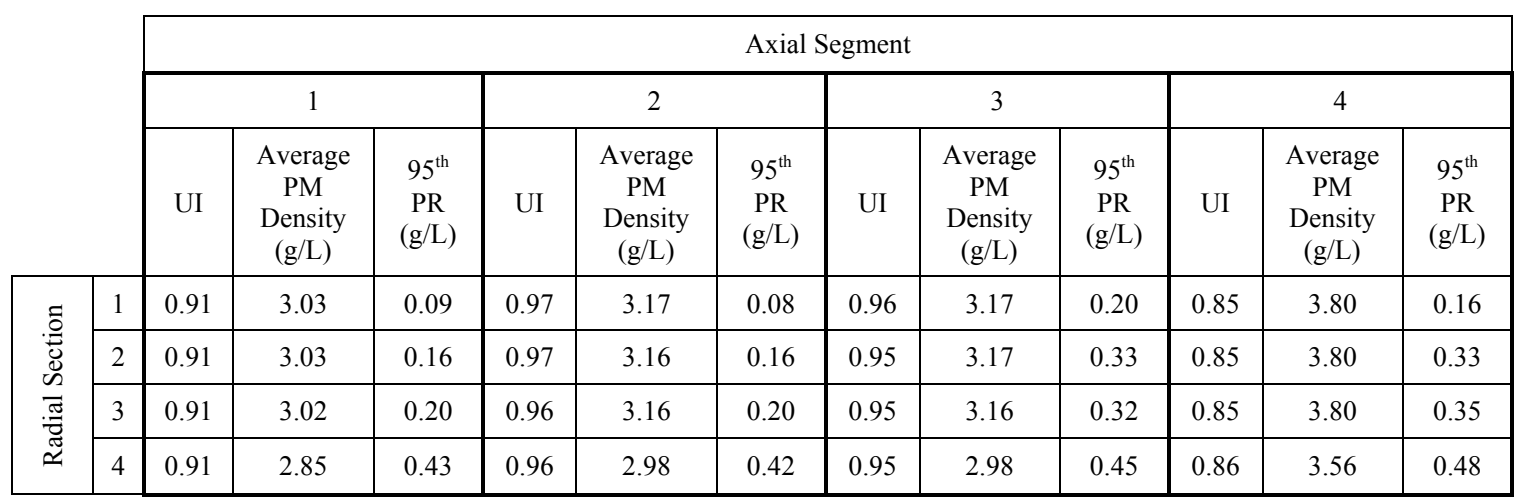

The overall radial UI for the passive oxidation scan from Test 8 of Phase 2 was 0.97 . The radial distribution data are given in Table 6.36. All of the quadrants in all of the axial segments had a UI above 0.94. The radial PM distributions shown in Figure 6.29 do not show significant variation, which agrees with the UIs. These results indicate that after this passive oxidation at EOC F, where $28 \%$ of the available PM was oxidized, a nonuniform radial distribution did not occur.

Table 6.36: Phase 2 Test 8- Passive Oxidation Radial PM Distribution

\begin{tabular}{|c|c|c|c|c|c|c|c|c|c|c|c|c|c|}
\hline & \multicolumn{12}{|c|}{ Axial Segment } \\
\hline & & \multicolumn{3}{|c|}{1} & \multicolumn{3}{|c|}{2} & \multicolumn{3}{|c|}{3} & \multicolumn{3}{|c|}{4} \\
\hline & & UI & $\begin{array}{l}\text { Average } \\
\text { PM } \\
\text { Density } \\
(g / L)\end{array}$ & $\begin{array}{l}95^{\text {th }} \\
\text { PR } \\
(\mathrm{g} / \mathrm{L})\end{array}$ & UI & $\begin{array}{l}\text { Average } \\
\text { PM } \\
\text { Density } \\
(g / L)\end{array}$ & $\begin{array}{l}95^{\text {th }} \\
\text { PR } \\
(\mathrm{g} / \mathrm{L})\end{array}$ & UI & $\begin{array}{l}\text { Average } \\
\text { PM } \\
\text { Density } \\
\text { (g/L) }\end{array}$ & $\begin{array}{l}95^{\text {th }} \\
\text { PR } \\
(\mathrm{g} / \mathrm{L})\end{array}$ & UI & $\begin{array}{l}\text { Average } \\
\text { PM } \\
\text { Density } \\
\text { (g/L) }\end{array}$ & $\begin{array}{l}95^{\text {th }} \\
\text { PR } \\
(\mathrm{g} / \mathrm{L})\end{array}$ \\
\hline \multirow{4}{*}{ 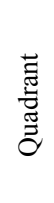 } & 1 & 0.97 & 2.90 & 0.14 & 0.97 & 3.08 & 0.13 & 0.97 & 3.12 & 0.14 & 0.96 & 3.81 & 0.13 \\
\hline & 2 & 0.97 & 2.94 & 0.20 & 0.97 & 3.11 & 0.11 & 0.97 & 3.11 & 0.13 & 0.96 & 3.80 & 0.10 \\
\hline & 3 & 0.97 & 2.99 & 0.09 & 0.98 & 3.14 & 0.15 & 0.98 & 3.12 & 0.19 & 0.97 & 3.86 & 0.16 \\
\hline & 4 & 0.98 & 2.97 & 0.09 & 0.98 & 3.14 & 0.10 & 0.98 & 3.13 & 0.20 & 0.97 & 3.80 & 0.23 \\
\hline
\end{tabular}

The overall angular UI for the passive oxidation scan in Test 8 of Phase 2 was 0.98 . The angular distribution data are given in Table 6.37. Each radial section in each axial segment had a UI above 0.94, indicating a uniform distribution. Radial section 4 had an average PM density that was 6\% lower than the substrate average, which is consistent with the other test data. These results indicate that after this passive oxidation at EOC F, where $28 \%$ of the available PM was oxidized, a non-uniform angular distribution did not occur. 
Table 6.37: Phase 2 Test 8- Passive Oxidation Angular PM Distribution

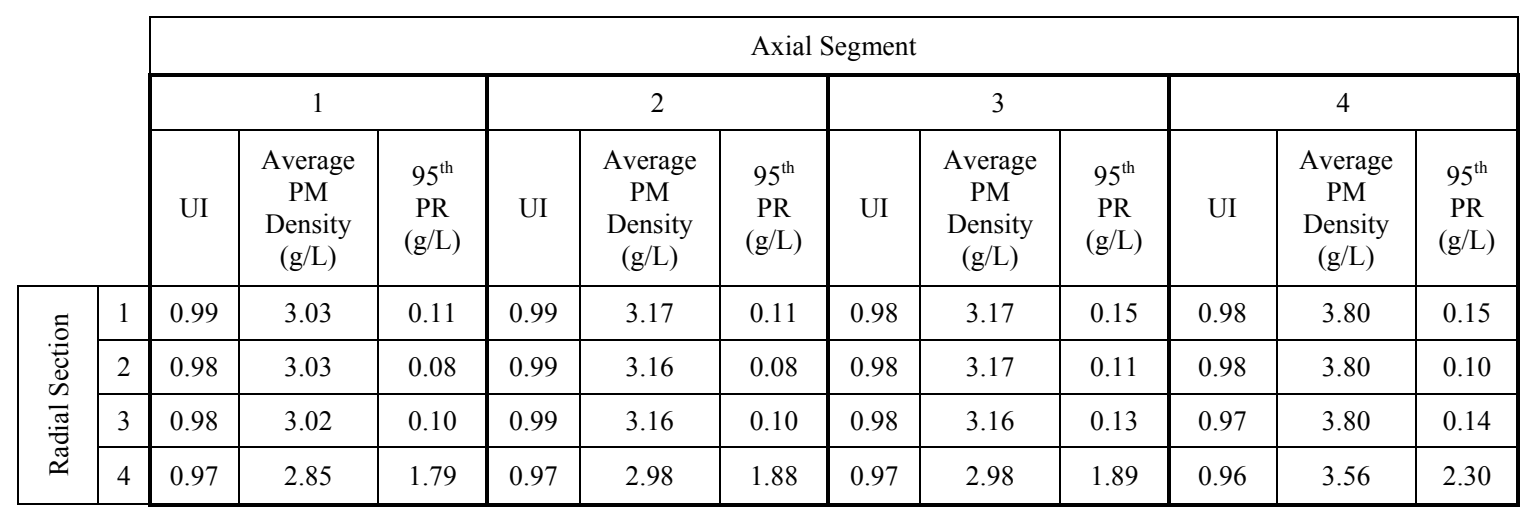

\section{Phase 2 Test 9: Passive Oxidation at a Balance Point}

The final passive oxidation result that will be presented is from Test 9 of Phase 2. This test used EOC A, from Table 5.8, which is a balance point. A balance point is a point where the pressure drop across the CPF is constant. At the balance point the PM oxidation rate in the substrate is equal to the PM loading rate, causing the PM loading in the substrate to be constant. The substrate was initially loaded to $3.13 \mathrm{~g} / \mathrm{L}$, and then EOC A was run for 131 minutes. This resulted in $6 \%$ of the available PM being oxidized. During the oxidation portion of the test, the mass of the substrate increased by 0.3 grams, which is within the measurement error of the scale used. The PM density in the substrate at the time of the scan was $3.51 \mathrm{~g} / \mathrm{L}$. The result of this scan is shown in Figure 6.30.

The overall axial UI was 0.95 for Test 9 of Phase 2. This is higher than the passive oxidation scans from Test 3 of Phase 1 (0.91), Test 1 of Phase 2 (0.89), and Test 8 of Phase 2 (0.91) where $22-45 \%$ of the available PM was oxidized. The axial PM distribution data are given in Table 6.38. The average PM densities in axial segments 1-3 are similar. The average among the three axial segments was $3.54 \mathrm{~g} / \mathrm{L}$ and the range of the average is $0.05 \mathrm{~g} / \mathrm{L}$, or $1 \%$. The average PM densities in axial segments $1-3$ were 0 $1 \%$ higher than the average PM density for the entire substrate. Axial segment 4 had an average PM density that was 8\% higher than axial segments $1-3$ and $9 \%$ higher than the substrate average. This axial distribution trend is similar to the trends found for the other passive oxidation scans from Phase 2. Axial segment 4 is the only axial segment to have a non-uniform axial distribution. The $95^{\text {th }} \mathrm{PR}$ is $1-60 \%$ lower for radial section 1 than radial sections 2 and 3. The temperature distribution plot shown in Figure I.18 shows that the temperature of the outer edges of the substrate was $25{ }^{\circ} \mathrm{C}$ lower than the centerline and there was minimal temperature variation in the axial direction. 


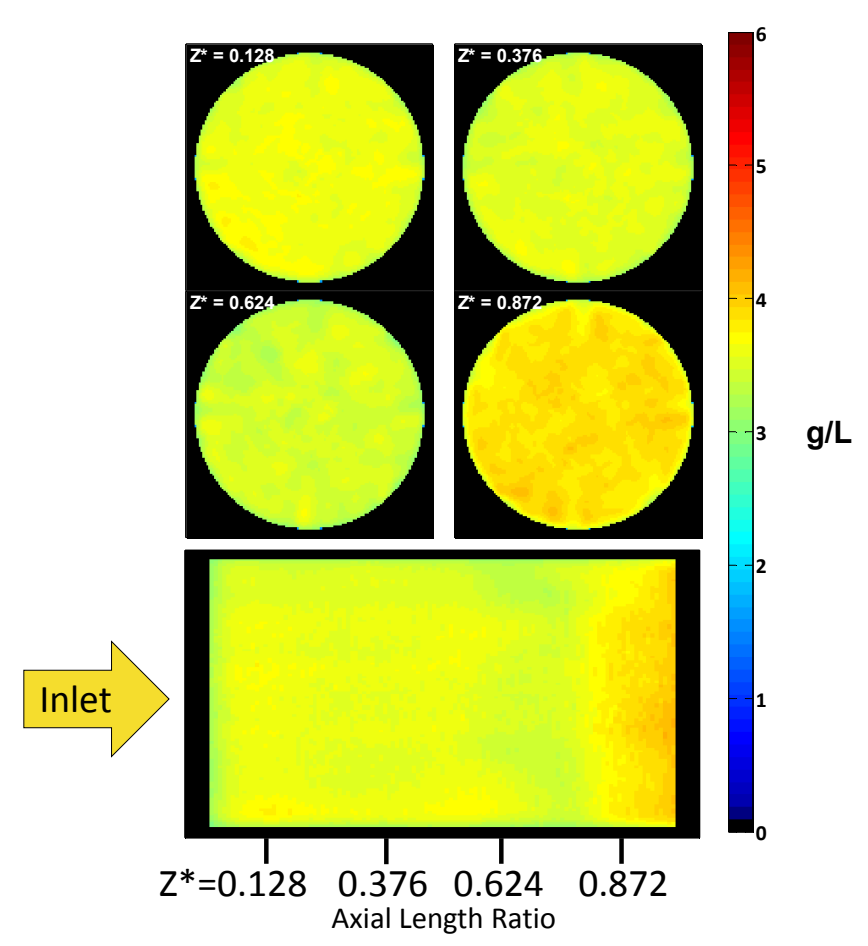

Figure 6.30: Phase 2 Test 9- Balance Point Scan Result

Table 6.38: Phase 2 Test 9- Balance Point Axial PM Distribution

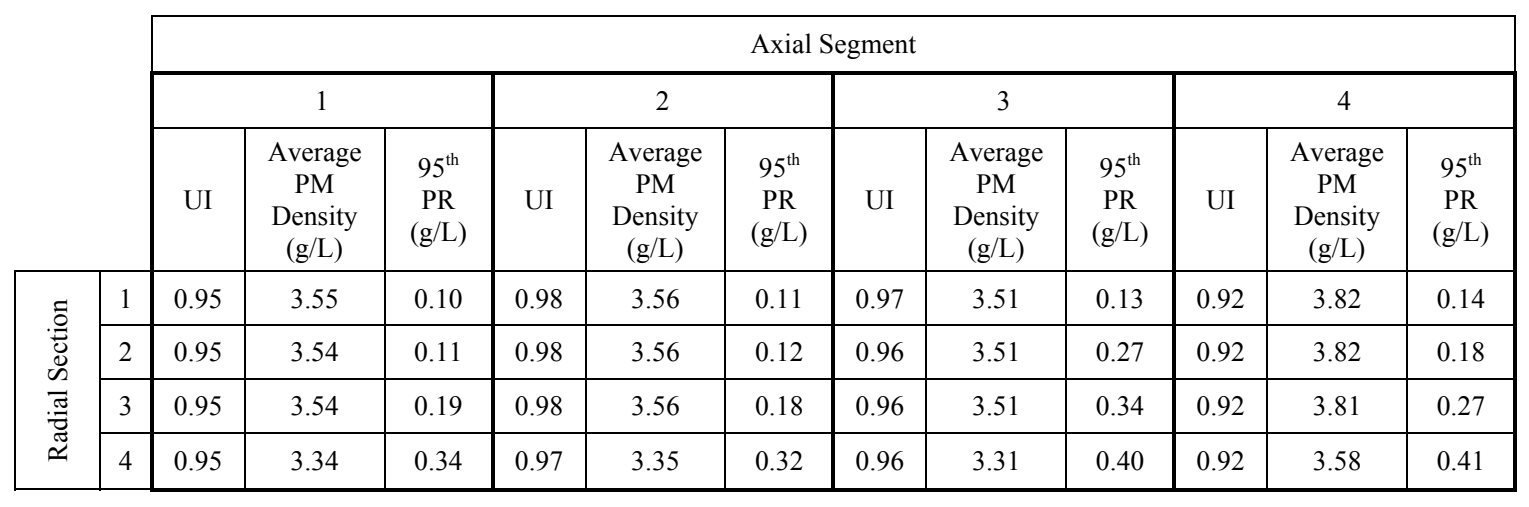

The overall radial UI for Test 9 of Phase 2 was 0.97 , which is similar to the overall radial UIs for the other passive oxidation tests from Phase 2. The radial PM distribution data are given in Table 6.39. Each quadrant in each axial segment had a UI above 0.94, indicating a uniform distribution. The result of the balance point scan, shown in Figure 6.30, did not show a lot of variation in the PM density, which is in agreement with the calculated UIs. 
Table 6.39: Phase 2 Test 9- Balance Point Radial PM Distribution

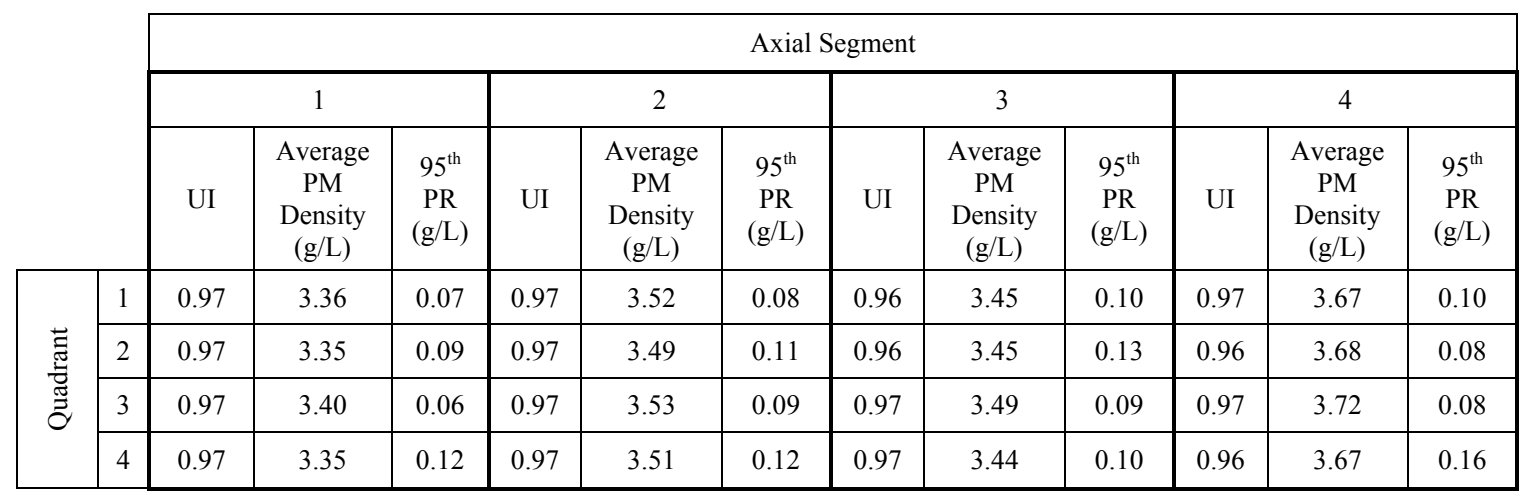

The overall angular UI was 0.98 for Test 9 of Phase 2, which is similar to the overall angular UIs for the other passive oxidation tests from Phase 2. The angular PM distribution data are given in Table 6.40. Each radial section in each axial segment had a UI above 0.94 , indicating a uniform distribution. Radial section 4 had an average PM density that was $6 \%$ lower than radial section 1 , which is consistent with previous findings.

Table 6.40: Phase 2 Test 9- Balance Point Angular PM Distribution

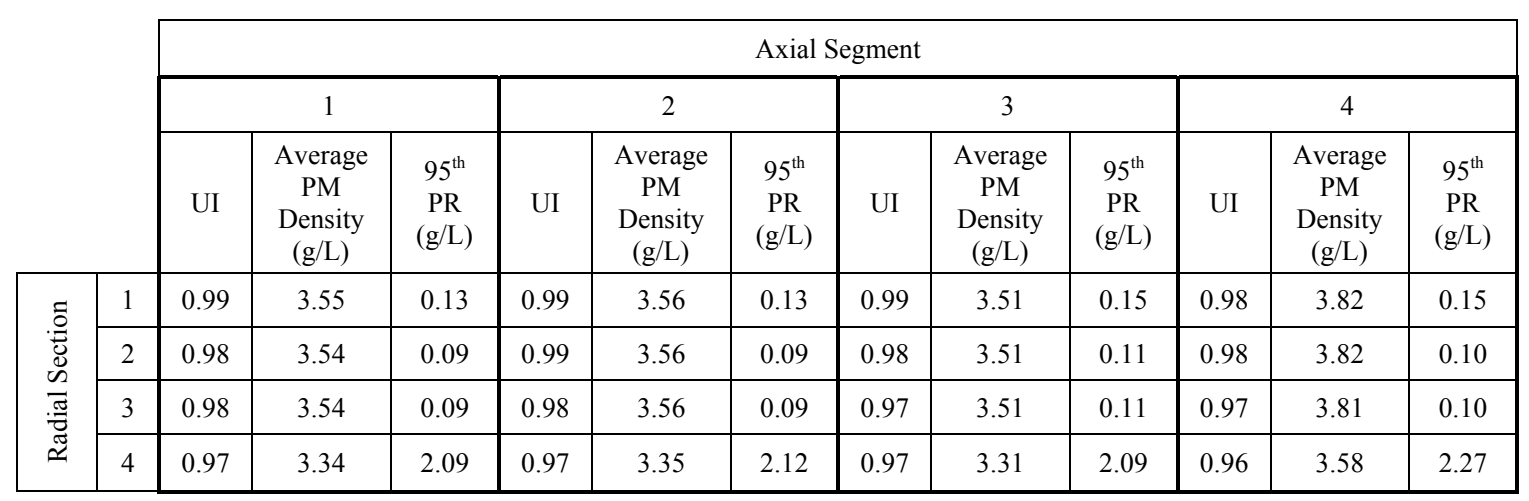

The axial PM distribution for all of the passive oxidation tests that were conducted are shown in Figure 6.31. A summary of each test was provided in Table 6.28. The axial PM distribution for Test 3 of Phase 1 does not follow the trends of the tests in Phase 2 . The increase in the PM density between 20 and 50\% of the axial length for Test 3 is not found for any other tests. This different distribution could be caused by a higher PM density prior to beginning the passive oxidation. The PM density in the substrate during Test 3 of Phase 1 was $5.81 \mathrm{~g} / \mathrm{L}$ which was, on average, $72 \%$ higher than the average PM density of the Phase 2 tests. The space velocity for Test 3 of Phase 1, shown in Table 6.28, was 17\% higher than EOC F used for Tests 1, 7, and 8 of Phase 2, also shown in Table 6.28. The increased velocity could alter the passive oxidation characteristics by changing the wall flow velocity distribution. Figure 2.5 shows the effect of changing the exhaust gas velocity on the wall velocity distribution. The modified engine calibration that was used for all PM loading during Phase 1 could have had an impact on passive 
oxidation as well, even though the PM distribution trend after PM loading was found to not be affected by the modified engine calibration in Figure 6.24. The use of stage 3 during the Phase 2 work to stabilize the CPF temperature, and return the CPF to a repeatable temperature, prior to weighing the substrate at the end of the test, after a passive oxidation, could have an impact on the PM distribution as well. In Phase 1, stage 3 loaded 0.3 grams $(0.03 \mathrm{~g} / \mathrm{L})$ of PM into the substrate. In Phase 2, stage 3 loaded 6.7 grams $(0.39 \mathrm{~g} / \mathrm{L})$, on average, of PM into the substrate. While the effect of the additional 6.7 grams should be minimal, it is unknown how the distribution was affected.

The axial PM distributions for the passive oxidation tests from Phase 2 are similar to the PM distribution trends found after loading the substrate for Tests 1 and 6 of Phase 2, as shown in Figure 6.24. Test 1 of Phase 2 had a PM distribution that was similar to the loading data that was collected during Test 1 of Phase 2. However, as discussed previously, more PM was oxidized near the inlet of the substrate. The PM distribution trends for Tests 7 and 9 of Phase 2 are similar even though the mass of PM oxidized was 65 and $6 \%$ respectively. The space velocity for Test 9 was $41 \%$ lower than Test 7 , as shown by the data in Table 6.28. This indicates that the space velocity did not have a noticeable impact on the PM distribution trends that resulted from these passive oxidation experiments. The average CPF temperature for Test 9 was $27 \%$ lower than the average CPF temperature for Test 7, as shown by the data in Table 6.28. This indicates that the CPF temperature did not have a noticeable impact on the PM distribution trends found for these passive oxidation experiments. The PM distribution trend for Test 8 of Phase 2 shows more PM oxidized in the first $75 \%$ of the axial length, with the last $25 \%$ being the same as Test 9. Since Tests 7 and 9 have similar distributions for two different amounts of PM oxidized, it would be expected that Test 8 would follow this trend. However, the results show that as the percentage of PM oxidized increases, the PM is oxidized in the first $75 \%$ of the axial length first, with the last $25 \%$ being oxidized later. Differences in the PM distribution between Test 1 of Phase 2 and the other tests from Phase 2 can be explained by the differences in the PM distribution after loading. The substrates used for Tests 7,8 , and 9 had previous loading done on them, so it is expected that they would load similar to Test 6 of Phase 2, which had a different PM distribution after loading than Test 1 of Phase 2.

The overall axial UI is plotted as a function of the amount of available PM oxidized in Figure 6.32. Only the axial UI is studied because the radial and angular UIs were all similar for all tests, and all were above 0.94 . The results showed a uniform axial PM distribution when the amount of PM oxidized during the passive oxidation was 6 and $65 \%$ (Tests 9 and 7 of Phase 2 respectively). The UI decreased by $6 \%$ to 0.89 as the amount of PM oxidized increased from $6 \%$ to $22 \%$. The UI increased by $2 \%$ to 0.91 as the amount of PM oxidized increased from $22 \%$ to between 28 and $45 \%$. Both Test 3 of Phase 1 and Test 8 of Phase 2 had a UI of 0.91 , even though the resulting distributions of the two tests were different. All of these data show that the passive oxidations that resulted in more than $6 \%$ but less than $65 \%$ had a non- uniform PM distribution. 


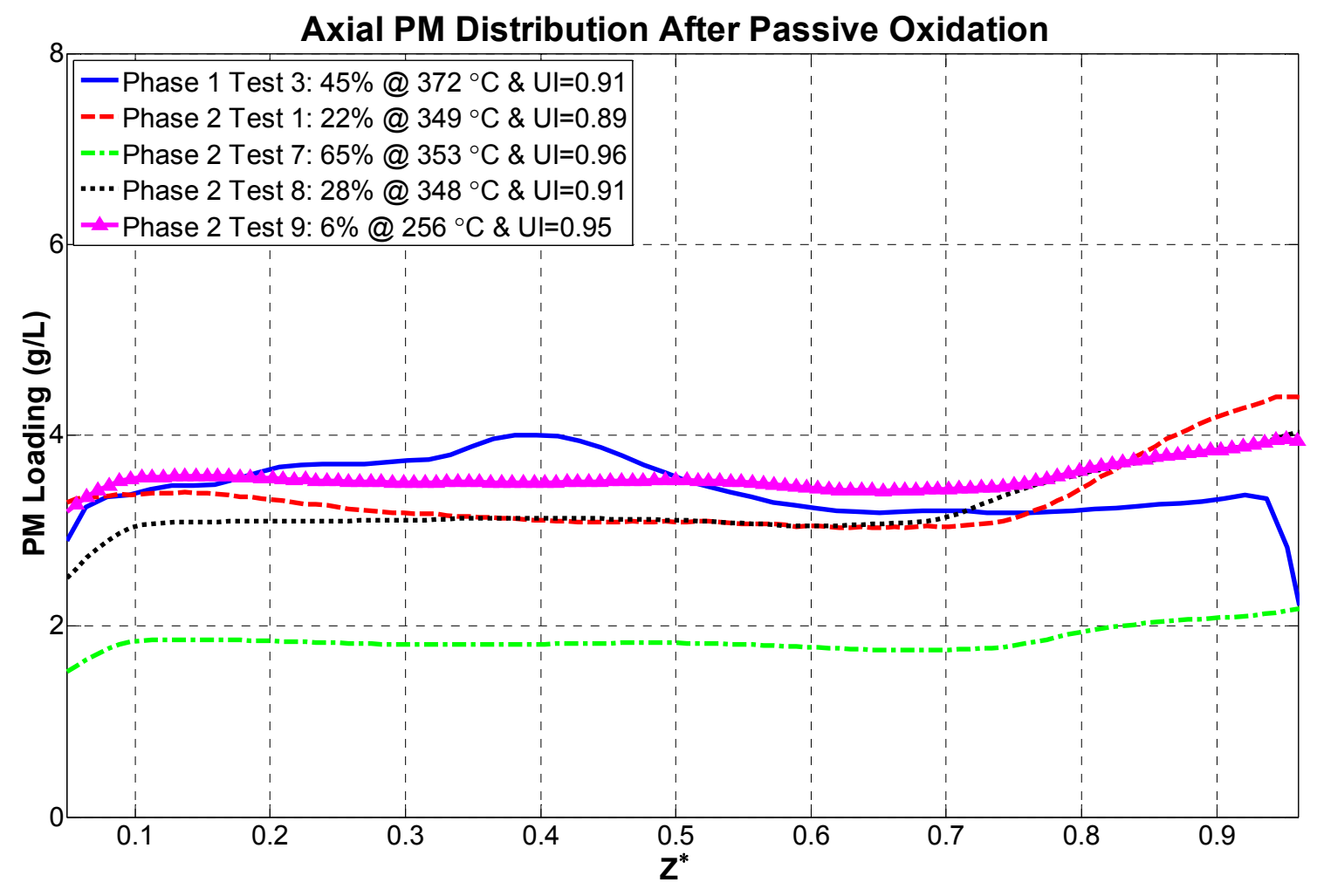

Figure 6.31: Axial PM Distribution for all Passive Oxidation Tests

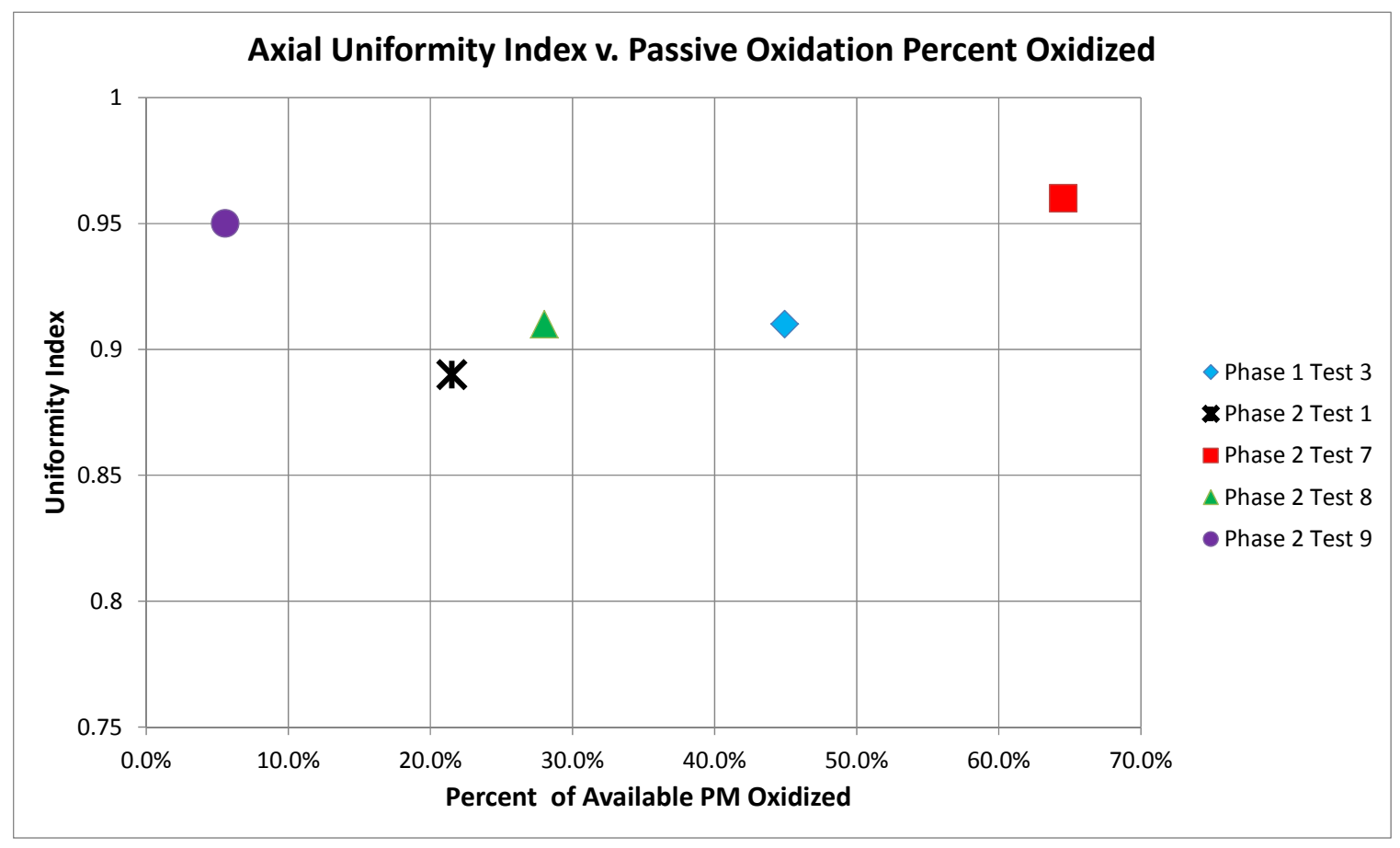

Figure 6.32: Axial Uniformity Index as a Function of Percent of PM Oxidized 


\subsection{PM Distribution Trends after Active Regeneration}

This section covers the active regeneration experiments that were completed as part of the experimental work. A total of seven active regeneration experiments were conducted as part of the experimental study on PM distribution trends. The first three active regeneration experiments are given in section 6.1, and the scan results are shown in Figures 6.4, 6.7, and 6.13. These experiments were active regenerations at 500 and $600{ }^{\circ} \mathrm{C}$ with $40-70 \%$ of the available PM oxidized using the "Oxidation" EOC from Table 5.2. Tests $2-5$ of Phase 2 will be presented in this section. The TAS7000 data for the Phase 2 tests were processed using the new PM density calculation method presented in section 4.1, so the resulting distribution data are for the PM density, including the carbonaceous and ash content. The Phase 2 tests were active regenerations at 525 and $600{ }^{\circ} \mathrm{C}$ with $26-81 \%$ of the available PM oxidized using EOC AR, found in Table 5.8. The space velocities in Tables 5.2 and 5.8 are calculated without any fuel dosing. Incylinder fuel dosing was used to accomplish all active regenerations. A summary of the main parameters of the active regeneration tests is provided in Table 6.41. The main parameters that were varied during the experiments were the temperature of the active regeneration, the mass of PM that was oxidized, and the PM density in the substrate prior to the active regeneration. The reaction rate was varied by changing the temperatures of the active regeneration. The full test descriptions are provided in Chapter 5 and the full test summaries are provided in Appendix E. The space velocities in Table 6.41 are calculated with fuel dosing. A comparison of the reaction rates listed in Table 6.41 to the reaction rates for other active regeneration tests can be found in Appendix F.

Table 6.41: Active Regeneration Test Summaries

\begin{tabular}{|c|c|c|c|c|c|c|c|c|c|c|}
\hline \multirow[t]{2}{*}{ Engine } & \multirow[t]{2}{*}{ Test } & $\begin{array}{c}\text { CPF } \\
\text { Space } \\
\text { Velocity }\end{array}$ & $\begin{array}{c}\text { Average } \\
\text { CPF } \\
\text { Temp. }\end{array}$ & $\begin{array}{c}\text { Engine } \\
\text { Out PM } \\
\text { Conc. }\end{array}$ & $\begin{array}{c}\mathrm{NO}_{2} \\
\text { Conc. } \\
\text { into the } \\
\text { CPF }\end{array}$ & $\begin{array}{c}\mathrm{O}_{2} \\
\text { Conc. } \\
\text { into } \\
\text { the } \\
\text { CPF }\end{array}$ & $\begin{array}{c}\text { PM } \\
\text { Density } \\
\text { before } \\
\text { Active } \\
\text { Regen. }\end{array}$ & $\begin{array}{l}\text { Percent } \\
\text { of } \\
\text { Available } \\
\text { PM } \\
\text { Oxidized }\end{array}$ & $\begin{array}{l}\text { PM } \\
\text { Density } \\
\text { at Time } \\
\text { of Scan }\end{array}$ & $\begin{array}{c}\text { Reaction } \\
\text { Rate }\end{array}$ \\
\hline & & $1 \mathrm{k} / \mathrm{hr}$ & ${ }^{\circ} \mathrm{C}$ & $\mathrm{mg} / \mathrm{scm}$ & $\mathrm{mg} / \mathrm{scm}$ & $\%$ & $\mathrm{~g} / \mathrm{L}$ & - & $\mathrm{g} / \mathrm{L}$ & $1 / \mathrm{s}$ \\
\hline \multirow{3}{*}{ ISB } & $\begin{array}{c}\text { Phase } 1 \\
\text { Test } 1\end{array}$ & 206 & 497 & 8.7 & 45.8 & 6.2 & 4.96 & $58.4 \%$ & 2.03 & $0.46 \mathrm{E}-03$ \\
\hline & $\begin{array}{c}\text { Phase } 1 \\
\text { Test } 2\end{array}$ & 206 & 491 & 8.5 & 98.5 & 6.4 & 5.23 & $41.0 \%$ & 2.99 & $0.52 \mathrm{E}-03$ \\
\hline & $\begin{array}{c}\text { Phase } 1 \\
\text { Test } 4\end{array}$ & 214 & 577 & 12.1 & 96.9 & 4.1 & 5.12 & $69.1 \%$ & 1.52 & $2.70 \mathrm{E}-03$ \\
\hline \multirow{4}{*}{ ISL } & $\begin{array}{c}\text { Phase } 2 \\
\text { Test } 2\end{array}$ & 206 & 611 & - & - & - & 4.88 & $81.4 \%$ & 1.12 & - \\
\hline & $\begin{array}{c}\text { Phase } 2 \\
\text { Test } 3\end{array}$ & 199 & 519 & 17.8 & 1.0 & 7.4 & 2.92 & $26.3 \%$ & 2.60 & $0.39 \mathrm{E}-03$ \\
\hline & $\begin{array}{c}\text { Phase } 2 \\
\text { Test } 4 \\
\end{array}$ & 197 & 526 & 16.2 & 0.4 & 7.5 & 3.06 & $45.0 \%$ & 2.22 & $0.39 \mathrm{E}-03$ \\
\hline & $\begin{array}{c}\text { Phase } 2 \\
\text { Test } 5\end{array}$ & 213 & 574 & 22.9 & 0.9 & 7.0 & 3.90 & $52.1 \%$ & 2.33 & $2.50 \mathrm{E}-03$ \\
\hline
\end{tabular}

"-" Denotes that the Parameter was not Measured 


\section{Phase 2 Test 2: Cleanout}

The fourth active regeneration test was Test 2 of Phase 2. Two substrate scans were taken during Test 2, one baseline scan and one scan after the cleanout. This test consisted of a $600{ }^{\circ} \mathrm{C}$ substrate cleanout after multiple loading, cleanout, and partial oxidation cycles. The substrate, substrate 2, was used for no more than 70 hours prior to the cleanout taking place. The PM density in the substrate prior to the cleanout was $4.88 \mathrm{~g} / \mathrm{L}$. The cleanout was performed for 26 minutes with an average CPF temperature of $611{ }^{\circ} \mathrm{C}$. The cleanout oxidized $81 \%$ of the available PM, resulting in a PM density of $1.12 \mathrm{~g} / \mathrm{L}$ at the time of the substrate scan. Approximately $0.8 \mathrm{~g} / \mathrm{L}$ was ash loading, which was discovered after all of the Phase 2 experiments were completed and is discussed in Appendix G. The other $0.3 \mathrm{~g} / \mathrm{L}$ is residual PM that was not cleaned out. The cleanout was performed until the pressure drop across the CPF was constant. The calculated slope of the pressure drop curve in the last 5 minutes of the cleanout was $-0.006 \mathrm{kPa} / \mathrm{min}$. Therefore, if the cleanout was carried out for an additional 5 minutes, the pressure drop across the CPF would decrease by $0.03 \mathrm{kPa}$, which is within the accuracy of the pressure sensor used to measure the pressure drop. This indicates that the pressure drop was nearly constant at the completion of the cleanout. The result of the cleanout scan is shown in Figure 6.33.

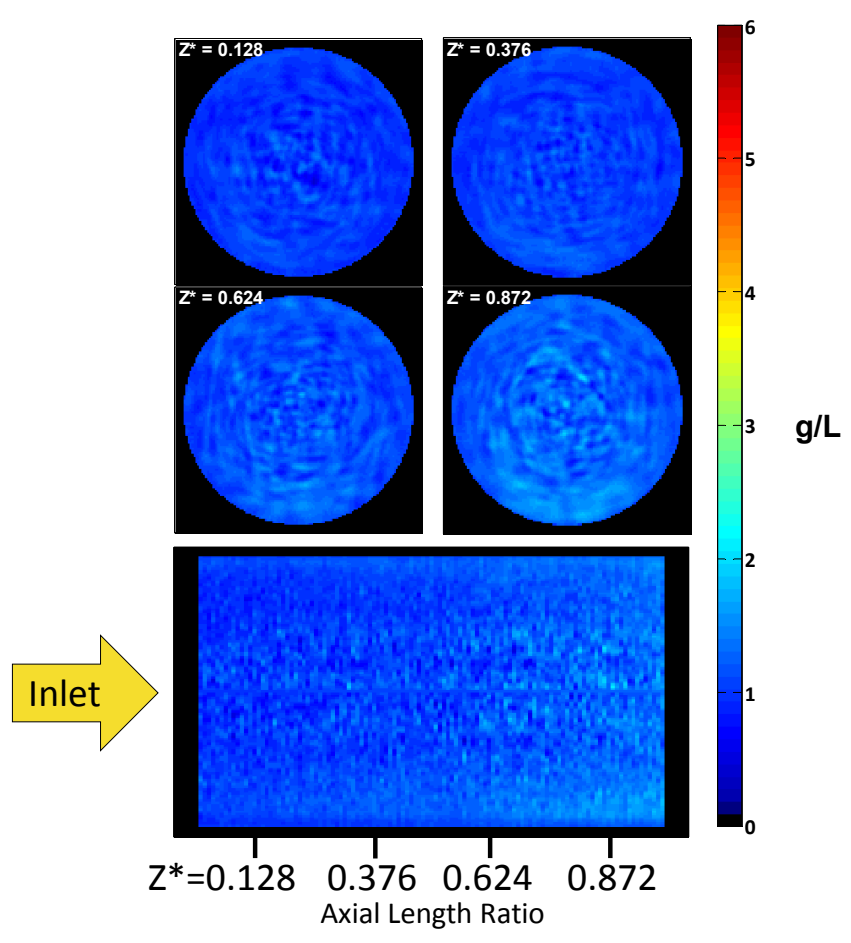

Figure 6.33: Phase 2 Test 2- Cleanout Scan Result

The overall axial UI was 0.97 for Test 2 of Phase 2. The axial PM distribution data are given in Table 6.42. Each radial section in each axial segment had an axial UI above 0.94, indicating a uniform distribution. Test 4 of Phase 1 had a similar amount of PM oxidized and was run at a similar temperature, but the axial distribution trends are 
significantly different. Test 4 had an average PM density near the outlet of the substrate that was $67 \%$ lower than the substrate average. The data in Table 6.42 show that the average PM density increased in each axial segment. In axial segments 3 and 4 for Test 2 of Phase 2, the average PM densities were 3-14\% higher than the substrate average. The average PM densities in axial segments 1 and 2 for Test 2 of Phase 2 were 6-9\% lower than the substrate average. The variation between the axial segments is within the $95 \%$ CI of the TAS7000 data, indicating the average PM density in each axial segment is similar. Test 4 of Phase 1 did not have as many hours of operation on it, which could be the cause of the difference. It should be noted that Test 4 did have an increase in the PM density in the last $10 \%$ of the axial length from 0.5 to $2.5 \mathrm{~g} / \mathrm{L}$. This increase may have been the start of a region with a higher PM density, which is difficult to clean out and may consist of ash, similar to what is found near the outlet for Test 2 of Phase 2. The temperature distribution plot in Figure I.8 shows that radial sections 1-3 had average CPF temperatures that were $25{ }^{\circ} \mathrm{C}$ higher at the outlet of the substrate than the inlet of the substrate, which is similar to the distribution found for Test 4 of Phase 1 (Figure I.5). The increase of $25^{\circ} \mathrm{C}$ near the outlet of the substrate would result in a PM reaction rate near the outlet that is $66 \%$ higher than the PM reaction rate near the inlet of the substrate, based on the data in [45] and assuming all other parameters were constant. This indicates that more PM should have been oxidized near the outlet of the substrate, than near the inlet. Therefore, any significant increase in the PM density is likely ash loading.

Table 6.42: Phase 2 Test 2- Cleanout Axial PM Distribution

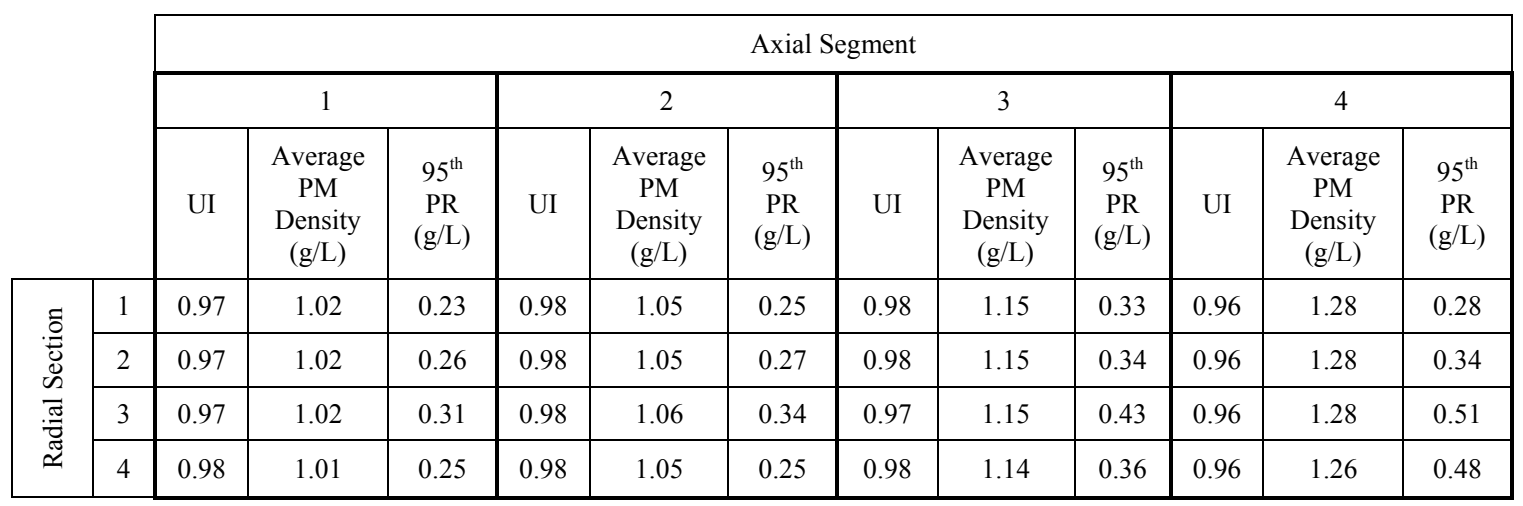

The overall radial UI was 0.99 for Test 2 of Phase 2, which is higher than the overall radial UI from Test 4 of Phase 1. The radial distribution data are given in Table 6.43. Each quadrant in each axial segment had a uniform distribution, with a UI above 0.94. The average PM density and $95^{\text {th }}$ PR is similar for each quadrant in each axial segment. The similarities in Table 6.43 are consistent with the data in Figure 6.33. 
Table 6.43: Phase 2 Test 2- Cleanout Radial PM Distribution

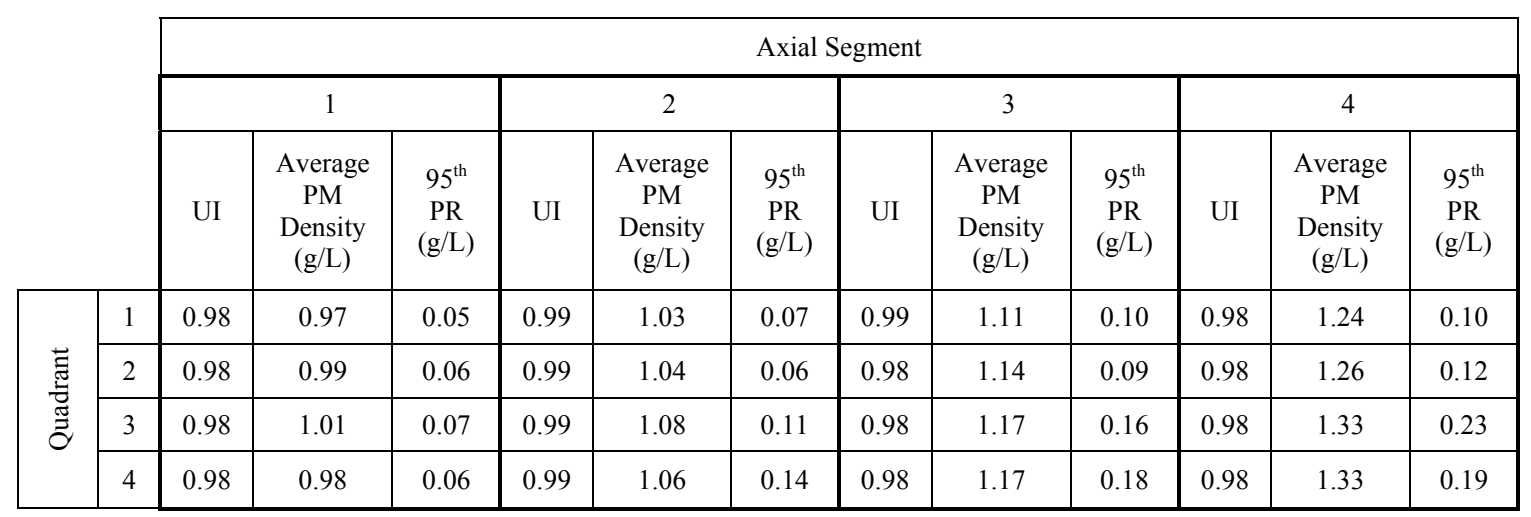

The overall angular UI was 0.98 for Test 2 of Phase 2. The angular PM distribution data are given in Table 6.44. All radial sections in each axial segment had a UI above 0.94, indicating a uniform distribution. This is consistent with the data in Figure 6.33. The average PM density in radial section 4 was a maximum of $2 \%$ lower than radial sections 1-3. This difference is less than the difference found for other data sets.

Table 6.44: Phase 2 Test 2- Cleanout Angular PM Distribution

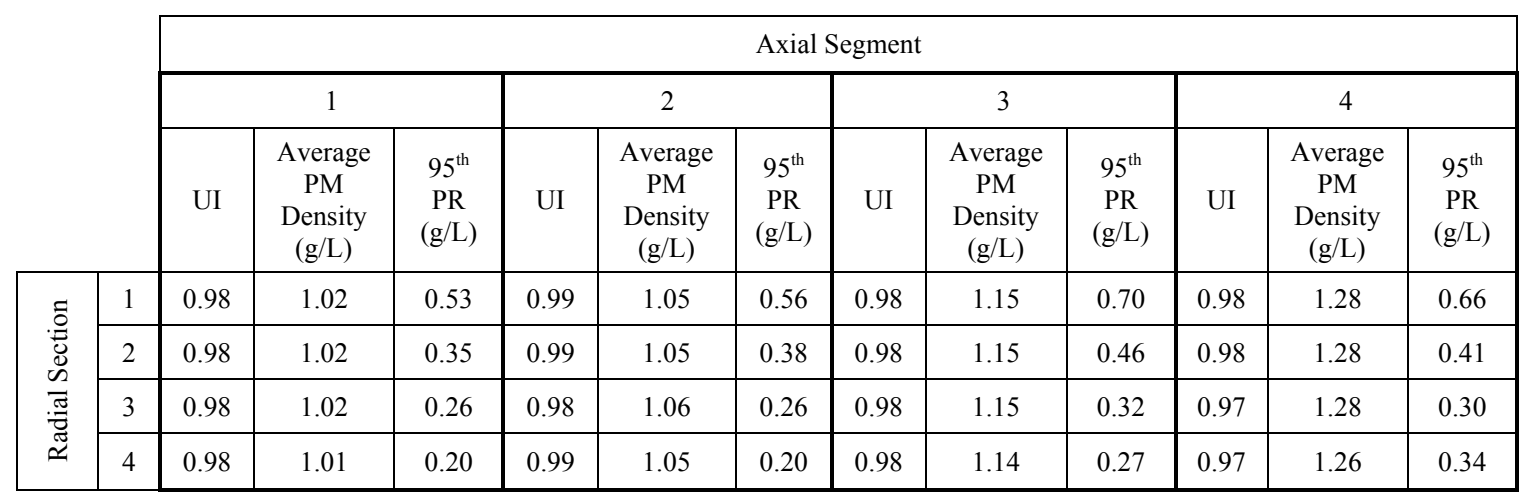

\section{Phase 2 Test 3: Active Regeneration}

The fifth active regeneration test was Test 3 of Phase 2. There were 2 substrate scans taken during Test 3. The first was a baseline scan that was taken before any loading of the substrate. The second scan was taken after the active regeneration. The substrate was loaded to an average PM density of $2.92 \mathrm{~g} / \mathrm{L}$. Then an active regeneration was performed for 16 minutes, with an average CPF temperature of $519{ }^{\circ} \mathrm{C}$. This oxidized $26 \%$ of the available PM. The PM density at the time of the substrate scan was $2.60 \mathrm{~g} / \mathrm{L}$. Table 6.41 presents the other parameters of Test 3 of Phase 2. The result of the scan is shown in Figure 6.34. Test 8 of Phase 2 was a passive oxidation test with a similar amount of PM oxidized, and those results are shown in Figure 6.29. The active regeneration scan from Test 2 of Phase 1 had a similar amount of PM oxidized at a similar active regeneration temperature. Those results are shown in Figure 6.7. 


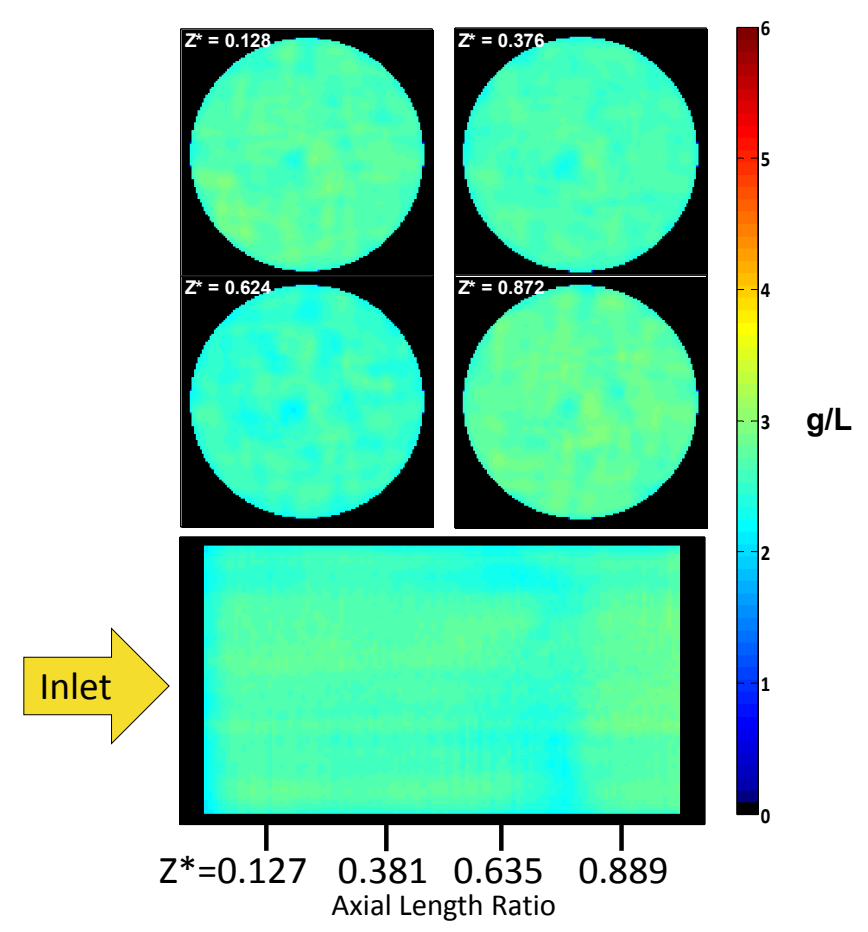

Figure 6.34: Phase 2 Test 3- Active Regeneration Scan Result

The overall axial UI was 0.96 for Test 3 of Phase 2, indicating a uniform distribution. For comparison, the overall axial UI for Test 8 of Phase 2 was 0.91 and 0.85 for Test 2 of Phase 1. The axial PM distribution data are given in Table 6.45. The average PM densities in axial segments 1-3 are within the 95\% CI of the TAS7000 data, so it can be said that they are similar. The actual average PM density was within -2 to $1 \%$ of the average PM density in the entire substrate. For comparison, the average PM densities in axial segments $1-3$ were $2-7 \%$ below the average PM density for Test 8 of Phase 2. Axial segment 4 had a PM density that was 5\% higher than the substrate average for Test 3 of Phase 2. In Test 8 of Phase 2, axial segment 4 had a PM density that was $17 \%$ higher than the average PM density. This shows that the active regeneration at a PM density near $3 \mathrm{~g} / \mathrm{L}$ produced a more uniform axial PM distribution than the passive oxidation performed at a similar PM density. The PM distribution found for Test 2 of Phase 1 was not at all similar to the trend found for Tests 3 and 8 of Phase 2. In Test 2 of Phase 1, axial segments 3 and 4 had an average PM density that was 10-31\% lower than the substrate average PM density and axial segments 1 and 2 had an average PM density that was $14-26 \%$ higher than the substrate average. The temperature distribution for Test 3 of Phase 2, shown in Figure I.9 of Appendix I, had a similar trend to the temperature distribution for Test 2 of Phase 1, shown in Figure I.3. The temperature near the inlet of the substrate was $25^{\circ} \mathrm{C}$ lower than the temperature near the outlet, and the temperature near the outer edges of the substrate was $25{ }^{\circ} \mathrm{C}$ lower than the temperature at the centerline of the substrate. These limited data indicate that the active regeneration at a PM density near $3 \mathrm{~g} / \mathrm{L}$ produced a more uniform axial distribution than the active regeneration at a PM density near $5 \mathrm{~g} / \mathrm{L}$. 
All axial segments for Test 3 of Phase 2 had a UI above 0.94 in each radial section. The $95^{\text {th }} \mathrm{PRs}$ were similar for all radial sections as well. The $95^{\text {th }} \mathrm{PR}$ for radial section 3 was $0-72 \%$ greater than the $95^{\text {th }} \mathrm{PR}$ for radial sections 1 and 2 . This indicates that the distribution near the centerline was more consistent than near the periphery of the filter, even though the UIs were identical for all radial sections in each axial segment.

Table 6.45: Phase 2 Test 3- Active Regeneration Axial PM Distribution

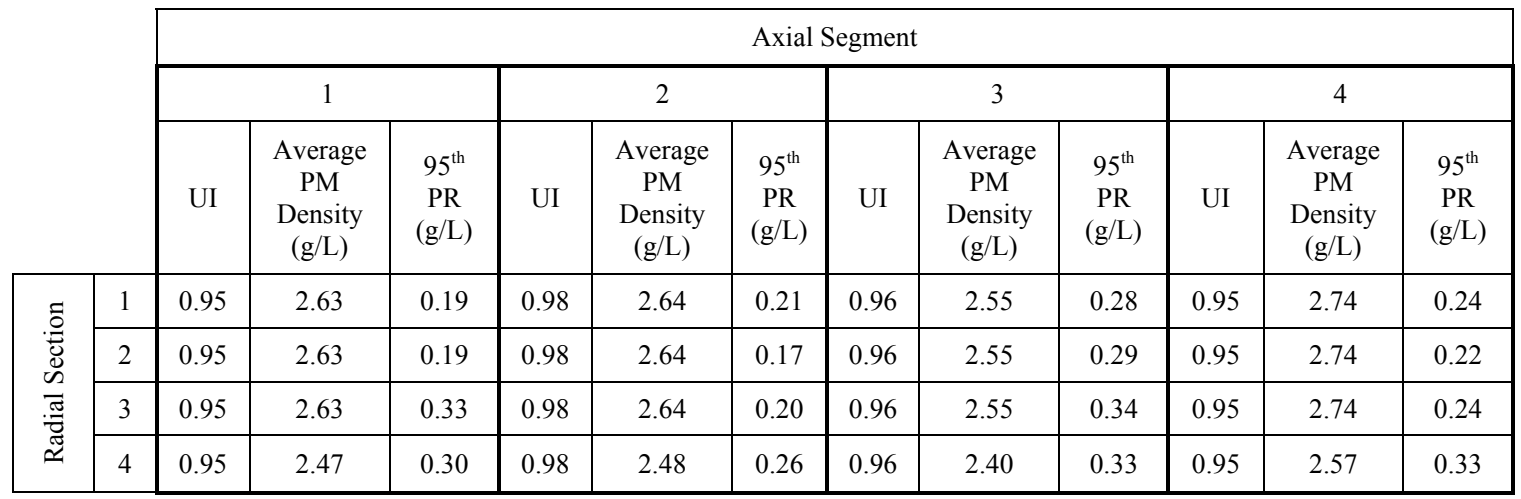

The overall radial UI was 0.97 for Test 3 of Phase 2, which is similar to Test 8 from Phase 2 and Test 2 of Phase 1. The radial PM distribution data are given in Table 6.46. Each quadrant in each axial segment had a UI above 0.94, indicating a uniform distribution. This is consistent with the data shown in Figure 6.34. All quadrants had a similar average PM density and $95^{\text {th }}$ PR in each axial segment as well. Test 8 of Phase 2 produced similar results. These data indicate that the active regeneration and passive oxidation experiments where $26-41 \%$ of the available PM was oxidized had similar radial distributions, regardless of the PM density in the substrate prior to the active regeneration or passive oxidation being performed.

Table 6.46: Phase 2 Test 3- Active Regeneration Radial PM Distribution

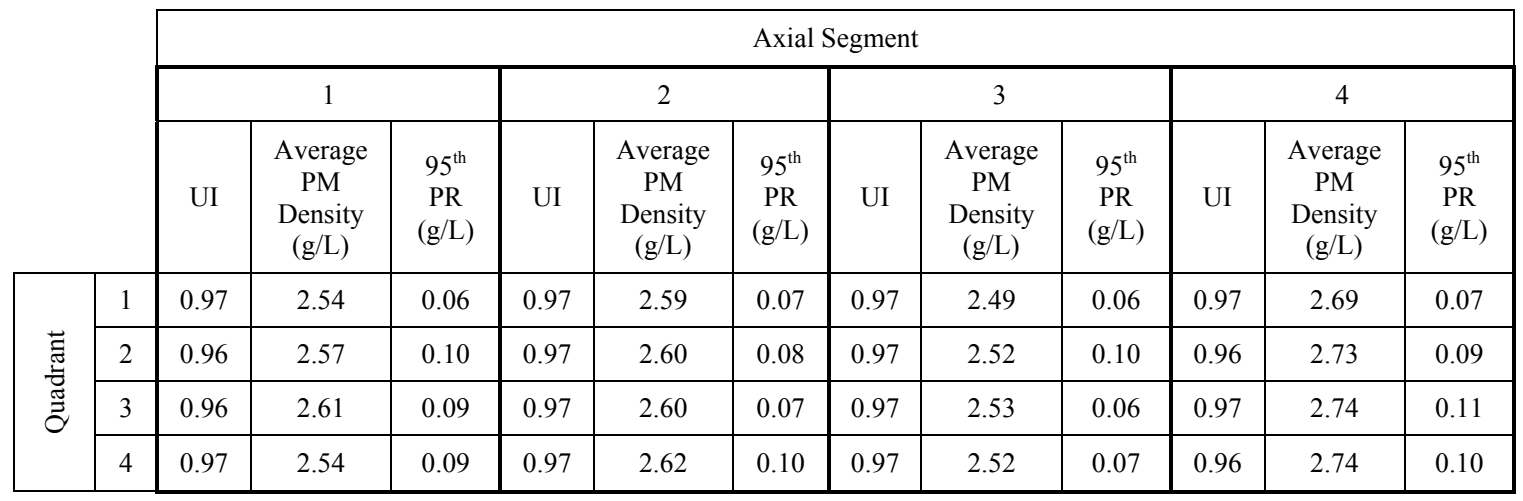

The overall angular UI for Test 3 of Phase 2 was 0.98 , which is similar to Test 8 of Phase 2. The overall angular UI for Test 2 of Phase 1 was 0.94 . The angular PM distribution data are given in Table 6.47. The average PM density in radial section 4 was $6 \%$ lower 
than the other radial sections, which is consistent with other data. All of the radial sections in each axial segment had UIs above 0.94. The angular PM distributions are similar for the active regeneration and passive oxidation experiments, where $26-41 \%$ of the available PM was oxidized, regardless of the PM density in the substrate prior to the active regeneration or passive oxidation being performed.

Table 6.47: Phase 2 Test 3- Active Regeneration Angular PM Distribution

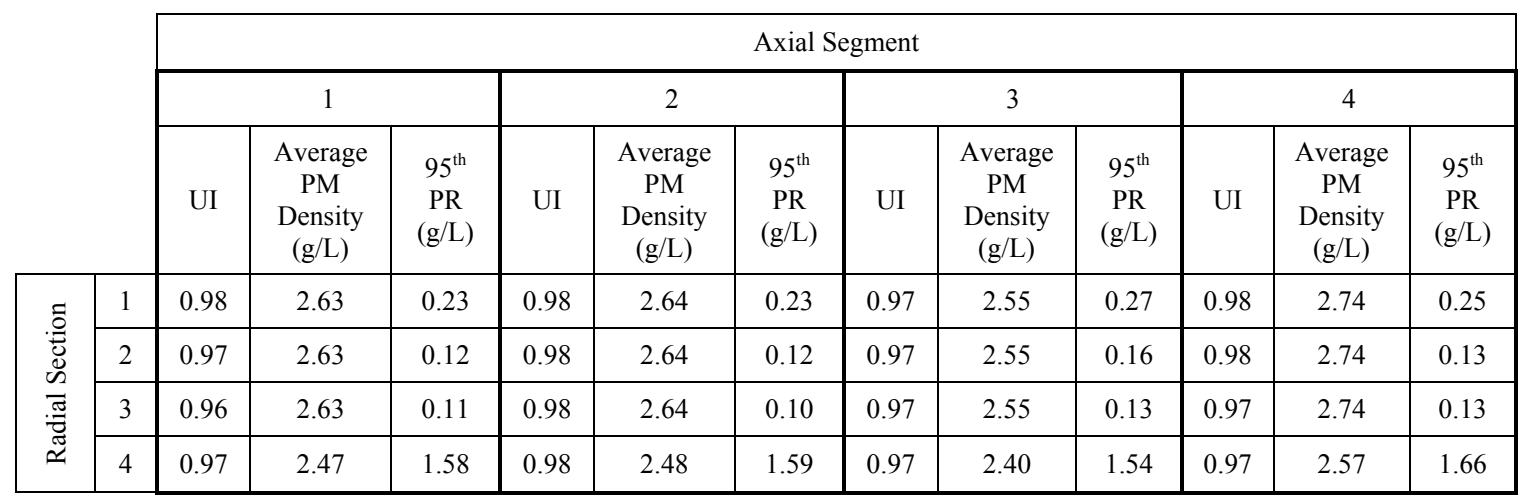

\section{Phase 2 Test 4: Active Regeneration}

The sixth active regeneration test was Test 4 of Phase 2. Three substrate scans were taken during this test, a baseline scan, a scan after an active regeneration, and a scan after post loading was performed on the substrate. The post loading results will be presented in section 6.8. For this test, a substrate was loaded to $3.06 \mathrm{~g} / \mathrm{L}$. Then an active regeneration was performed for 34 minutes with an average $\mathrm{CPF}$ temperature of $526{ }^{\circ} \mathrm{C}$. This oxidized $45 \%$ of the available PM, resulting in a PM density at the time of the substrate scan of $2.22 \mathrm{~g} / \mathrm{L}$. The result of this scan is shown in Figure 6.35. The active regeneration scan from Test 1 of Phase 1 was performed at a similar temperature and had a similar amount of PM oxidized. The results of that test were presented in section 6.1 and Figure 6.4 .

The overall axial UI for Test 4 of Phase 2 was 0.97 . For comparison, the active regeneration scan from Test 1 of Phase 1 had an overall axial UI of 0.84 . The axial PM distribution data for Test 4 of Phase 2 are given in Table 6.48. All of the radial sections in each axial segment had a similar axial UI. The $95^{\text {th }}$ PR for radial section 3 was 19$129 \%$ greater than the $95^{\text {th }} \mathrm{PRs}$ in radial sections 1 and 2 . This indicates that the data near the centerline were more consistent. Axial segments 1-3 had average PM densities that were between -5 and $1 \%$ different than the substrate average. This is similar to the results found in Test 3 of Phase 2. In Test 1 of Phase 1, axial segments 1-3 had average PM densities that were between -12 and $34 \%$ different than the substrate average. In Table 6.48 axial segment 4 had an average PM density that was 5\% greater than the substrate average, similar to Test 3 of Phase 2. For Test 1 of Phase 1, axial segment 4 had an average PM density that was 53\% lower than the substrate average. The axial PM distribution is not similar between Test 4 of Phase 2 and Test 1 of Phase 1 . Test 4 of Phase 2 had a more uniform axial PM distribution than Test 1 of Phase 1. This indicates 
that the axial PM distribution is more uniform when the PM density in the substrate is 3 $\mathrm{g} / \mathrm{L}$ prior to the regeneration instead of $5 \mathrm{~g} / \mathrm{L}$ prior to the regeneration, for these active regenerations where $45-58 \%$ of the PM was oxidized. The temperature distribution for Test 4 of Phase 2, as shown in Figure I.10, shows that the outlet of the substrate had a temperature that was $25{ }^{\circ} \mathrm{C}$ higher than the inlet, and the outer edges of the substrate had a temperature that was $25^{\circ} \mathrm{C}$ lower than the centerline. The axial temperature distribution for Test 1 of Phase 1 was similar, but the difference in the radial temperature distribution was $50{ }^{\circ} \mathrm{C}$, rather than $25^{\circ} \mathrm{C}$, as shown in Figure I.2.

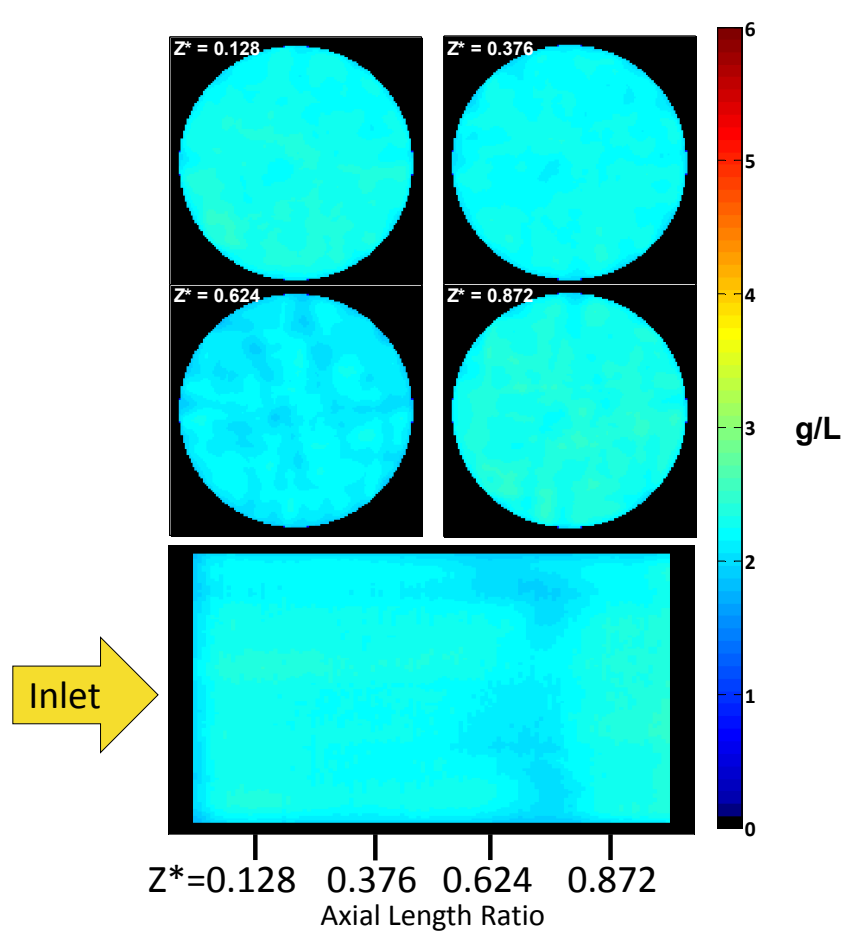

Figure 6.35: Phase 2 Test 4- Active Regeneration Scan Result

Table 6.48: Phase 2 Test 4- Active Regeneration Axial PM Distribution

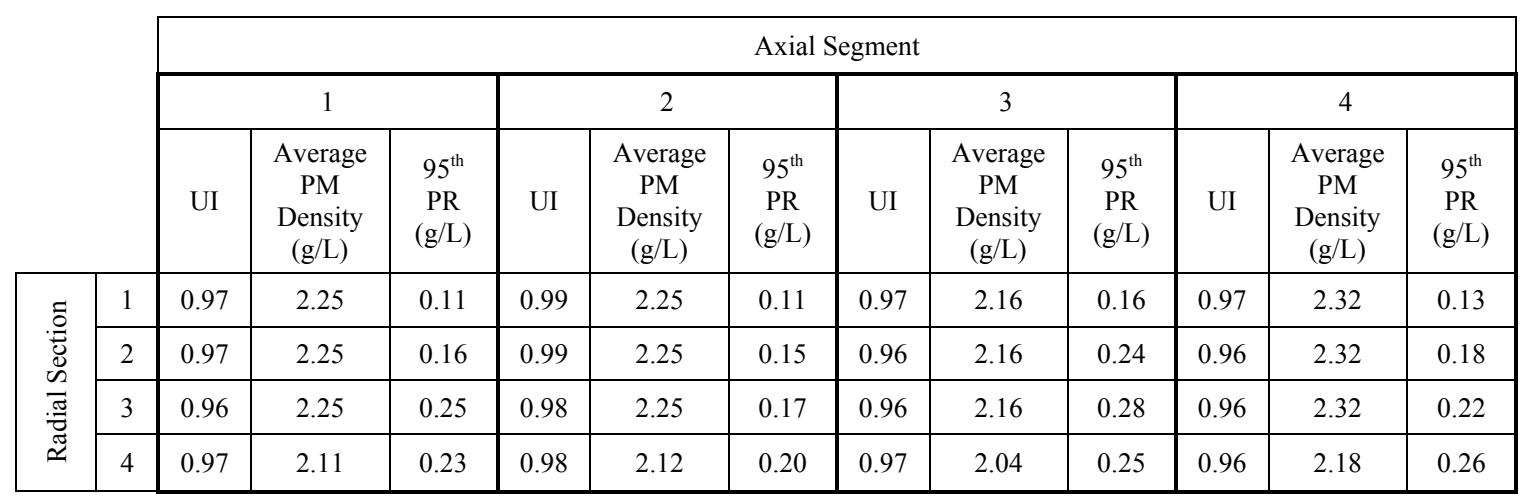


The overall radial UI for Test 4 of Phase 2 was 0.98 , which is similar to the overall radial UI for Test 3 of Phase 2. For comparison, the overall radial UI for the active regeneration scan from Test 1 of Phase 1 was 0.93 . This indicates that active regeneration experiments, where $45-58 \%$ of the available PM was oxidized, had a more uniform radial distribution when the regeneration was performed with a PM density in the substrate prior to the regeneration of $3 \mathrm{~g} / \mathrm{L}$ instead of $5 \mathrm{~g} / \mathrm{L}$. The radial PM distribution data are given in Table 6.49. All of the quadrants in each axial segment had a radial UI above 0.94 , indicating a uniform distribution. This is in agreement with the data shown in Figure 6.35.

Table 6.49: Phase 2 Test 4- Active Regeneration Radial PM Distribution

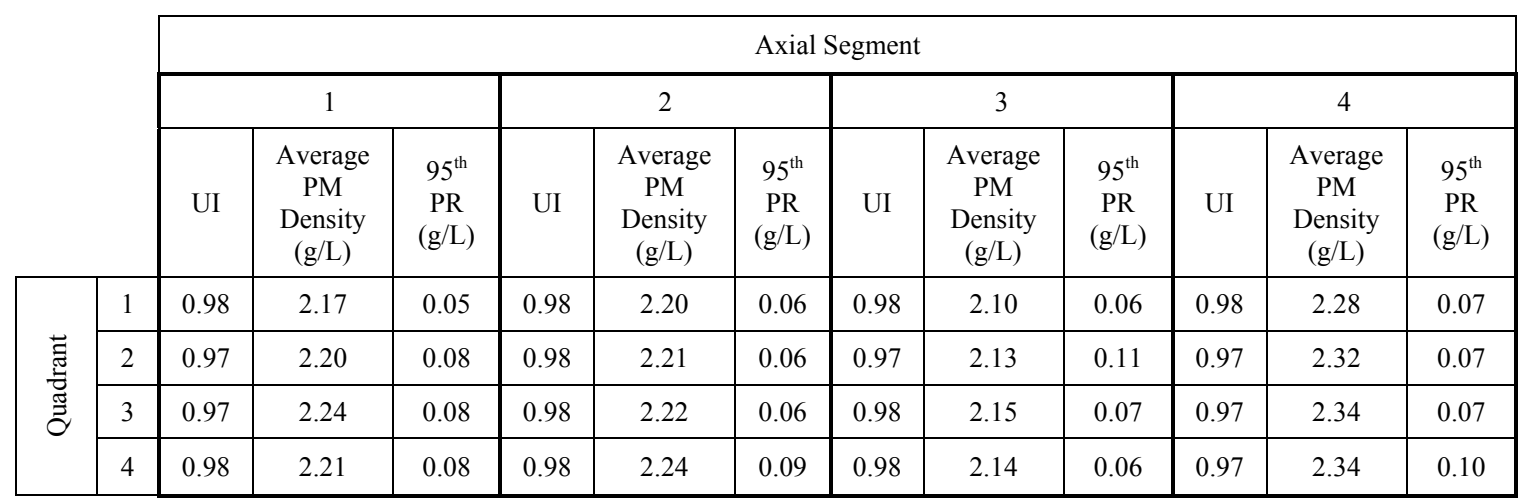

The overall angular UI for Test 4 of Phase 2 was 0.98 , which is similar to the overall angular UI from Test 3 of Phase 2. The overall angular UI from the active regeneration scan for Test 1 of Phase 1 was 0.91. This indicates that the active regeneration experiments, where $45-58 \%$ of the available PM was oxidized, had a more uniform angular PM distribution when the PM density prior to the active regeneration was $3 \mathrm{~g} / \mathrm{L}$, as compared to $5 \mathrm{~g} / \mathrm{L}$. The angular PM distribution data are given in Table 6.50. All of the radial sections in each axial segment had a UI above 0.94, indicating a uniform distribution. Radial section 4 had an average PM density that was 6\% lower than the other radial sections, which is consistent with the other data.

Table 6.50: Phase 2 Test 4- Active Regeneration Angular PM Distribution

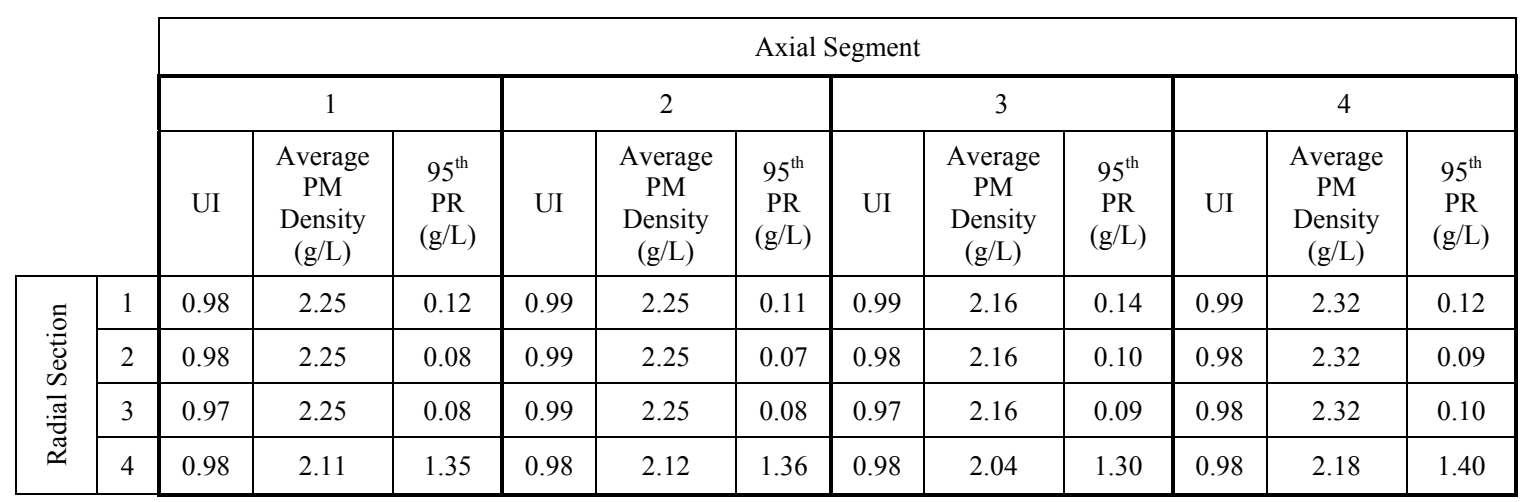




\section{Phase 2 Test 5: Active Regeneration}

The seventh and final active regeneration scan was taken during Test 5 of Phase 2. For this test, the substrate was loaded to $3.90 \mathrm{~g} / \mathrm{L}$. This test achieved a higher PM density because of the ash loading in substrate number 2, as discussed previously and in Appendix G. The substrate had approximately $0.8 \mathrm{~g} / \mathrm{L}$ of ash. An active regeneration was then performed for 8.5 minutes with an average $\mathrm{CPF}$ temperature of $574{ }^{\circ} \mathrm{C}$. This oxidized $52 \%$ of the available PM, resulting in a PM density in the substrate of $2.33 \mathrm{~g} / \mathrm{L}$ at the time the substrate was scanned. The result of this scan is shown in Figure 6.36. For comparison, Test 4 of Phase 1 was an active regeneration performed at a similar temperature and resulted in a similar amount of PM oxidized, and Test 7 of Phase 2 was a passive oxidation test with a similar amount of PM oxidized. The results from those tests are shown in Figures 6.13 and 6.28 respectively.

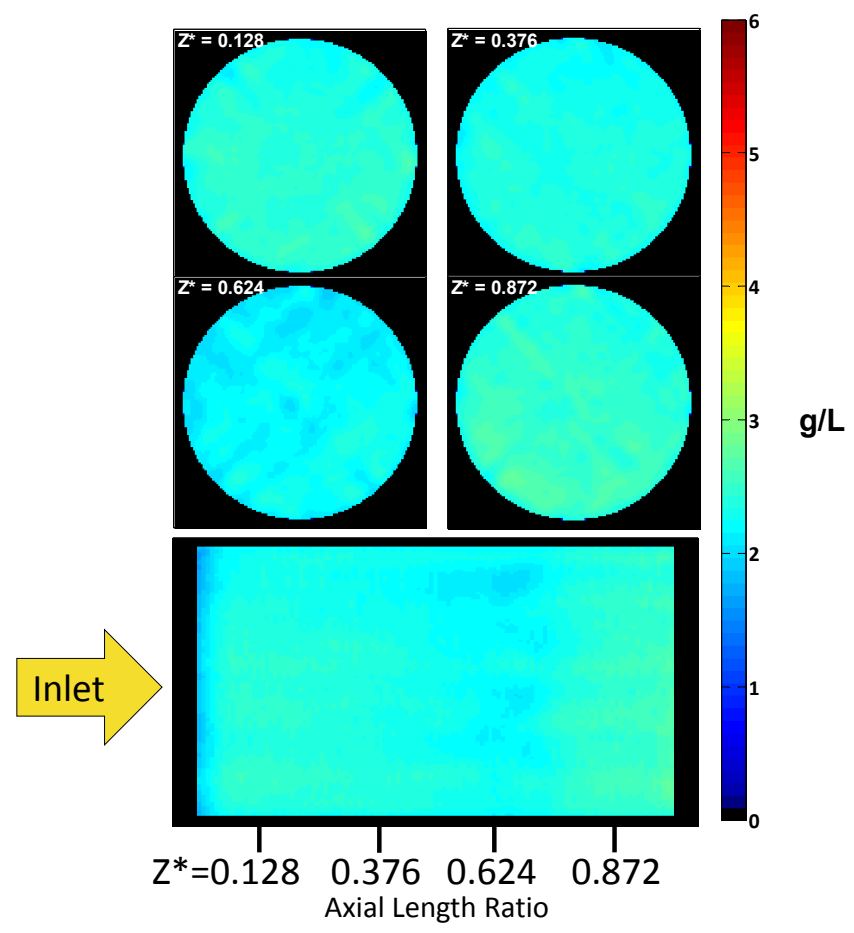

Figure 6.36: Phase 2 Test 5- Active Regeneration Scan Result

The overall axial UI for Test 5 of Phase 2 was 0.96 , which is similar to the overall axial UI for the passive oxidation scan from Tests 2 and 7 of Phase 2. This indicates that the active regeneration and passive oxidation experiments that resulted in $52-81 \%$ of the available PM being oxidized had a uniform axial PM distribution with a similar amount of variation in the data, during Phase 2. The overall axial UI for Test 4 of Phase 1 was 0.80. The temperature distribution plots for Tests 2 and 5 of Phase 2 and Test 4 of Phase 1, which are presented in Figures I.8, I.12, and I.5, respectively, show that all of the tests had a temperature increase between 25 and $50{ }^{\circ} \mathrm{C}$ from the inlet to the outlet of the substrate. This indicates that the active regeneration experiments, where $52-81 \%$ of the available PM was oxidized, produced a more uniform axial distribution during Phase 2, 
which used the ISL engine and corresponding aftertreatment. The axial PM distribution data are presented in Table 6.51. Axial segments 1-3 had average PM densities that were between -3 and $1 \%$ different than the average PM density in the substrate. This is a result similar to the data from the passive oxidation scan taken during Test 7 of Phase 2 where axial segments 1-3 had average PM densities that were between -3 and $-1 \%$ different than the average substrate PM density. Test 4 of Phase 1 had average PM densities in axial segments 1-3 that were between -66 and $64 \%$ different than the substrate average. In Table 6.51 axial segment 4 had an average PM density that was $7 \%$ higher than the substrate average, which is similar to the results from Tests 3, 4, and 7 of Phase 2 . Test 4 of Phase 1 had an average PM density in axial segment 4 that was 35\% lower than the substrate average. The data in Table 6.51 also show that each radial section in each axial segment had a UI above 0.94, indicating the axial distribution was uniform everywhere. Radial section 3 had a $95^{\text {th }}$ PR that was $1-212 \%$ larger than radial sections 1 and 2 . This indicates that there is more variation in the PM density near the edge of the substrate.

Table 6.51: Phase 2 Test 5- Active Regeneration Axial PM Distribution

\begin{tabular}{|c|c|c|c|c|c|c|c|c|c|c|c|c|c|}
\hline & \multicolumn{12}{|c|}{ Axial Segment } \\
\hline & & \multicolumn{3}{|c|}{1} & \multicolumn{3}{|c|}{2} & \multicolumn{3}{|c|}{3} & \multicolumn{3}{|c|}{4} \\
\hline & & UI & $\begin{array}{l}\text { Average } \\
\text { PM } \\
\text { Density } \\
\text { (g/L) }\end{array}$ & $\begin{array}{l}95^{\text {th }} \\
\text { PR } \\
(\mathrm{g} / \mathrm{L})\end{array}$ & UI & $\begin{array}{l}\text { Average } \\
\text { PM } \\
\text { Density } \\
(g / L)\end{array}$ & $\begin{array}{l}95^{\text {th }} \\
\text { PR } \\
\text { (g/L) }\end{array}$ & UI & $\begin{array}{c}\text { Average } \\
\text { PM } \\
\text { Density } \\
(\mathrm{g} / \mathrm{L})\end{array}$ & $\begin{array}{l}95^{\text {th }} \\
\text { PR } \\
(\mathrm{g} / \mathrm{L})\end{array}$ & UI & $\begin{array}{l}\text { Average } \\
\text { PM } \\
\text { Density } \\
\text { (g/L) }\end{array}$ & $\begin{array}{c}95^{\text {th }} \\
\text { PR } \\
\text { (g/L) }\end{array}$ \\
\hline \multirow{4}{*}{ 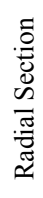 } & 1 & 0.95 & 2.34 & 0.10 & 0.99 & 2.36 & 0.10 & 0.97 & 2.25 & 0.19 & 0.96 & 2.49 & 0.15 \\
\hline & 2 & 0.95 & 2.34 & 0.22 & 0.98 & 2.36 & 0.20 & 0.96 & 2.25 & 0.32 & 0.96 & 2.49 & 0.29 \\
\hline & 3 & 0.95 & 2.34 & 0.31 & 0.98 & 2.35 & 0.27 & 0.96 & 2.25 & 0.32 & 0.95 & 2.49 & 0.42 \\
\hline & 4 & 0.95 & 2.21 & 0.37 & 0.98 & 2.23 & 0.33 & 0.97 & 2.13 & 0.34 & 0.96 & 2.35 & 0.34 \\
\hline
\end{tabular}

The overall radial UI for Test 5 of Phase 2 was 0.98 , which is similar to Tests 2 and 7 of Phase 2. This indicates that the active regeneration and passive oxidation experiments, where $52-81 \%$ of the available PM was oxidized, resulted in uniform radial distributions, during Phase 2. Test 4 of Phase 1 had an overall radial UI of 0.91. The temperature distribution plots show that Test 5 of Phase 2, Figure I.12, Test 2 of Phase 2, Figure I.8, and Test 4 of Phase 1, Figure I.5, had a temperature near the edge of the substrate that was $25-100{ }^{\circ} \mathrm{C}$ lower than the temperatures near the centerline. Test 2 had the smallest amount of variation at $25{ }^{\circ} \mathrm{C}$. This indicates that a more uniform radial distribution exists after the active regeneration events in Phase 2, which used the ISL engine and corresponding aftertreatment system. The radial distribution data are given in Table 6.52. Each quadrant in each axial segment was 0.98 , indicating a consistent and uniform distribution. This is consistent with the data plotted in Figure 6.36. 
Table 6.52: Phase 2 Test 5- Active Regeneration Radial PM Distribution

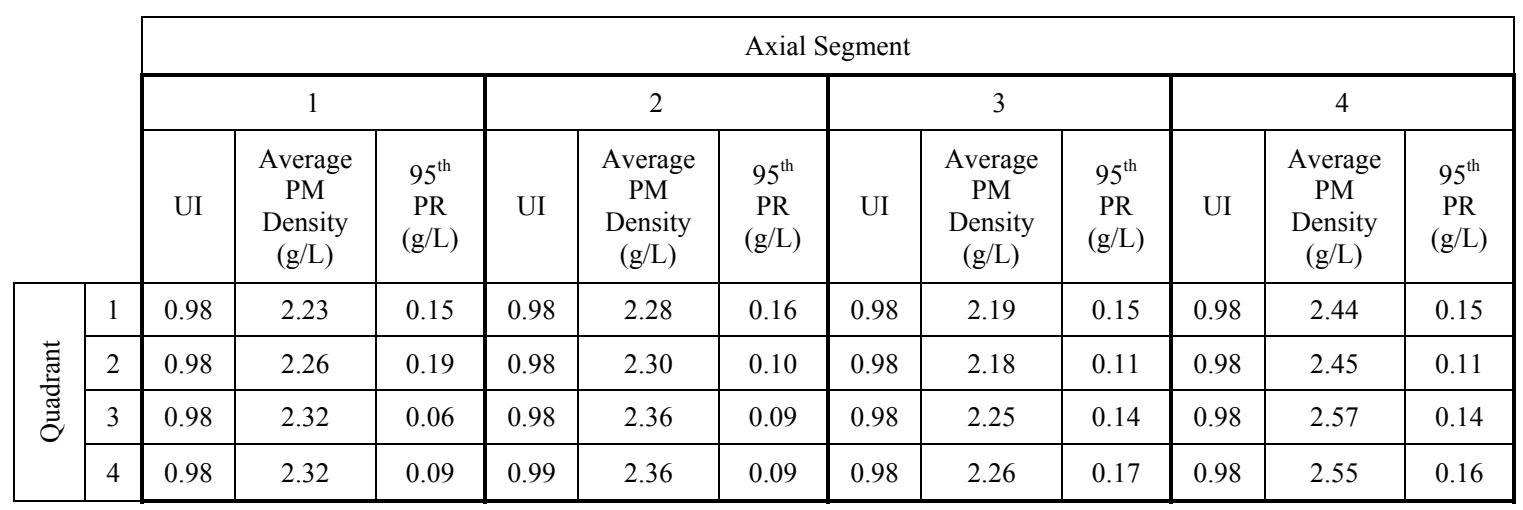

The overall angular UI for Test 5 of Phase 2 was 0.98 , which is similar to the overall angular UI from Tests 2 and 7 of Phase 2. Test 4 of Phase 1 had an overall angular UI of 0.89 . This indicates that the angular distribution is more uniform for passive oxidation and active regeneration events that used the ISL engine and aftertreatment system instead of the ISB engine and aftertreatment system. The angular PM distribution data are given in Table 6.53. All of the radial sections in each axial segment had a UI above 0.94. Radial section 4 had an average PM density that was $6 \%$ lower than the other radial sections, which is consistent with other data.

Table 6.53: Phase 2 Test 5- Active Regeneration Angular PM Distribution

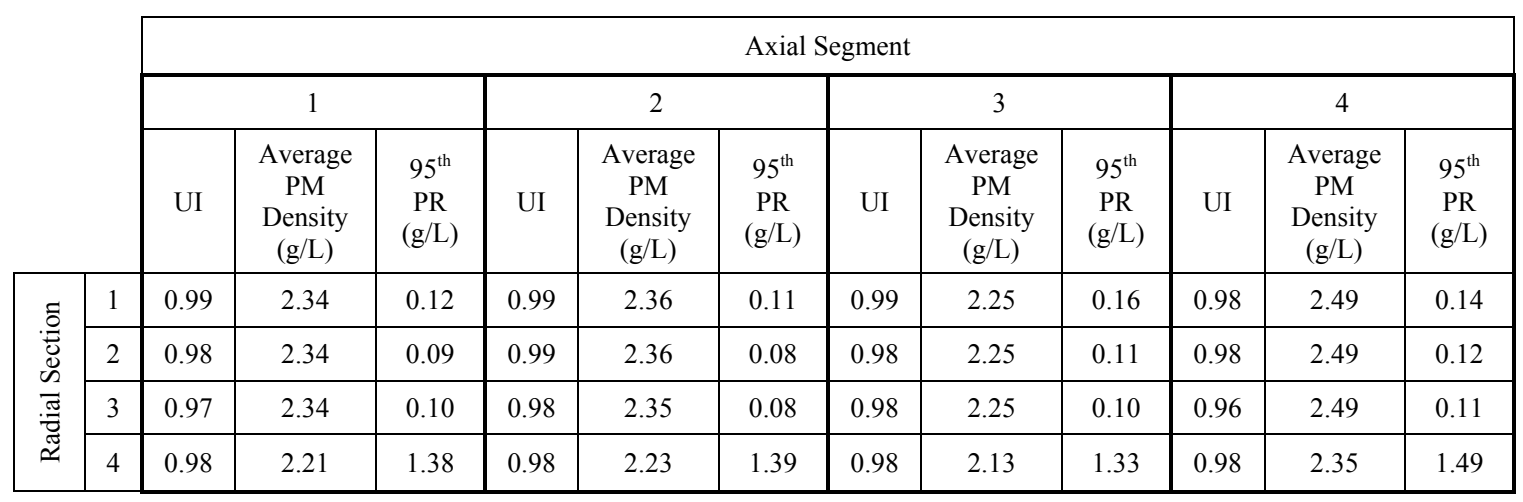

The axial PM distribution for each active regeneration test is plotted in Figure 6.37. A summary of each test was provided in Table 6.41. The distributions from Phase 1 have a different shape than the distributions from Phase 2. The Phase 2 PM distributions for active regenerations conditions follow similar trends as the PM distributions for the loading and passive oxidation conditions. The PM distribution trends for all of the Phase 2 active regeneration tests are similar as well, indicating that the 525 and $600{ }^{\circ} \mathrm{C}$ active regenerations produced similar PM distributions. The Phase 1 tests all show a decreased PM density in the last $50 \%$ of the axial length. There are a few possible explanations as to what causes the differences between the tests. The first is that the modified engine calibration that was used during Phase 1 could have had an impact on the axial PM 
distribution after active regeneration, even though it was found to have no effect on the distribution after loading. The second possible explanation is that the use of stage 3 to stabilize the CPF temperature before weighing the substrate could have an effect on the PM distribution, similar to the passive oxidation experiments. During stage 3 of the Phase 2 tests, 6.7 grams of PM were loaded into the CPF on average. The third possible explanation is that the oxygen concentration into the CPF during Phase 1 active regenerations was $5.6 \%$ on average and $7.3 \%$ on average during Phase 2 active regenerations. The oxygen concentration affects the reaction rate of active regenerations, as discussed by Premchand, et al. [45], but the effect on the PM distribution is unknown.

Test 2 of Phase 2 had a similar average PM density prior to the cleanout as the tests from Phase 1. However, the axial PM distribution was not similar to the other tests from Phase 1, indicating that the PM density in the substrate prior to the active regeneration during Phase 1 was not the only factor that caused a different PM distribution. The average PM density in the substrate prior the active regeneration during Phase 1 was $67 \%$ higher than the average PM density in the substrate prior to the other active regenerations performed during Phase 2. The higher PM density could have had an effect on the axial distribution when less PM was oxidized. The space velocities for Phase 1 and Phase 2 were similar, so the axial wall flow distribution trend should have been similar. Test 2 of Phase 2 was exposed to multiple loading, cleanout, and partial oxidation cycles, but the axial PM distribution trend was still similar to the other axial PM distribution trends after active regenerations in Phase 2. This indicates that the repeated testing on this part did not affect the PM distribution for this test.

Figure 2.11 gives the axial PM distribution after different levels of PM oxidation, as measured using a scaled down substrate and SEM imaging. The measurements are of PM cake thickness, so the density of PM cannot be directly determined. The results in Figure 2.11 show that the PM cake thickness increased until $45 \%$ of the PM was oxidized. After $45 \%$ of the PM was oxidized, the PM cake thickness in the front half of the substrate is $5-33 \%$ lower than the back half. One similarity between the data collected by Pinturaud, et al, and the Phase 2 data presented in this section is that the PM distributions after loading and after active regenerations were similar. In Figure 2.11, the distribution shown for $83 \%$ PM oxidation is similar to the axial PM distribution trend for Test 2 of Phase 2 where $81 \%$ of the PM was oxidized. In general, the other distribution trends do not agree with the trends collected in this experimental work, as the PM density was either $52-200 \%$ higher in the front half of the substrate or constant throughout the substrate. The differences between the experimental data presented in Figure 2.11 and the data presented in Figure 6.37 could be due to how the Pinturaud, et al. [19] performed the tests, loaded the substrate, conducted the active regenerations, or scaled the particulate filter down. No other experimental work on the PM distribution after PM oxidation was found for comparison. 


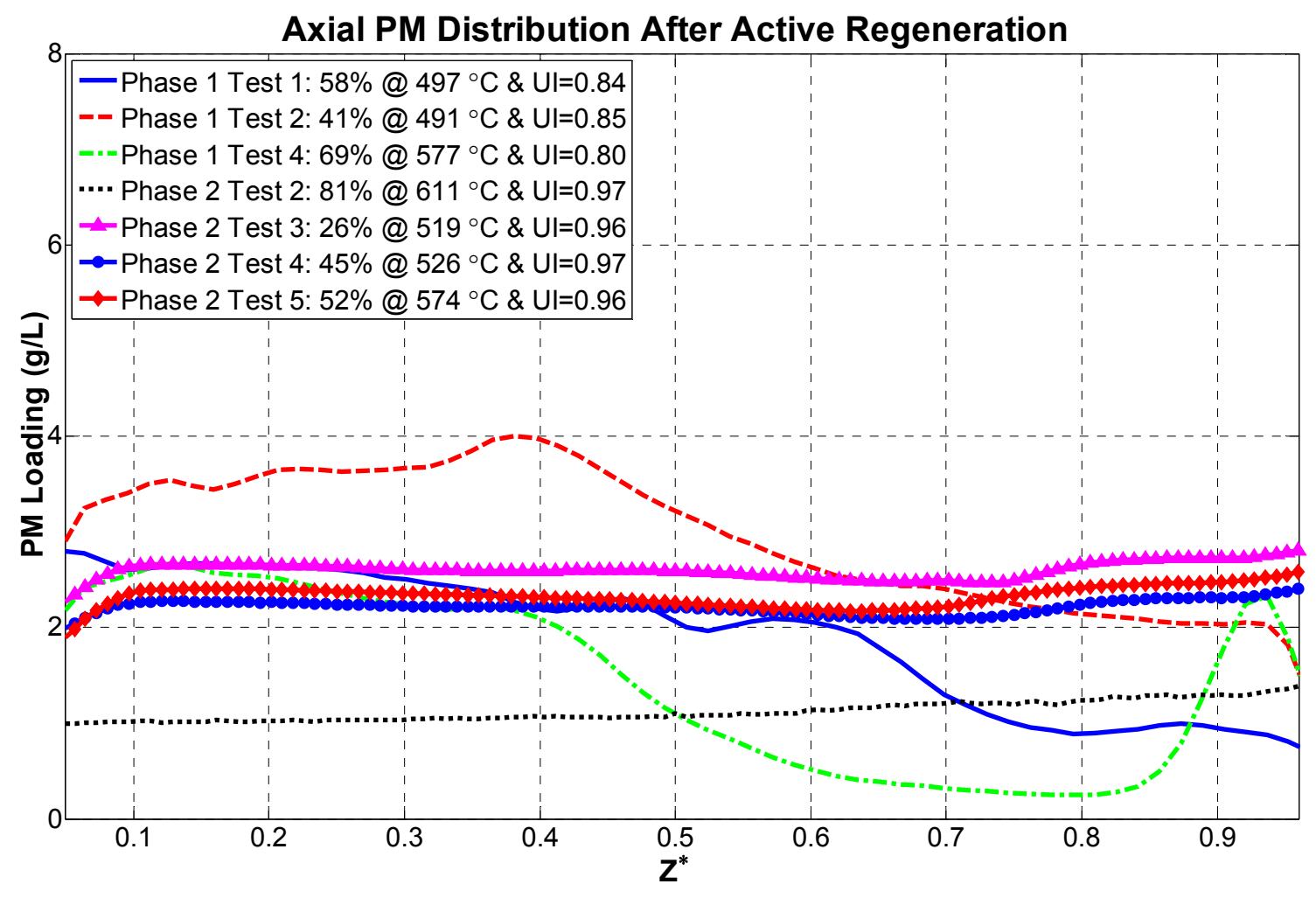

Figure 6.37: Axial PM Distribution for all Active Regeneration Tests

The overall axial UI that was calculated for each active regeneration test is plotted against the amount of PM that was oxidized for that test in Figure 6.38. The Phase 2 tests, where standard loading conditions were used show a uniform axial distribution, regardless of the amount of PM oxidized. The Phase 1 tests show a decreasing axial UI as the amount of PM oxidized increases. These data shows that the active regeneration performed at average PM densities near $5 \mathrm{~g} / \mathrm{L}$, and resulted in $41-69 \%$ of the PM being oxidized had a non-uniform distribution. The data suggests that modified engine conditions used to load the substrates during Phase 1 should be avoided to avoid non-uniform PM distributions.

Active regeneration tests were the only tests shown to heavily impact the radial and angular PM distribution and show a significant trend. The overall radial UI that was calculated for each active regeneration test is plotted against the amount of PM that was oxidized for that test and is shown in Figure 6.39. The overall angular UI that was calculated for each test is plotted against the amount of PM oxidized for each test in Figure 6.40. The results shown in these plots are similar to the results in Figure 6.38. The one large difference is that Test 2 from Phase 1 has a uniform radial and angular PM distribution. This indicates that for active regenerations to cause a non-uniform radial and angular PM distribution, the PM density in the substrate prior the regeneration needs to be near $5 \mathrm{~g} / \mathrm{L}$ and the active regeneration needs to oxidize more than 41 and less than $81 \%$ of the available PM. When the PM density in the substrate was above $4 \mathrm{~g} / \mathrm{L}$, the radial and angular UI decreased as the amount of PM being oxidized increased until $81 \%$ 
of the PM was oxidized. After $81 \%$ of the available PM was oxidized, the angular and radial distributions were uniform. Under $4 \mathrm{~g} / \mathrm{L}$, the UI remained stable or increased slightly as the amount of PM being oxidized increased.

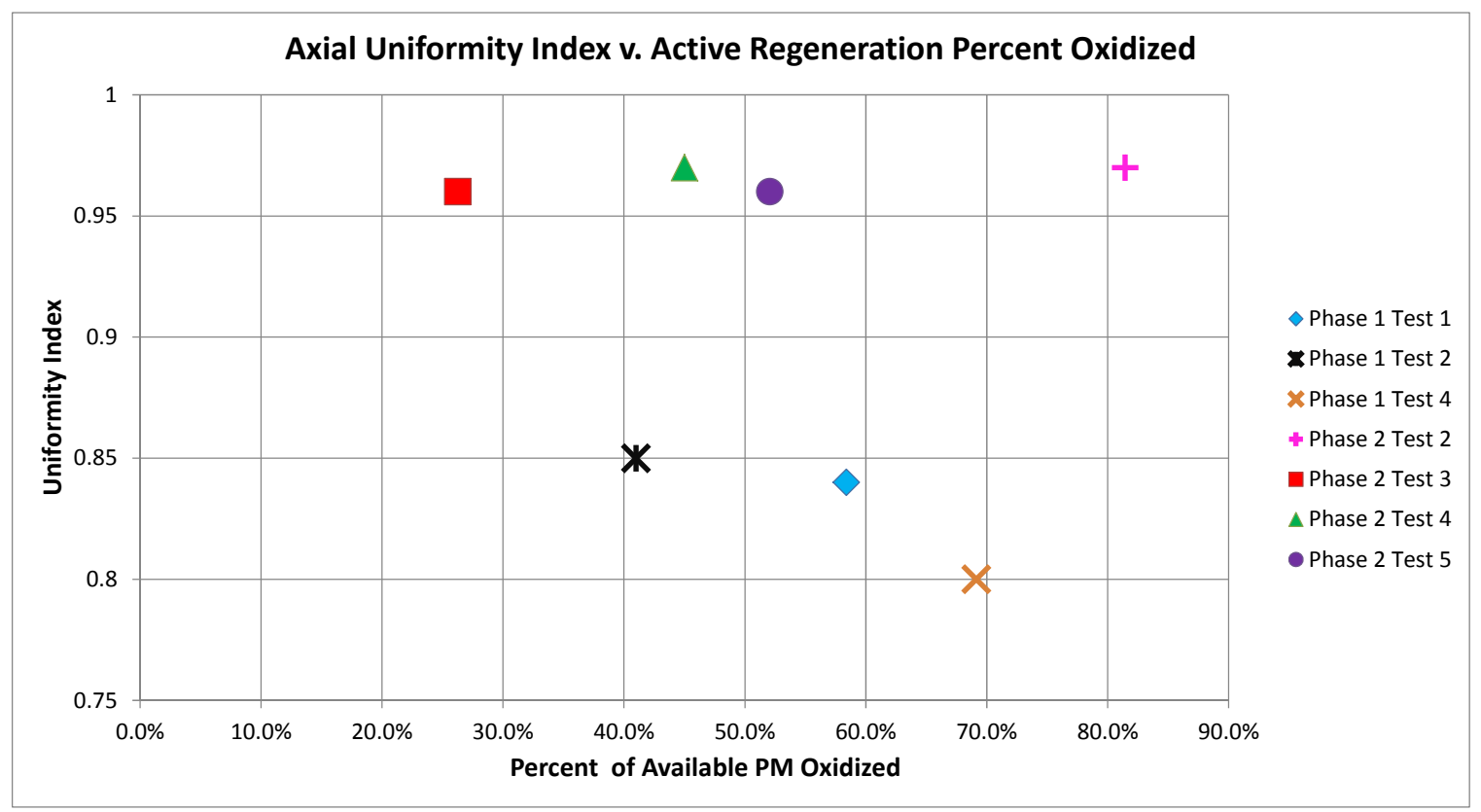

Figure 6.38: Axial Uniformity Index as a Function of Percent of PM Oxidized

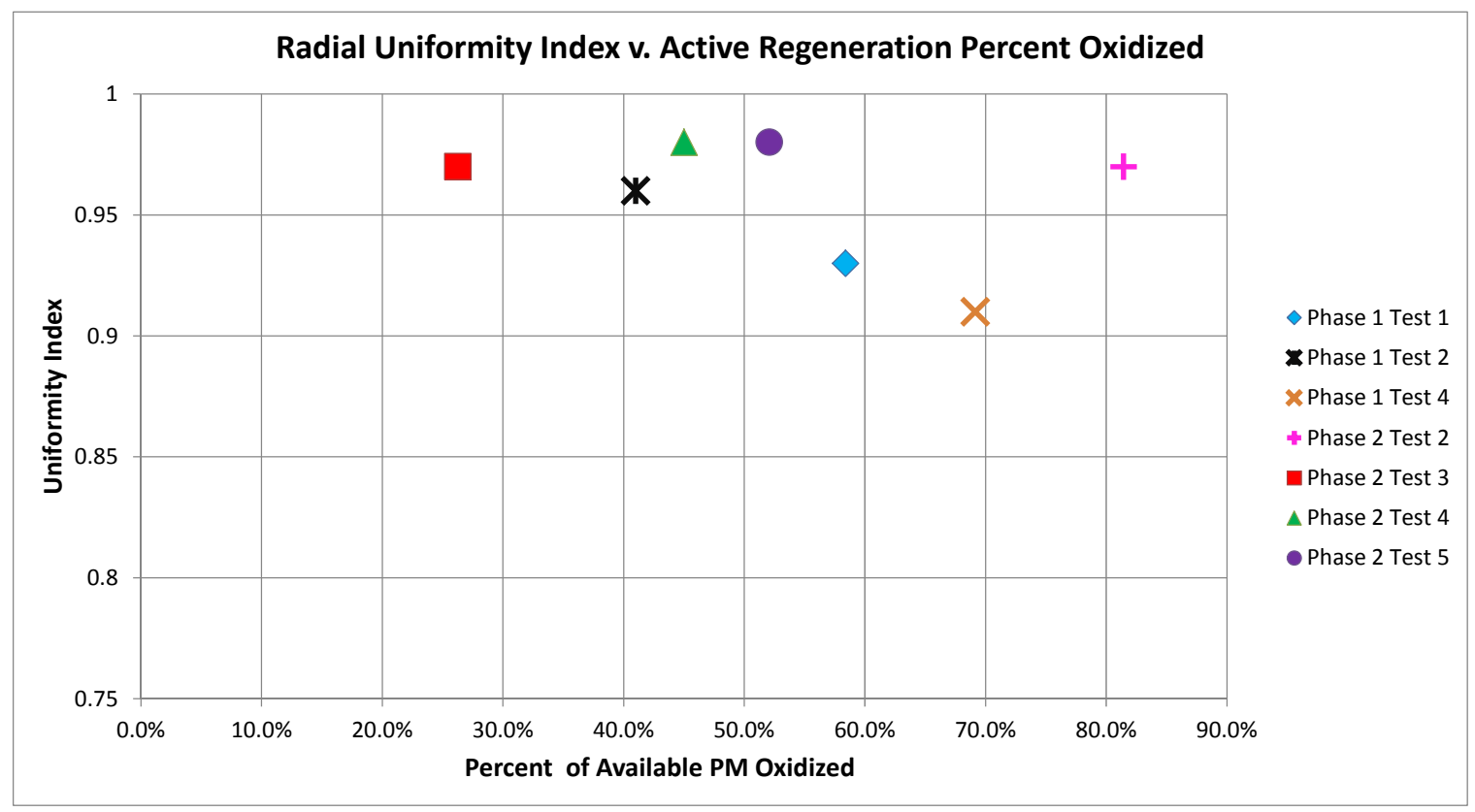

Figure 6.39: Radial Uniformity Index as a Function of Percent of PM Oxidized 


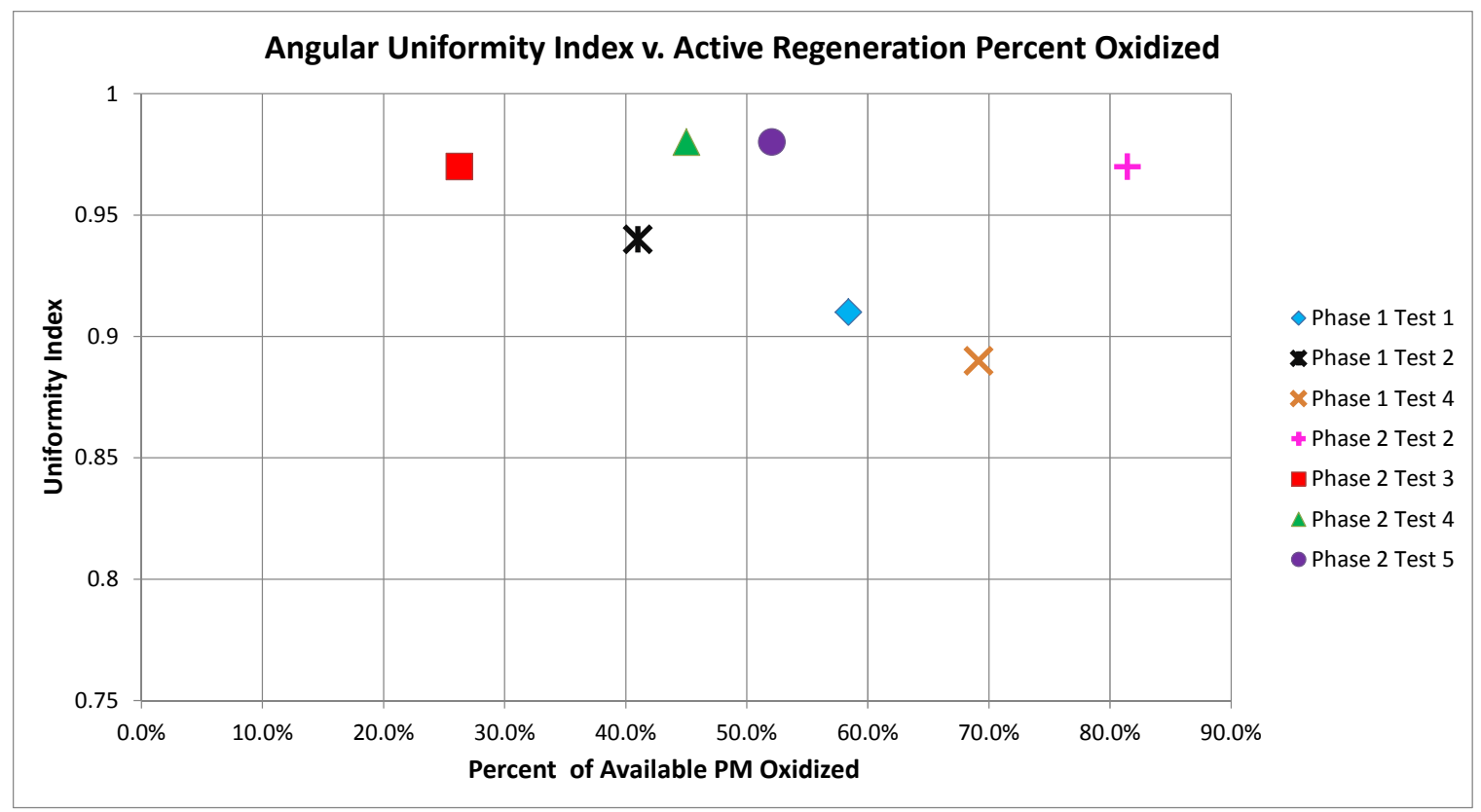

Figure 6.40: Angular Uniformity Index as a Function of Percent of PM Oxidized

\subsection{PM Distribution Trends after Post Loading}

This section discusses the PM distribution trends that existed after post loading was performed on the substrates. Post loading is PM loading that occurs after PM is oxidized from the substrate. Two post loading tests were performed during Phase 2. The data from both of these tests were processed using the new PM density calculation method, which was described in 4.1. Therefore, TAS7000 data presented in this section is for the PM density distribution in the substrate, including the carbonaceous and ash content. The first post loading test occurred during Test 4 , after an active regeneration oxidized $45 \%$ of the available PM. The second post loading test was after the passive oxidation that oxidized $65 \%$ of the available PM in Test 7. All PM loading was accomplished using EOC B from Table 5.8, which was the same EOC used during the loading tests of Phase 2. A summary of the two post loading tests is found in Table 6.54. The full test descriptions can be found in Chapter 5 and the full summaries of the tests are provided in Appendix E. The main parameter that was varied for the post loading tests was the type of PM oxidation (passive or active) that occurred before the post loading. Both tests were carried out for a similar amount of time, around 97-101 minutes, with Test 4 from Phase 2 being five minutes longer than Test 7 of Phase 2 . 
Table 6.54: Post Loading Test Summaries

\begin{tabular}{|c|c|c|c|c|c|c|c|c|c|c|}
\hline \multirow[t]{2}{*}{ Engine } & \multirow[t]{2}{*}{ Test } & \multirow[t]{2}{*}{$\begin{array}{l}\text { Post Loading } \\
\text { Occurred } \\
\text { After }\end{array}$} & Time & $\begin{array}{c}\text { CPF } \\
\text { Space } \\
\text { Velocity }\end{array}$ & $\begin{array}{c}\text { Average } \\
\text { CPF } \\
\text { Temp. }\end{array}$ & $\begin{array}{c}\text { Engine } \\
\text { Out PM } \\
\text { Conc. }\end{array}$ & $\begin{array}{c}\mathrm{NO}_{2} \\
\text { Conc. } \\
\text { into the } \\
\text { CPF }\end{array}$ & $\begin{array}{c}\mathrm{O}_{2} \\
\text { Conc. } \\
\text { into } \\
\text { the } \\
\mathrm{CPF}\end{array}$ & $\begin{array}{c}\text { PM } \\
\text { Density } \\
\text { before } \\
\text { Post } \\
\text { Loading }\end{array}$ & $\begin{array}{c}\text { PM } \\
\text { Density } \\
\text { After } \\
\text { Post } \\
\text { Loading }\end{array}$ \\
\hline & & & $\min$. & $1 \mathrm{k} / \mathrm{hr}$ & ${ }^{\circ} \mathrm{C}$ & $\mathrm{mg} / \mathrm{scm}$ & $\mathrm{mg} / \mathrm{scm}$ & $\%$ & $\mathrm{~g} / \mathrm{L}$ & $g / L$ \\
\hline \multirow{2}{*}{ ISL } & $\begin{array}{c}\text { Phase } 2 \\
\text { Test } 4\end{array}$ & $\begin{array}{c}\text { Active } \\
\text { Regeneration }\end{array}$ & 101.4 & 212 & 260 & 22.2 & 52.7 & 13.8 & 2.22 & 3.47 \\
\hline & $\begin{array}{c}\text { Phase } 2 \\
\text { Test } 7\end{array}$ & $\begin{array}{l}\text { Passive } \\
\text { Oxidation }\end{array}$ & 96.5 & 211 & 263 & 21.8 & 32.9 & 13.6 & 1.85 & 2.99 \\
\hline
\end{tabular}

\section{Phase 2 Test 4: Post Loading}

The third substrate scan from Test 4 was the post loading scan. This test occurred after $45 \%$ of the available PM was oxidized during a $526{ }^{\circ} \mathrm{C}$ active regeneration. After the active regeneration scan was completed, the substrate was loaded for 101 minutes. This resulted in a PM density in the substrate of $3.47 \mathrm{~g} / \mathrm{L}$ at the time the of the post loading substrate scan. This is $1.25 \mathrm{~g} / \mathrm{L}$ higher than the substrate average PM density at the time of the active regeneration scan $(2.22 \mathrm{~g} / \mathrm{L})$. The result of this scan is shown in Figure 6.41 .

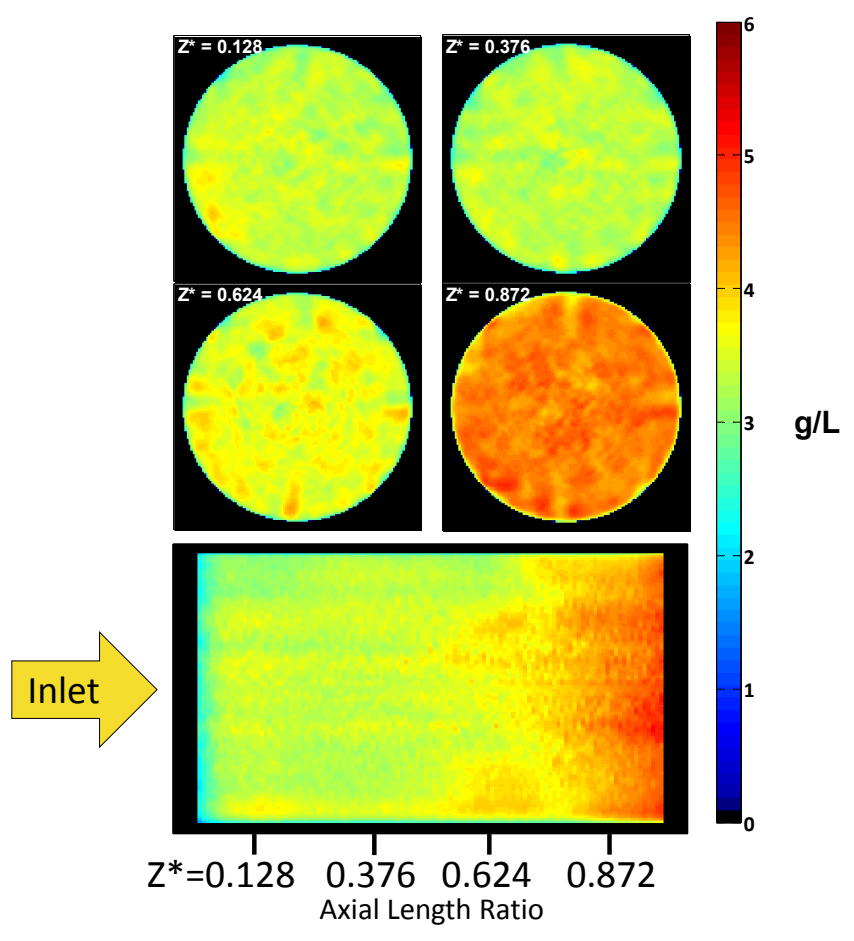

Figure 6.41: Phase 2 Test 4- Post Loading Scan Result

The overall axial UI for the post loading scan from Test 4 of Phase 2 was 0.84 . For comparison, the overall axial UIs for the loading scans from Tests 1 and 6 of Phase 2 were 0.90 and 0.94 , respectively. The overall axial UI after the active regeneration in 
Test 4 of Phase 2 was 0.97 . The overall axial UI for Test 4 was $7-11 \%$ lower than the overall axial UIs for Tests 1 and 6, and 13\% lower than the overall axial UI for the active regeneration scan from Test 4 . This indicates that the post loading that occurred after the active regeneration experiment that oxidized $45 \%$ of the available PM decreased the uniformity of the axial PM distribution. The axial PM distribution data are given in Table 6.55. Radial sections 1 and 2 in axial segment 3 were the only radial sections to have a uniform PM distribution. All other radial sections had a UI below 0.94. The axial PM distribution trend is different than the loading data sets studied in section 6.5. The average PM density increased in each axial segment. Axial segments 1-3 were within -7 to $5 \%$ of the average PM density in the substrate. In axial segment 4 , the average PM density was $26 \%$ higher than the substrate average. Prior to this test the second loading scan from Test 6 , which was presented in section 6.5, had the highest PM density in axial segment 4 , at $19 \%$ higher than the substrate average. The loading data sets showed a PM density that decreased or was nearly constant in axial segments 1-3 and a PM density in axial segment 4 that was $7-15 \%$ greater than the substrate average. The axial PM distribution from the post loading scan for Test 4 of Phase 2 was different than the active regeneration scan for Test 4 of Phase 2 as well. This indicates that a post loading that added $1.25 \mathrm{~g} / \mathrm{L}$ after an active regeneration affects the axial PM distribution and more PM is loaded near the outlet of the substrate. The temperature distribution plot shown in Figure I.11 shows that the temperature of the outer edges of the substrate was $25^{\circ} \mathrm{C}$ lower than the centerline and there was minimal temperature variation in the axial direction.

Table 6.55: Phase 2 Test 4- Post Loading Axial PM Distribution

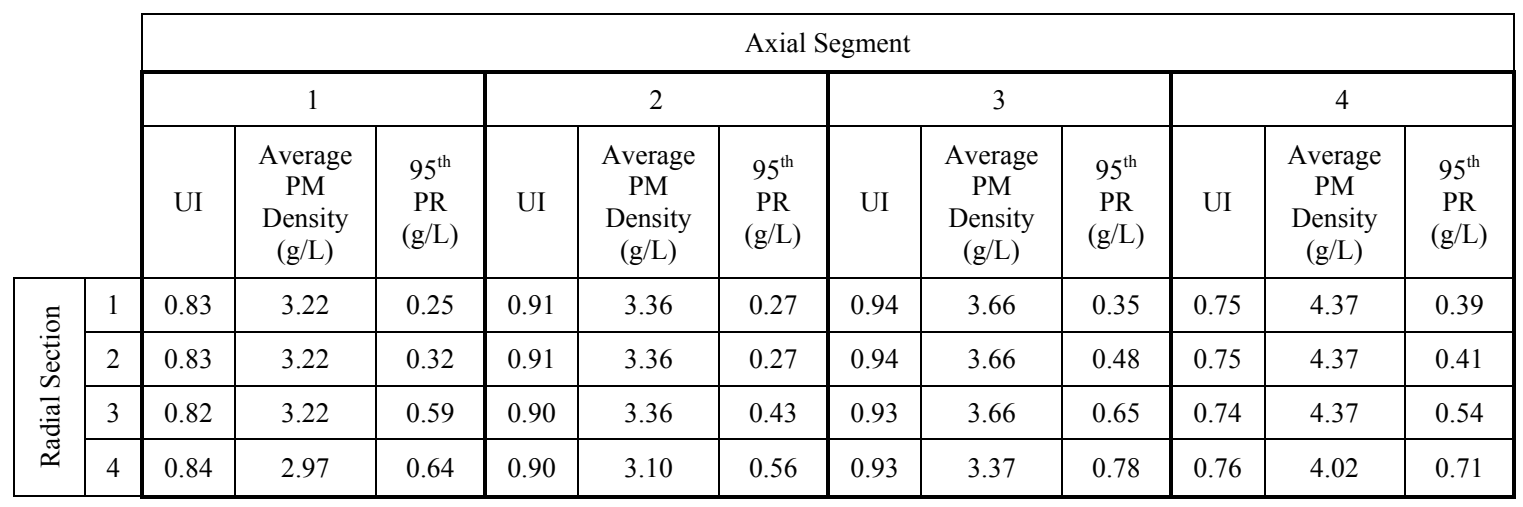

The overall radial UI for the post loading scan from Test 4 of Phase 2 was 0.95 , which is uniform. The active regeneration scan from Test 4 of Phase 2 and the loading scans from Tests 1 and 6 of Phase 2 had overall radial UIs above 0.94 as well. This indicates that for this test, post loading did not impact the radial PM distribution, when the PM density after the post loading is $1.25 \mathrm{~g} / \mathrm{L}$ higher than the PM density after the active regeneration. The radial PM distribution data are given in Table 6.56. Non-uniform PM distributions were found in axial segment 1 and quadrant 2, axial segment 3 and quadrant 2, and axial segment 4 and quadrants 1,2 , and 4 . These non-uniformities are not visible in the data in Figure 6.41. 
Table 6.56: Phase 2 Test 4- Post Loading Radial PM Distribution

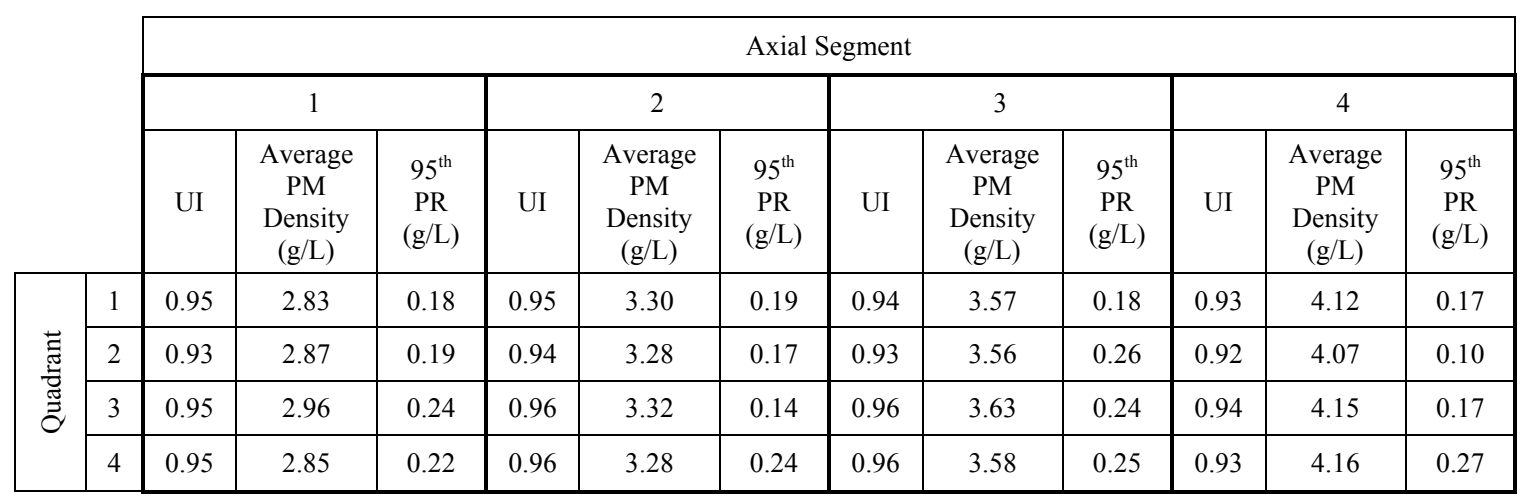

The overall angular UI for the post loading scan from Test 4 of Phase 2 was 0.95 . The overall angular UIs for the active regeneration scan from Test 4 of Phase 2 and the loading scans from Tests 1 and 6 of Phase 2 where above 0.94 as well. This indicates for this post loading, which resulted in an increase in the PM density of $1.25 \mathrm{~g} / \mathrm{L}$ after an active regeneration, the uniformity of the PM distribution in the angular direction was not affected. The angular PM distribution data are given in Table 6.57. Radial sections 1 and 2 have a UI that is a maximum of $3 \%$ greater than radial sections 3 and 4 , indicating that the PM distribution is more uniform near the centerline of the substrate after post loading. The only non-uniform angular distributions are found in radial sections 3 and 4 .

Table 6.57: Phase 2 Test 4- Post Loading Angular PM Distribution

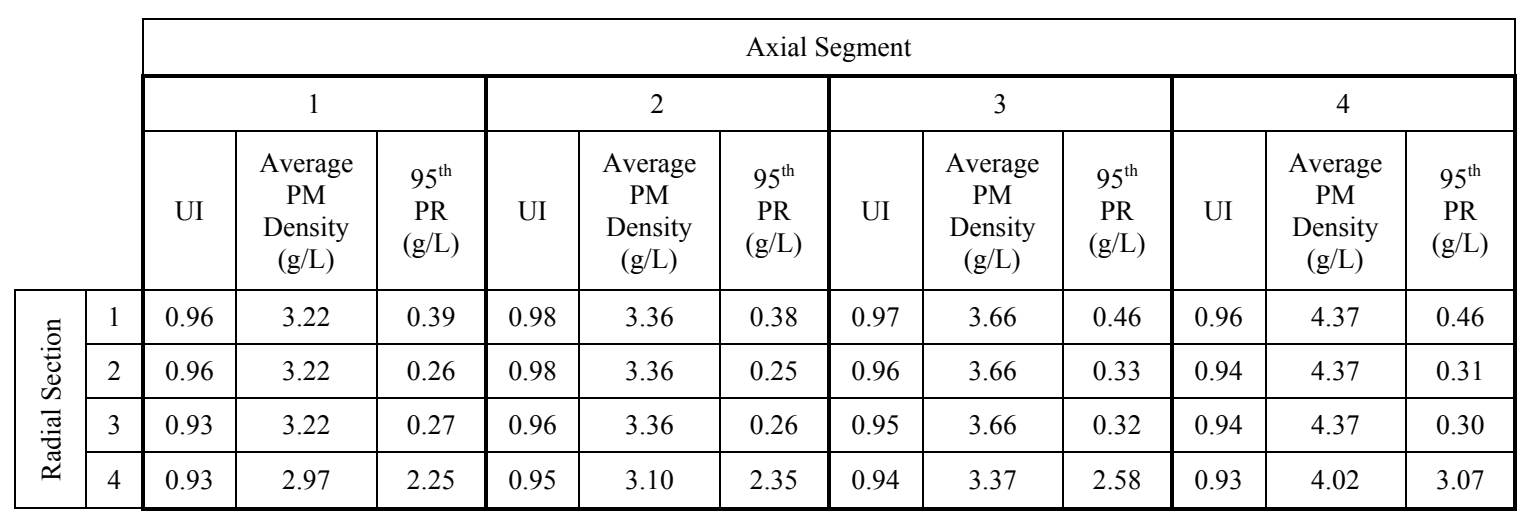

\section{Phase 2 Test 7: Post Loading}

The third substrate scan from Test 7 of Phase 2 was taken after post loading a substrate. After a 227 minute passive oxidation was completed, which oxidized $65 \%$ of the available PM, and after the passive oxidation substrate scan was taken, an additional 97 minute post loading was performed. The post loading resulted in a PM density of $2.99 \mathrm{~g} / \mathrm{L}$ at the time of the post loading substrate scan. This is $1.14 \mathrm{~g} / \mathrm{L}$ higher than the PM density at the time of the passive oxidation substrate scan. The result of the post loading substrate scan is shown in Figure 6.42. 


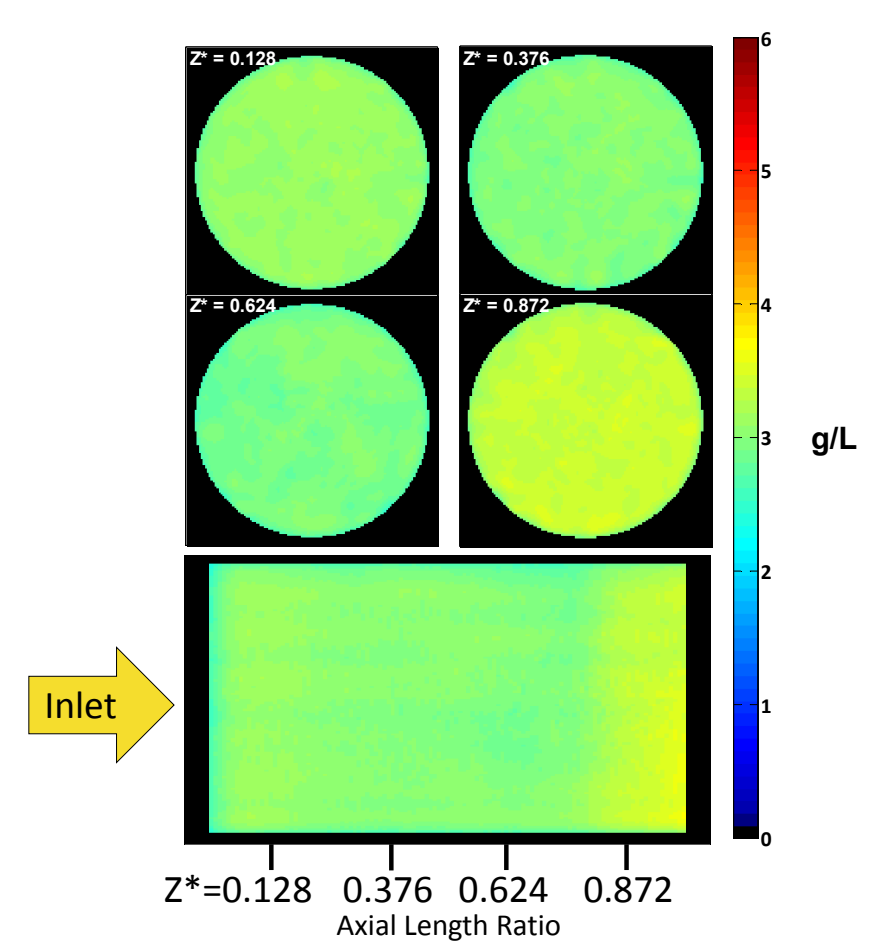

Figure 6.42: Phase 2 Test 7- Post Loading Scan Results

The overall axial UI for the post loading scan from Test 7 of Phase 2 was 0.95 . This is similar to the overall axial UI for the passive oxidation scan from Test 7 of Phase 2 and the loading scan from Test 6 of Phase 2. The axial PM distribution data are given in Table 6.58. The post loading axial PM distribution trend for Test 7 of Phase 2 is different than the post loading axial distribution for Test 4 of Phase 2. The temperature distribution plot shown in Figure I.16 shows that the temperature of the outer edges of the substrate was $25{ }^{\circ} \mathrm{C}$ lower than the centerline and there was minimal temperature variation in the axial direction for Test 7 of Phase 2, which is similar to Test 4 of Phase 2. Tests 4 and 7 had similar axial PM distributions at the completion of the oxidation (passive or active) stage of the test. Instead of the average PM density increasing in each axial segment, as it did for the post loading scan from Test 4 of Phase 2, the average PM density was nearly constant in axial segments $1-3$. The variation in the average PM density in axial segments 1-3 was within the $95 \%$ CI for the TAS7000 data, and the values are within -1 to $1 \%$ of the average PM density in the substrate. Axial segment 4 had an average PM density that was $11 \%$ greater than the average PM density in the substrate. Axial segment 4 was the only axial segment to have a non-uniform distribution. This axial PM distribution is similar to the axial PM distribution after the passive oxidation was performed. This indicates that post loading $1.14 \mathrm{~g} / \mathrm{L}$ after a passive oxidation event at EOC F, which oxidized $65 \%$ of the available PM, did not affect the axial PM distribution. 
Table 6.58: Phase 2 Test 7- Post Loading Axial PM Distribution

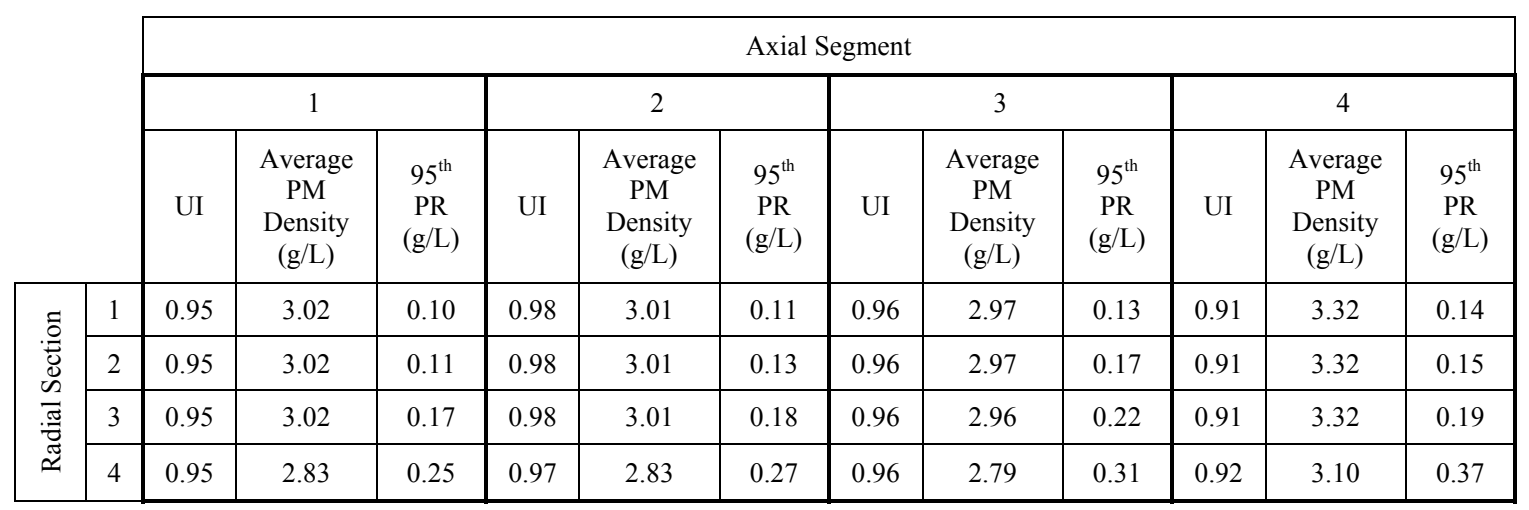

The overall radial UI for the post loading scan from Test 7 of Phase 2 was 0.97 . This is similar to the passive oxidation scan from Test 7 of Phase 2, the loading scans from Tests 1 and 6 of Phase 2, and the post loading scan from Test 4 of Phase 2. The radial PM distribution data are given in Table 6.59. All of the quadrants in each axial segment had a UI above 0.94, indicating a uniform distribution. The data in Figure 6.42 agree with this conclusion. These results indicate that the radial PM distribution is not affected by post loading $1.14 \mathrm{~g} / \mathrm{L}$ after a passive oxidation event at EOC F, which oxidized $65 \%$ of the available PM.

Table 6.59: Phase 2 Test 7- Post Loading Radial PM Distribution

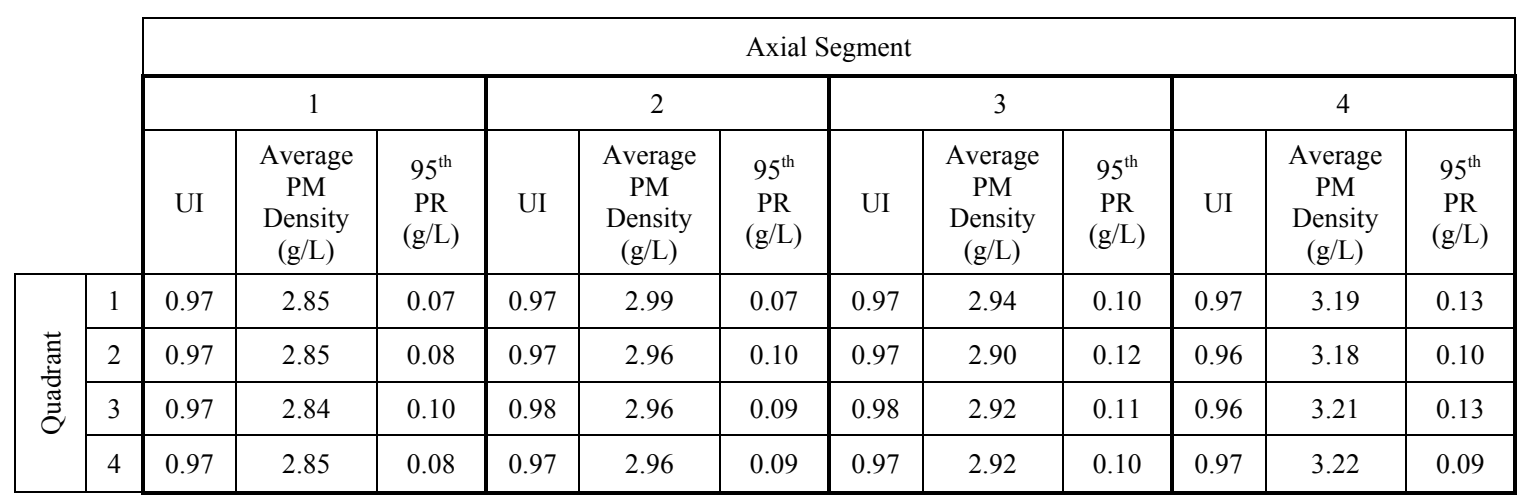

The overall angular UI for the post loading scan from Test 7 of Phase 2 was 0.98 . This is similar to the overall angular UI for the passive oxidation scan from Test 7 of Phase 2, the loading scans from Tests 1 and 6 of Phase 2, and the post loading scan from Test 4 of Phase 2. The angular PM distribution data are given in Table 6.60. Each radial section in each axial segment had a UI above 0.94, indicating a uniform angular PM distribution. The results of the angular PM distribution analysis indicate that the angular PM distribution is not affected by post loading $1.14 \mathrm{~g} / \mathrm{L}$ after a passive oxidation event at EOC F, which oxidized $65 \%$ of the available PM. 
Table 6.60: Phase 2 Test 7- Post Loading Angular PM Distribution

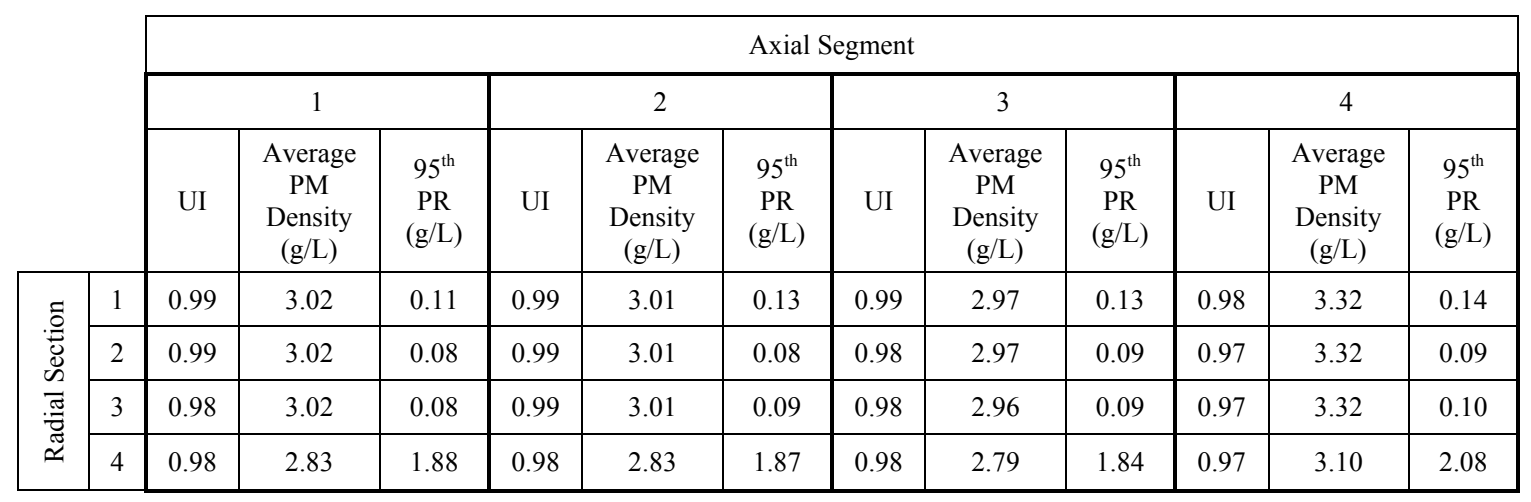

The axial PM distributions for both post loading tests, Tests 4 and 7 of Phase 2 are shown in Figure 6.43. A summary of both tests was given in Table 6.54. The results of the post loading tests indicate that the angular and radial PM distributions were not affected by post loading performed on the substrate. The post loading for Tests 4 and 7 resulted in an increase of 56 and $62 \%$, respectively, in the average PM density in the substrate. The axial PM distribution after the oxidation stage was similar for both tests. However, the axial PM distribution after the post loading portion of the tests resulted in different PM distributions. Test 7 of Phase 2 has a PM distribution similar to what was found after loading, passive oxidation, and active regeneration. Axial segments 1-3 were near the average PM density in the substrate, and axial segment 4 had a PM density that was $11 \%$ higher than the substrate average. The post loading axial PM distribution from Test 4 of Phase 2 did not match any of the previous data from Phase 2, except for Test 2 of Phase 2. In section 6.5, it was discussed that the axial PM distribution after loading generally followed the trends of the wall flow velocity distribution. Therefore, for the post loading scan from Test 4 of Phase 2 to have a different PM distribution trend, the wall flow velocity distribution would have had to change. In section 2.1 it is shown that the wall flow velocity distribution is affected by changes in the permeability of the substrate wall and PM cake, and changes in the exhaust flow rate. The exhaust flow rates were identical for the post loading portion of Tests 4 and 7 of Phase 2. This means that the permeability of the PM cake layer and substrate wall would have to change to achieve a different axial PM distribution. Therefore the permeability of the substrate and PM cake layer may be different after active regeneration events than the permeability after passive oxidation events. The overall axial UI for the post loading from Test 4 of Stage 2 was 0.84 and the overall axial UI for the post loading from Test 7 of Stage 2 was 0.95 . Both tests had a similar amount of PM loaded in the substrate after the oxidation stage, indicating that the post loading after the active regeneration test produced a more non-uniform PM distribution than the post loading after the passive oxidation test. 


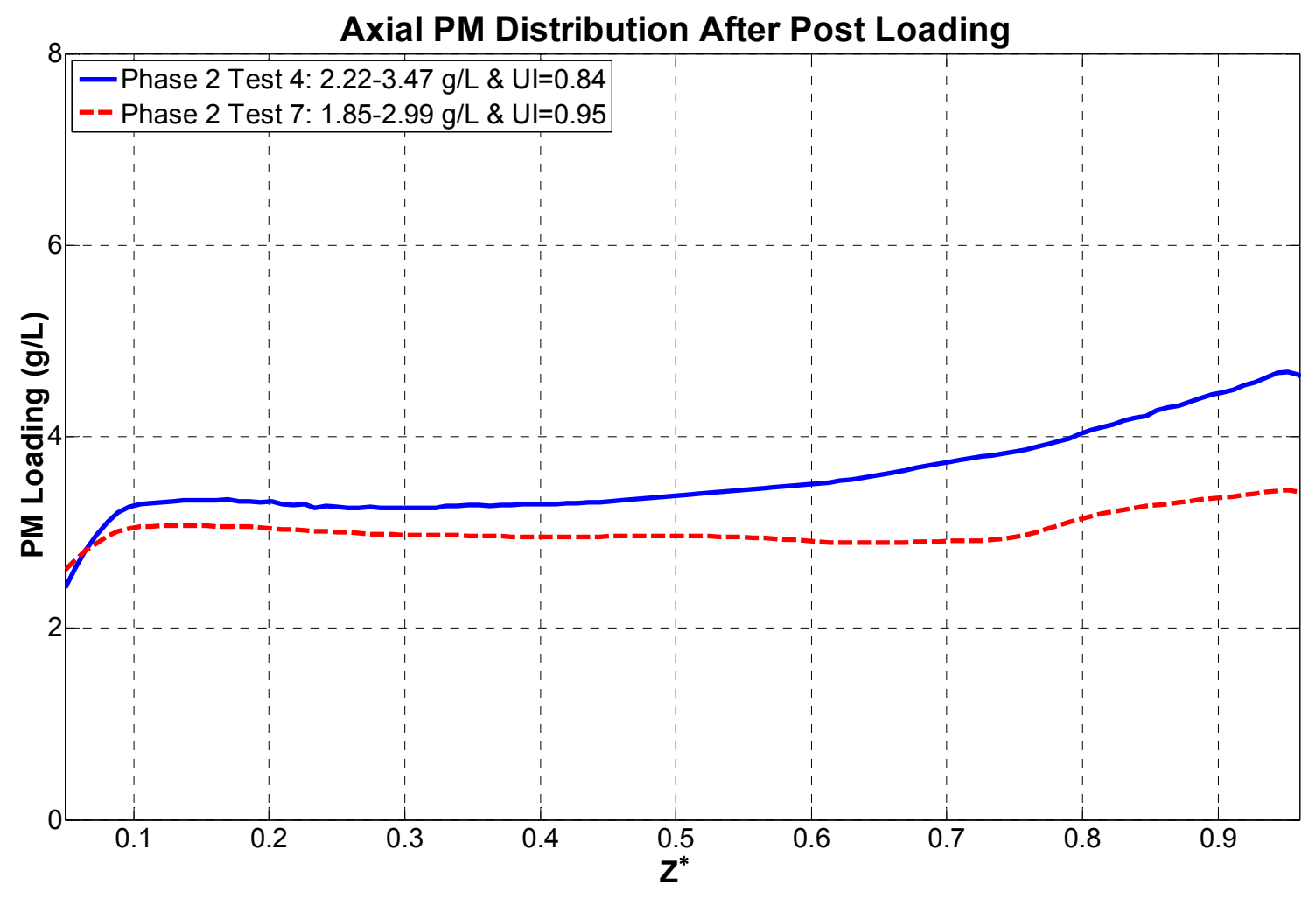

Figure 6.43: Axial PM Distribution for all Post Loading Tests

The axial PM distribution shown for the post loading from Test 4 of Phase 2 is similar to the axial PM distribution shown for Test 2 of Phase 2 in Figure 6.37. The active regeneration results in section 6.7 also show that the active regenerations performed during Phase 2 did not alter the PM distribution that was found after PM loading significantly. The passive oxidation results shown in section 6.6 indicate that partial passive oxidations oxidize more PM near the inlet of the substrate, until $65 \%$ of the available PM was oxidized. Since Test 2 of Phase 2 was exposed to multiple loading, partial active regeneration, and partial passive oxidation cycles, the PM distribution prior to the cleanout likely looked similar to the result from the post loading scan from Test 4 of Phase 2. Since active regenerations were found to not change the PM distribution significantly during Phase 2, the cleanout that was performed during Test 2 may have maintained the axial PM distribution trend. This, combined with the ash loading, is what may have caused the increased PM loading found in axial segments 3 and 4 after the cleanout. In order to prevent an increased PM density near the outlet of the substrate, which is difficult to remove and could potentially damage the substrate should a runaway regeneration start, the operation of the CPF should depend on more passive oxidations, for longer periods of time, at lower PM densities, and the ability of the cleanout strategy used with CPFs to remove all of the PM from the substrate needs to be investigated. 


\section{Chapter 7. Summary and Conclusions ${ }^{5}$}

The PM distribution trends that were measured after PM loading, passive oxidation, active regeneration, and post loading were discussed. The substrates were scanned with a TAS7000 3D Imaging Analysis System, a terahertz wave scanner, manufactured by Advantest Corporation. A total of 24 substrate scans were performed. Six substrate scans were for baseline, unloaded, data sets, four substrate scans were taken after loading conditions, five substrate scans were taken after passive oxidation conditions, seven substrate scans were taken after active regeneration conditions, and two substrate scans were taken after post loading conditions. The data collected from these scans provide a better understanding of the effect of loading, passive oxidation, active regeneration, and post loading on the PM distribution in the CPFs. These data are needed to minimize the fuel consumption and emissions associated with the $\mathrm{CPF}$, and prevent damage from a non-uniform PM distribution. The scan data is also useful in the development of accurate CPF models. This chapter presents a summary of the findings from Chapter 6. Each section in Chapter 6 will be summarized independently. Finally, the conclusions from this research are listed.

\subsection{Summary of Experiments Conducted}

\section{Analysis Method Functionality}

The results from five substrate scans, taken during Phase 1, were presented in section 6.1. The analysis method that was developed in sections 4.3-4.6 was applied to the data and the results were analyzed. The loading scan result had a PM density that was $12 \%$ higher than the substrate average density near the outlet, indicating more PM was loaded near the outlet of the substrate. The active regeneration results showed a PM density that was $26-67 \%$ lower than the substrate average, indicating more PM was oxidized near the outlet of the substrate. The passive oxidation result had a PM density near the middle of the axial length that was $28 \%$ higher than the substrate average, indicating less PM was oxidized near the middle of the substrate. The analysis method found that the axial PM distribution was non-uniform (less than 0.94) for all five test cases. The radial and angular PM distributions were non-uniform for active regeneration cases where $58 \%$ or more of the available PM was oxidized. The data analysis method showed that both a UI and a $95^{\text {th }}$ PR quantified the amount of variation in the data. The UI measures the consistency in the average PM density and the $95^{\text {th }}$ PR measures the range of the data used to calculate the average PM density. Through the use of the UI and the $95^{\text {th }} \mathrm{PR}$, the data nearest the centerline of the substrate was found to have a more uniform distribution. Lastly, the developed analysis method was found to quantify PM distribution trends that were not visible in plots of the data.

\footnotetext{
${ }^{5}$ Parts of the material contained in this chapter have been submitted, or are being considered for submission, to SAE International for publication consideration.
} 


\section{Changes in the PM Density Calculation Process}

The difference between the original TAS7000 PM density calculation process and the developed PM density calculation process (section 4.1) was found for the 9 data sets in section 6.2. The new PM density calculation process does not have the ability to distinguish between the PM and ash loading, whereas the old PM density calculation method did. Therefore, the results from the new PM density calculation method give the PM data trends for the carbonaceous and ash content. The ash loading in the parts that were used to analyze the functionality of the new PM density calculation method was below $0.3 \mathrm{~g} / \mathrm{L}$. The results show that the new PM density calculation process produced results that were 0.05 to $0.50 \mathrm{~g} / \mathrm{L}$, or 1 to $19 \%$, different than the original data processing method for each of the axial segments analyzed for the data sets from Test 1 of Phase 2. The largest differences were typically found in radial section 4 . The differences were 0.06 to $0.15 \mathrm{~g} / \mathrm{L}$, or 1 to $6 \%$, when radial section 4 was excluded. The increase in the difference in radial section 4 is likely caused by additional error that is due to how the terahertz wave enters the substrate, which may have been accounted for in the original PM density calculation process. The data sets from the shipping orientation and repeatability tests had differences between -1.11 and $0.57 \mathrm{~g} / \mathrm{L}$, or -34 to $147 \%$, throughout the substrate. The larger differences may be caused by a different number of axial sections used in the original and new PM density calculations, 64 and 63 axial sections respectively. The differences between the new and original PM density calculation methods are thought to be caused in part by the absence of a blending factor in the new data processing method. The effect of the blending factor may also be dependent on the average density of the PM in the substrate at the time of the substrate scan. The original PM density calculation method used by the TAS7000 system software applied a blending factor to the data, and it is unknown how that affected the processed scan data. The results of the new PM density calculation method are acceptable for the work being performed within this thesis.

\section{Advantest TAS7000 Repeatability}

The results of three repeatability tests were discussed in section 6.3. The repeatability tests were performed on the substrates used for the active regeneration scan from Test 4 of Phase 1, the passive oxidation scan from Test 1 of Phase 2, and the passive oxidation scan from Test 7 of Phase 2. The repeatability of the TAS7000 was quantified using a $95 \%$ CI. The $95 \%$ CIs for the three repeatability tests were $\pm 0.11,0.19,0.62 \mathrm{~g} / \mathrm{L}$, which were $6-19 \%$ of the PM loading in the substrate. The average $95 \%$ CI was $\pm 0.31 \mathrm{~g} / \mathrm{L}$. This was used as the lower limit for detecting significant changes in the PM distribution. Differences in the PM distribution that are within the $95 \%$ CI are within the measurement variability and therefore, are not significant.

\section{Effect of Substrate Shipping Orientation}

The results from the substrate shipping test were presented in section 6.4. The substrate from Test 4 of Phase 1, which was an active regeneration experiment, was shipped in three different orientations, and a scan was taken after each shipping orientation, to 
determine if the shipping orientation had an impact on the PM density distribution. The substrate was shipped in the following order: inlet pointed up, inlet pointed to the side, and inlet pointed down. The difference in the PM distribution was calculated for the inlet to the side and inlet up orientation and the inlet down and inlet to the side orientation. The results indicated that the differences between the inlet to the side and inlet up and inlet down and inlet to the side orientations were within the $95 \%$ CI of the TAS7000 data, indicating that there was no significant change in the PM distribution. The orientation of the substrate during shipping is not critical, but should remain consistent. The effect of shipping the substrate with the inlet up could not be determined from these tests, due to the order in which the test had to be conducted. It is recommended that the substrate be shipped with the inlet up or to the side to prevent any possible loss of PM.

\section{PM Distribution Trends after Loading}

There were four loading tests discussed in section 6.5. The average PM density in the substrates ranged from 2.81 to $5.18 \mathrm{~g} / \mathrm{L}$. Three different PM distribution trends were found. For a clean substrate loaded to $3.08 \mathrm{~g} / \mathrm{L}$ with the ISL engine in Phase 2, axial segments 1 and 4 had average PM densities that were $12-15 \%$ higher than the substrate average. Axial segments 2 and 3 had average PM densities that were 0-7\% lower than the substrate average. A substrate that had previous loading performed on it, and was cleaned out afterward, had a different PM distribution after loading when loaded to $2.81 \mathrm{~g} / \mathrm{L}$, with the ISL engine in Phase 2, than the clean substrate mentioned previously. Axial segments 1-3 had average PM densities within $0-2 \%$ of the substrate average. Axial segment 4 had an average PM density that was $7 \%$ higher than the substrate average. When the same substrate was loaded to $5.18 \mathrm{~g} / \mathrm{L}$ with the ISL engine in Phase 2, the trend was similar to the $2.81 \mathrm{~g} / \mathrm{L}$ loading as well as the $4.96 \mathrm{~g} / \mathrm{L}$ loading conducted as part of Phase 1 with the ISB engine. The average PM densities in axial segments 1-3 were within -1 and $3 \%$ of the average PM density in the substrate. Axial segment 4 had an average PM density that was 19\% higher than the substrate average.

The results indicate that the two different sized substrates loaded in a similar fashion and the modified engine calibration that was used with the ISB engine during Phase 1 to load the substrates did not affect the PM loading trends. There was a difference in the PM distribution that occurred in a new substrate and a previously loaded substrate, with the previously loaded substrate having a PM density in axial segment 1 that was near the average PM density in the substrate instead of being $12 \%$ higher than the average. As the PM density in the substrate increased, the axial trend remained similar, but the PM density in axial segment 4 increased from being $7 \%$ higher than the substrate average to $19 \%$ higher. In general the axial PM distribution followed the axial wall flow velocity distributions shown in section 2.1. The radial and angular PM distributions were uniform for all loading tests. In general, the PM distribution near the centerline was more uniform than the PM distribution near the edge of the substrate. Published experimental and modeling results were found to generally agree with the measured PM distributions. Lastly, as the PM density in the substrate increased, the axial UI decreased. The only uniform axial PM distribution occurred at a PM loading of $2.81 \mathrm{~g} / \mathrm{L}$. The PM densities above $3 \mathrm{~g} / \mathrm{L}$ had non-uniform distributions. This indicates that the upper limit of the PM 
density in a substrate should be no more than $3 \mathrm{~g} / \mathrm{L}$ to maintain a uniform PM distribution. The PM distributions for the four loading tests are summarized in Table 7.1. For each test, the overall UI in the axial, radial, and angular direction is given, along with comments on the PM distribution in each direction. Any percentage that is given is a comparison between the local PM density in a particular axial segment and quadrant or radial section and the average PM density in the substrate.

Table 7.1: Loading Tests PM Distribution Summary

\begin{tabular}{|c|c|c|c|}
\hline Test & Axial Distribution & Radial Distribution & Angular Distribution \\
\hline \multirow[b]{2}{*}{$\begin{array}{c}\text { Phase } 1 \text { Test } 1: \\
4.96 \mathrm{~g} / \mathrm{L}\end{array}$} & 0.89 & 0.96 & 0.96 \\
\hline & $\begin{array}{l}\text { - Axial Segment 1: 5\% Lower } \\
\text { - Axial Segment 2: 2\% Lower } \\
\text { - Axial Segment 3: } 0 \% \text { Different } \\
\text { - Axial Segment 4: 3\% Higher (Peak } \\
\text { was 12\% Higher) }\end{array}$ & $\begin{array}{l}\text { - Quadrant } 2 \text { in Axial } \\
\text { Segment } 1 \text { is Non- } \\
\text { Uniform }\end{array}$ & $\begin{array}{l}\text { - Radial Sections } 3 \text { and } 4 \text { in } \\
\text { Axial Segment } 1 \text { are Non- } \\
\text { Uniform }\end{array}$ \\
\hline \multirow[b]{2}{*}{$\begin{array}{c}\text { Phase } 2 \text { Test } 1: \\
3.08 \mathrm{~g} / \mathrm{L}\end{array}$} & 0.90 & 0.96 & 0.97 \\
\hline & $\begin{array}{l}\text { - Axial Segment 1: 12\% Higher } \\
\text { - Axial Segment 2: } 0 \% \text { Different } \\
\text { - Axial Segment 3: 7\% Lower } \\
\text { - Axial Segment 4: 15\% Higher }\end{array}$ & $\begin{array}{l}\text { - All Quadrants are } \\
\text { Uniform }\end{array}$ & $\begin{array}{l}\text { - All Radial Sections are } \\
\text { Uniform }\end{array}$ \\
\hline \multirow[b]{2}{*}{$\begin{array}{c}\text { Phase } 2 \text { Test } 6: \\
2.81 \mathrm{~g} / \mathrm{L}\end{array}$} & 0.94 & 0.97 & 0.97 \\
\hline & $\begin{array}{l}\text { - Axial Segment 1: 1\% Lower } \\
\text { - Axial Segment 2: 0\% Different } \\
\text { - Axial Segment 3: 2\% Lower } \\
\text { - Axial Segment 4: 7\% Higher }\end{array}$ & $\begin{array}{l}\text { - All Quadrants are } \\
\text { Uniform }\end{array}$ & $\begin{array}{l}\text { - All Radial Sections are } \\
\text { Uniform }\end{array}$ \\
\hline \multirow[b]{2}{*}{$\begin{array}{l}\text { Phase } 2 \text { Test } 6 \\
\text { Second } \\
\text { Loading: } 5.18 \\
\text { g/L }\end{array}$} & 0.89 & 0.95 & 0.95 \\
\hline & $\begin{array}{l}\text { - Axial Segment 1: 1\% Lower } \\
\text { - Axial Segment 2: 3\% Higher } \\
\text { - Axial Segment 3: 1\% Higher } \\
\text { - Axial Segment 4: 19\% Higher }\end{array}$ & $\begin{array}{l}\text { - Quadrants } 1 \text { and } 2 \text { in } \\
\text { Axial Segment } 4 \text { are Non- } \\
\text { Uniform }\end{array}$ & $\begin{array}{l}\text { - Radial Sections } 2 \text { and } 3 \text { in } \\
\text { Axial Segment } 4 \text { are Non- } \\
\text { Uniform }\end{array}$ \\
\hline
\end{tabular}

\section{PM Distribution Trends after Passive Oxidation}

Five passive oxidation tests were discussed in section 6.6. During Phase 2, which used the ISL engine, it was found that when the substrate had a PM density near $3 \mathrm{~g} / \mathrm{L}$ prior to the passive oxidation being performed, and the passive oxidation oxidized $22-28 \%$ of the available PM, more PM was oxidized near the inlet of the substrate. When $22 \%$ of the available PM was oxidized, axial segment 1 was $4 \%$ higher than the substrate average. For comparison, axial segment 1 was $12 \%$ higher than the substrate average after loading. Axial segment 4 went from having an average PM density that was $15 \%$ higher than the substrate average after loading to having a PM density that was $22 \%$ higher than the substrate average after passive oxidation. Axial segments 2 and 3 had similar trends after loading and after passive oxidation. When $28 \%$ of the available PM was oxidized, axial segment 1 had an average PM density that was 7\% lower than the substrate average. At the completion of loading, the average PM density in axial segment 1 should have been within $2 \%$ of the average PM density, based on the loading results. Axial segment 4 should have had a PM density that was $7 \%$ higher than the substrate average after loading, based on previous results. After passive oxidation, axial segment 4 had an 
average PM density that was $17 \%$ higher than the substrate average. During Phase 2, which used the ISL engine, it was found that when $6 \%$ or $65 \%$ of the available PM was oxidized, and the PM density in the substrate was around $3 \mathrm{~g} / \mathrm{L}$ prior to the oxidation taking place, the axial PM distribution was similar to the axial PM distribution after loading. The engine condition that was used for the test that resulted in $6 \%$ of the PM being oxidized had space velocity that was $41 \%$ lower than the engine condition that resulted in $65 \%$ of the PM being oxidized, indicating that the space velocity did not have an effect on the PM distribution trends after passive oxidation for this test. During Phase 1 , which used the ISB engine, it was found that when $45 \%$ of the available PM was oxidized, and the PM density in the substrate was near $6 \mathrm{~g} / \mathrm{L}$ prior to the oxidation, more PM was oxidized near the inlet and outlet of the substrate, with a PM density in axial segment 2 that was $17 \%$ higher than the substrate average. This distribution was not similar to any other passive oxidation distributions.

The results of the passive oxidation tests indicate that when the PM density in the substrate is near $3 \mathrm{~g} / \mathrm{L}$ prior to the passive oxidation, as it was during the Phase 2 experiments with the ISL engine, the axial PM distribution after passive oxidation followed trends similar to those found after loading. When $22-28 \%$ of the PM is oxidized, more PM was found to be oxidized near the inlet of the substrate. When $6 \%$ or $65 \%$ of the PM was oxidized, the axial distribution after passive oxidation was found unchanged from the axial distribution after loading. When the PM density in the substrate is near $6 \mathrm{~g} / \mathrm{L}$ prior to the passive oxidation, as it was during Phase 1 with the ISB engine, the axial PM distribution did not match the trends that were found after PM loading, which is a different result than the Phase 2 tests. The difference could be caused the modified engine condition used during PM loading to achieve $6 \mathrm{~g} / \mathrm{L}$ during Phase 1 , the increased PM density prior to the passive oxidation, a difference in the space velocity for the passive oxidation condition, or PM loading that occurred in the substrate during stage 3 for the $3 \mathrm{~g} / \mathrm{L}$ tests during Phase 2. The radial and angular PM distributions were uniform for all passive oxidation tests. In general, the PM distribution near the centerline of the substrate was more uniform. The uniformity of the axial PM distribution was found to be affected by the amount of PM oxidized, and not the PM density in the substrate prior to the passive oxidation being performed. When the amount of PM oxidized during the passive oxidation was $6 \%$ or $65 \%$, the axial PM distribution was uniform. The uniformity of the axial PM distribution was the lowest with a UI of 0.89 when $22 \%$ of the PM was passively oxidized. The axial UI increased to 0.91 when 28 $45 \%$ of the available PM was passively oxidized. These data indicate that passive oxidations need to be performed until more than $45 \%$ of the available PM is oxidized in order to prevent non-uniform PM distributions. Additionally, these data indicate that the PM density in the substrate prior to the passive oxidation does not have an impact on the uniformity of the PM distribution after oxidation. The only possible impact from the PM density prior to the passive oxidation is the shape axial distribution. The PM distributions for the five passive oxidation tests are summarized in Table 7.2. For each test, the overall UI in the axial, radial, and angular direction is given, along with comments on the PM distribution in each direction. Any percentage that is given is a 
comparison between the local PM density in a particular axial segment and quadrant or radial section and the average PM density in the substrate.

Table 7.2: Passive Oxidation Tests PM Distribution Summary

\begin{tabular}{|c|c|c|c|}
\hline Test & Axial Distribution & Radial Distribution & Angular Distribution \\
\hline \multirow[b]{2}{*}{$\begin{array}{l}\text { Phase } 1 \text { Test } 3: \\
45 \% \text { Oxidized } \\
\text { @ } 372{ }^{\circ} \mathrm{C}\end{array}$} & 0.91 & 0.96 & 0.94 \\
\hline & $\begin{array}{l}\text { - Axial Segment 1: } 2 \% \text { Higher } \\
\text { - Axial Segment 2: } 17 \% \text { Higher (Peak was } \\
\text { 28\% Higher) } \\
\text { - Axial Segment 3: } 0 \% \text { Different } \\
\text { - Axial Segment 4: } 4 \% \text { Lower }\end{array}$ & $\begin{array}{l}\text { - All Quadrants are } \\
\text { Uniform }\end{array}$ & $\begin{array}{l}\text { - Radial Sections 2-4 in Axial } \\
\text { Segment } 1 \text { and 3-4 in Axial } \\
\text { Segment } 4 \text { are Non-Uniform }\end{array}$ \\
\hline \multirow[b]{2}{*}{$\begin{array}{l}\text { Phase } 2 \text { Test } 1: \\
22 \% \text { Oxidized } \\
\text { (a) } 349{ }^{\circ} \mathrm{C}\end{array}$} & 0.89 & 0.97 & 0.98 \\
\hline & $\begin{array}{l}\text { - Axial Segment 1: } 4 \% \text { Higher } \\
\text { - Axial Segment 2: 2\% Lower } \\
\text { - Axial Segment 3: } 4 \% \text { Lower } \\
\text { - Axial Segment 4: } 22 \% \text { Higher }\end{array}$ & $\begin{array}{l}\text { - All Quadrants are } \\
\text { Uniform }\end{array}$ & $\begin{array}{l}\text { - All Radial Sections are } \\
\text { Uniform }\end{array}$ \\
\hline \multirow[b]{2}{*}{$\begin{array}{l}\text { Phase } 2 \text { Test } 7: \\
65 \% \text { Oxidized } \\
\text { @ } 353{ }^{\circ} \mathrm{C}\end{array}$} & 0.96 & 0.98 & 0.99 \\
\hline & $\begin{array}{l}\text { - Axial Segment 1: 2\% Lower } \\
\text { - Axial Segment 2: 0\% Different } \\
\text { - Axial Segment 3: 3\% Lower } \\
\text { - Axial Segment 4: } 11 \% \text { Higher }\end{array}$ & $\begin{array}{l}\text { - All Quadrants are } \\
\text { Uniform }\end{array}$ & $\begin{array}{l}\text { - All Radial Sections are } \\
\text { Uniform }\end{array}$ \\
\hline \multirow[b]{2}{*}{$\begin{array}{l}\text { Phase } 2 \text { Test } 8: \\
28 \% \text { Oxidized } \\
\text { @ } 348^{\circ} \mathrm{C}\end{array}$} & 0.91 & 0.97 & 0.98 \\
\hline & $\begin{array}{l}\text { - Axial Segment 1: 7\% Lower } \\
\text { - Axial Segment 2: 2\% Lower } \\
\text { - Axial Segment 3: 2\% Lower } \\
\text { - Axial Segment 4: } 17 \% \text { Higher }\end{array}$ & $\begin{array}{l}\text { - All Quadrants are } \\
\text { Uniform }\end{array}$ & $\begin{array}{l}\text { - All Radial Sections are } \\
\text { Uniform }\end{array}$ \\
\hline \multirow[b]{2}{*}{$\begin{array}{l}\text { Phase } 2 \text { Test } 9 \text { : } \\
6 \% \text { Oxidized @ } \\
256{ }^{\circ} \mathrm{C}\end{array}$} & 0.95 & 0.97 & 0.98 \\
\hline & $\begin{array}{l}\text { - Axial Segment 1: 1\% Higher } \\
\text { - Axial Segment 2: 1\% Higher } \\
\text { - Axial Segment 3: 0\% Different } \\
\text { - Axial Segment 4: } 9 \% \text { Higher }\end{array}$ & $\begin{array}{l}\text { - All Quadrants are } \\
\text { Uniform }\end{array}$ & $\begin{array}{l}\text { - All Radial Sections are } \\
\text { Uniform }\end{array}$ \\
\hline
\end{tabular}

\section{PM Distribution Trends after Active Regeneration}

Seven active regeneration tests were discussed in section 6.7. The results from Phase 2, which used the ISL engine, indicate that active regenerations that were performed when the PM density in the substrate prior to the active regeneration was 3-4 g/L had an axial PM distribution similar to the axial PM distribution after loading. Axial segments 1-3 had average PM densities that were within -5 to $1 \%$ of the average PM density in the substrate. Axial segment 4 had average PM densities that were 5-7\% greater than the substrate average. The results from Phase 1, which used the ISB engine, indicate that active regenerations that were performed when the PM density in the substrate prior to the active regeneration was $5 \mathrm{~g} / \mathrm{L}$ had an axial PM distribution that did not match the other axial PM distributions found for loading or active regenerations. Axial segments 1 and 2 had average PM densities that were $20-50 \%$ higher than the substrate average and axial segments 3 and 4 had average PM densities 10-66\% lower than the substrate average. A scan taken after a substrate cleanout was performed during Phase 2 had an axial PM distribution that was similar to the other Phase 2 experiments. Axial segments 1-3 had average PM densities that were -9 to $3 \%$ different than the substrate average and 
axial segment 4 had an average PM density that was 14\% higher than the substrate average. The data indicate that multiple loading, partial passive oxidation, and partial active regeneration cycles did not significantly change the axial PM distribution that existed after an active regeneration. The cleanout, where the pressure drop across the CPF became constant, did not remove all of the PM though, and a region with a higher PM density that is near the outlet of the substrate likely contains ash.

The active regeneration results indicate that active regenerations that are performed when the PM density in the substrate is 3-4 g/L, as it was during Phase 2 with the ISL engine, result in axial PM distribution that are similar to the axial PM distribution after loading. If the active regeneration occurred after the PM density in the substrate is near $5 \mathrm{~g} / \mathrm{L}$, as it was during Phase 1 with the ISB engine, more PM was oxidized near the outlet of the substrate. This difference could be caused by the modified engine calibration used to load the substrates to $5 \mathrm{~g} / \mathrm{L}$ during the Phase 1 experiments with the ISB engine, the use of stage 3 to stabilize the CPF temperature prior to weighing the CPF which loaded 6.7 grams of PM during Phase 2 with the ISL engine, or the oxygen concentration in the exhaust. The average density of the PM in the substrate prior to the active regeneration may have had an effect as well. When the PM density in the substrate prior to the active regeneration was $5 \mathrm{~g} / \mathrm{L}$ and $41-69 \%$ of the PM is actively regenerated, non-uniform PM distribution resulted. When $81 \%$ of the PM was actively regenerated, as it was during Test 2 of Phase 2 with the ISL engine, a uniform PM distribution was found. The temperature gradients in the CPFs used for the Phase 1 and Phase 2 tests were similar. The space velocity for the $5 \mathrm{~g} / \mathrm{L}$ tests from Phase 1 with the ISB engine tests was similar to the space velocity for the 3-4 g/L tests from Phase 2 with the ISL engine. Previously published PM distribution data for an active regeneration that resulted in $83 \%$ of the PM being oxidized had a similar axial distribution trend as the axial trend found during this study where $81 \%$ of the PM was actively regenerated. The previously published work also shows that the active regenerations did not change the axial PM distribution significantly, which agrees with this data collected during this study. In general though, the axial PM distribution in that study did not match the distribution trends found in this work. Performing multiple loading, partial passive oxidation and partial active regeneration cycles on a substrate did not significantly impact the axial PM distribution trend after an active regeneration. The substrate was not completely clean after a cleanout where the pressure drop across the substrate became constant.

The uniformity of the axial, radial, and angular PM distributions was affected by the PM density in the substrate prior to the active regeneration being performed and the amount of PM that was oxidized during the active regeneration. The PM distribution near the centerline of the substrate was still more consistent than the PM distribution near the edge of the substrate though. When the amount of PM loaded in the substrate prior to the active regeneration was $5 \mathrm{~g} / \mathrm{L}$, and $41-69 \%$ of the PM was oxidized, as it was during Phase 1 with the ISB engine, the axial PM distribution was non-uniform. The uniformity of the axial distribution decreased as the amount of PM that was oxidized during the active regeneration increased, until $81 \%$ of the PM was oxidized, as it was during Test 2 of Phase 2. When the PM density in the substrate prior to the active regeneration was 3-4 g/L, as it was during Phase 2 with the ISL engine, the axial PM distribution was 
uniform and remained uniform as the amount of PM that was oxidized during the active regeneration increased. The radial and angular PM distributions were uniform when the PM density in the substrate prior to the active regeneration was 3-4 g/L, as it was with during Phase 2 with the ISL engine, and the uniformity slightly increased as the amount of PM that was oxidized increased. When the PM density in the substrate prior to the active regeneration was $5 \mathrm{~g} / \mathrm{L}$, as it was during Phase 1 with the ISB engine, the radial and angular PM distributions were only uniform when the amount of PM oxidized was $41 \%$ or $81 \%$. As the amount of PM that was oxidized increased, the uniformity of the radial and angular PM distribution decreased, until more than $69 \%$ of the PM was oxidized. This trend indicates that active regenerations should be performed before the PM density in the substrate is above $4 \mathrm{~g} / \mathrm{L}$ or until more than $81 \%$ of the PM is oxidized, to avoid non-uniform PM distributions. These data are for two different engines, the ISB for Phase 1 and the ISL for Phase 2, and the ISB engine calibration was modified during the PM loading, which could have an impact on the PM distribution after active regeneration. Additionally, when a substrate has been used for 70 hours, a higher PM density was found near the outlet of the substrate after an active regeneration that oxidized $81 \%$ of the PM was performed. The higher PM density consists mainly of ash. The PM distributions for the seven active regeneration tests are summarized in Table 7.3. For each test, the overall UI in the axial, radial, and angular direction is given, along with comments on the PM distribution in each direction. Any percentage that is given is a comparison between the local PM density in a particular axial segment and quadrant or radial section and the average PM density in the substrate. 
Table 7.3: Active Regeneration Tests PM Distribution Summary

\begin{tabular}{|c|c|c|c|}
\hline Test & Axial Distribution & Radial Distribution & Angular Distribution \\
\hline \multirow[b]{2}{*}{$\begin{array}{l}\text { Phase } 1 \text { Test 1: } \\
58 \% \text { Oxidized } \\
\text { @ } 497{ }^{\circ} \mathrm{C}\end{array}$} & 0.84 & 0.93 & 0.91 \\
\hline & $\begin{array}{l}\text { - Axial Segment 1: } 34 \% \text { Higher } \\
\text { - Axial Segment 2: 19\% Higher } \\
\text { - Axial Segment 3: 12\% Lower } \\
\text { - Axial Segment 4: 53\% Lower (Peak was } \\
\text { 55\% Lower) }\end{array}$ & $\begin{array}{l}\text { - Quadrant } 3 \text { in Axial } \\
\text { Segments } 3 \text { and } 4 \text { are the } \\
\text { only Uniform Quadrants }\end{array}$ & $\begin{array}{l}\text { - Radial Section } 1 \text { is the } \\
\text { only Uniform Radial } \\
\text { Section }\end{array}$ \\
\hline \multirow[b]{2}{*}{$\begin{array}{l}\text { Phase } 1 \text { Test } 2 \text { : } \\
41 \% \text { Oxidized } \\
\text { @ } 491{ }^{\circ} \mathrm{C}\end{array}$} & 0.85 & 0.96 & 0.94 \\
\hline & $\begin{array}{l}\text { - Axial Segment 1: } 14 \% \text { Higher } \\
\text { - Axial Segment 2: 26\% Higher } \\
\text { - Axial Segment 3: 10\% Lower } \\
\text { - Axial Segment 4: 31\% Lower }\end{array}$ & $\begin{array}{l}\text { - Quadrant } 2 \text { in Axial } \\
\text { Segment } 1 \text { is Non- } \\
\text { Uniform }\end{array}$ & $\begin{array}{l}\text { - Radial Sections 2-4 in } \\
\text { Axial Segment } 1 \text { and 3-4 } \\
\text { in Axial Segment } 4 \text { are } \\
\text { Non-Uniform }\end{array}$ \\
\hline \multirow[b]{2}{*}{$\begin{array}{l}\text { Phase } 1 \text { Test } 4: \\
69 \% \text { Oxidized } \\
\text { @ } 577^{\circ} \mathrm{C}\end{array}$} & 0.80 & 0.91 & 0.89 \\
\hline & $\begin{array}{l}\text { - Axial Segment 1: } 62 \% \text { Higher } \\
\text { - Axial Segment 2: 32\% Higher } \\
\text { - Axial Segment 3: 66\% Lower } \\
\text { - Axial Segment 4: 35\% Lower }\end{array}$ & $\begin{array}{l}\text { - Quadrants in Axial } \\
\text { Segment } 3 \text { are the only } \\
\text { Uniform Quadrants }\end{array}$ & $\begin{array}{l}\text { - Radial Sections } 3 \text { and } 4 \\
\text { are Non-Uniform in Every } \\
\text { Axial Segment } \\
\text { - Radial Section } 2 \text { is } \\
\text { Uniform in Axial } \\
\text { Segment } 3 \text { Only }\end{array}$ \\
\hline \multirow[b]{2}{*}{$\begin{array}{l}\text { Phase } 2 \text { Test } 2: \\
81 \% \text { Oxidized } \\
\text { @ } 611{ }^{\circ} \mathrm{C}\end{array}$} & 0.97 & 0.99 & 0.98 \\
\hline & $\begin{array}{l}\text { - Axial Segment 1: 9\% Lower } \\
\text { - Axial Segment 2: 6\% Lower } \\
\text { - Axial Segment 3: 3\% Higher } \\
\text { - Axial Segment 4: 14\% Higher }\end{array}$ & $\begin{array}{l}\text { - All Quadrants are } \\
\text { Uniform }\end{array}$ & $\begin{array}{l}\text { - All Radial Sections are } \\
\text { Uniform }\end{array}$ \\
\hline \multirow[b]{2}{*}{$\begin{array}{l}\text { Phase } 2 \text { Test } 3 \text { : } \\
26 \% \text { Oxidized } \\
\text { @ } 519^{\circ} \mathrm{C}\end{array}$} & 0.96 & 0.97 & 0.97 \\
\hline & $\begin{array}{l}\text { - Axial Segment 1: 1\% Higher } \\
\text { - Axial Segment 2: 2\% Higher } \\
\text { - Axial Segment 3: } 2 \% \text { Lower } \\
\text { - Axial Segment 4: 5\% Higher }\end{array}$ & $\begin{array}{l}\text { - All Quadrants are } \\
\text { Uniform }\end{array}$ & $\begin{array}{l}\text { - All Radial Sections are } \\
\text { Uniform }\end{array}$ \\
\hline \multirow[b]{2}{*}{$\begin{array}{l}\text { Phase } 2 \text { Test } 4: \\
45 \% \text { Oxidized } \\
\text { @ } 526^{\circ} \mathrm{C}\end{array}$} & 0.97 & 0.98 & 0.98 \\
\hline & $\begin{array}{l}\text { - Axial Segment 1: } 1 \% \text { Higher } \\
\text { - Axial Segment 2: 1\% Higher } \\
\text { - Axial Segment 3: 3\% Lower } \\
\text { - Axial Segment 4: 5\% Higher }\end{array}$ & $\begin{array}{l}\text { - All Quadrants are } \\
\text { Uniform }\end{array}$ & $\begin{array}{l}\text { - All Radial Sections are } \\
\text { Uniform }\end{array}$ \\
\hline \multirow[b]{2}{*}{$\begin{array}{l}\text { Phase } 2 \text { Test } 5 \text { : } \\
52 \% \text { Oxidized } \\
\text { @ } 574{ }^{\circ} \mathrm{C}\end{array}$} & 0.96 & 0.98 & 0.98 \\
\hline & $\begin{array}{l}\text { - Axial Segment 1: } 0 \% \text { Different } \\
\text { - Axial Segment 2: 1\% Higher } \\
\text { - Axial Segment 3: 3\% Lower } \\
\text { - Axial Segment 4: 7\% Higher }\end{array}$ & $\begin{array}{l}\text { - All Quadrants are } \\
\text { Uniform }\end{array}$ & $\begin{array}{l}\text { - All Radial Sections are } \\
\text { Uniform }\end{array}$ \\
\hline
\end{tabular}

\section{PM Distribution Trends after Post Loading}

Two post loading tests were discussed in section 6.8, both of which were from Phase 2 and used the ISL engine. One was completed after an active regeneration that oxidized $45 \%$ of the available PM and the other was completed after a passive oxidation that oxidized $65 \%$ of the available PM. The post loading tests increased the PM density in the substrate by 56 and $62 \%$, from $2.22 \mathrm{~g} / \mathrm{L}$ to $3.47 \mathrm{~g} / \mathrm{L}$ (a difference of $1.25 \mathrm{~g} / \mathrm{L}$ ) and $1.85 \mathrm{~g} / \mathrm{L}$ to $2.99 \mathrm{~g} / \mathrm{L}$ (a difference of $1.14 \mathrm{~g} / \mathrm{L}$ ), respectively. The post loading after the active regeneration caused the PM density in each axial segment to increase and the axial PM distribution was different than the axial PM distribution found after active 
regeneration or loading tests. Axial segments 1-3 had average PM densities within -7 to $5 \%$ of the substrate average. Axial segment 4 had a PM density that was $26 \%$ higher than the substrate average. This distribution trend caused the axial UI to decrease from 0.97 after the active regeneration, to 0.84 after the post loading. The post loading after the passive oxidation test had an axial PM distribution that was similar to the axial PM distribution found after passive oxidation and loading tests. Axial segments 1-3 had average PM densities within -1 to $1 \%$ of the substrate average, and axial segment 4 had an average PM density that was $11 \%$ higher than the substrate average. This distribution trend caused the axial UI to remain nearly constant. After the passive oxidation, the axial UI was 0.96 and after the post loading the axial UI was 0.95 .

These results indicate that PM loading that takes place after an active regeneration produces a different axial PM distribution trend than PM loading that takes place after passive oxidations. Since the PM loading distribution was found to generally follow the axial wall flow velocity distribution, the different loading distribution would be caused by a different axial wall flow velocity distribution for the active regeneration post loading. The exhaust gas velocities were identical for both post loading tests, so the permeability of the substrate and PM cake layer would have to change in order for the wall flow velocity distribution to change for the active regeneration post loading. This indicates that the permeability of the substrate wall and PM cake may be different after active regenerations than the permeability after PM loading and passive oxidations. The axial PM distribution was uniform, and nearly constant, for the post loading after the passive oxidation. The axial PM distribution became non-uniform (a decrease from 0.970.84 ) after the post loading after the active regeneration. This indicates that post loading that is performed after partial active regenerations can cause non-uniform axial PM distribution. The radial and angular PM distributions were uniform for both post loading tests. In general, the PM distribution near the centerline was more uniform than the PM distribution near the edge of the substrate. The PM distributions for the two post loading tests are summarized in Table 7.4. For each test, the overall UI in the axial, radial, and angular direction is given, along with comments on the PM distribution in each direction. Any percentage that is given is a comparison between the local PM density in a particular axial segment and quadrant or radial section and the average PM density in the substrate.

Table 7.4: Post Loading Tests PM Distribution Summary

\begin{tabular}{|c|c|c|c|}
\hline Test & Axial Distribution & Radial Distribution & Angular Distribution \\
\hline \multirow[b]{2}{*}{$\begin{array}{l}\text { Phase } 2 \text { Test } 4 \text { : } \\
2.22 \text { to } 3.47 \mathrm{~g} / \mathrm{L} \\
\text { Increase of } 1.25 \\
\mathrm{~g} / \mathrm{L}\end{array}$} & 0.84 & 0.95 & 0.95 \\
\hline & $\begin{array}{l}\text { - Axial Segment 1: } 7 \% \text { Lower } \\
\text { - Axial Segment 2: 3\% Lower } \\
\text { - Axial Segment 3: 5\% Higher } \\
\text { - Axial Segment 4: 26\% Higher }\end{array}$ & $\begin{array}{l}\text { - Non-Uniform Distributions in: } \\
\circ \text { Quadrant } 2 \text { in Axial Segment } 1 \\
\circ \text { Quadrant } 2 \text { in Axial Segment } 3 \\
\circ \text { Quadrants 1, 2, and } 4 \text { in Axial } \\
\text { Segment } 4 \\
\end{array}$ & $\begin{array}{l}\text { - Radial Sections } 3 \text { and } 4 \\
\text { had Non-Uniform } \\
\text { Distributions in Axial } \\
\text { Segments } 1 \text { and } 4\end{array}$ \\
\hline \multirow[b]{2}{*}{$\begin{array}{l}\text { Phase } 2 \text { Test } 7: \\
1.85 \text { to } 2.99 \mathrm{~g} / \mathrm{L} \\
\text { Increase of } 1.14 \\
\mathrm{~g} / \mathrm{L}\end{array}$} & 0.95 & 0.97 & 0.98 \\
\hline & $\begin{array}{l}\text { - Axial Segment 1: } 1 \% \text { Higher } \\
\text { - Axial Segment 2: } 1 \% \text { Higher } \\
\text { - Axial Segment 3: } 1 \% \text { Lower } \\
\text { - Axial Segment 4: } 11 \% \text { Higher }\end{array}$ & - All Quadrats are Uniform & $\begin{array}{l}\text { - All Radial Sections are } \\
\text { Uniform }\end{array}$ \\
\hline
\end{tabular}


The axial PM distribution that was found after the post loading that was performed after the active regeneration test (Test 4 of Phase 2) had a trend that was similar to the axial PM distribution found for the substrate exposed to multiple loading, partial passive oxidation, and partial active regeneration cycles. Active regenerations performed on substrates with PM densities between 3 and $4 \mathrm{~g} / \mathrm{L}$ were found to not alter the axial PM distribution, during Phase 2. The axial trend found after a substrate cleanout that oxidized $81 \%$ of the PM, which was performed on the substrate used for multiple loading, partial passive oxidation, and partial active regeneration cycles, may be caused by the axial PM distribution trend that was found after post loading on a partial active regeneration. Due to this, the cleanout strategy used with CPFs may have to be modified to ensure that the substrate is clean at the completion of the cleanout.

\subsection{Conclusions}

The main conclusions of this work, which are based on the goals stated in Chapter 1 and the results presented in Chapter 6, are as follows.

\section{Methodology for Data Collection and Analysis}

- The experimental methods that are required to perform PM distribution experiments have been developed. These include the procedures for weighing, scanning, and canning the substrates, along with the procedures used to conduct the experiments.

- The methodology was developed to:

- Calculate the PM density in the substrate using the TAS7000 scan data.

- Convert the data from units of grams per liter to units of grams per unit surface area.

- Analyze the PM distribution trends in the TAS7000 data in multiple directions.

- Analyze the integrity of the data from the TAS7000 and detect for shifting or other noise in the data sets.

- The TAS7000 was able to measure the PM distribution trends for two different size substrates, with average PM densities between 1 and $6 \mathrm{~g} / \mathrm{L}$. Differences in the PM density in the substrate were identifiable.

- A method that allowed for the analysis of the PM distribution trends in the axial, radial, and angular direction was developed. The method used the average PM density, a $95^{\text {th }}$ PR for the average PM density, and a UI to quantify the PM distribution. The method was shown to provide data trends that could not be deduced from observing the plots of the data. 
- A UI value of 0.94 or higher indicates that the distribution is uniform.

- A method to calculate the PM density in the substrate based on the raw data from the TAS7000 was developed. The method was validated against the original method used by the TAS7000 to calculate the PM density in the substrate. The new method produced PM density values that were on average within 0.05 to $0.50 \mathrm{~g} / \mathrm{L}$, or 4 to $19 \%$ of the average PM density at the time of the scan when the number of axial sections used for the comparison was the same for both PM density calculation methods. When the number of axial sections was different for the two methods, the differences were to -1.11 to $0.57 \mathrm{~g} / \mathrm{L}$, or -33 to $147 \%$. The differences are thought to be caused by a blending factor that was used in the original PM density calculation process.

- The $95 \%$ CI of the TAS7000 data was found to be $\pm 0.31 \mathrm{~g} / \mathrm{L}$.

- The shipping orientation of the substrate does not matter. The variations in the PM distribution were less than the $95 \%$ CI of the TAS 7000 data for three shipping orientations.

\section{Distribution Trends after PM Loading}

- The axial PM distribution trends after loading were found to follow the predicted wall flow velocity distribution profiles and are non-uniform for substrate average PM densities above $3 \mathrm{~g} / \mathrm{L}$. The PM density near the outlet of the substrate was 7$19 \%$ higher than the average PM density in the substrate. A clean substrate has a different axial PM distribution than a previously loaded substrate.

- The radial and angular PM distributions are uniform after loading, and the PM distribution was more uniform near the centerline of the substrate.

- The two different size substrates had a similar axial PM distribution trend after loading.

- The modified engine calibration that was used during Phase 1 had a similar axial PM distribution as the standard engine calibration that was used during Phase 2.

\section{Distribution Trends after Passive Oxidation}

- When passive oxidations occurred at PM densities near $3 \mathrm{~g} / \mathrm{L}$, the axial PM distribution was similar to the loading axial PM distribution. When $22-28 \%$ of the available PM was oxidized, for the experiments conducted, more PM was oxidized near the inlet of the substrate and the axial PM distribution was nonuniform. For one test the PM density near the inlet of the substrate was $12 \%$ higher than the substrate average after loading, and $4 \%$ higher than the substrate average after passive oxidation. The other regions of the substrate had a similar PM distribution trend before and after oxidation. 
- When passive oxidations occurred at PM densities near $3 \mathrm{~g} / \mathrm{L}$, and when 6 or $65 \%$ of the available PM was oxidized, the axial PM distribution was uniform and followed the trends found after loading.

- As the mass of PM that was passively oxidized increased above $22 \%$, for the experiments conducted, the uniformity of the axial distribution increased.

- For the experiment conducted where a passive oxidation occurred at a PM density near $6 \mathrm{~g} / \mathrm{L}$ and $45 \%$ of the available PM was oxidized, more PM was oxidized near the inlet and outlet of the substrate, with less PM being oxidized near the middle of the substrate. The PM density in axial segment 2 was $17 \%$ higher than the substrate average. This was a non-uniform axial PM distribution.

- The radial and angular PM distributions were uniform for all passive oxidation experiments.

- The differences in the axial PM distribution trends for the two different average PM density test cases could be caused by the higher loading, the modified engine calibration used to load the substrate, a different space velocity, or additional PM loading that occurred for the $3 \mathrm{~g} / \mathrm{L}$ passive oxidation cases from Phase 2 that could change the axial PM distribution trend.

\section{Distribution Trends after Active Regeneration}

- When active regenerations occurred at PM densities near 3-4 g/L, the axial, radial, and angular PM distributions were uniform, regardless of the mass of PM oxidized or the temperature of the active regeneration. The axial PM distribution trend after active regeneration was similar to the axial PM distribution trend after loading.

- When active regenerations occurred at PM densities near $5 \mathrm{~g} / \mathrm{L}$, the axial PM distribution was non-uniform until $81 \%$ of the PM was oxidized. The uniformity decreased as the mass of PM oxidized increased, until after $69 \%$ of the PM was oxidized. The axial PM distribution trend showed more PM being oxidized near the outlet of the substrate when 41-69\% of the PM was oxidized, which does not match the axial PM distribution trends found after loading or the other active regeneration experiments performed. The PM density near the outlet of the substrate was $21-50 \%$ lower than the substrate average when $41-69 \%$ of the PM was oxidized.

- For the experiments conducted where active regenerations occurred at PM densities near $5 \mathrm{~g} / \mathrm{L}$, the radial and angular PM distributions were uniform when 41 or $81 \%$ of the available PM was oxidized, but non-uniform between those values. 
- The differences in the axial PM distribution trends for the two different average PM density test cases could be caused by the modified engine calibration used to load the substrate, the PM loading that occurred during stage 3 from the 3-4 g/L active regeneration experiments from Phase 2, differences in the oxygen concentration in the exhaust.

- The PM density in the substrate prior to the active regeneration could also affect the axial PM distribution trend when $41-69 \%$ of the PM is oxidized.

- When a substrate experiences multiple PM loading, partial active regeneration, and partial passive oxidation cycles, the substrate cleanout used did not remove all of the PM, and the resulting axial PM distribution trend is not significantly different than the trends found for other active regenerations.

\section{Distribution Trends after Post Loading}

- The post loading that occurred after an active regeneration where $45 \%$ of the available PM was oxidized resulted in a non-uniform axial PM distribution. The axial PM distribution trend showed an increased PM density in each axial segment and a PM density near the outlet that was $26 \%$ higher than the substrate average. This does not match the axial PM distribution trends found after active regeneration or loading experiments, where axial segments 1-3 had a similar PM density and the PM density near the outlet was $5-19 \%$ higher than the substrate average. The difference may be caused by a change in permeability of the substrate and PM cake, causing the wall flow velocity distribution to change.

- The axial PM distribution trend that was found for post loading after an active regeneration was similar to the axial PM distribution found after a cleanout was performed on the substrate that experienced multiple PM loading, partial active regeneration, and partial passive oxidation cycles.

- The post loading that occurred after a passive oxidation where $65 \%$ of the available PM was oxidized resulted in a uniform axial PM distribution trend that is similar to the loading and passive oxidation axial PM distribution trends. 


\section{Chapter 8. Recommendations}

The data that were collected in this study give trends of the PM distribution that can be expected after PM loading, passive oxidation, active regeneration, and post loading conditions. The PM density in the substrate prior to oxidation, amount of PM that was oxidized in the substrate, and the type of oxidation that occurred prior to the post loading were the three main parameters that were varied. Additionally, the temperatures at which active regenerations were performed and the engine conditions used for passive oxidation were varied. The results presented PM distribution trends that were previously unknown. This chapter discusses recommendations that should be considered for future work. There are three main areas of recommendations: 1) process improvement, 2) physical understanding, and 3) future tests. Since there is limited experimental data on the PM distribution trends after loading, passive oxidation, and active regeneration conditions, there is still a need for additional tests and analysis that can be used to develop a fundamental understanding, and to develop better CPF models. It is important that the data be collected in an efficient way and be of high quality.

\section{Process Improvement}

The work presented in this thesis was the first experimental study into PM distribution data for CPFs performed at MTU. The test procedures and test conditions that were used were adapted from the work performed by Shiel [4] and Pidgeon [5]. However, stage 3, which was used to stabilize the CPF temperature after passive oxidations and active regenerations, and return the $\mathrm{CPF}$ to a repeatable temperature prior to weighing it, should be reconsidered. During Phase 1 of the maldistribution study, stage 3 was a weighing condition that loaded $0.2-0.3$ grams, or $0.02-0.03 \mathrm{~g} / \mathrm{L}$, of PM into the substrate. During Phase 2, stage 3 loaded between 5 and 8 grams, or $0.29-0.47 \mathrm{~g} / \mathrm{L}$, of $\mathrm{PM}$ into the substrate. The effect of this loading on the PM distribution is not known. Therefore, if the loading engine condition that is used during stage 2 loads more than $0.05 \mathrm{~g} / \mathrm{L}$ into the substrate in 30 minutes, a different weighing condition should be used. This will minimize the effect of stage 3 on the PM distribution. The other option would be to remove stage 3 from PM distribution studies. The substrate is weighed after each test is completed, and it was found that those weights correlated to in- test weights. Using the substrate only mass that is taken after the test is completed will still allow the mass balance to be completed. The one problem with not performing stage 3 is that if there was residual PM at the start of the test, which was not cleaned out, it could not be identified. By running stage 3 and comparing the in-test substrate mass to the substrate only mass measurements, residual PM that was not cleaned out can be identified by the difference between the two measurements. Either way, care should be taken to measure the PM distribution at the completion of the oxidation stage of the test and limit additional PM loading.

It is also recommended to re-run the shipping orientation test. The original test was completed with the goal of keeping the shipping orientation the same for all initial substrate scans. Therefore, the first shipping was done with the inlet of the substrate pointed up. The test should be repeated with the inlet of the substrate pointed to the side 
as the first shipping orientation. That would allow the effect of shipping the substrate with the inlet pointed up to be identified. The shipping orientation test should also be completed on a recently loaded substrate. The substrate that was used for the shipping test that was conducted had not been tested on for nine months and the effect of that time on the PM is not known.

In keeping with process improvement, additional repeat scans and tests should be conducted. The repeat scans that were performed with the TAS7000 showed a large amount of variation. Two of the repeat tests had a 95\% CI of $\pm 0.11-0.19 \mathrm{~g} / \mathrm{L}$, with the third repeat test having a $95 \% \mathrm{CI}$ around $\pm 0.62 \mathrm{~g} / \mathrm{L}$. This is wide range of $95 \% \mathrm{CIs}$, and it would be useful to know of the $\pm 0.62 \mathrm{~g} / \mathrm{L}$ was an outlier. As the need for more accurate PM distribution information arises, it would be useful to know the actual repeatability of the TAS7000 so that the distribution trends can be discussed with certainty. Repeat tests on PM loading, passive oxidation, and active regenerations cases should be conducted in the MTU test cell as well to verify the repeatability of the PM distribution trends. The two loading scans that were taken as part of this work had different axial PM distributions. Knowing the repeatability of the PM distribution trends would allow the results to be discussed with greater certainty.

\section{Physical Understanding}

The PM density measurements that are taken by the TAS7000 should be verified against physical measurements to ensure their accuracy. This would be done by first scanning a PM loaded substrate, and then performing a destructive test to analyze the PM density in regions of the substrate. The PM density in different regions of the substrate could be measured by weighing the section before and after performing an oven regeneration of the sectioned part. The mass difference, divided by the volume of the sectioned part would give the local PM density measurement. The physical data points would not have to represent the same resolution as the TAS7000, but an effort should be made to find data within the axial segments, radial sections, and quadrants. By comparing the measurements of the TAS7000 and the destructive physical measurements, the accuracy of the PM density calculation method that was developed in section 4.1 can be verified. The physical correlation of the TAS7000 data to the actual PM density distribution in the substrate is not known at this time.

The threshold of the UI that indicates a uniform/ non-uniform distribution should be verified using physical data. Right now the threshold is correlated to an amount of variation in the data. Tests should be conducted with the intent of finding the value of the UI that correlates to a PM distribution that causes damage to a substrate. Based on conversations with Cummins, they have a general idea of what PM distributions should be avoided, but there has been not been any data collected that support this idea. Understanding the correlation between damaging distributions and the UI would give the threshold of the UI a stronger physical meaning and would allow PM distributions to be classified as damaging. Classifying a PM distribution as damaging would allow for engineers to make decisions about how to prevent PM distributions that may damage substrates. 


\section{Possible Future PM Distribution Tests}

The data collected in this study provided preliminary data on the PM distribution for a $\mathrm{CPF}$ after loading, passive oxidation, and active regeneration conditions. Due to the lack of experimental data in this area, there is still a lot of work that can be accomplished. A list is provided below of possible tests that could be conducted based on the results found for this research.

- Active regenerations and passive oxidations should be performed on clean substrates at PM densities near $5 \mathrm{~g} / \mathrm{L}$. The PM should be loaded without modifying the engine calibration. This would allow the results from the Phase 1 tests, which were obtained with a modified engine calibration, to be compared to standard engine calibration results at similar loadings. This would allow the effect of the modified engine calibration on the PM distribution to be studied. This would also provide the results of passive oxidation and active regenerations at a different PM density that could be compared to the results found in Phase 2.

- Multiple engine operating conditions should be used for PM loading, active regeneration, and passive oxidation tests. The exhaust space velocity, $\mathrm{NO}_{2}$ concentration into the CPF, and exhaust temperature should all be varied. The current results are for one or two engine conditions and rather limited in scope.

- The evolution of the PM distribution as a function of loading should be studied closer. This work analyzed the PM distribution near 3 and $5 \mathrm{~g} / \mathrm{L}$. Additional data points could show the exact evolution of the PM distribution and provide a better understanding of the resulting uniformity. The evolution of the PM distribution would assist in the development of accurate CPF models. The MTU modeling team is interested in this test.

- Based on the results found for the post loading studies in Phase 2, it would be useful to examine the post loading after different levels of oxidation. This is especially true for passive oxidation, where achieving $65 \%$ oxidation may be difficult in real world driving scenarios. For active regenerations, it would be important to see how the substrate loads after the substrate is cleaned out.

- The results from Test 2 of Phase 2 are the only results that were taken after multiple loading and oxidation cycles. The results showed an increasing PM density in the axial direction for the inlet to the outlet of the substrate which is slightly different than other active regeneration results. A test should be designed that would investigate how PM loading and incomplete PM oxidation affect the PM distribution. Post loading should be included as part of this study, since the post loading after an active regeneration showed a significant increase in the PM density near the outlet of the substrate.

- The effect of transient operation on the PM distribution would be another test that could be conducted. This would provide a more real world PM loading and oxidation scenario and possibly open other areas for investigation.

- The effect of ash loading on the PM distribution in the substrate should also be studied in the future, using tests similar to what is listed above. 


\section{References}

[1] Premchand, K. C., Johnson, J., and Yang, S.-L., 2013, "Development of a 1-D Catalyzed Diesel Particulate Filter Model for Simulation of the Oxidation of Particulate Matter and Gaseous Species During Passive Oxidation and Active Regeneration," 201301-1574, SAE International.

[2] Zhan, R., Huang, Y., and Khair, M., 2006, "Methodologies to Control DPF Uncontrolled Regenerations," 2006-01-1090, SAE International.

[3] Hutton, C. R., 2010, "An Experimental Investigation Into The Passive Oxidation Of Particulate Matter In A Catalyzed Particulate Filter," Master of Science, Michigan Technological University, Houghton, MI.

[4] Shiel, K. L., 2012, "A Study of the Effect of Biodiesel Fuel on Passive Oxidation in a Catalyzed Particulate Filter," Master of Science, Michigan Technological University, Houghton, MI.

[5] Pidgeon, J. M., 2013, "An Experimental Investigation into the Effects of Biodiesel Blends on Particulate Matter Oxidation in a Catalyzed Particulate Filter During Active Regeneration," Master of Science, Michigan Technological University, Houghton, MI. [6] Konstandopoulos, A. G., and Johnson, J. H., 1989, "Wall-Flow Diesel Particulate Filters-Their Pressure Drop and Collection Efficiency," 890405, SAE International. [7] Ohara, E., Mizuno, Y., Miyairi, Y., Mizutani, T., Yuuki, K., Noguchi, Y., Hiramatsu, T., Makino, M., Takahashi, A., Sakai, H., Tanaka, M., Martin, A., Fujii, S., Busch, P., Toyoshima, T., Ito, T., Lappas, I., and Vogt, C. D., 2007, "Filtration Behavior of Diesel Particulate Filters (1)," 2007-01-0921, SAE International.

[8] Opris, C. N., and Johnson, J. H., 1998, "A 2-D Computational Model Describing the Flow and Filtration Characteristics of a Ceramic Diesel Particulate Trap," 980545, SAE International.

[9] Liu, Y., Gong, J., Fu, J., Cai, H., and Long, G., 2009, "Nanoparticle motion trajectories and deposition in an inlet channel of wall-flow diesel particulate filter," Journal of Aerosol Science, 40(4), pp. 307-323.

[10] Konstandopoulos, A. G., Skaperdas, E., and Masoudi, M., 2002, "Microstructural Properties of Soot Deposits in Diesel Particulate Traps," 2002-01-1015, SAE International.

[11] Masoudi, M., 2002, "Hydrodynamics of Diesel Particulate Filters," 2002-01-1016, SAE International.

[12] Liu, Z. G., and Miller, R. K., 2002, "Flow Distributions and Pressure Drops of WallFlow Diesel Particulate Filters," 2002-01-1311, SAE International.

[13] Piscaglia, F., Rutland, C. J., and Foster, D. E., 2005, "Development of a CFD Model to Study the Hydrodynamic Characteristics and the Soot Deposition Mechanism on the Porous Wall of a Diesel Particulate Filter," 2005-01-0963, SAE International. [14] Yi, Y., 2006, "Simulating the Soot Loading in Wall-flow DPF Using a ThreeDimensional Macroscopic Model," 2006-01-0264, SAE International.

[15] Bensaid, S., Marchisio, D. L., Fino, D., Saracco, G., and Specchia, V., 2009, "Modelling of diesel particulate filtration in wall-flow traps," Chemical Engineering Journal, 154(1-3), pp. 211-218. 
[16] Nagata, M., Banno, Y., Kimura, R., and Maki, K., 2002, "Observation of Soot Accumulation Conditions in Diesel Particulate Filter and Gas Flow Analysis," 2002-011013, SAE International.

[17] Koltsakis, G. C., Konstantinou, A., Haralampous, O. A., and Samaras, Z. C., 2006, "Measurement and Intra-Layer Modeling of Soot Density and Permeability in Wall-flow Filters," 2006-01-0261, SAE International.

[18] Bensaid, S., Marchisio, D. L., Russo, N., and Fino, D., 2009, "Experimental investigation of soot deposition in diesel particulate filters," Catalysis Today, 147, pp. S295-S300.

[19] Pinturaud, D., Charlet, A., Caillol, C., Higelin, P., Girot, P., and Briot, A., 2007, "Experimental Study of DPF Loading and Incomplete Regeneration," 2007-24-0094, Consiglio Nazionale delle Ricerche.

[20] Choi, S., and Lee, K., 2013, "Detailed Investigation of Soot Deposition and Oxidation Characteristics in a Diesel Particulate Filter Using Optical Visualization," 2013-01-0528, SAE International.

[21] Subbu, G., and Collings, N., 2003, "A Novel Technique to Measure Wall Flow in Diesel Particulate Filters," 2003-01-1390, SAE International.

[22] Ranalli, M., Hossfeld, C., Kaiser, R., Schmidt, S., and Elfinger, G., 2002, "Soot Loading Distribution as a Key Factor for a Reliable DPF System: An Innovative Development Methodology," 2002-01-2158, SAE International.

[23] Stratakis, G. A., and Stamatelos, A. M., 2004, "Flow maldistribution measurements in wall-flow diesel filters," Proceedings of the Institution of Mechanical Engineers Part D: Journal of Automobile Engineering, 218(9), p. 15.

[24] Stratakis, G. A., and Stamatelos, A. M., 2004, "Flow distribution effects in the loading and catalytic regeneration of wall-flow diesel particulate filters," Proceedings of the Institution of Mechanical Engineers Part D: Journal of Automobile Engineering, $218(2)$, p. 14.

[25] Stratakis, G. A., Psarianos, D. L., and Stamatelos, A. M., 2002, "Experimental investigation of the pressure drop in porous ceramic diesel particulate filters," Proceedings of the Institution of Mechanical Engineers Part D: Journal of Automobile Engineering, 216(9), p. 12.

[26] Zandhuis, J. A., Finney, C. E. A., Toops, T. J., Partridge, W. P., Daw, C. S., and Fox, T., 2009, "Nondestructive X-ray Inspection of Thermal Damage, Soot and Ash Distributions in Diesel Particulate Filters," 2009-01-0289, SAE International. [27] Harvel, G., Chang, J.-S., Ewing, D., Fanson, P., and Kakinohana, M., 2009, "Measurement of Multi-Dimension Soot Distribution in Diesel Particulate Filters by a Dynamic Neutron Radiography," 2009-01-1263, SAE International.

[28] Nishina, S., Takeuchi, K., Shinohara, M., Imamura, M., Shibata, M., Hashimoto, Y., and Watanabe, F., 2011, "Novel Nondestructive Imaging Analysis for Catalyst Washcoat Loading and DPF Soot Distribution Using Terahertz Wave Computed Tomography," SAE Int. J. Fuels Lubr., 5(1), pp. 343-351.

[29] Zhang, X.-C., 2002, "Terahertz wave imaging: horizons and hurdles," Physics in Medicine and Biology, 47(21), p. 11.

[30] Hu, B. B., and Nuss, M. C., 1995, "Imaging with Terahertz Waves," Optics Letters, 20(16), p. 3. 
[31] Ranalli, M., Klement, J., Hoehnen, M., and Rosenberger, R., 2004, "Soot Distribution in DPF Systems. A Simple and Cost Effective Measurement Method for Series Development," 2004-01-1432, SAE International.

[32] Harvel, G. D., Chang, J.-S., Tung, A., Fanson, P., and Watanabe, M., 2011, "ThreeDimension Deposited Soot Distribution Measurement in Silicon Carbide Diesel Particulate Filters by Dynamic Neutron Radiography," 2011-01-0599, SAE International. [33] Kost, R., 2012, 4WD Product Validaton Manager at CNH America, LLC, private communication.

[34] Lakkireddy, V. R., 2004, "The Effect on an Advanced Oxidation Catalytic Converter and a Catalyzed Particulate Filter on the Emissions from a Heavy Duty Diesel Engine," Masters of Science, Michigan Technological University, Houghton, MI.

[35] Chilumukuru, K. P., 2008, "An Experimental Study of Particulate Thermal Oxidation in a Catalyzed Filter during Active Regeneration," Master of Science, Michigan Technological University, Houghton, MI.

[36] Austin, G. T., 2010, "Effects of Biodiesel Blends on Particulate Matter Oxidation in a Catalyzed Particulate Filter during Active Regeneration," Master of Science, Michigan Technological University, Houghton, MI.

[37] Rogoski, L., 2013, Senior Engineer Catalyst Technology Group at Cummins Inc., private communication.

[38] Self, G., 2012, EVP Marketing at Advantest America, Inc, private communication.

[39] Sappok, A. G., and Wong, V. W., 2007, "Detailed Chemical and Physical

Characterization of Ash Species in Diesel Exhaust Entering Aftertreatment Systems," 2007-01-0318, SAE International.

[40] Tao, H.-G., Chen, H.-X., Xie, J.-L., and Hu, Y.-P., 2012, "An alternative approach to quantifying fluid flow uniformity based on area-weighted average velocity and massweighted average velocity," Energy and Buildings, 45, pp. 116-123.

[41] Levine, D. M., Ramsey, P. P., and Smidt, R. K., 2001, Applied Statistics for Engineers and Scientists: Using Microsoft Excel and Minitab, Prentice Hall, Upper Saddle River, New Jersey.

[42] Hutton, C., Johnson, J., Naber, J., and Keith, J., 2012, "Procedure Development and Experimental Study of Passive Particulate Matter Oxidation in a Diesel Catalyzed Particulate Filter," 2012-01-0851, SAE International.

[43] Shiel, K. L., Naber, J., Johnson, J., and Hutton, C., 2012, "Catalyzed Particulate

Filter Passive Oxidation Study with ULSD and Biodiesel Blended Fuel," 2012-01-0837, SAE International.

[44] Pidgeon, J., Johnson, J., and Naber, J., 2013, "An Experimental Investigation into Particulate Matter Oxidation in a Catalyzed Particulate Filter with Biodiesel Blends on an Engine during Active Regeneration," 2013-01-0521, SAE International.

[45] Premchand, K., Surenahalli, H., and Johnson, J., 2013, "Particulate Matter and Nitrogen Oxides Kinetics Based on Engine Experimental Data for a Catalyzed Diesel Particulate Filter," 14PFL-0792, Manuscript submitted for publication to SAE International.

[46] Powers, J., 2013, Director Technology \& Expert Engineering at Advantest North America, SE, private communication. 
[47] Premchand, K. C., 2013, "Development of a 1-D Catalyzed Diesel Particulate Filter Model Using Engine Experimental Data for Simulation of the Performance and the Oxidation of Particulate Matter and Oxides of Nitrogen During Passive Oxidation and Active Regeneration," Doctor of Philosophy, Michigan Technological University, Houghton, MI.

[48] Aravelli, K., and Heibel, A., 2007, "Improved Lifetime Pressure Drop Management for Robust Cordierite (RC) Filters with Asymmetric Cell Technology (ACT)," 2007-010920, SAE International.

[49] Manni, M., and Pedicillo, A., 2009, "An Engine Test to Assess the Effect of Fuels and Lubricating Oils on Soot Loading of Diesel Particulate Filters," 2009-01-1871, SAE International. 


\section{Appendix A. CPF Canning Procedures}

The CPFs that were used for the research discussed in this thesis had a removable can, as discussed in Chapter 3. Due to this, the CPF had to be canned before every test. This appendix describes the procedure that is used to can the CPF. The procedure for the ISB and ISL substrates is identical, only the dimensions of the parts are different.

Prior to the first time a substrate is canned, there are markings that need to be made. Place all markings on the side of the substrate so that they will be visible after loading the substrate.

1. Label the substrate with the phase and substrate number. For example substrate 3 of Phase 2 would be labeled P2S3. See Figure A.1.

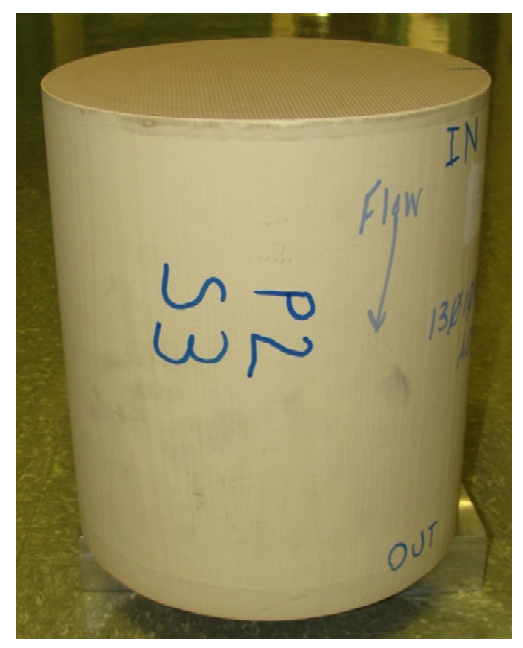

Figure A.1: Substrate Labeling

2. Mark the direction of flow through the substrate. Cummins will typically mark the inlet of the substrate, but after the substrate is loaded for the first time, this will be difficult to see. A flow arrow can be seen in Figure A.1 as well.

3. Cummins will typically place alignment marks on the substrate for how they orientate the substrate during scanning. Make sure the alignment marks are on the inlet face of the substrate, on the side of the substrate towards the inlet and outlet, and on the outlet face of the substrate. This way the alignment marks can be seen from any direction. An example of this is shown in Figure A.2. 


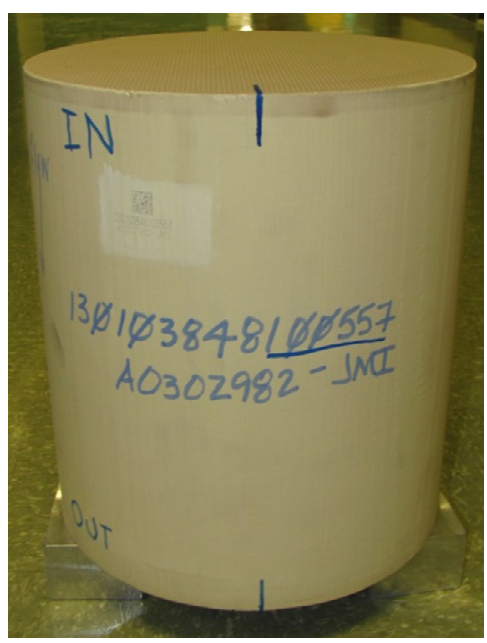

Figure A.2: Substrate Alignment Marks

4. The last required marking is for the radial locations of the thermocouples to be placed within the substrate. This may have to be done after the substrate is canned for the first time, if a substrate of similar size hasn't been done already. If a substrate of similar size has been done already, the markings can be transferred by measurements. An example of the markings used for the thermocouple locations is shown in Figure A.3.

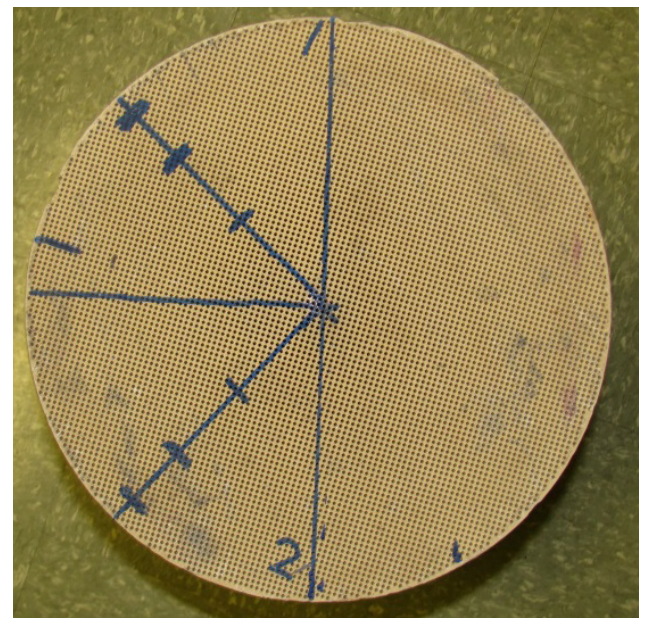

Figure A.3: Thermocouple Location Markings

After a substrate is marked completely, it is ready to be canned. The canning process follows.

1. Place the substrate on two blocks of equal height. The blocks need to be tall enough to center the substrate inside the can during the canning process. For the ISB substrate the required height is $57.15 \mathrm{~mm}$ and for the ISL substrate the required height is $46.04 \mathrm{~mm}$. When the substrate is first placed on the blocks, the edges of the blocks should stick out past the edges of the substrate to allow the 
matting to rest on the blocks while it is being wrapped around the substrate. The blocks can be seen underneath the substrate in Figure A.4.

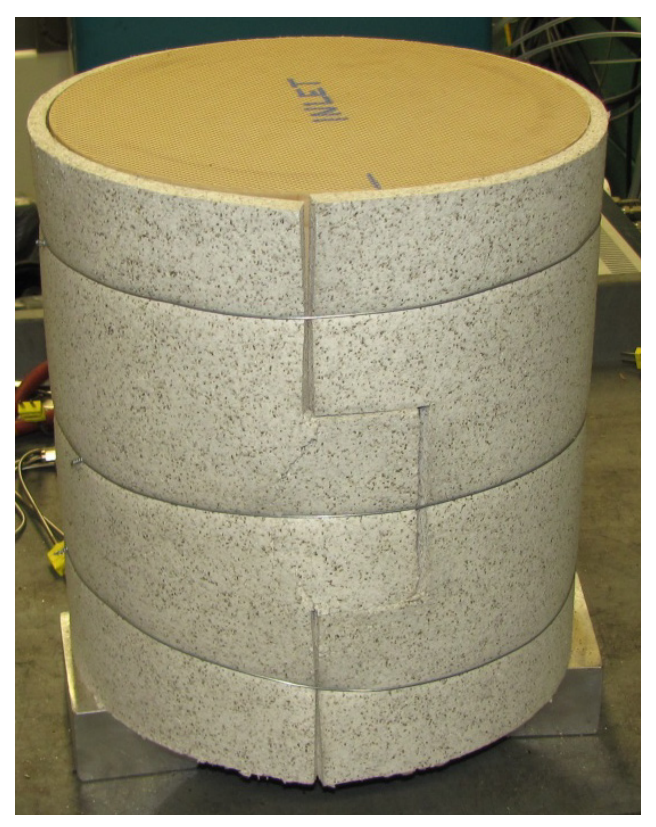

Figure A.4: Matting Alignment

2. Wrap a new piece of matting around the substrate. Rest the matting on the blocks underneath the substrate while positioning it. The matting is aligned with the substrate using the alignment mark on the face of the substrate. Since the matting has an offset cut in it, the alignment mark is placed halfway between the innermost and outer-most edges of the cut. This is shown in Figure A.4.

3. Once the matting is positioned with the substrate correctly, uses steel wire to hold the matting against the substrate. Wrap the wire in around the matting in three locations, as shown in Figure A.4, and twist the ends together to tighten it down. Trim off the excess wire.

4. The blocks underneath the substrate will now have to be moved closer to the center of the substrate to allow the can to fit correctly.

5. Place the can around the substrate. The can has alignment marks that need to be aligned with the substrate, as shown in Figure A.5. The alignment mark on the substrate is aligned with the left side of the bolt flange. There is also an inner sleeve used in the can that has to be aligned with the alignment mark on the rim of the can. Both the inner sleeve and rim of the can are scratched to show the alignment position. The alignment of the substrate, inner sleeve, and can are checked before the can is tightened around the substrate. This is accomplished by squeezing the two bolt flanges together by hand, and checking all of the alignment marks. It was found during assembly that the left bolt flange tends to not move during the tightening of the can around the substrate, and only the right flange will move. Squeezing the bolt flanges together by hand will provide enough pressure to check the alignment. However, during the tightening of the can, adjustments may have to be made if the left bolt flange moves. 


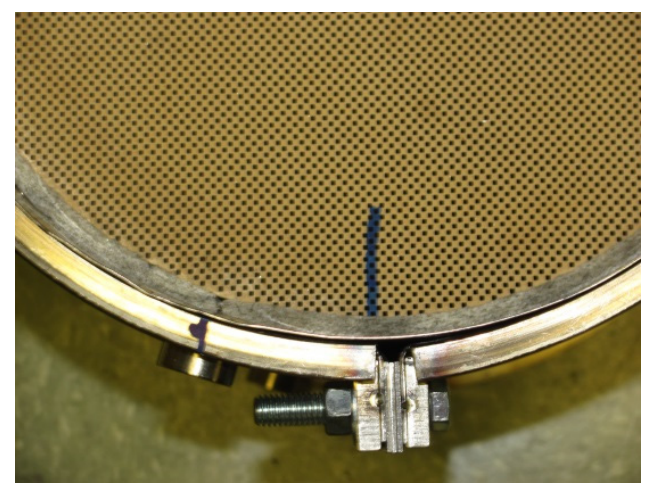

Figure A.5: Substrate and Can Alignment

6. After the alignment is checked, two C-clamps are placed on the bolt flange to begin the tightening process. The $\mathrm{C}$-clamps are tightened slowly and uniformly to avoid misalignment in the can. Once the bolt flanges are close enough, insert the bolts in the seven bolt holes, and place the washers and nuts on the bolts to hold the can closed. The C-clamps can then be released.

7. Tighten the bolt flange slowly and evenly, to prevent distortion of the can, using the bolts. The recommending bolt tightening sequence is given in Figure A.6. Continue to tighten the bolts until the two flanges are compressed together. The bolts will not tighten anymore at that point, due to the metal on metal contact.

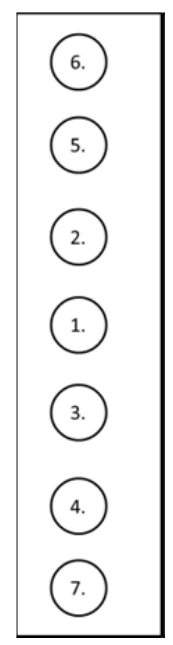

Figure A.6: Bolt Tightening Sequence

8. Instrument the now canned CPF.

a. The ISB has 28 internal thermocouples, 1 thermocouple in the outlet, 2 thermocouples at the inlet, 1 emissions probe, 2 pressure drop probes, and the OEM thermistors and pressure drop probes.

b. The ISL has 28 internal thermocouples, 3 thermocouples in the inlet, and 3 thermocouples in the outlet. All of the other instrumentation is the other components of the exhaust. 


\section{Appendix B. CPF Weighing Procedure}

The substrate is weighed multiple times during the testing process. This appendix discusses the different procedures that are used to weigh the substrate. The in- test procedure is used to weigh the substrate during the on-engine testing. The substrate only measurement procedure is used to weigh the substrate after it has been removed from the can and before it is shipped, or after it has been shipped and before it is canned. The Cummins' procedure is used to weigh the substrate before it is scanned with the TAS7000. Details on the scales used in these procedures can be found in Chapter 3.

\section{B.1. Procedure During a Test}

The CPF needs to be weighed in the middle of on-engine tests to allow for the calculation of the PM mass balance. Weighing the CPF provides a measure of the amount of PM retained in the CPF. Figure B.1 shows the ISL CPF as it is installed in the exhaust line. The clamp labeled 1 is the clamp that connects the DOC to the CPF. The clamp labeled 2 connects the CPF to the outlet cone. Although Figure B.1 is for the ISL CPF, the ISB $\mathrm{CPF}$ is installed in the exhaust in a similar fashion. The steps taken to weigh the CPF during testing are given below.

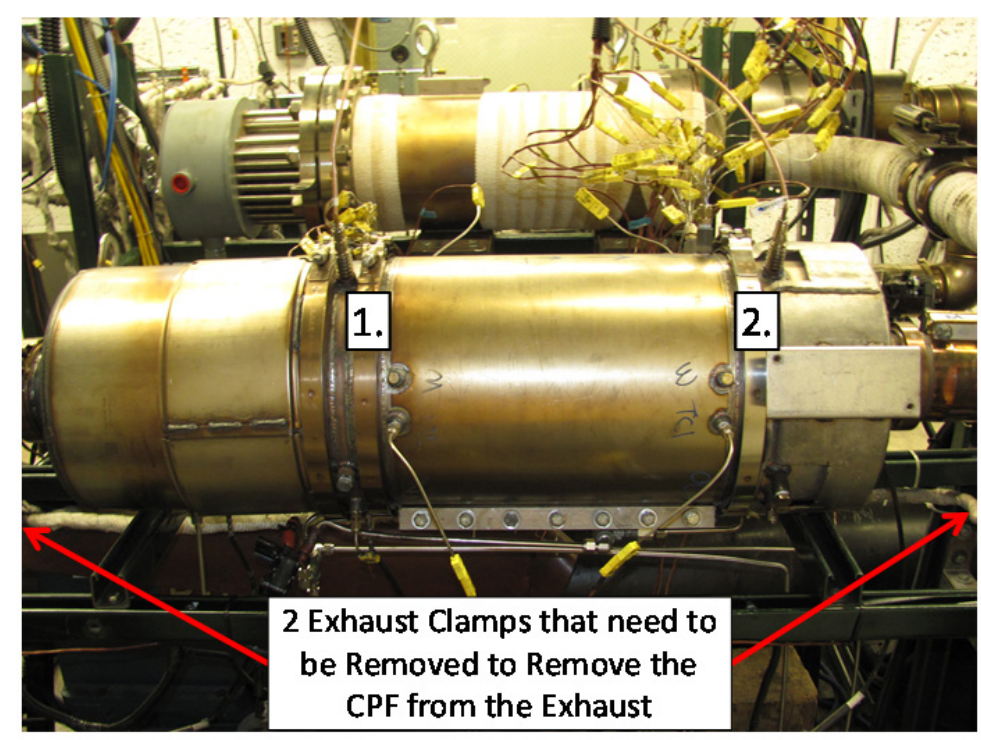

Figure B.1: CPF Clamp Removal

1. Run the engine at a repeatable engine point for 30 minutes to let the $\mathrm{CPF}$ temperature stabilize. For the Phase 1 work, this was the "Weighing" EOC from Table 5.2. For the Phase 2 work, this was EOC B, from Table 5.8. Running a repeatable engine condition and letting the $\mathrm{CPF}$ temperature stabilize will help to maintain consistent CPF mass measurements. Austin, in reference [36], describes the effect of temperature on the mass of the substrate. Weighing the $\mathrm{CPF}$ hot is important because water will not be adsorbed into the PM of the substrate, which would decrease the accuracy of the measurement. 
2. Switch from the Trapline to the Baseline, to route the engine exhaust around the aftertreatment system. This allows the engine to be cooled down before being stopped.

3. After cooling down the engine, shut the engine down.

4. Remove the two exhaust clamps that connect the DOC, CPF, outlet cone assembly to the rest of the exhaust. Disconnect all of the thermocouples, pressure sensor lines, emissions lines, and other sensor wires from the assembly.

5. Lift the assembly out of the exhaust line and set it down vertically with the outlet cone down and the inlet to the DOC pointed up.

6. Remove clamp 1 and lift the DOC off. Set the DOC off to the side.

7. Remove clamp 2 and lift the CPF off. Take the CPF over towards the scale.

Note: Both Austin [36] and Hutton [3] discuss the need to cap the ends of the CPF during the weighing process to eliminate the chance of having airflow through the substrate, which would generate lift. However, caps were not available during the Phase 1 work, and the caps that were available for the Phase 2 work did not fit properly. Instead the focus was on being consistent in the amount of time it took to remove the substrate and weigh it, and on maintaining a consistent $\mathrm{CPF}$ temperature during mass measurements. Hutton, in reference [3], recommends maintaining CPF temperatures within $\pm 15{ }^{\circ} \mathrm{C}$ for mass measurements taken during different stages of testing. Mass measurements that were completed in- test agreed with mass measurements that were completed post-test using this method.

8. Record the temperature of the thermocouples C1-C4 and Q1-1- Q1-12. These thermocouple measurements should be within $\pm 15{ }^{\circ} \mathrm{C}$ each time the substrate is weighed [3].

9. Place the $17.9 \mathrm{~kg}$ calibration weight on the scale and record the measurement. Repeat this for a total of three measurements. Ensure that the weight is placed in a similar location for all three measurements.

10. Place the CPF on the scale and record the measurements. Repeat this for a total of three measurements. Ensure that the CPF is placed in a similar location for all three measurements.

11. Place the CPF on the outlet cone, align the alignment marks, and attach clamp 2.

12. Place the DOC on the CPF, align the alignment marks, and attach clamp 1.

13. Lift the assembly back into the engine exhaust system and attach the two exhaust clamps that connect the DOC and outlet cone to the rest of the exhaust system. Connect all of the sensor wiring, emissions lines, pressure sensor lines, and thermocouples.

14. Start the engine, with the exhaust still routed through the Baseline. Allow the engine to warm up.

15. Once the engine is at the desired operating condition, and is warm and stabilized, switch from the Baseline to the Trapline and continue the test. 


\section{B.2. Substrate Only Measurement Procedure}

It is important to be able to measure the mass of the substrate without the can and instrumentation occasionally. This is usually done when the substrate is new (before any testing has been done), after each test that was conducted, and before a test that was stopped in the middle is started again. All of these measurements need to be taken hot to avoid water adsorption by the PM.

Substrates are weighed when they are clean to establish a baseline weight. Substrates are weighed after tests are completed for two reasons. The first is that it is important to verify the mass measurements that were taken during testing, especially when using specialized aftertreatment systems that have a lot of components that are changing on them. The second reason is that the matting that is wrapped around the substrate contains volatile material. The volatiles will be baked off during testing, causing mass measurements to be erratic. Typically, a substrate cleanout at the beginning of a test will remove the volatiles. However, if a test is not started from the beginning the volatiles cannot be baked off before any mass measurements are taken. In that case, the substrate only measurements are used to determine the amount of PM retained in the substrate. For this same reason, the substrate is weighed before testing if the test is not being started from the beginning. The procedure used to obtain substrate only measurements is given below.

1. Turn on the Photoelastic P-2307M oven. Set the temperature to $232{ }^{\circ} \mathrm{C}$. Wait 1 hour for the oven to warm up.

2. Remove the substrate from the can, if the substrate was canned.

3. Insert 1 thermocouple into the center outlet channel of the substrate. The thermocouple should measure the temperature near the middle of the axial length.

4. Place the substrate in the oven. Every 30 minutes, rotate the substrate $90^{\circ}$. Repeat this until the internal temperature of the substrate is near $200{ }^{\circ} \mathrm{C}$.

5. Once the substrate temperature reaches $200{ }^{\circ} \mathrm{C}$, use a second thermocouple to record the internal temperatures at four other locations. The locations should be near the edge of the substrate and $90^{\circ}$ apart. Each measurement should be taken near the middle of the axial length. A diagram indicating the general location of the temperature measurements is shown in Figure B.2.
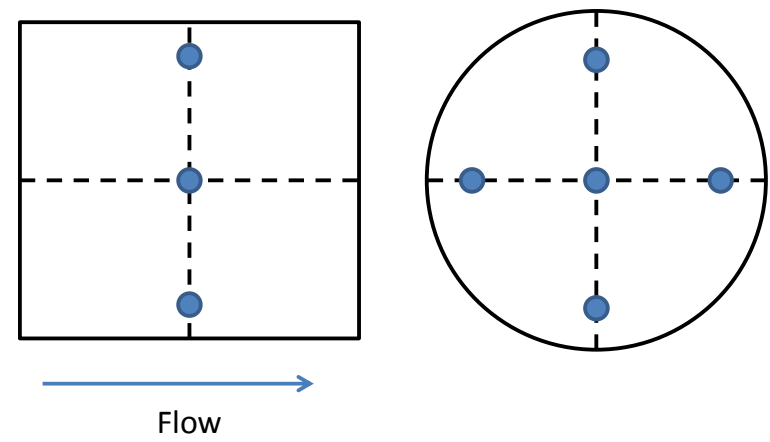

Figure B.2: Temperature Measurement Locations for Substrate Baking 
6. Remove the substrate from the oven and remove all thermocouples.

7. Place the $4.5 \mathrm{~kg}$ calibration weight on the scale and record the measurement. Repeat this for a total of three measurements. Ensure that the weight is placed in a similar location for all three measurements.

8. Place the $6.8 \mathrm{~kg}$ calibration weight on the scale and record the measurement. Repeat this for a total of three measurements. Ensure that the weight is placed in a similar location for all three measurements.

9. Place the substrate on the scale and record the measurement. Repeat this for a total of three measurements. Ensure that the substrate is placed in a similar location for all three measurements.

Note: If the substrate is in a clean state and is being weighed for the first time, record the mass at room temperature. Insert a thermocouple along the centerline to the middle of the substrate and measure the internal temperature. Remove the thermocouple and perform steps 7-9 after completing step 1. Then start at step 3 and finish the rest of the procedure.

\section{B.3. Cummins' Procedure}

After a test has been completed on a substrate, it is shipped to the Cummins Technical Center in Columbus, IN for a scan to be taken. Prior to the scan being taken, Cummins measures the mass of the substrate using their procedure. The mass measurement that results from this process is the value that is used in the calculation of the PM density using the raw TAS7000 data. Thus, this is the measurement that is considered the most critical for the purposes of this work, since it is part of the accuracy of the TAS7000 system. Overall, the measurements made at MTU and at Cummins were no more than 2 grams different. This procedure was provided by Rogoski in reference [37].

1. Heat the substrate to $150{ }^{\circ} \mathrm{C}$.

2. Let the part cool to $50{ }^{\circ} \mathrm{C}$.

3. Measure the mass of the substrate.

4. Place the substrate in the TAS7000 for scanning. 


\section{Appendix C. Substrate Shipping Procedure}

The substrate has to be shipped to the Cummins Technical Center in Columbus, IN for it to be scanned by the TAS7000. This appendix details the process used to ship the substrate between MTU and Cummins. This process occurs after the substrate is weighed at MTU at the completion of the test. See Appendix B.2.

1. Place the substrate inside a plastic shipping bag.

2. Slip the substrate, in the bag, into the foam inserts that are placed within the shipping crate. There are four foam inserts.

3. Place the foam inserts, with the substrate inside, into the wood shipping crate.

4. Place a cardboard cover over the substrate inlet, inside the bag, to add additional protection. The cover is designed to fit over top the substrate, without touching the inlet.

5. Close the plastic bag over the substrate cover, and tape the edges of the bag down to seal it off. Figure C.1 shows the substrate in the foam inserts, with the cardboard cover, and the edges of the bag taped down.

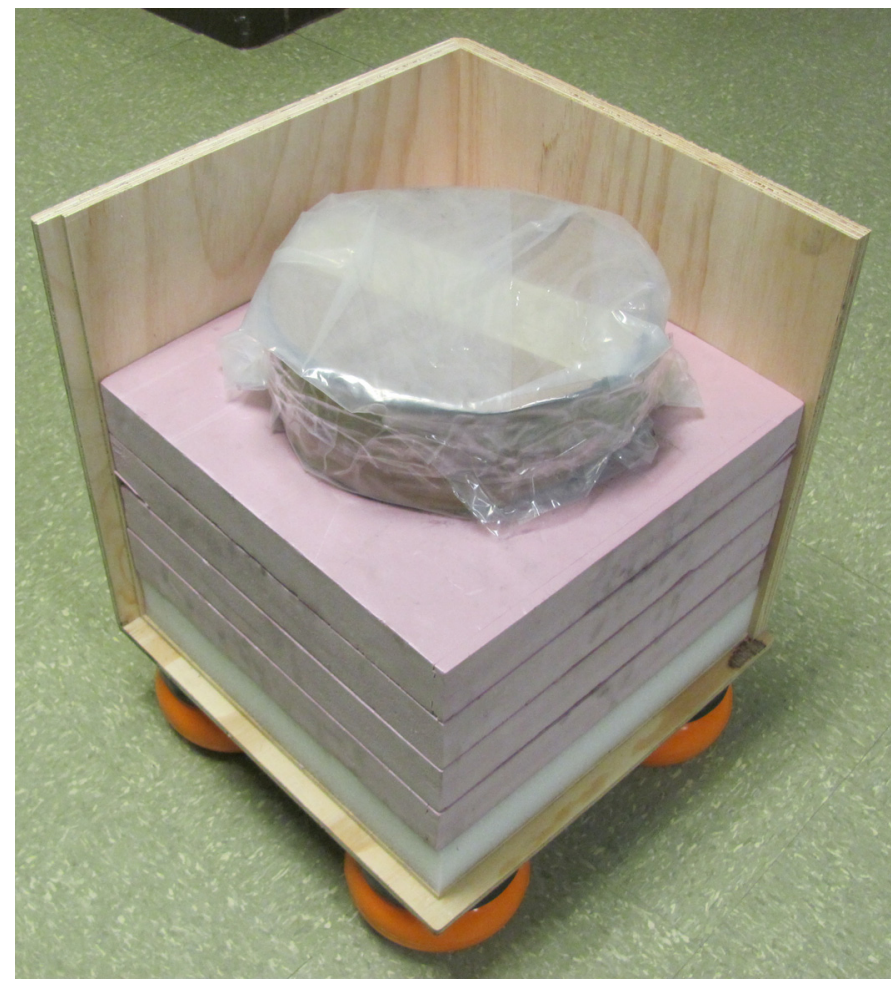

Figure C.1: Substrate in Shipping Crate

6. Close the shipping crate.

7. Strap the shipping crate to a pallet. This will ensure the orientation of the shipping crate is maintained throughout the shipping process. All shipping was done with the inlet in the upright position. 


\section{Appendix D. Test Plan Measurements}

This appendix shows the type of measurements that were taken during each stage. This is the general test plan that was followed for every Phase 2 experiment.

\section{Test Plan for Part 1 (6 PM Filters are Needed)}

1. Warm Engine Up

2. Verify Passive Oxidation Condition/ Active Regeneration Condition (If not stopping the test for substrate scan after loading.)

a. Collect Dilution Ratio Data at Passive Oxidation/ Active Regeneration Condition

b. Collect 3 UDOC PM Samples if an Active Regeneration Test is being Conducted

c. Collect UDOC, DDOC, DCPF Emissions if an Active Regeneration Test is being Conducted

d. Verify Fuel Dosing Rate if running an Active Regeneration Test

3. Run a Substrate Cleanout

a. $600{ }^{\circ} \mathrm{C}$

b. 30 minutes

\section{Stage 1- 30 Min}

1. DDOC Emissions- 7 Min. Sample

2. UDOC PSD

3. 1 PM UDOC Sample- 5 Min.

4. DCPF Emissions- 7 Min. Sample

5. DDOC PSD

6. UDOC Emissions- 7 Min. Sample

7. DCPF PSD

Weigh the Substrate at this Point

\section{Stage 2- 3 Hours}

1. DDOC Emission- 15 Min. Sample

2. UDOC PSD Sample

3. DCPF PSD Sample

4. 2 UDOC PM Samples- 5 Min. Each

5. DCPF Emissions- 15 Min. Sample

6. DDOC PSD Sample

7. 1 DCPF PM Sample- 1 Hour

8. UDOC Emissions- 15 Min. Sample 
9. DDOC Dilution Ratio- 10 Min.

10. UDOC Dilution Ratio- 10 Min.

11. DCPF Dilution Ratio- 10 Min.

12. DDOC Emission- 15 Min. Sample

13. UDOC PSD Sample

14. DCPF PSD Sample

15. 2 UDOC PM Samples- 5 Min. Each

16. DCPF Emissions- 15 Min. Sample

17. DDOC PSD Sample

18. UDOC Emissions- 15 Min. Sample

Weigh the Substrate at this Point

\section{Test Plan for Part 2 (8 PM Samples are Needed)}

Start with the stock CPF in exhaust if a substrate scan was taken after Stage 2.

If no substrate scan was taken after Stage 2, skip to Ramp Up.

1. Warm Engine Up

2. Verify Passive Oxidation Condition/ Active Regeneration Condition

a. Collect Dilution Ratio Data at Passive Oxidation/ Active Regeneration Condition

b. Collect 3 UDOC PM Samples if an Active Regeneration Test is being Conducted

c. Collect UDOC, DDOC, DCPF Emissions if an Active Regeneration Test is being Conducted

d. Verify Fuel Dosing Rate if running an Active Regeneration Test

3. Collect Dilution Ratio Data at Loading Condition

Install Removable Can CPF in Exhaust

\section{Pre Ramp Up-}

\section{Minutes for Point F and A / 1 Hour for Point $H$ and AR}

1. DDOC Emissions- 7 Min. Sample

2. UDOC PSD

3. DDOC PSD (DCPF for $\mathrm{H}$ and $\mathrm{AR}$ )

4. 2 PM UDOC Samples- 5 Min. Each

5. DCPF Emissions- 7 Min. Sample

6. 1 PM DCPF Sample- 30 Min. (Point H and AR only)

7. DCPF PSD (DDOC for $\mathrm{H}$ and $\mathrm{AR}$ ) 
8. UDOC Emissions- 7 Min. Sample

Weigh the Substrate at this Point

\section{Ramp Up- 15 Min}

\section{Pre-Active Regeneration Ramp-10 min}

\section{Passive Oxidation}

Point A- Run for 130 Min: 2\% Oxidation (20 min samples for emissions)

Point F- Run for 111 Min: 40\% Oxidation

Point F- Run for 227 Min: 70\% Oxidation

Point H- Run for 40 Min: 40\% Oxidation

1. DDOC Emission- 10 Min. Sample

2. UDOC PSD Sample

3. DCPF PSD Sample

4. 3 UDOC PM Samples- 5 Min. Each

5. DCPF Emissions- 10 Min. Sample

6. 1 DCPF PM Sample- 30 Min. (Point F and A only)

7. DDOC PSD Sample

8. UDOC Emissions- 10 Min. Sample

\section{Active Regeneration}

$525^{\circ} \mathrm{C}$ Test- 34 Min of Dosing: 52\% Oxidation

$525^{\circ} \mathrm{C}$ Test- 16 Min of Dosing: 30\% Oxidation (5 Min Emission Samples)

1. DDOC Emissions- 10 Min. Sample

2. DCPF PSD

3. DCPF Emissions- 10 Min. Sample

4. DDOC PSD

$600{ }^{\circ} \mathrm{C}$ Test- 9 min of Dosing: $70 \%$ Oxidation

1. DDOC- 2 Min. Samples- $\mathrm{NO}_{\mathrm{X}}$ Only

2. DCPF- 2 Min. Samples- $\mathrm{NO}_{\mathrm{X}}$ Only

\section{Stage 3- 30 minutes}

1. DDOC Emissions- 7 Min. Sample 
2. UDOC PSD

3. 2 PM UDOC Sample- 5 Min. Each

4. DCPF Emissions- 7 Min. Sample

5. DDOC PSD

6. UDOC Emissions- 7 Min. Sample

7. DCPF PSD

Weigh the Substrate at this Point

\section{Test Plan for Part 3 (5 PM Samples Needed)}

Start with a stock CPF in exhaust if a substrate scan was taken after Stage 3.

If no substrate scan was taken after Stage 3, skip to Stage 4.

1. Warm Engine Up

2. Collect Loading Dilution Ratio Data UDOC, DDOC, DCPF for 10 Minutes Each

Install Removable Can CPF in Exhaust

\section{Pre Stage 4- 30 Min}

1. DDOC Emissions- 7 Min. Sample

2. UDOC PSD

3. 2 PM UDOC Sample- 5 Min. Each

4. DCPF Emissions- 7 Min. Sample

5. DDOC PSD

6. UDOC Emissions- 7 Min. Sample

7. DCPF PSD

Weigh the Substrate at this Point

\section{Stage 4- 60 Min}

1. DDOC Emissions- 10 Min. Sample

2. UDOC PSD Sample

3. DCPF PSD Sample

4. 2 PM UDOC Sample- 5 Min. Each

5. DCPF Emissions- 10. Min Sample

6. 1 PM DCPF Sample- 30 Min.

7. DDOC PSD Sample

8. UDOC Emissions- 10 Min. Sample

Weigh the Substrate at this Point 


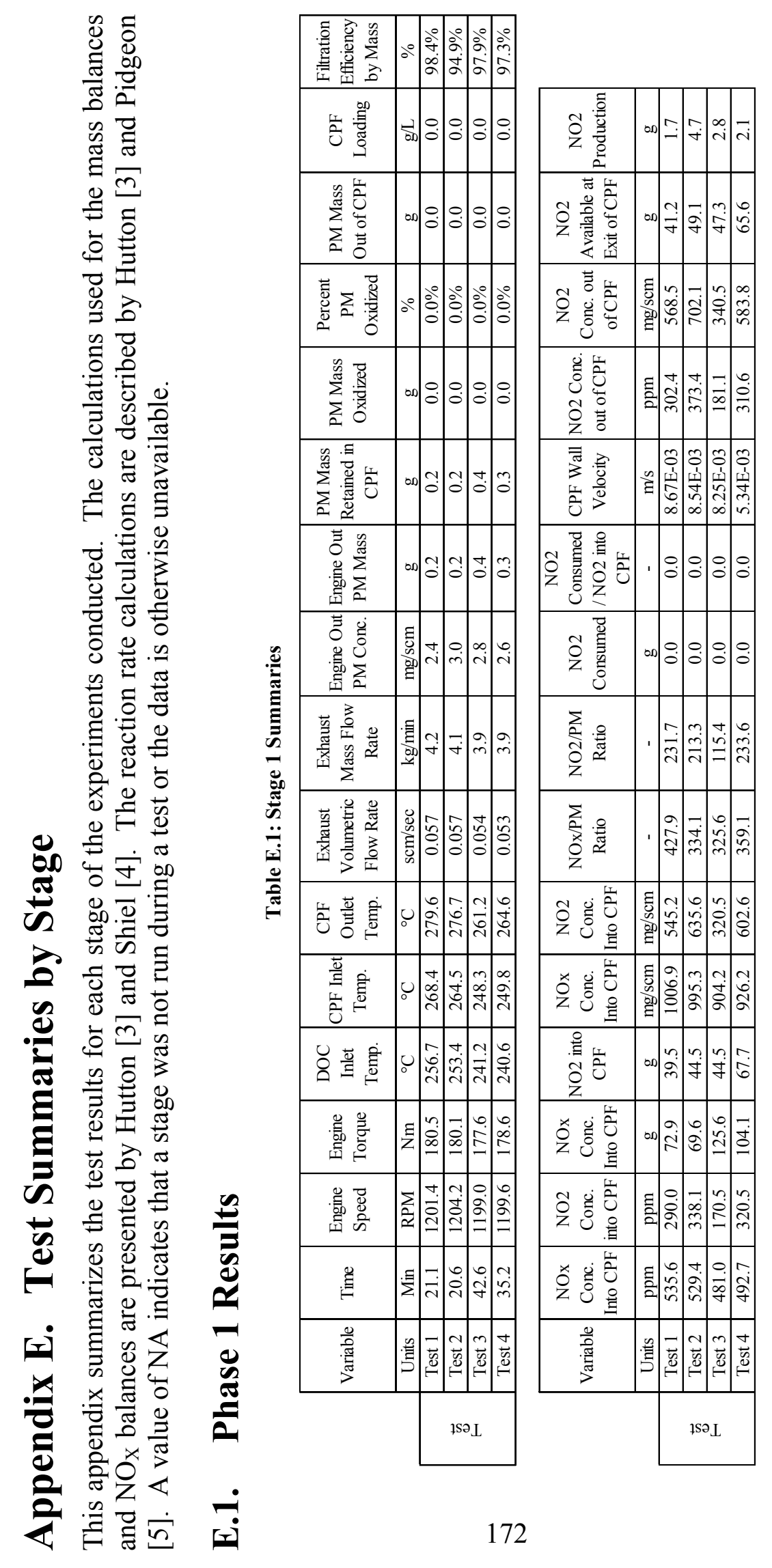



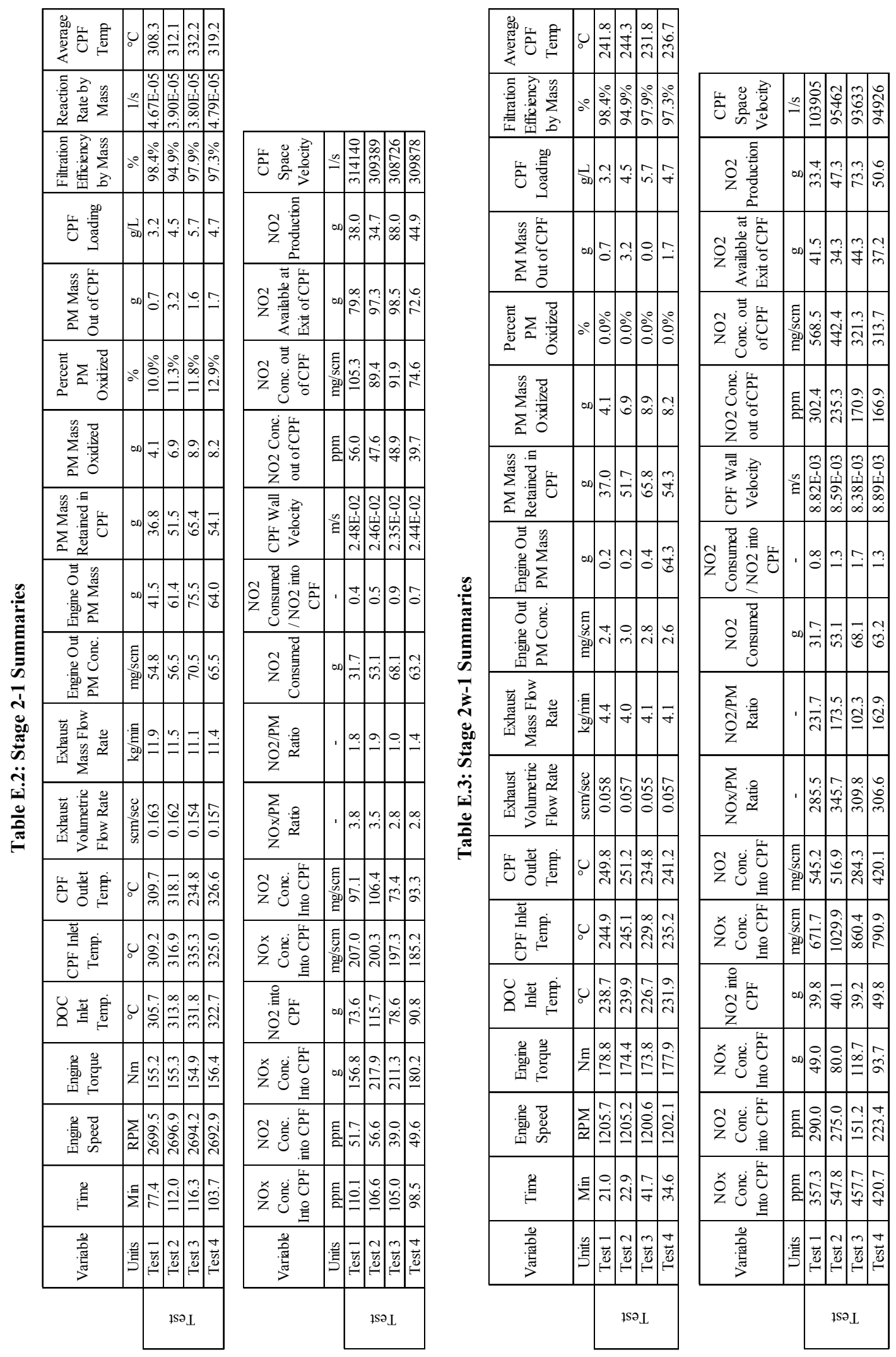

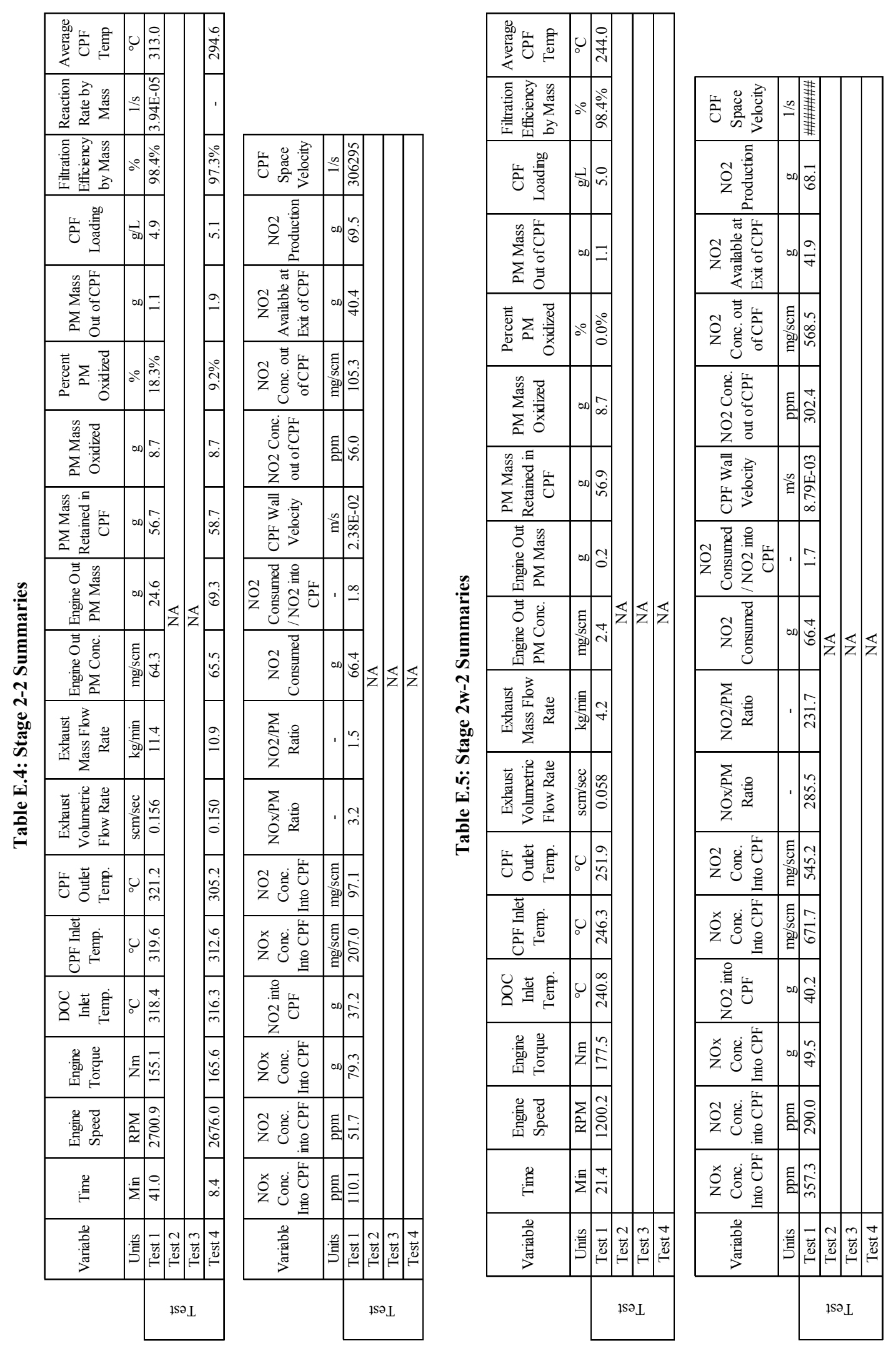

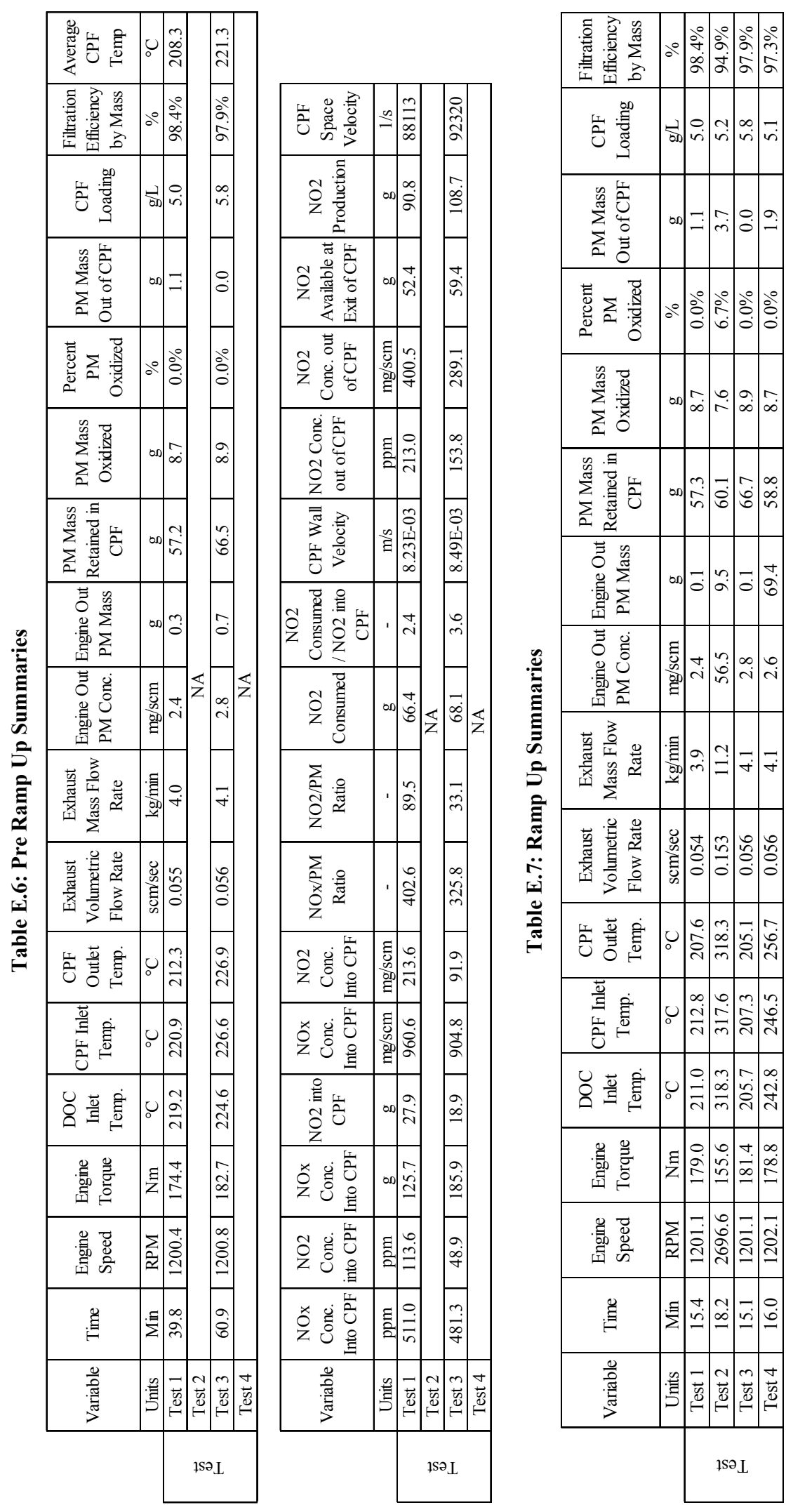

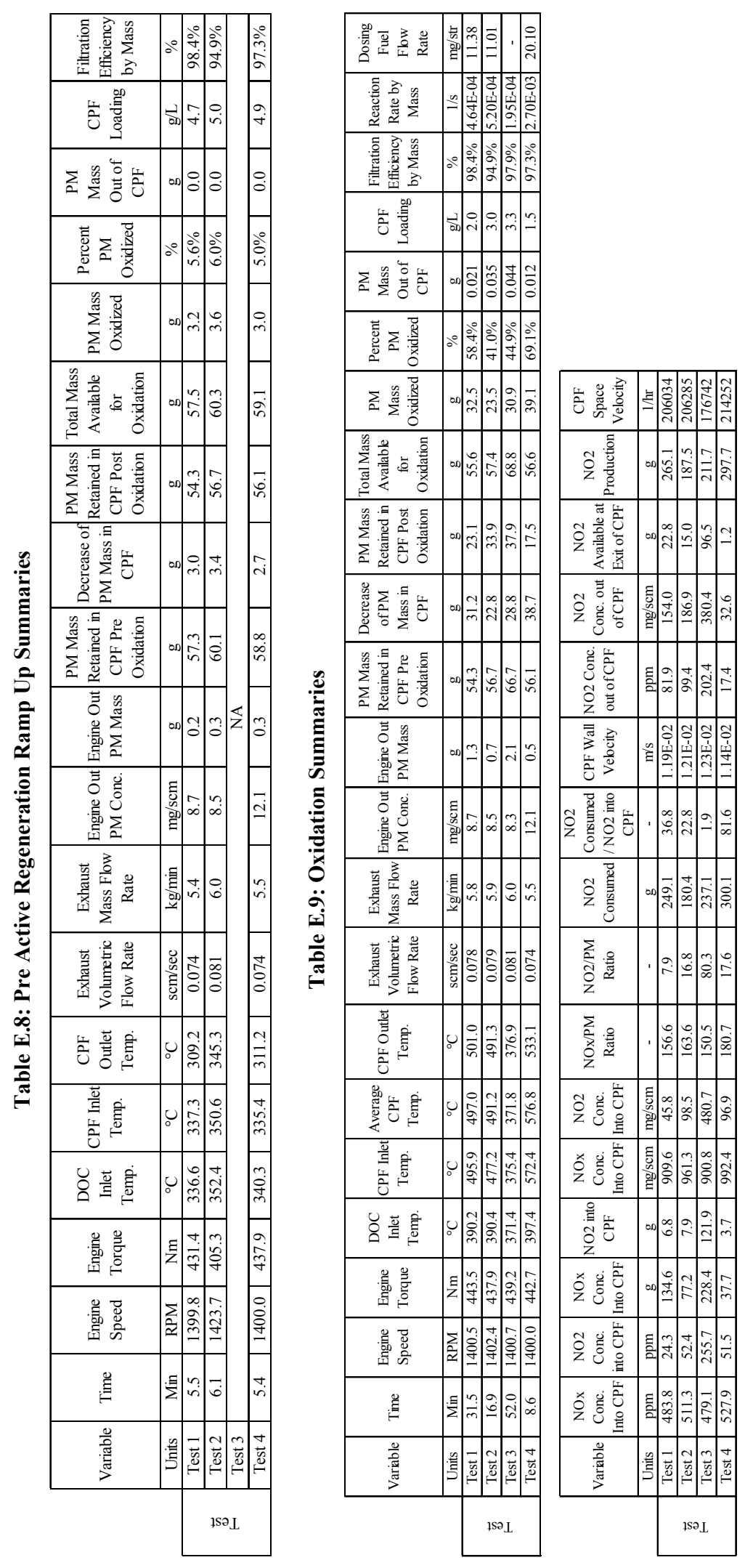


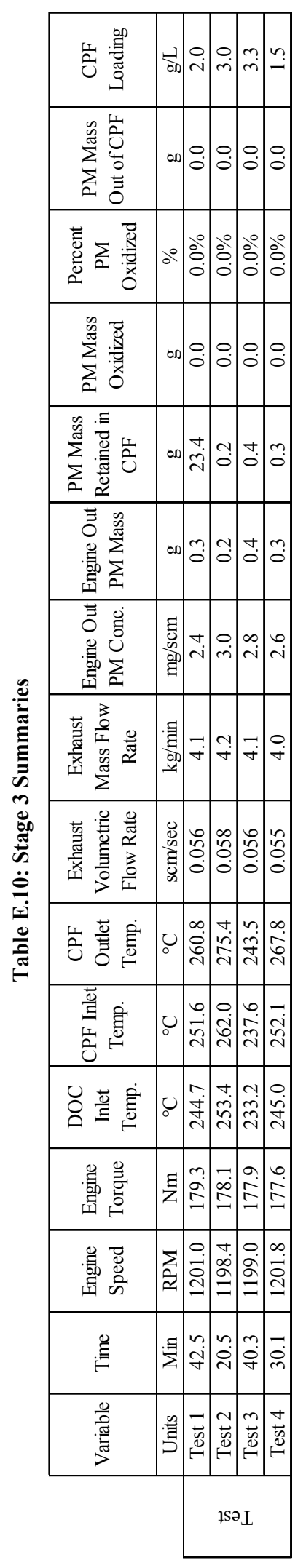

\begin{tabular}{|c|c|c|c|c|}
\hline 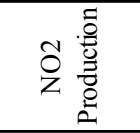 & $\infty 00=$ & : & & $\begin{array}{l}\infty \\
\dot{n}\end{array}$ \\
\hline 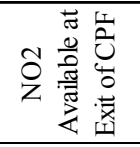 & $|00| \delta$ & $\begin{array}{ll}\dot{0} \\
\dot{\infty}\end{array}$ & $\hat{n}$ & $\stackrel{f}{\dot{n}}$ \\
\hline 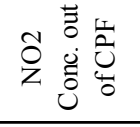 & 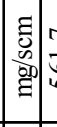 & $\begin{aligned} 0 \\
\vdots \\
i \\
n\end{aligned}$ & $\frac{\partial}{\partial}$ & $\begin{array}{l}n \\
\dot{0} \\
\dot{b} \\
i n\end{array}$ \\
\hline 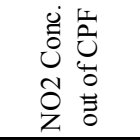 & 常: & 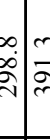 & & $\begin{array}{l}\infty \\
\vdots \\
\vdots \\
\grave{2}\end{array}$ \\
\hline 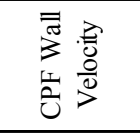 & 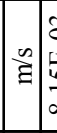 & 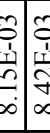 & & 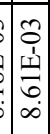 \\
\hline 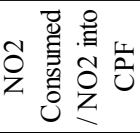 & & $\because:$ & 0 & $\dot{0}$ \\
\hline 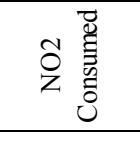 & $\infty$ & $\bullet:$ & $\because$ & $\dot{0}$ \\
\hline 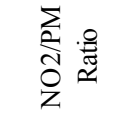 & & 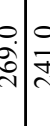 & & 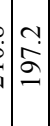 \\
\hline 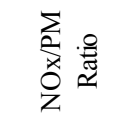 & & & & $\begin{array}{c}\tilde{c} \\
\infty \\
\infty \\
m\end{array}$ \\
\hline 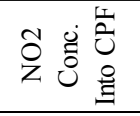 & \begin{tabular}{|c|c} 
\\
0 \\
0 \\
0 \\
0 \\
$\vdots$ \\
\end{tabular} & $\frac{\infty}{7}$ & & $\begin{array}{l}0 \\
0 \\
0 \\
0 \\
n \\
n\end{array}$ \\
\hline 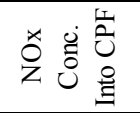 & 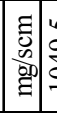 & $\begin{array}{lll}0 \\
0 \\
0 \\
0\end{array}$ & & 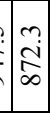 \\
\hline 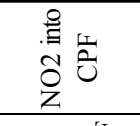 & 0 & $\begin{array}{l}\frac{1}{n} \\
\end{array}$ & & 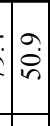 \\
\hline 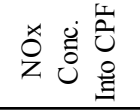 & bol? & $\vec{\square}$ & & 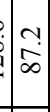 \\
\hline 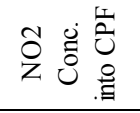 & 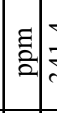 & $\vec{m}$ & & $\mid \begin{array}{l}n \\
\stackrel{2}{\hat{N}}\end{array}$ \\
\hline 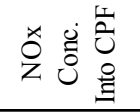 & 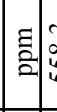 & 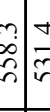 & & $\begin{array}{l}0 \\
\dot{b} \\
\dot{q}\end{array}$ \\
\hline 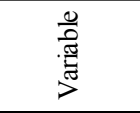 & 5 & $\overrightarrow{\breve{s}}$ & & 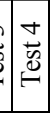 \\
\hline & & & & \\
\hline
\end{tabular}




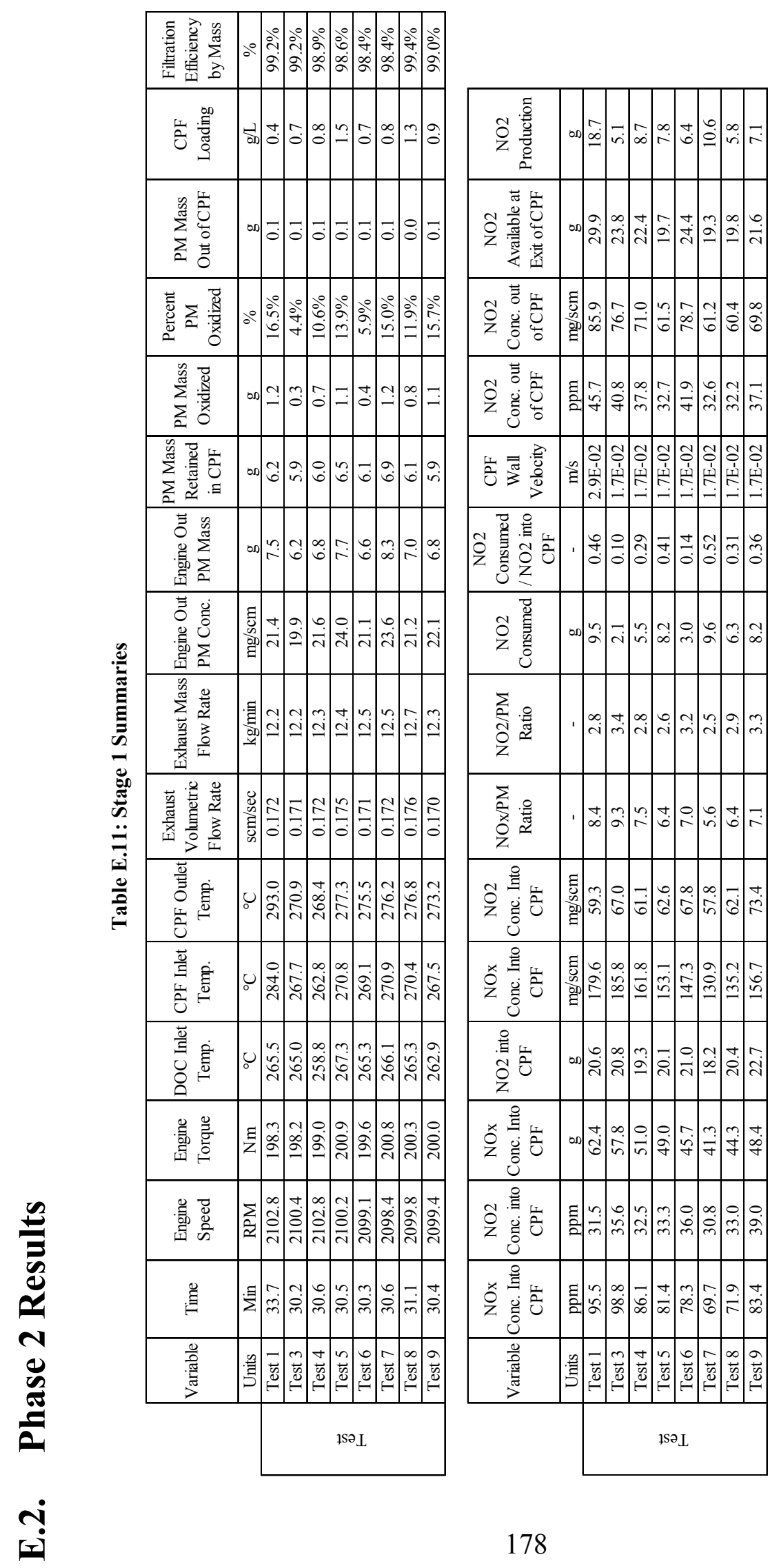



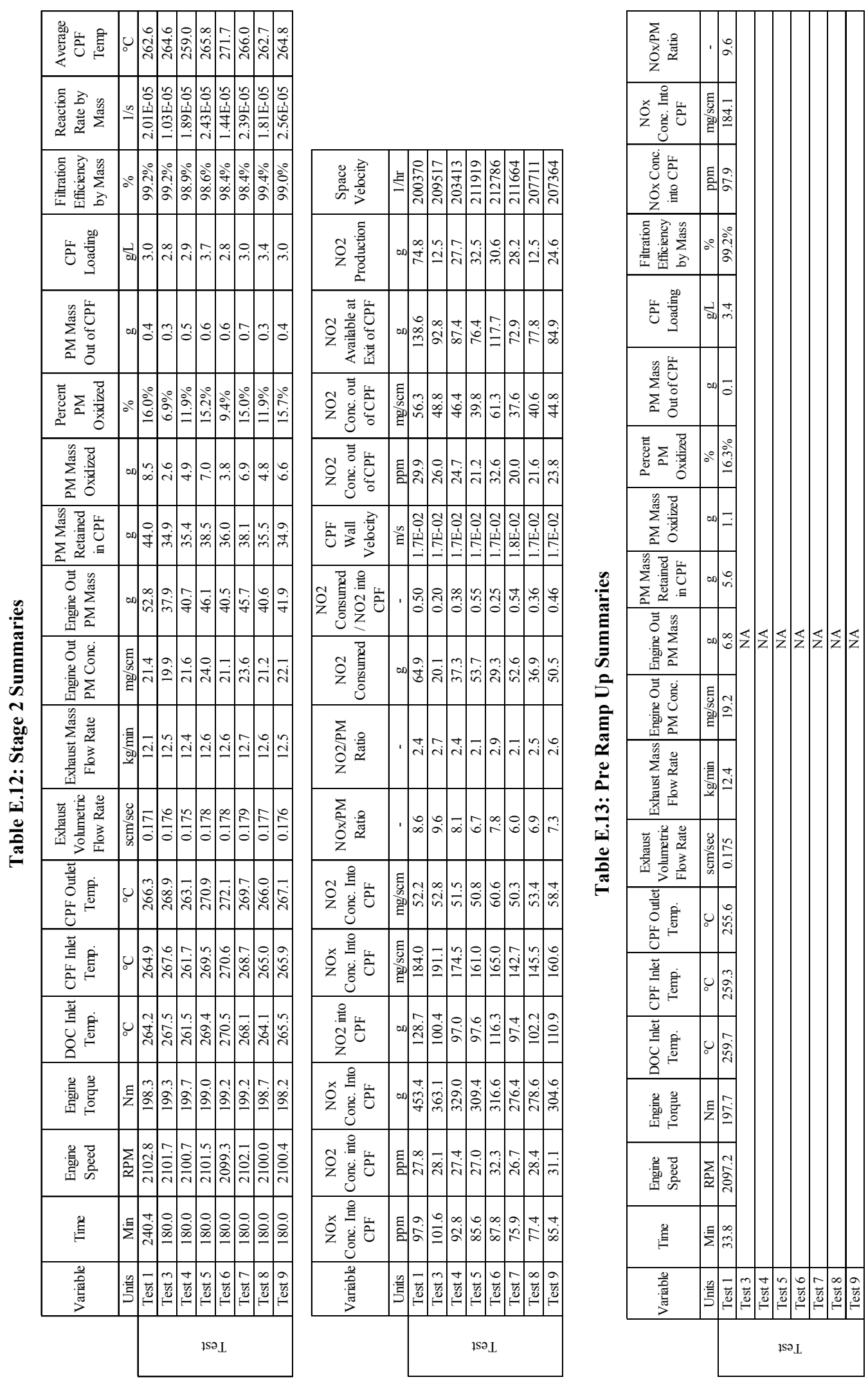

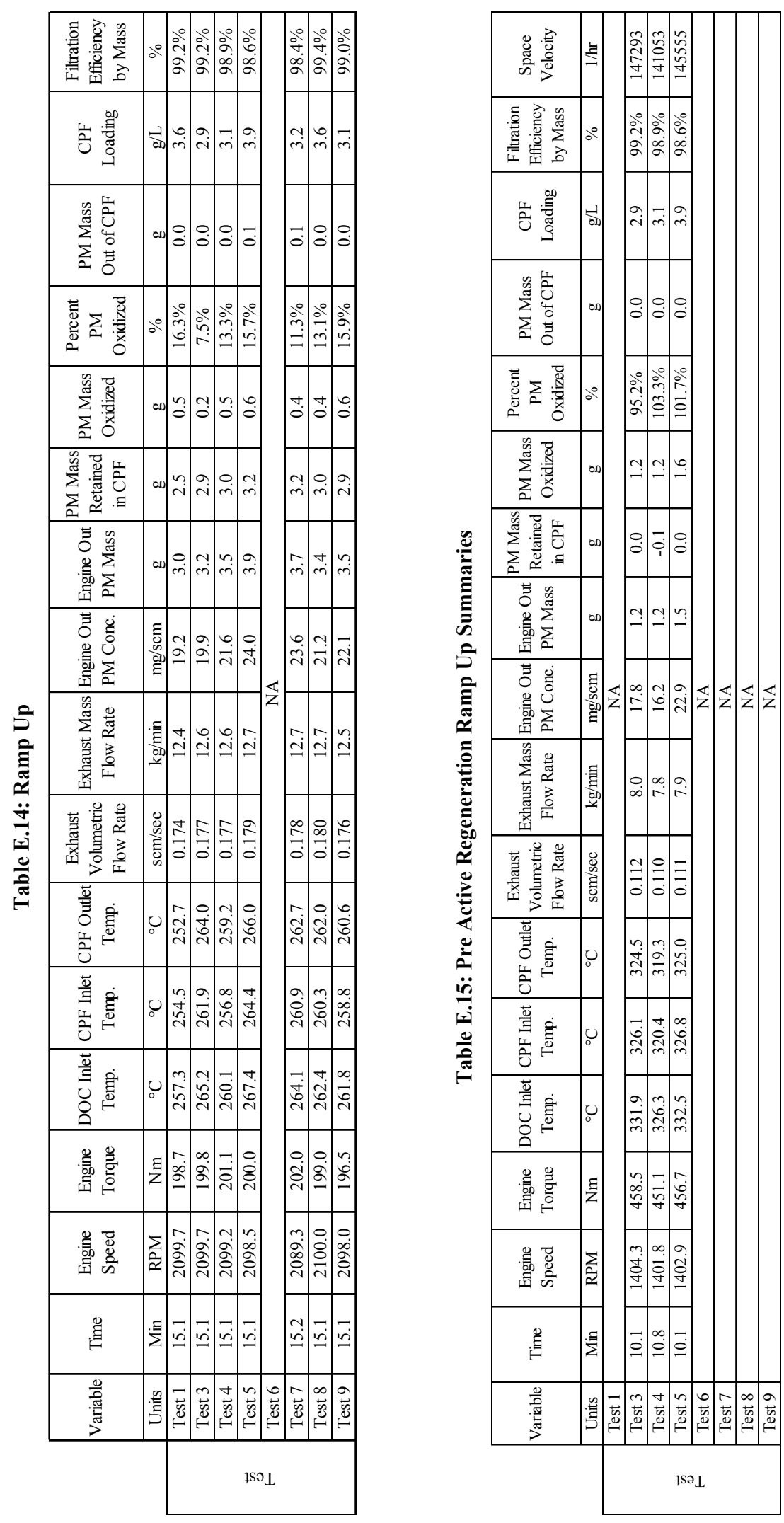


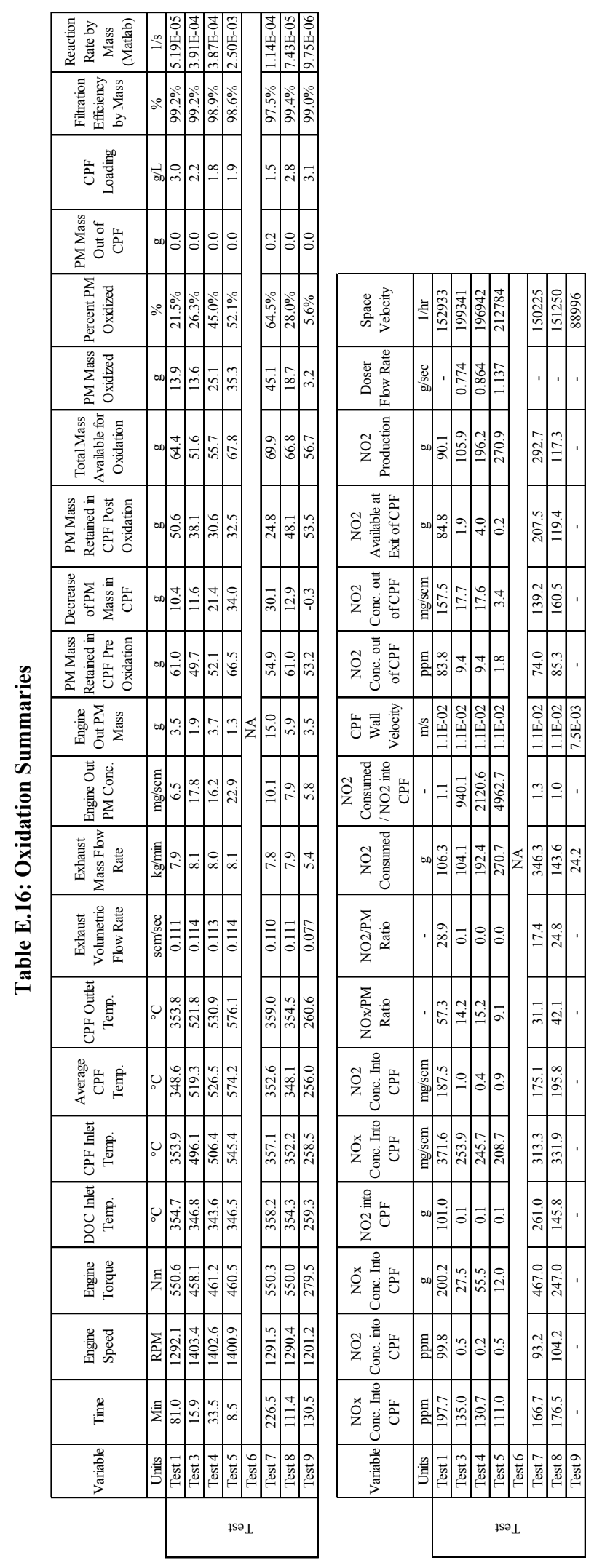




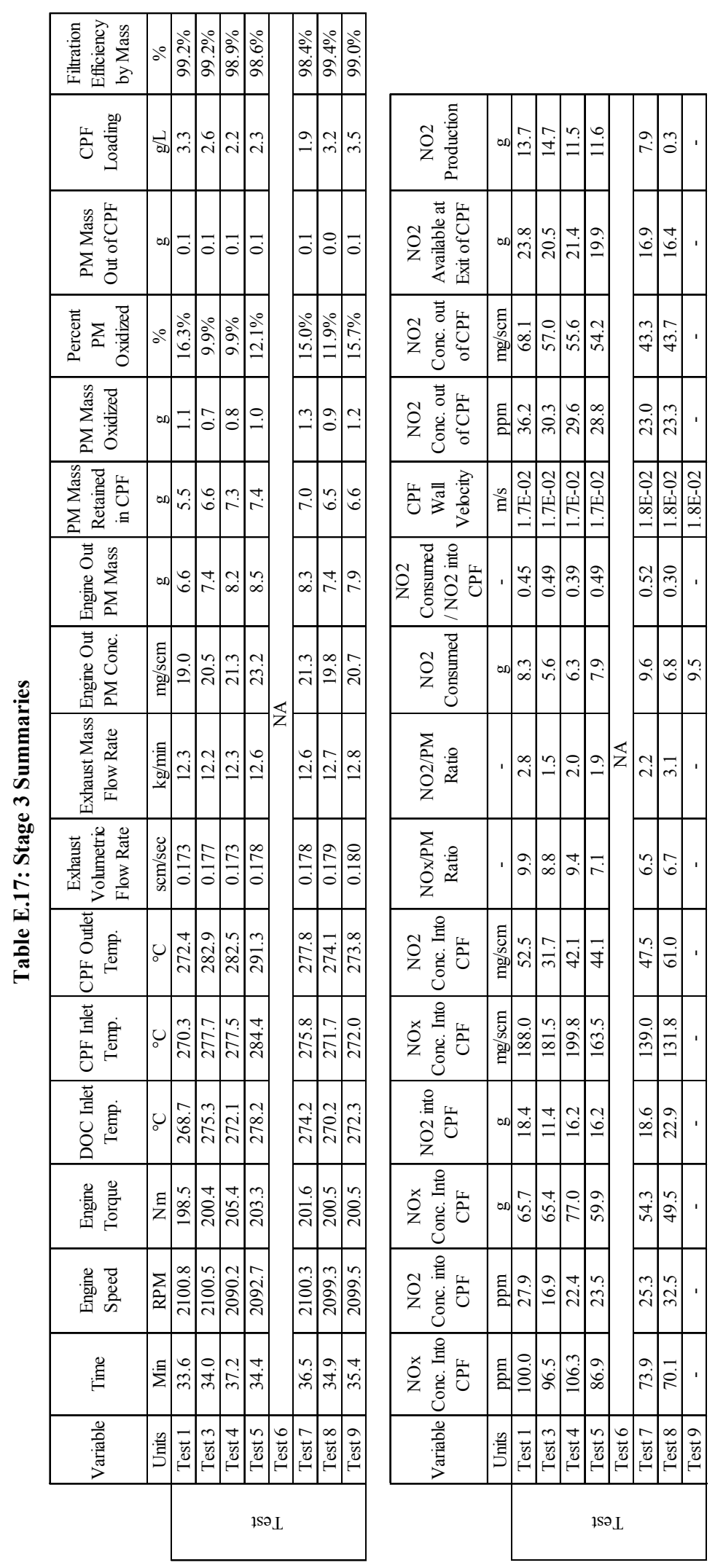




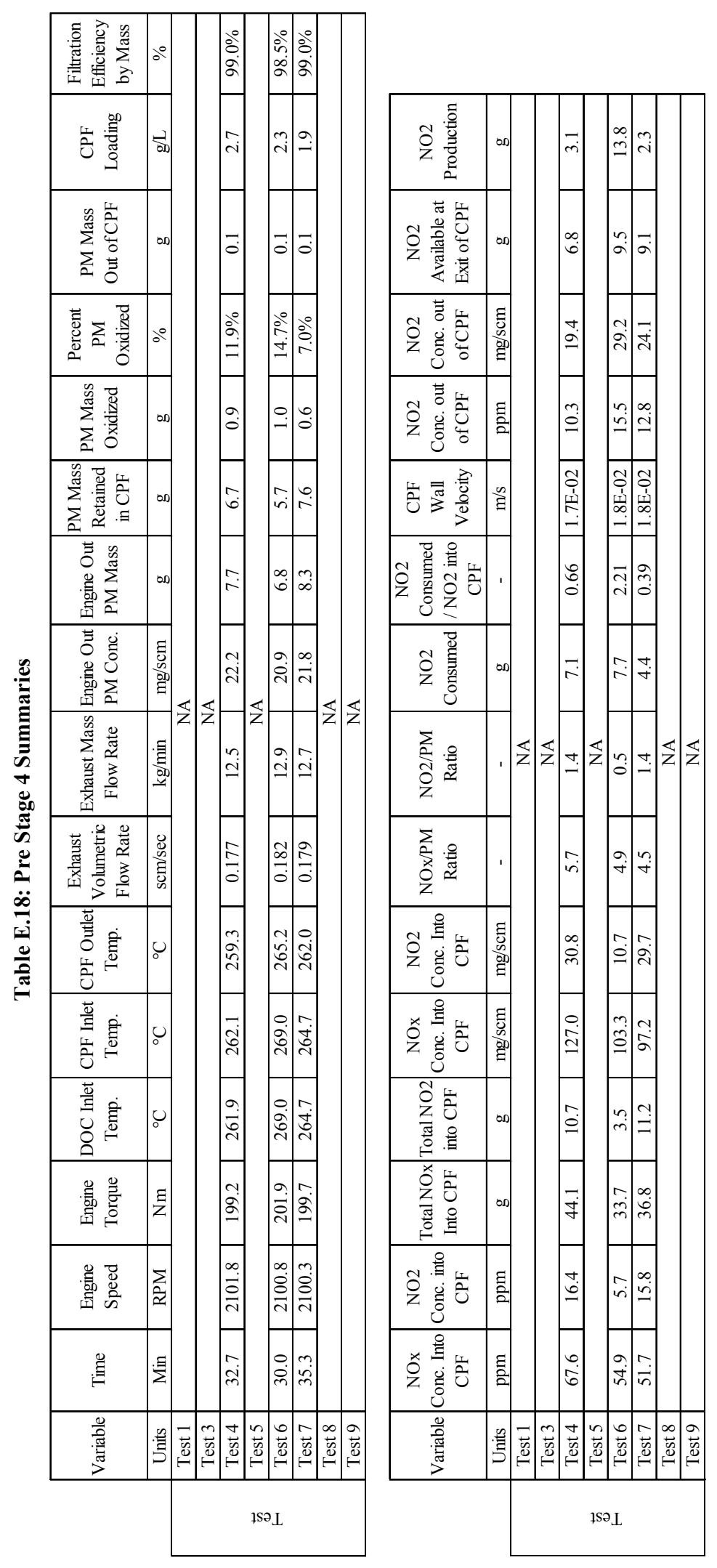




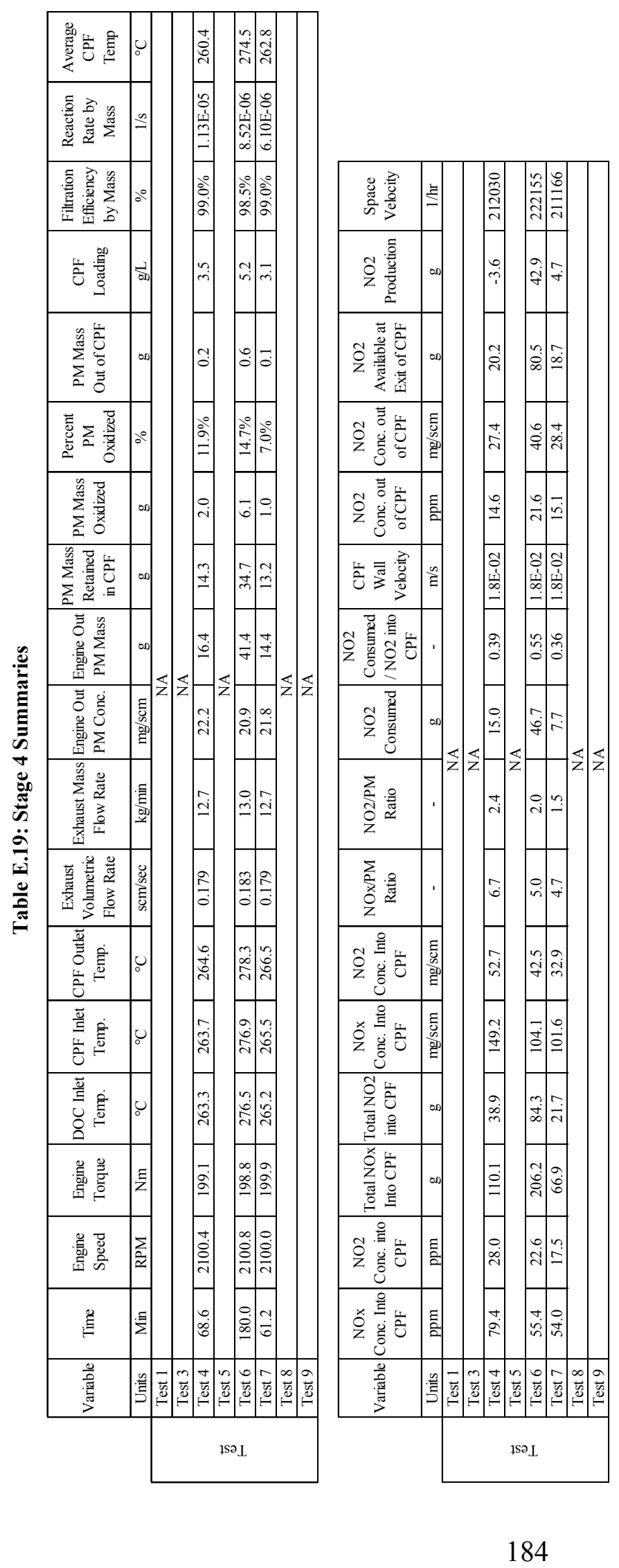




\section{Appendix F. PM Reaction Rate}

The experiments that were conducted during the PM distribution study were designed to be similar to the tests conducted by Hutton [3], Shiel [4], and Pidgeon [5]. The PM reaction rates that were found for the passive oxidation and active regeneration tests that were conducted were plotted with the PM reaction rates from the tests conducted by Hutton, Shiel, and Pidgeon, the result of which is shown in Figure F.1. The reaction rate calculations are explained by Hutton [3] and Pidgeon [5]. In Figure F.1, the " $X$ " data points represent the PM distribution tests that were discussed. The reaction rates for the PM distribution tests were similar to the reaction rates for the tests conducted by Hutton, Shiel, and Pidgeon. This indicates that the CPFs used for the PM distribution tests, that utilized a removable can, had PM oxidation kinetics that were similar to the production CPFs used for the tests conducted by Hutton, Shiel, and Pidgeon. Additionally, the test conducted on the ISB engine had similar reaction rates as the tests conducted on the ISL engine.

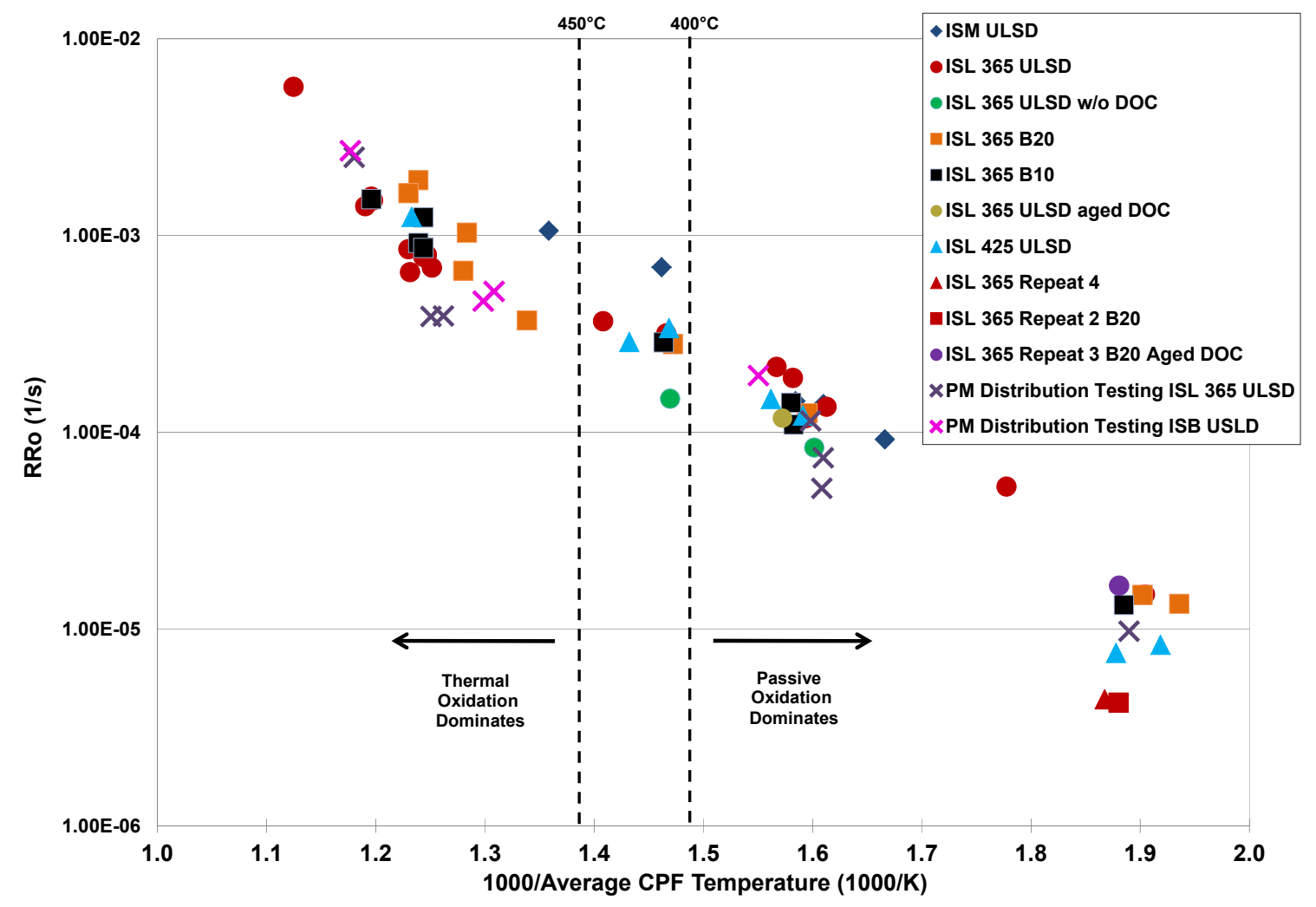

Figure F.1: PM Reaction Rate Plot

The reaction rates that were found during six PM distribution tests are compared against the model predicted reaction rates in Figure F.2. The plot shows the $\mathrm{NO}_{2}$ concentration on the $\mathrm{x}$-axis, and the average $\mathrm{CPF}$ temperature on the $\mathrm{y}$-axis. The contour lines shown represent the reaction rates as calculated by the model. The model was calibrated with data collected by Shiel [4] and Pidgeon [5]. Near each data point (the "X" points) is the 
calculated reaction rate, based on the experimental data, and the model predicted value. The data points near or above $0.0001 \mathrm{~s}^{-1}$ are passive oxidation or active regeneration experiments and the data points below $0.0001 \mathrm{~s}^{-1}$ are loading experiments. In general, the model predicted reaction rates are similar to the experimental reaction rates. Experimental variation is likely responsible for the differences between the experimental and modeled reaction rates. There was limited ULSD data available for the calibration of the model, which may also be the cause of the differences. The similarity between the experimental and modeled reaction rates provides further evidence that the PM oxidation kinetics for the PM distribution study were similar to the PM oxidation kinetics for the tests conducted by Shiel [4] and Pidgeon [5].

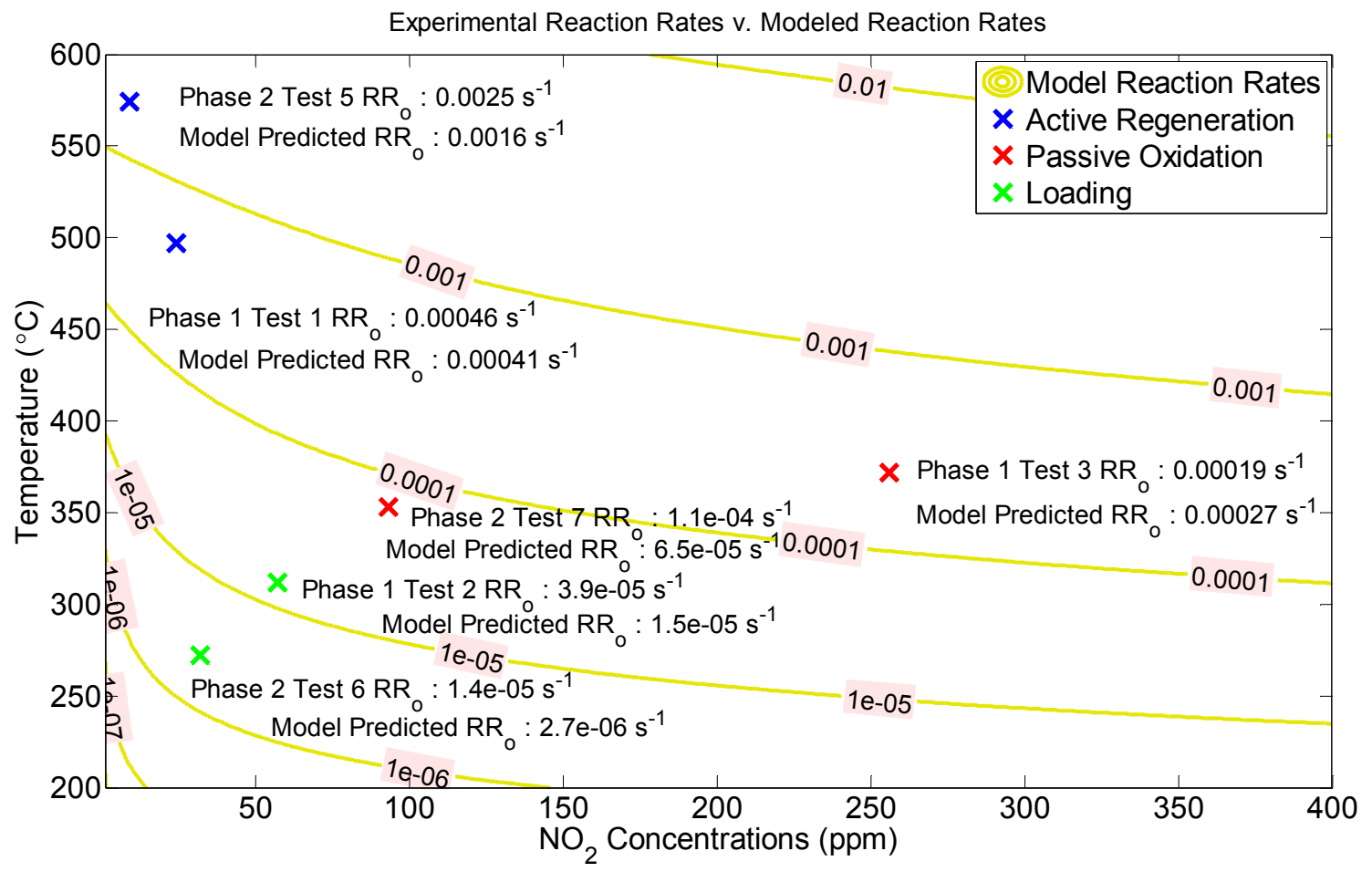

Figure F.2: Experimental Versus Model Predicted Reaction Rates 


\section{Appendix G. Substrate Mass Discrepancies}

Throughout the course of the PM distribution experiments, it was found that the substrates used in the experiments were not being completely cleaned out between tests. Table G.1 lists the differences that were found between the in- test measurements taken at the completion of the stage listed, and substrate only measurement taken prior to shipping the substrate. A positive value indicates that the substrate only measurement was higher than the in- test measurement. For Phase 1, the largest difference in the measurements was found during Test 4, with a value of 3.6 grams. The cause of this increase in mass is not known, but it was either residual PM that was not cleaned out between tests or ash loading. During Phase 1, the differences in the substrate mass measurements were not investigated since the residual PM was minimal. During Phase 2, differences in the mass measurement were between 1 and 18 grams. Substrate 2 from Phase 2 had the largest difference between the in- test and substrate only measurements, with differences between 15 and 18 grams. The large amount of difference in the mass measurements prompted an investigation of the cause of the difference.

Table G.1: Difference Between In- Test and Substrate Only Mass Measurements

\begin{tabular}{|c|c|c|c|c|c|}
\hline \multirow{2}{*}{\multicolumn{2}{|c|}{ Tests }} & \multirow{3}{*}{$\begin{array}{c}\text { Substrate } \\
1\end{array}$} & \multirow{3}{*}{$\begin{array}{c}\text { Stage } \\
\text { Loading }\end{array}$} & \multicolumn{2}{|c|}{ Difference } \\
\hline & & & & \multirow{2}{*}{$\frac{\text { grams }}{0.4}$} & \multirow{2}{*}{$\frac{\mathrm{g} / \mathrm{L}}{0.0}$} \\
\hline \multirow{4}{*}{ Phase 1} & Test 1 & & & & \\
\hline & Test 2 & 2 & Active Regeneration & 0.5 & 0.0 \\
\hline & Test 3 & 2 & Passive Oxidation & 2.1 & 0.2 \\
\hline & Test 4 & 2 & Active Regeneration & 3.6 & 0.3 \\
\hline \multirow{9}{*}{ Phase 2} & Test 1 & 1 & Loading & 1.4 & 0.1 \\
\hline & Test 2 & 2 & Cleanout & 15.5 & 0.9 \\
\hline & Test 3 & 3 & Active Regeneration & 6.0 & 0.4 \\
\hline & Test 4 & 4 & Active Regeneration & 7.7 & 0.5 \\
\hline & Test 5 & 2 & Active Regeneration & 18.3 & 1.1 \\
\hline & Test 6 & 1 & Loading & 5.4 & 0.3 \\
\hline & Test 7 & 3 & Passive Oxidation & 6.7 & 0.4 \\
\hline & Test 8 & 2 & Passive Oxidation & 16.4 & 1.0 \\
\hline & Test 9 & 4 & Balance Point & 9.5 & 0.6 \\
\hline
\end{tabular}

The differences in the substrate mass were investigated by performing an off-engine substrate cleanout. This was done by baking the substrates, at Cummins, using a transient cycle that has temperatures between 150 and $625^{\circ} \mathrm{C}$ and lasts for a total of 20 hours [37]. After the cleanout was completed, the substrates were weighed at Cummins, following the procedure in Appendix B.3. The differences between the initial and final masses of the four substrates used during Phase 2 are shown in Table G.2. 
Table G.2: Difference Between Initial and Final Mass at Cummins

\begin{tabular}{|c|c|c|c|}
\cline { 2 - 4 } \multicolumn{1}{c|}{} & Initial Mass $(\mathrm{g})$ & Final Mass $(\mathrm{g})$ & Difference $(\mathrm{g})$ \\
\hline Substrate 1 & 8369.5 & 8371.5 & 2.0 \\
\hline Substrate 2 & 8377.5 & 8390.9 & 13.4 \\
\hline Substrate 3 & 7497.9 & 7491.8 & -6.1 \\
\hline Substrate 4 & 7451.6 & 7454.8 & 3.2 \\
\hline
\end{tabular}

After weighing the substrates, Leigh Rogoski and her team at Cummins used a borescope to check each substrate for residual carbonaceous PM and ash loading. Each substrate was found to have no carbonaceous PM left after the cleanout. Substrates 1, 3, and 4 had a small amount of ash, if any, near the channel plugs. Substrate 1 had a difference of 2.0 grams, which is likely the ash loading combined with experimental error. Substrate 3 had a difference of -6.1 grams, which was caused by some damage to the edges of the substrate. Without the damage, substrate 3 would be expected to have a difference similar to substrates 1 and 4, since it was used for approximately the same amount of time. Substrate 4 had a difference of 3.2 grams, which is the combined effect of ash loading and experimental variation. Substrate 2 had a difference of 13.4 grams which is caused by ash loading. The borescope images showed ash loading throughout substrate 2. The additional ash loading in substrate 2 was caused by the 70 hours of research performed on the substrate prior to the first PM distribution experiment being performed.

A literature search was conducted to determine if 13 grams of ash loading is reasonable for 70 hours of CPF operation. In reference [48], Aravelli, et al., used a 1992 Cummins N14 engine to perform ash loading on a substrate. They correlated the amount of oil consumed during testing with the amount of ash loaded in a substrate, and a linear trend was found. Based on their work, after 70 hours of operation approximately 3000 grams of oil was consumed. This correlates to 28 grams of ash accumulation in the particulate filter. For this test, the crankcase blowby gases were routed into the intake of the engine which will increase the engine out ash concentration. Another study by Manni, et al., [49], used a 1.9 L passenger car engine to study ash loading on a substrate. Their work shows that after 70 hours of operation, 900 grams of oil would be consumed. This correlates to 4 grams of ash loading. Both of these engines are drastically different than the 2007 Cummins ISL that was used for the Phase 2 research, but the data shows that between 4 and 28 grams of ash loading can be expected after 70 hours of operation. This indicates that the 13 grams of ash in substrate 2 is reasonable for the number of hours it was used.

The data in Table G.2 suggest that the differences in Table G.1 are caused by both residual PM that was not cleaned out between tests, and ash loading. The difference between the initial and final mass for each of the four substrates were subtracted from the differences found between the in-test and substrate only measurements, and the results are shown in Table G.3. For the tests using substrate 3, the differences between the intest and substrate only measurements were corrected using a value of 2.6 grams, which is the average of the initial and final mass differences for substrates 1 and 4 . The 
differences between the in- test and substrate only mass measurements were between -0.6 and 6.3 grams, which is a result similar to the results found in Table G.1 for the Phase 1 experiments. This indicates that all of the substrates had a similar amount of residual PM that was not cleaned out between tests.

Table G.3: Corrected Differences Between In-Test and Substrate Only Measurements

\begin{tabular}{|c|c|c|c|c|c|}
\hline \multicolumn{2}{|c|}{ Tests } & \multirow{2}{*}{ Substrate } & \multirow{2}{*}{ Stage } & \multicolumn{2}{c|}{ Difference } \\
\cline { 5 - 6 } & & & grams & $\mathrm{g} / \mathrm{L}$ \\
\hline \multirow{4}{*}{ Phase 2 } & Test 1 & 1 & Loading & -0.6 & 0.0 \\
\cline { 2 - 6 } & Test 2 & 2 & Cleanout & 2.1 & 0.1 \\
\cline { 2 - 6 } & Test 3 & 3 & Active Regeneration & 3.4 & 0.2 \\
\cline { 2 - 6 } & Test 4 & 4 & Active Regeneration & 4.5 & 0.3 \\
\cline { 2 - 6 } & Test 5 & 2 & Active Regeneration & 4.9 & 0.3 \\
\cline { 2 - 6 } & Test 6 & 1 & Loading & 3.4 & 0.2 \\
\cline { 2 - 6 } & Test 7 & 3 & Passive Oxidation & 4.1 & 0.2 \\
\cline { 2 - 6 } & Test 8 & 2 & Passive Oxidation & 3.0 & 0.2 \\
\cline { 2 - 6 } & Test 9 & 4 & Balance Point & 6.3 & 0.4 \\
\hline
\end{tabular}

The substrate mass measurements that were used to calculate the PM density in the Phase 2 data from the TAS7000 were not adjusted to account for the ash loading. This is because the TAS7000 was measuring the total amount of constituent retained in the substrate, which includes PM and ash. In future tests, care will have to be taken to only measure the PM loading in the substrate, if direct PM density measurements are to be taken. This is especially important if a substrate is used for more than 50 hours of research, when the ash loading may be more than 2 or 3 grams. 


\section{Appendix H. Test Pressure Drop Curves}

This appendix presents the pressure drop curves for each test that was conducted. Although the pressure drop across the CPF was not of critical importance during this research, it is a good measure of the repeatability of the tests. The peak loading in the $\mathrm{CPF}$ should result in similar pressure drops.

\section{Phase 1 Test 1}

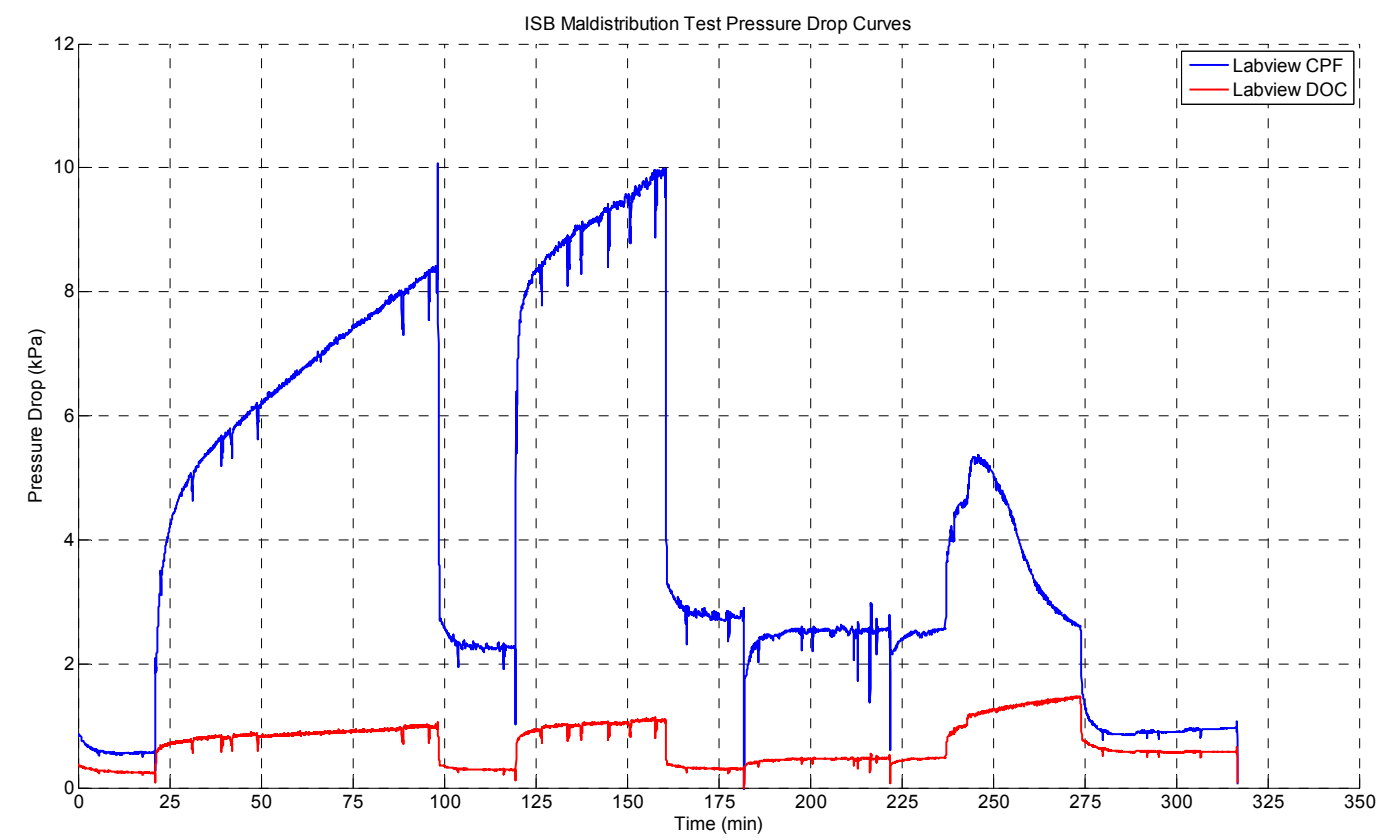

Figure H.1: Phase 1 Test 1 Pressure Drop Curves 


\section{Phase 1 Test 2}

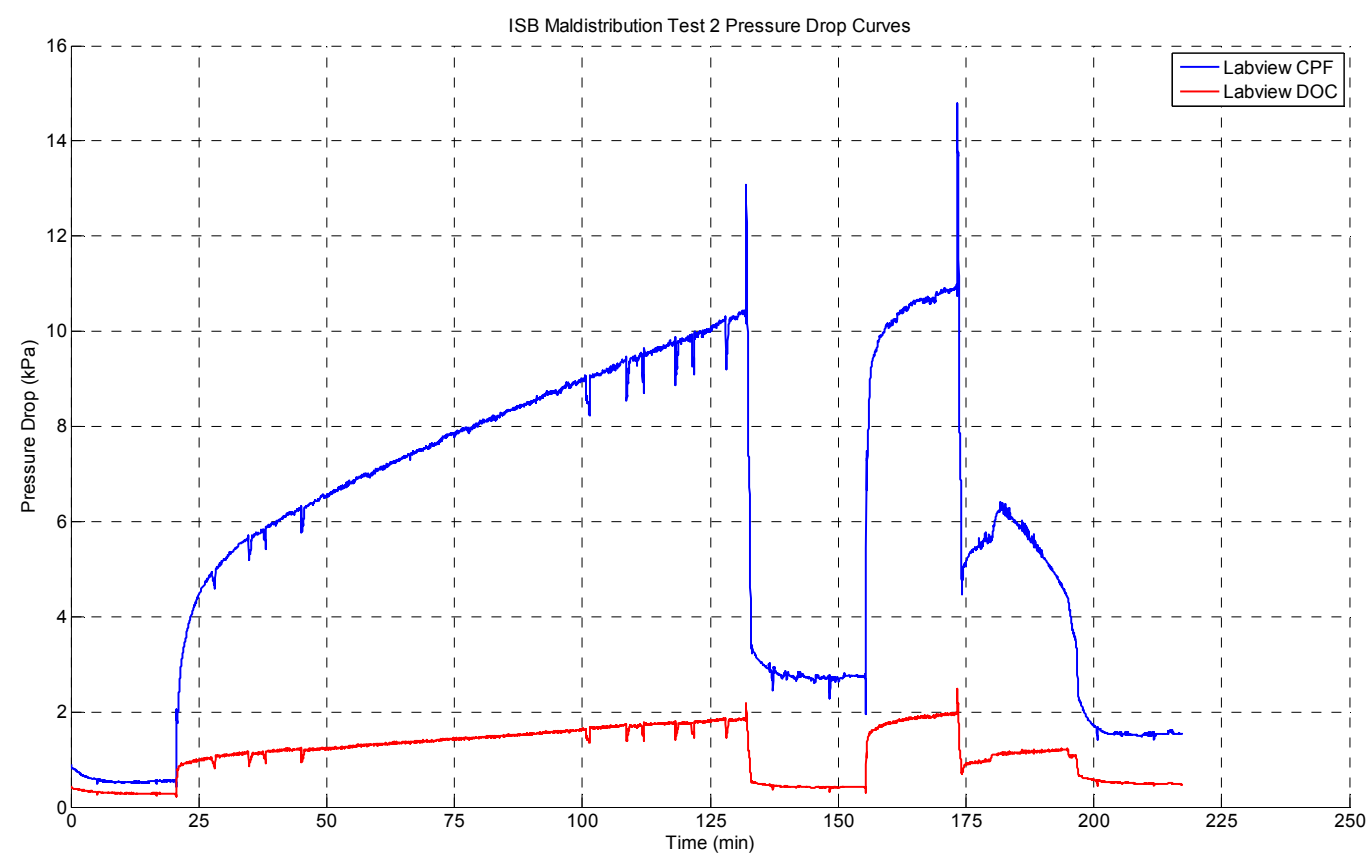

Figure H.2: Phase 1 Test 2 Pressure Drop Curves

\section{Phase 1 Test 3}

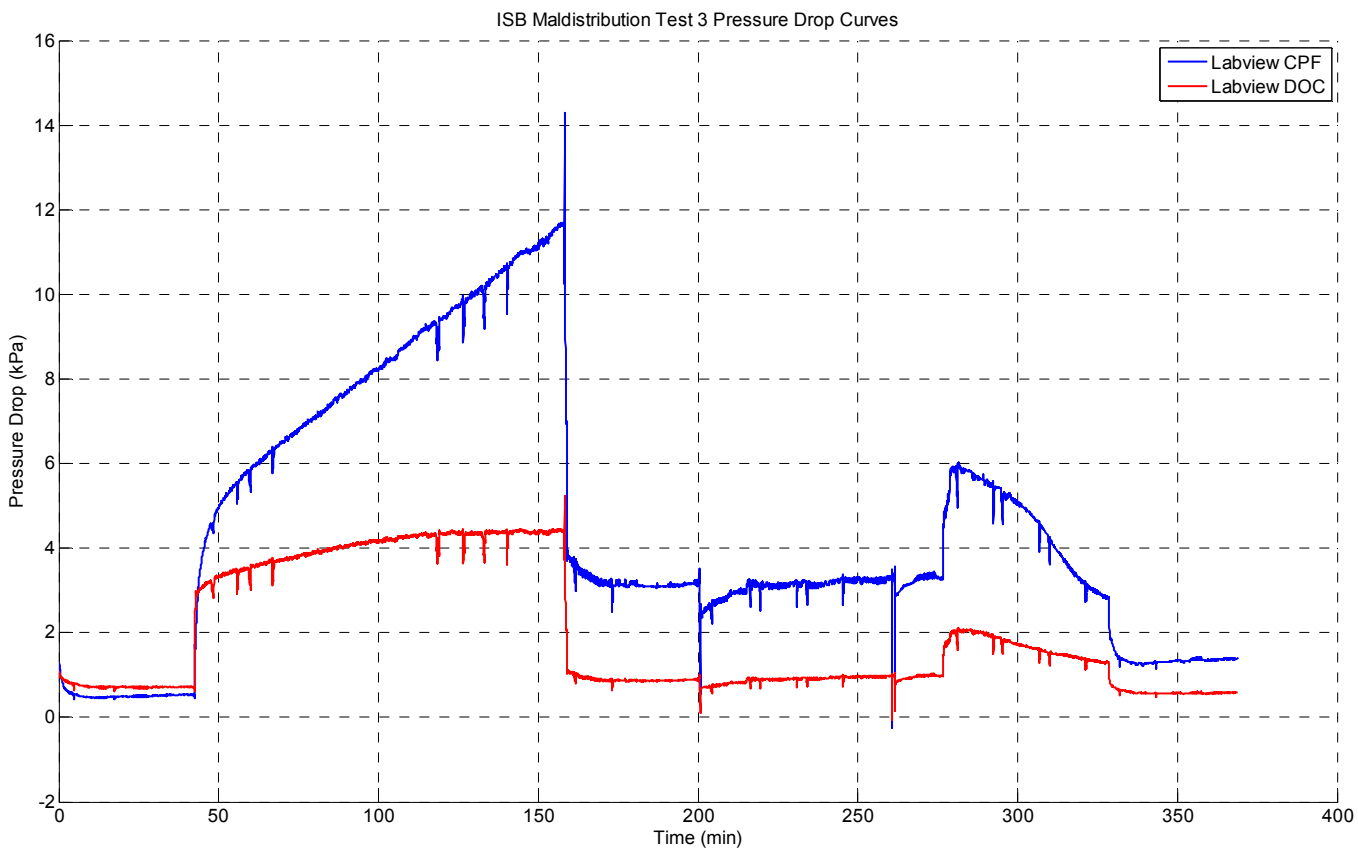

Figure H.3: Phase 1 Test 3 Pressure Drop Curves 


\section{Phase 1 Test 4}

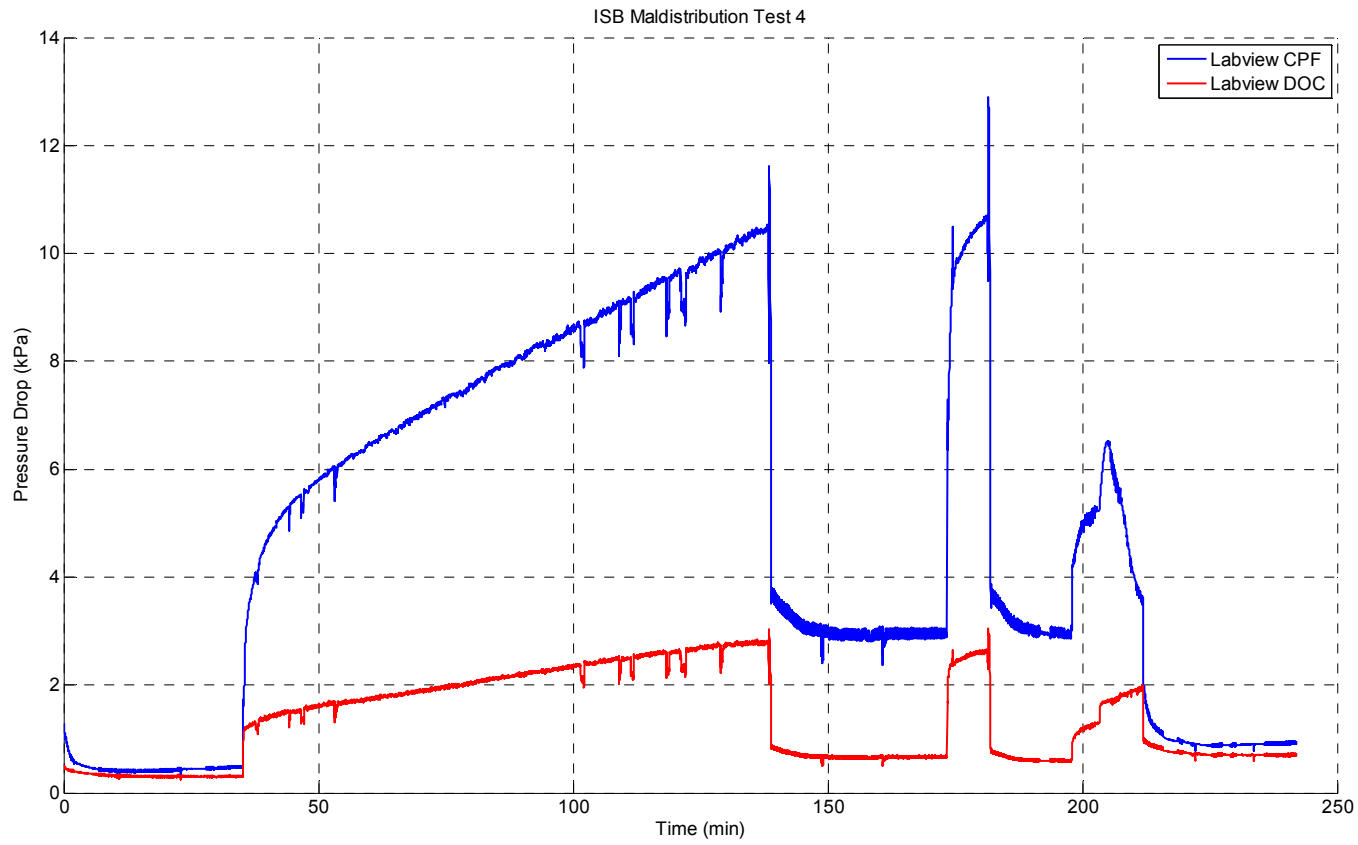

Figure H.4: Phase 1 Test 4 Pressure Drop Curves

\section{Phase 2 Test 1}

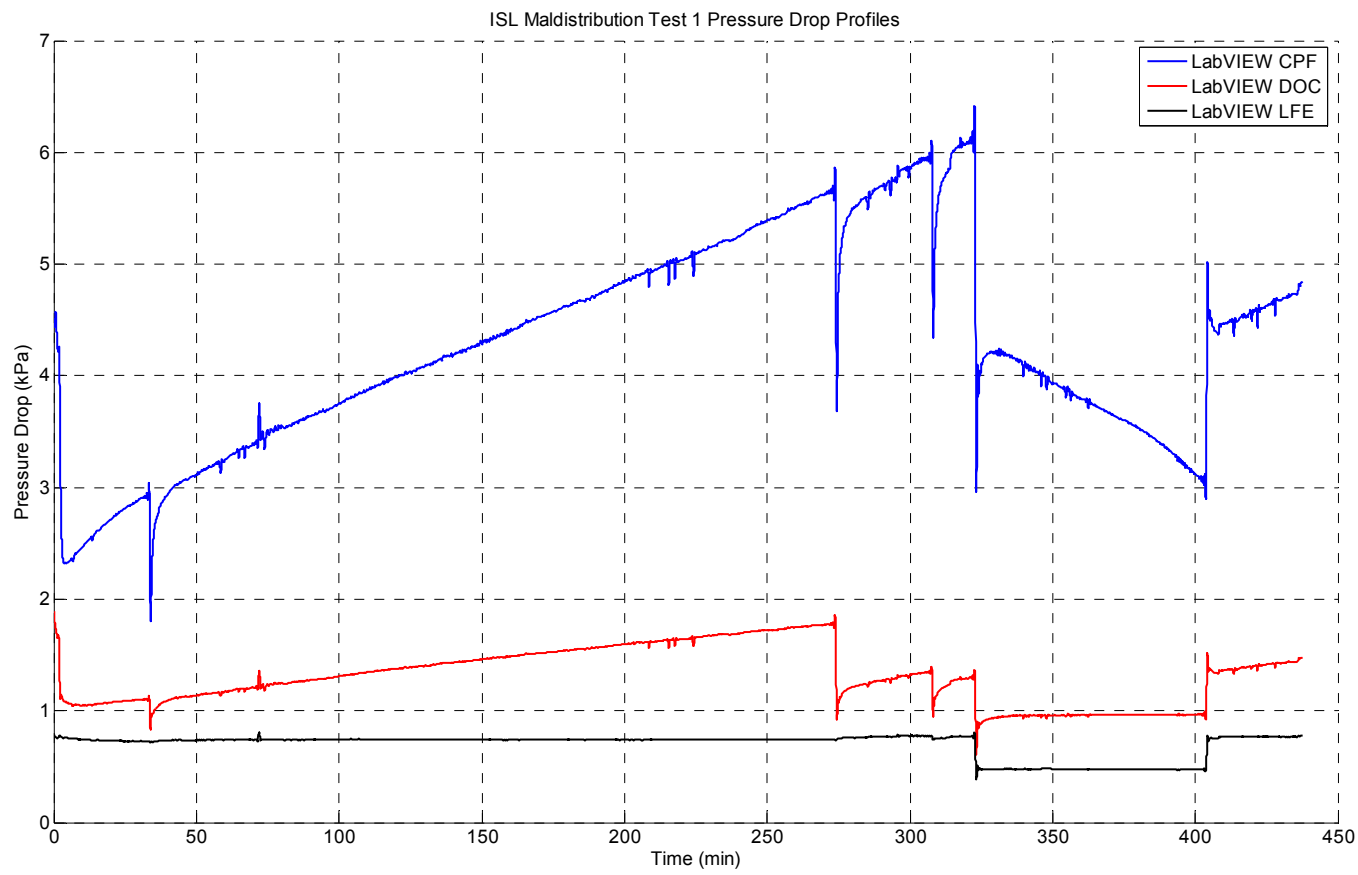

Figure H.5: Phase 2 Test 1 Pressure Drop Curves 


\section{Phase 2 Test 2}

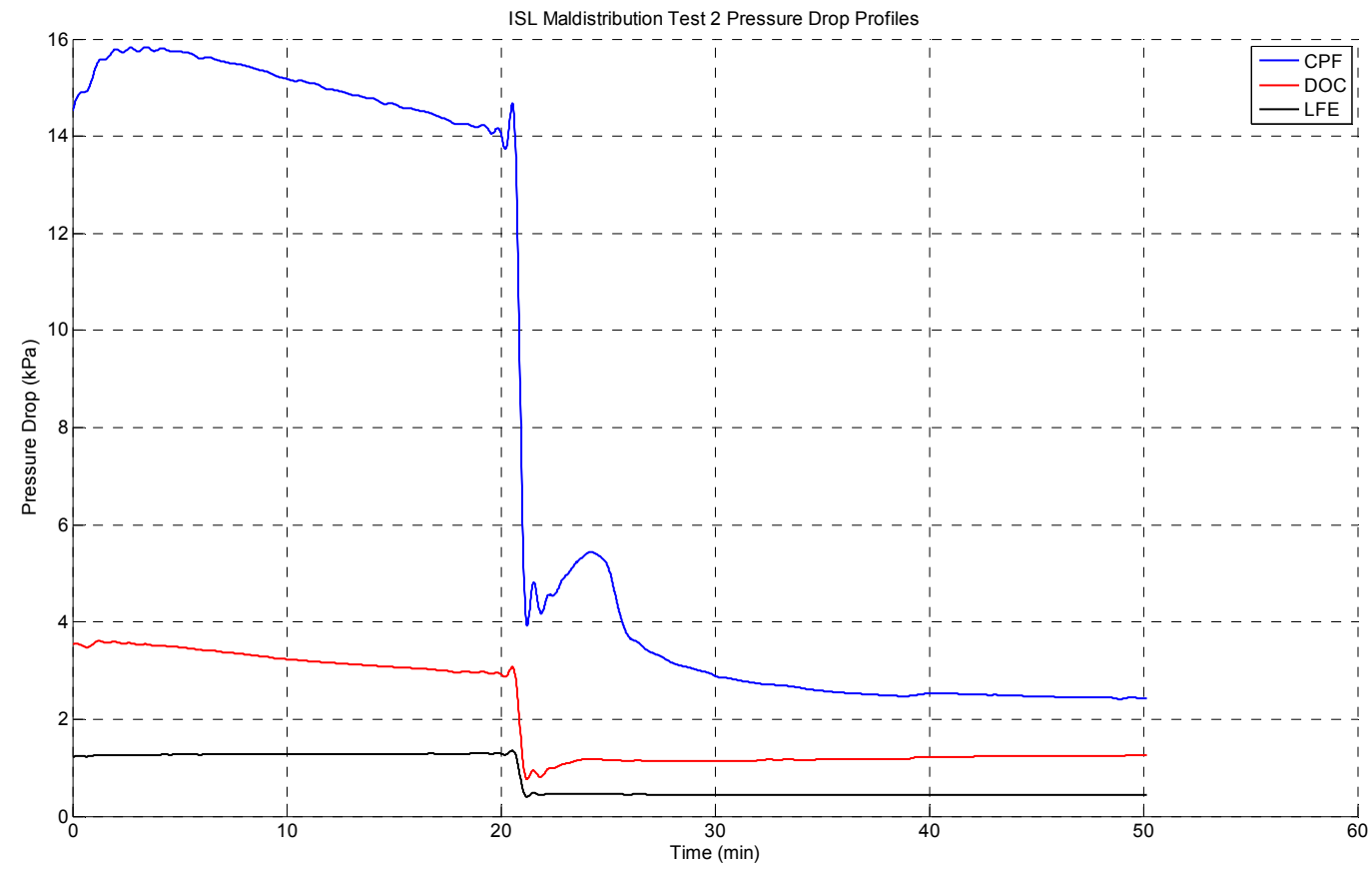

Figure H.6: Phase 2 Test 2 Pressure Drop Curves

\section{Phase 2 Test 3}

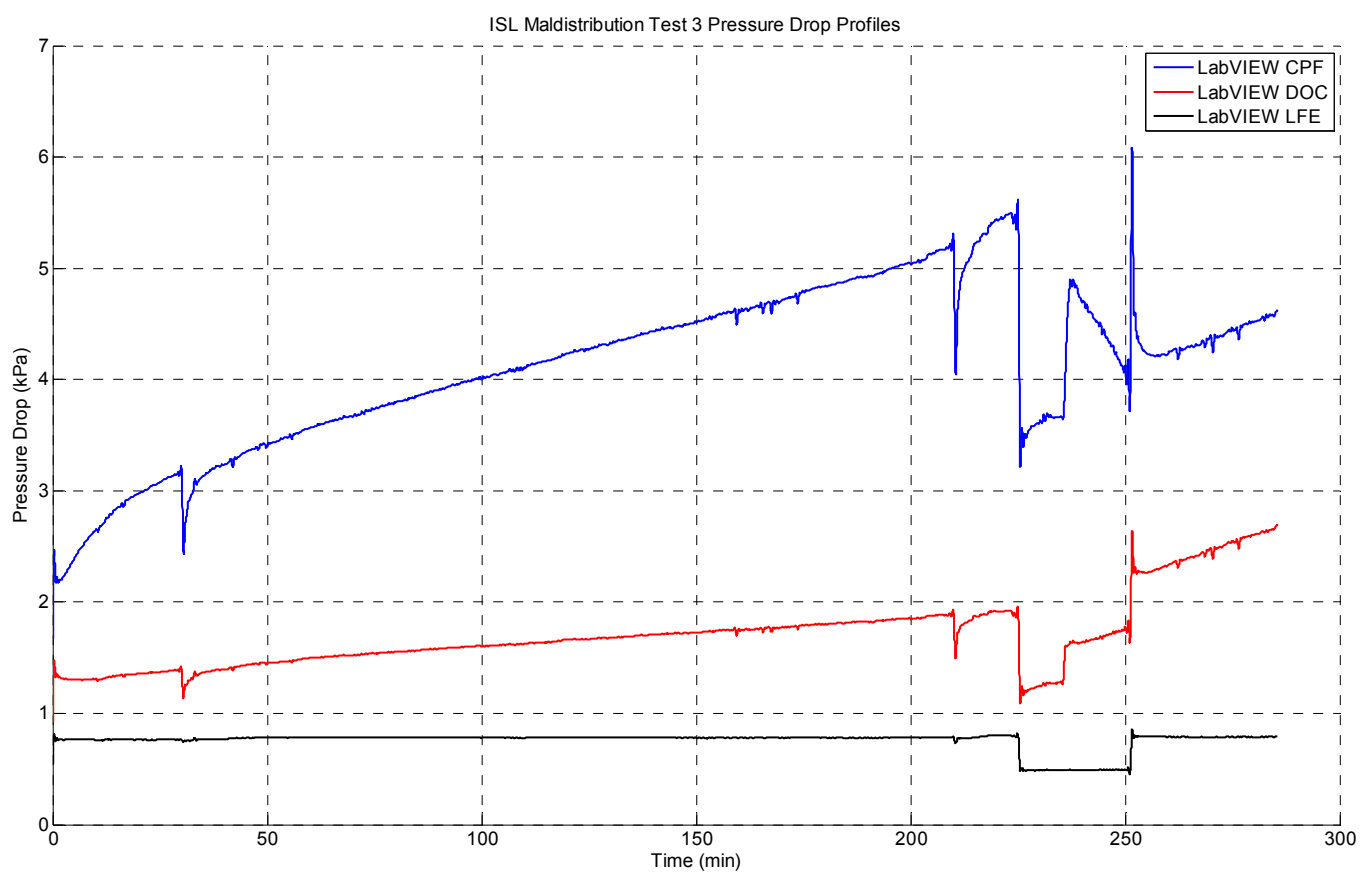

Figure H.7: Phase 2 Test 3 Pressure Drop Curves 


\section{Phase 2 Test 4}

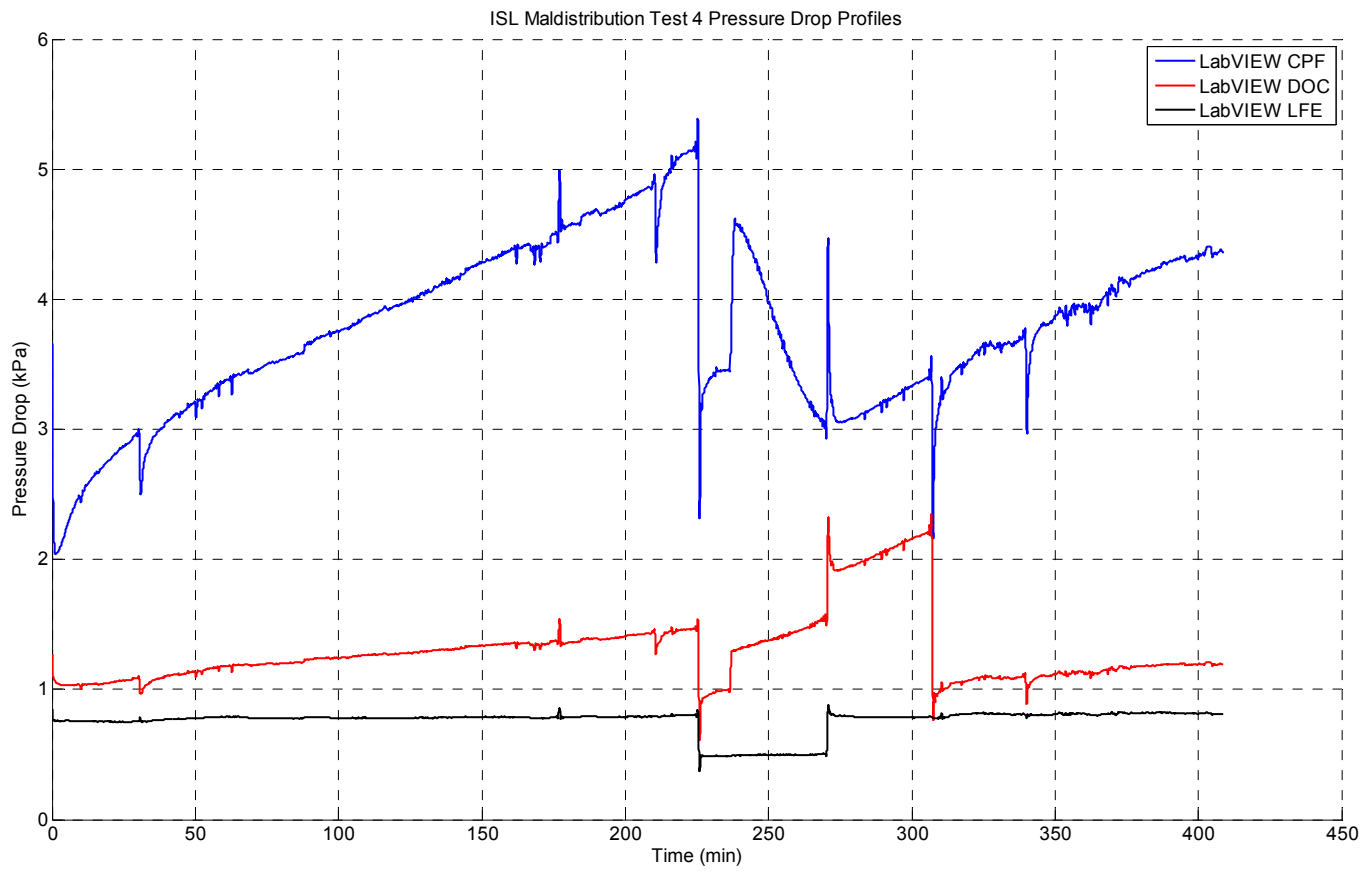

Figure H.8: Phase 2 Test 4 Pressure Drop Curves

\section{Phase 2 Test 5}

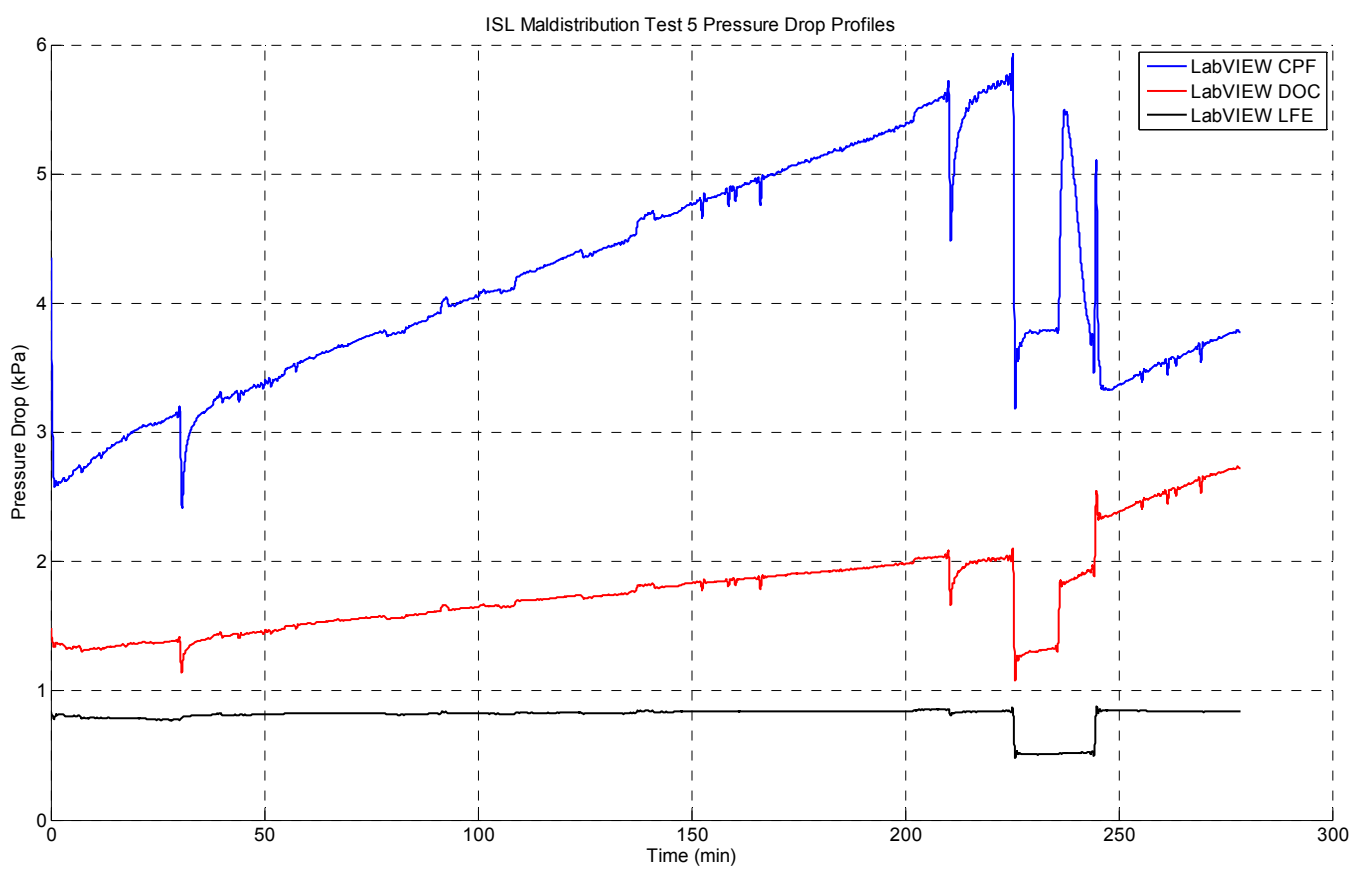

Figure H.9: Phase 2 Test 5 Pressure Drop Curves 


\section{Phase 2 Test 6}

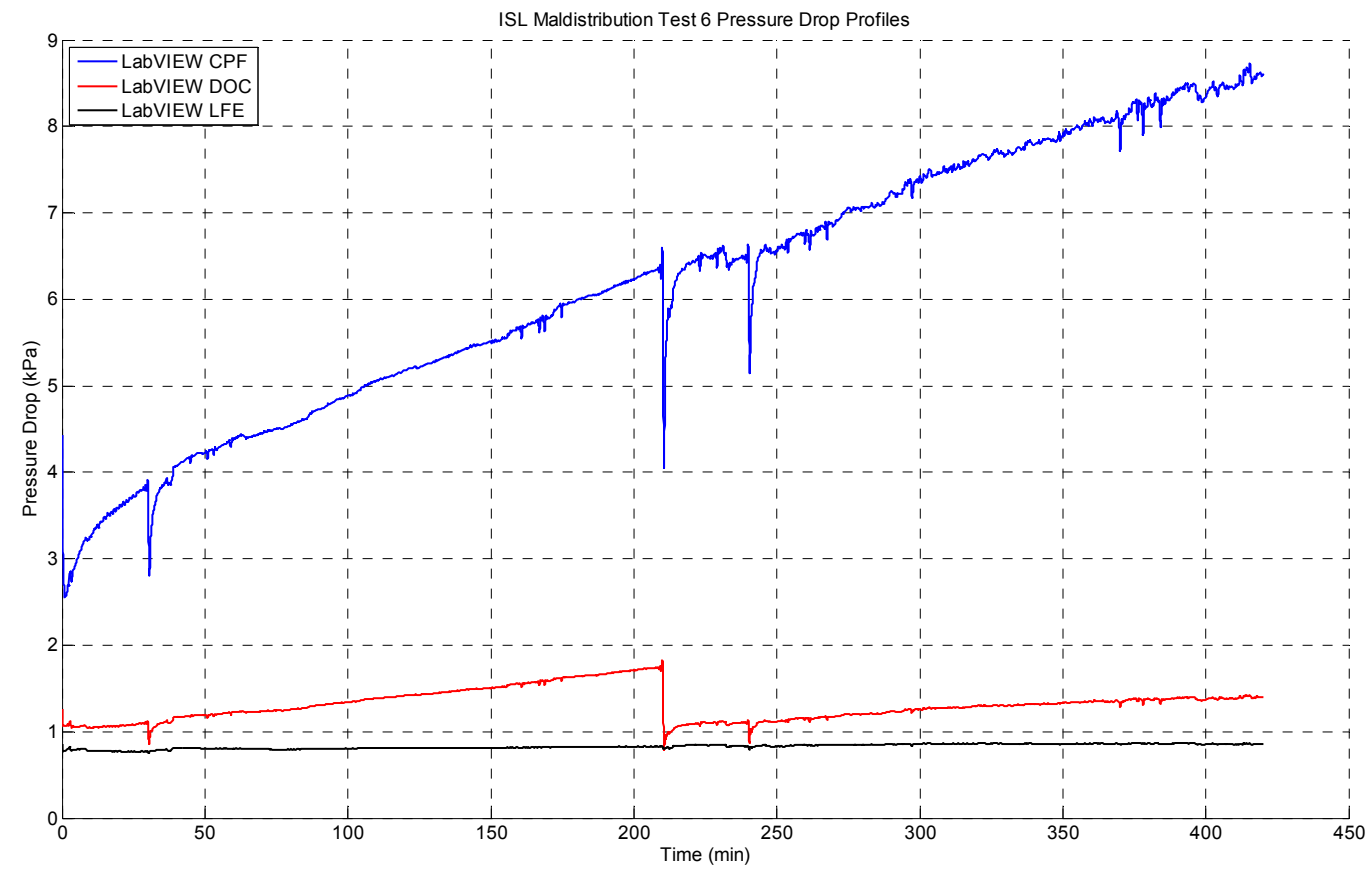

Figure H.10: Phase 2 Test 6 Pressure Drop Curves

\section{Phase 2 Test 7}

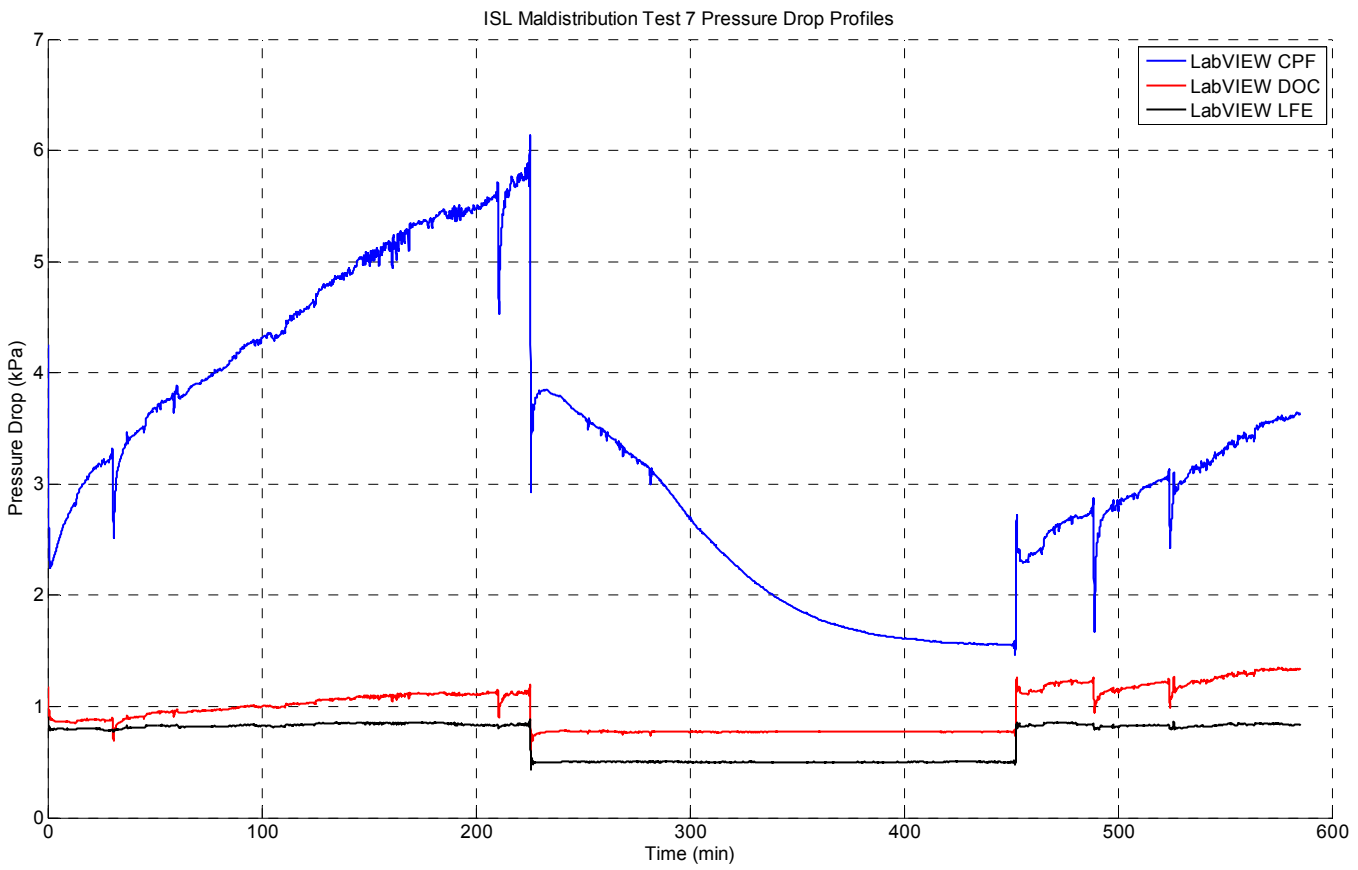

Figure H.11: Phase 2 Test 7 Pressure Drop Curves 


\section{Phase 2 Test 8}

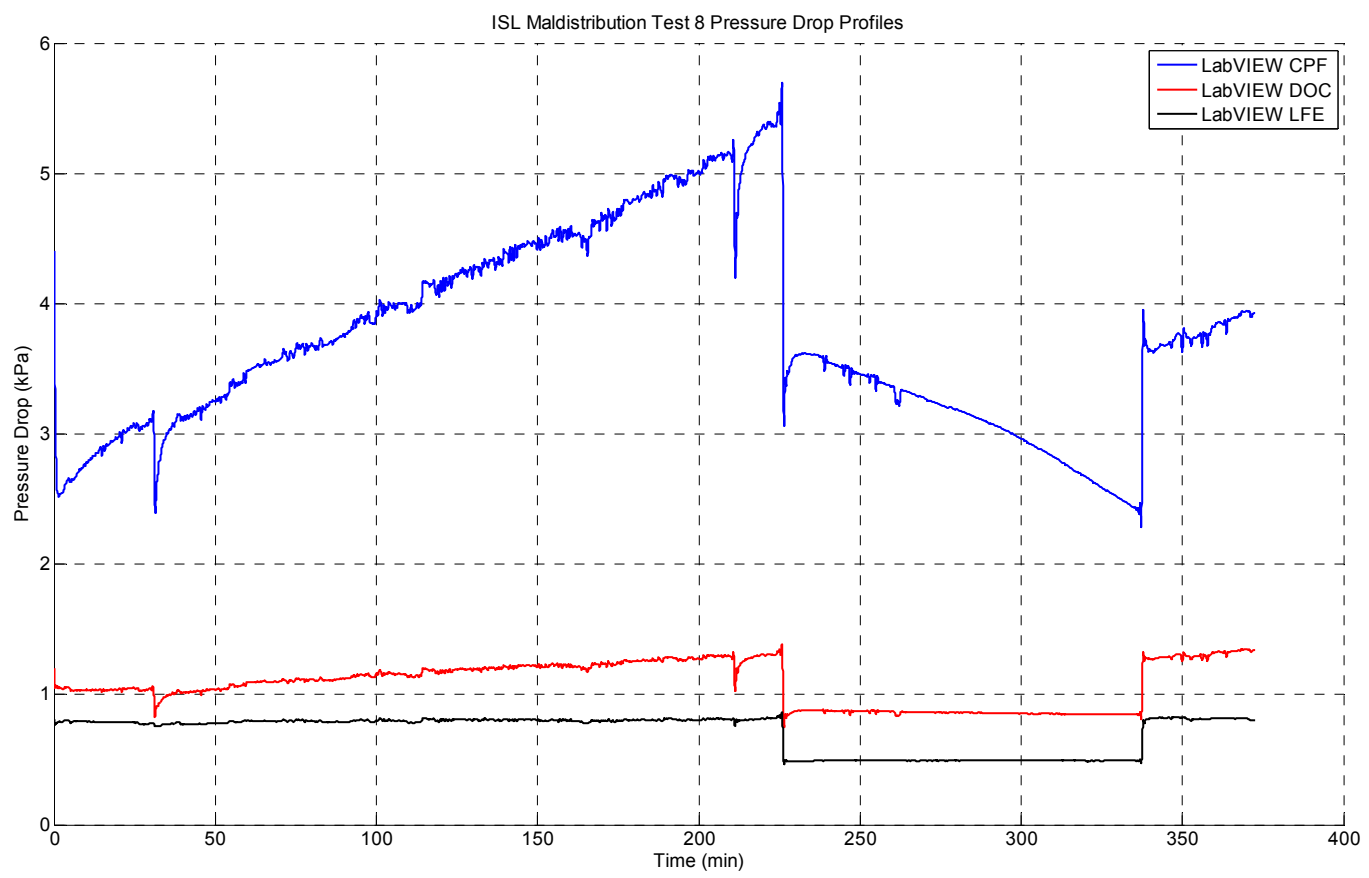

Figure H.12: Phase 2 Test 8 Pressure Drop Curves

Phase 2 Test 9

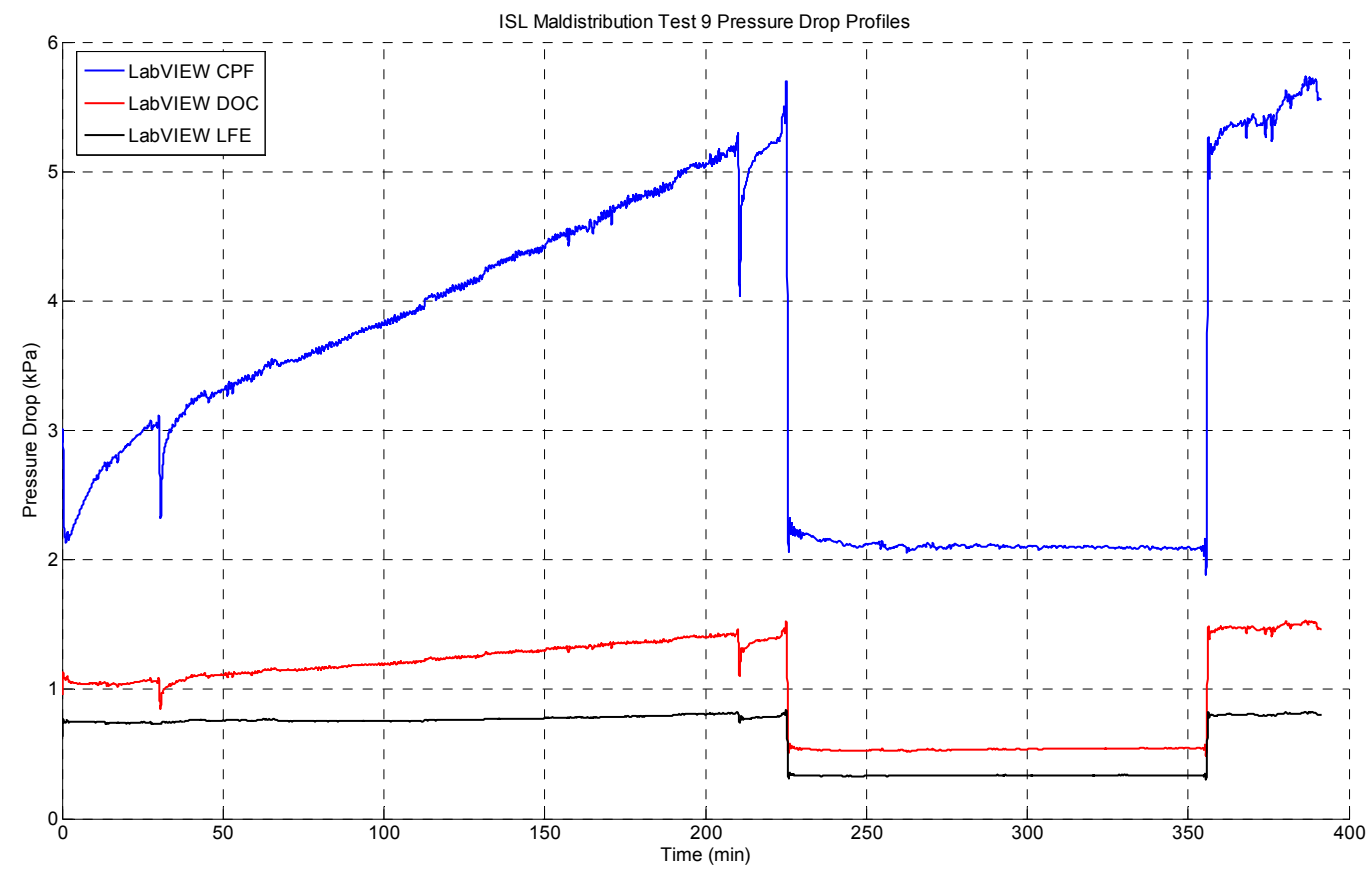

Figure H.13: Phase 2 Test 9 Pressure Drop Curves 


\section{Appendix I. Average CPF Temperatures}

This appendix presents the average temperature distribution in the CPF. The data in the plots is the average of the CPF temperatures at each axial and radial location for the last 5 minutes of a particular stage of the test. Any missing data points are caused by broken thermocouples. Only the plots for the stages that represent the goals of the individual test are shown. For example, if the test was designed to investigate passive oxidation trends, only the temperature distribution for the passive oxidation event is shown.

\section{Phase 1 Test 1}

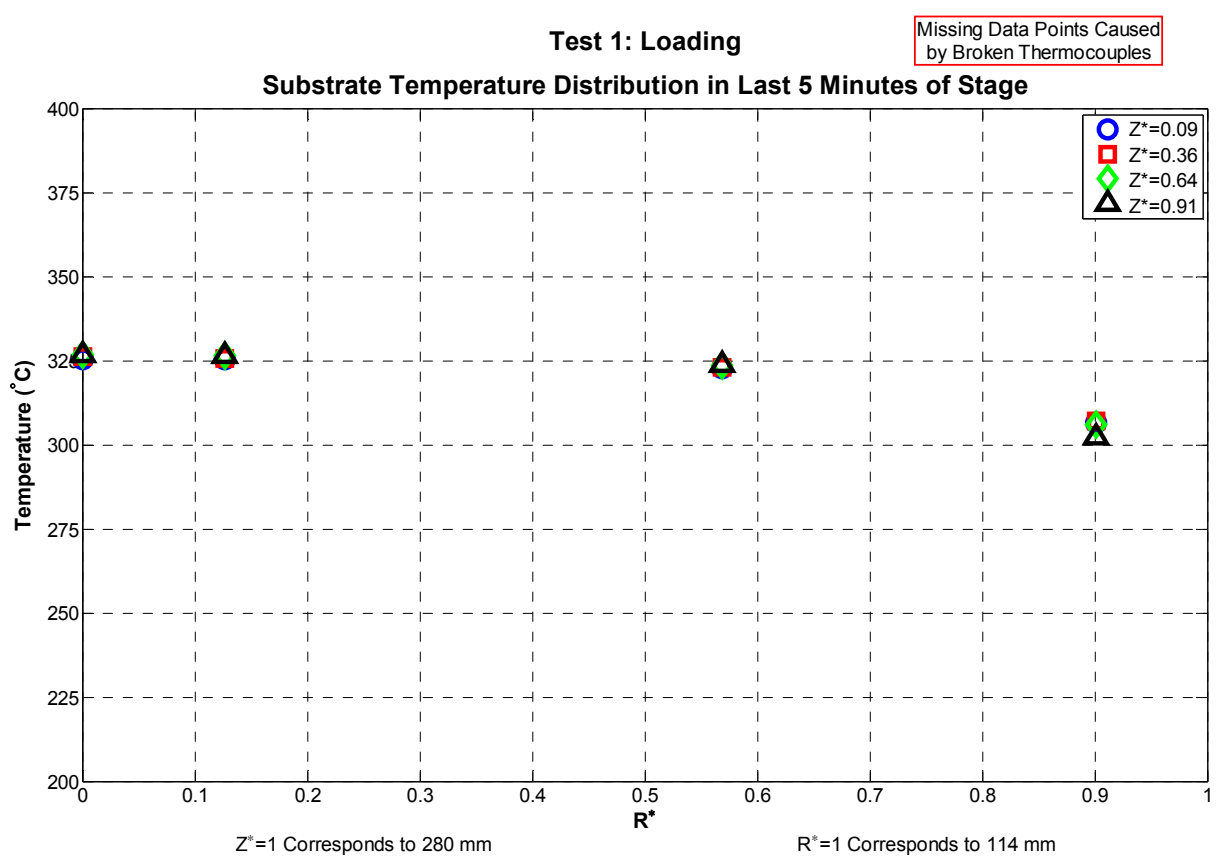

Figure I.1: Radial Temperature Distribution for Phase 1 Test 1 Loading 


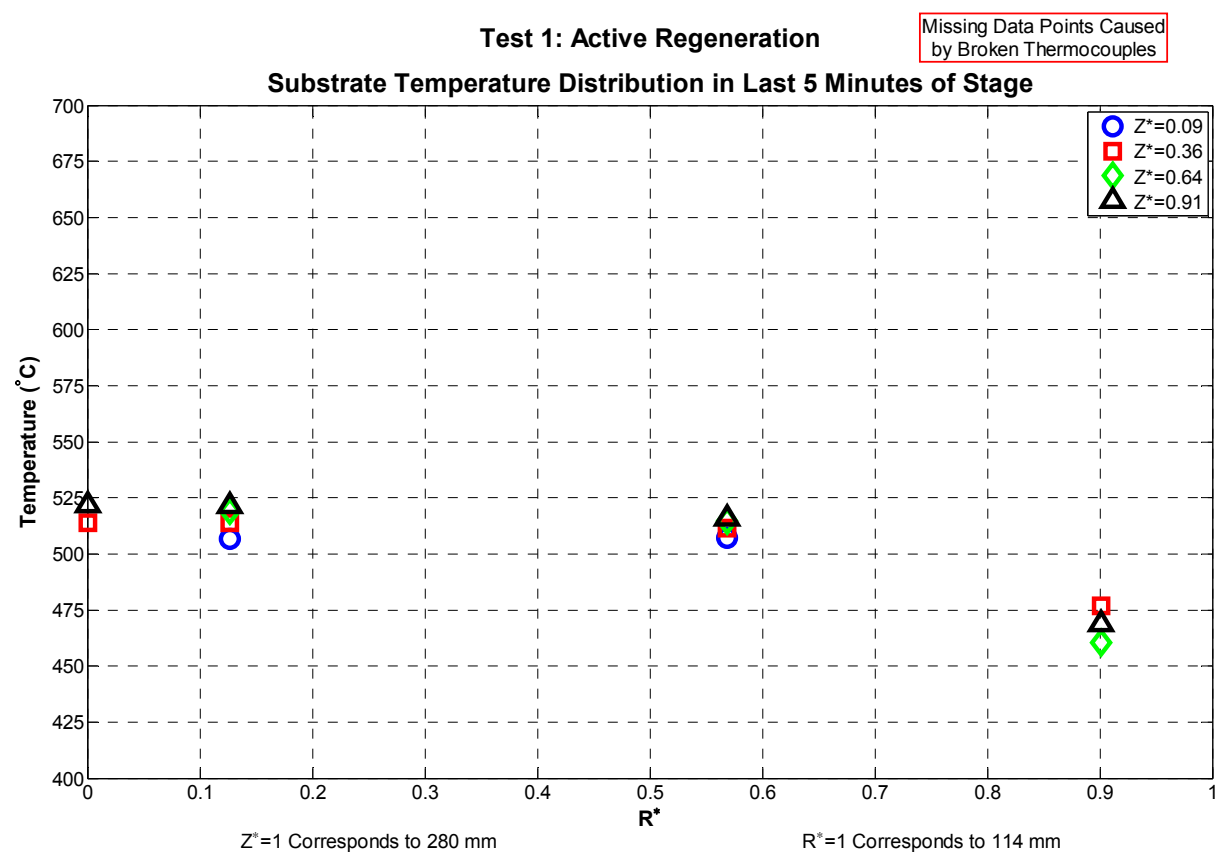

Figure I.2: Radial Temperature Distribution for Phase 1 Test 1 Active Regeneration

\section{Phase 1 Test 2}

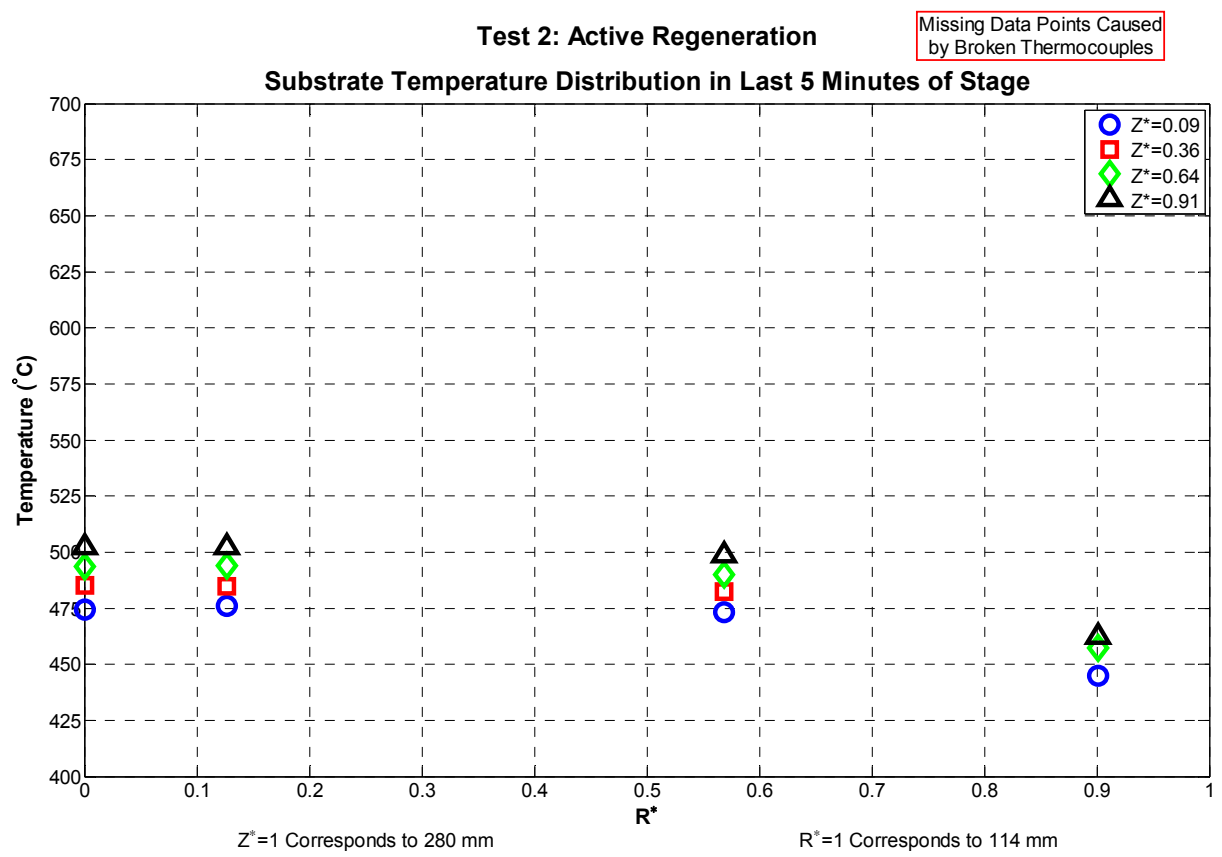

Figure I.3: Radial Temperature Distribution for Phase 1 Test 2 Active Regeneration 


\section{Phase 1 Test 3}

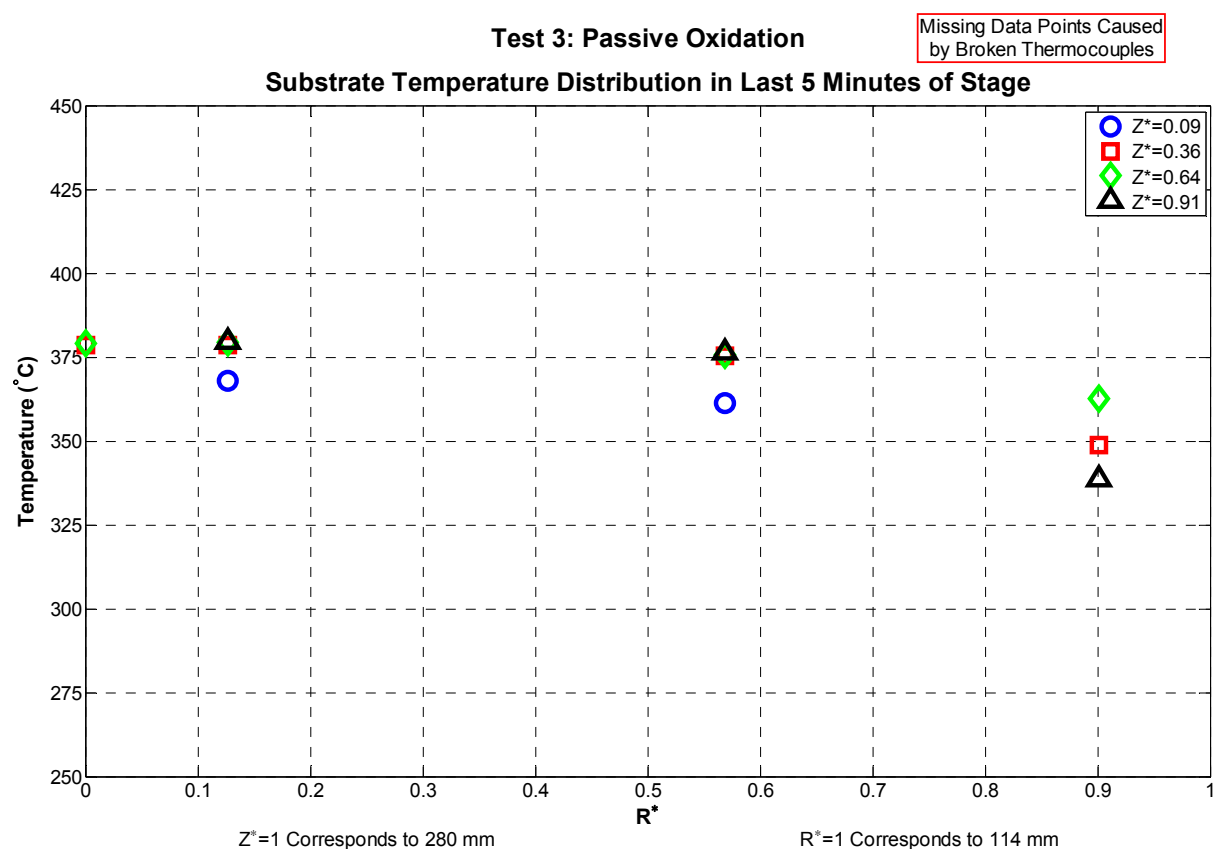

Figure I.4: Radial Temperature Distribution for Phase 1 Test 3 Passive Oxidation

\section{Phase 1 Test 4}

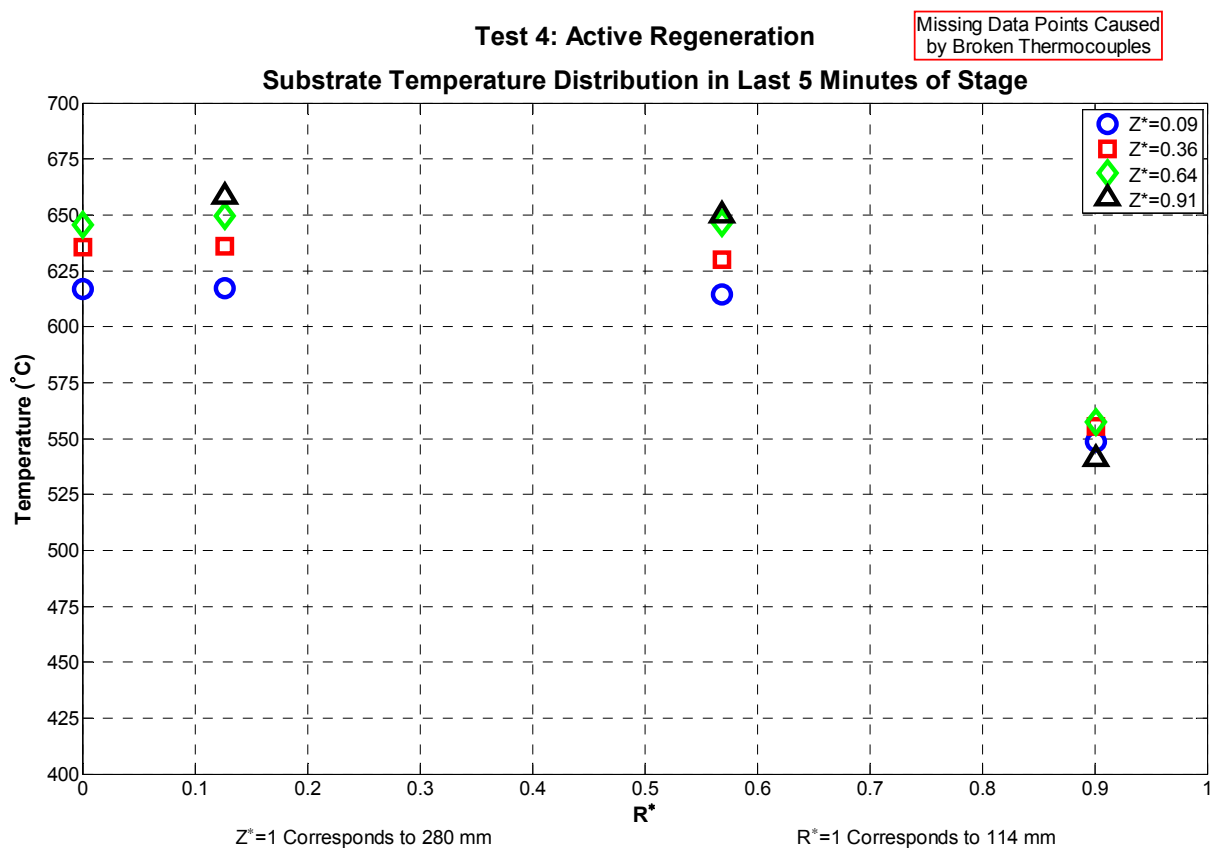

Figure I.5: Radial Temperature Distribution for Phase 1 Test 4 Active Regeneration 


\section{Phase 2 Test 1}

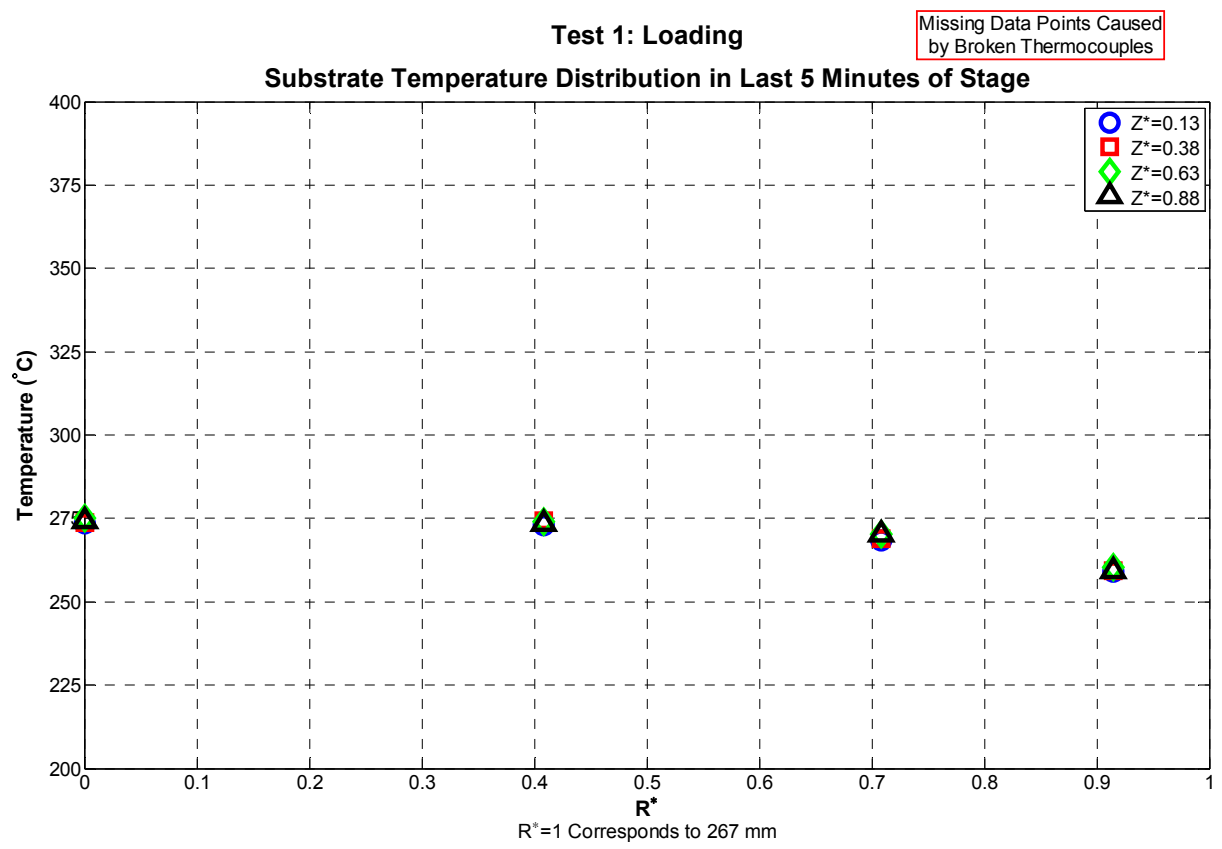

Figure I.6: Radial Temperature Distribution for Phase 2 Test 1 Loading

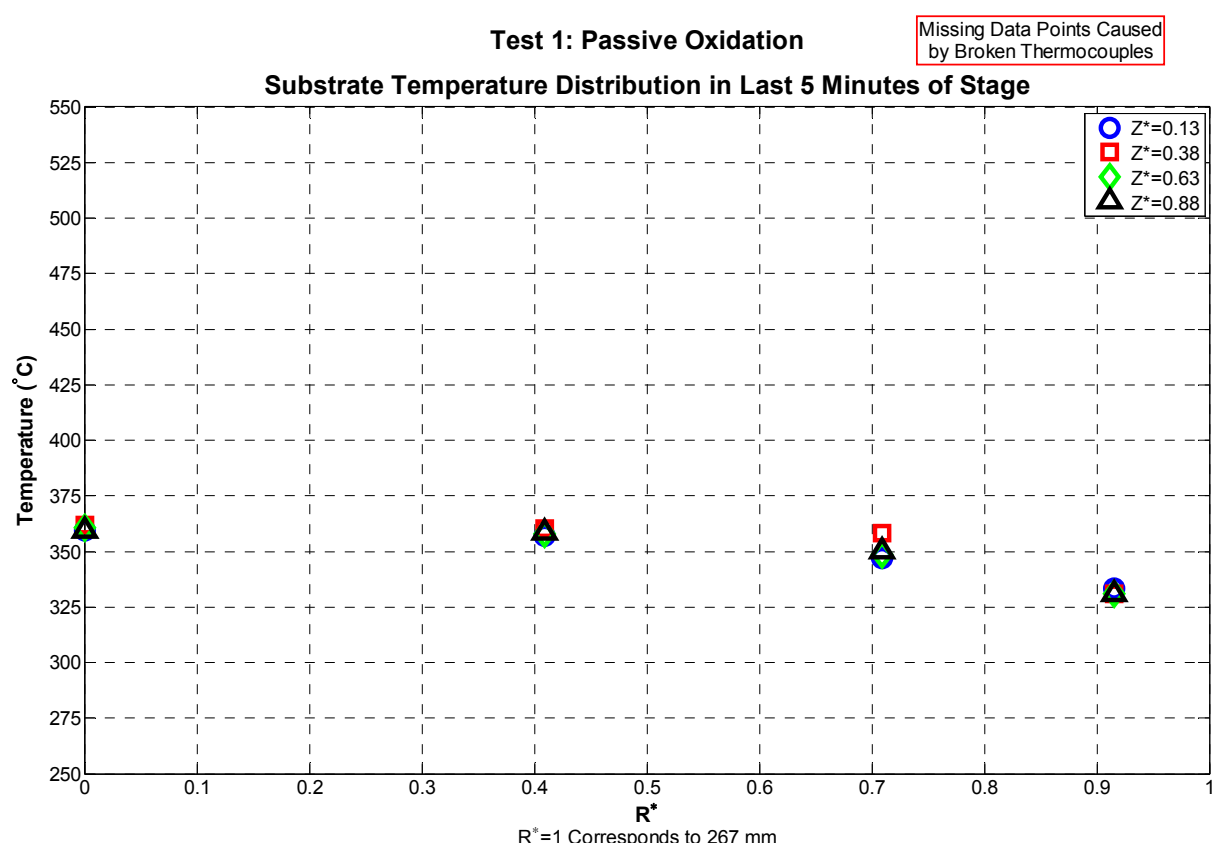

Figure I.7: Radial Temperature Distribution for Phase 2 Test 1 Passive Oxidation 


\section{Phase 2 Test 2}

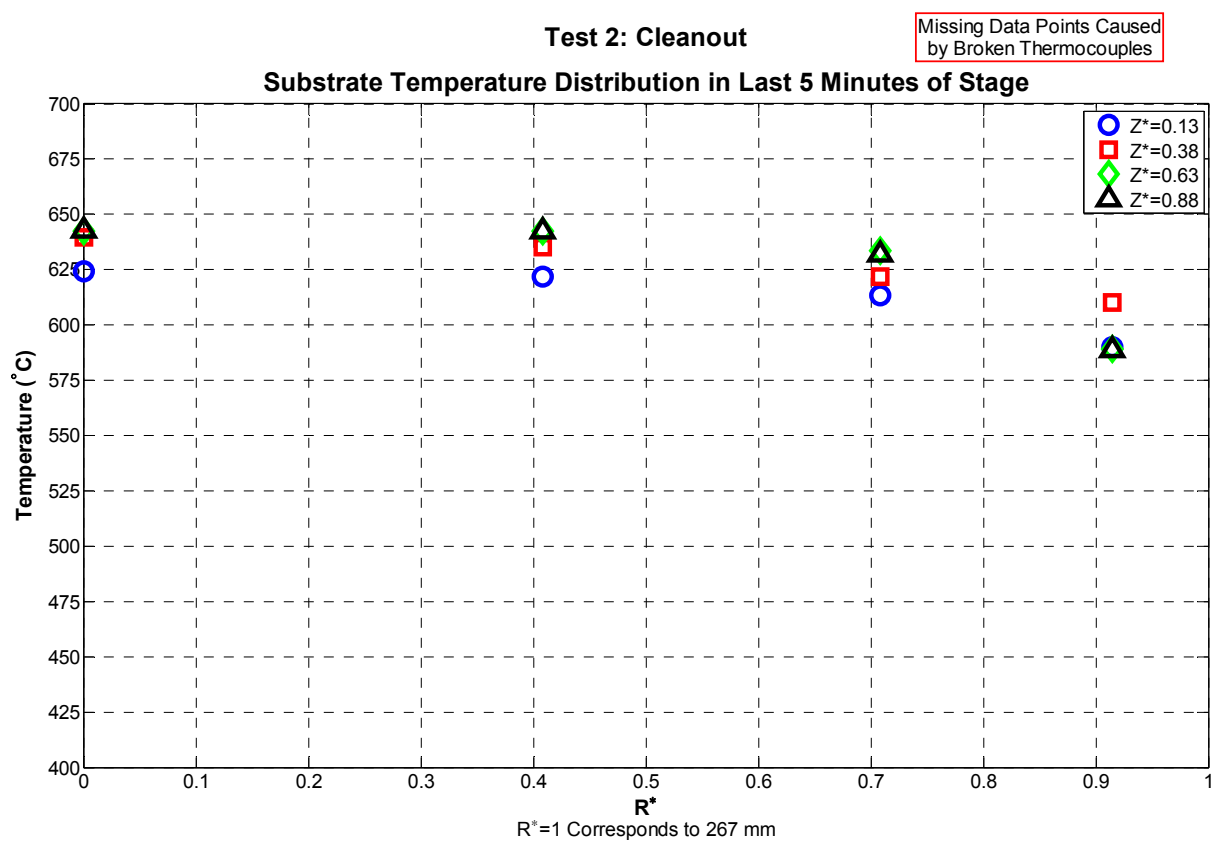

Figure I.8: Radial Temperature Distribution for Phase 2 Test 2 Cleanout

\section{Phase 2 Test 3}

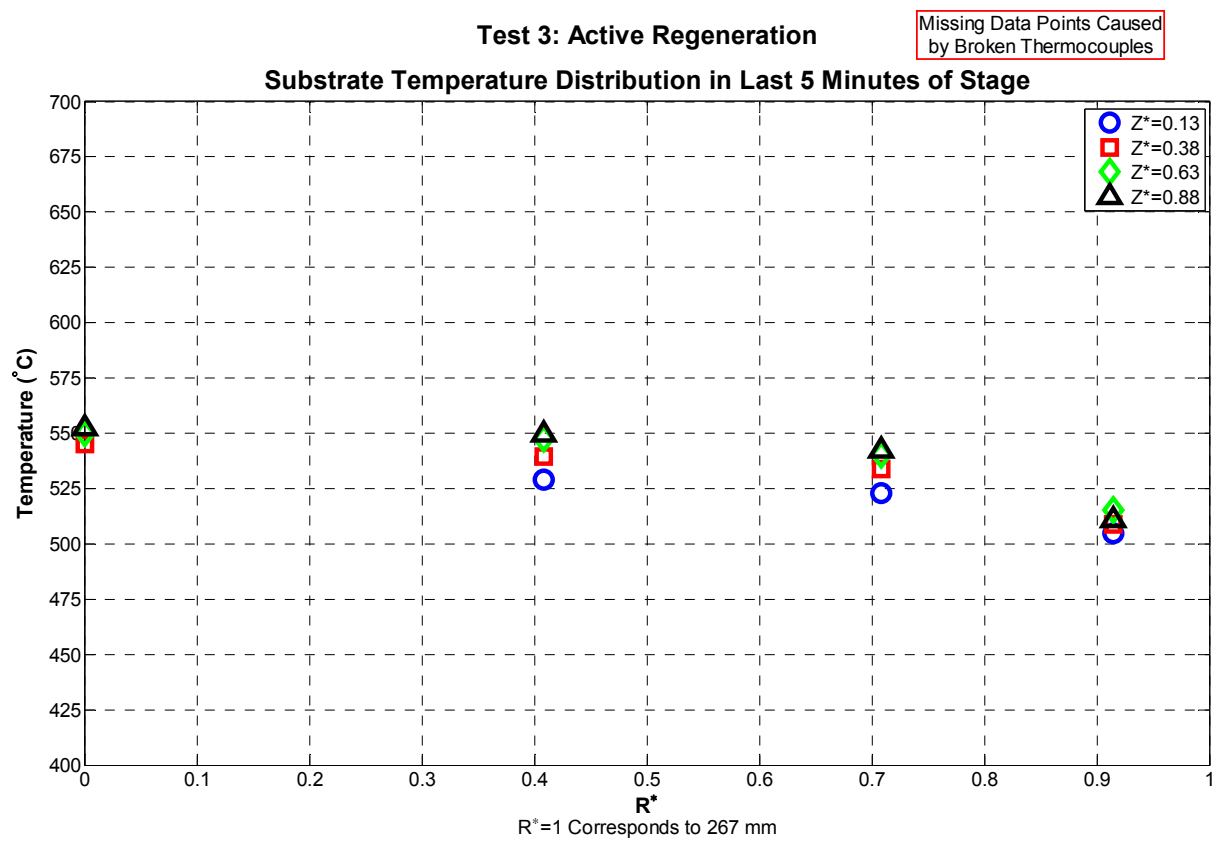

Figure I.9: Radial Temperature Distribution for Phase 2 Test 3 Active Regeneration 


\section{Phase 2 Test 4}

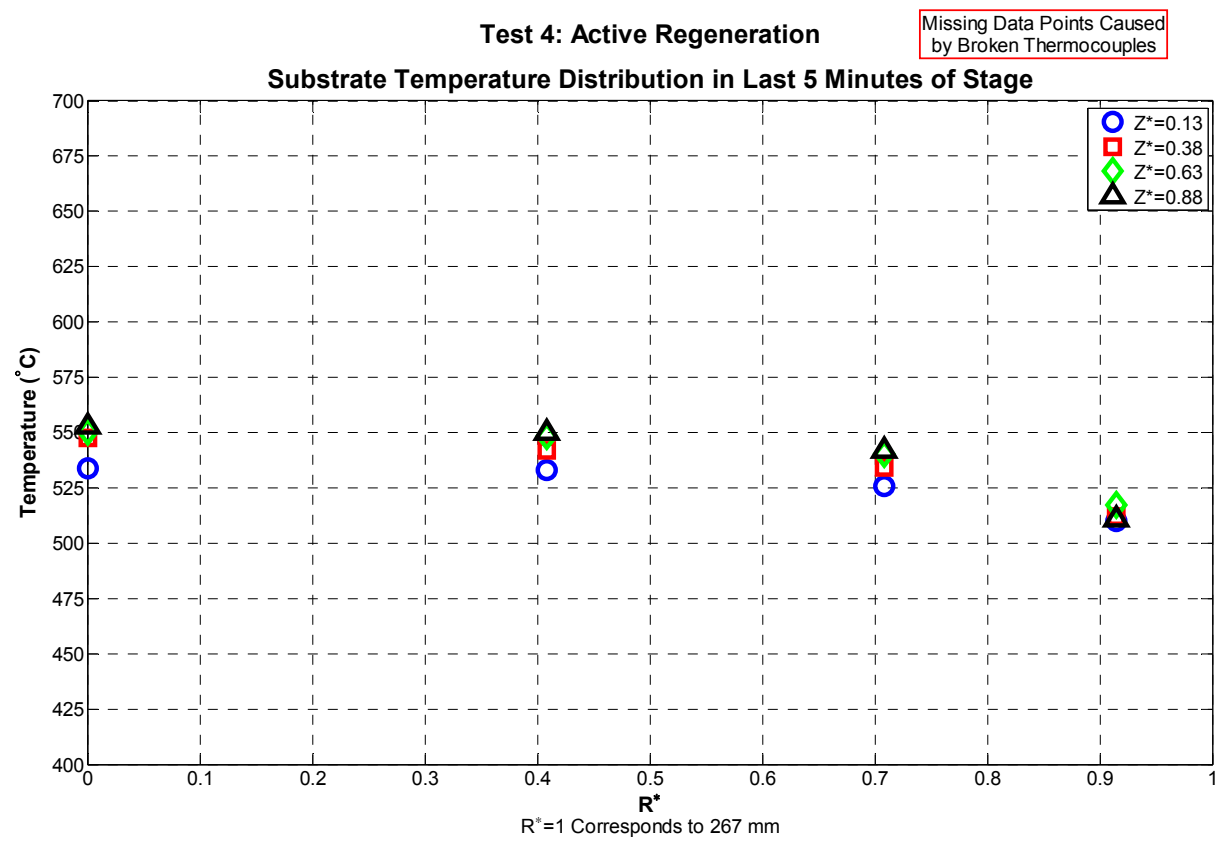

Figure I.10: Radial Temperature Distribution for Phase 2 Test 4 Active Regeneration

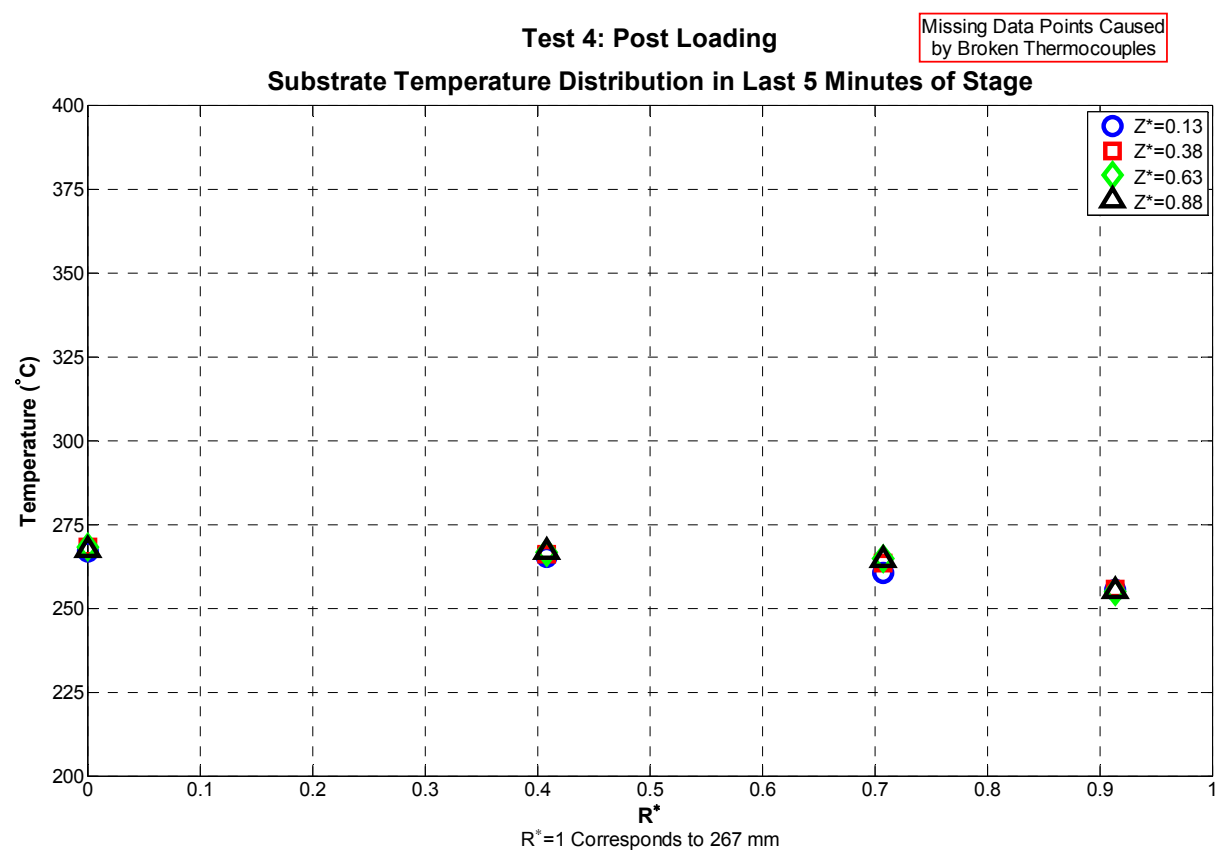

Figure I.11: Radial Temperature Distribution for Phase 2 Test 4 Post Loading 


\section{Phase 2 Test 5}

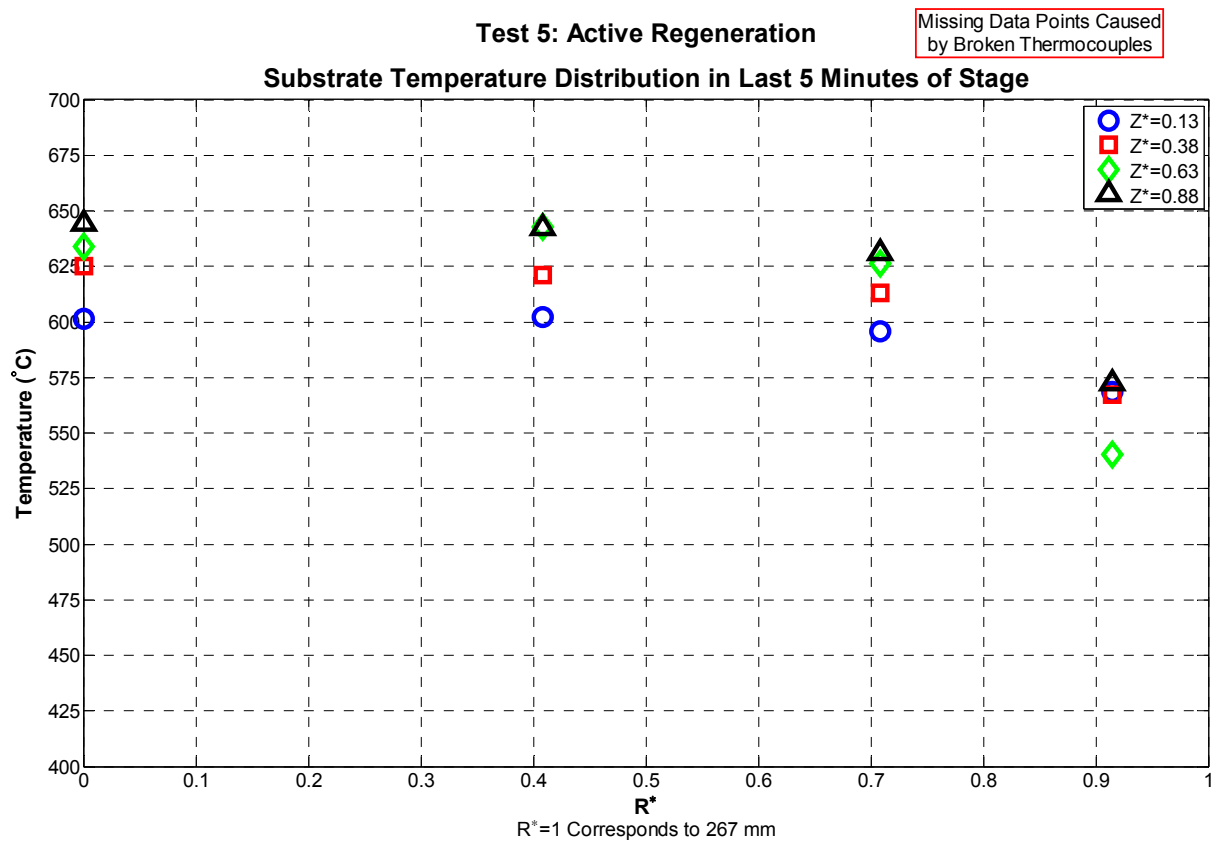

Figure I.12: Radial Temperature Distribution for Phase 2 Test 5 Active Regeneration

\section{Phase 2 Test 6}

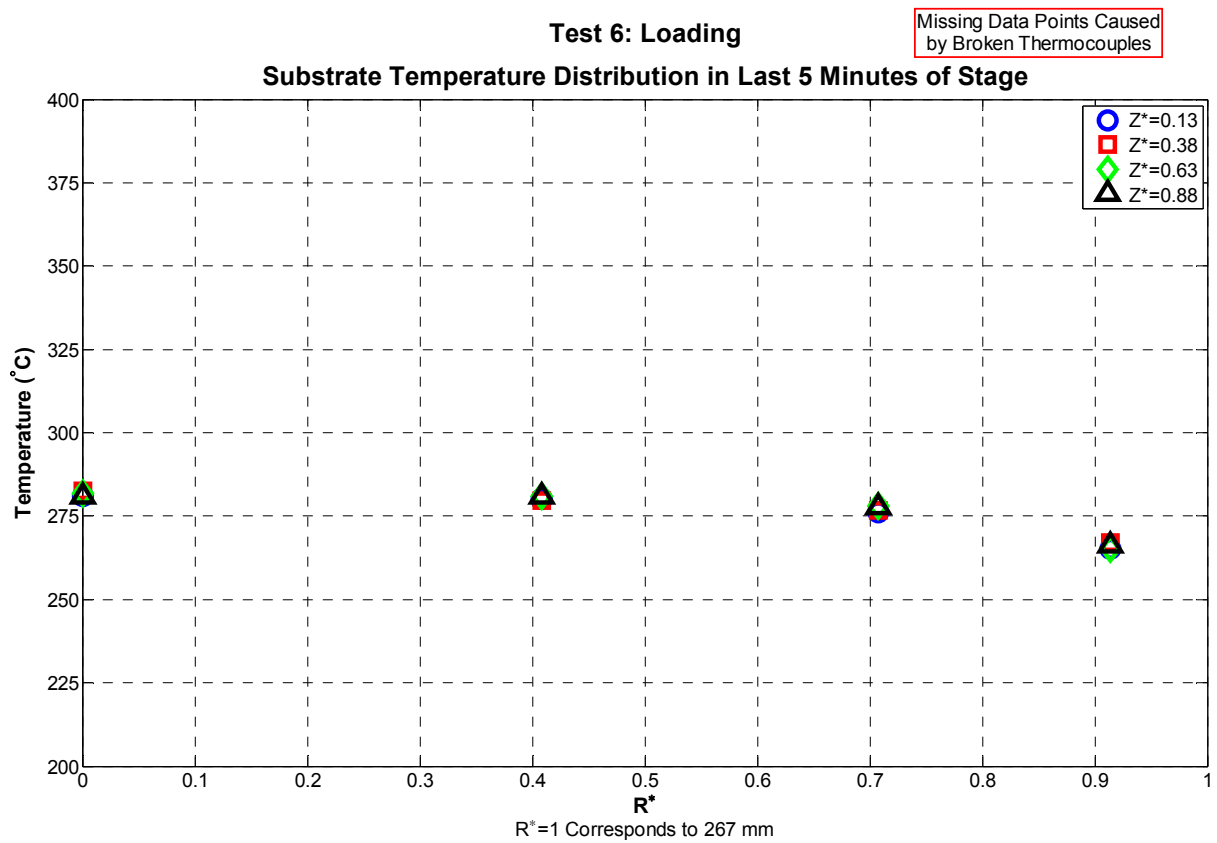

Figure I.13: Radial Temperature Distribution for Phase 2 Test 6 Loading 


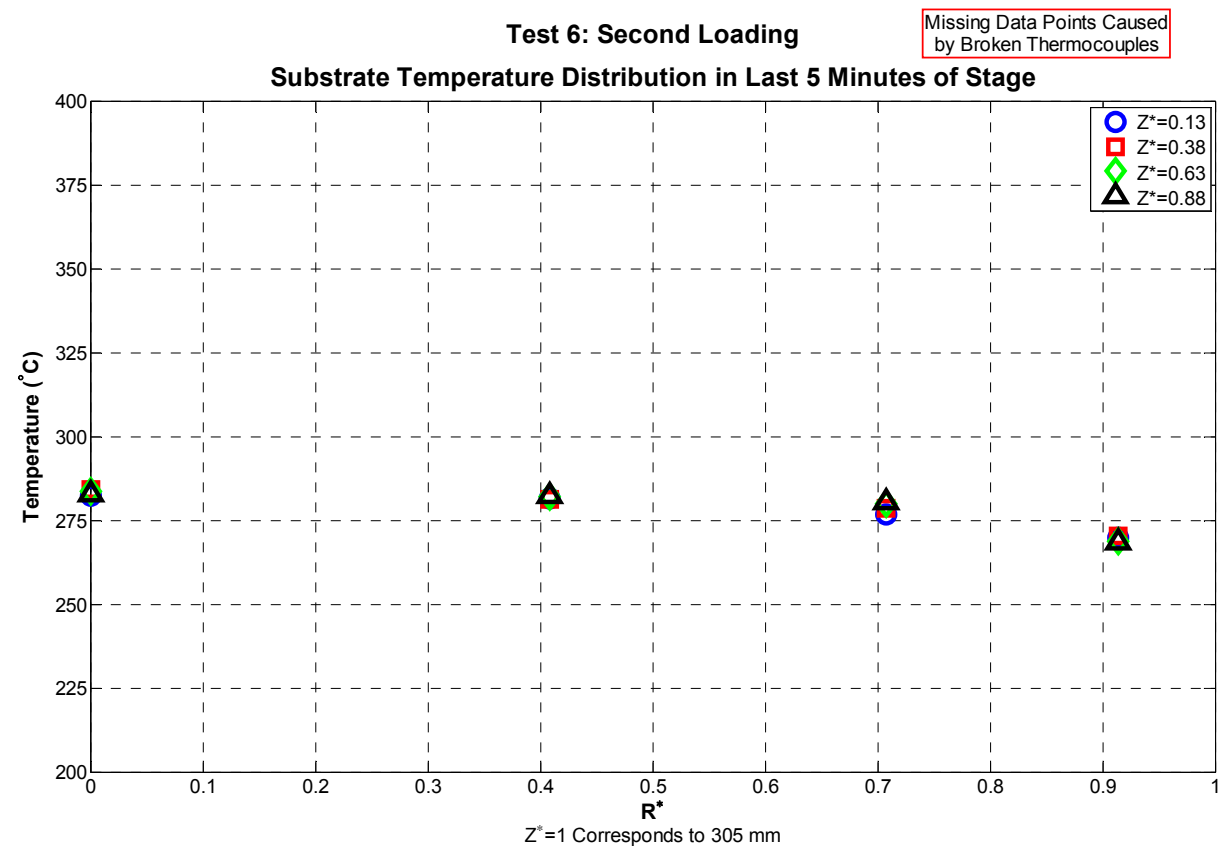

Figure I.14: Radial Temperature Distribution for Phase 2 Test 6 Second Loading

\section{Phase 2 Test 7}

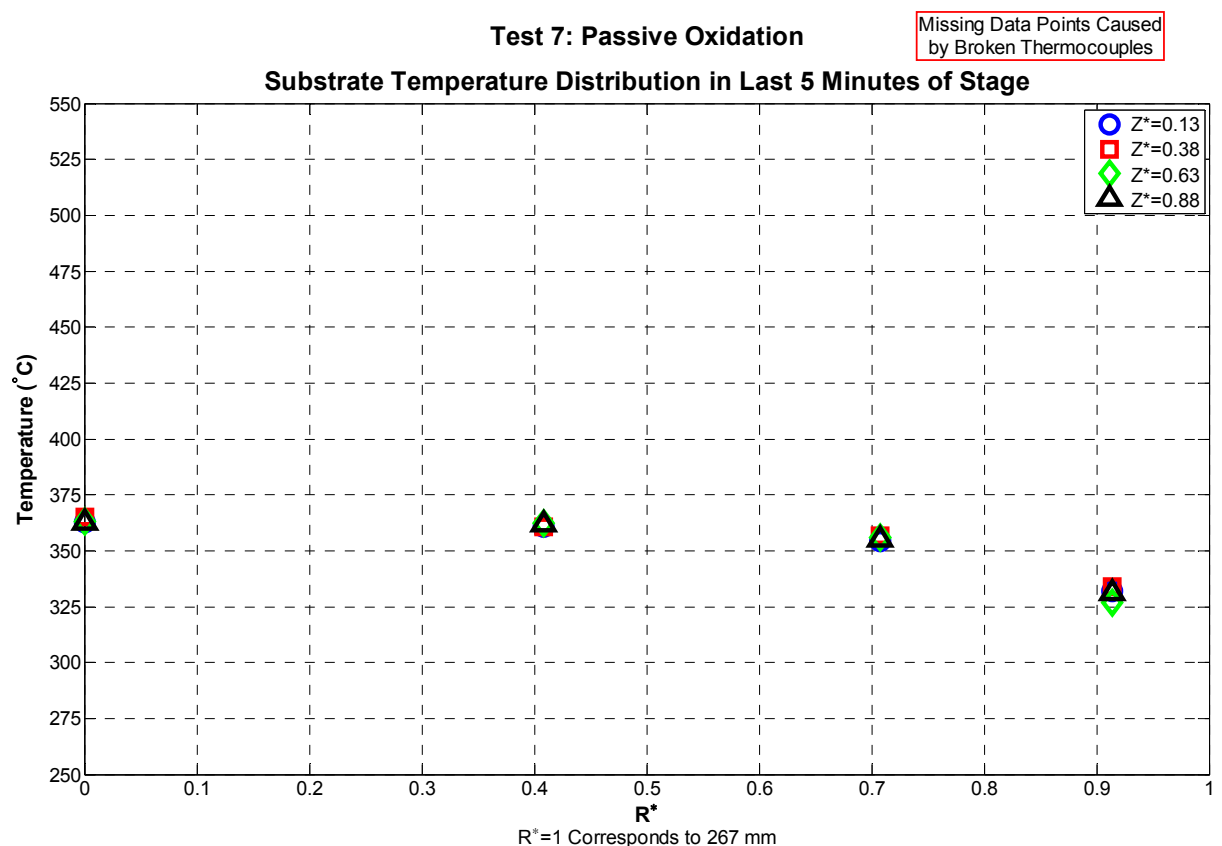

Figure I.15: Radial Temperature Distribution for Phase 2 Test 7 Passive Oxidation 


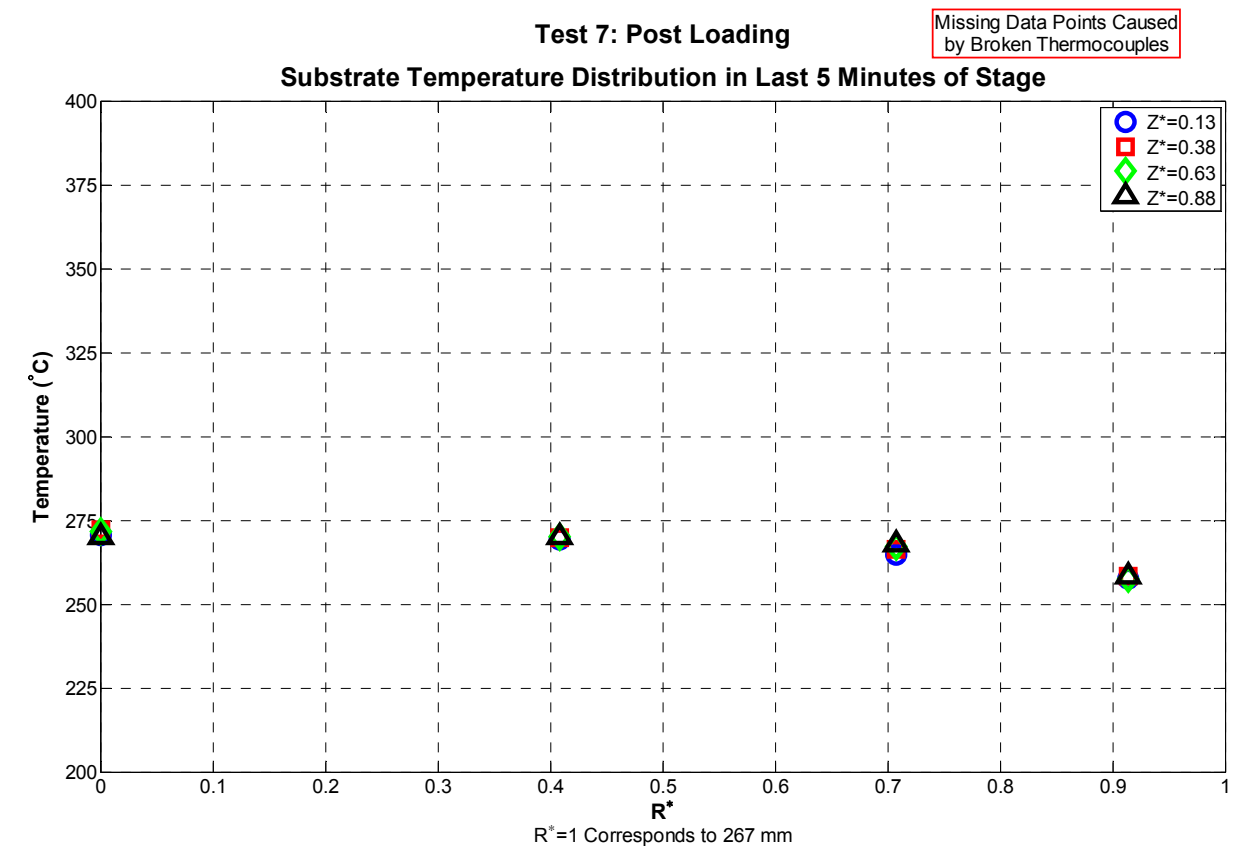

Figure I.16 Radial Temperature Distribution for Phase 2 Test 7 Post Loading

\section{Phase 2 Test 8}

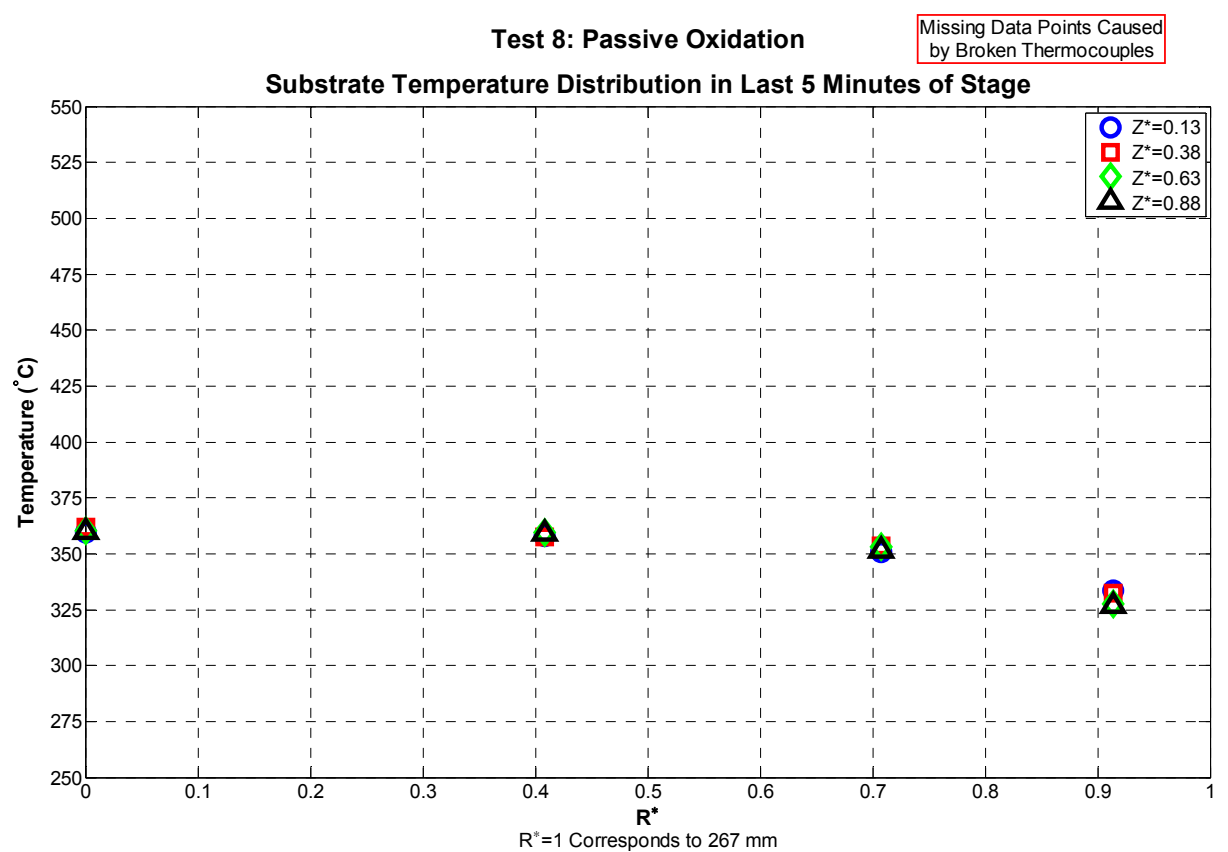

Figure I.17: Radial Temperature Distribution for Phase 2 Test 8 Passive Oxidation 


\section{Phase 2 Test 9}

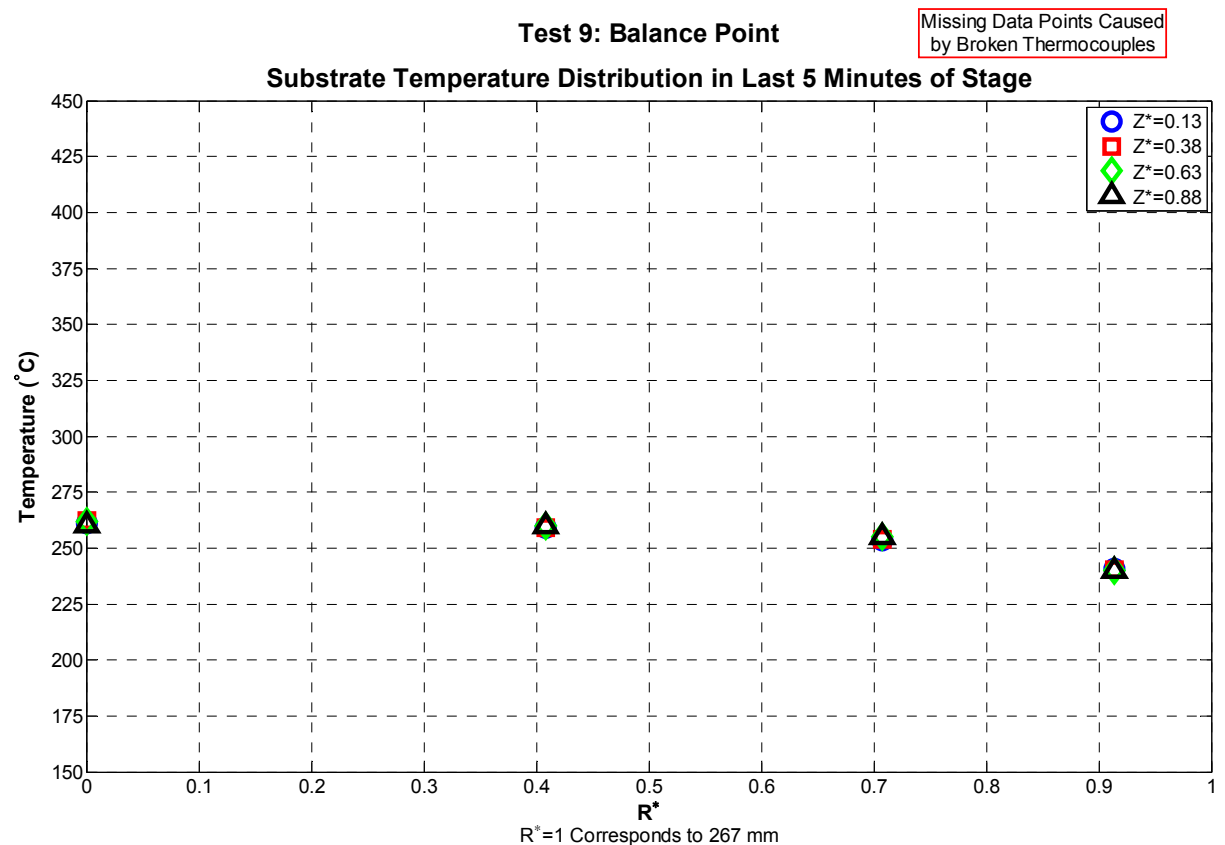

Figure I.18: Radial Temperature Distribution for Phase 2 Test 9 Balance Point 


\section{Appendix J. Emissions Measurements by Stage}

This appendix presents the average emissions for each stage of the experiments conducted. Some of the THC measurements were calculated using a method presented by Pidgeon in reference [5]. Comments regarding the data are made below each table. A value of NA indicates that a stage was not run during a test or the data is otherwise unavailable.

\section{J.1. Phase 1 Results}

Table J.1: Stage 1 Emission Summaries

\begin{tabular}{|c|c|c|c|c|c|c|c|c|}
\cline { 3 - 9 } \multicolumn{2}{c|}{} & $\begin{array}{c}\mathrm{THC} \\
(\mathrm{ppmC})\end{array}$ & $\begin{array}{c}\mathrm{NO}_{\mathrm{X}} \\
(\mathrm{ppm})\end{array}$ & $\begin{array}{c}\mathrm{NO} \\
(\mathrm{ppm})\end{array}$ & $\begin{array}{c}\mathrm{NO}_{2} \\
(\mathrm{ppm})\end{array}$ & $\begin{array}{c}\mathrm{CO} \\
(\mathrm{ppm})\end{array}$ & $\begin{array}{c}\mathrm{CO}_{2} \\
(\%)\end{array}$ & $\begin{array}{c}\mathrm{O}_{2} \\
(\%)\end{array}$ \\
\hline \multirow{4}{*}{ Test 1 } & UDOC & 70 & 563 & 521 & 42 & 84 & 5.46 & 13.43 \\
\cline { 2 - 9 } & DDOC & 4 & 536 & 246 & 290 & 0 & 5.47 & 13.44 \\
\cline { 2 - 9 } & DCPF & 3 & 561 & 258 & 302 & 0 & 5.42 & 13.48 \\
\hline \multirow{4}{*}{ Test 2 } & UDOC & 95 & 556 & 525 & 31 & 83 & 5.47 & 13.60 \\
\cline { 2 - 9 } & DDOC & 7 & 529 & 191 & 338 & 0 & 5.48 & 13.56 \\
\cline { 2 - 9 } & DCPF & 4 & 552 & 178 & 373 & 0 & 5.63 & 13.36 \\
\hline \multirow{4}{*}{ Test 3 } & UDOC & 48 & 522 & 464 & 58 & 94 & 5.33 & 13.80 \\
\cline { 2 - 9 } & DDOC & 6 & 481 & 310 & 170 & 0 & 5.23 & 13.95 \\
\cline { 2 - 9 } & DCPF & 0 & 510 & 329 & 181 & 0 & 5.36 & 13.77 \\
\hline \multirow{3}{*}{ Test 4 } & UDOC & 82 & 506 & 467 & 39 & 84 & 5.46 & 12.75 \\
\cline { 2 - 9 } & DDOC & 5 & 493 & 172 & 321 & 0 & 5.48 & 13.03 \\
\cline { 2 - 9 } & DCPF & 3 & 520 & 209 & 311 & 0 & 5.52 & 12.83 \\
\hline
\end{tabular}

Table J.2: Stage 2-1 Emission Summaries

\begin{tabular}{|c|c|c|c|c|c|c|c|c|}
\cline { 2 - 8 } \multicolumn{2}{c|}{} & $\begin{array}{c}\mathrm{THC} \\
(\mathrm{ppmC})\end{array}$ & $\begin{array}{c}\mathrm{NO}_{\mathrm{X}} \\
(\mathrm{ppm})\end{array}$ & $\begin{array}{c}\mathrm{NO} \\
(\mathrm{ppm})\end{array}$ & $\begin{array}{c}\mathrm{NO}_{2} \\
(\mathrm{ppm})\end{array}$ & $\begin{array}{c}\mathrm{CO} \\
(\mathrm{ppm})\end{array}$ & $\begin{array}{c}\mathrm{CO}_{2} \\
(\%)\end{array}$ & $\begin{array}{c}\mathrm{O}_{2} \\
(\%)\end{array}$ \\
\hline \multirow{3}{*}{ Test 1 } & UDOC & 41 & 107 & 91 & 16 & 171 & 5.56 & 13.31 \\
\cline { 2 - 9 } & DDOC & 14 & 110 & 58 & 52 & 0 & 5.50 & 13.45 \\
\cline { 2 - 9 } & DCPF & 2 & 109 & 53 & 56 & 0 & 5.56 & 13.34 \\
\hline \multirow{4}{*}{ Test 2 } & UDOC & 43 & 109 & 92 & 17 & 171 & 5.63 & 13.20 \\
\cline { 2 - 9 } & DDOC & 10 & 109 & 61 & 48 & 0 & 5.52 & 13.37 \\
\cline { 2 - 9 } Test 3 & DCPF & 0 & 107 & 50 & 57 & 0 & 5.61 & 13.24 \\
\hline & UDOC & 66 & 102 & 86 & 17 & 192 & 6.08 & 12.82 \\
\cline { 2 - 9 } & DDOC & 19 & 105 & 66 & 39 & 3 & 5.98 & 12.99 \\
\hline \multirow{4}{*}{ Test 4 } & DCPF & 1 & 104 & 55 & 49 & 0 & 6.08 & 12.83 \\
\hline & UDOC & 43 & 95 & 88 & 7 & 144 & 6.93 & 12.49 \\
\cline { 2 - 9 } & DDOC & 16 & 98 & 49 & 50 & 0 & 5.63 & 12.67 \\
\cline { 2 - 9 } & DCPF & 3 & 93 & 53 & 40 & 0 & 5.69 & 12.49 \\
\hline
\end{tabular}


Table J.3: Stage 2w-1 Emission Summaries

\begin{tabular}{|c|c|c|c|c|c|c|c|c|}
\cline { 3 - 9 } \multicolumn{2}{c|}{} & $\begin{array}{c}\mathrm{THC} \\
(\mathrm{ppmC})\end{array}$ & $\begin{array}{c}\mathrm{NO}_{\mathrm{x}} \\
(\mathrm{ppm})\end{array}$ & $\begin{array}{c}\mathrm{NO} \\
(\mathrm{ppm})\end{array}$ & $\begin{array}{c}\mathrm{NO}_{2} \\
(\mathrm{ppm})\end{array}$ & $\begin{array}{c}\mathrm{CO} \\
(\mathrm{ppm})\end{array}$ & $\begin{array}{c}\mathrm{CO}_{2} \\
(\%)\end{array}$ & $\begin{array}{c}\mathrm{O}_{2} \\
(\%)\end{array}$ \\
\hline \multirow{3}{*}{ Test 11 } & UDOC & 77 & 563 & 521 & 42 & 91 & 5.39 & 13.47 \\
\cline { 2 - 9 } & DDOC & 5 & 536 & 246 & 290 & 0 & 5.27 & 13.64 \\
\cline { 2 - 9 } & DCPF & 3 & 561 & 258 & 302 & 0 & 5.27 & 13.63 \\
\hline \multirow{3}{*}{ Test 2 2} & UDOC & 30 & 580 & 533 & 48 & 91 & 5.36 & 13.50 \\
\cline { 2 - 9 } & DDOC & 0 & 548 & 273 & 275 & 0 & 5.33 & 13.52 \\
\cline { 2 - 9 } & DCPF & 0 & 576 & 341 & 235 & 0 & 5.32 & 13.55 \\
\hline \multirow{3}{*}{ Test 4 } & UDOC & 48 & 531 & 475 & 56 & 95 & 5.25 & 13.93 \\
\cline { 2 - 9 } & DDOC & 4 & 458 & 306 & 151 & 0 & 5.03 & 14.22 \\
\cline { 2 - 9 } & DCPF & 0 & 521 & 350 & 171 & 0 & 5.22 & 13.97 \\
\cline { 2 - 9 } & DDOC & 62 & 499 & 460 & 39 & 91 & 5.37 & 12.65 \\
\cline { 2 - 9 } & DCPF & 2 & 421 & 197 & 223 & 0 & 5.31 & 13.39 \\
\hline
\end{tabular}

Table J.4: Stage 2-2 Emission Summaries

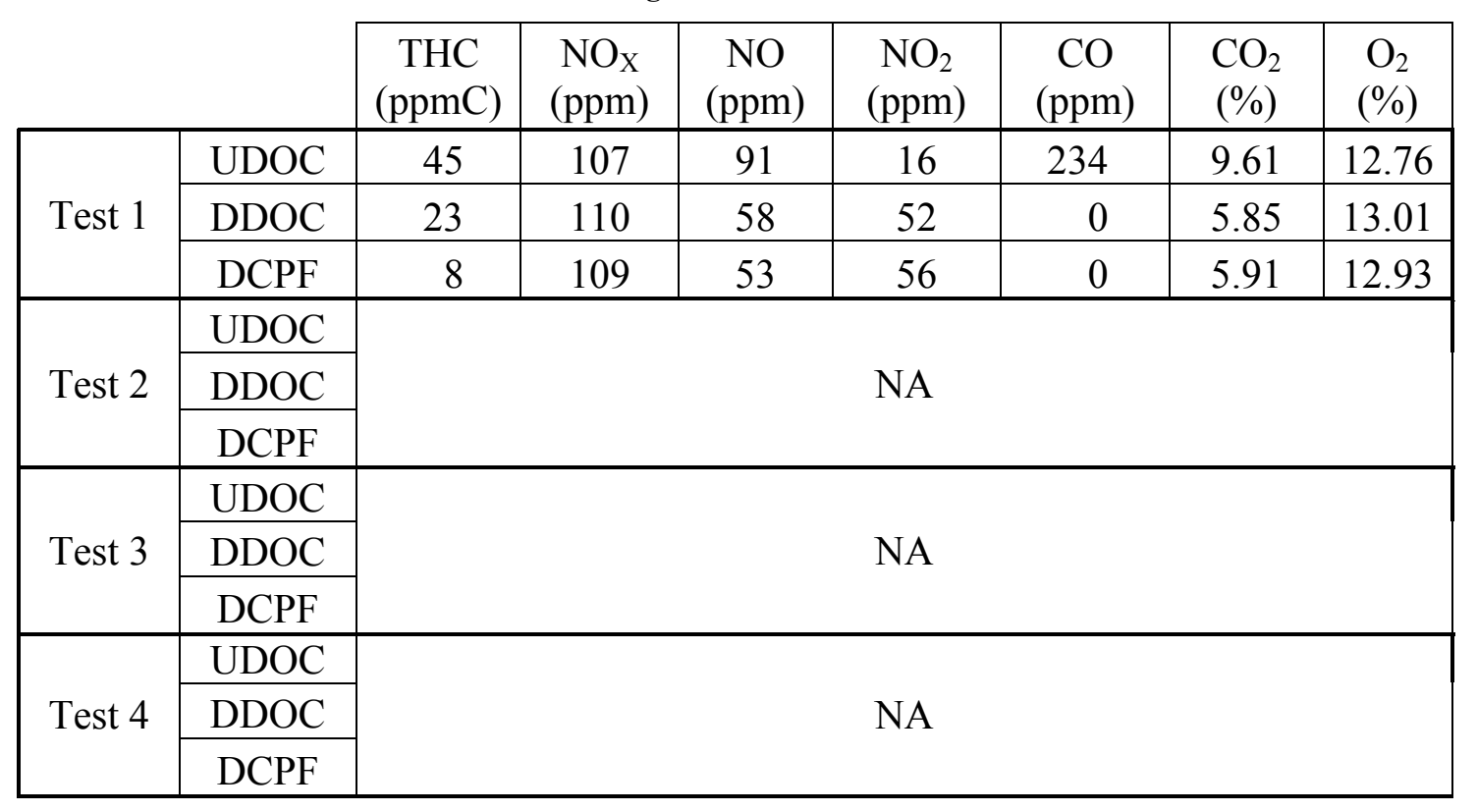


Table J.5: Stage 2w-2 Emission Summaries

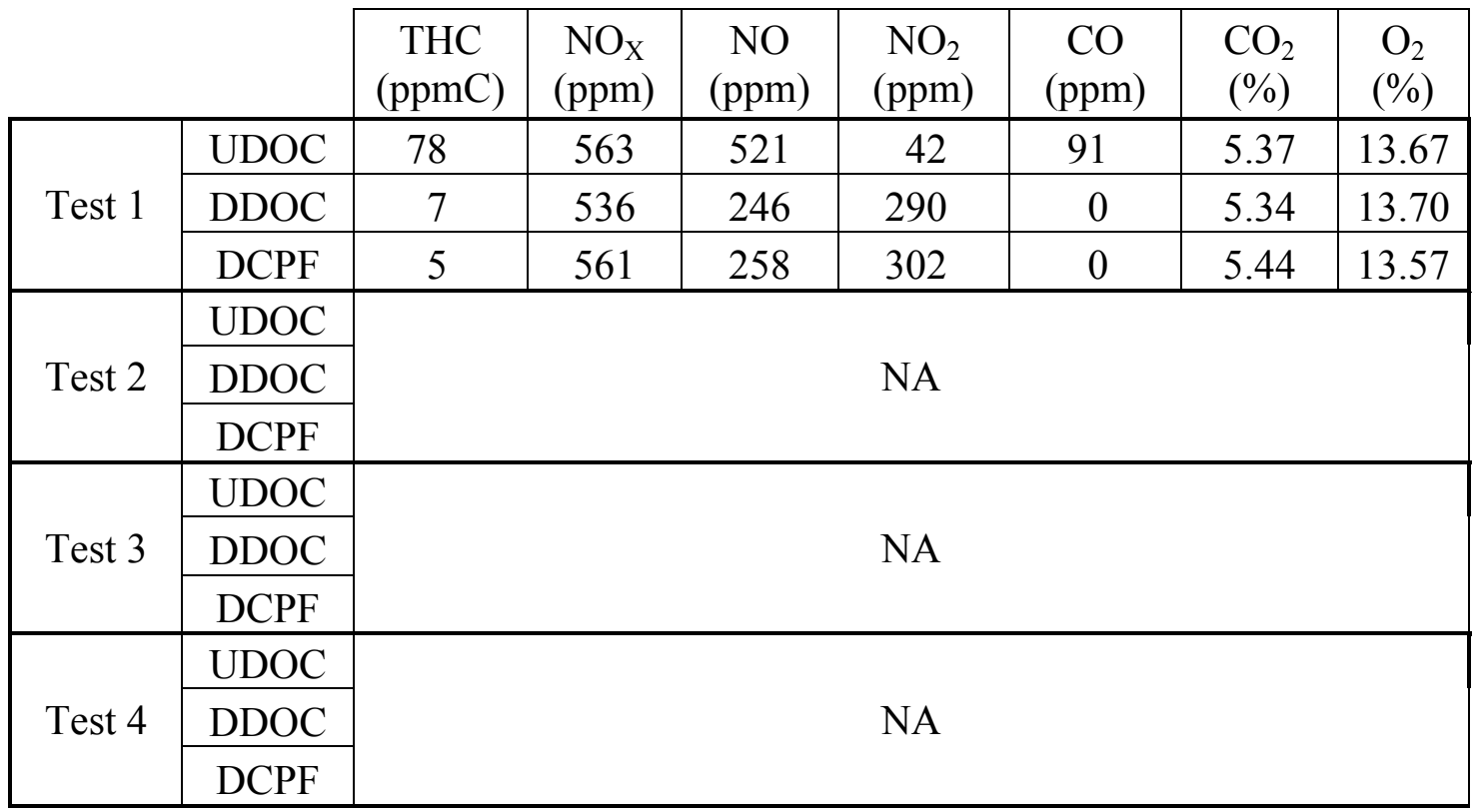

Table J.6: Pre Ramp Up Emission Summaries

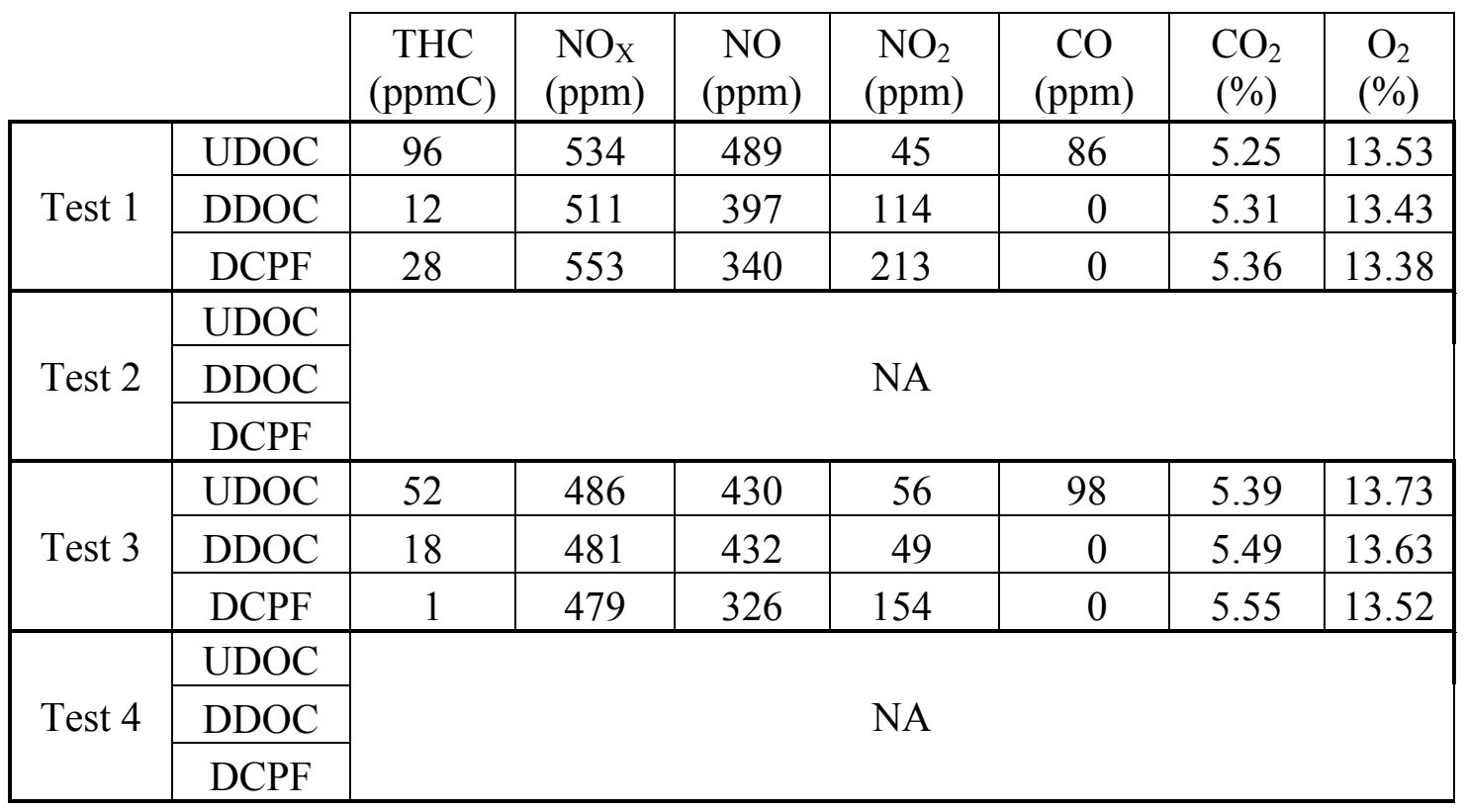


Table J.7: Oxidation Emission Summaries

\begin{tabular}{|c|c|c|c|c|c|c|c|c|}
\hline & & $\begin{array}{c}\text { THC } \\
\text { (ppmC) }\end{array}$ & $\begin{array}{c}\mathrm{NO}_{\mathrm{X}} \\
(\mathrm{ppm})\end{array}$ & $\begin{array}{l}\mathrm{NO} \\
(\mathrm{ppm})\end{array}$ & $\begin{array}{c}\mathrm{NO}_{2} \\
(\mathrm{ppm})\end{array}$ & $\begin{array}{c}\mathrm{CO} \\
(\mathrm{ppm})\end{array}$ & $\begin{array}{l}\mathrm{CO}_{2} \\
(\%)\end{array}$ & $\begin{array}{l}\mathrm{O}_{2} \\
(\%)\end{array}$ \\
\hline \multirow{3}{*}{ Test 1} & UDOC* $^{*}$ & 16663 & 479 & 473 & 7 & 107 & 9.91 & 7.43 \\
\hline & DDOC & 392 & 484 & 459 & 24 & 0 & 10.32 & 6.24 \\
\hline & DCPF & 20 & 498 & 416 & 82 & 0 & 10.77 & 6.21 \\
\hline \multirow{3}{*}{ Test 2} & UDOC $^{*}$ & 16145 & 515 & 504 & 10 & 94 & 9.86 & 7.56 \\
\hline & DDOC & 52 & 511 & 459 & 52 & 0 & 10.69 & 6.43 \\
\hline & DCPF & 8 & 501 & 402 & 99 & 0 & 10.79 & 6.32 \\
\hline \multirow{3}{*}{ Test 3} & UDOC & 24 & 436 & 420 & 16 & 97 & 9.38 & 8.30 \\
\hline & DDOC & 5 & 479 & 223 & 256 & 0 & 9.72 & 7.92 \\
\hline & DCPF & 0 & 449 & 246 & 202 & 0 & 9.61 & 8.00 \\
\hline \multirow{3}{*}{ Test 4} & UDOC* & 30650 & 447 & 443 & 3 & 100 & 9.88 & 6.65 \\
\hline & DDOC & 410 & 528 & 476 & 52 & 0 & 10.51 & 4.12 \\
\hline & DCPF & 42 & 446 & 429 & 17 & 0 & 10.78 & 3.79 \\
\hline
\end{tabular}

Table J.8: Stage 3 Emission Summaries

\begin{tabular}{|c|c|c|c|c|c|c|c|c|}
\cline { 2 - 9 } \multicolumn{2}{c|}{} & $\begin{array}{c}\mathrm{THC} \\
(\mathrm{ppmC})\end{array}$ & $\begin{array}{c}\mathrm{NO}_{\mathrm{X}} \\
(\mathrm{ppm})\end{array}$ & $\begin{array}{c}\mathrm{NO} \\
(\mathrm{ppm})\end{array}$ & $\begin{array}{c}\mathrm{NO}_{2} \\
(\mathrm{ppm})\end{array}$ & $\begin{array}{c}\mathrm{CO} \\
(\mathrm{ppm})\end{array}$ & $\begin{array}{c}\mathrm{CO}_{2} \\
(\%)\end{array}$ & $\begin{array}{c}\mathrm{O}_{2} \\
(\%)\end{array}$ \\
\hline \multirow{3}{*}{ Test 1 } & UDOC & 100 & 571 & 527 & 45 & 83 & 5.42 & 13.27 \\
\cline { 2 - 9 } & DDOC & 10 & 558 & 217 & 341 & 0 & 5.64 & 12.39 \\
\cline { 2 - 9 } & DCPF & 2 & 572 & 273 & 299 & 0 & 5.46 & 13.23 \\
\hline \multirow{3}{*}{ Test 2 } & UDOC & 36 & 575 & 532 & 44 & 82 & 5.39 & 13.53 \\
\cline { 2 - 9 } & DDOC & 1 & 531 & 149 & 382 & 1 & 5.38 & 13.51 \\
\cline { 2 - 9 } & DCPF & 0 & 550 & 159 & 391 & 0 & 5.29 & 13.64 \\
\hline \multirow{3}{*}{ Test 3 } & UDOC & 43 & 549 & 488 & 61 & 89 & 5.37 & 13.75 \\
\cline { 2 - 9 } & DDOC & 0 & 504 & 193 & 311 & 0 & 5.16 & 14.00 \\
\cline { 2 - 9 } & DCPF & 0 & 530 & 311 & 219 & 0 & 5.31 & 13.83 \\
\hline \multirow{3}{*}{ Test 4 } & UDOC & 68 & 498 & 458 & 40 & 83 & 5.40 & 12.63 \\
\cline { 2 - 9 } & DDOC & 5 & 464 & 193 & 271 & 0 & 5.51 & 12.75 \\
\cline { 2 - 9 } & DCPF & 1 & 508 & 217 & 291 & 0 & 5.37 & 12.63 \\
\hline
\end{tabular}




\section{J.2. Phase 2 Results}

Table J.9: Stage 1 Emission Summaries

\begin{tabular}{|c|c|c|c|c|c|c|c|c|}
\hline & \multirow{3}{*}{$\begin{array}{c}\begin{array}{c}\mathrm{THC} \\
(\mathrm{ppmC})\end{array} \\
80\end{array}$} & \multirow{3}{*}{$\begin{array}{c}\begin{array}{c}\mathrm{NO}_{\mathrm{X}} \\
(\mathrm{ppm})\end{array} \\
102 \\
\end{array}$} & \multirow{3}{*}{$\begin{array}{c}\begin{array}{c}\mathrm{NO} \\
(\mathrm{ppm})\end{array} \\
79 \\
\end{array}$} & \multirow{3}{*}{$\begin{array}{c}\begin{array}{c}\mathrm{NO}_{2} \\
(\mathrm{ppm})\end{array} \\
23 \\
\end{array}$} & \multirow{3}{*}{$\begin{array}{c}\begin{array}{c}\mathrm{CO} \\
(\mathrm{ppm})\end{array} \\
121 \\
\end{array}$} & \multirow{3}{*}{$\begin{array}{l}\mathrm{CO}_{2} \\
(\%) \\
5.24 \\
\end{array}$} & \multirow{3}{*}{$\begin{array}{c}\begin{array}{c}\mathrm{O}_{2} \\
(\%)\end{array} \\
13.72 \\
\end{array}$} \\
\hline & & & & & & & & \\
\hline \multirow{3}{*}{ Test 1} & UDOC & & & & & & & \\
\hline & DDOC & 28 & 96 & 64 & 32 & 13 & 5.18 & 13.83 \\
\hline & DCPF & 7 & 89 & 43 & 46 & 0 & 4.62 & 14.57 \\
\hline \multirow{3}{*}{ Test 3} & UDOC & 67 & 104 & 78 & 27 & 117 & 5.22 & 13.57 \\
\hline & DDOC & 12 & 99 & 63 & 36 & 0 & 5.25 & 13.58 \\
\hline & DCPF & 3 & 102 & 61 & 41 & 0 & 5.24 & 13.57 \\
\hline \multirow{3}{*}{ Test 4} & UDOC & 78 & 96 & 70 & 26 & 122 & 4.93 & 13.71 \\
\hline & DDOC & 9 & 86 & 54 & 33 & 0 & 4.93 & 13.71 \\
\hline & DCPF & 2 & 95 & 57 & 38 & 0 & 4.91 & 13.73 \\
\hline \multirow{3}{*}{ Test 5} & UDOC & 62 & 92 & 65 & 28 & 129 & 5.36 & 13.29 \\
\hline & DDOC & 2 & 81 & 48 & 33 & 0 & 5.37 & 13.32 \\
\hline & DCPF & 0 & 89 & 56 & 33 & 0 & 5.35 & 13.31 \\
\hline \multirow{3}{*}{ Test 6} & UDOC & 91 & 87 & 61 & 26 & 127 & 5.36 & 13.61 \\
\hline & DDOC & 17 & 78 & 42 & 36 & 0 & 5.39 & 13.59 \\
\hline & DCPF & 8 & 86 & 44 & 42 & 0 & 5.39 & 13.58 \\
\hline \multirow{3}{*}{ Test 7} & UDOC & 76 & 76 & 55 & 22 & 132 & 5.31 & 13.48 \\
\hline & DDOC & 14 & 70 & 39 & 31 & 0 & 5.30 & 13.52 \\
\hline & DCPF & 4 & 75 & 42 & 33 & 0 & 5.28 & 13.53 \\
\hline \multirow{3}{*}{ Test 8} & UDOC & 85 & 80 & 54 & 26 & 126 & 5.23 & 13.56 \\
\hline & DDOC & 19 & 72 & 39 & 33 & 0 & 5.22 & 13.60 \\
\hline & DCPF & 8 & 77 & 45 & 32 & 0 & 5.23 & 13.57 \\
\hline \multirow{3}{*}{ Test 9} & UDOC & 47 & 86 & 61 & 25 & 120 & 5.19 & 13.68 \\
\hline & DDOC & 3 & 83 & 44 & 39 & 0 & 5.23 & 13.66 \\
\hline & DCPF & 0 & 86 & 48 & 37 & 0 & 5.27 & 13.58 \\
\hline
\end{tabular}


Table J.10: Stage 2 Emission Summaries

\begin{tabular}{|c|c|c|c|c|c|c|c|c|}
\hline & & $\begin{array}{c}\text { THC } \\
(\mathrm{ppmC})\end{array}$ & $\begin{array}{c}\mathrm{NO}_{\mathrm{X}} \\
(\mathrm{ppm})\end{array}$ & $\begin{array}{c}\mathrm{NO} \\
(\mathrm{ppm})\end{array}$ & $\begin{array}{c}\mathrm{NO}_{2} \\
(\mathrm{ppm})\end{array}$ & $\begin{array}{c}\mathrm{CO} \\
(\mathrm{ppm})\end{array}$ & $\begin{array}{l}\mathrm{CO}_{2} \\
(\%)\end{array}$ & $\begin{array}{l}\mathrm{O}_{2} \\
(\%)\end{array}$ \\
\hline \multirow{3}{*}{ Test 1} & UDOC & 52 & 104 & 80 & 24 & 118 & 5.25 & 13.58 \\
\hline & DDOC & 13 & 98 & 70 & 28 & 0 & 4.99 & 13.97 \\
\hline & DCPF & 2 & 89 & 59 & 30 & 0 & 4.59 & 14.53 \\
\hline \multirow{3}{*}{ Test 3} & UDOC & 50 & 106 & 80 & 26 & 116 & 5.49 & 13.58 \\
\hline & DDOC & 17 & 102 & 74 & 28 & 0 & 5.47 & 13.64 \\
\hline & DCPF & 2 & 103 & 77 & 26 & 0 & 5.48 & 13.61 \\
\hline \multirow{3}{*}{ Test 4} & UDOC & 74 & 104 & 78 & 26 & 121 & 5.06 & 13.67 \\
\hline & DDOC & 15 & 93 & 65 & 27 & 0 & 5.05 & 13.69 \\
\hline & DCPF & 4 & 99 & 74 & 25 & 0 & 5.08 & 13.64 \\
\hline \multirow{3}{*}{ Test 5} & UDOC & 55 & 96 & 68 & 28 & 130 & 5.38 & 13.29 \\
\hline & DDOC & 22 & 86 & 59 & 27 & 0 & 5.37 & 13.31 \\
\hline & DCPF & 5 & 90 & 69 & 21 & 0 & 5.39 & 13.27 \\
\hline \multirow{3}{*}{ Test 6} & UDOC & 72 & 97 & 70 & 27 & 127 & 5.46 & 13.38 \\
\hline & DDOC & 18 & 88 & 56 & 32 & 0 & 5.48 & 13.40 \\
\hline & DCPF & 5 & 94 & 62 & 33 & 0 & 5.49 & 13.36 \\
\hline \multirow{3}{*}{ Test 7} & UDOC* $^{*}$ & 68 & 80 & 60 & 20 & 64 & 5.08 & 13.36 \\
\hline & DDOC* & 11 & 76 & 49 & 27 & 0 & 5.33 & 13.48 \\
\hline & DCPF* & 7 & 77 & 57 & 20 & 0 & 4.98 & 12.85 \\
\hline \multirow{3}{*}{ Test 8} & UDOC & 67 & 86 & 60 & 26 & 120 & 5.16 & 13.64 \\
\hline & DDOC & 17 & 77 & 49 & 28 & 0 & 5.19 & 13.62 \\
\hline & DCPF & 4 & 81 & 59 & 22 & 0 & 5.19 & 13.61 \\
\hline \multirow{3}{*}{ Test 9} & UDOC & 29 & 86 & 63 & 24 & 121 & 5.23 & 13.65 \\
\hline & DDOC & 4 & 85 & 54 & 31 & 0 & 5.25 & 13.66 \\
\hline & DCPF & 0 & 87 & 63 & 24 & 0 & 5.25 & 13.64 \\
\hline
\end{tabular}


Table J.11: Pre Ramp Up Emission Summaries

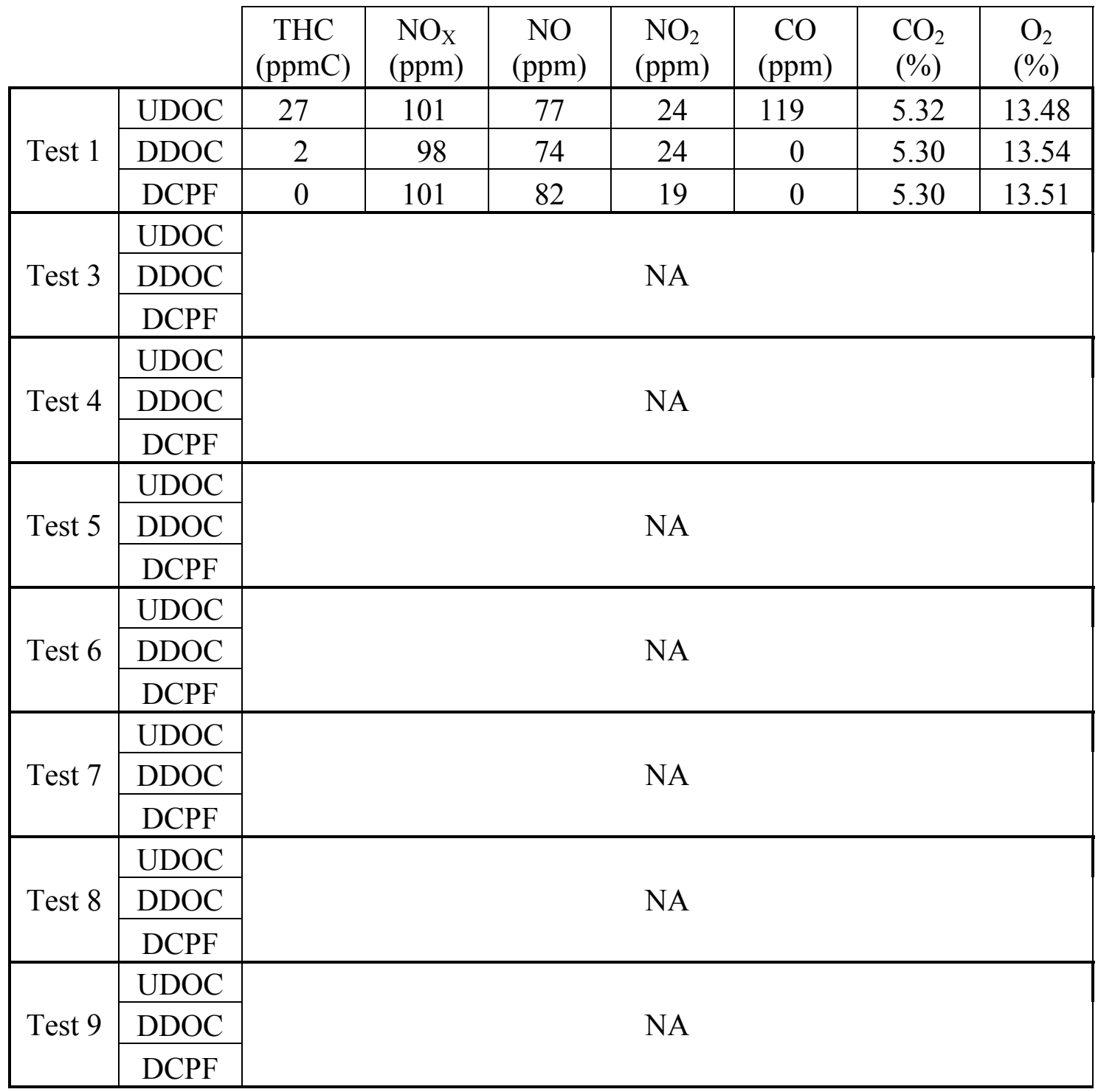


Table J.12: Oxidation Stage Emission Summaries

\begin{tabular}{|c|c|c|c|c|c|c|c|c|}
\hline & & $\begin{array}{c}\mathrm{THC} \\
(\mathrm{ppmC})\end{array}$ & $\begin{array}{r}\mathrm{NO}_{\mathrm{X}} \\
(\mathrm{ppm})\end{array}$ & $\begin{array}{c}\mathrm{NO} \\
(\mathrm{ppm})\end{array}$ & $\begin{array}{c}\mathrm{NO}_{2} \\
(\mathrm{ppm}) \\
\end{array}$ & $\begin{array}{c}\mathrm{CO} \\
(\mathrm{ppm})\end{array}$ & $\begin{array}{l}\mathrm{CO}_{2} \\
(\%)\end{array}$ & $\begin{array}{l}\mathrm{O}_{2} \\
(\%) \\
\end{array}$ \\
\hline \multirow{3}{*}{ Test 1} & UDOC & 19 & 202 & 193 & 10 & 65 & 8.87 & 8.76 \\
\hline & DDOC & 0 & 198 & 98 & 100 & 0 & 8.97 & 8.66 \\
\hline & DCPF & 0 & 200 & 116 & 84 & 0 & 8.91 & 8.72 \\
\hline \multirow{3}{*}{ Test 3} & UDOC* & 11724 & 142 & 127 & 15 & 89 & 8.42 & 9.34 \\
\hline & DDOC & 792 & 135 & 134 & 1 & 38 & 10.20 & 7.44 \\
\hline & DCPF & 36 & 134 & 124 & 9 & 0 & 10.31 & 7.29 \\
\hline \multirow{3}{*}{ Test 4} & UDOC* & 13188 & 151 & 136 & 15 & 87 & 7.93 & 9.66 \\
\hline & DDOC & 629 & 131 & 130 & 0 & 29 & 9.51 & 7.46 \\
\hline & DCPF & 23 & 138 & 129 & 9 & 0 & 9.57 & 7.36 \\
\hline \multirow{3}{*}{ Test 5} & UDOC** & 17107 & 125 & 108 & 17 & 101 & 8.45 & 9.18 \\
\hline & DDOC $* *$ & - & 111 & 111 & 1 & 51 & 9.55 & 6.99 \\
\hline & $\mathrm{DCPF}^{* *}$ & - & 116 & 114 & 2 & 0 & 10.58 & 6.42 \\
\hline \multirow{3}{*}{ Test 6} & UDOC & \multirow{3}{*}{\multicolumn{7}{|c|}{ NA }} \\
\hline & DDOC & & & & & & & \\
\hline & DCPF & & & & & & & \\
\hline \multirow{3}{*}{ Test 7} & UDOC & 32 & 165 & 156 & 10 & 74 & 8.83 & 8.76 \\
\hline & DDOC & 0 & 167 & 74 & 93 & 0 & 8.93 & 8.67 \\
\hline & DCPF & 0 & 172 & 98 & 74 & 0 & 8.86 & 8.72 \\
\hline \multirow{3}{*}{ Test 8} & UDOC & 38 & 171 & 159 & 11 & 69 & 8.74 & 8.84 \\
\hline & DDOC & 6 & 177 & 72 & 104 & 0 & 8.78 & 8.82 \\
\hline & DCPF & 1 & 186 & 101 & 85 & 0 & 8.76 & 8.83 \\
\hline \multirow{3}{*}{ Test 9} & $\mathrm{UDOC}^{* * *}$ & - & - & - & - & - & - & - \\
\hline & $\mathrm{DDOC}^{* * *}$ & - & - & - & - & - & - & - \\
\hline & $\mathrm{DCPF}^{* * *}$ & - & - & - & - & - & - & - \\
\hline
\end{tabular}

* THC is calculated using James' method. Other values are taken from the Pre-Test ** THC Analyzer was not Working Properly *** The Software did not Record any Data 
Table J.13: Stage 3 Emission Summaries

\begin{tabular}{|c|c|c|c|c|c|c|c|c|}
\hline & & $\begin{array}{c}\mathrm{THC} \\
(\mathrm{ppmC})\end{array}$ & $\begin{array}{l}\mathrm{NO}_{\mathrm{X}} \\
(\mathrm{ppm})\end{array}$ & $\begin{array}{c}\mathrm{NO} \\
(\mathrm{ppm})\end{array}$ & $\begin{array}{c}\mathrm{NO}_{2} \\
\text { (ppm) }\end{array}$ & $\begin{array}{c}\mathrm{CO} \\
(\mathrm{ppm})\end{array}$ & $\begin{array}{l}\mathrm{CO}_{2} \\
(\%)\end{array}$ & $\begin{array}{l}\mathrm{O}_{2} \\
(\%)\end{array}$ \\
\hline \multirow{3}{*}{ Test 1} & UDOC & 27 & 101 & 74 & 26 & 119 & 5.29 & 13.40 \\
\hline & DDOC & 2 & 100 & 72 & 28 & 0 & 5.36 & 13.36 \\
\hline & DCPF & 0 & 101 & 65 & 36 & 0 & 5.34 & 13.35 \\
\hline \multirow{3}{*}{ Test 3} & UDOC & 67 & 102 & 78 & 24 & 119 & 5.52 & 13.50 \\
\hline & DDOC & 32 & 97 & 80 & 17 & 1 & 5.54 & 13.47 \\
\hline & DCPF & 6 & 100 & 70 & 30 & 0 & 5.54 & 13.46 \\
\hline \multirow{3}{*}{ Test 4} & UDOC & 90 & 110 & 84 & 26 & 120 & 5.03 & 13.65 \\
\hline & DDOC & 36 & 106 & 84 & 22 & 1 & 5.13 & 13.52 \\
\hline & DCPF & 11 & 107 & 78 & 30 & 0 & 5.09 & 13.56 \\
\hline \multirow{3}{*}{ Test 5} & UDOC* & - & 99 & 73 & 26 & 128 & 5.38 & 13.20 \\
\hline & DDOC* & - & 87 & 63 & 23 & 1 & 5.38 & 13.22 \\
\hline & DCPF* & - & 94 & 65 & 29 & 0 & 5.44 & 13.13 \\
\hline \multirow{3}{*}{ Test 6} & UDOC & \multirow{3}{*}{\multicolumn{7}{|c|}{ NA }} \\
\hline & DDOC & & & & & & & \\
\hline & DCPF & & & & & & & \\
\hline \multirow{3}{*}{ Test 7} & UDOC & 84 & 80 & 58 & 23 & 130 & 5.36 & 13.36 \\
\hline & DDOC & 17 & 74 & 49 & 25 & 0 & 5.33 & 13.39 \\
\hline & DCPF & 9 & 78 & 55 & 23 & 0 & 5.36 & 13.35 \\
\hline \multirow{3}{*}{ Test 8} & UDOC & 58 & 86 & 59 & 27 & 119 & 5.14 & 13.67 \\
\hline & DDOC & 9 & 70 & 38 & 32 & 0 & 5.27 & 13.52 \\
\hline & DCPF & 3 & 81 & 57 & 23 & 0 & 5.15 & 13.66 \\
\hline \multirow{3}{*}{ Test 9} & UDOC** & - & - & - & - & - & - & - \\
\hline & $\mathrm{DDOC}^{* *}$ & - & - & - & - & - & - & - \\
\hline & $\mathrm{DCPF}^{* *}$ & - & - & - & - & - & - & - \\
\hline
\end{tabular}

* THC Analyzer was not Working Properly

** The Software did not Record any Data 
Table J.14: Pre Stage 4 Emission Summaries

\begin{tabular}{|c|c|c|c|c|c|c|c|c|}
\hline & & $\begin{array}{c}\text { THC } \\
(\mathrm{ppmC})\end{array}$ & $\begin{array}{c}\mathrm{NO}_{\mathrm{X}} \\
(\mathrm{ppm})\end{array}$ & $\begin{array}{c}\mathrm{NO} \\
(\mathrm{ppm})\end{array}$ & $\begin{array}{c}\mathrm{NO}_{2} \\
(\mathrm{ppm})\end{array}$ & $\begin{array}{c}\mathrm{CO} \\
(\mathrm{ppm})\end{array}$ & $\begin{array}{l}\mathrm{CO}_{2} \\
(\%)\end{array}$ & $\begin{array}{c}\mathrm{O}_{2} \\
(\%)\end{array}$ \\
\hline \multirow{3}{*}{ Test 1} & UDOC & \multirow{3}{*}{\multicolumn{7}{|c|}{ NA }} \\
\hline & DDOC & & & & & & & \\
\hline & DCPF & & & & & & & \\
\hline \multirow{3}{*}{ Test 3} & UDOC & \multirow{3}{*}{\multicolumn{7}{|c|}{ NA }} \\
\hline & DDOC & & & & & & & \\
\hline & DCPF & & & & & & & \\
\hline \multirow{3}{*}{ Test 4} & UDOC & 74 & 84 & 61 & 24 & 123 & 5.25 & 13.68 \\
\hline & DDOC & 14 & 68 & 51 & 16 & 0 & 5.29 & 13.67 \\
\hline & DCPF & 24 & 75 & 65 & 10 & 0 & 5.29 & 13.64 \\
\hline \multirow{3}{*}{ Test 5} & UDOC & \multirow{3}{*}{\multicolumn{7}{|c|}{ NA }} \\
\hline & DDOC & & & & & & & \\
\hline & DCPF & & & & & & & \\
\hline \multirow{3}{*}{ Test 6} & UDOC* & - & 59 & 43 & 15 & - & - & 13.28 \\
\hline & DDOC* & - & 55 & 49 & 6 & - & - & 13.31 \\
\hline & DCPF* & - & 60 & 45 & 16 & - & - & 13.26 \\
\hline \multirow{3}{*}{ Test 7} & UDOC $^{* *}$ & - & 58 & 37 & 21 & 130 & 5.31 & 13.57 \\
\hline & DDOC $^{* *}$ & - & 52 & 36 & 16 & 0 & 5.36 & 13.55 \\
\hline & $\mathrm{DCPF}^{* *}$ & - & 55 & 42 & 13 & 0 & 5.32 & 13.57 \\
\hline \multirow{3}{*}{ Test 8} & UDOC & \multirow{3}{*}{\multicolumn{7}{|c|}{ NA }} \\
\hline & DDOC & & & & & & & \\
\hline & DCPF & & & & & & & \\
\hline \multirow{3}{*}{ Test 9} & UDOC & \multirow{3}{*}{\multicolumn{7}{|c|}{ NA }} \\
\hline & DDOC & & & & & & & \\
\hline & DCPF & & & & & & & \\
\hline
\end{tabular}

* The Pump and Cooler for the $\mathrm{CO}, \mathrm{CO}_{2}$, and $\mathrm{O}_{2}$ Analyzers Failed

** THC Analyzer was not Working 
Table J.15: Stage 4 Emission Summaries

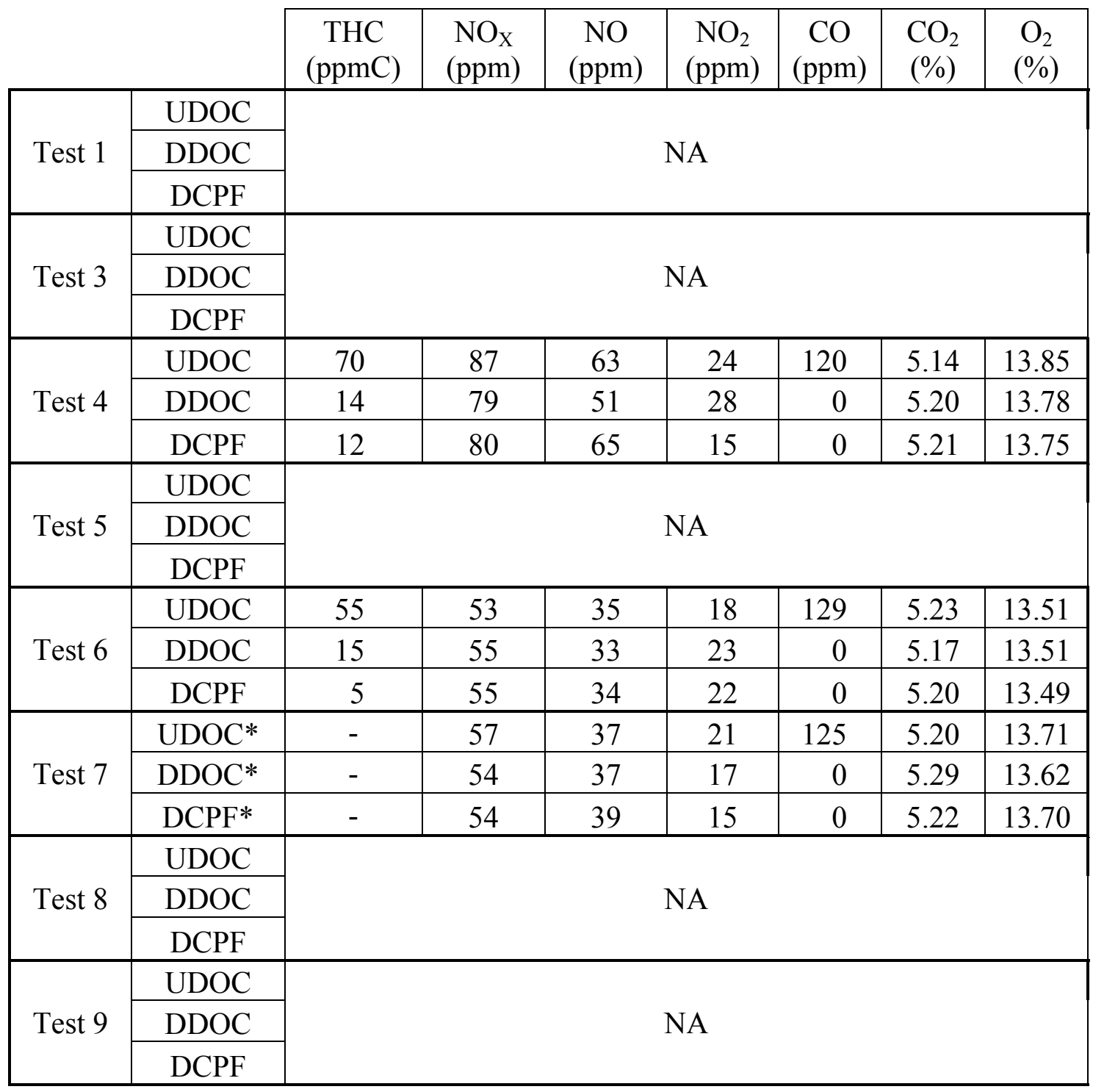

*THC Analyzer was not Working 


\section{Appendix K. Additional PM Distribution Plots}

This appendix contains additional PM distribution plots for each test.

\section{Phase 1 Test 1 Loading Scan}

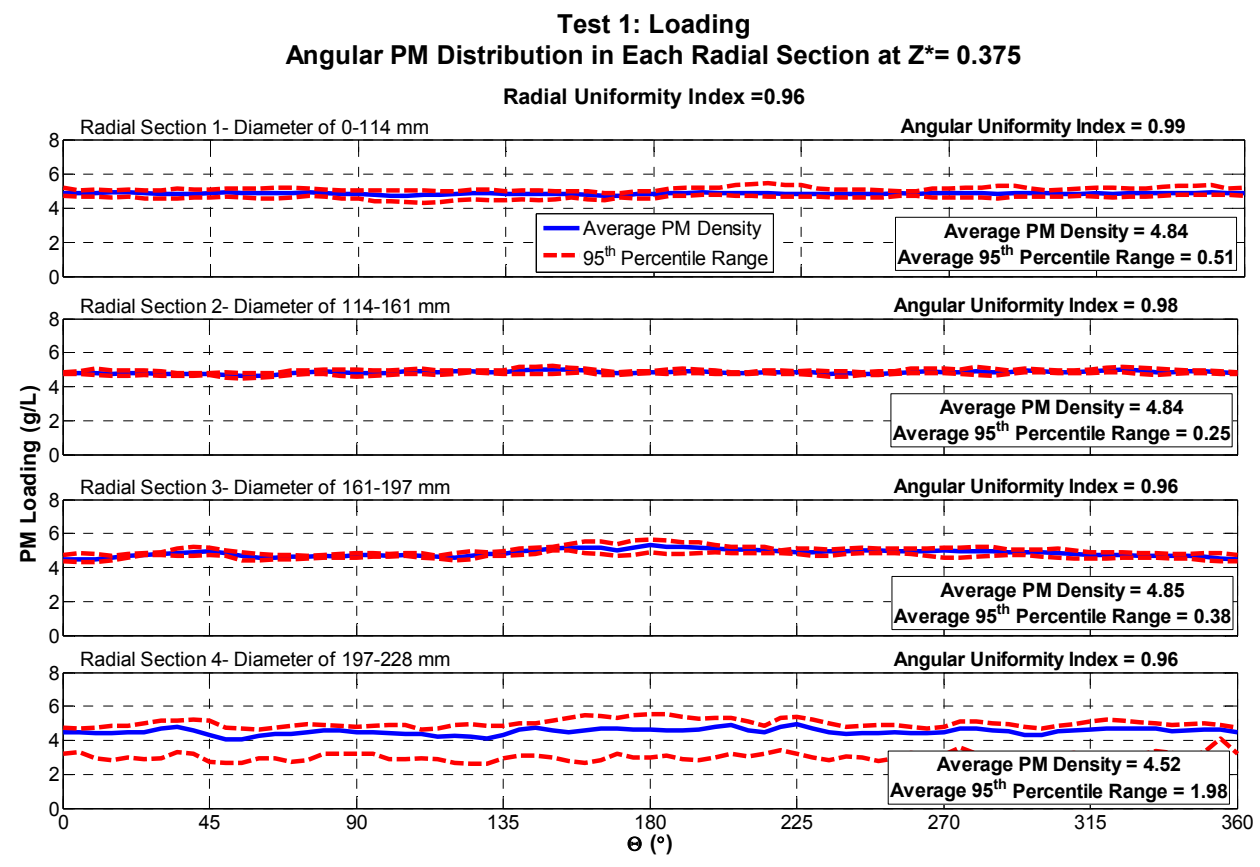

Figure K.1: Phase 1 Test 1- Loading $Z *=0.375$

Test 1: Loading

Angular PM Distribution in Each Radial Section at $Z^{*}=\mathbf{0 . 6 2 5}$

Radial Uniformity Index $=0.96$

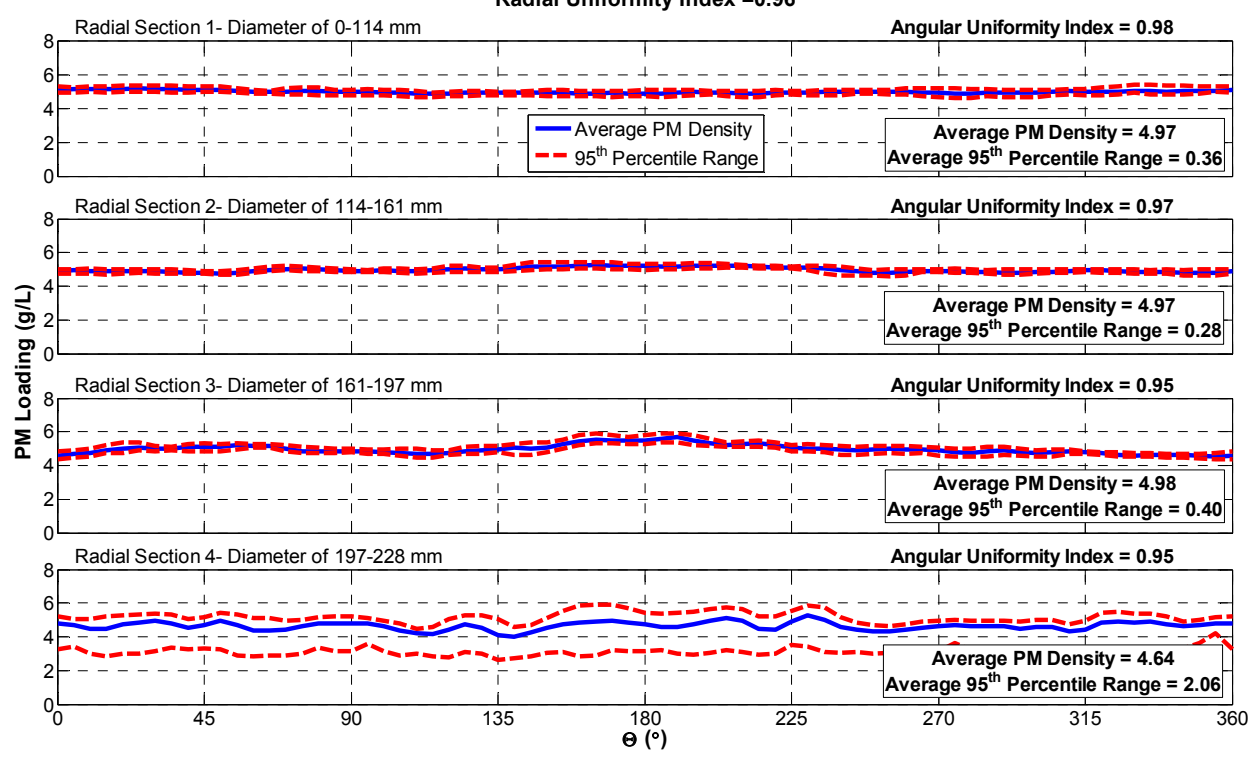

Figure K.2: Phase 1 Test 1- Loading $Z^{*}=0.625$ 
Test 1: Loading

Angular PM Distribution in Each Radial Section at $Z^{*}=\mathbf{0 . 8 7 5}$

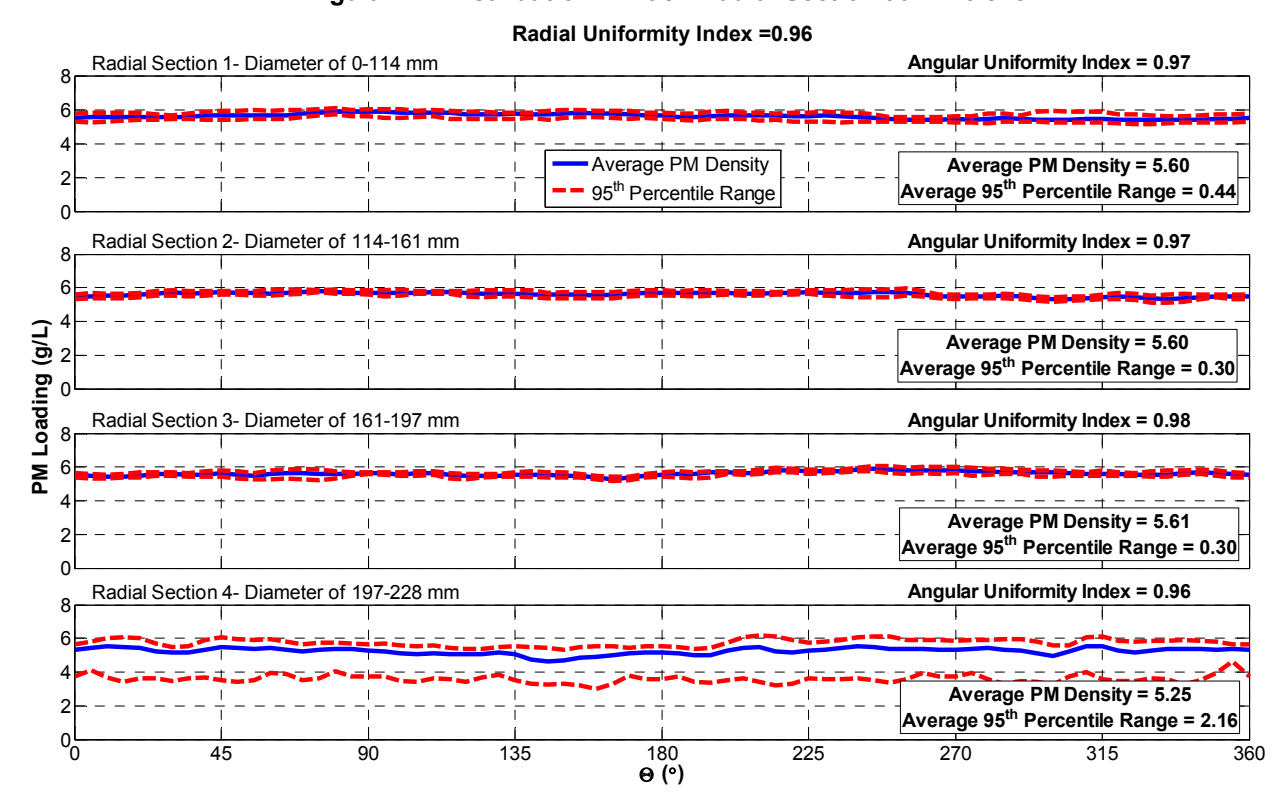

Figure K.3: Phase 1 Test 1- Loading $Z^{*}=0.875$

\section{Phase 1 Test 1 Active Regeneration Scan}

Test 1: Active Regeneration

Angular PM Distribution in Each Radial Section at $Z^{*}=\mathbf{0 . 3 7 5}$

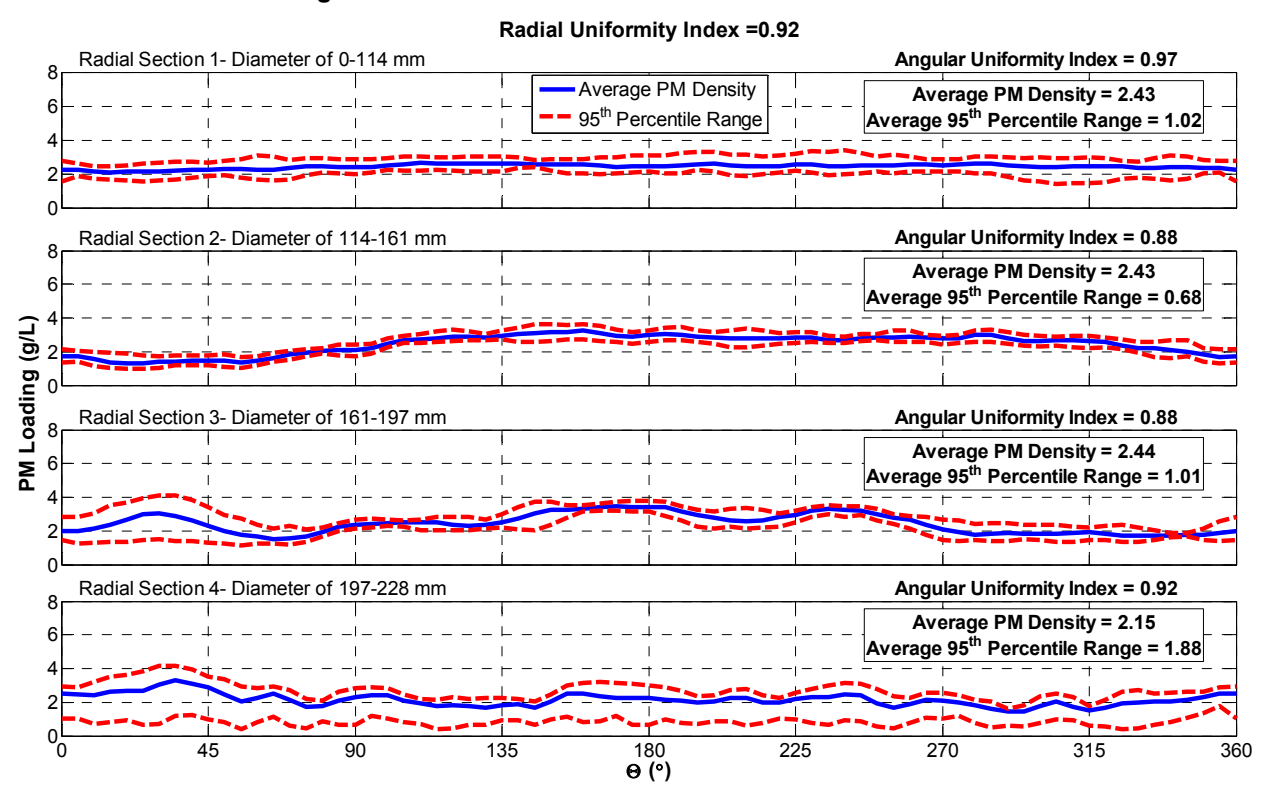

Figure K.4: Phase 1 Test 1- Active Regeneration $Z^{*}=0.375$ 
Test 1: Active Regeneration

Angular PM Distribution in Each Radial Section at $Z^{*}=0.625$

Radial Uniformity Index $=0.93$

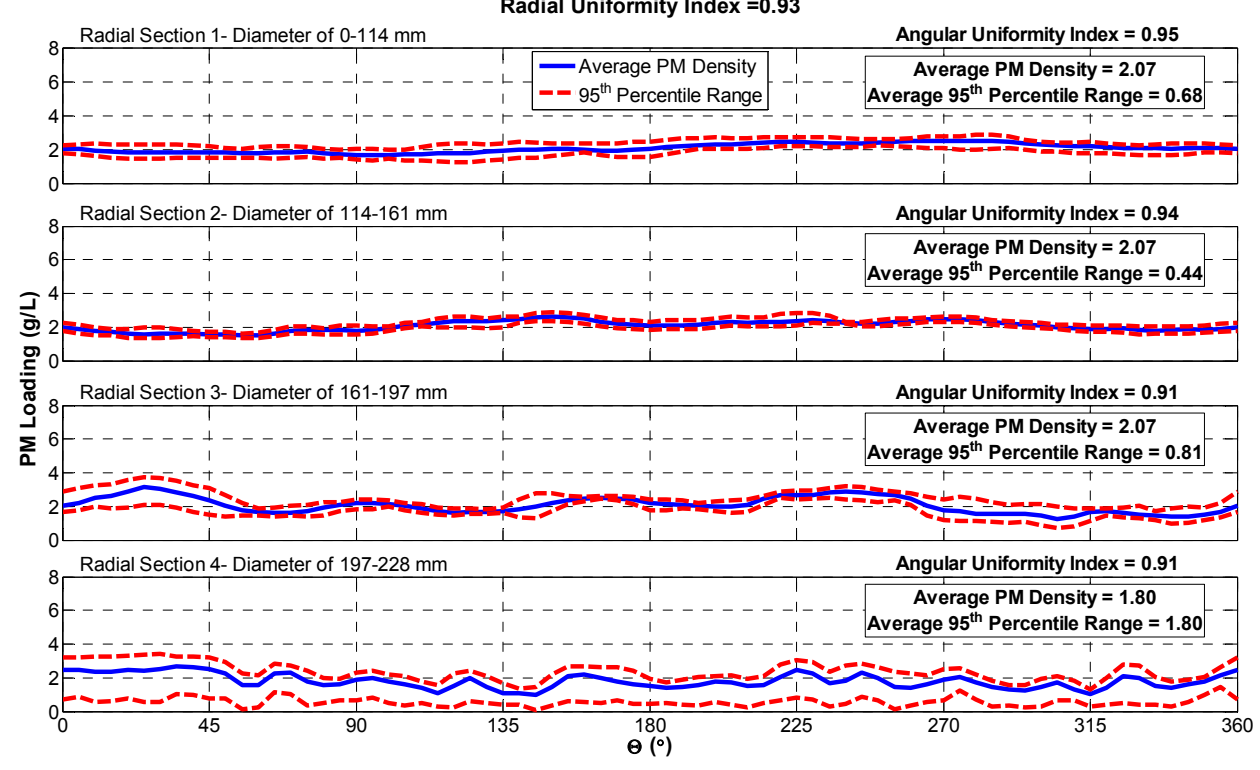

Figure K.5: Phase 1 Test 1- Active Regeneration $Z^{*}=0.625$

Test 1: Active Regeneration

Angular PM Distribution in Each Radial Section at $Z^{*}=\mathbf{0 . 8 7 5}$

Radial Uniformity Index $=\mathbf{0 . 9 3}$
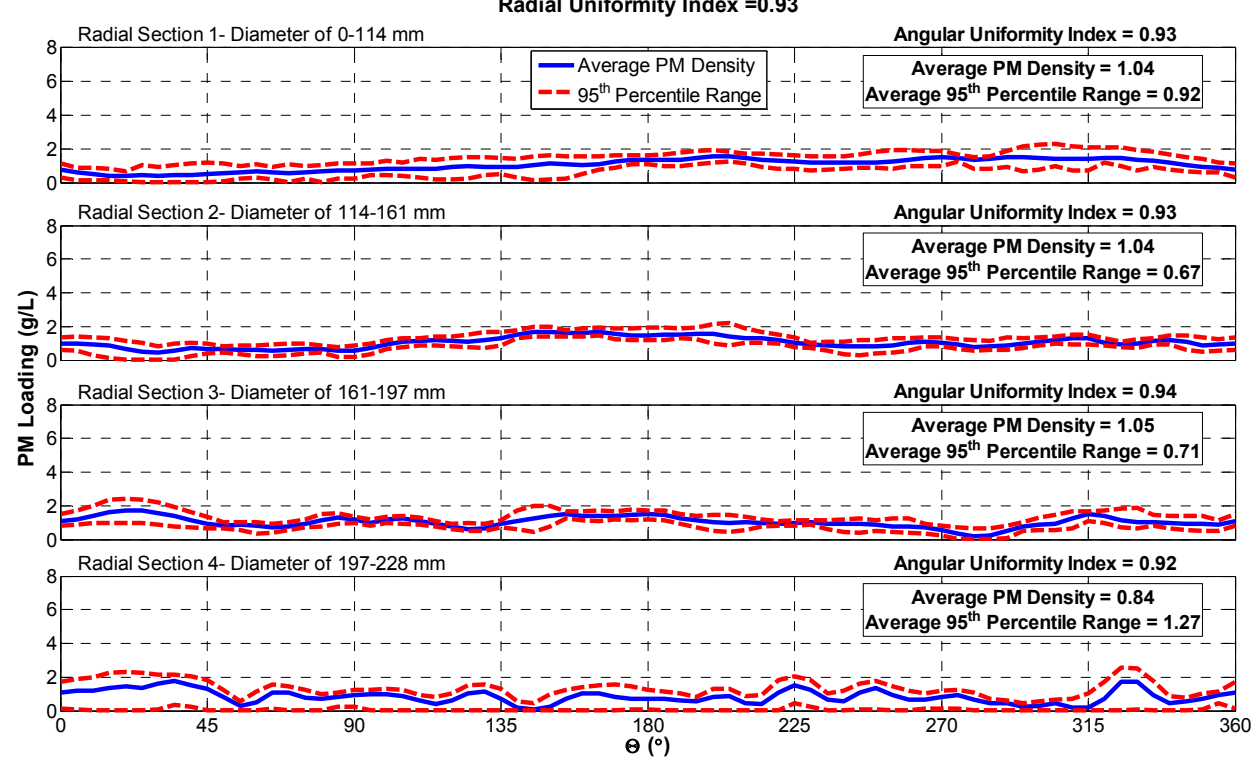

Figure K.6: Phase 1 Test 1- Active Regeneration $Z^{*}=0.875$ 


\section{Phase 1 Test 2 Active Regeneration Scan}

Test 2: Active Regeneration

Angular PM Distribution in Each Radial Section at $Z^{*}=\mathbf{0 . 3 7 5}$

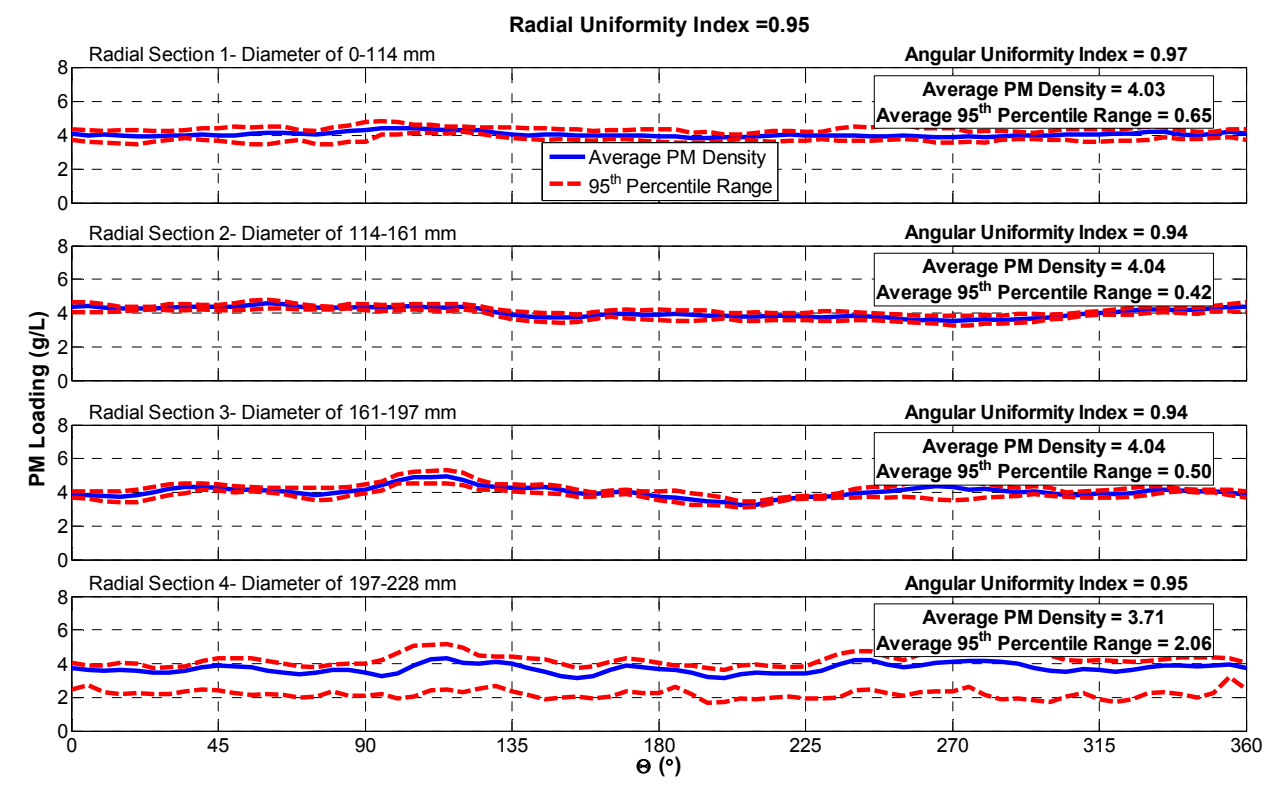

Figure K.7: Phase 1 Test 2- Active Regeneration $Z^{*}=0.375$

Test 2: Active Regeneration

Angular PM Distribution in Each Radial Section at $Z^{*}=0.625$

Radial Uniformity Index $=0.96$

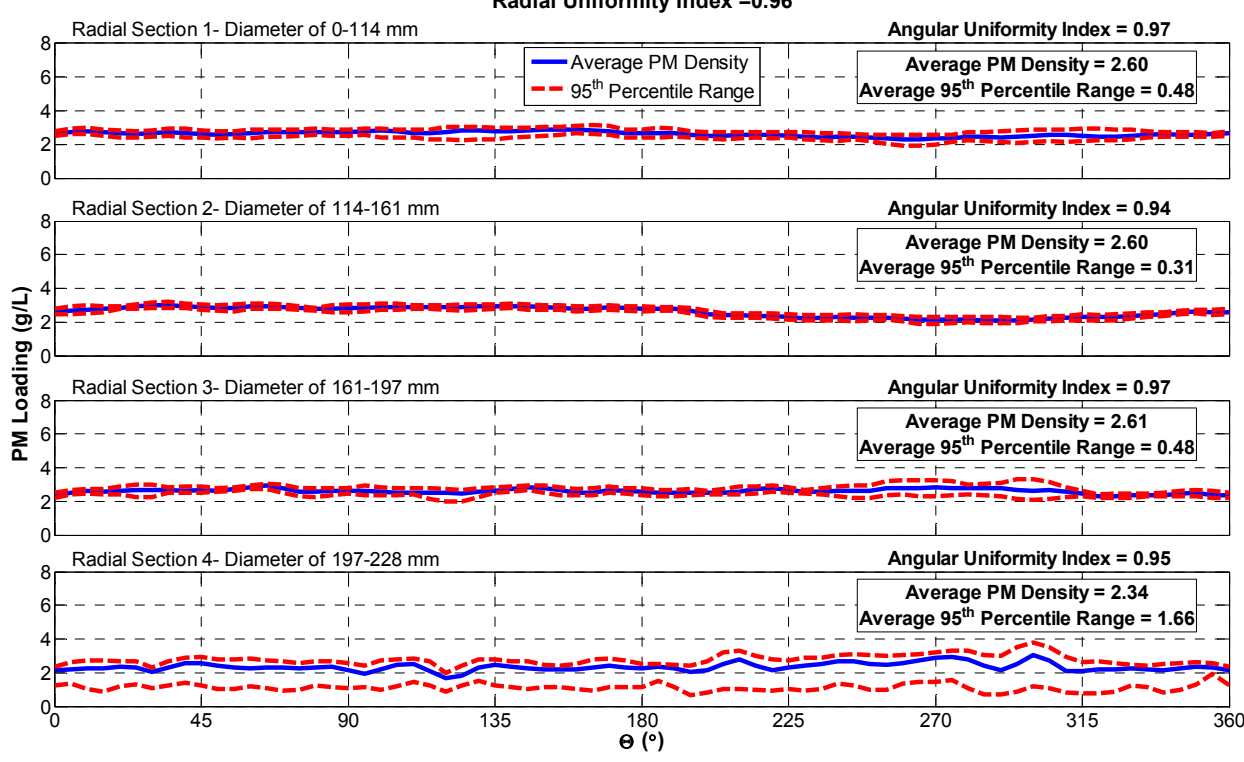

Figure K.8: Phase 1 Test 2- Active Regeneration $Z^{*}=0.625$ 
Test 2: Active Regeneration

Angular PM Distribution in Each Radial Section at $Z^{*}=\mathbf{0 . 8 7 5}$

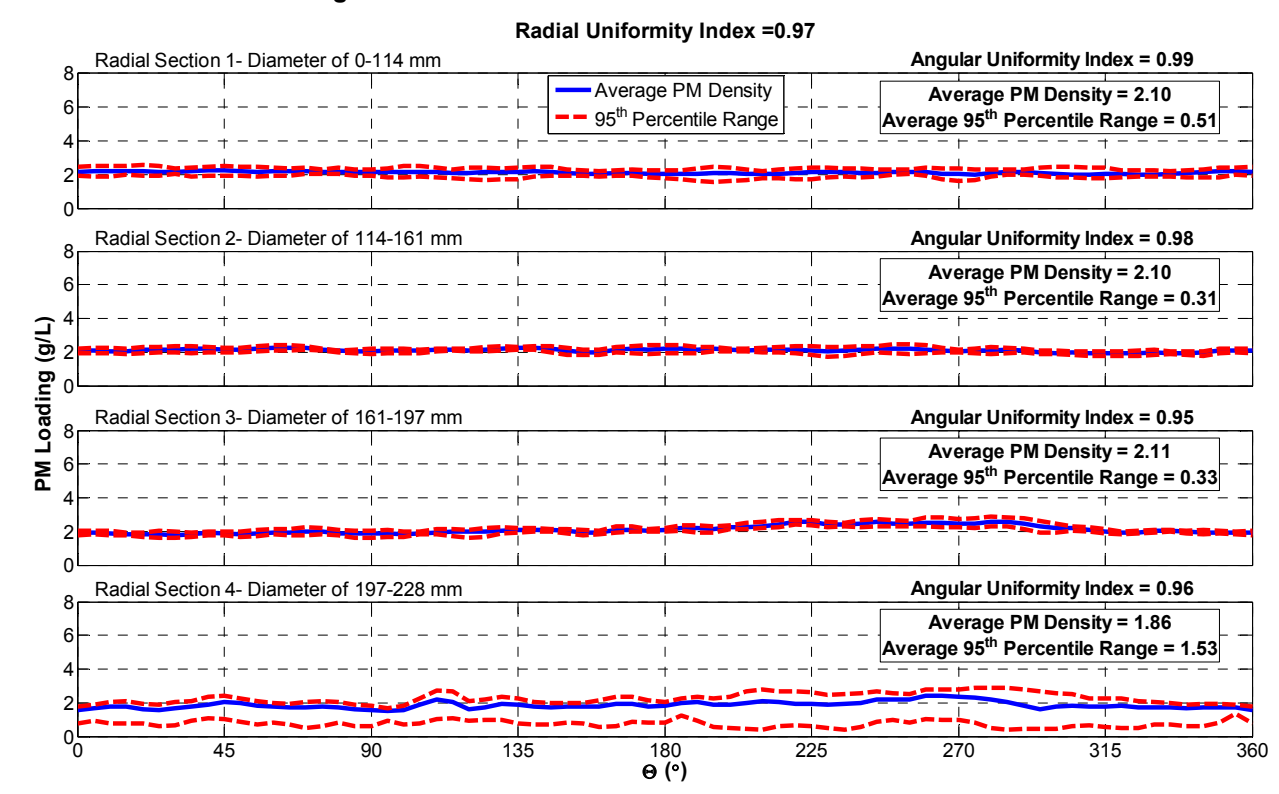

Figure K.9: Phase 1 Test 2- Active Regeneration $Z^{*}=0.875$

\section{Phase 1 Test 3 Passive Oxidation Scan}

Test 3: Passive Oxidation

Angular PM Distribution in Each Radial Section at $Z^{*}=0.375$

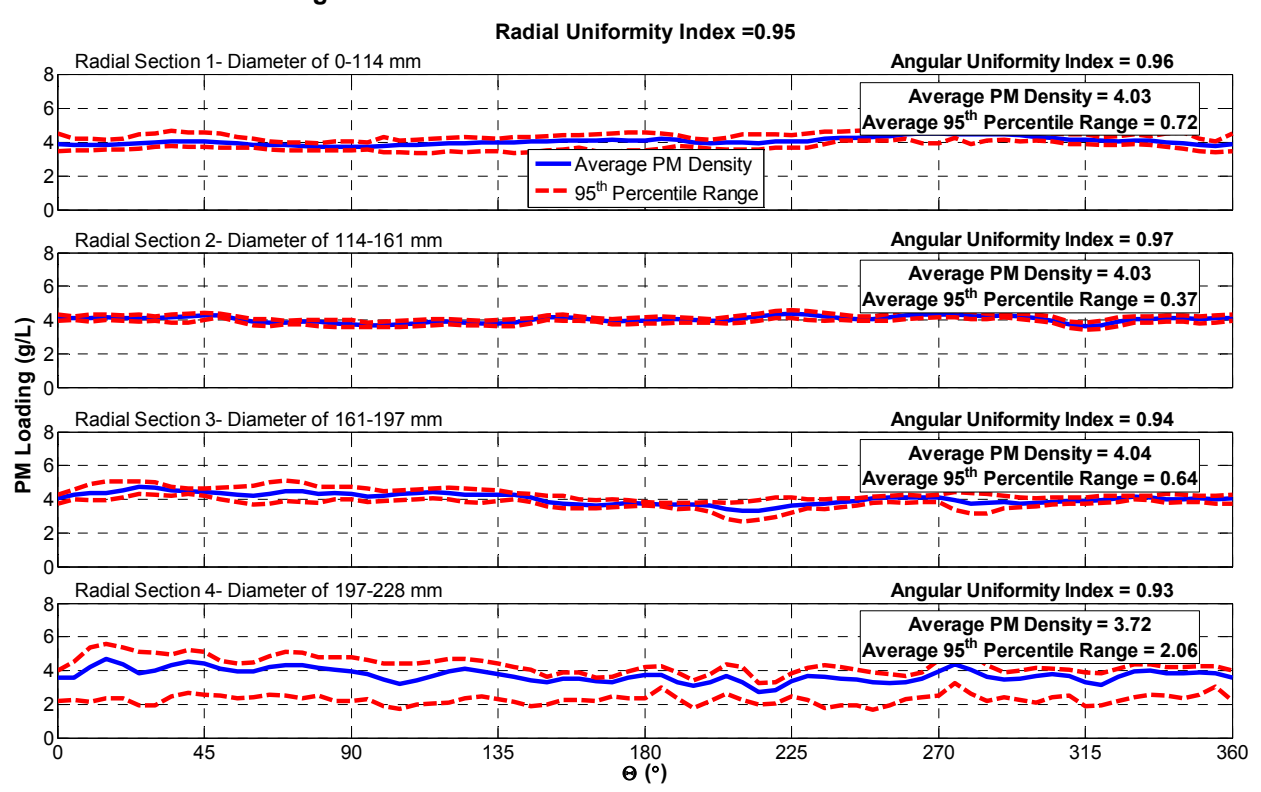

Figure K.10: Phase 1 Test 3- Passive Oxidation $Z^{*}=0.375$ 
Test 3: Passive Oxidation

Angular PM Distribution in Each Radial Section at Z*=0.625

Radial Uniformity Index $=0.96$

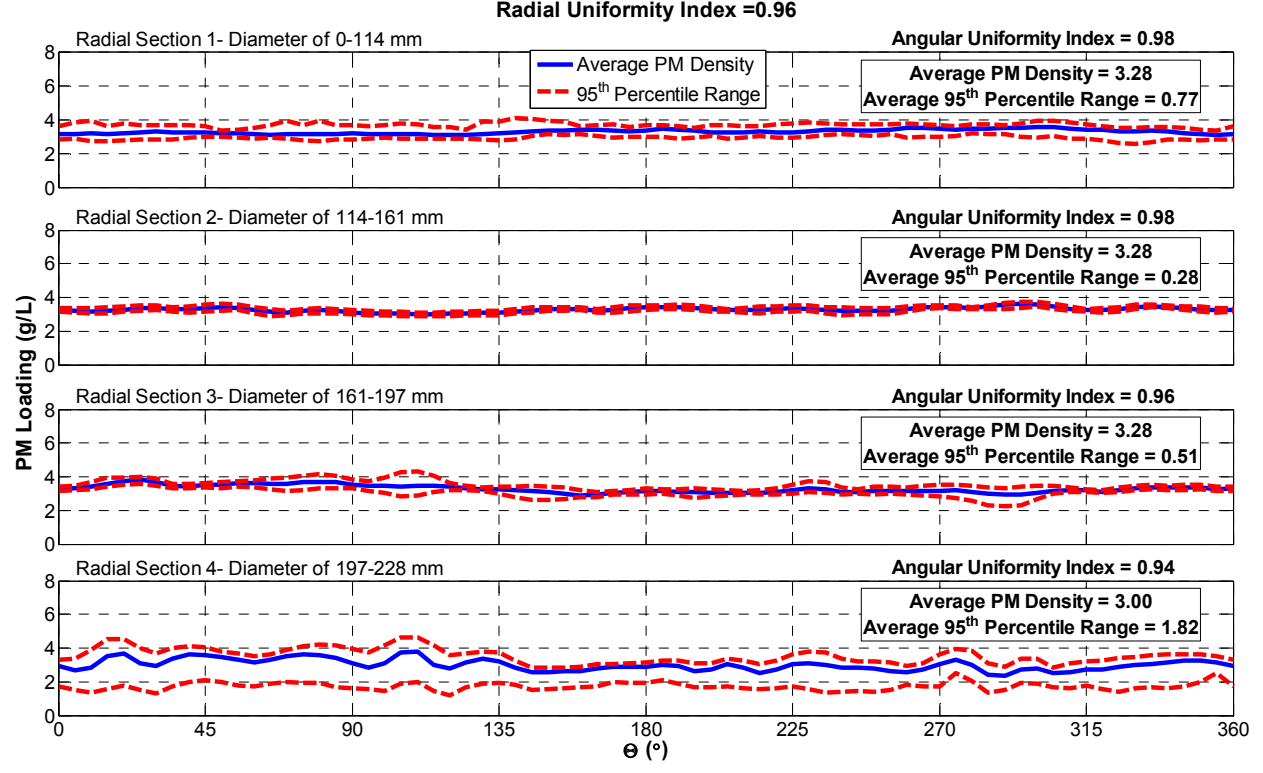

Figure K.11: Phase 1 Test 3- Passive Oxidation $Z^{*}=0.625$

Test 3: Passive Oxidation

Angular PM Distribution in Each Radial Section at $Z^{*}=\mathbf{0 . 8 7 5}$

Radial Uniformity Index $=0.96$

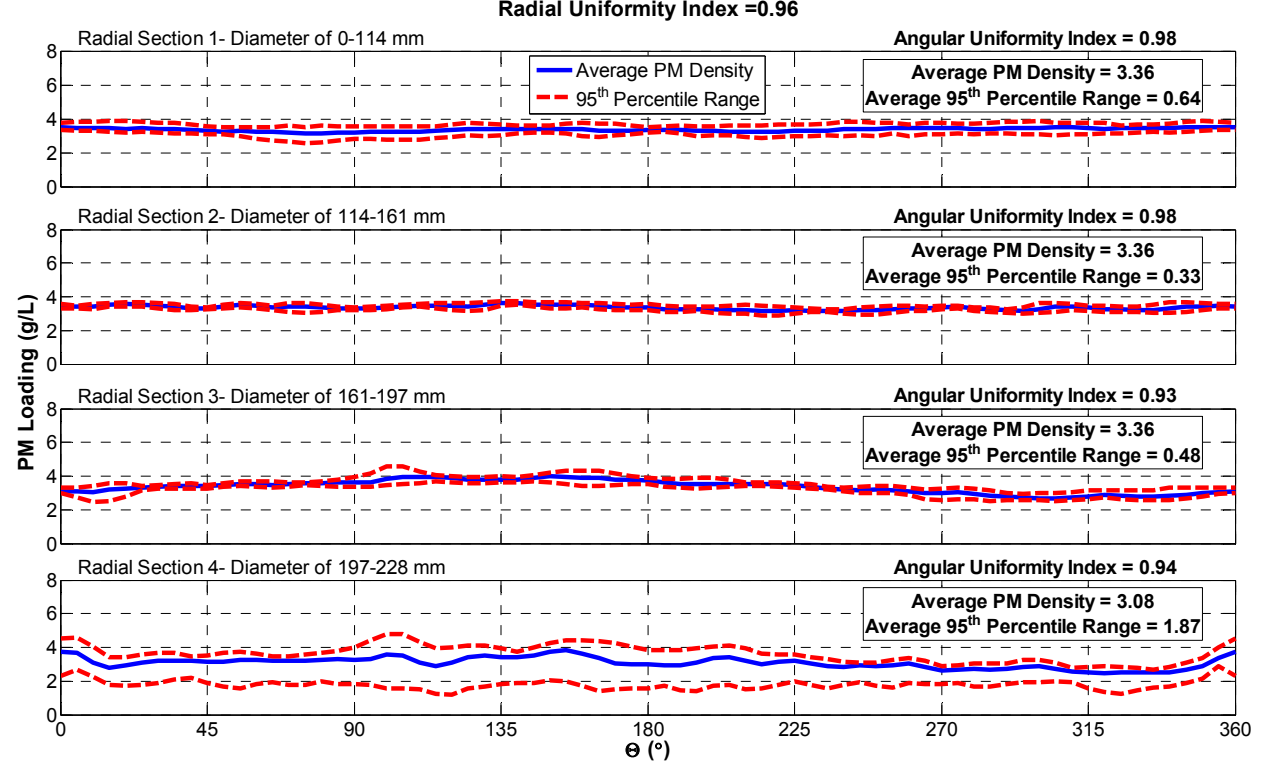

Figure K.12: Phase 1 Test 3- Passive Oxidation $Z^{*}=0.875$ 


\section{Phase 1 Test 4 Active Regeneration Scan}

Test 4: Active Regeneration

Angular PM Distribution in Each Radial Section at $Z^{*}=\mathbf{0 . 3 7 5}$

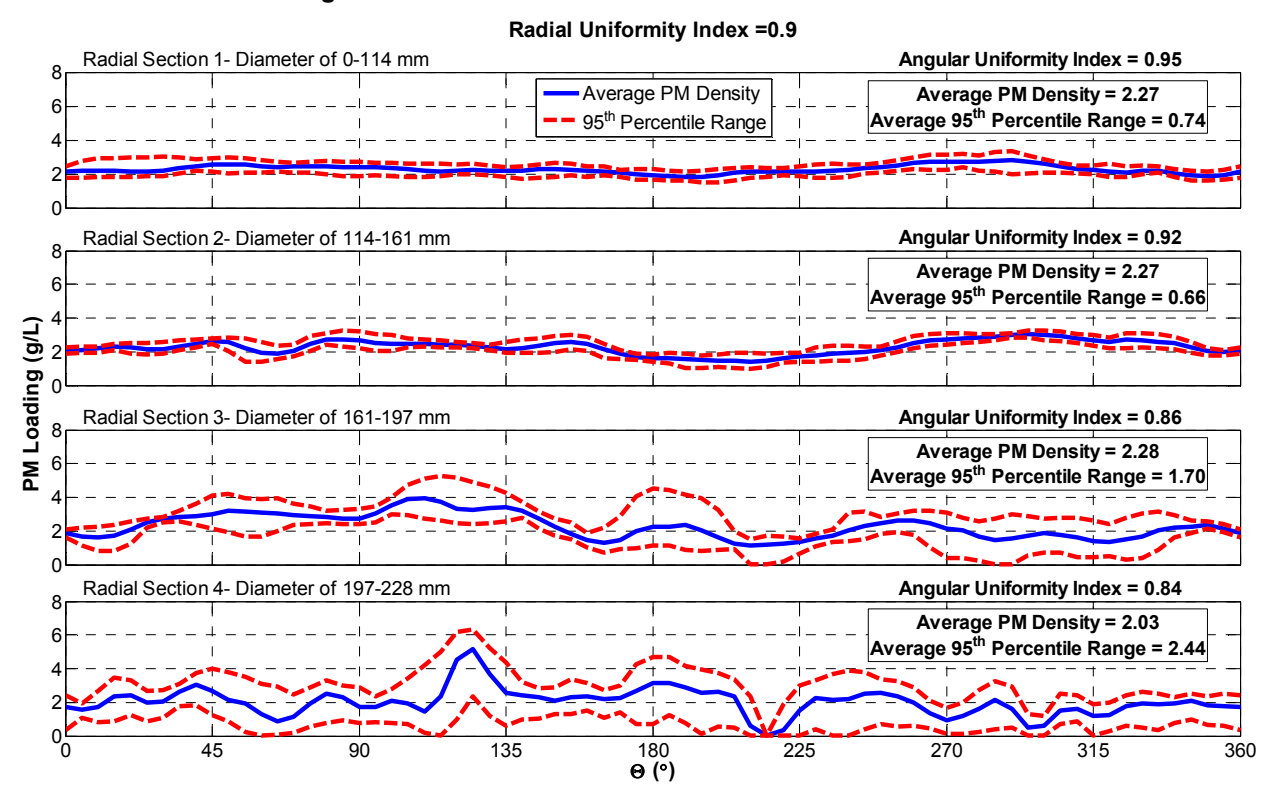

Figure K.13: Phase 1 Test 4- Active Regeneration $Z^{*}=0.375$

Test 4: Active Regeneration

Angular PM Distribution in Each Radial Section at $Z^{*}=0.625$

Radial Uniformity Index $=0.96$

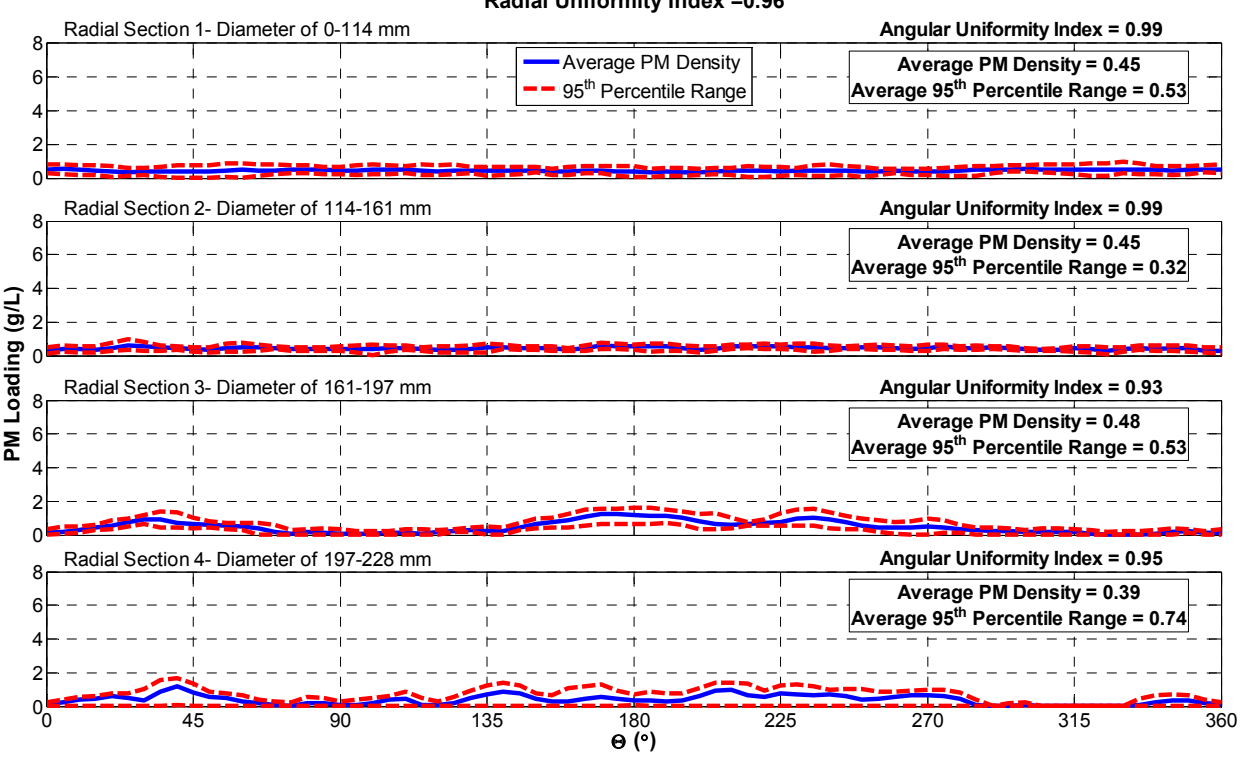

Figure K.14: Phase 1 Test 4- Active Regeneration $Z^{*}=0.625$ 
Test 4: Active Regeneration

Angular PM Distribution in Each Radial Section at $Z^{*}=\mathbf{0 . 8 7 5}$

Radial Uniformity Index $=0.96$

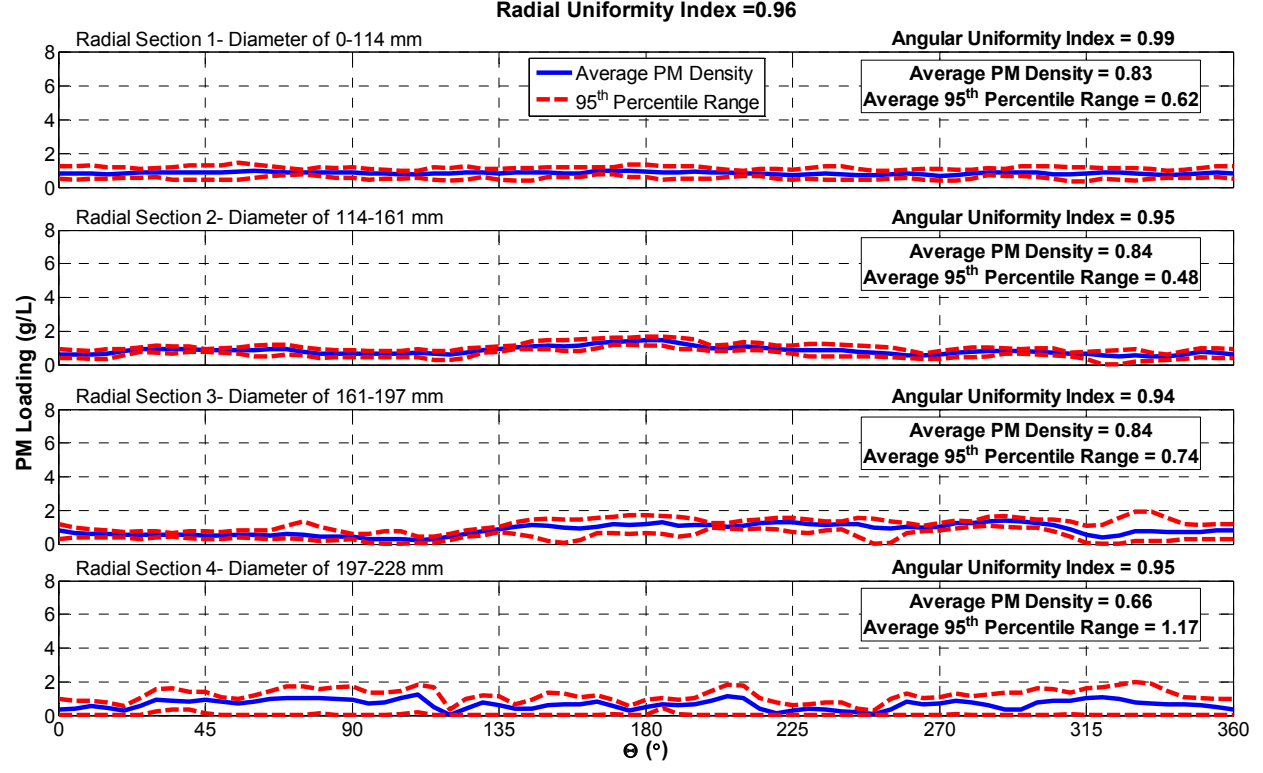

Figure K.15: Phase 1 Test 4- Active Regeneration $Z^{*}=0.875$

\section{Phase 2 Test 1 Loading Scan}

Test 1: Loading

Axial PM Distribution in Each Radial Section

Average Radial Uniformity Index $=0.96$
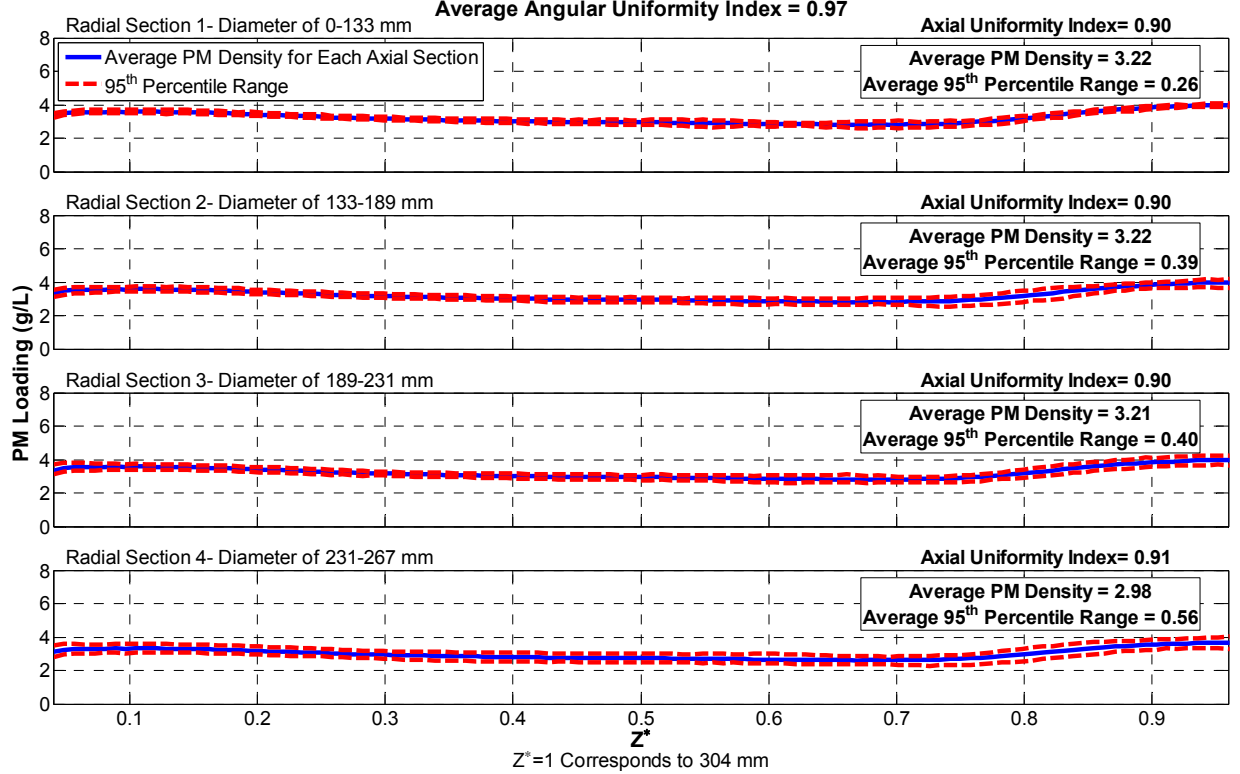

Figure K.16: Phase 2 Test 1- Loading Axial Distribution 
Test 1: Loading

Angular PM Distribution in Each Radial Section at $Z^{*}=\mathbf{0 . 1 2 8}$

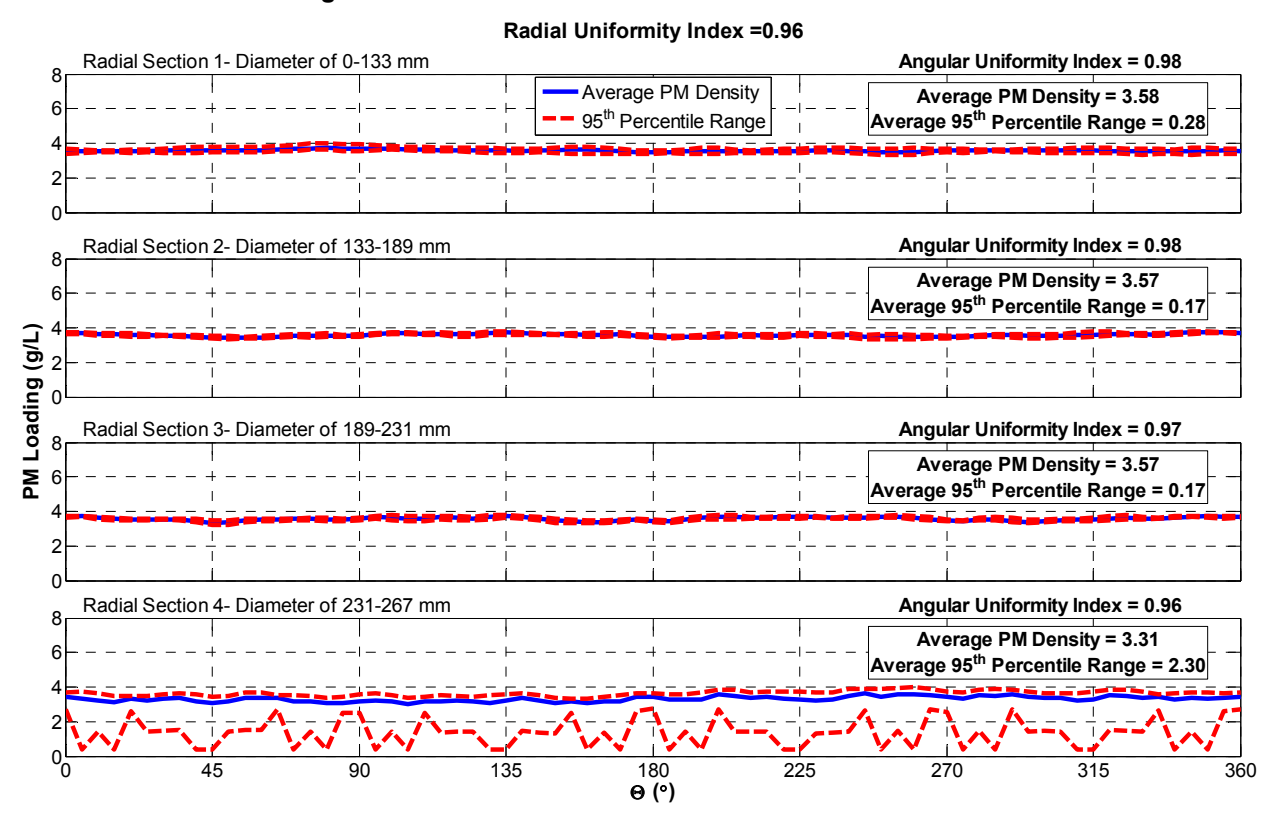

Figure K.17: Phase 2 Test 1- Loading $Z^{*}=0.128$

\section{Angular PM Distribution in Each Radial Section at $Z^{*}=\mathbf{0 . 3 7 6}$}
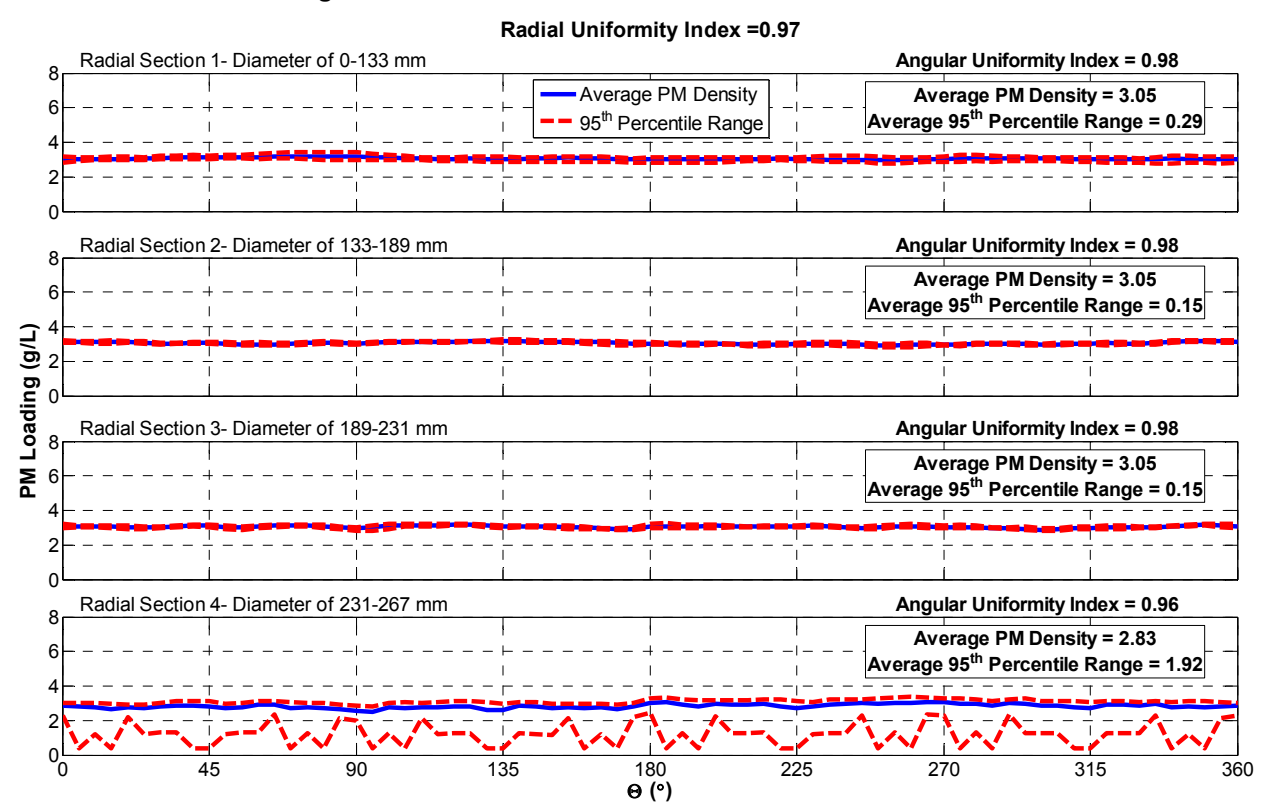

Figure K.18: Phase 2 Test 1- Loading $Z^{*}=0.376$ 
Test 1: Loading

Angular PM Distribution in Each Radial Section at $Z^{*}=\mathbf{0 . 6 2 4}$

Radial Uniformity Index $=0.97$
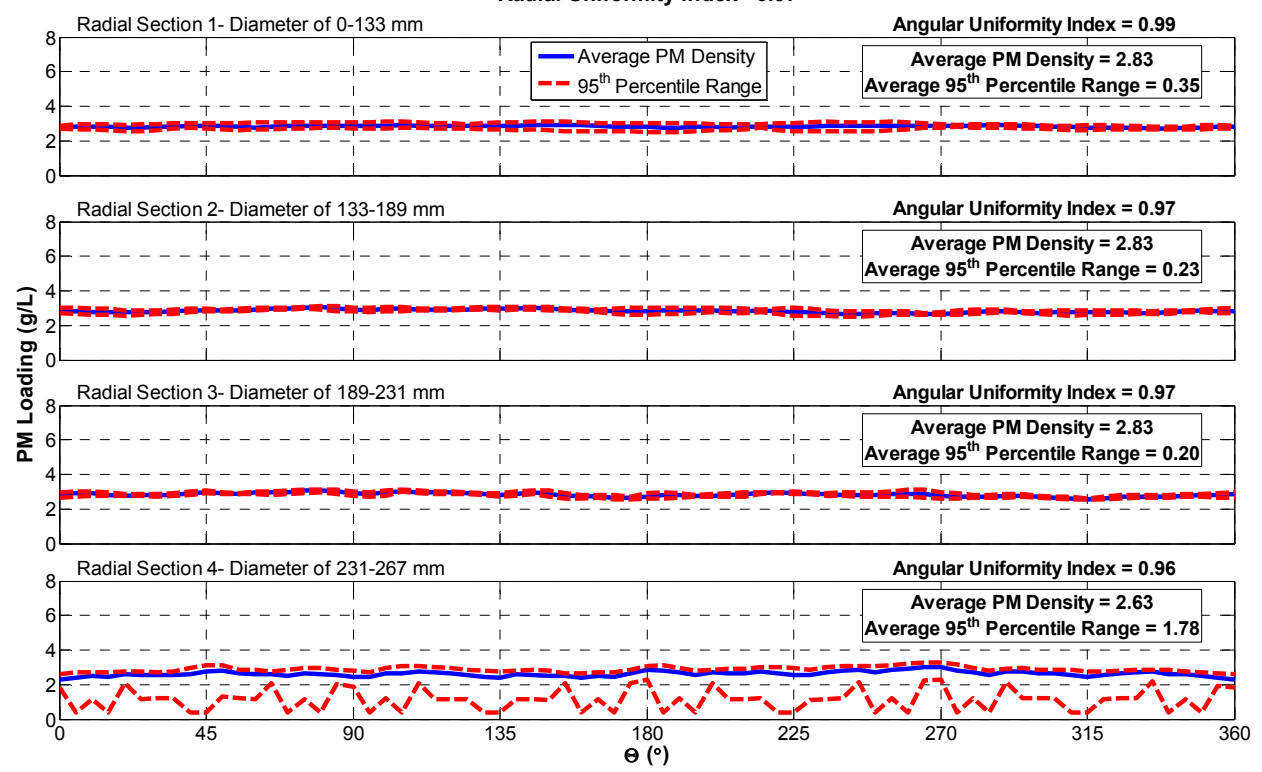

Figure K.19: Phase 2 Test 1- Loading $Z^{*}=0.624$

Test 1: Loading
Angular PM Distribution in Each Radial Section at $Z^{*}=0.872$

Radial Uniformity Index $=0.96$
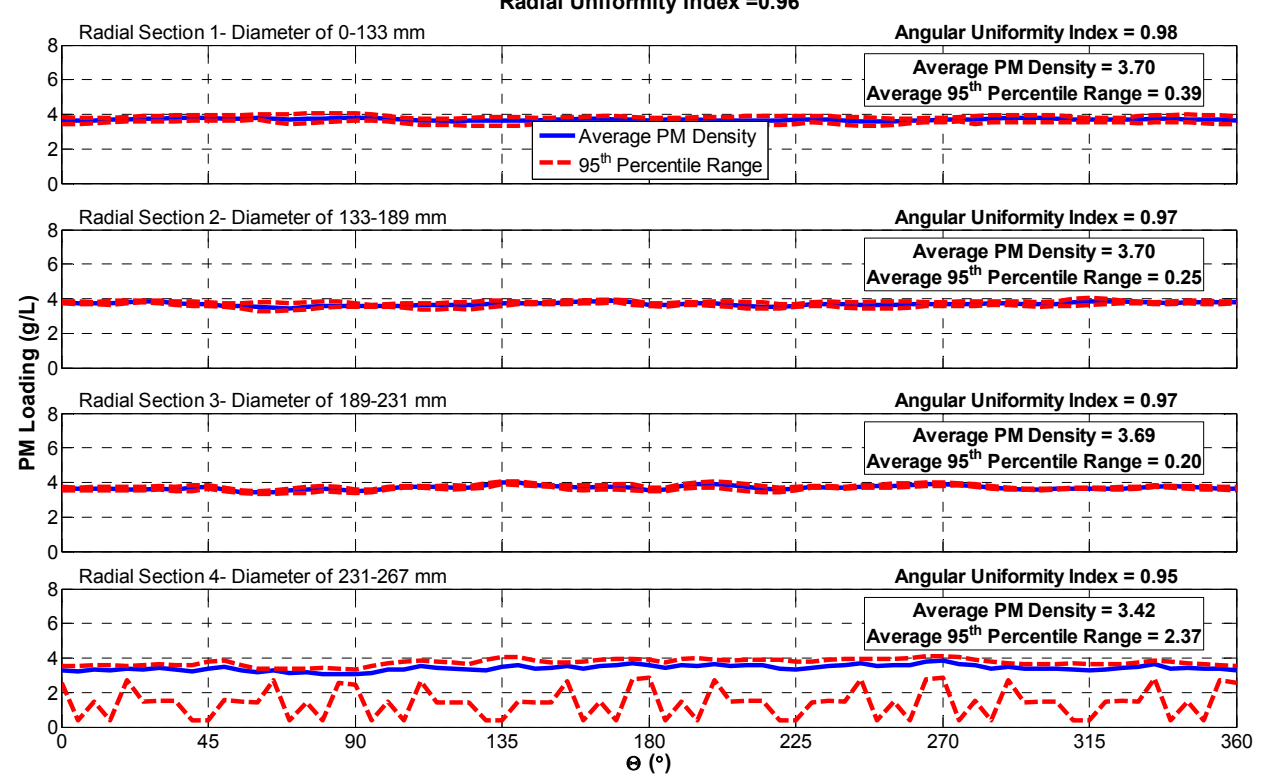

Figure K.20: Phase 2 Test 1- Loading $Z^{*}=0.872$ 


\section{Phase 2 Test 1 Passive Oxidation Scan}

Test 1: Passive Oxidation

Axial PM Distribution in Each Radial Section

Average Radial Uniformity Index $=0.97$

Average Angular Uniformity Index $=0.98$
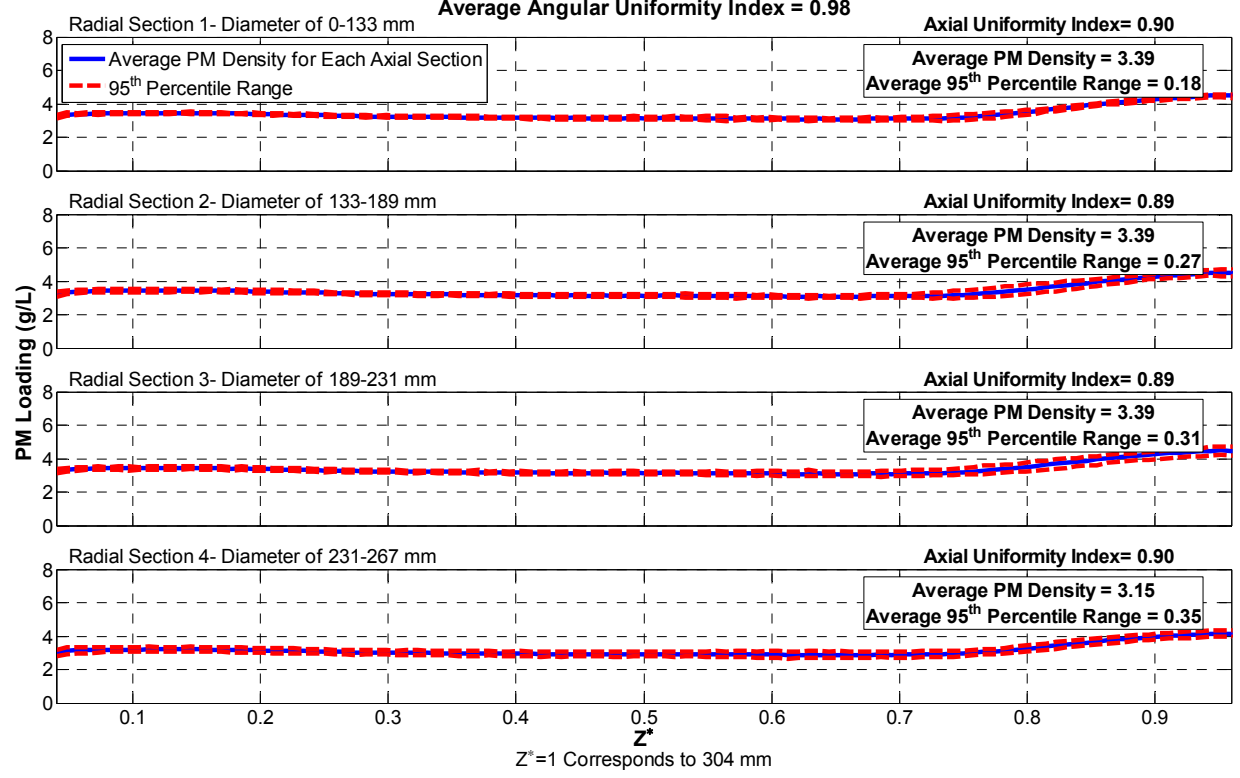

Figure K.21: Phase 2 Test 1- Passive Oxidation Axial Distribution

Test 1: Passive Oxidation

Angular PM Distribution in Each Radial Section at $Z^{*}=0.128$

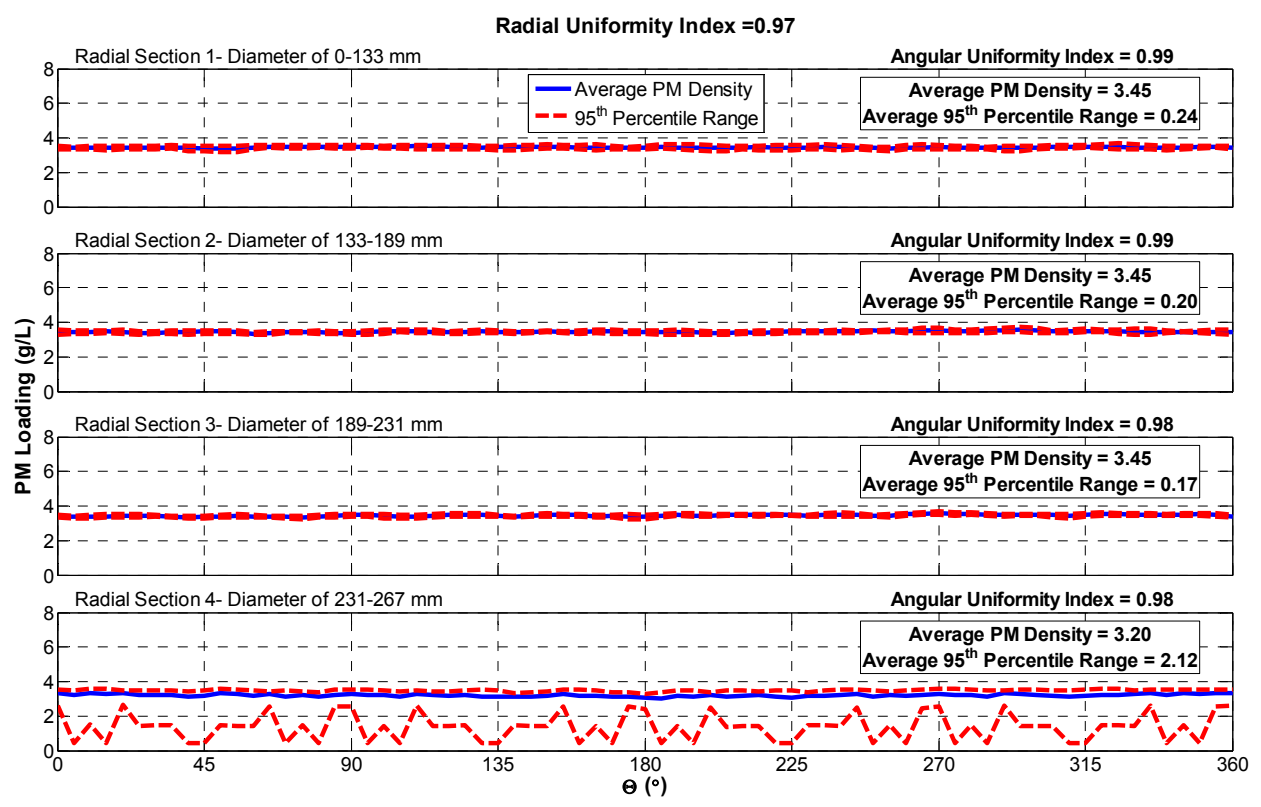

Figure K.22: Phase 2 Test 1- Passive Oxidation $Z^{*}=0.128$ 
Test 1: Passive Oxidation

Angular PM Distribution in Each Radial Section at $Z^{*}=0.376$

Radial Uniformity Index $=0.97$
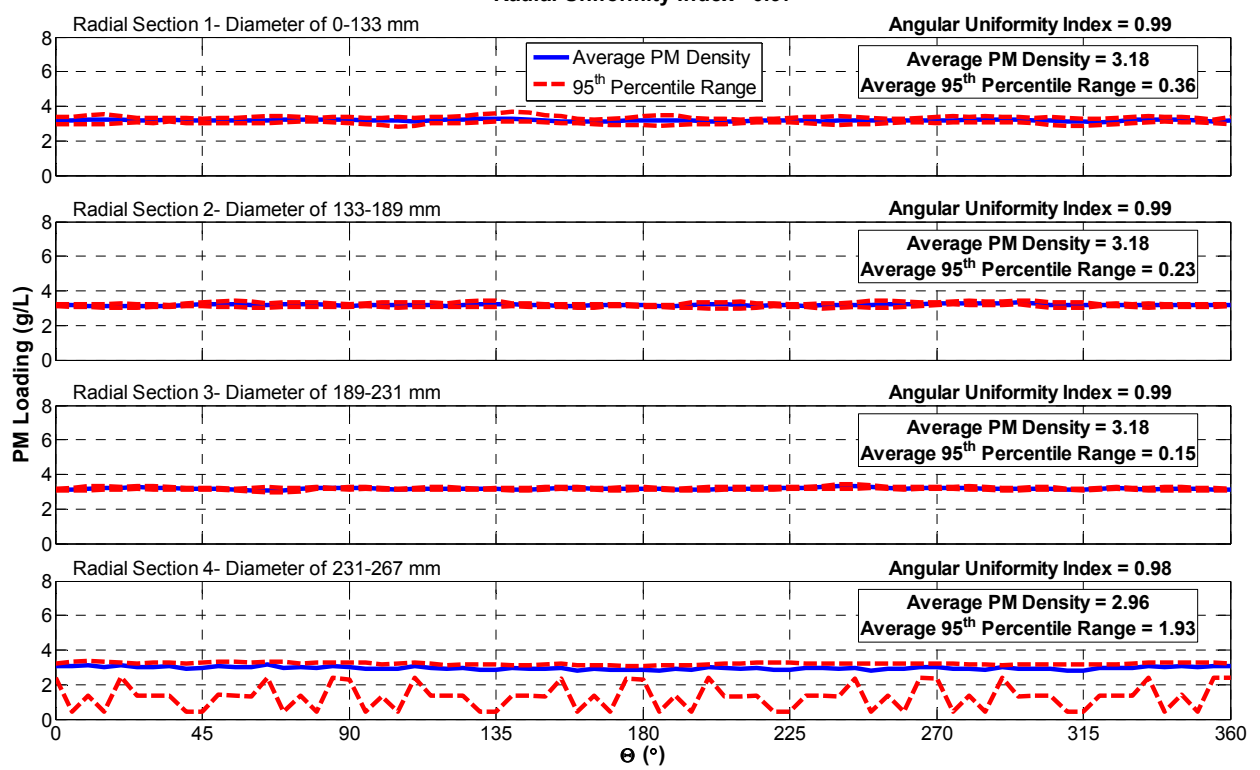

Figure K.23: Phase 2 Test 1- Passive Oxidation $Z^{*}=0.376$

Test 1: Passive Oxidation

Angular PM Distribution in Each Radial Section at $Z^{*}=\mathbf{0 . 6 2 4}$

Radial Uniformity Index $=0.97$
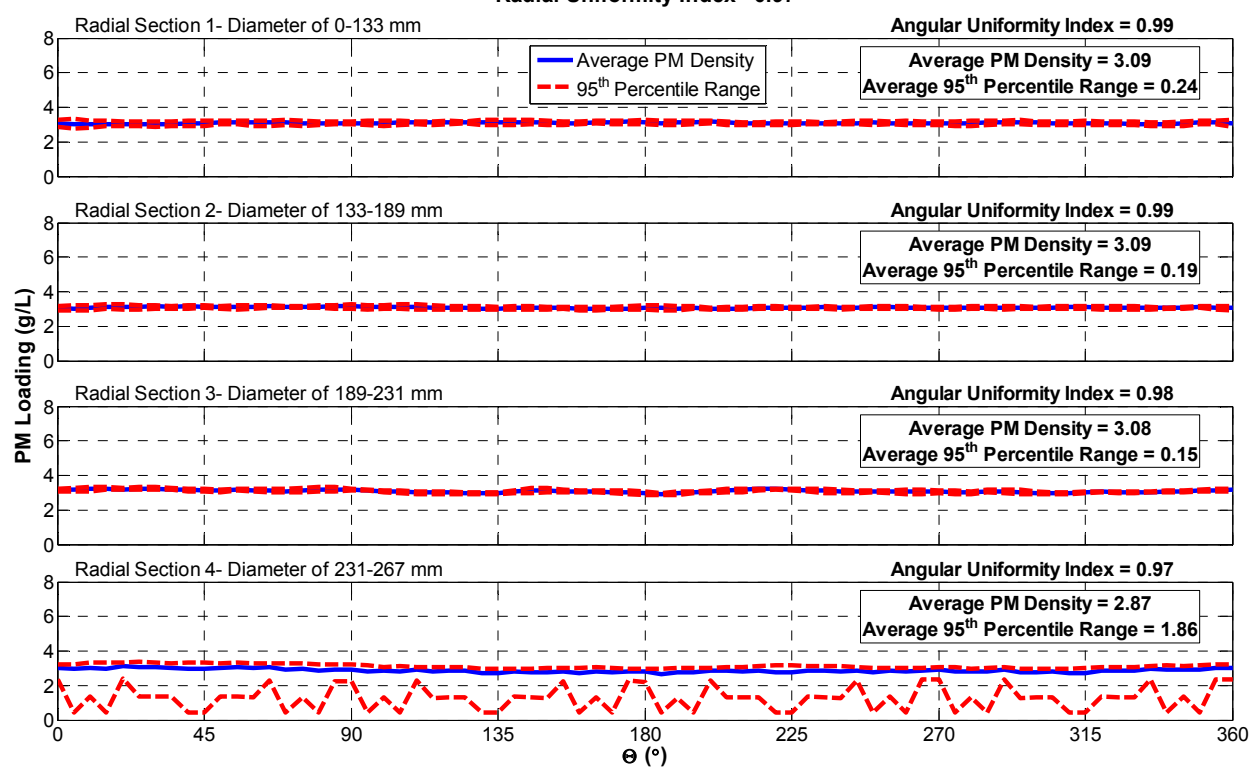

Figure K.24: Phase 2 Test 1- Passive Oxidation $Z^{*}=0.624$ 
Test 1: Passive Oxidation

Angular PM Distribution in Each Radial Section at $Z^{*}=0.872$

Radial Uniformity Index $=0.96$
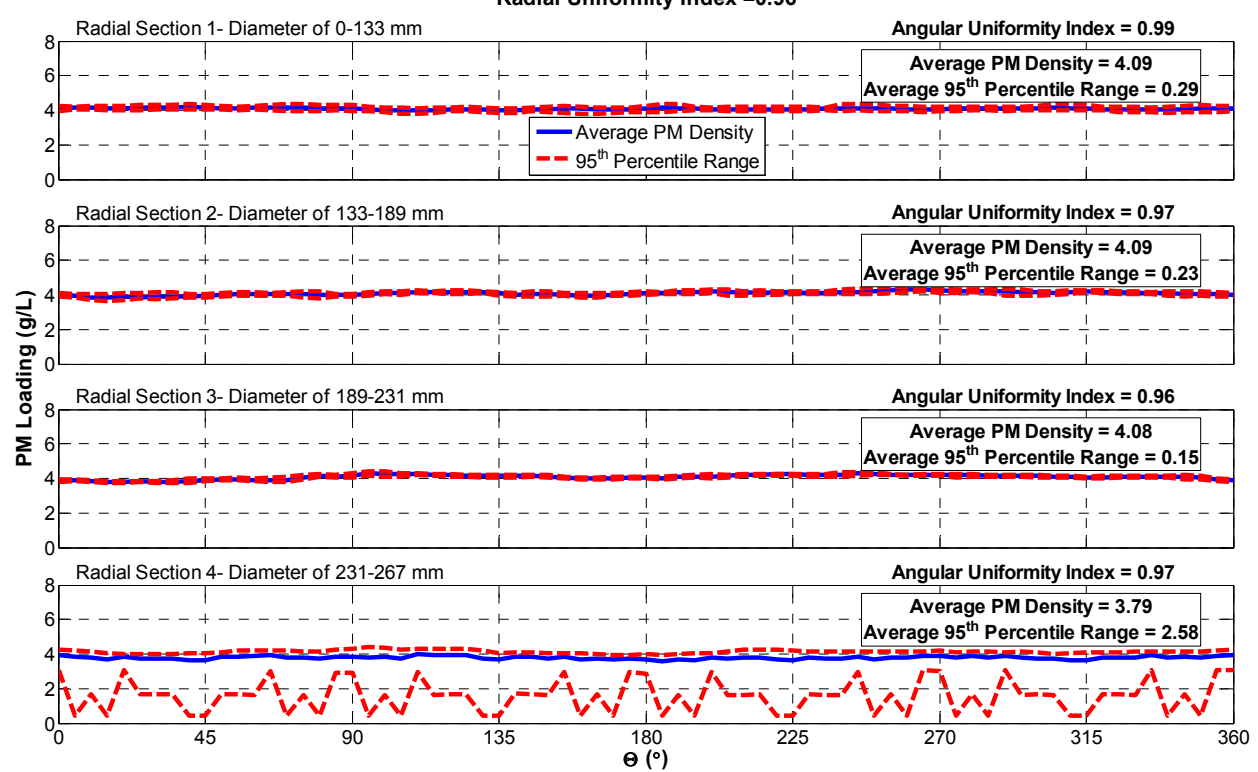

Figure K.25: Phase 2 Test 1- Passive Oxidation $Z^{*}=0.872$

\section{Phase 2 Test 2 Cleanout Scan}

Test 2: Cleanout

Axial PM Distribution in Each Radial Section

Average Radial Uniformity Index $=0.99$

Average Angular Uniformity Index $=0.98$
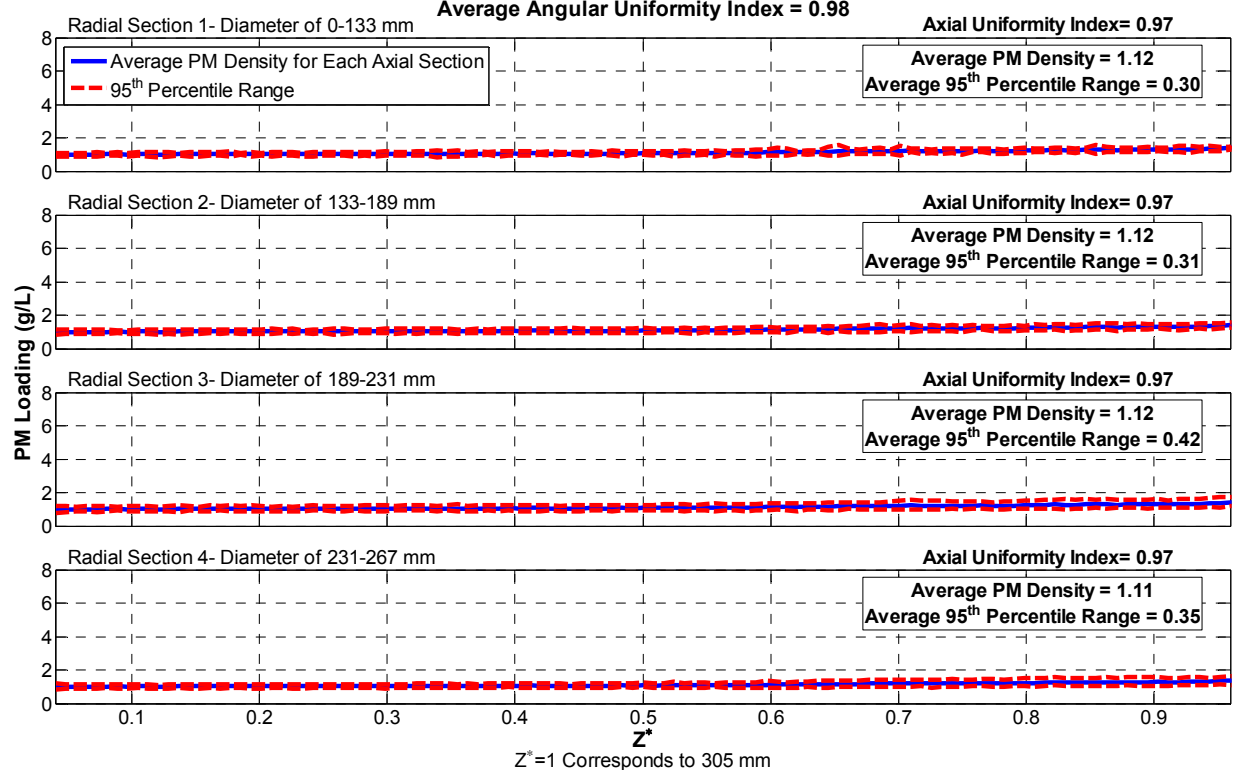

Figure K.26: Phase 2 Test 2- Cleanout Axial Distribution 
Test 2: Cleanout

Angular PM Distribution in Each Radial Section at $Z^{*}=0.128$

Radial Uniformity Index $=0.99$
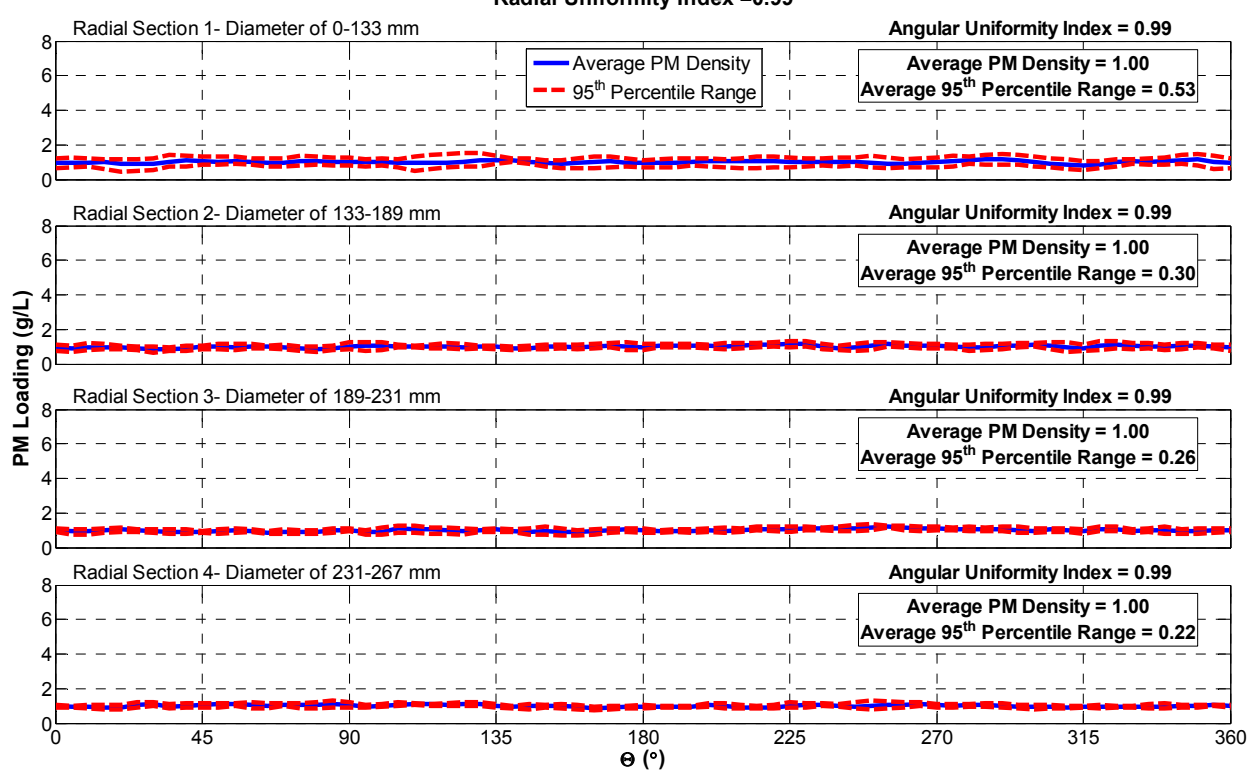

Figure K.27: Phase 2 Test 2- Cleanout $Z^{*}=0.128$

Test 2: Cleanout

Angular PM Distribution in Each Radial Section at $Z^{*}=0.376$

Radial Uniformity Index $=0.99$
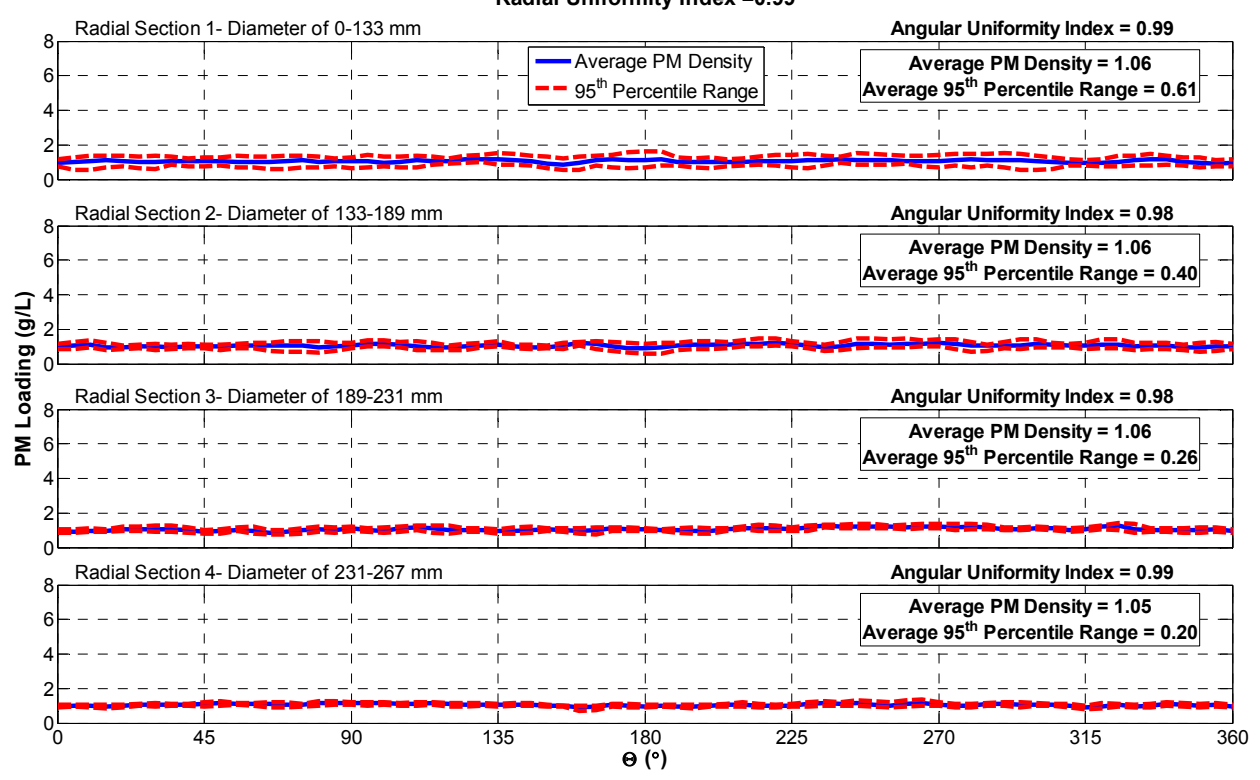

Figure K.28: Phase 2 Test 2- Cleanout $Z^{*}=0.376$ 
Test 2: Cleanout

Angular PM Distribution in Each Radial Section at $Z^{*}=0.624$

Radial Uniformity Index $=0.99$
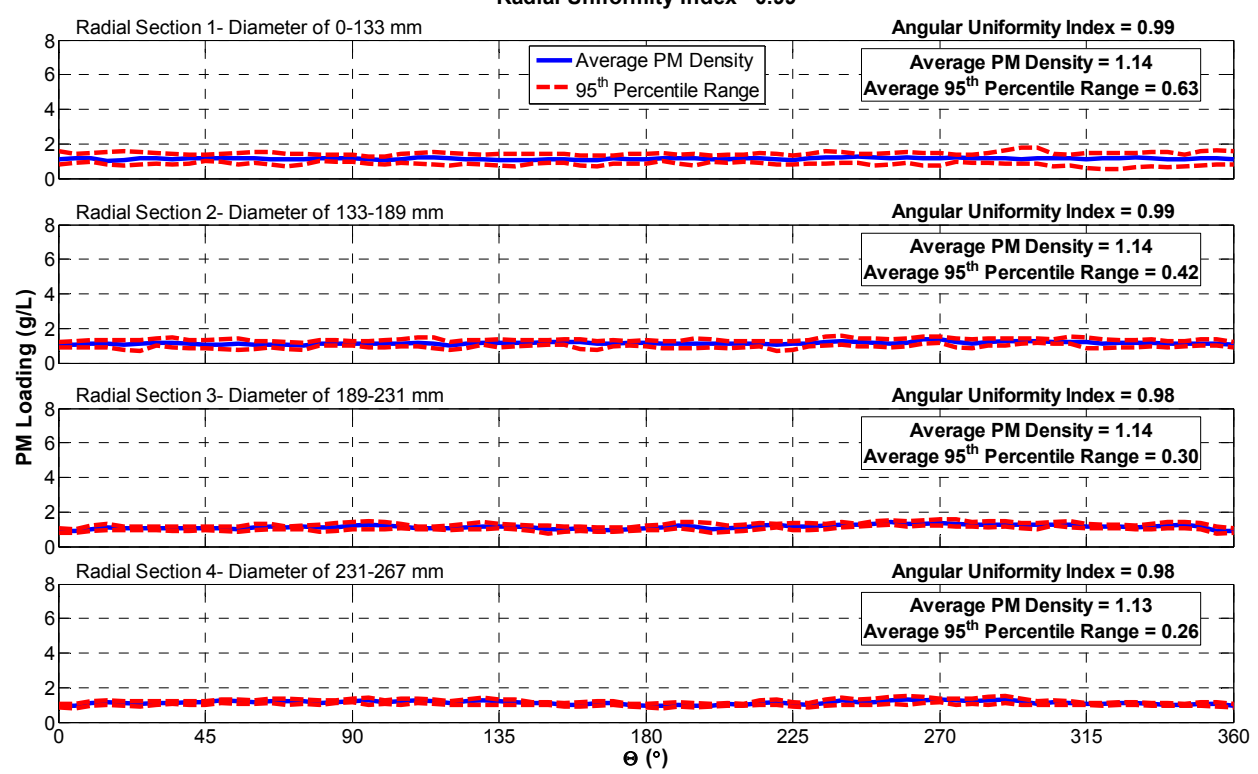

Figure K.29: Phase 2 Test 2- Cleanout $Z^{*}=0.624$

Test 2: Cleanout

Angular PM Distribution in Each Radial Section at $Z^{*}=0.872$
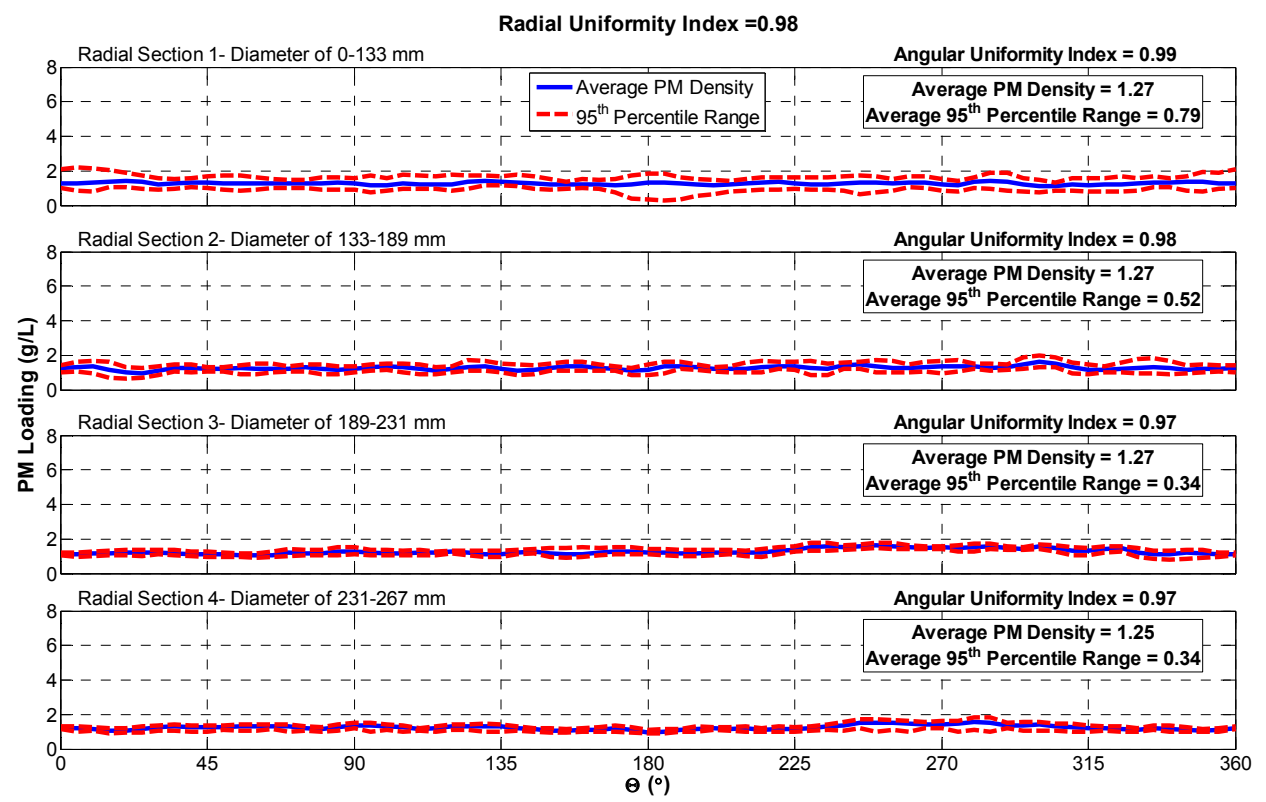

Figure K.30: Phase 2 Test 2- Cleanout $Z^{*}=0.872$ 


\section{Phase 2 Test 3 Active Regeneration Scan}

Test 3: Active Regeneration

Axial PM Distribution in Each Radial Section

Average Radial Uniformity Index $=0.97$

8 Radial Section 1- Diameter of 0-133 mm Average Angular Uniformity Index $=0.97$
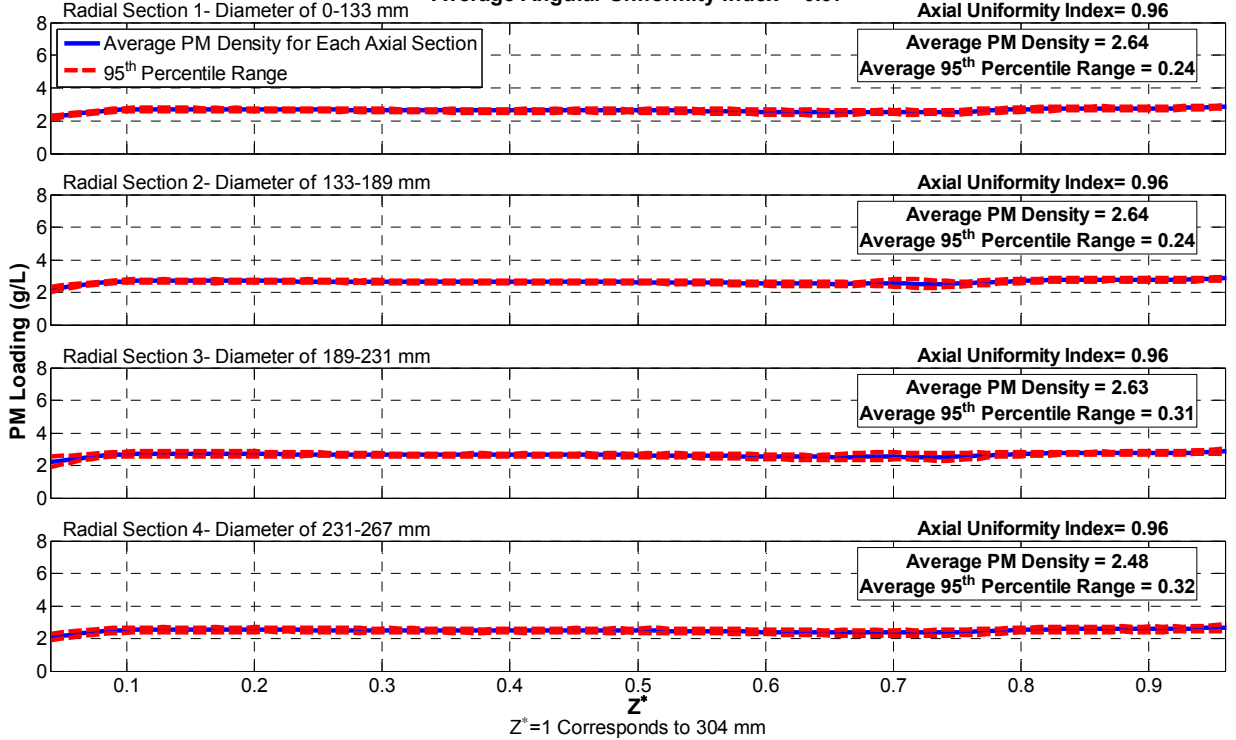

Figure K.31: Phase 2 Test 3- Active Regeneration Axial Distribution

Test 3: Active Regeneration

Angular PM Distribution in Each Radial Section at $Z^{*}=0.128$

Radial Uniformity Index $=0.97$

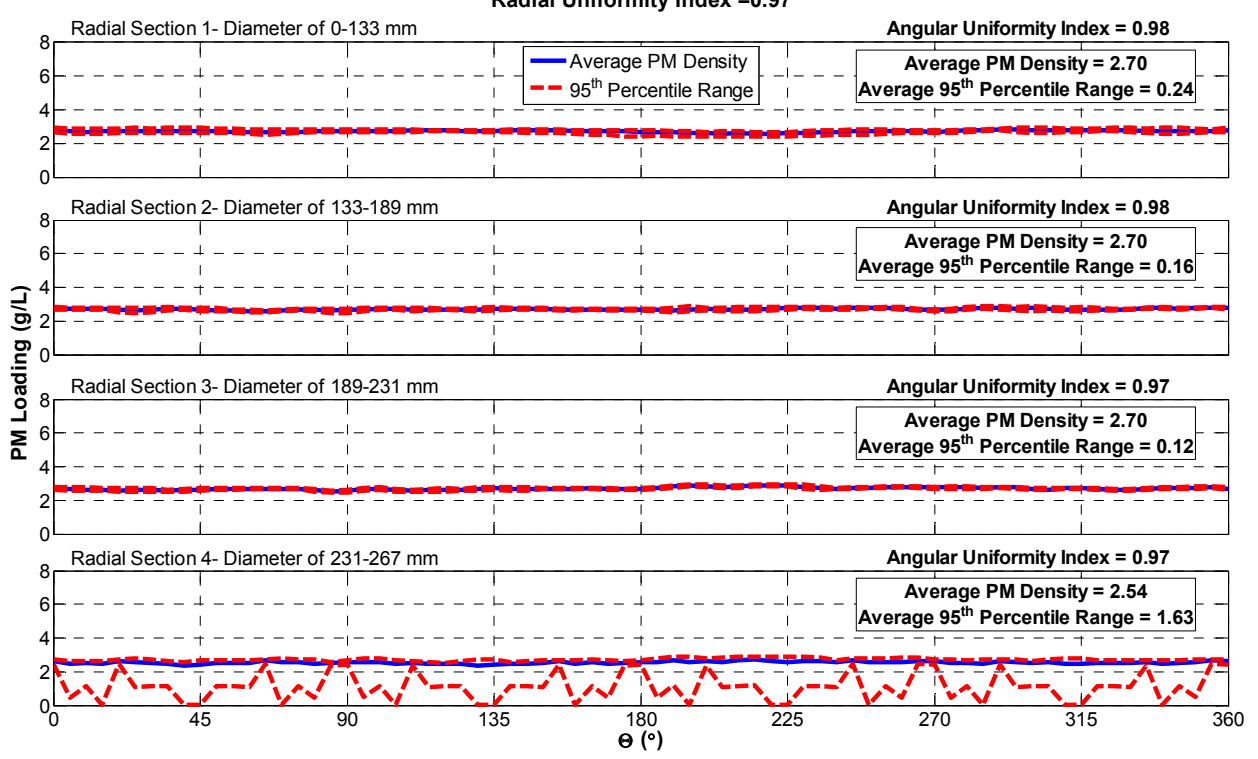

Figure K.32: Phase 2 Test 3- Active Regeneration $Z^{*}=0.128$ 
Test 3: Active Regeneration

Angular PM Distribution in Each Radial Section at $Z^{*}=\mathbf{0 . 3 7 6}$

Radial Uniformity Index $=0.97$

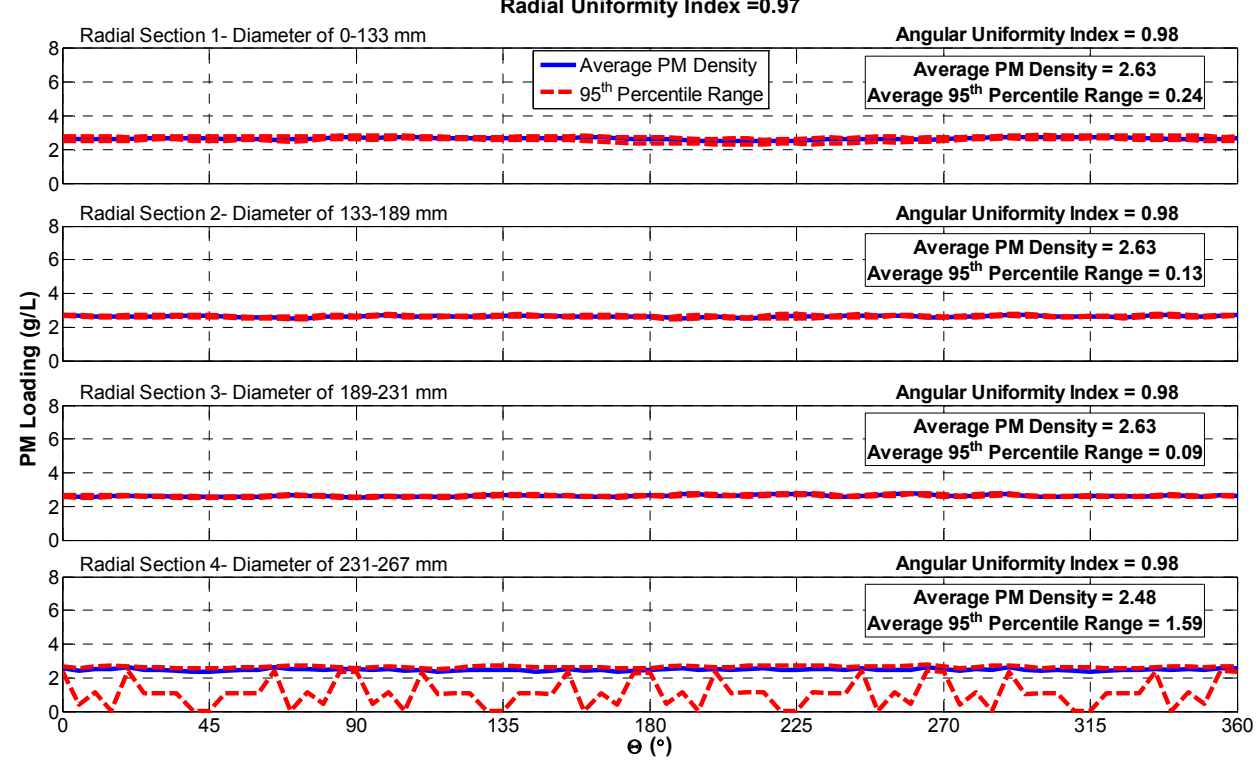

Figure K.33: Phase 2 Test 3- Active Regeneration $Z^{*}=0.376$

Test 3: Active Regeneration

Angular PM Distribution in Each Radial Section at $Z^{*}=\mathbf{0 . 6 2 4}$

Radial Uniformity Index $=0.97$

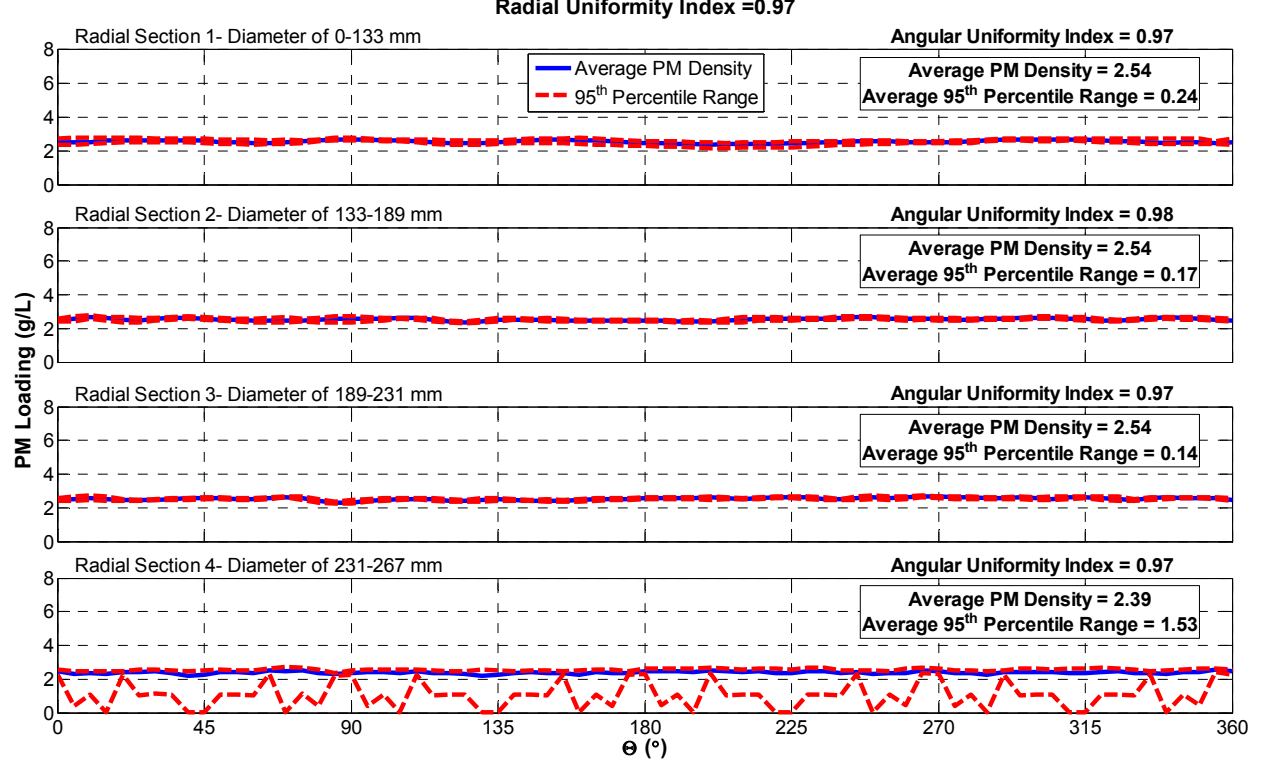

Figure K.34: Phase 2 Test 3- Active Regeneration $Z^{*}=0.624$ 
Test 3: Active Regeneration

Angular PM Distribution in Each Radial Section at $Z^{*}=\mathbf{0 . 8 7 2}$

Radial Uniformity Index $=0.97$

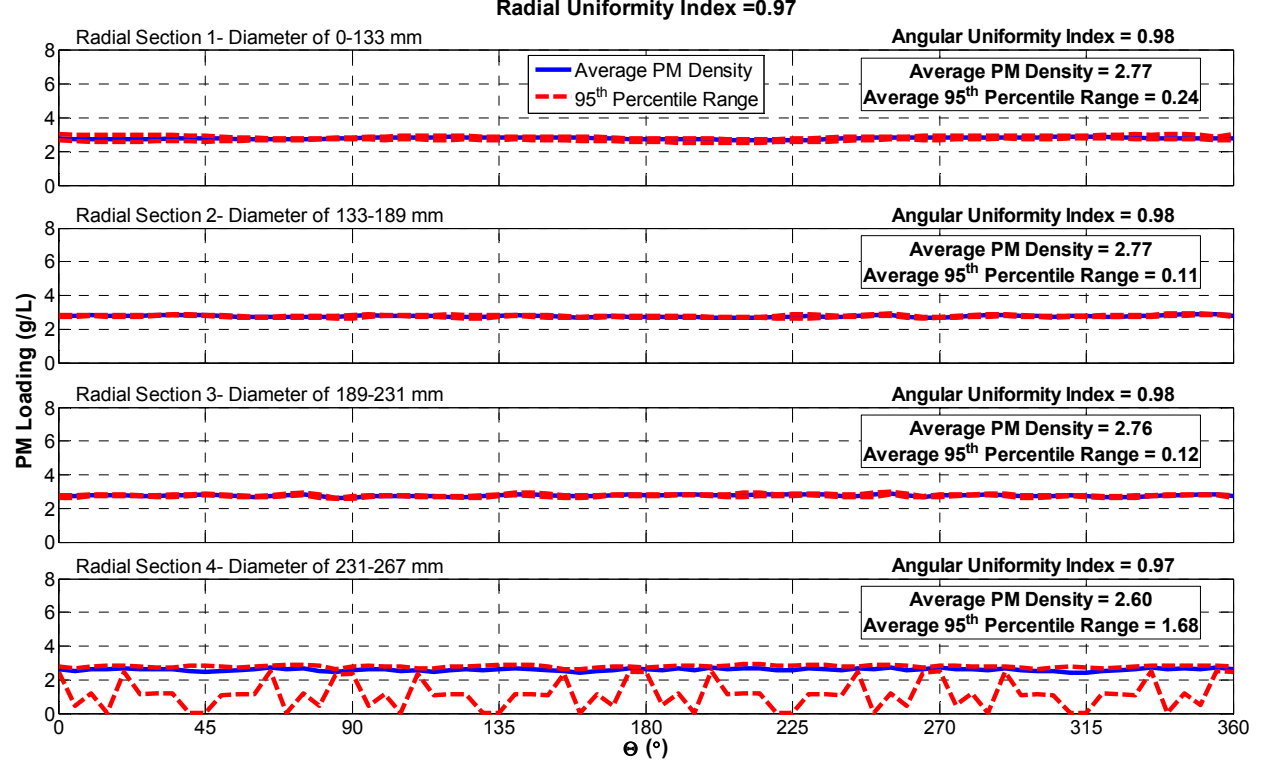

Figure K.35: Phase 2 Test 3- Active Regeneration $Z^{*}=0.872$

\section{Phase 2 Test 4 Active Regeneration Scan}

Test 4: Active Regeneration

Axial PM Distribution in Each Radial Section

Average Radial Uniformity Index $=0.98$
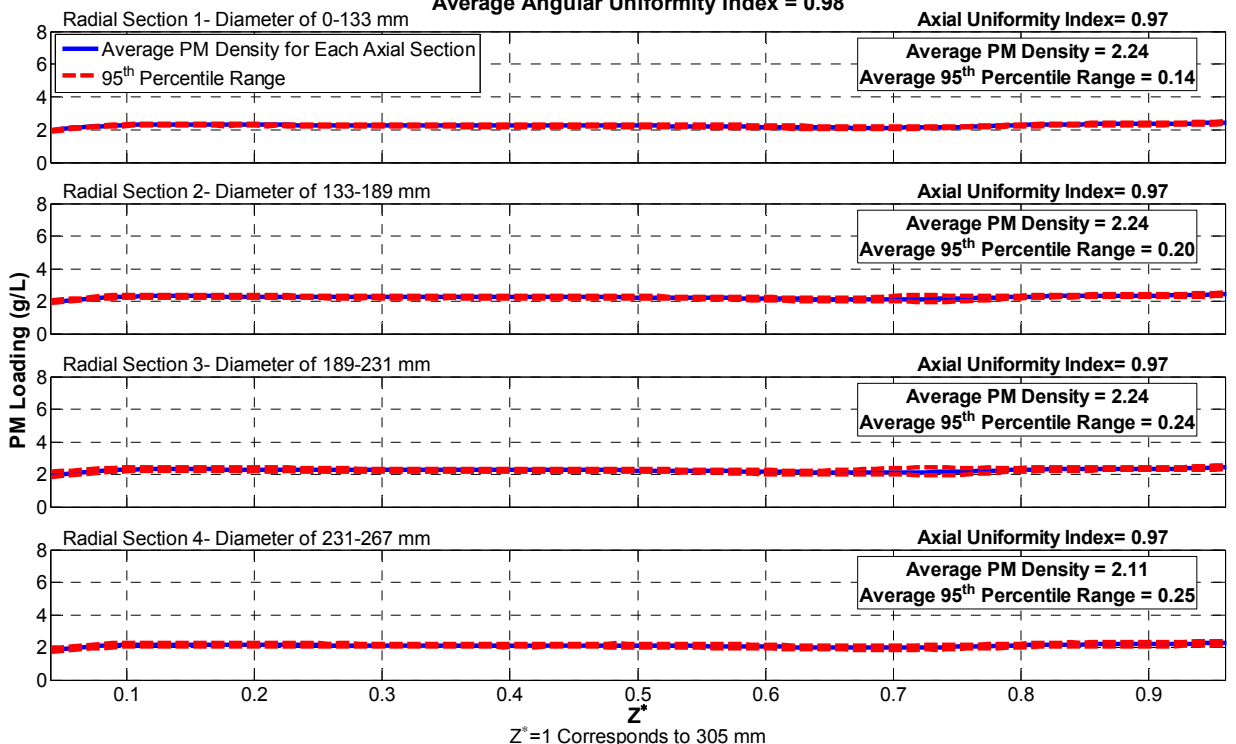

Figure K.36: Phase 2 Test 4- Active Regeneration Axial Distribution 
Test 4: Active Regeneration

Angular PM Distribution in Each Radial Section at $Z^{*}=0.128$

Radial Uniformity Index $=0.98$

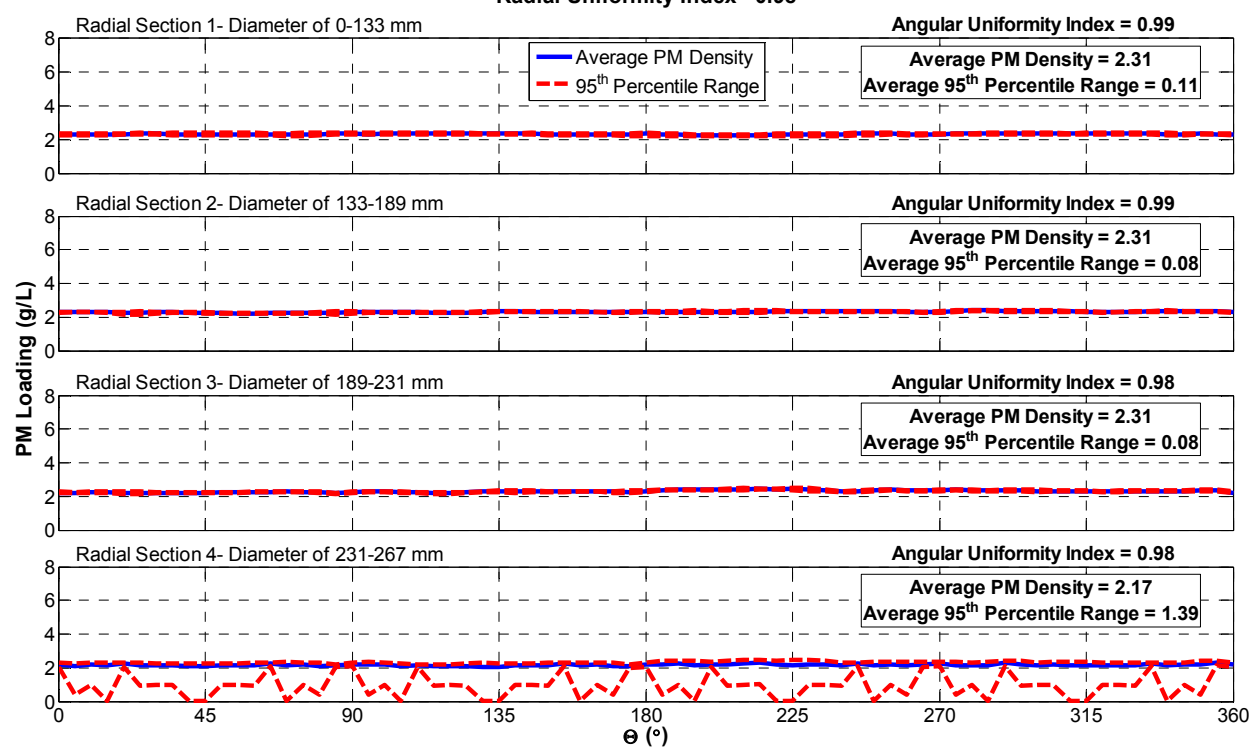

Figure K.37: Phase 2 Test 4- Active Regeneration $Z^{*}=0.128$

Test 4: Active Regeneration

Angular PM Distribution in Each Radial Section at $Z^{*}=\mathbf{0 . 3 7 6}$

Radial Uniformity Index $=0.98$

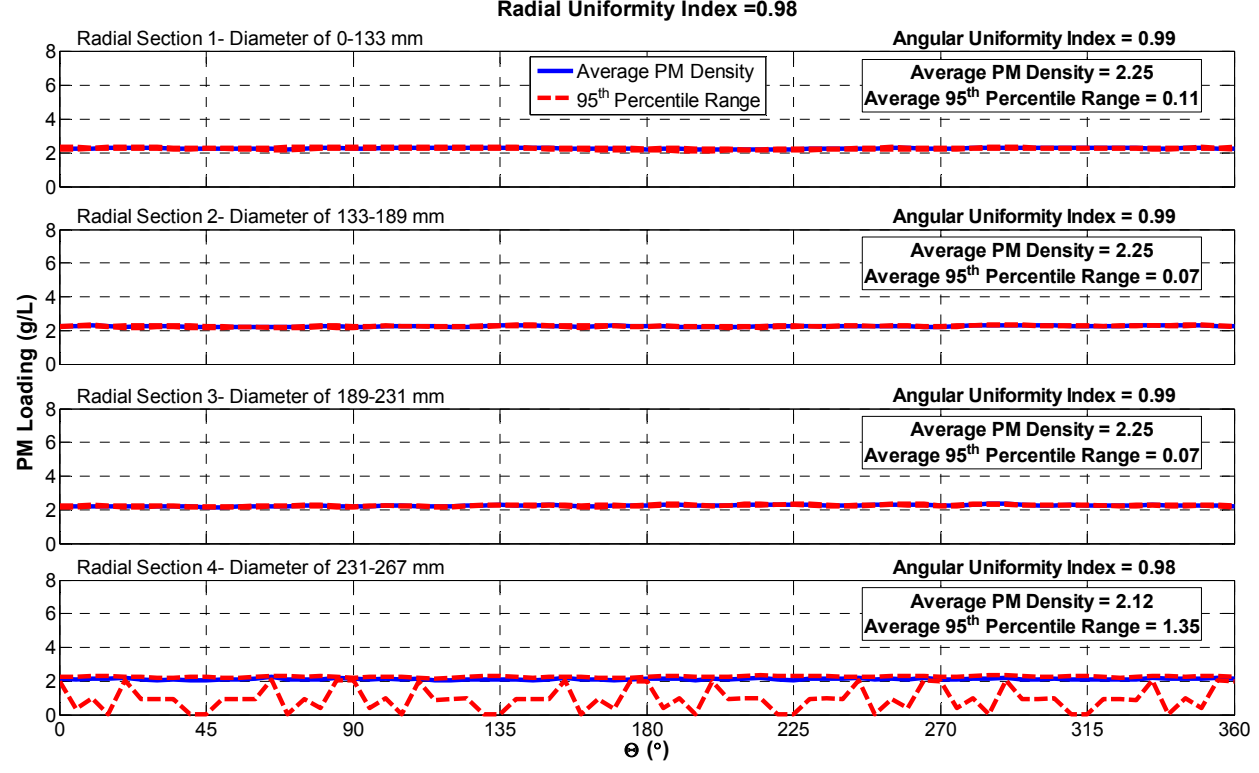

Figure K.38: Phase 2 Test 4- Active Regeneration $Z^{*}=0.376$ 
Test 4: Active Regeneration

Angular PM Distribution in Each Radial Section at $Z^{*}=\mathbf{0 . 6 2 4}$

Radial Uniformity Index $=0.98$

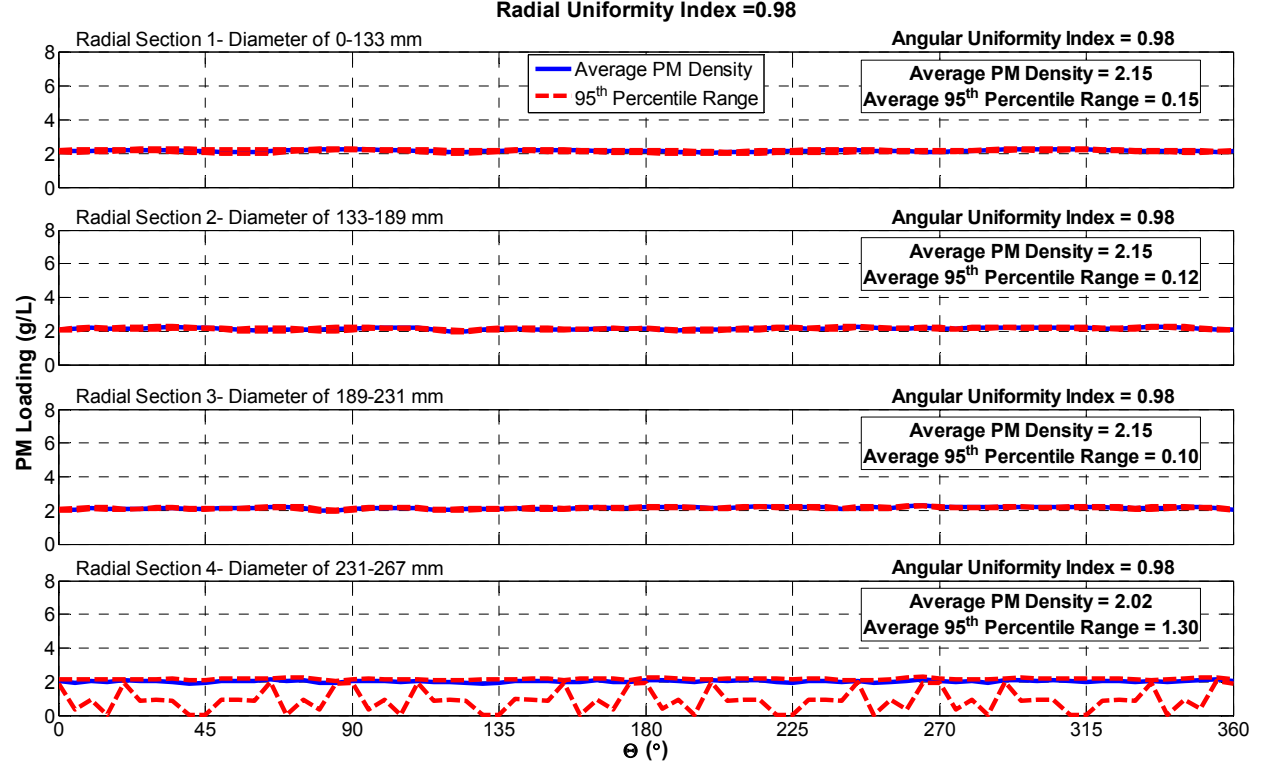

Figure K.39: Phase 2 Test 4- Active Regeneration $Z^{*}=0.624$

Test 4: Active Regeneration

Angular PM Distribution in Each Radial Section at $Z^{*}=\mathbf{0 . 8 7 2}$

Radial Uniformity Index $=0.98$

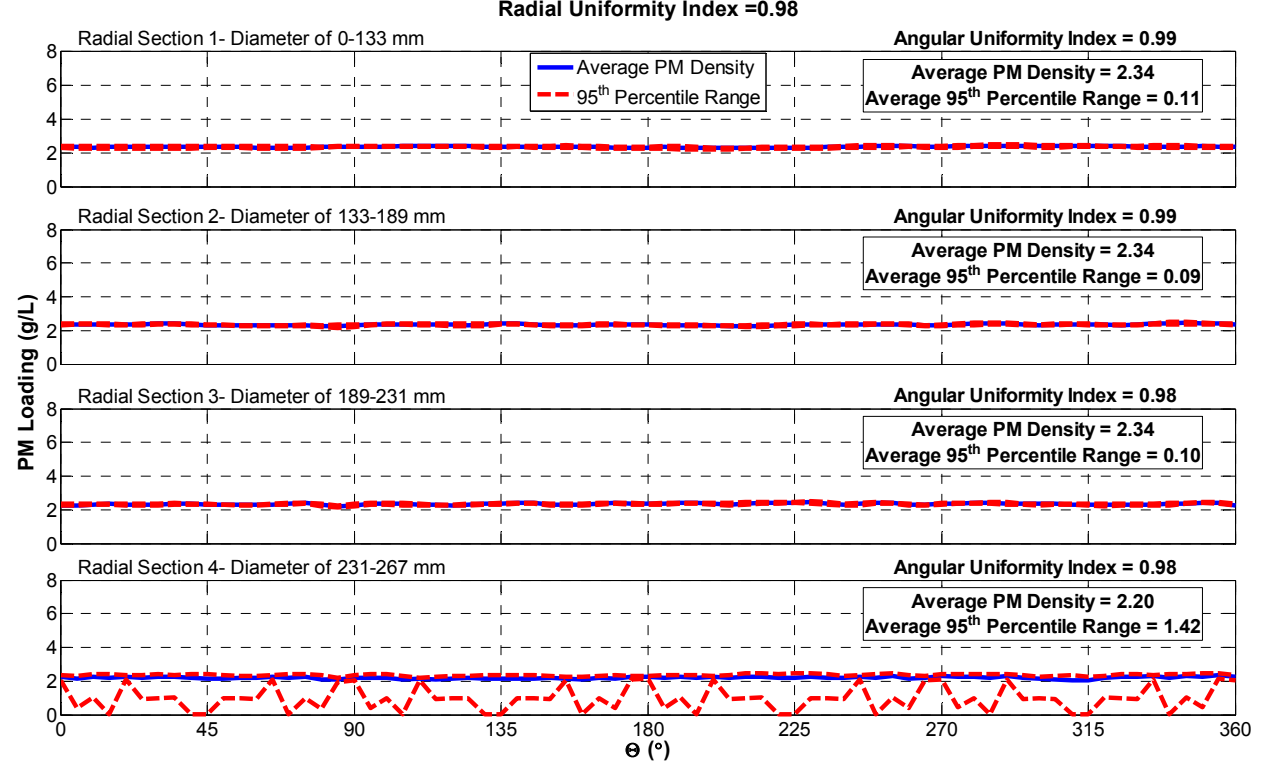

Figure K.40: Phase 2 Test 4- Active Regeneration $Z^{*}=0.872$ 


\section{Phase 2 Test 4 Post Loading Scan}

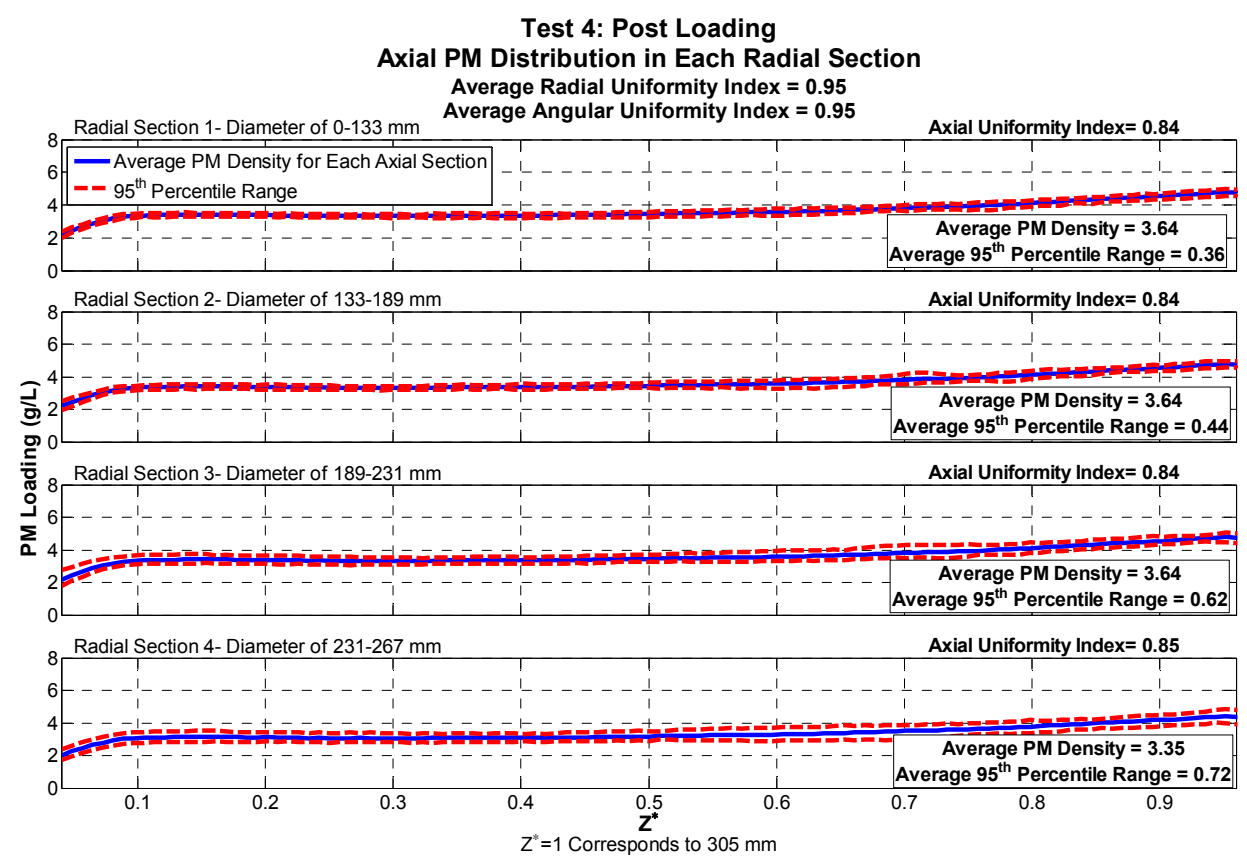

Figure K.41: Phase 2 Test 4- Post Loading Axial Distribution

Test 4: Post Loading

Angular PM Distribution in Each Radial Section at $Z^{*}=0.128$

Radial Uniformity Index $=0.95$

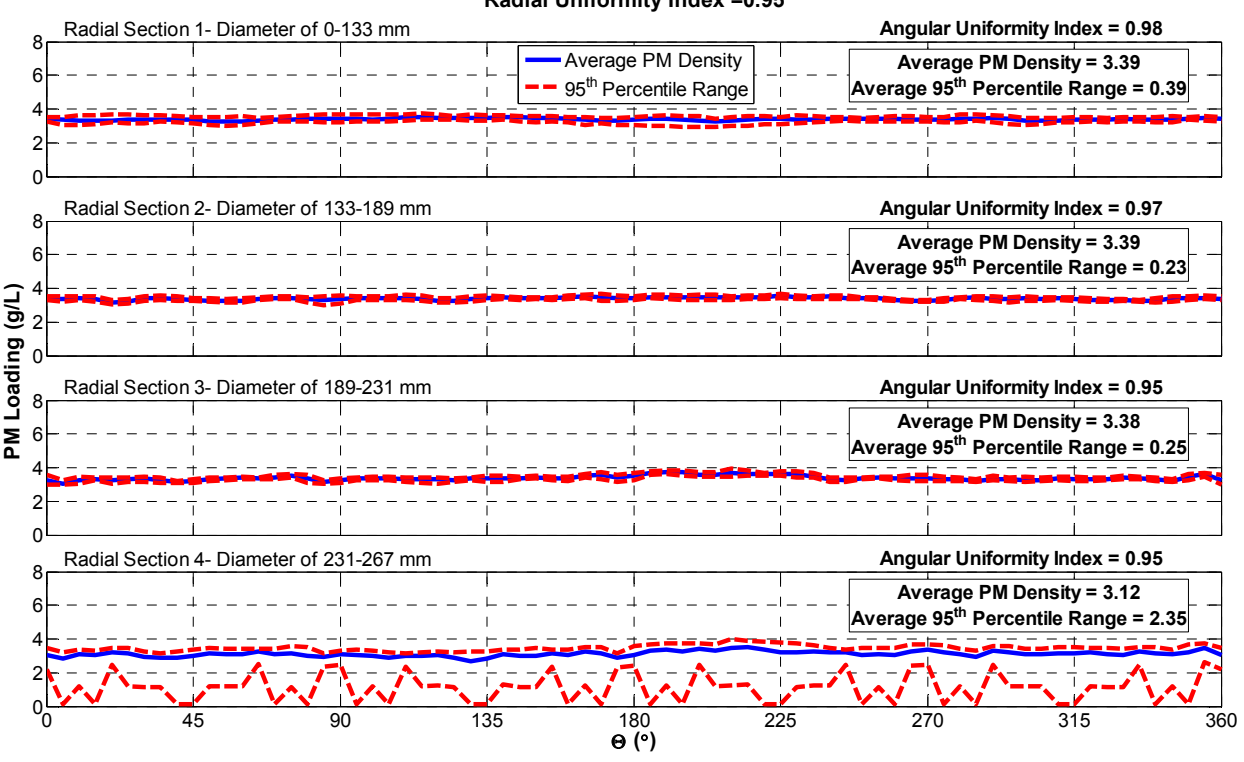

Figure K.42: Phase 2 Test 4- Post Loading $Z^{*}=0.128$ 
Test 4: Post Loading

Angular PM Distribution in Each Radial Section at $Z^{*}=\mathbf{0 . 3 7 6}$

Radial Uniformity Index $=0.95$

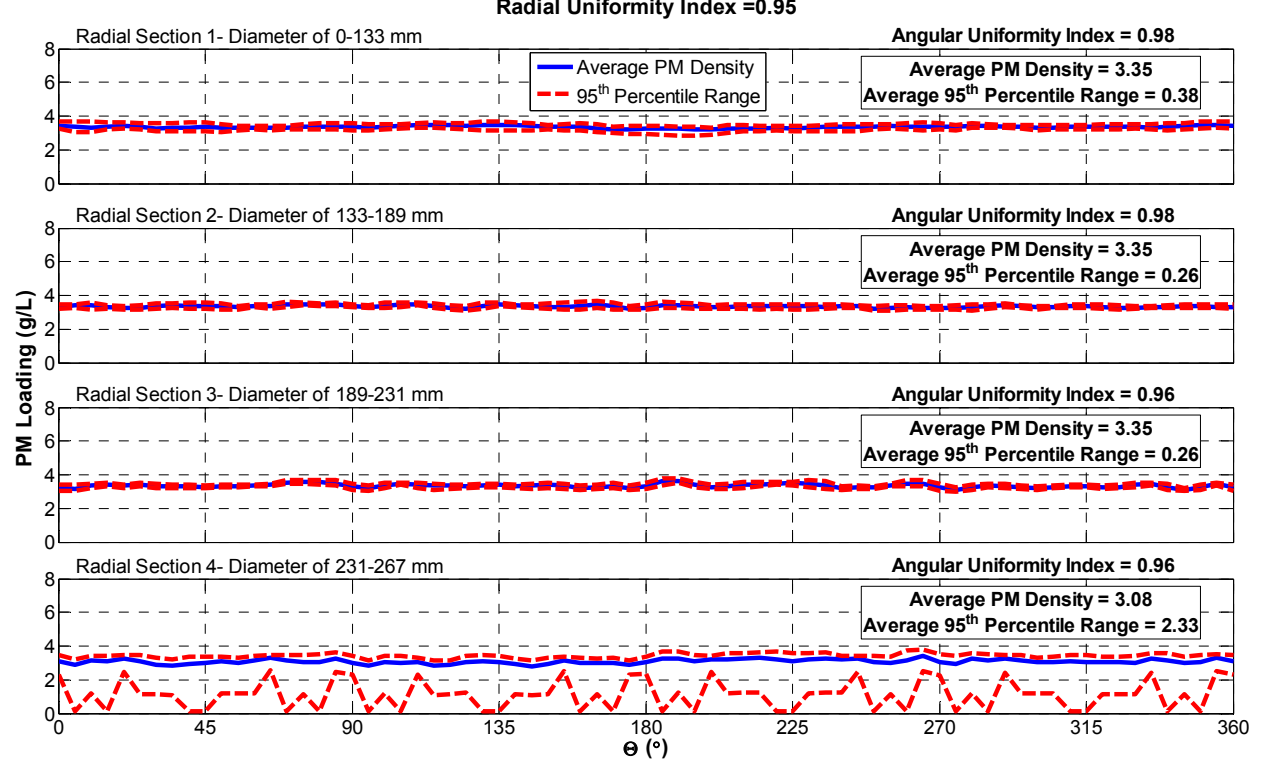

Figure K.43: Phase 2 Test 4- Post Loading $Z^{*}=0.376$

Test 4: Post Loading

Angular PM Distribution in Each Radial Section at $Z^{*}=\mathbf{0 . 6 2 4}$

Radial Uniformity Index $=0.94$

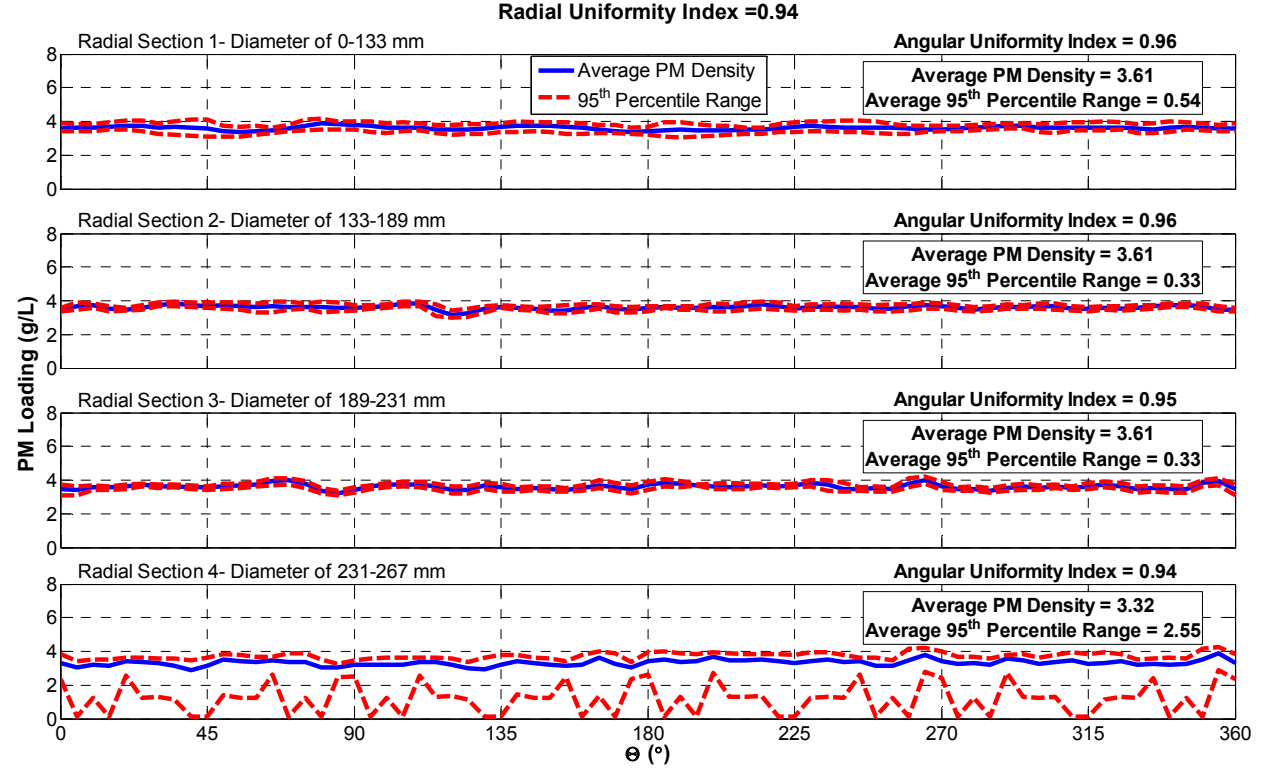

Figure K.44: Phase 2 Test 4- Post Loading $Z^{*}=0.624$ 
Test 4: Post Loading

Angular PM Distribution in Each Radial Section at $Z^{*}=0.872$

Radial Uniformity Index $=0.94$

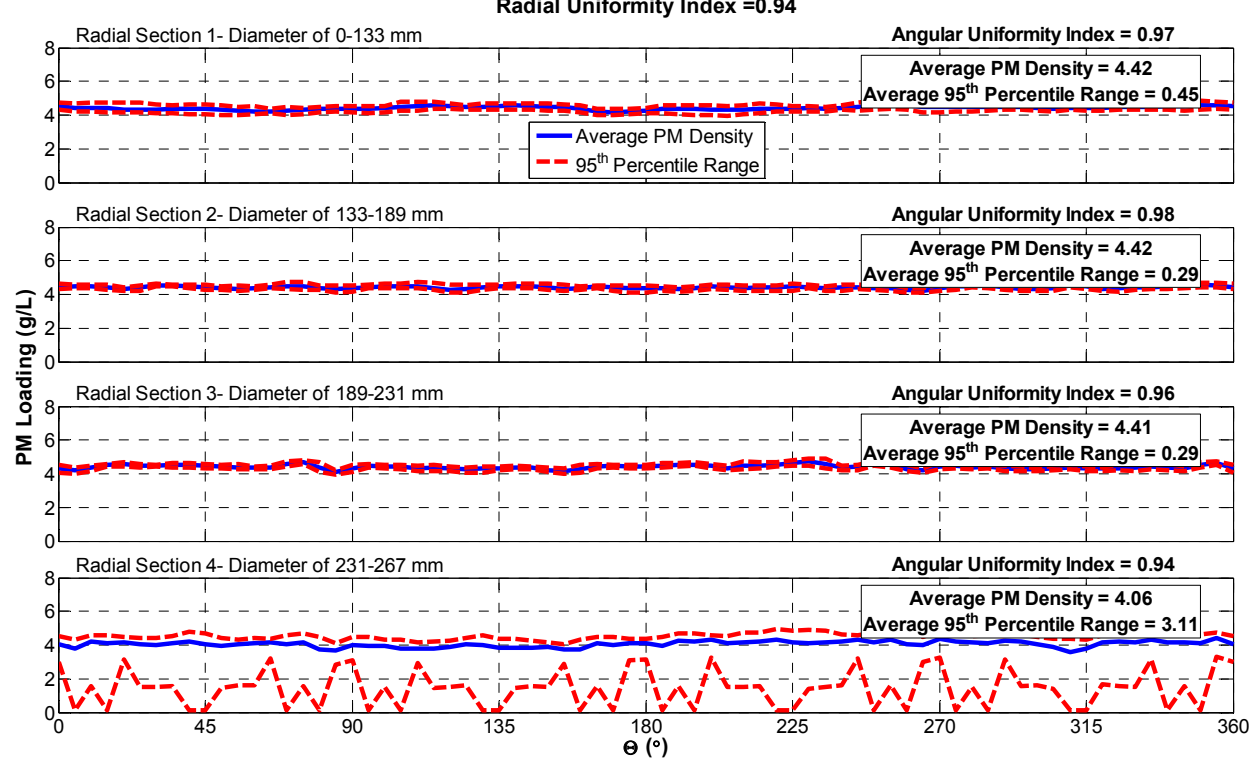

Figure K.45: Phase 2 Test 4- Post Loading $Z^{*}=0.872$

\section{Phase 2 Test 5 Active Regeneration Scan}

Test 5: Active Regeneration

Axial PM Distribution in Each Radial Section

Average Radial Uniformity Index $=0.98$

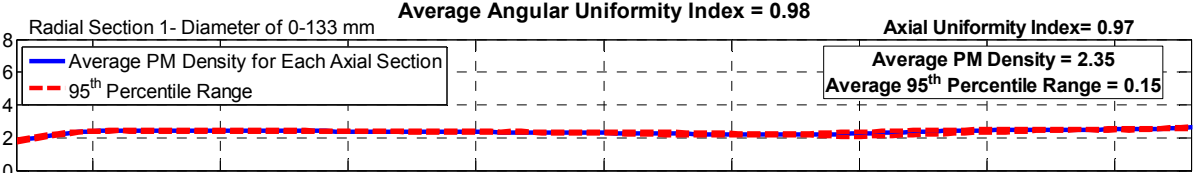
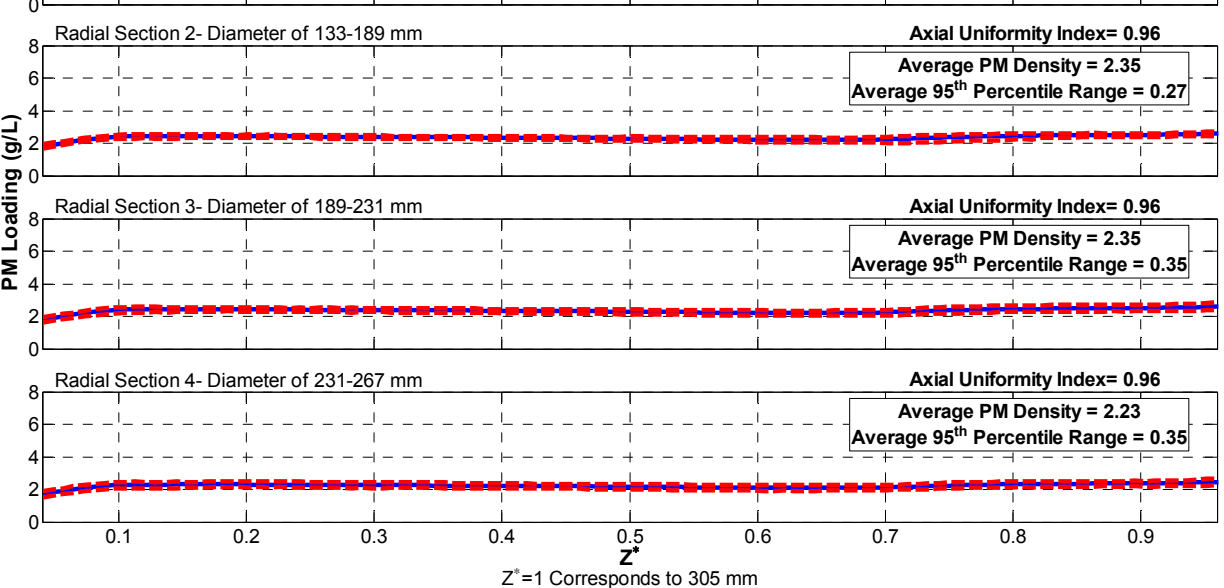

Figure K.46: Phase 2 Test 5- Active Regeneration Axial Distribution 
Test 5: Active Regeneration

Angular PM Distribution in Each Radial Section at $Z^{*}=0.128$

Radial Uniformity Index $=0.98$

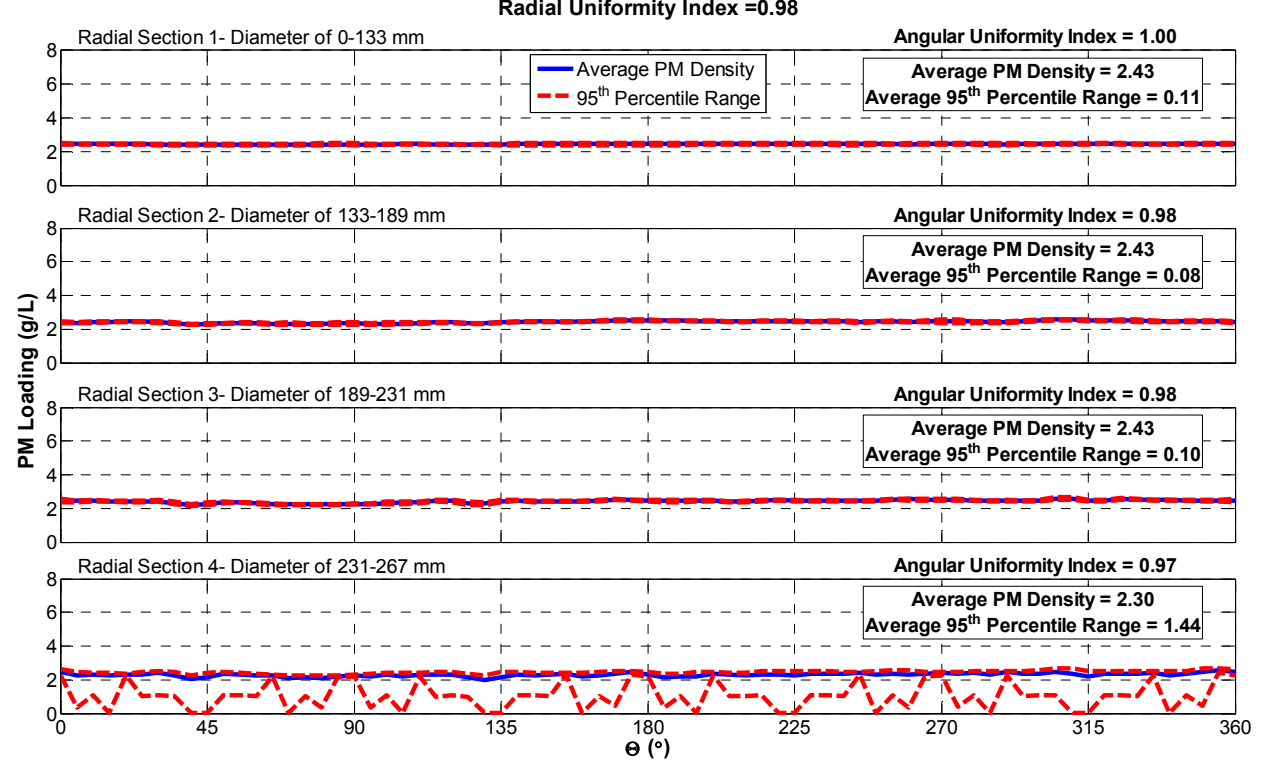

Figure K.47: Phase 2 Test 5- Active Regeneration $Z^{*}=0.128$

Test 5: Active Regeneration

Angular PM Distribution in Each Radial Section at $Z^{*}=0.376$

Radial Uniformity Index $=0.98$

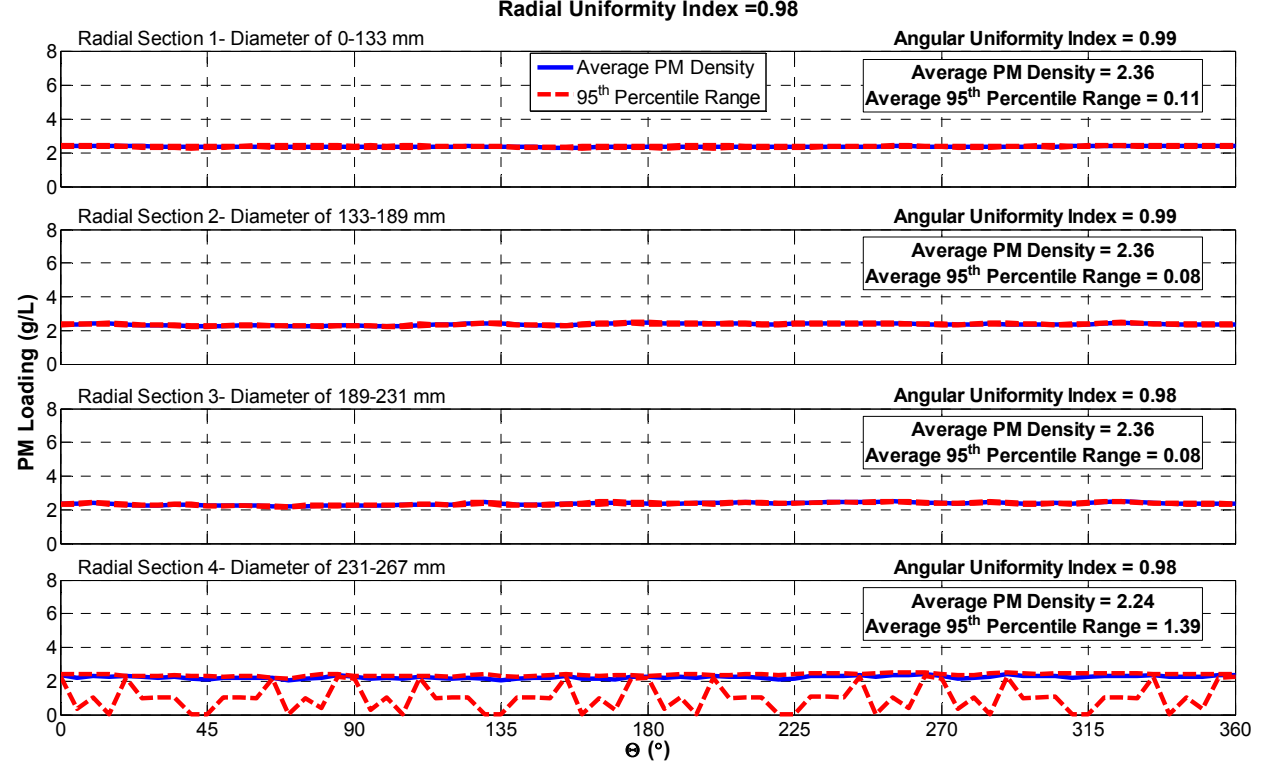

Figure K.48: Phase 2 Test 5- Active Regeneration $Z^{*}=0.376$ 
Test 5: Active Regeneration

Angular PM Distribution in Each Radial Section at $Z^{*}=\mathbf{0 . 6 2 4}$

Radial Uniformity Index $=0.98$

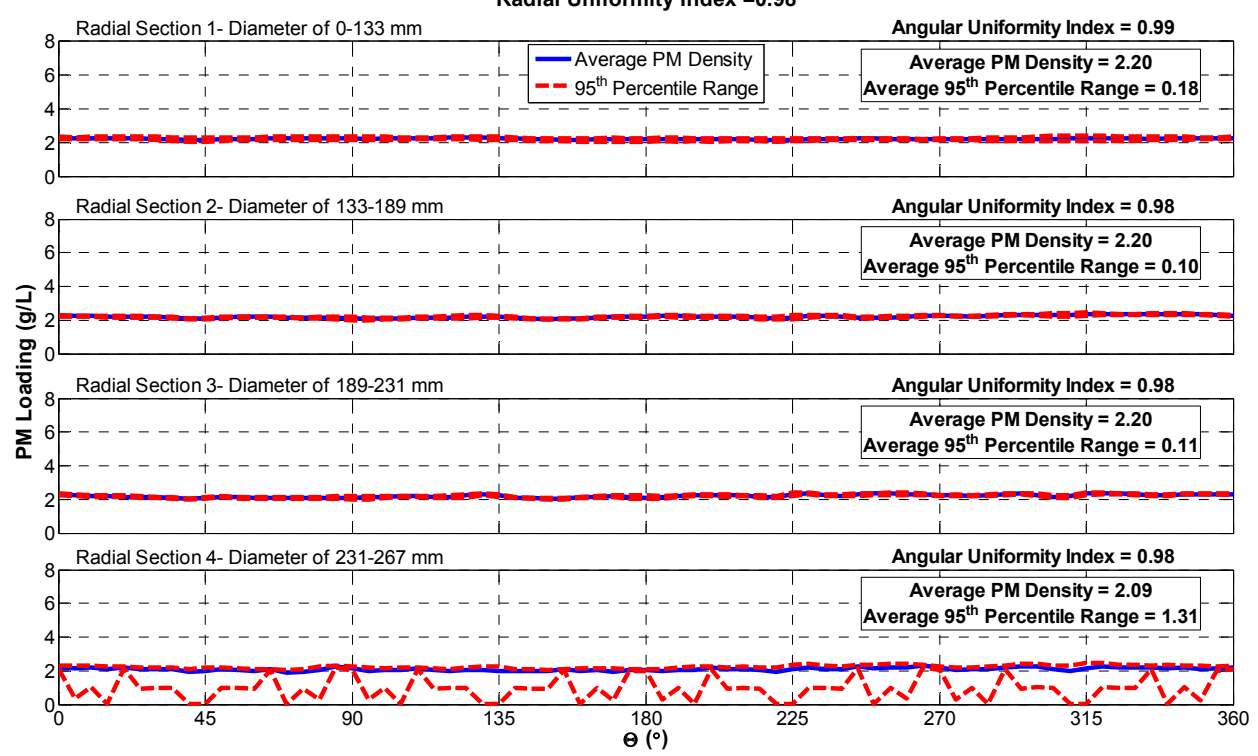

Figure K.49: Phase 2 Test 5- Active Regeneration $Z^{*}=0.624$

Test 5: Active Regeneration

Angular PM Distribution in Each Radial Section at $Z^{*}=\mathbf{0 . 8 7 2}$

Radial Uniformity Index $=0.98$

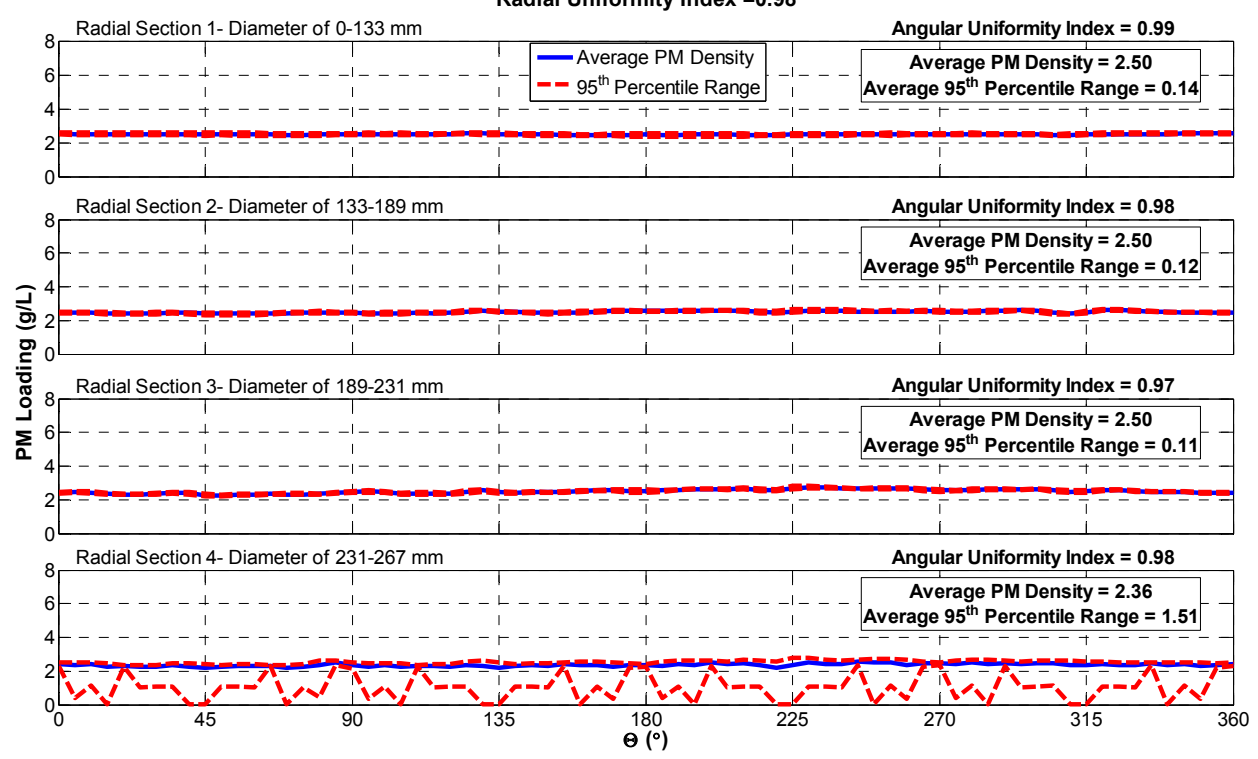

Figure K.50: Phase 2 Test 5- Active Regeneration $Z^{*}=0.872$ 
Phase 2 Test 6 Loading Scan

Test 6: Loading

Axial PM Distribution in Each Radial Section

Average Radial Uniformity Index $=0.97$

Radial Section 1- Diameter of 0-133 mm

Average Angular Uniformity Index $=0.97$

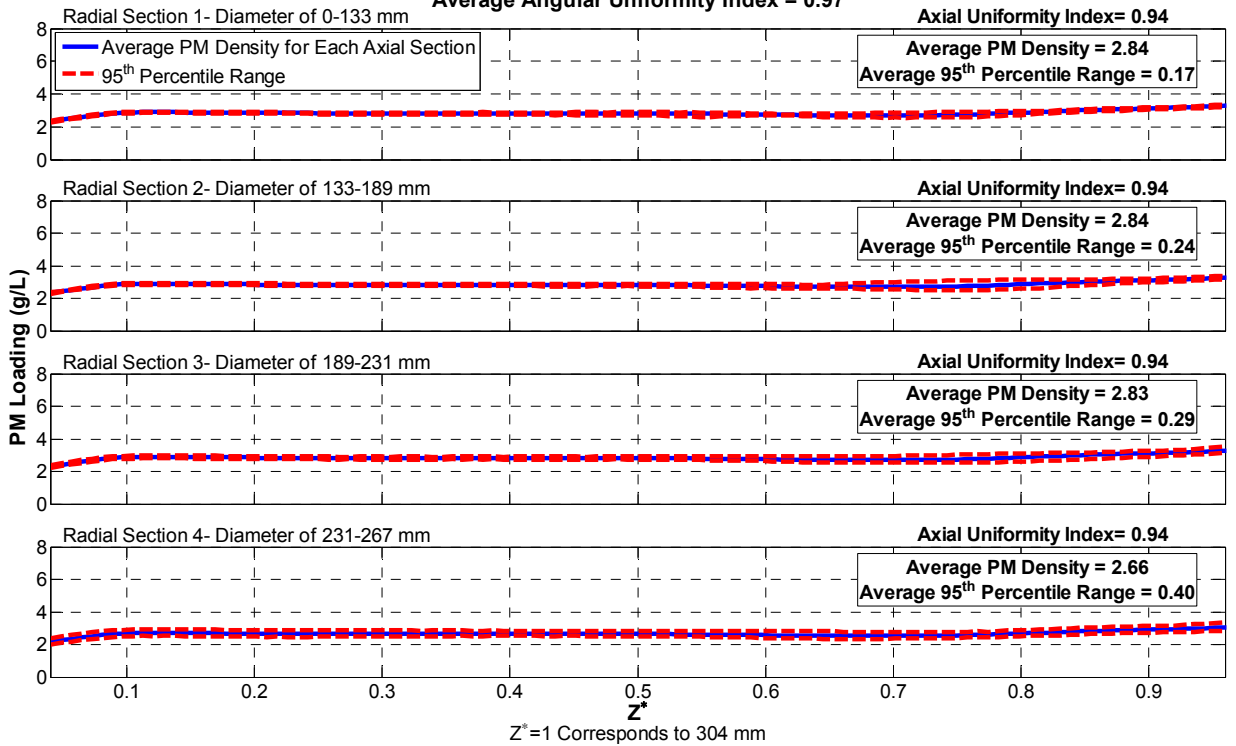

Figure K.51: Phase 2 Test 6- Loading Axial Distribution

Test 6: Loading

Angular PM Distribution in Each Radial Section at $Z^{*}=0.128$

Radial Uniformity Index $=0.97$

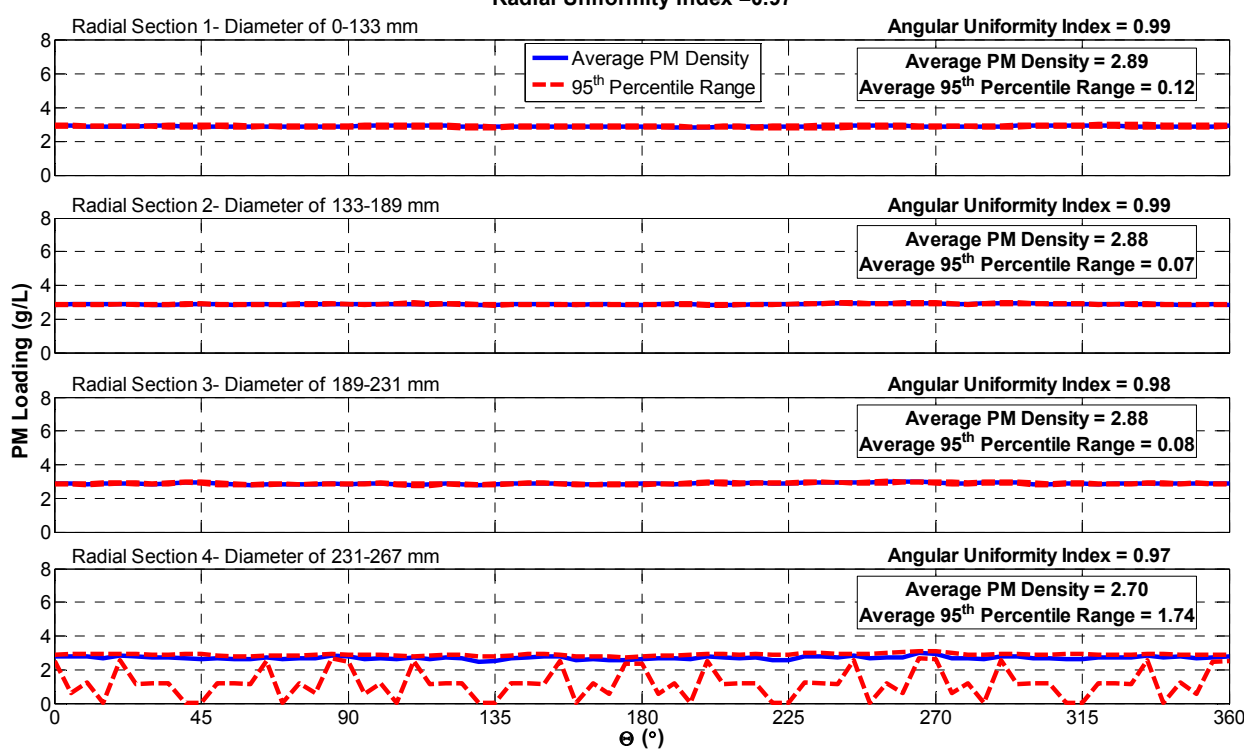

Figure K.52: Phase 2 Test 6- Loading $Z^{*}=0.128$ 
Test 6: Loading

Angular PM Distribution in Each Radial Section at $Z^{*}=\mathbf{0 . 3 7 6}$

Radial Uniformity Index $=0.97$

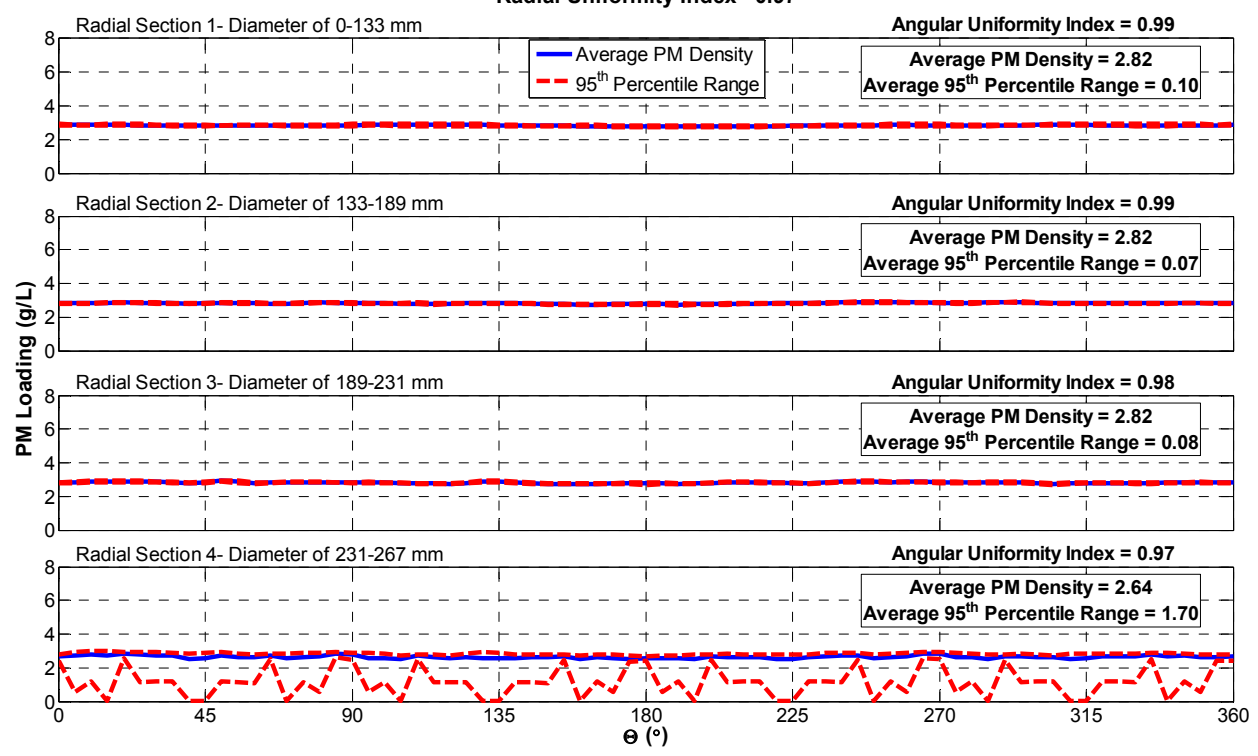

Figure K.53: Phase 2 Test 6- Loading $Z^{*}=0.376$

Test 6: Loading

Angular PM Distribution in Each Radial Section at $Z^{*}=0.624$

Radial Uniformity Index $=0.97$

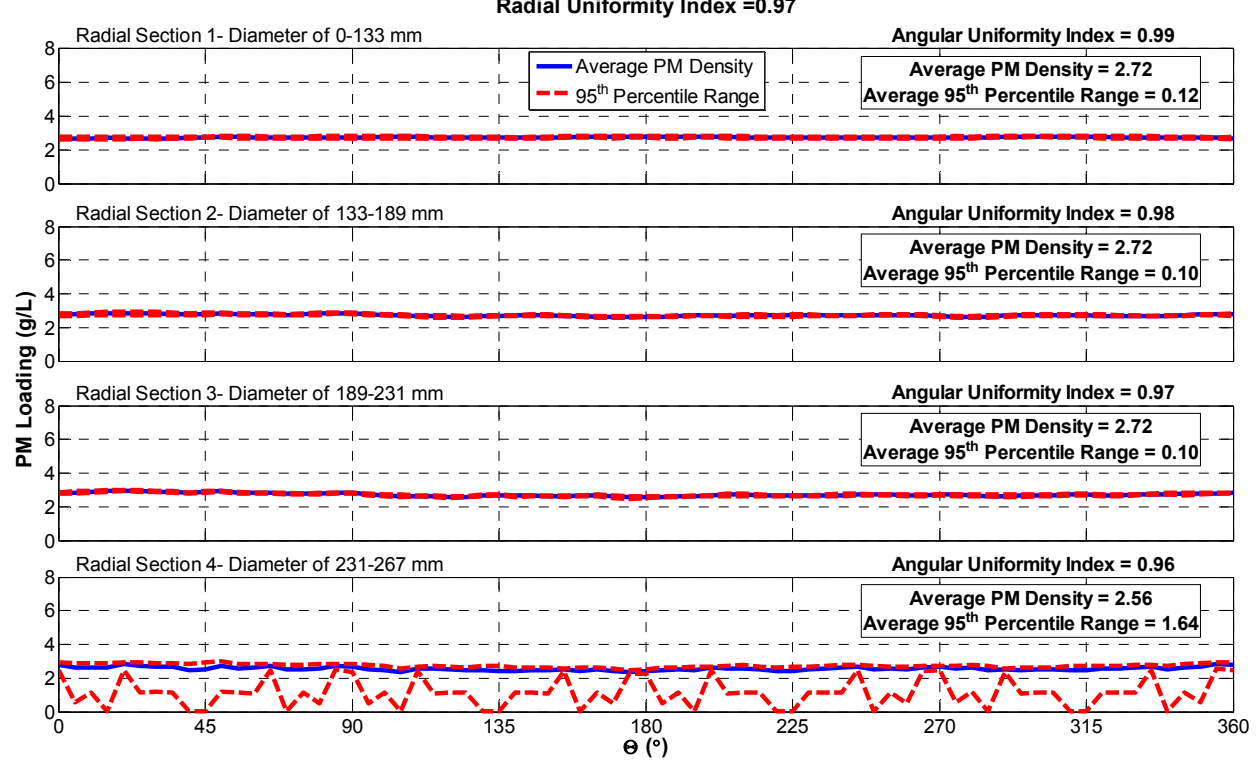

Figure K.54: Phase 2 Test 6- Loading $Z^{*}=0.624$ 
Test 6: Loading

Angular PM Distribution in Each Radial Section at $Z^{*}=\mathbf{0 . 8 7 2}$

Radial Uniformity Index $=0.96$

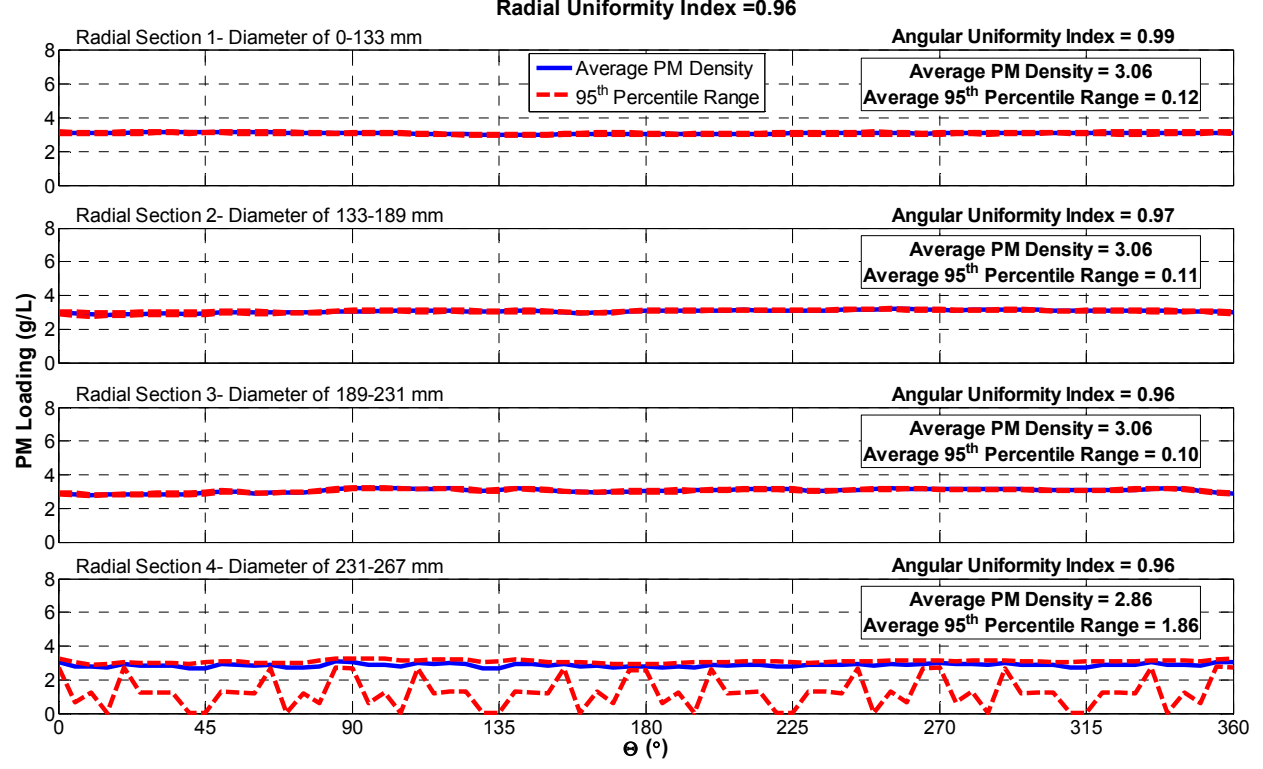

Figure K.55: Phase 2 Test 6- Loading $Z^{*}=0.872$

\section{Phase 2 Test 6 Second Loading}

Test 6: Second Loading

Axial PM Distribution in Each Radial Section

Average Radial Uniformity Index $=0.95$

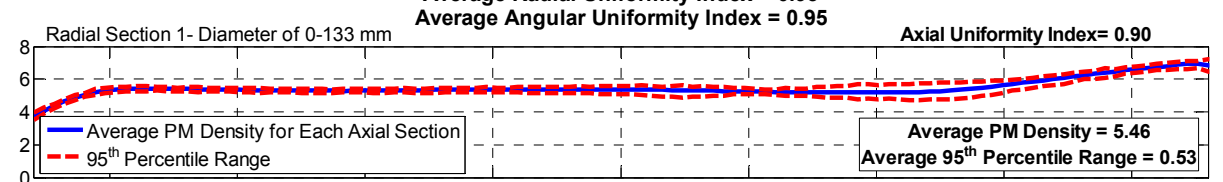

8 Radial Section 2- Diameter of 133-189 mm Axial Uniformity Index $=0.89$
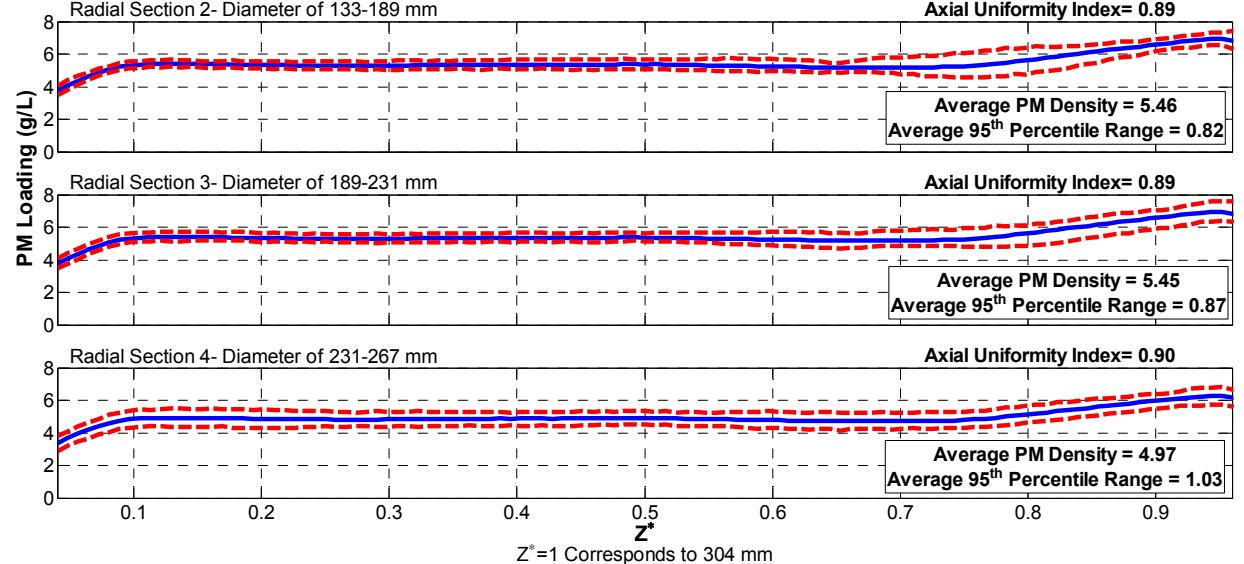

Figure K.56: Phase 2 Test 6- Second Loading Axial Distribution 
Test 6: Second Loading

Angular PM Distribution in Each Radial Section at $Z^{*}=\mathbf{0 . 1 2 8}$

Radial Uniformity Index $=0.95$

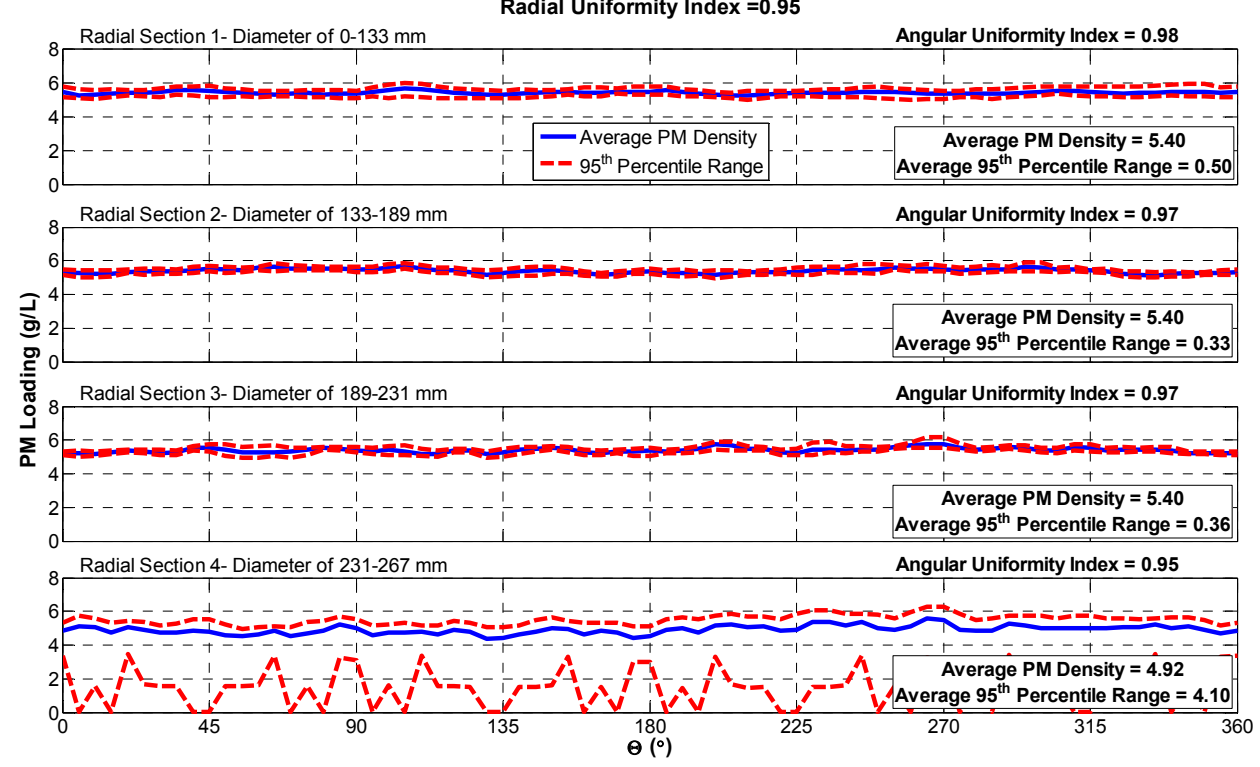

Figure K.57: Phase 2 Test 6- Second Loading $Z *=0.128$

Test 6: Second Loading

Angular PM Distribution in Each Radial Section at $Z^{*}=\mathbf{0 . 3 7 6}$

Radial Uniformity Index $=0.95$

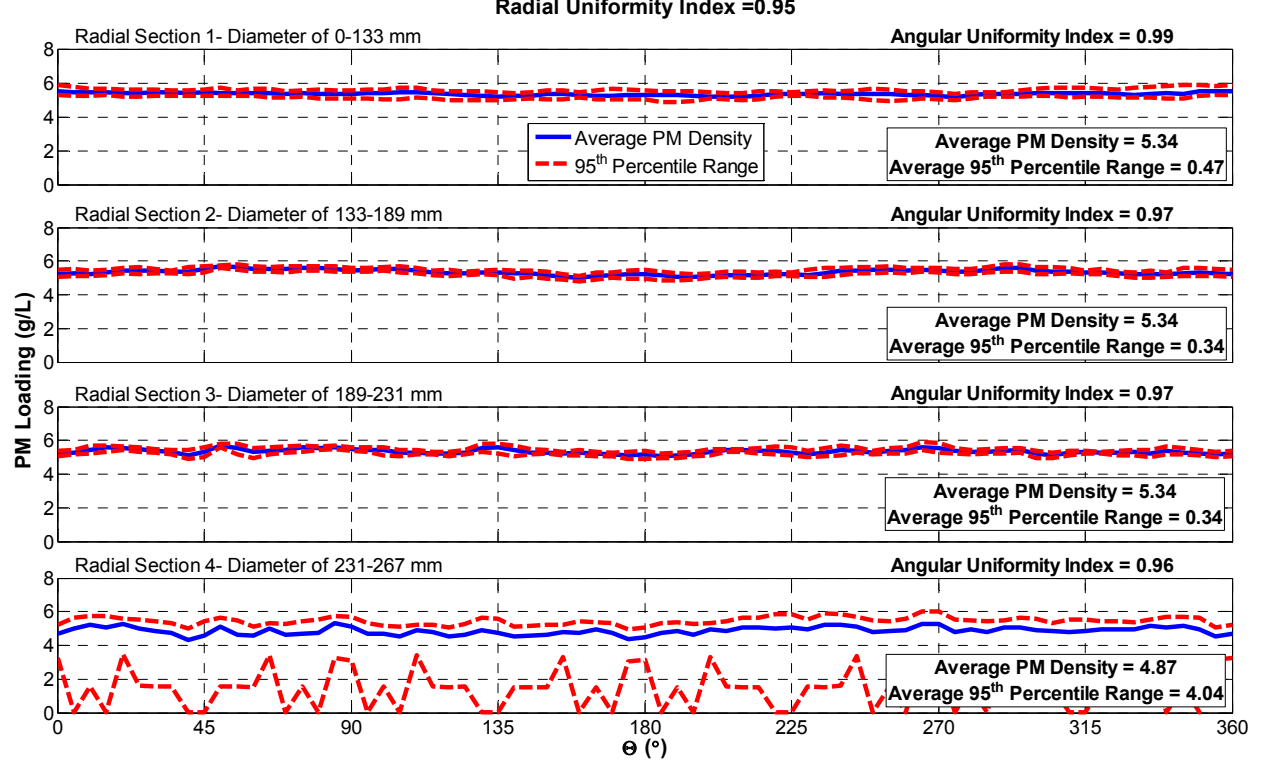

Figure K.58: Phase 2 Test 6- Second Loading $Z^{*}=0.376$ 
Test 6: Second Loading

Angular PM Distribution in Each Radial Section at $Z^{*}=0.624$

Radial Uniformity Index $=0.95$

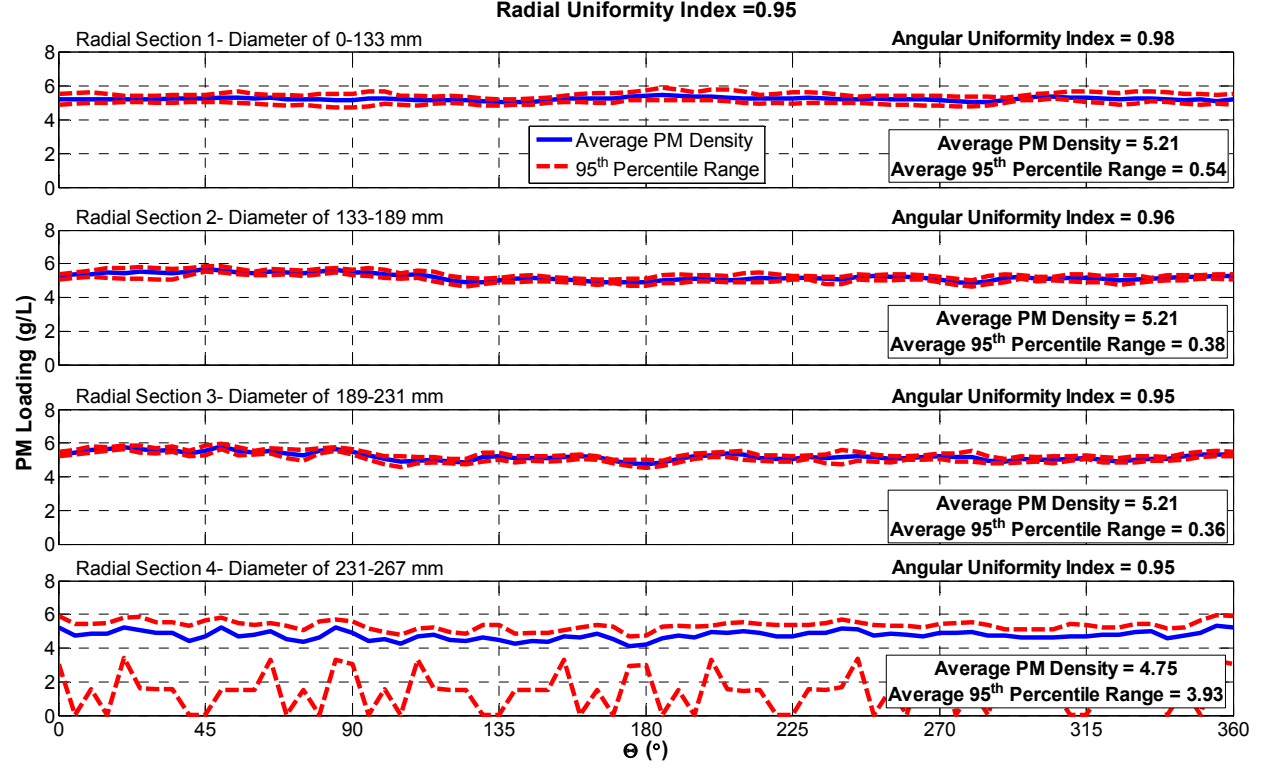

Figure K.59: Phase 2 Test 6- Second Loading $Z^{*}=0.624$

Test 6: Second Loading

Angular PM Distribution in Each Radial Section at $Z^{*}=\mathbf{0 . 8 7 2}$

Radial Uniformity Index $=0.94$

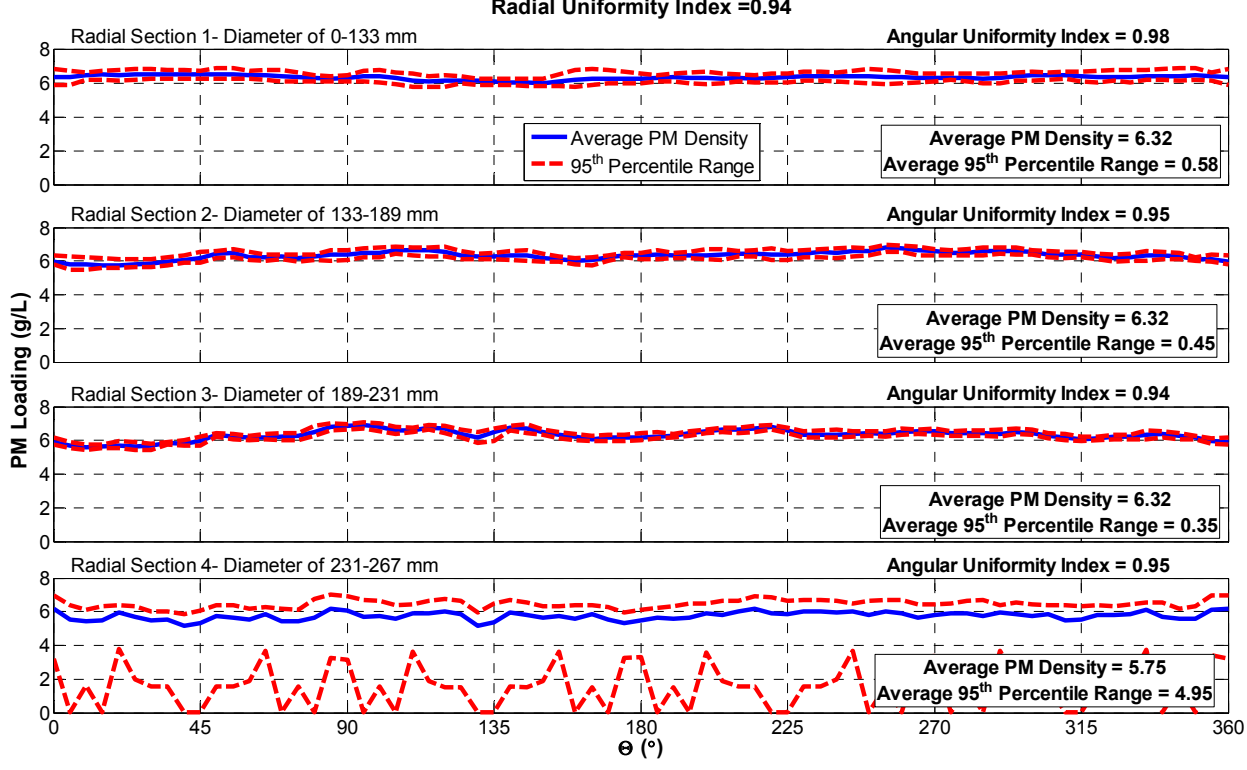

Figure K.60: Phase 2 Test 6- Second Loading $Z^{*}=0.872$ 


\section{Phase 2 Test 7 Passive Oxidation Scan}

Test 7: Passive Oxidation

Axial PM Distribution in Each Radial Section

Average Radial Uniformity Index $=0.98$

8 Radial Section 1- Diameter of 0-133 mm

Average Angular Uniformity Index $=0.99$
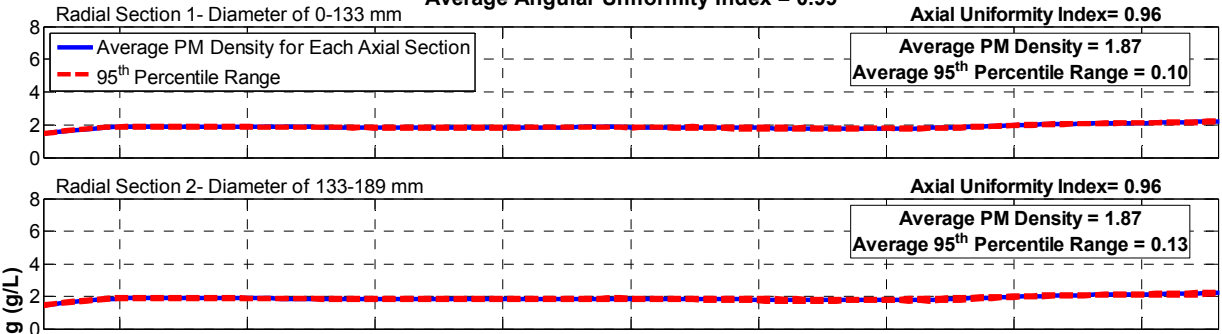

Radial Section 3- Diameter of $189-231 \mathrm{~mm}$ Axial Uniformity Index $=0.96$

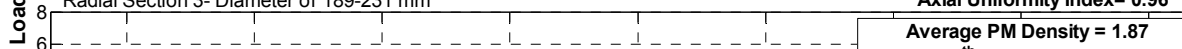

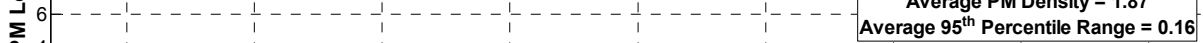
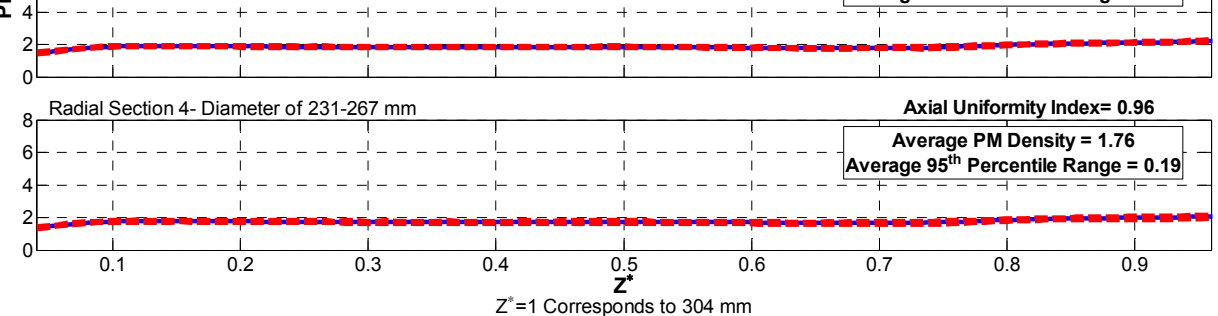

Figure K.61: Phase 2 Test 7- Passive Oxidation Axial Distribution

Test 7: Passive Oxidation

Angular PM Distribution in Each Radial Section at $Z^{*}=0.128$

Radial Uniformity Index $=0.98$

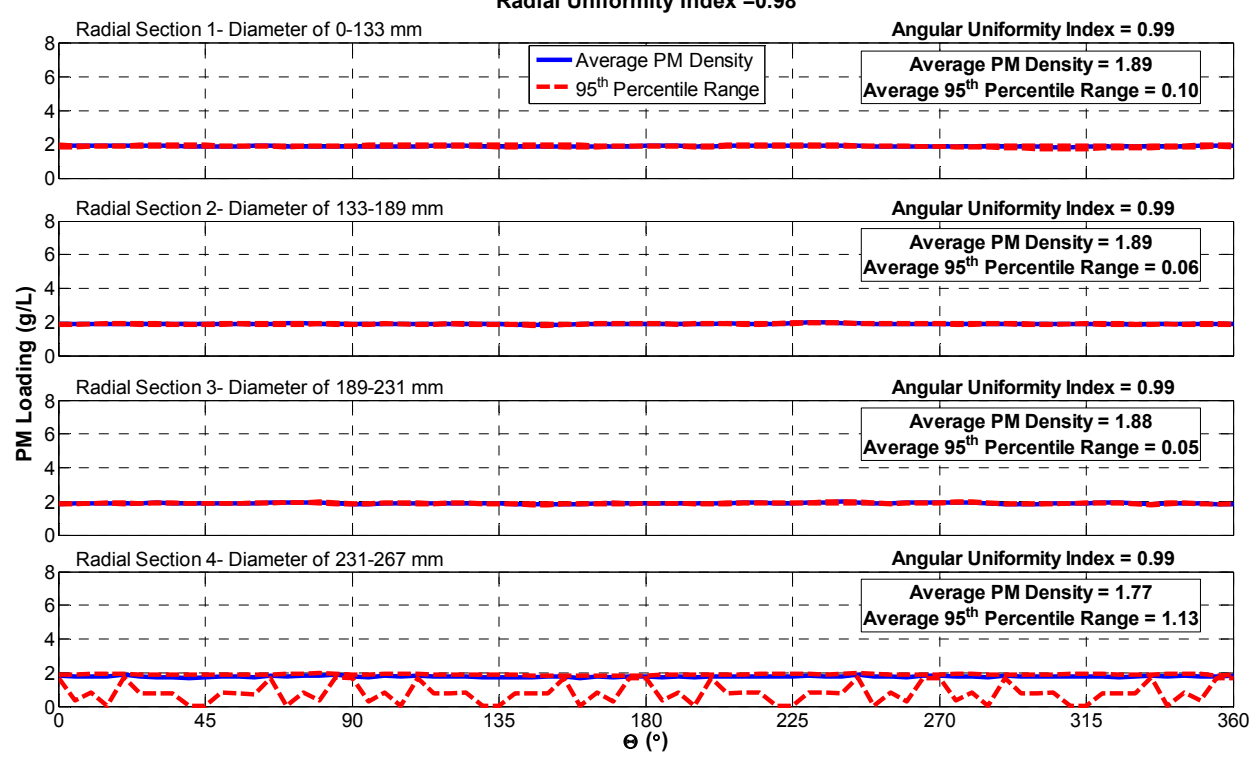

Figure K.62: Phase 2 Test 7- Passive Oxidation $Z^{*}=0.128$ 
Test 7: Passive Oxidation

Angular PM Distribution in Each Radial Section at $Z^{*}=0.376$

Radial Uniformity Index $=0.98$

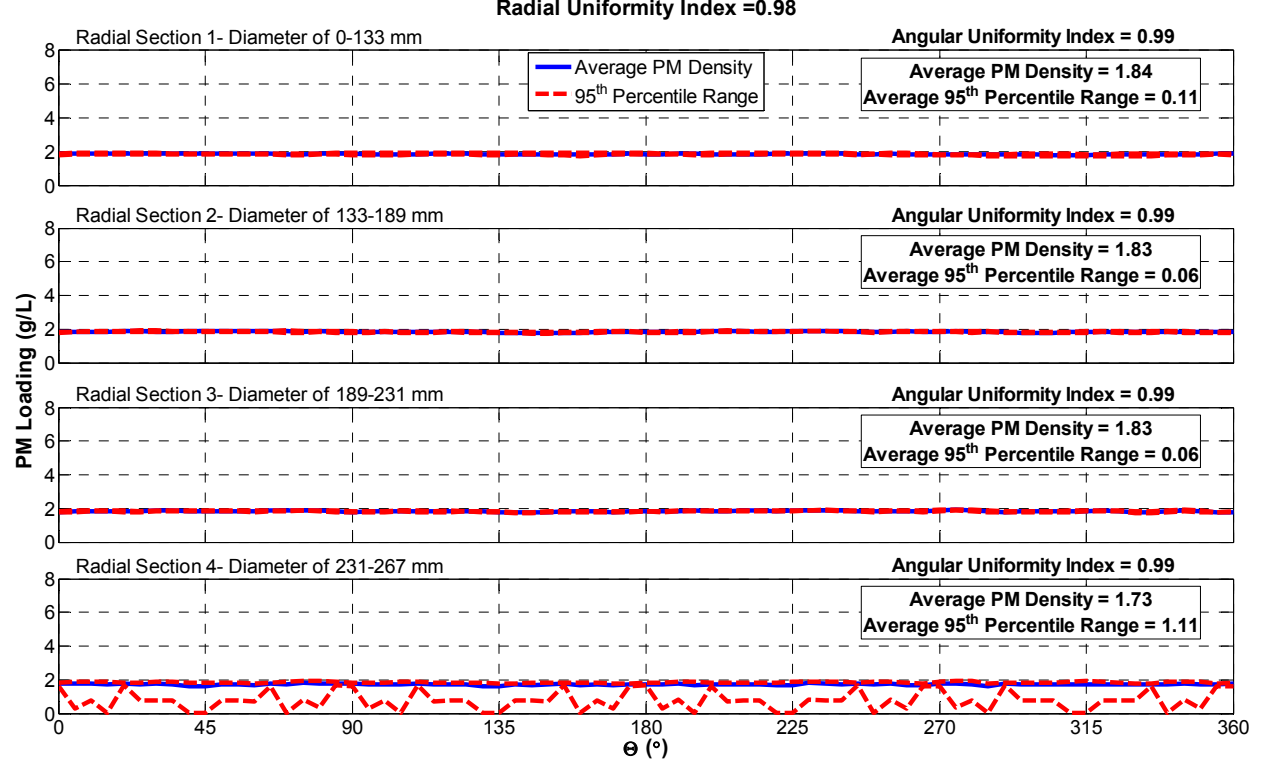

Figure K.63: Phase 2 Test 7- Passive Oxidation $Z *=0.376$

Test 7: Passive Oxidation

Angular PM Distribution in Each Radial Section at $Z^{*}=0.624$

Radial Uniformity Index $=0.98$

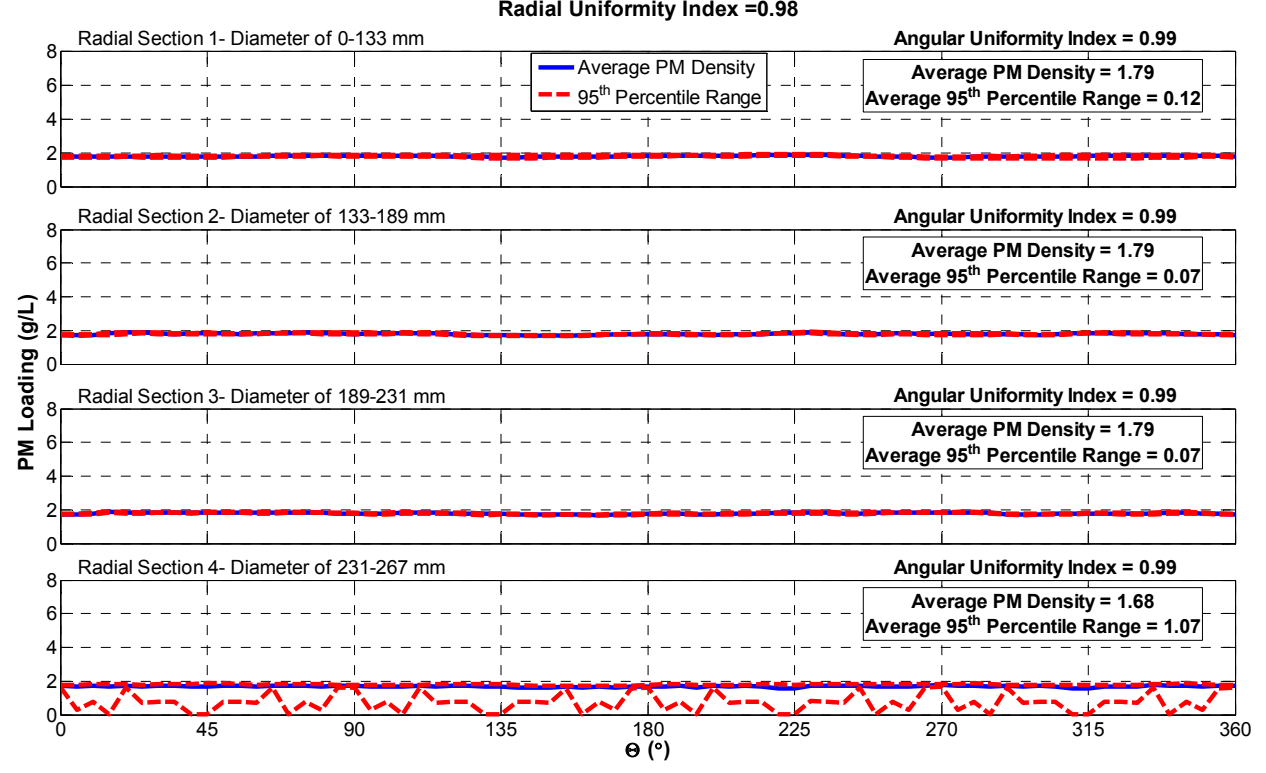

Figure K.64: Phase 2 Test 7- Passive Oxidation $Z^{*}=0.624$ 
Test 7: Passive Oxidation

Angular PM Distribution in Each Radial Section at $Z^{*}=0.872$

Radial Uniformity Index $=0.98$

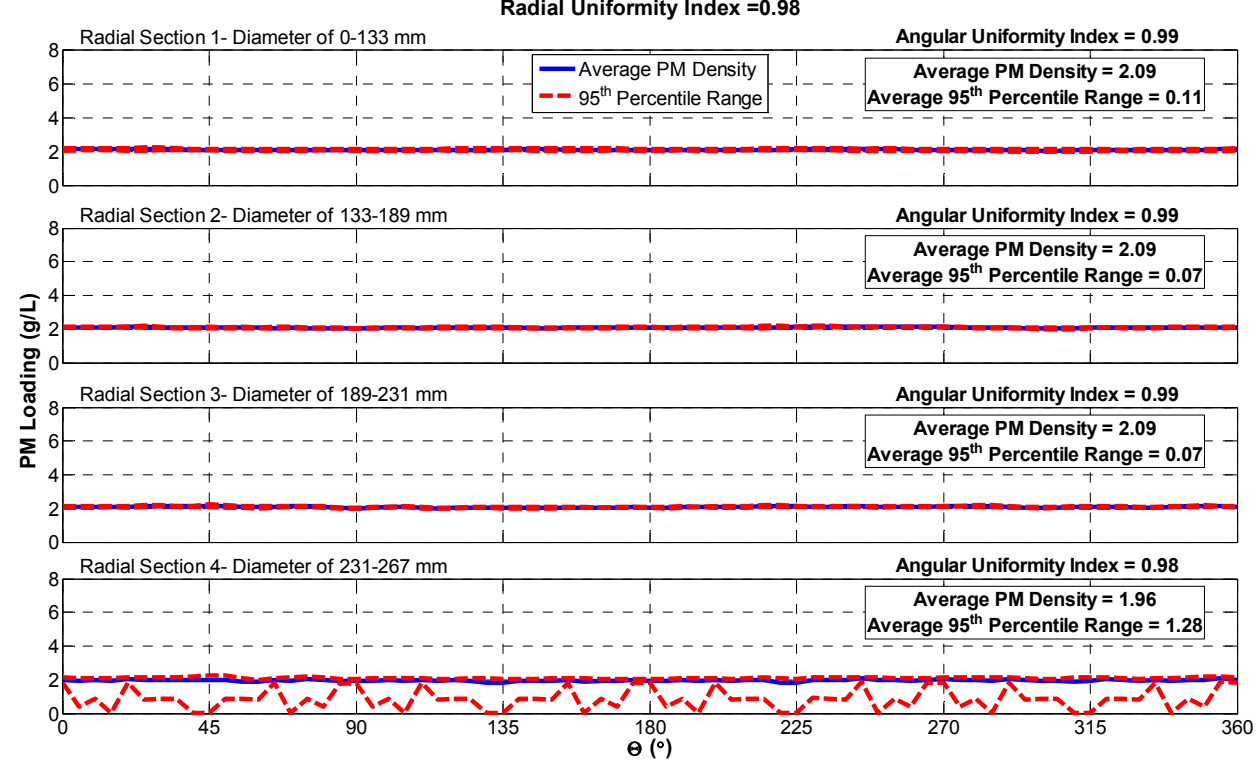

Figure K.65: Phase 2 Test 7- Passive Oxidation $Z^{*}=0.872$

\section{Phase 2 Test 7 Post Loading Scan}

Test 7: Post Loading

Axial PM Distribution in Each Radial Section Average Radial Uniformity Index $=0.97$
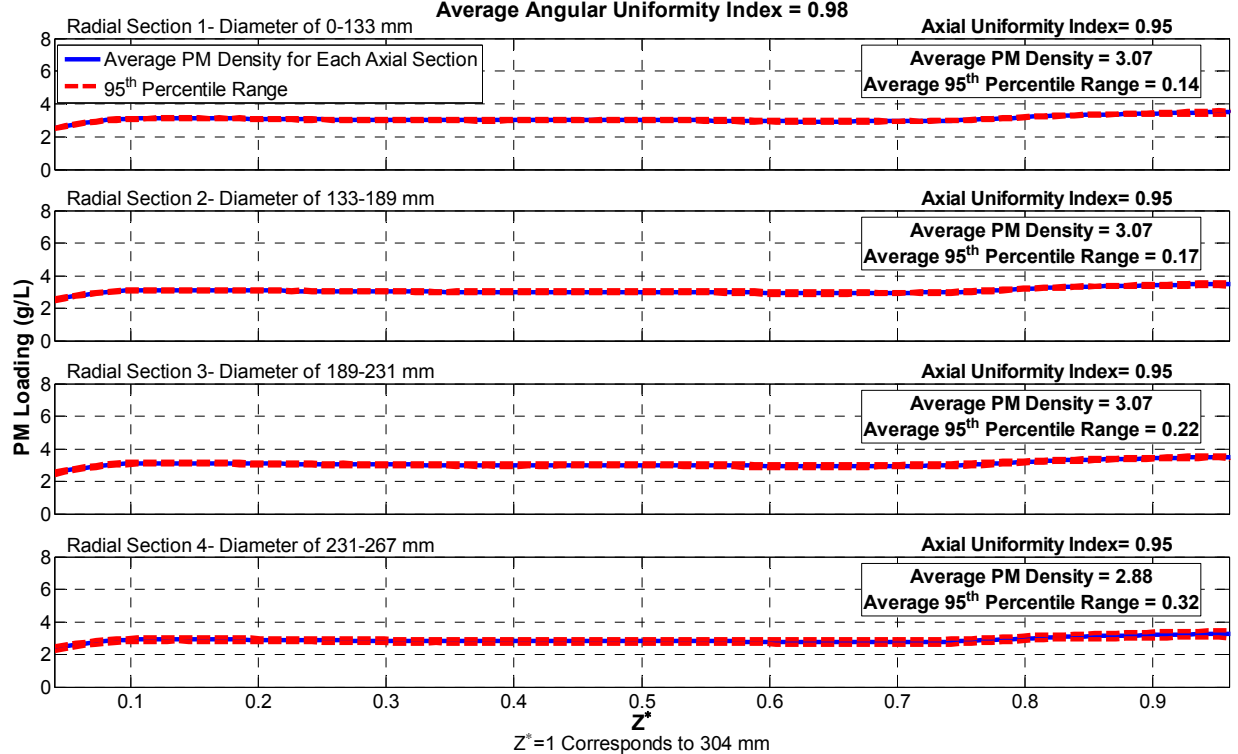

Figure K.66: Phase 2 Test 7- Post Loading Axial Distribution 
Test 7: Post Loading

Angular PM Distribution in Each Radial Section at $Z^{*}=\mathbf{0 . 1 2 8}$

Radial Uniformity Index $=0.97$

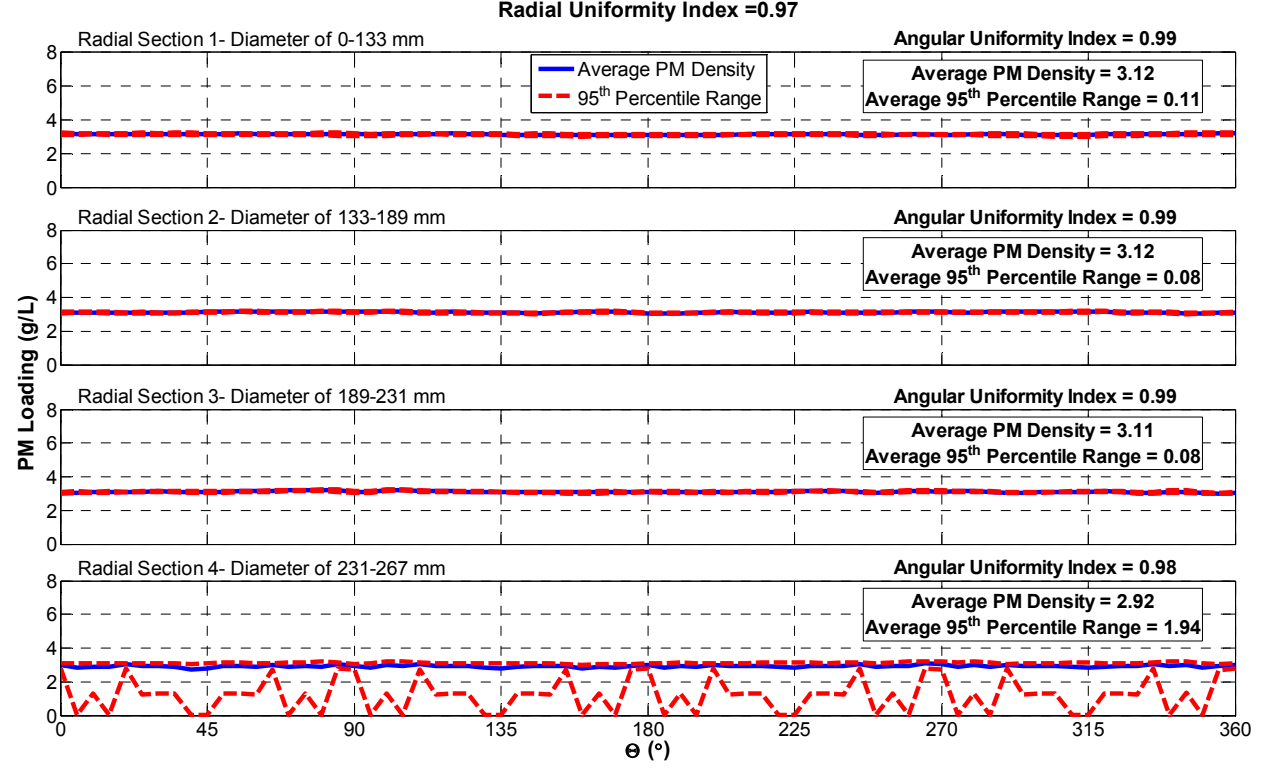

Figure K.67: Phase 2 Test 7- Post Loading $Z^{*}=0.128$

Test 7: Post Loading

Angular PM Distribution in Each Radial Section at $Z^{*}=\mathbf{0 . 3 7 6}$

Radial Uniformity Index $=0.97$

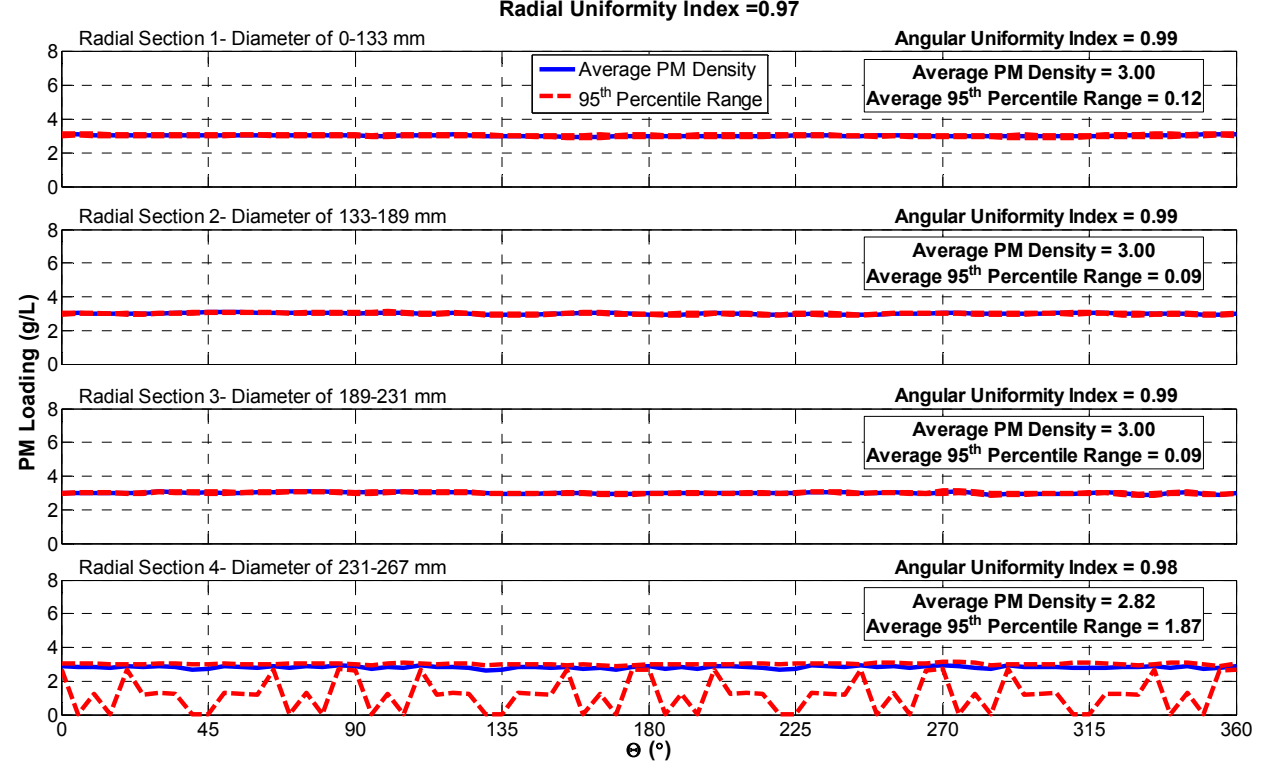

Figure K.68: Phase 2 Test 7- Post Loading $Z^{*}=0.376$ 
Test 7: Post Loading

Angular PM Distribution in Each Radial Section at $Z^{*}=\mathbf{0 . 6 2 4}$

Radial Uniformity Index $=0.97$

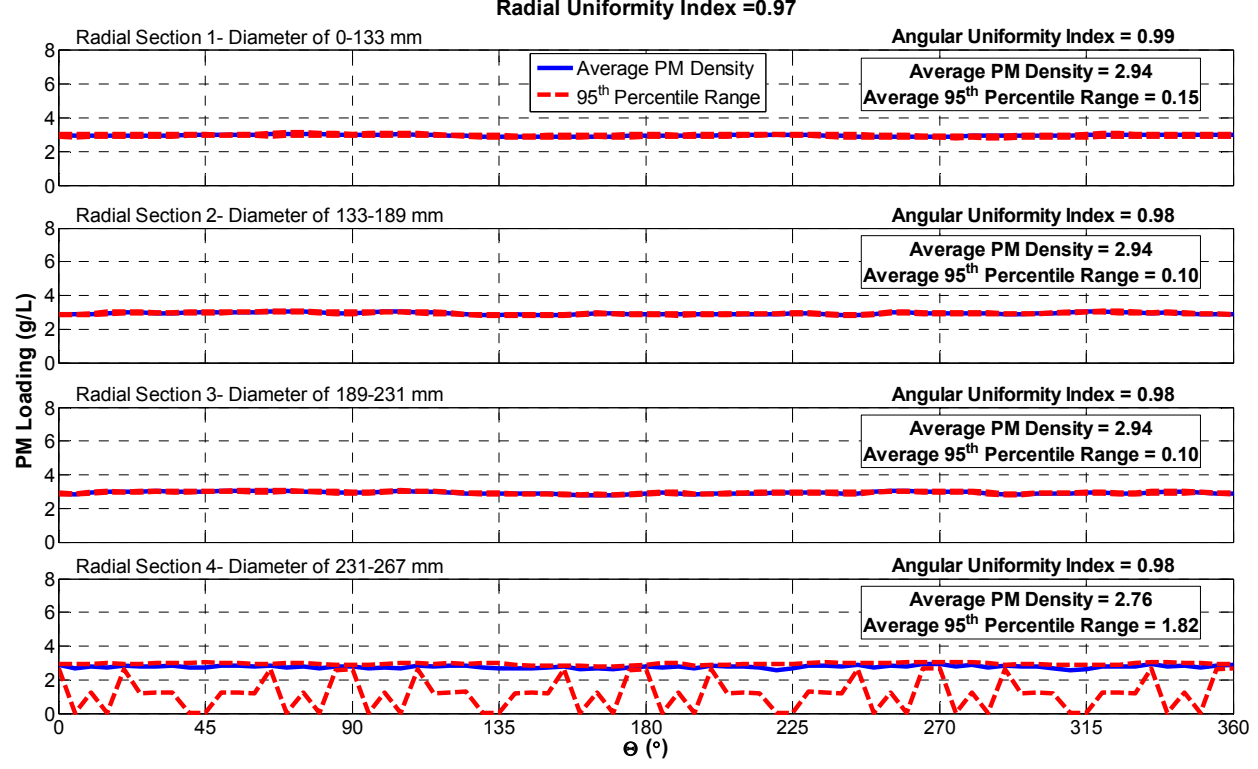

Figure K.69: Phase 2 Test 7- Post Loading $Z^{*}=0.624$

Test 7: Post Loading

Angular PM Distribution in Each Radial Section at $Z^{*}=0.872$

Radial Uniformity Index $=0.97$

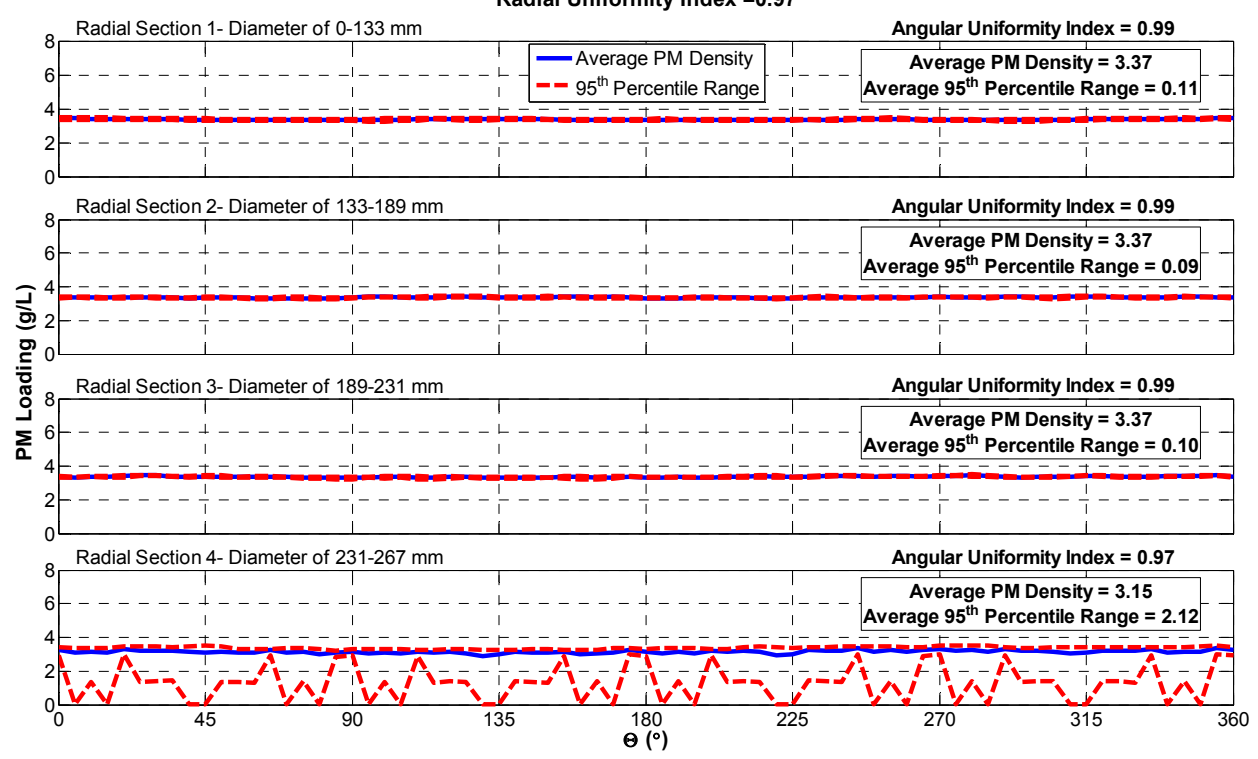

Figure K.70: Phase 2 Test 7- Post Loading $Z^{*}=0.872$ 


\section{Phase 2 Test 8 Passive Oxidation Scan}

Test 8: Passive Oxidation

Axial PM Distribution in Each Radial Section

Average Radial Uniformity Index $=0.97$

8 Radial Section 1- Diameter of 0-133 mm Average Angular Uniformity Index $=0.98$
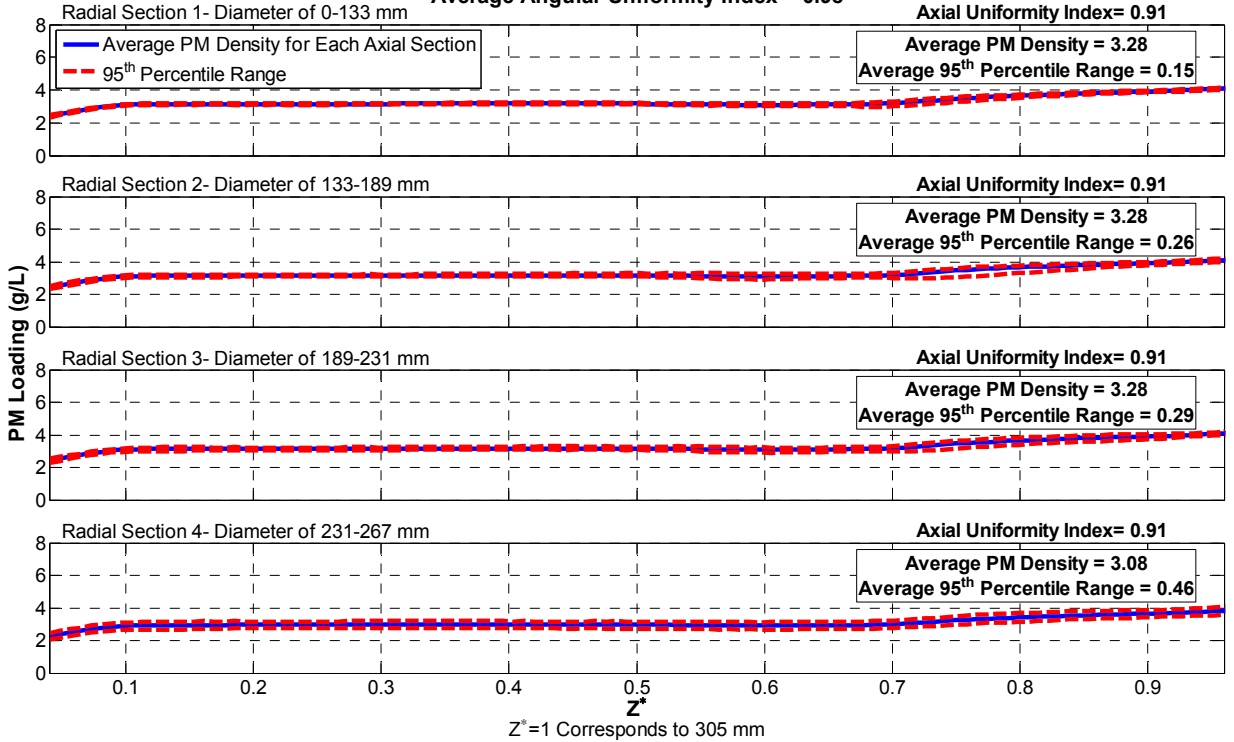

Figure K.71: Phase 2 Test 8- Passive Oxidation Axial Distribution

Test 8: Passive Oxidation

Angular PM Distribution in Each Radial Section at $Z^{*}=0.128$

Radial Uniformity Index $=0.97$

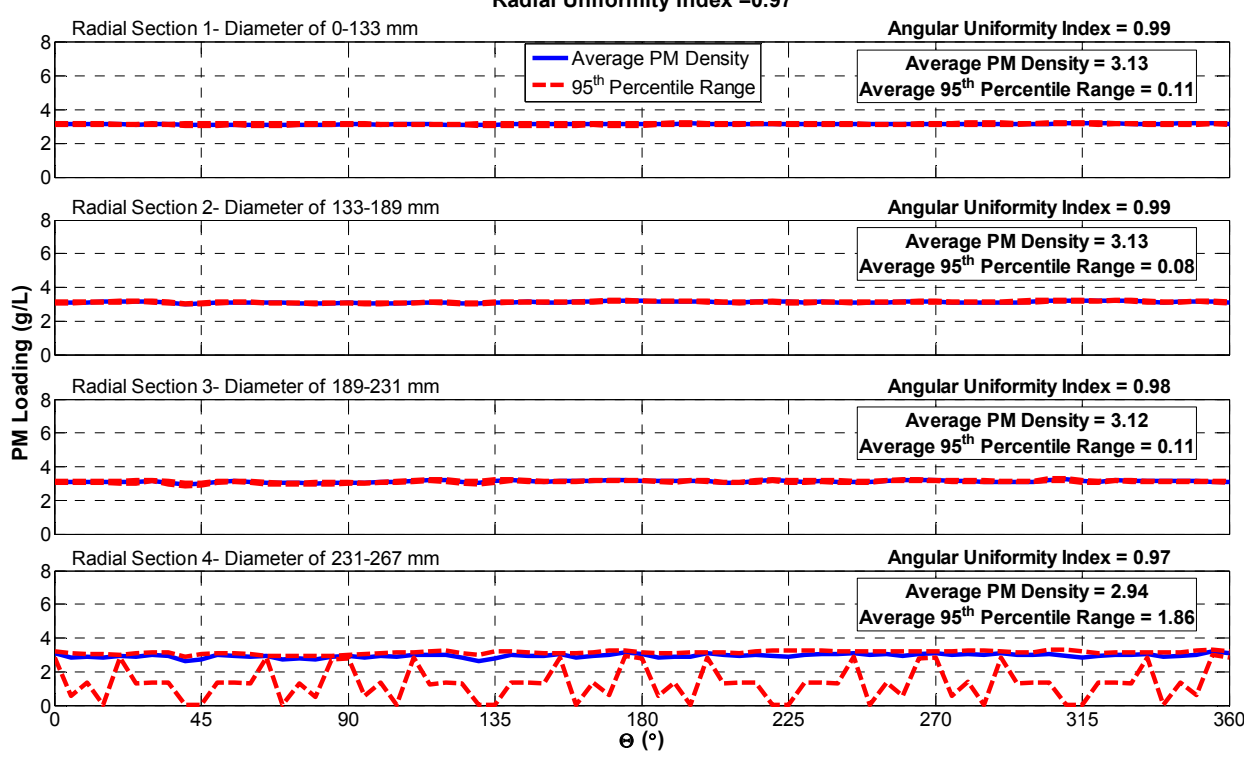

Figure K.72: Phase 2 Test 8- Passive Oxidation $Z^{*}=0.128$ 
Test 8: Passive Oxidation

Angular PM Distribution in Each Radial Section at $Z^{*}=\mathbf{0 . 1 2 8}$

Radial Uniformity Index $=0.97$

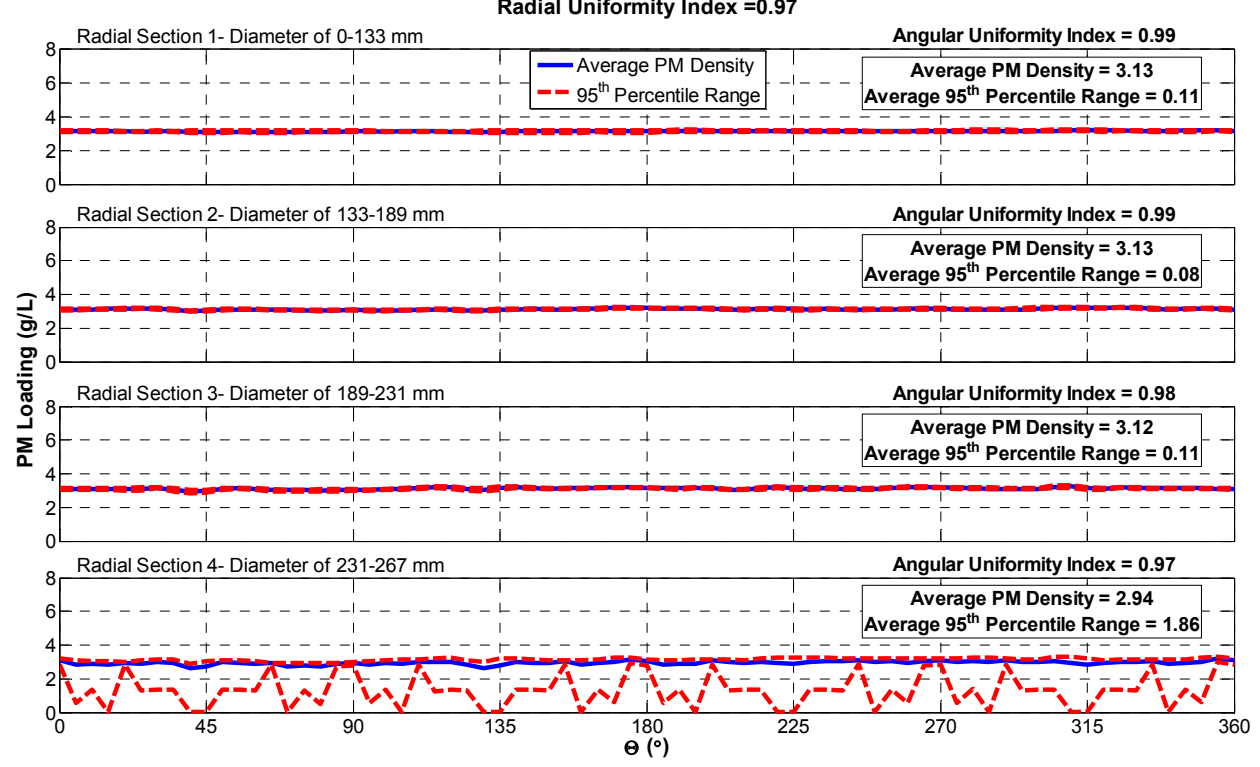

Figure K.73: Phase 2 Test 8- Passive Oxidation $Z^{*}=0.376$

Test 8: Passive Oxidation

Angular PM Distribution in Each Radial Section at $Z^{*}=0.624$

Radial Uniformity Index $=0.97$

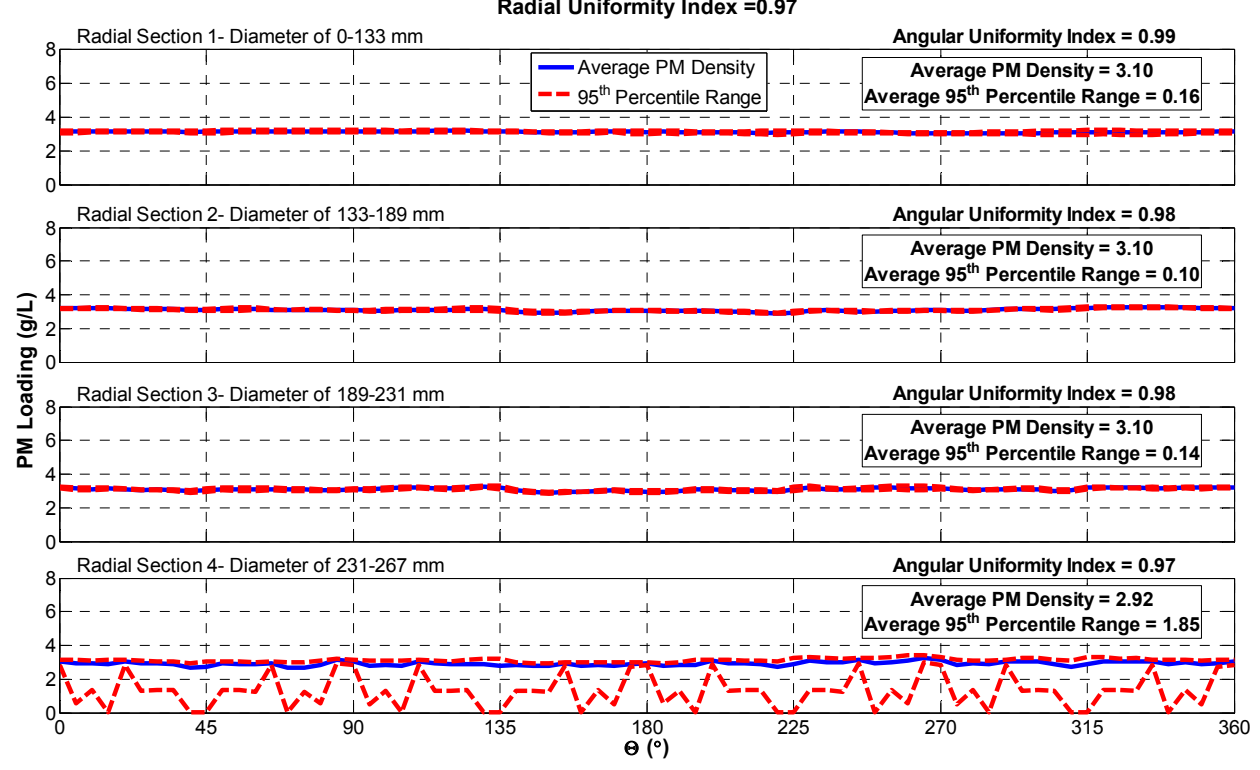

Figure K.74: Phase 2 Test 8- Passive Oxidation $Z^{*}=0.624$ 
Test 8: Passive Oxidation

Angular PM Distribution in Each Radial Section at $Z^{*}=0.872$

Radial Uniformity Index $=\mathbf{0 . 9 7}$

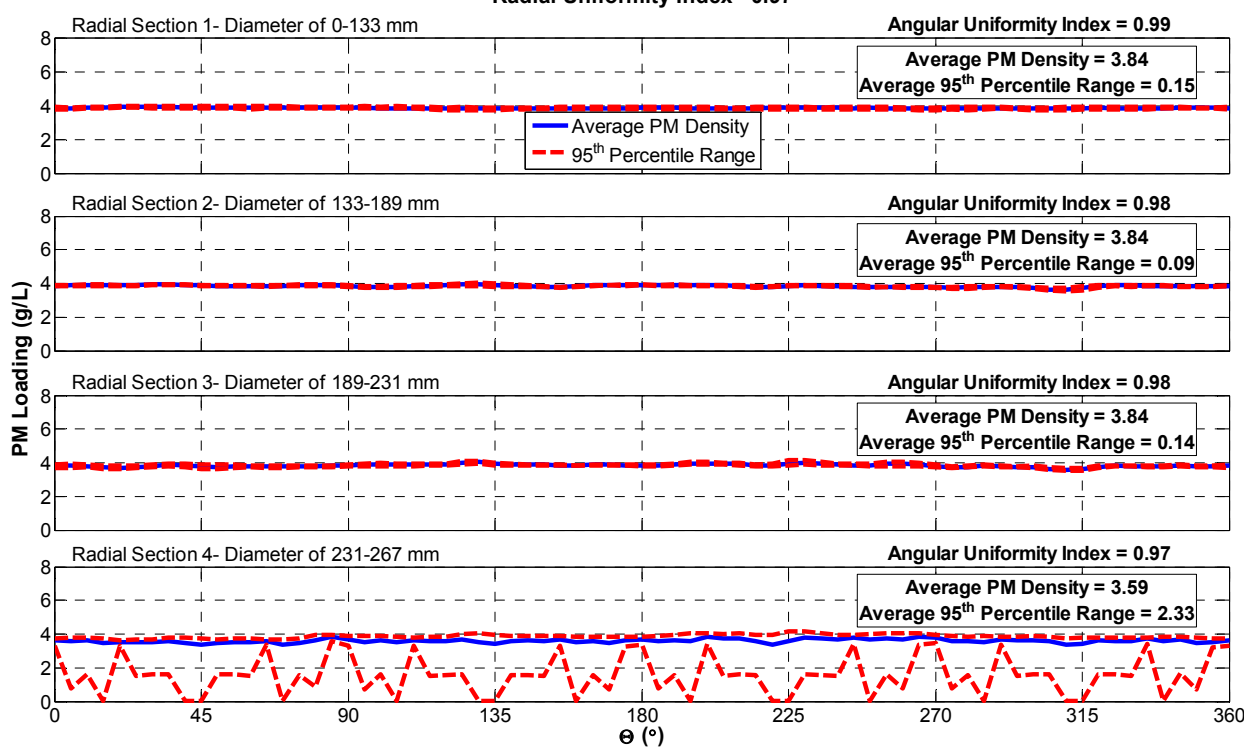

Figure K.75: Phase 2 Test 8- Passive Oxidation $Z^{*}=0.872$

\section{Phase 2 Test 9 Balance Point Scan}

Test 9: Balance Point

Axial PM Distribution in Each Radial Section

Average Radial Uniformity Index $=0.97$

Average Angular Uniformity Index $=0.98$
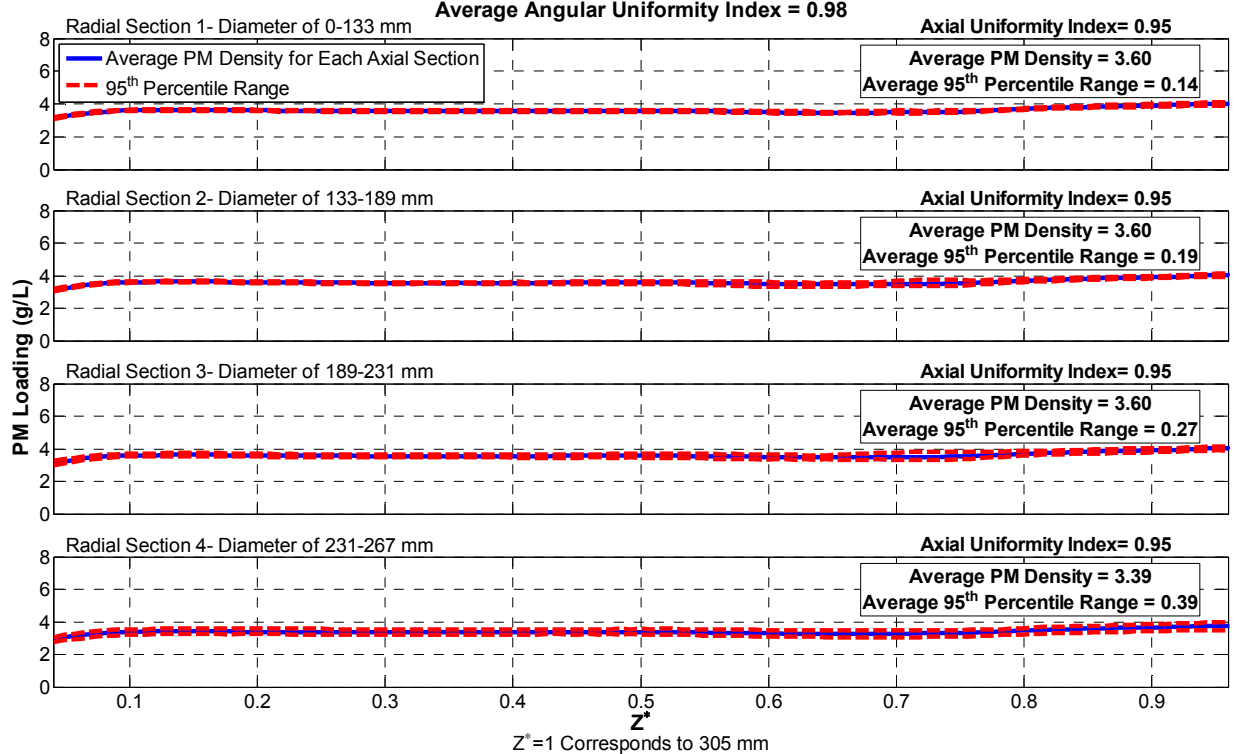

Figure K.76: Phase 2 Test 9- Balance Point Axial Distribution 
Test 9: Balance Point

Axial PM Distribution in Each Radial Section

Average Radial Uniformity Index $=0.97$

Average Angular Uniformity Index $=0.98$
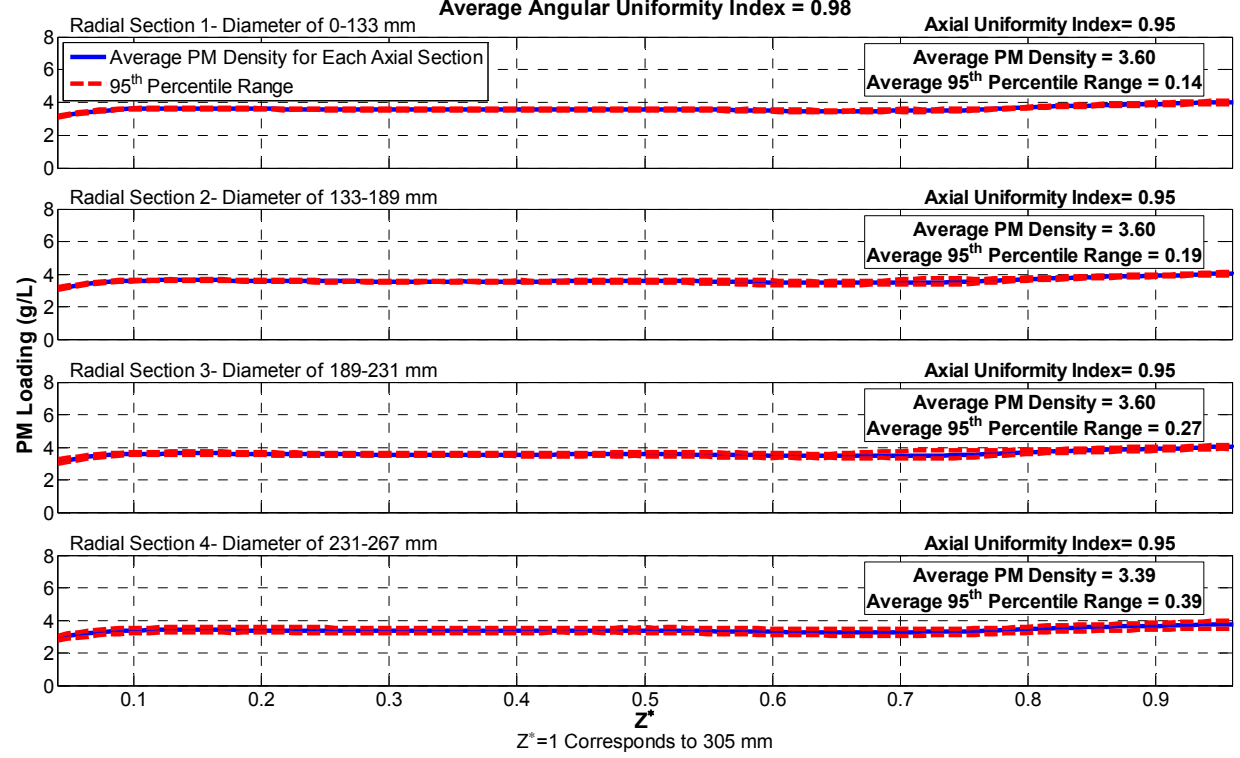

Figure K.77: Phase 2 Test 9- Balance Point $Z^{*}=0.128$

Test 9: Balance Point

Angular PM Distribution in Each Radial Section at $Z^{*}=\mathbf{0 . 3 7 6}$

Radial Uniformity Index $=0.97$

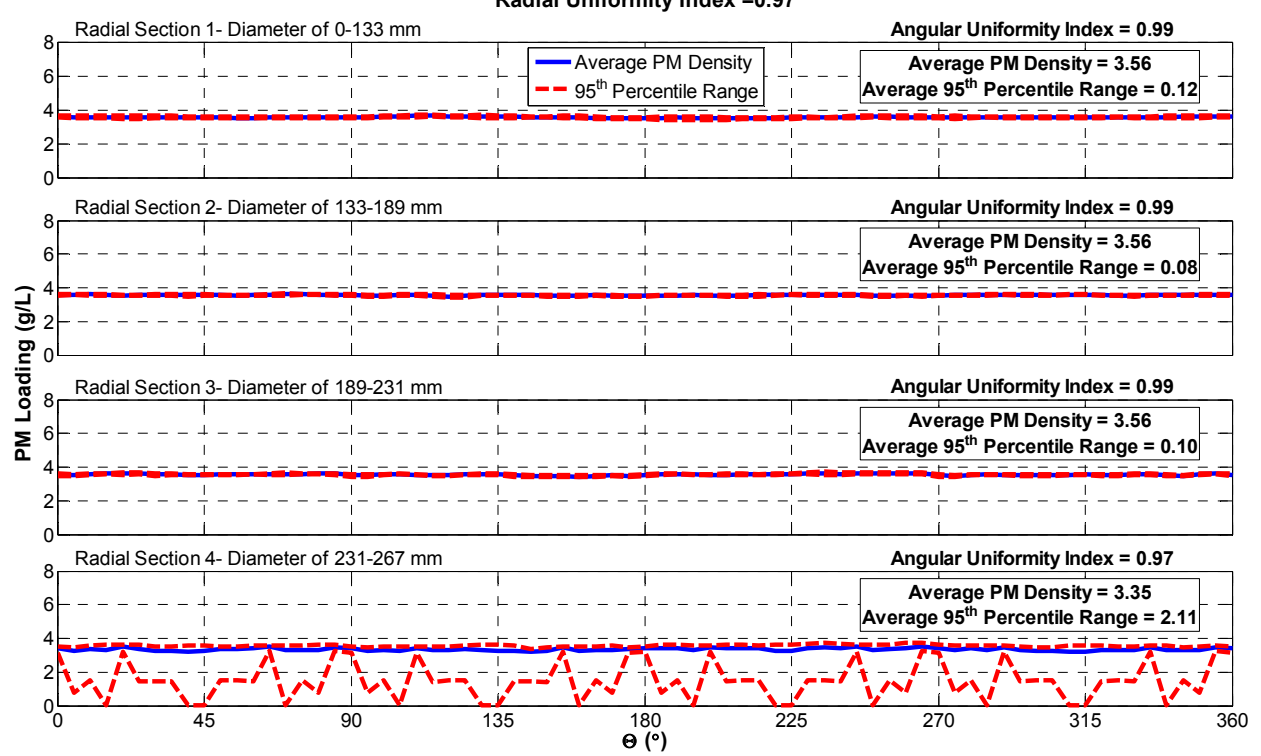

Figure K.78: Phase 2 Test 9- Balance Point $Z^{*}=0.376$ 
Test 9: Balance Point

Angular PM Distribution in Each Radial Section at $Z^{*}=\mathbf{0 . 6 2 4}$

Radial Uniformity Index $=0.97$

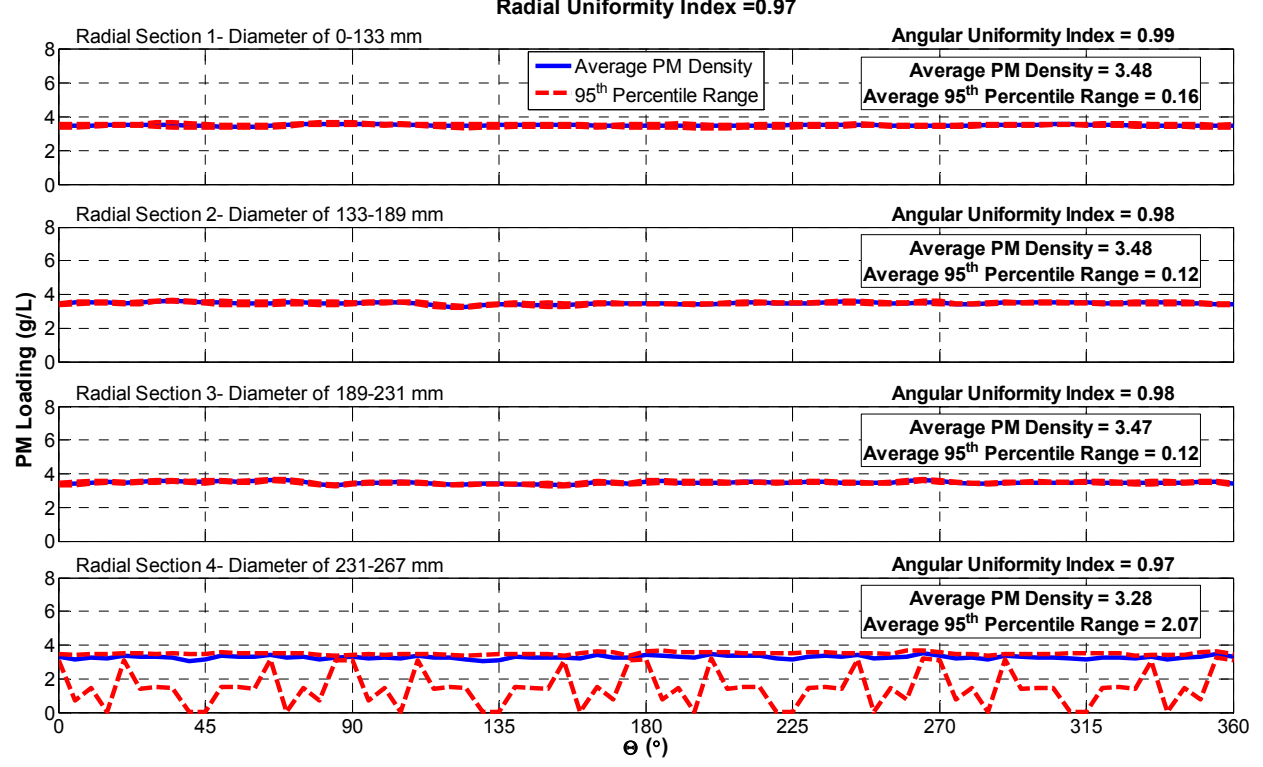

Figure K.79: Phase 2 Test 9- Balance Point $Z^{*}=0.624$

Test 9: Balance Point

Angular PM Distribution in Each Radial Section at $Z^{*}=\mathbf{0 . 8 7 2}$

Radial Uniformity Index $=0.96$

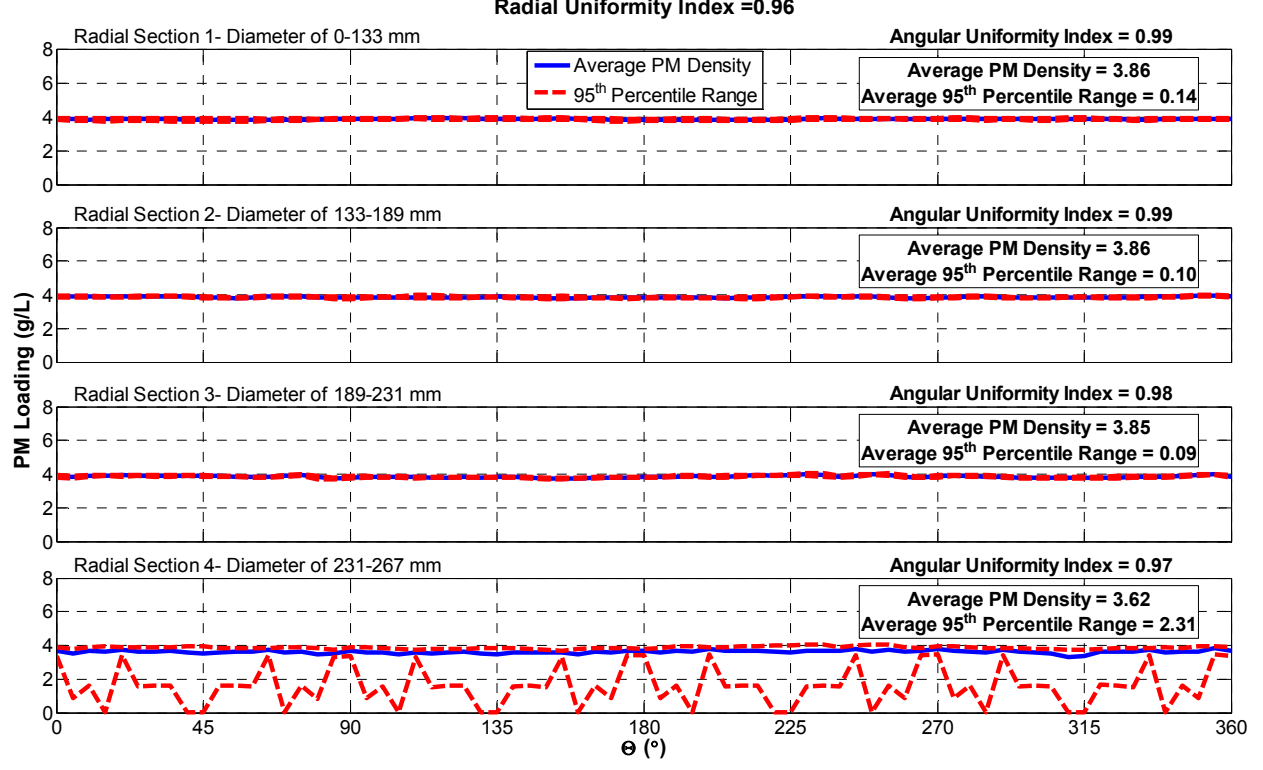

Figure K.80: Phase 2 Test 9- Balance Point $Z^{*}=0.872$ 


\section{Appendix L. Data Correlation Check Results by Test}

This appendix shows the data correlation check results for each test that was conducted. A consistent coefficient of correlation indicates that the data is good. A sudden change in the coefficient of correlation indicates that there is a shift in the data, or some other problem exists.

\section{Phase 1 Test 1 Loading Scan}
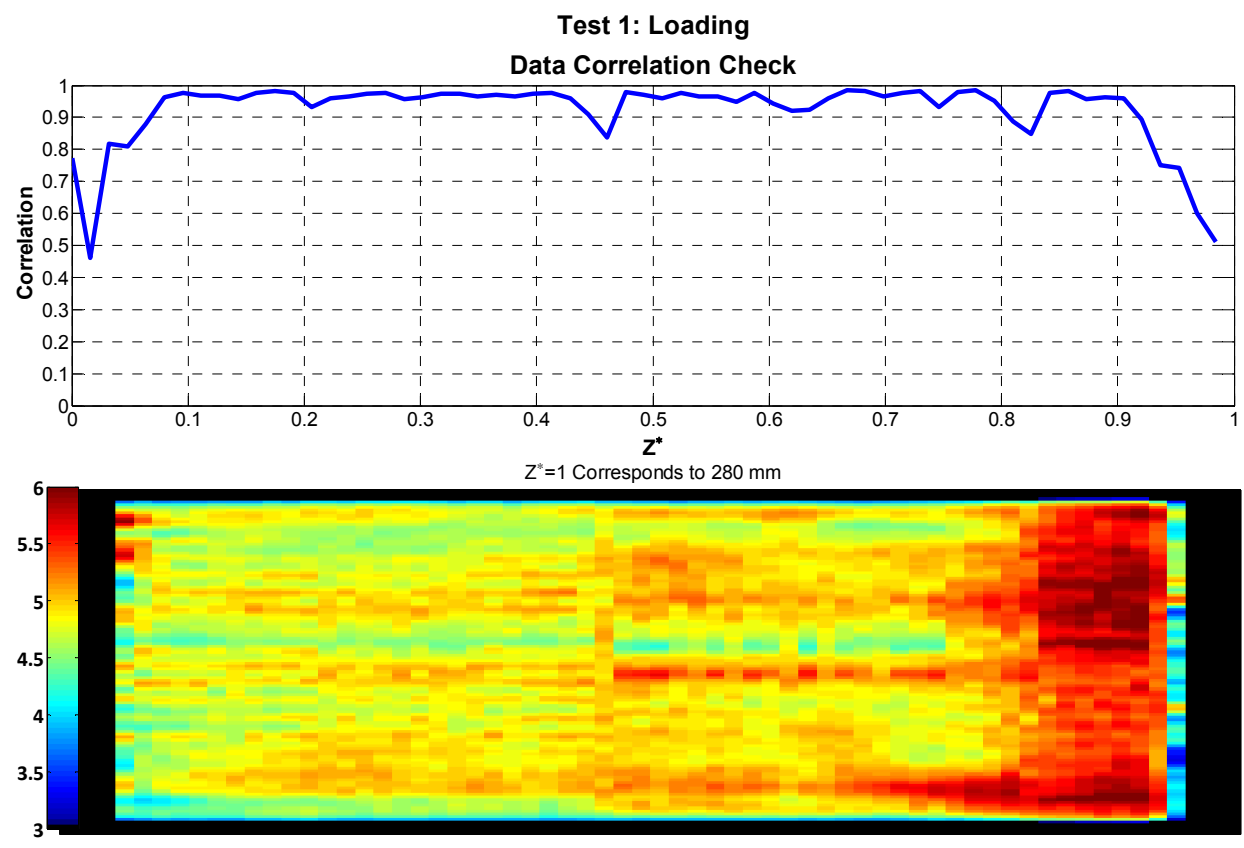

Figure L.1: Data Correlation for Phase 1 Test 1 Loading Scan 


\section{Phase 1 Test 1 Active Regeneration Scan}
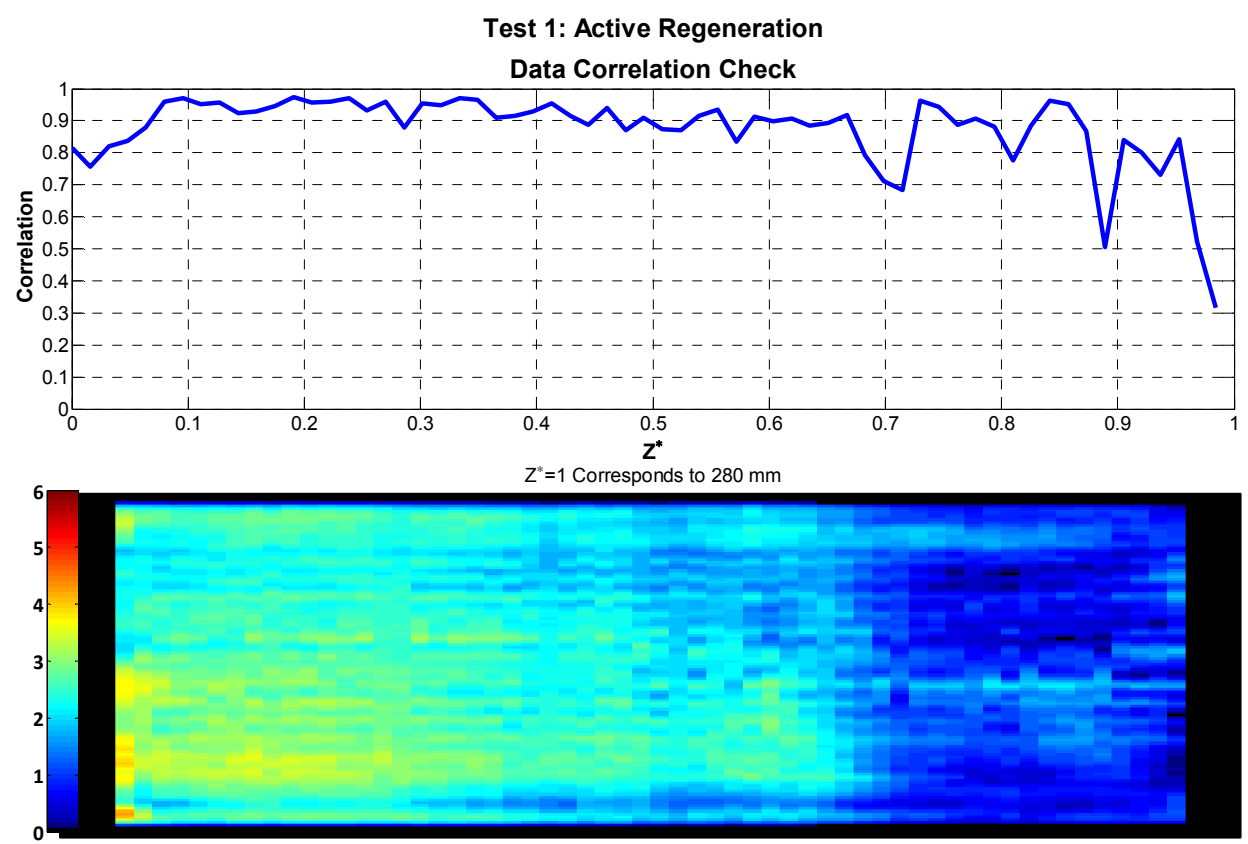

Figure L.2: Data Correlation for Phase 1 Test 1 Active Regeneration Scan

\section{Phase 1 Test 2 Active Regeneration Scan}
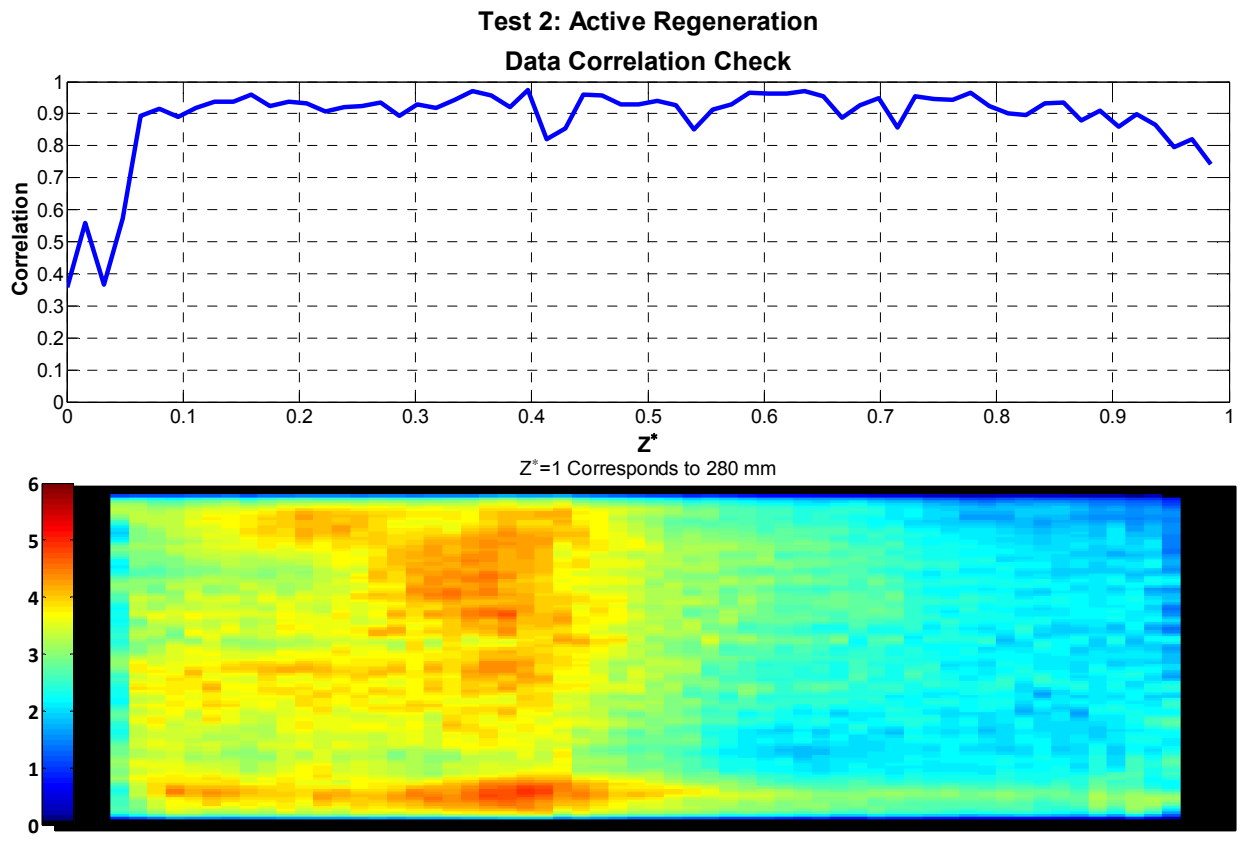

Figure L.3: Data Correlation for Phase 1 Test 2 Active Regeneration Scan 


\section{Phase 1 Test 3 Passive Oxidation Scan}
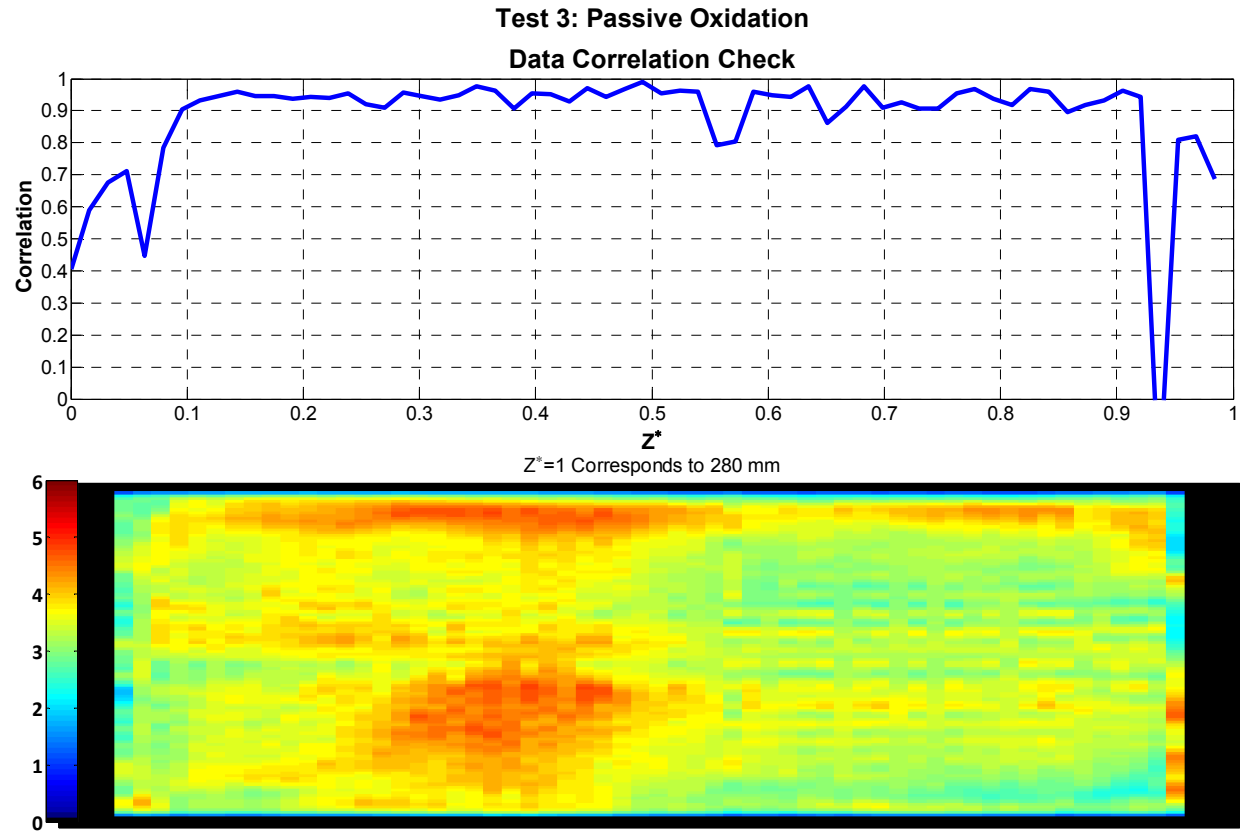

Figure L.4: Data Correlation for Phase 1 Test 3 Passive Oxidation Scan

\section{Phase 1 Test 4 Active Regeneration Scan}
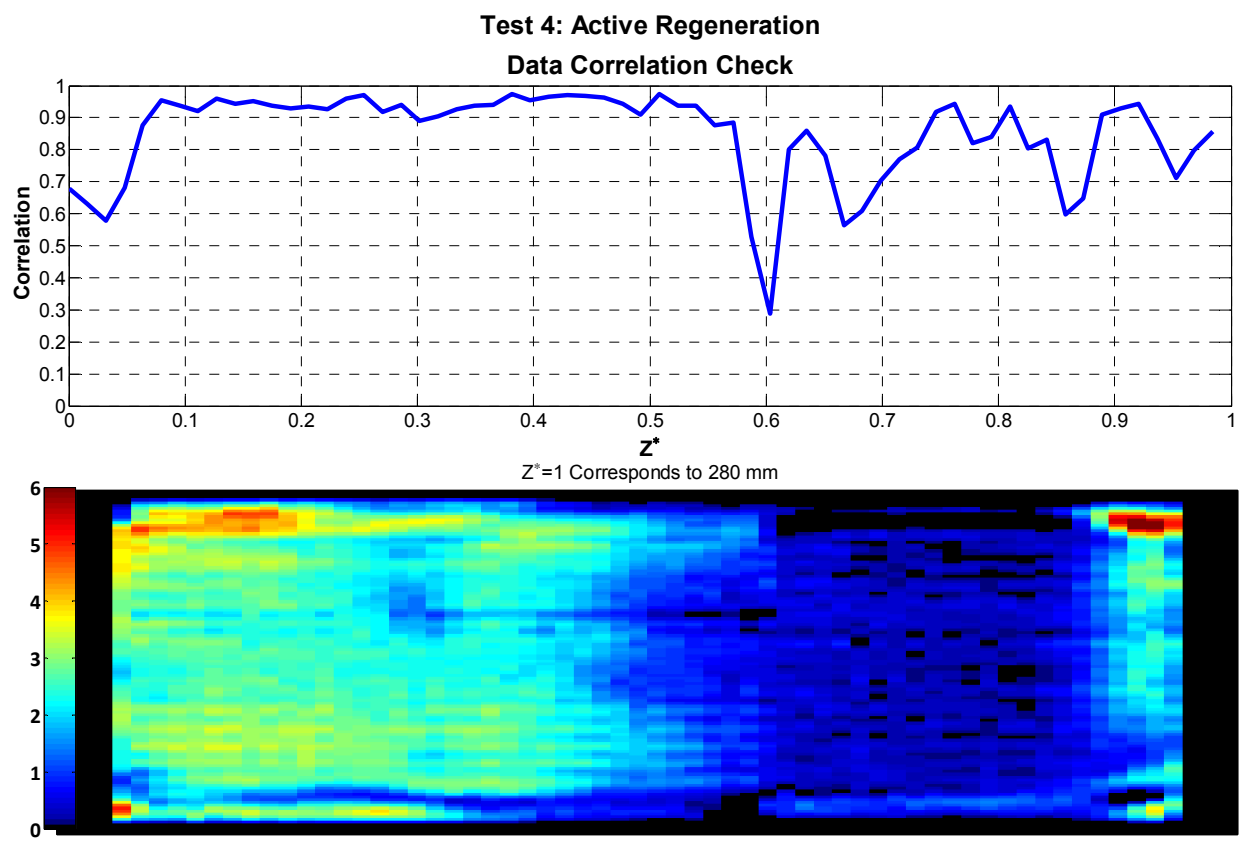

Figure L.5: Data Correlation for Phase 1 Test 4 Active Regeneration Scan 
Phase 2 Test 1 Loading Scan
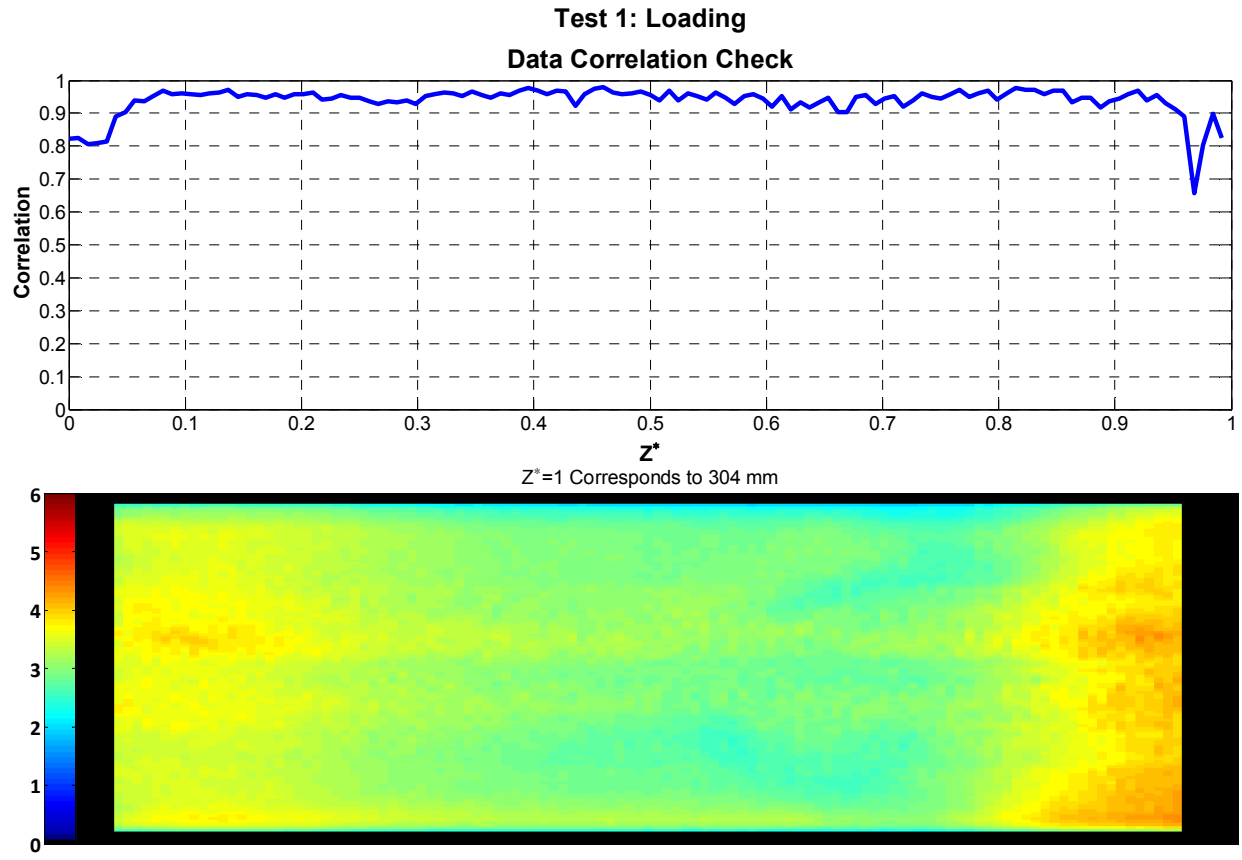

Figure L.6: Data Correlation for Phase 2 Test 1 Loading Scan

Phase 2 Test 1 Passive Oxidation Scan
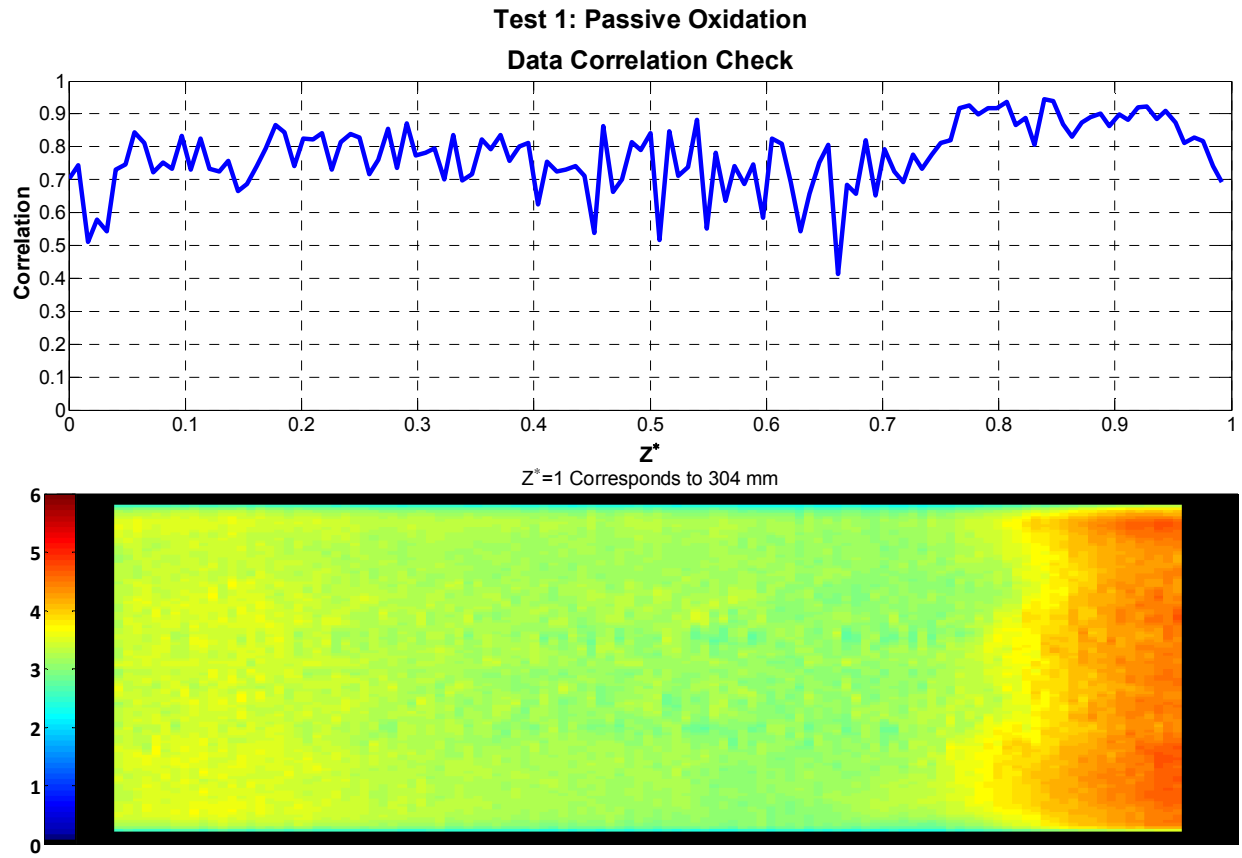

Figure L.7: Data Correlation for Phase 2 Test 1 Passive Oxidation Scan 
Phase 2 Test 2 Cleanout Scan

Test 2: Cleanout

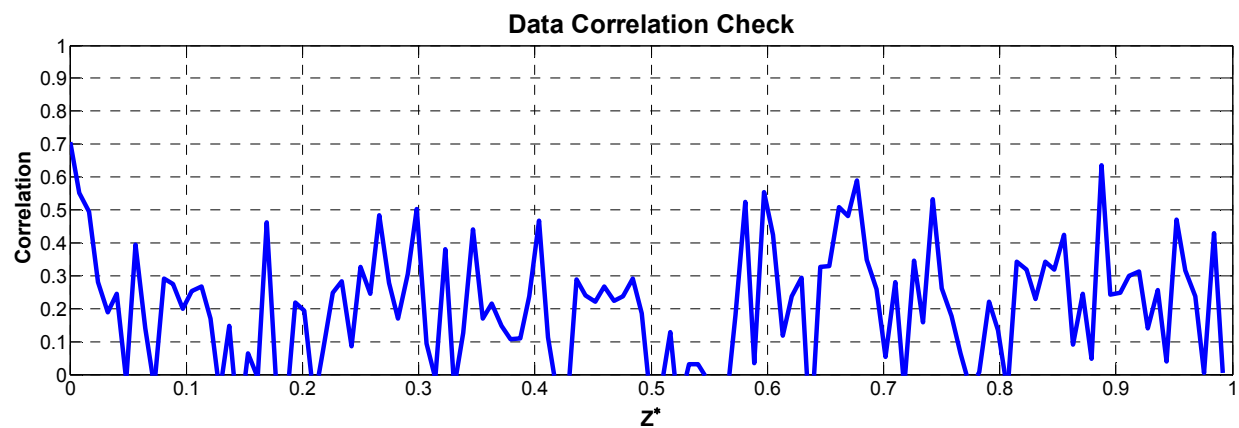

$Z^{*}=1$ Corresponds to $305 \mathrm{~mm}$

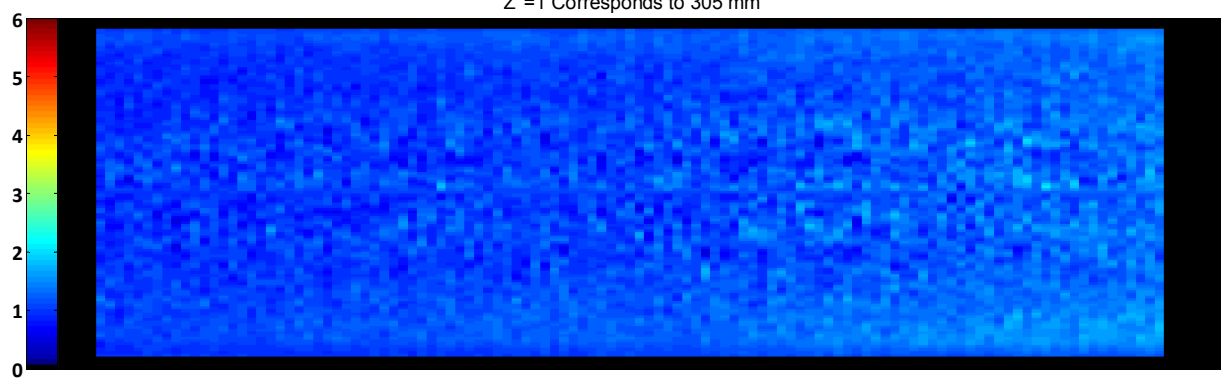

Figure L.8: Data Correlation for Phase 2 Test 2 Cleanout Scan

Phase 2 Test 3 Active Regeneration Scan
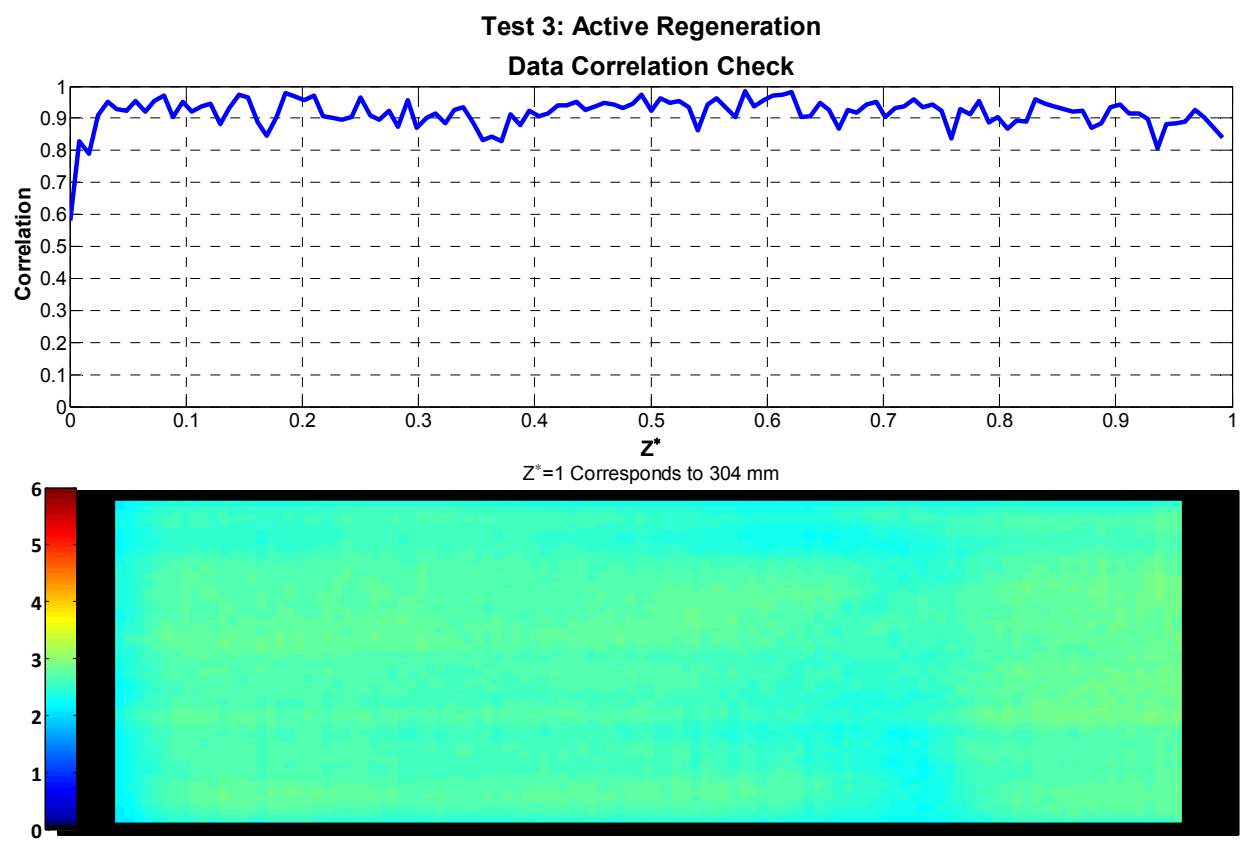

Figure L.9: Data Correlation for Phase 2 Test 3 Active Regeneration 
Phase 2 Test 4 Active Regeneration Scan
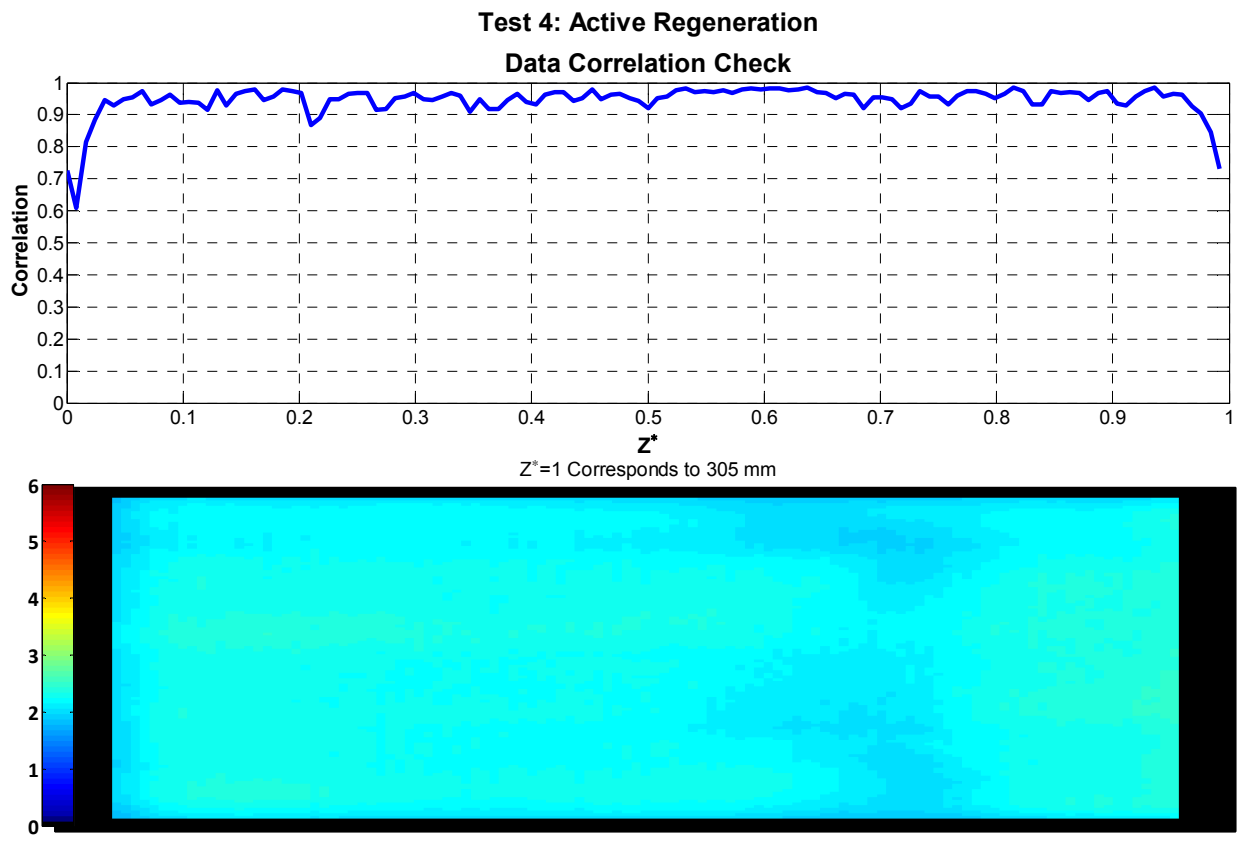

Figure L.10: Data Correlation for Phase 2 Test 4 Active Regeneration Scan

\section{Phase 2 Test 4 Post Loading Scan}
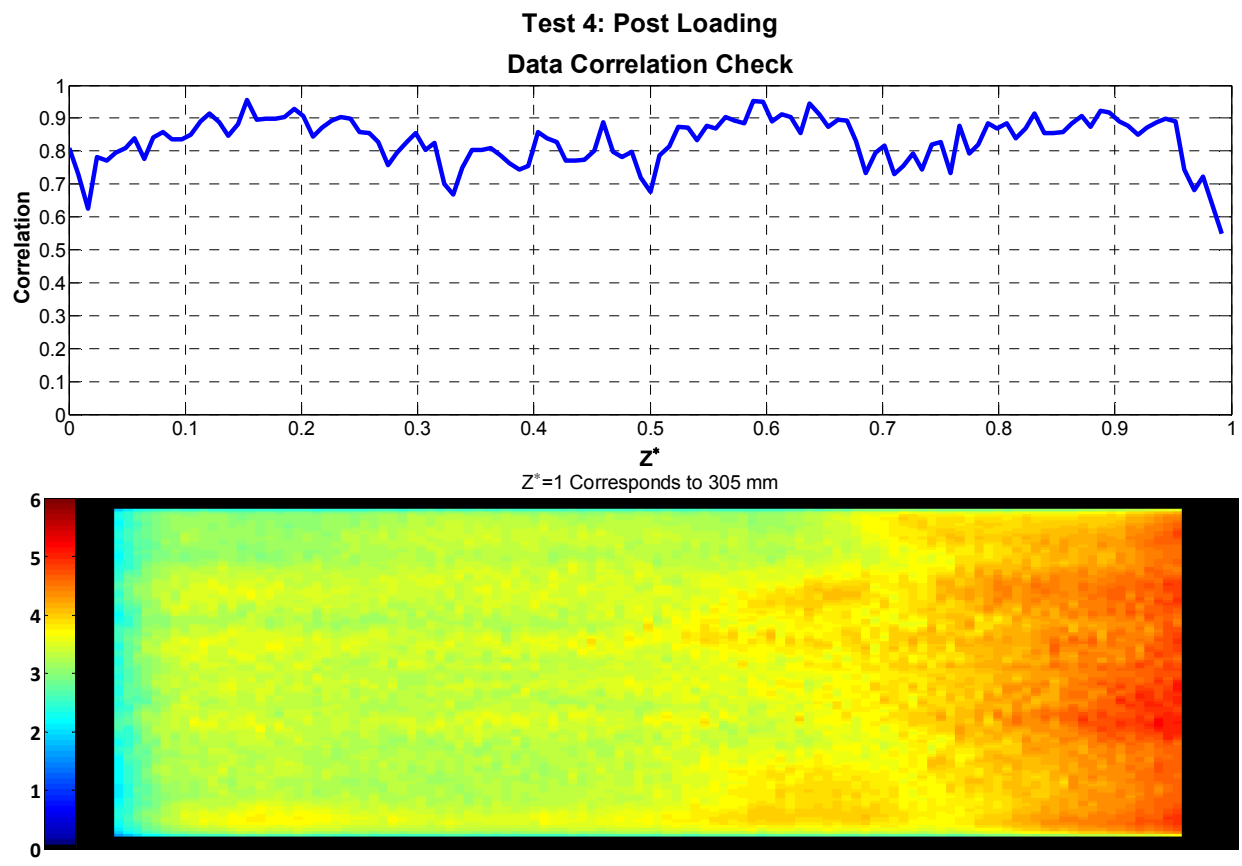

Figure L.11: Data Correlation for Phase 2 Test 4 Post Loading Scan 


\section{Phase 2 Test 5 Active Regeneration Scan}
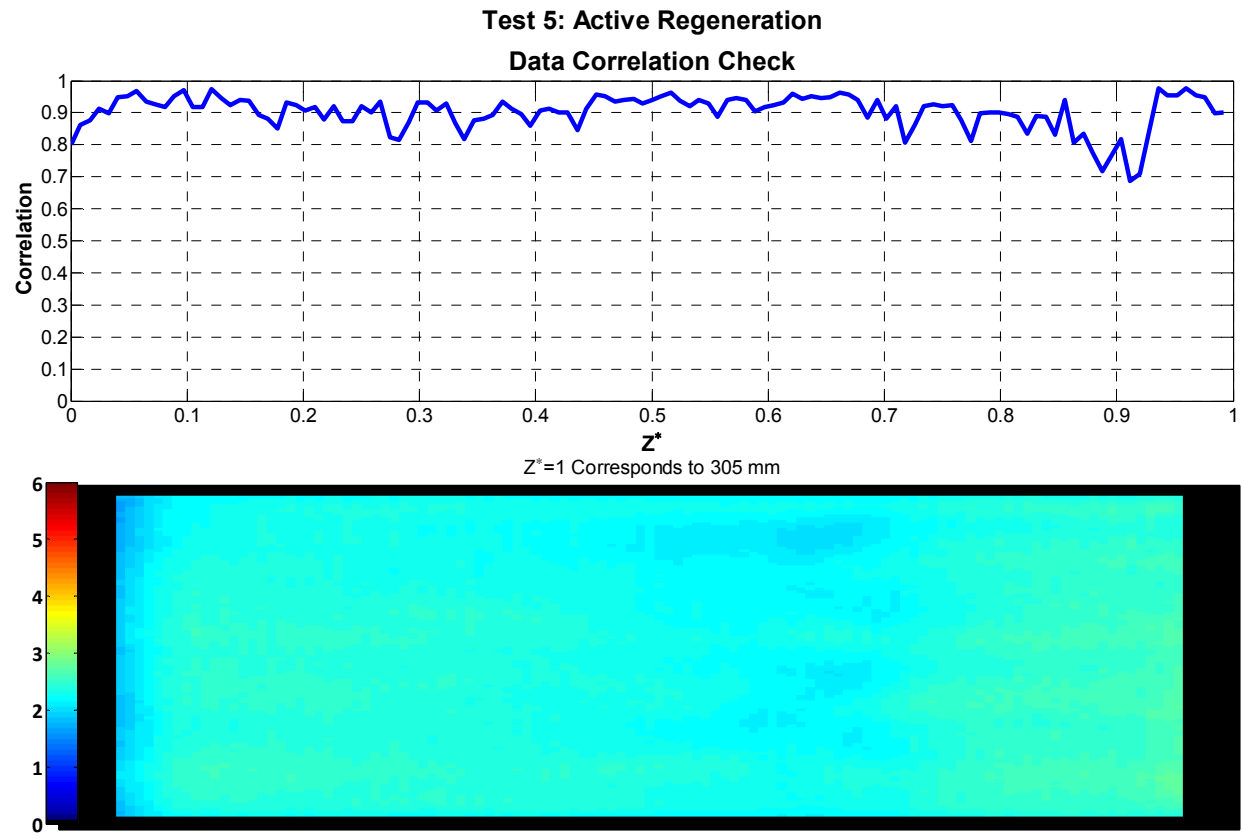

Figure L.12: Data Correlation for Phase 2 Test 5 Active Regeneration Scan

Phase 2 Test 6 Loading Scan
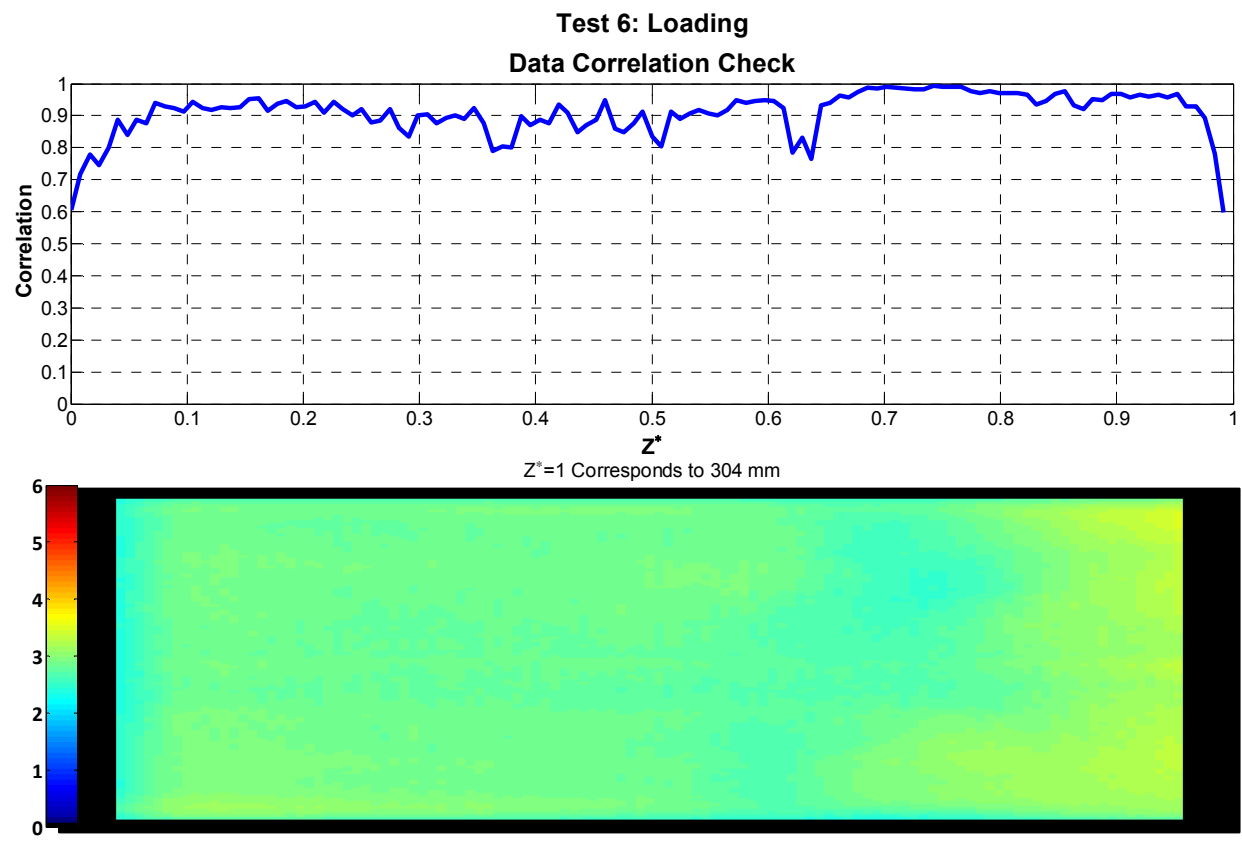

Figure L.13: Data Correlation for Phase 2 Test 6 Loading Scan 


\section{Phase 2 Test 6 Second Loading Scan}
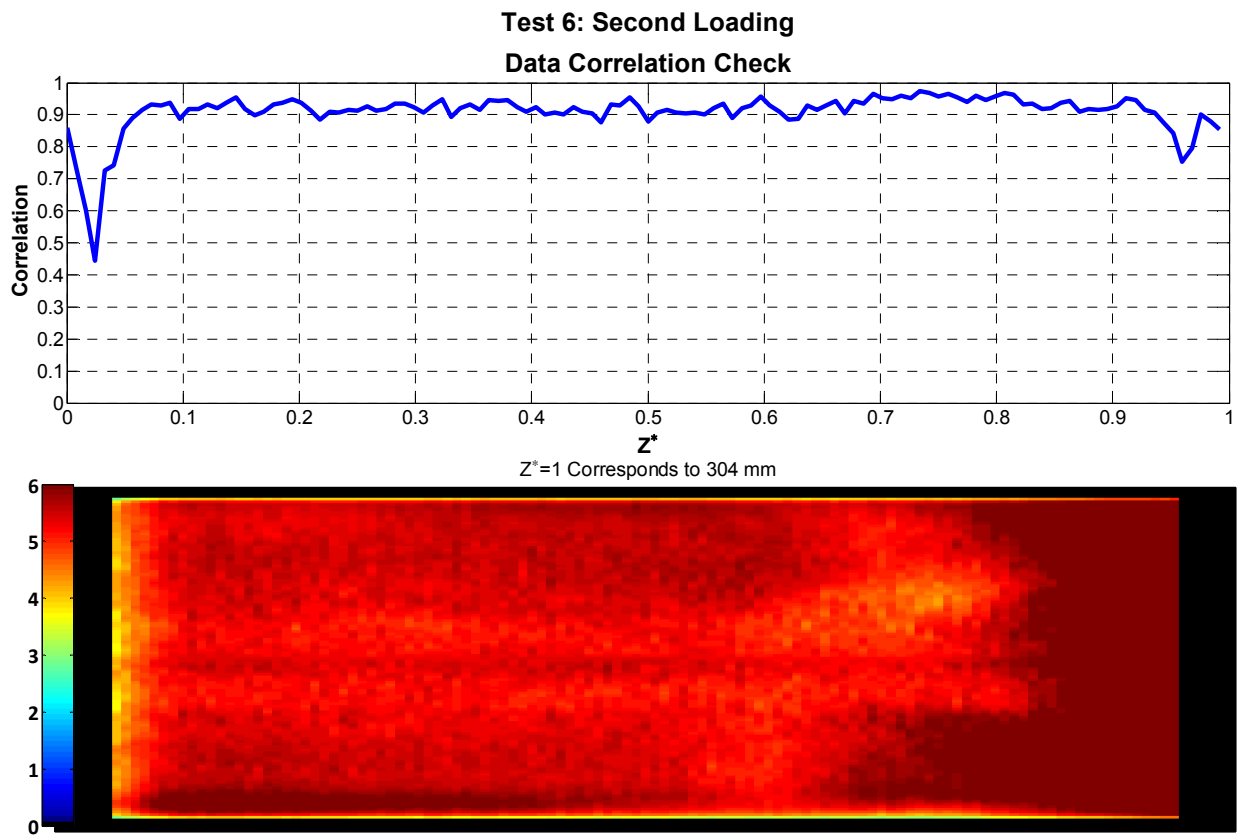

Figure L.14: Data Correlation for Phase 2 Test 6 Second Loading Scan

\section{Phase 2 Test 7 Passive Oxidation Scan}
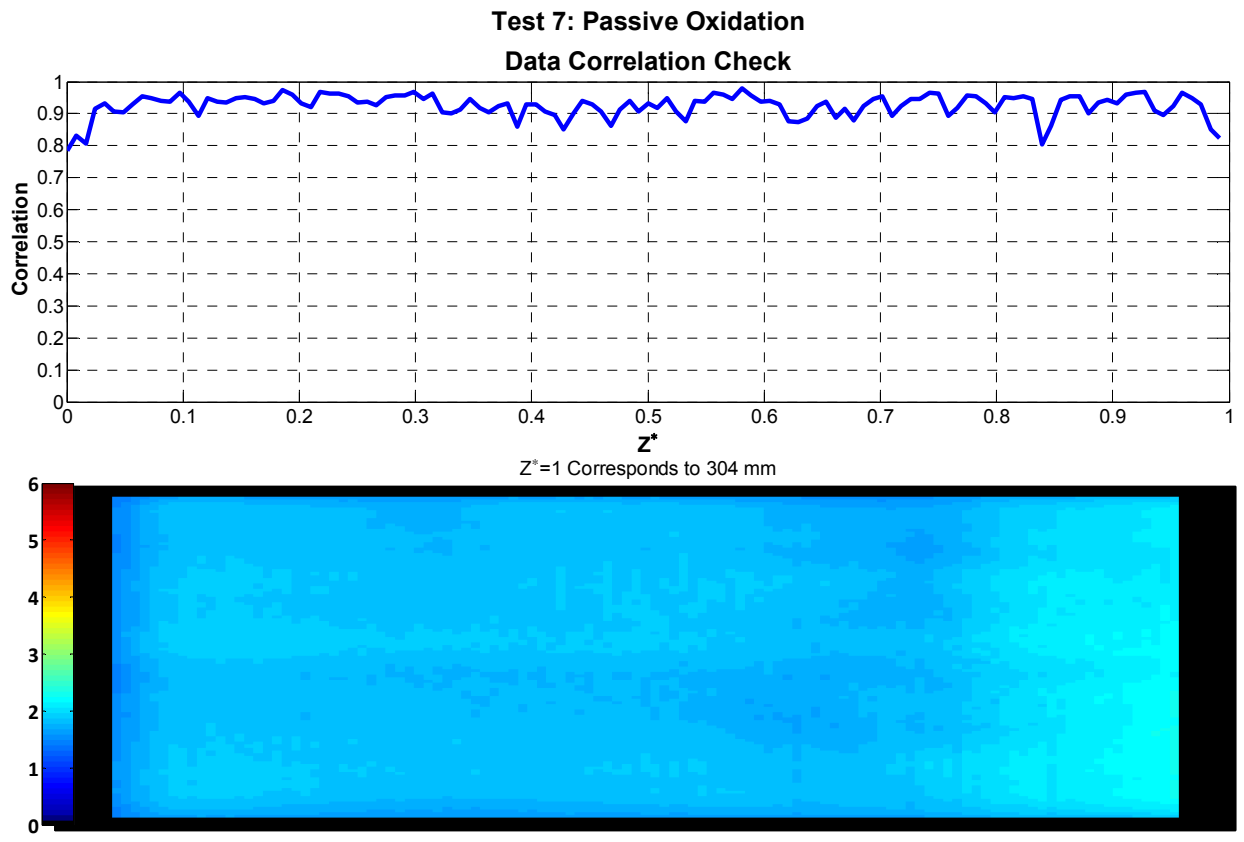

Figure L.15: Data Correlation for Phase 2 Test 7 Passive Oxidation Scan 


\section{Phase 2 Test 7 Post Loading Scan}
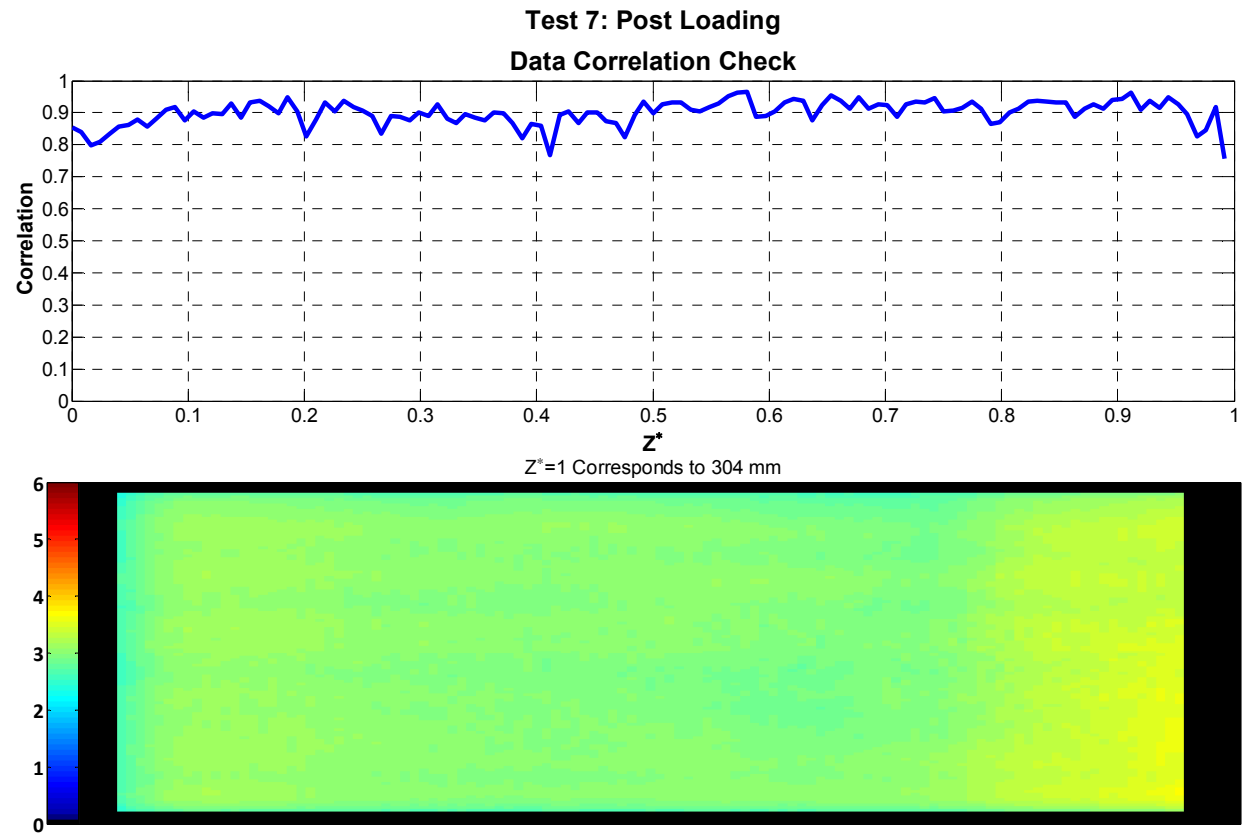

Figure L.16: Data Correlation for Phase 2 Test 7 Post Loading Scan

\section{Phase 2 Test 8 Passive Oxidation Scan}
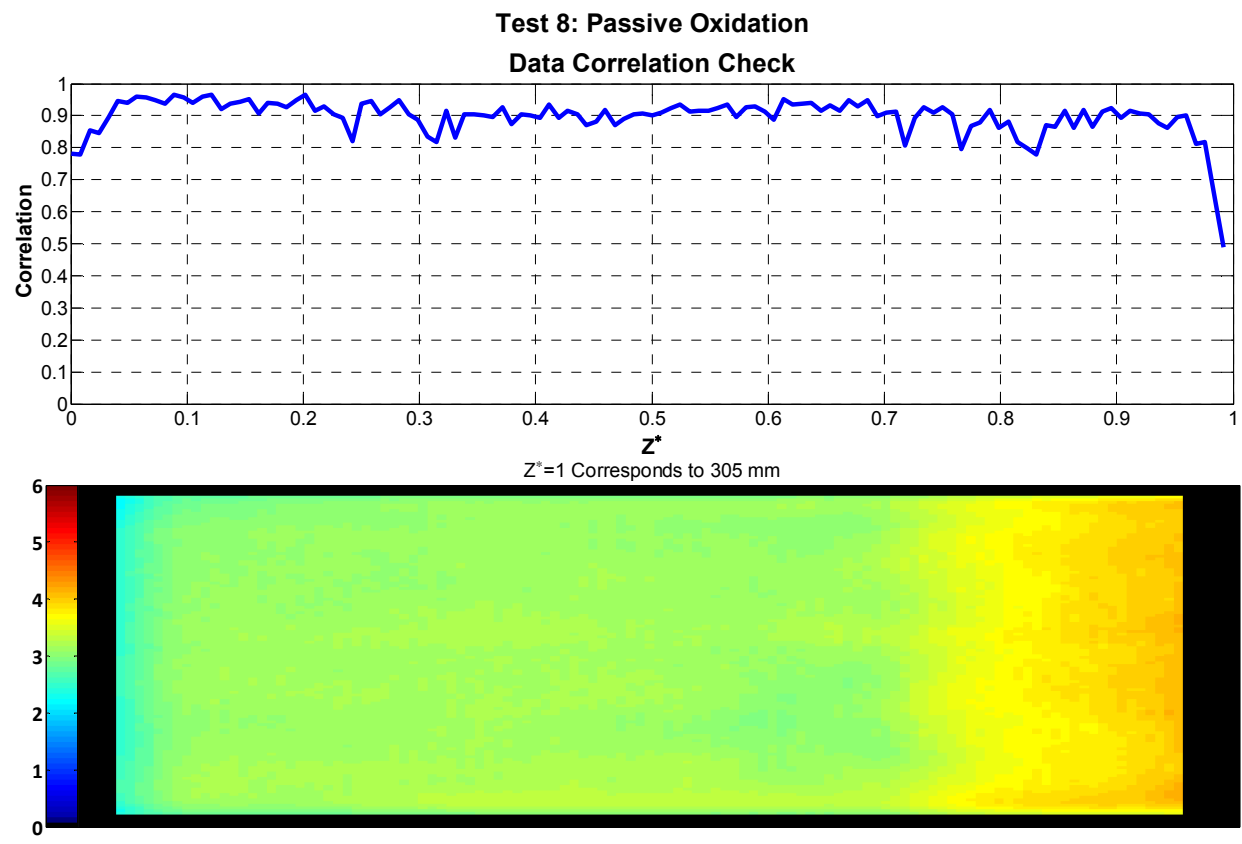

Figure L.17: Data Correlation for Phase 2 Test 8 Passive Oxidation Scan 
Phase 2 Test 9 Balance Point Scan
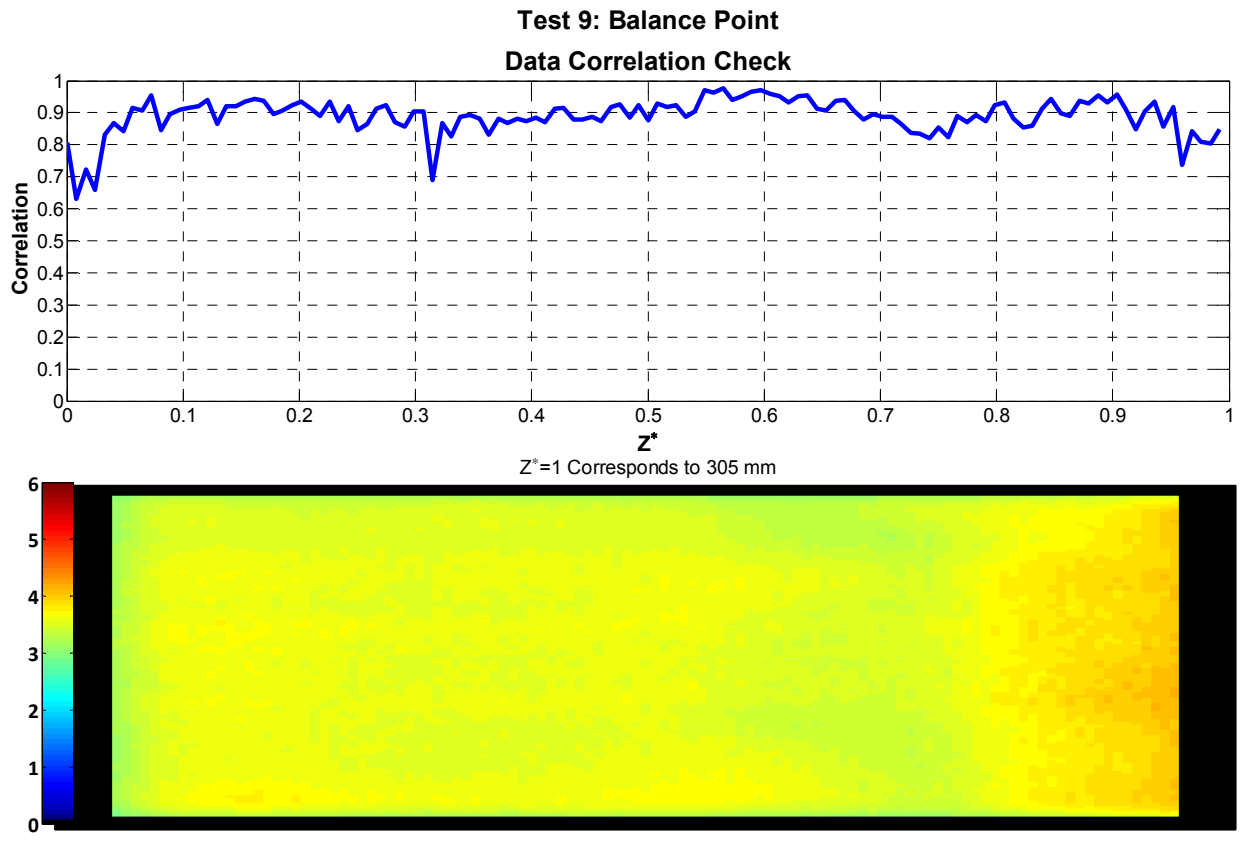

Figure L.18: Data Correlation for Phase 2 Test 9 Balance Point Scan 


\section{Appendix M. Permission to Use Copyrighted Material}

For Figure 3.6:

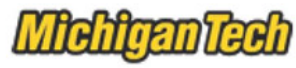

Ryan Foley <rkfoley@mtu.edu>

\section{Figure for my Thesis}

christopher.hutton@cummins.com <christopher.hutton@cummins.com>

To: Ryan Foley <rkfoley@mtu.edu>

Mon, Sep 30, 2013 at 7:26 AM

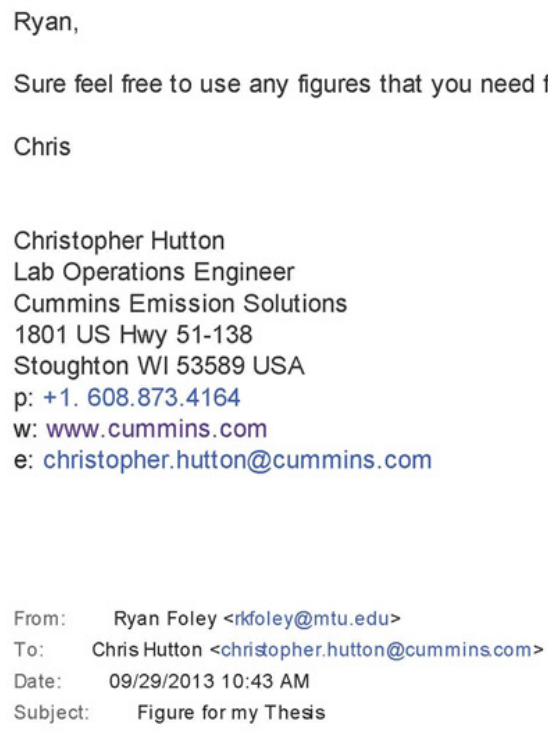

Chris,

I hope things are well. I am writing you today to request permission to use the figure you developed for the ISB thermocouple layout for Phase 1 of the maldistribution study. Please leave my original text in your reply and thank you for your time.

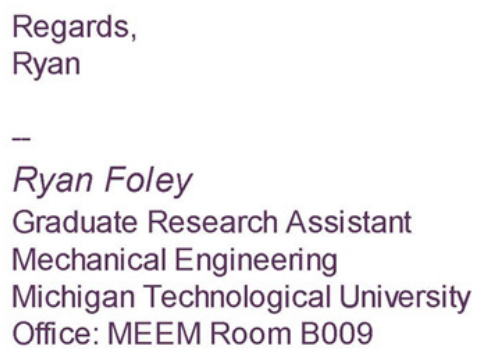

This email has been scanned for Malware. 


\section{For Figure 6.25:}

\section{Modeled PM Distribution v. Experimental}

Kiran Premchand $<$ kcpremch@mtu.edu>

Sun, Nov 24, 2013 at 4:46 PM

To: Ryan Foley <rkfoley@mtu.edu>

Hi Ryan,

You have my permission to use whatever material you need from my thesis. Please find attached a draft copy of the same and the comparison figure (.fig) that you need (Figure 5.33 in the thesis).

Regards,

Kiran

Kiran Premchand

Graduate Student

Mechanical Engineering - Engineering Mechanics Dept.

Michigan Technological University

1400 Townsend Drive

Houghton, MI - 49931

On Sun, Nov 24, 2013 at 4:07 PM, Ryan Foley <rkfoley@mtu.edu> wrote:

Kiran,

Would it be possible to get a plot from you showing my axial PM distribution data versus your axial PM distribution data for 1 loading case? There is interest in having that in my defense presentation.

Would I have permission to use that in my thesis as well, if required? If yes, could I get the citation information for your dissertation?

Regards,

Ryan

Ryan Foley

Graduate Research Assistant

Mechanical Engineering

Michigan Technological University

Office: MEEM Room B009

2 attachments

72 KP_PhD_thesis_2013_11212013_for_JHJ_SLY_review.pdf

$4466 \mathrm{~K}$

] comp_with_ryan.fig

$12 \mathrm{~K}$ 
For Figure 2.9:

\begin{abstract}
ELSEVIER LICENSE TERMS AND CONDITIONS
\end{abstract}

Sep 21, 2013

This is a License Agreement between Ryan K Foley ("You") and Elsevier ("Elsevier") provided by Copyright Clearance Center ("CCC"). The license consists of your order details, the terms and conditions provided by Elsevier, and the payment terms and conditions.

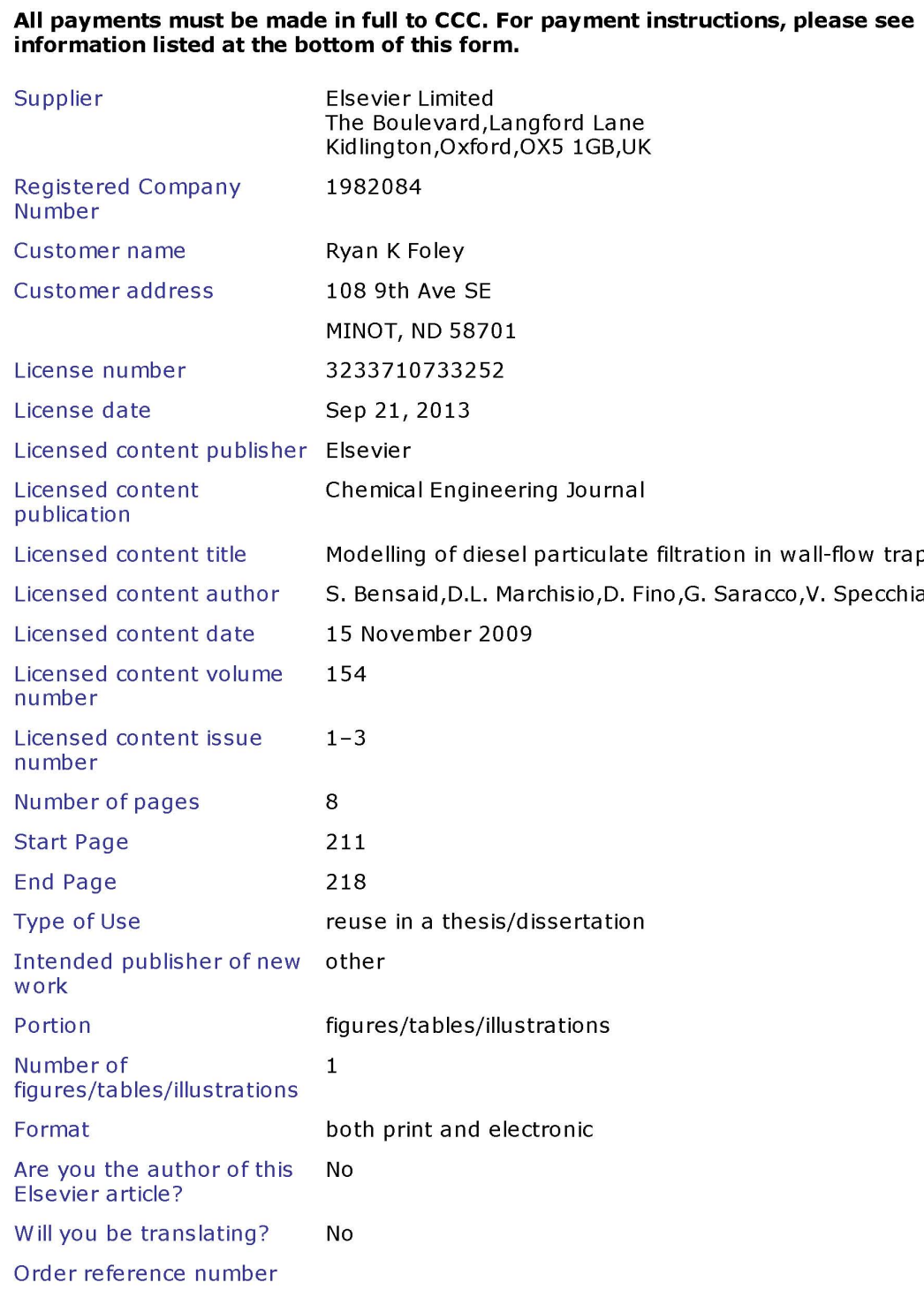




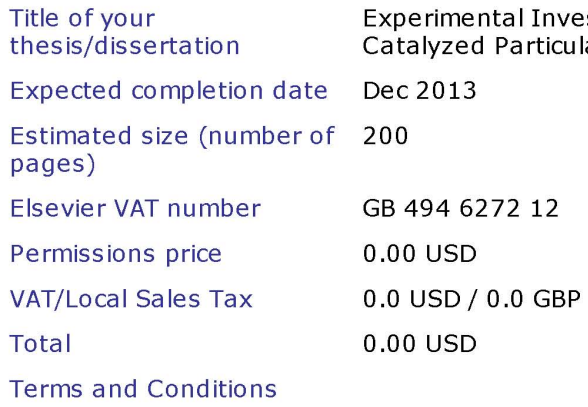

\section{INTRODUCTION}

1. The publisher for this copyrighted material is Elsevier. By clicking "accept" in connection with completing this licensing transaction, you agree that the following terms and conditions apply to this transaction (along with the Billing and Payment terms and conditions established by Copyright Clearance Center, Inc. ("CCC"), at the time that you opened your Rightslink account and that are available at any time at http://myaccount copyright.com).

\section{GENERAL TERMS}

2. Elsevier hereby grants you permission to reproduce the aforementioned material subject to the terms and conditions indicated.

3. Acknowledgement: If any part of the material to be used (for example, figures) has appeared in our publication with credit or acknowledgement to another source, permission must also be sought from that source. If such permission is not obtained then that material may not be included in your publication/copies. Suitable acknowledgement to the source must be made, either as a footnote or in a reference list at the end of your publication, as follows:

"Reprinted from Publication title, Vol /edition number, Author(s), Title of article / title of chapter, Pages No., Copyright (Year), with permission from Elsevier [OR APPLICABLE SOCIETY COPYRIGHT OWNER]." Also Lancet special credit - "Reprinted from The Lancet, Vol. number, Author(s), Title of article, Pages No., Copyright (Year), with permission from Elsevier."

4. Reproduction of this material is confined to the purpose and/or media for which permission is hereby given.

5. Altering/Modifying Material: Not Permitted. However figures and illustrations may be altered/adapted minimally to serve your work. Any other abbreviations, additions, deletions and/or any other alterations shall be made only with prior written authorization of Elsevier Ltd. (Please contact Elsevier at permissions@elsevier.com)

6. If the permission fee for the requested use of our material is waived in this instance, please be advised that your future requests for Elsevier materials may attract a fee.

7. Reservation of Rights: Publisher reserves all rights not specifically granted in the combination of (i) the license details provided by you and accepted in the course of this licensing transaction, (ii) these terms and conditions and (iii) CCC's Billing and Payment terms and conditions.

8. License Contingent Upon Payment: While you may exercise the rights licensed immediately upon 
issuance of the license at the end of the licensing process for the transaction, provided that you have disclosed complete and accurate details of your proposed use, no license is finally effective unless and until full payment is received from you (either by publisher or by CCC) as provided in CCC's Billing and Payment terms and conditions. If full payment is not received on a timely basis, then any license preliminarily granted shall be deemed automatically revoked and shall be void as if never granted. Further, in the event that you breach any of these terms and conditions or any of CCC's Billing and Payment terms and conditions, the license is automatically revoked and shall be void as if never granted. Use of materials as described in a revoked license, as well as any use of the materials beyond the scope of an unrevoked license, may constitute copyright infringement and publisher reserves the right to take any and all action to protect its copyright in the materials.

9. Warranties: Publisher makes no representations or warranties with respect to the licensed material

10. Indemnity: You hereby indemnify and agree to hold harmless publisher and $\mathrm{CCC}$, and their respective officers, directors, employees and agents, from and against any and all claims arising out of your use of the licensed material other than as specifically authorized pursuant to this license.

11. No Transfer of License: This license is personal to you and may not be sublicensed, assigned, or transferred by you to any other person without publisher's written permission.

12. No Amendment Except in Writing: This license may not be amended except in a writing signed by both parties (or, in the case of publisher, by CCC on publisher's behalf).

13. Objection to Contrary Terms: Publisher hereby objects to any terms contained in any purchase order, acknowledgment, check endorsement or other writing prepared by you, which terms are inconsistent with these terms and conditions or $\mathrm{CCC}^{\prime}$ s Billing and Payment terms and conditions. These terms and conditions, together with CCC's Billing and Payment terms and conditions (which are incorporated herein), comprise the entire agreement between you and publisher (and CCC) concerning this licensing transaction. In the event of any conflict between your obligations established by these terms and conditions and those established by CCC's Billing and Payment terms and conditions, these terms and conditions shall control.

14. Revocation: Elsevier or Copyright Clearance Center may deny the permissions described in this License at their sole discretion, for any reason or no reason, with a full refund payable to you. Notice of such denial will be made using the contact information provided by you. Failure to receive such notice will not alter or invalidate the denial. In no event will Elsevier or Copyright Clearance Center be responsible or liable for any costs, expenses or damage incurred by you as a result of a denial of your permission request, other than a refund of the amount(s) paid by you to Elsevier and/or Copyright Clearance Center for denied permissions.

\section{LIMITED LICENSE}

The following terms and conditions apply only to specific license types:

15. Translation: This permission is granted for non-exclusive world English rights only unless your license was granted for translation rights. If you licensed translation rights you may only translate this content into the languages you requested. A professional translator must perform all translations and reproduce the content word for word preserving the integrity of the article. If this license is to re-use 1 or 2 figures then permission is granted for non-exclusive world rights in all languages 
16. Website: The following terms and conditions apply to electronic reserve and author websites: Electronic reserve: If licensed material is to be posted to website, the web site is to be password-protected and made available only to bona fide students registered on a relevant course if:

This license was made in connection with a course,

This permission is granted for 1 year only. You may obtain a license for future website posting, All content posted to the web site must maintain the copyright information line on the bottom of each image,

A hyper-text must be included to the Homepage of the journal from which you are licensing at http $/ /$ www. sciencedirect.com/science/journal/xxxxx or the Elsevier homepage for books at http $/ /$ www. elsevier.com, and Central Storage: This license does not include permission for a scanned version of the material to be stored in a central repository such as that provided by Heron/XanEdu.

17. Author website for journals with the following additional clauses:

All content posted to the web site must maintain the copyright information line on the bottom of each image, and the permission granted is limited to the personal version of your paper. You are not allowed to download and post the published electronic version of your article (whether PDF or HTML, proof or final version), nor may you scan the printed edition to create an electronic version. A hyper-text must be included to the Homepage of the journal from which you are licensing at http//www.sciencedirect.com/science/journal/xxxxx. As part of our normal production process, you will receive an e-mail notice when your article appears on Elsevier's online service ScienceDirect (www. sciencedirect.com). That e-mail will include the article's Digital Object Identifier (DOI). This number provides the electronic link to the published article and should be included in the posting of your personal version. We ask that you wait until you receive this e-mail and have the DOI to do any posting.

Central Storage: This license does not include permission for a scanned version of the material to be stored in a central repository such as that provided by Heron/XanEdu.

18. Author website for books with the following additional clauses: Authors are permitted to place a brief summary of their work online only. A hyper-text must be included to the Elsevier homepage at http//www.elsevier.com. All content posted to the web site must maintain the copyright information line on the bottom of each image. You are not allowed to download and post the published electronic version of your chapter, nor may you scan the printed edition to create an electronic version.

Central Storage: This license does not include permission for a scanned version of the material to be stored in a central repository such as that provided by Heron/XanEdu.

19. Website (regular and for author): A hyper-text must be included to the Homepage of the journal from which you are licensing at $\mathrm{http} / / \mathrm{www}$.sciencedirect.com/science/journal/xxxxx. or for

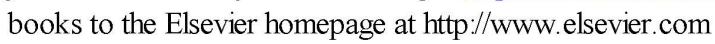

20. Thesis/Dissertation: If your license is for use in a thesis/dissertation your thesis may be submitted to your institution in either print or electronic form. Should your thesis be published commercially, please reapply for permission. These requirements include permission for the Library and Archives of Canada to supply single copies, on demand, of the complete thesis and include permission for UMI to supply single copies, on demand, of the complete thesis. Should your thesis 
be published commercially, please reapply for permission.

21. Other Conditions:

v1.6

If you would like to pay for this license now, please remit this license along with your payment made payable to "COPYRIGHT CLEARANCE CENTER" otherwise you will be invoiced within 48 hours of the license date. Payment should be in the form of a check or money order referencing your account number and this invoice number RLNK501118122. Once you receive your invoice for this order, you may pay your invoice by credit card. Please follow instructions provided at that time.

Make Payment To:

Copyright Clearance Center

Dept 001

P.O. Box 843006

Boston, MA 02284-3006

For suggestions or comments regarding this order, contact RightsLink Customer Support: customercare@copyright.com or +1-877-622-5543 (toll free in the US) or +1-978-6462777.

Gratis licenses (referencing $\mathbf{\$ 0}$ in the Total field) are free. Please retain this printable license for your reference. No payment is required. 
For Figures 2.4 and 2.5:

ELSEVIER LICENSE

TERMS AND CONDITIONS

Sep 21, 2013

This is a License Agreement between Ryan K Foley ("You") and Elsevier ("Elsevier") provided by Copyright Clearance Center ("CCC"). The license consists of your order details, the terms and conditions provided by Elsevier, and the payment terms and conditions.

\begin{tabular}{|c|c|}
\hline \multicolumn{2}{|c|}{$\begin{array}{l}\text { All payments must be made in full to CCC. For payment instructions, please see } \\
\text { information listed at the bottom of this form. }\end{array}$} \\
\hline Supplier & $\begin{array}{l}\text { Elsevier Limited } \\
\text { The Boulevard,Langford Lane } \\
\text { Kidlington,Oxford,OX5 1GB,UK }\end{array}$ \\
\hline $\begin{array}{l}\text { Registered Company } \\
\text { Number }\end{array}$ & 1982084 \\
\hline Customer name & Ryan K Foley \\
\hline \multirow[t]{2}{*}{ Customer address } & 1089 th Ave SE \\
\hline & MINOT, ND 58701 \\
\hline License number & 3233710617449 \\
\hline License date & Sep 21, 2013 \\
\hline Licensed content publisher & Elsevier \\
\hline $\begin{array}{l}\text { Licensed content } \\
\text { publication }\end{array}$ & Journal of Aerosol Science \\
\hline Licensed content title & $\begin{array}{l}\text { Nanoparticle motion trajectories and deposition in an inlet } \\
\text { channel of wall-flow diesel particulate filter }\end{array}$ \\
\hline Licensed content author & Yunqing Liu,Jinke Gong,Jun Fu,Hao Cai,Gang Long \\
\hline Licensed content date & April 2009 \\
\hline $\begin{array}{l}\text { Licensed content volume } \\
\text { number }\end{array}$ & 40 \\
\hline $\begin{array}{l}\text { Licensed content issue } \\
\text { number }\end{array}$ & 4 \\
\hline Number of pages & 17 \\
\hline Start Page & 307 \\
\hline End Page & 323 \\
\hline Type of Use & reuse in a thesis/dissertation \\
\hline Portion & figures/tables/illustrations \\
\hline $\begin{array}{l}\text { Number of } \\
\text { figures/tables/illustrations }\end{array}$ & 2 \\
\hline Format & both print and electronic \\
\hline $\begin{array}{l}\text { Are you the author of this } \\
\text { Elsevier article? }\end{array}$ & No \\
\hline Will you be translating? & No \\
\hline \multicolumn{2}{|l|}{ Order reference number } \\
\hline Title of your & Experimental Investigation into Particulate Matter Distribu \\
\hline
\end{tabular}


Estimated size (number of 200

pages)

Elsevier VAT number GB 494627212

Permissions price $\quad 0.00$ USD

VAT/Local Sales Tax $\quad 0.0$ USD / 0.0 GBP

Total

0.00 USD

Terms and Conditions

\section{INTRODUCTION}

1. The publisher for this copyrighted material is Elsevier. By clicking "accept" in connection with completing this licensing transaction, you agree that the following terms and conditions apply to this transaction (along with the Billing and Payment terms and conditions established by Copyright Clearance Center, Inc. ("CCC"), at the time that you opened your Rightslink account and that are available at any time at http://myaccount.copyright.com).

\section{GENERAL TERMS}

2. Elsevier hereby grants you permission to reproduce the aforementioned material subject to the terms and conditions indicated.

3. Acknowledgement: If any part of the material to be used (for example, figures) has appeared in our publication with credit or acknowledgement to another source, permission must also be sought from that source. If such permission is not obtained then that material may not be included in your publication/copies. Suitable acknowledgement to the source must be made, either as a footnote or in a reference list at the end of your publication, as follows:

"Reprinted from Publication title, Vol /edition number, Author(s), Title of article / title of chapter, Pages No., Copyright (Year), with permission from Elsevier [OR APPLICABLE SOCIETY COPYRIGHT OWNER]." Also Lancet special credit - "Reprinted from The Lancet, Vol. number, Author(s), Title of article, Pages No., Copyright (Year), with permission from Elsevier."

4. Reproduction of this material is confined to the purpose and/or media for which permission is hereby given.

5. Altering/Modifying Material: Not Permitted. However figures and illustrations may be altered/adapted minimally to serve your work. Any other abbreviations, additions, deletions and/or any other alterations shall be made only with prior written authorization of Elsevier Ltd. (Please contact Elsevier at permissions@elsevier.com)

6. If the permission fee for the requested use of our material is waived in this instance, please be advised that your future requests for Elsevier materials may attract a fee.

7. Reservation of Rights: Publisher reserves all rights not specifically granted in the combination of (i) the license details provided by you and accepted in the course of this licensing transaction, (ii) these terms and conditions and (iii) CCC's Billing and Payment terms and conditions.

8. License Contingent Upon Payment: While you may exercise the rights licensed immediately upon issuance of the license at the end of the licensing process for the transaction, provided that you have 
disclosed complete and accurate details of your proposed use, no license is finally effective unless and until full payment is received from you (either by publisher or by CCC) as provided in CCC's Billing and Payment terms and conditions. If full payment is not received on a timely basis, then any license preliminarily granted shall be deemed automatically revoked and shall be void as if never granted. Further, in the event that you breach any of these terms and conditions or any of CCC's Billing and Payment terms and conditions, the license is automatically revoked and shall be void as if never granted. Use of materials as described in a revoked license, as well as any use of the materials beyond the scope of an unrevoked license, may constitute copyright infringement and publisher reserves the right to take any and all action to protect its copyright in the materials.

9. Warranties: Publisher makes no representations or warranties with respect to the licensed material.

10. Indemnity: You hereby indemnify and agree to hold harmless publisher and $\mathrm{CCC}$, and their respective officers, directors, employees and agents, from and against any and all claims arising out of your use of the licensed material other than as specifically authorized pursuant to this license

11. No Transfer of License: This license is personal to you and may not be sublicensed, assigned, or transferred by you to any other person without publisher's written permission.

12. No Amendment Except in Writing: This license may not be amended except in a writing signed by both parties (or, in the case of publisher, by CCC on publisher's behalf).

13. Objection to Contrary Terms: Publisher hereby objects to any terms contained in any purchase order, acknowledgment, check endorsement or other writing prepared by you, which terms are inconsistent with these terms and conditions or CCC's Billing and Payment terms and conditions. These terms and conditions, together with CCC's Billing and Payment terms and conditions (which are incorporated herein), comprise the entire agreement between you and publisher (and CCC) concerning this licensing transaction. In the event of any conflict between your obligations established by these terms and conditions and those established by CCC's Billing and Payment terms and conditions, these terms and conditions shall control.

14. Revocation: Elsevier or Copyright Clearance Center may deny the permissions described in this License at their sole discretion, for any reason or no reason, with a full refund payable to you. Notice of such denial will be made using the contact information provided by you. Failure to receive such notice will not alter or invalidate the denial. In no event will Elsevier or Copyright Clearance Center be responsible or liable for any costs, expenses or damage incurred by you as a result of a denial of your permission request, other than a refund of the amount(s) paid by you to Elsevier and/or Copyright Clearance Center for denied permissions.

\section{LIMITED LICENSE}

The following terms and conditions apply only to specific license types:

15. Translation: This permission is granted for non-exclusive world English rights only unless your license was granted for translation rights. If you licensed translation rights you may only translate this content into the languages you requested. A professional translator must perform all translations and reproduce the content word for word preserving the integrity of the article. If this license is to re-use 1 or 2 figures then permission is granted for non-exclusive world rights in all languages. 
16. Website: The following terms and conditions apply to electronic reserve and author websites: Electronic reserve: If licensed material is to be posted to website, the web site is to be password-protected and made available only to bona fide students registered on a relevant course if:

This license was made in connection with a course,

This permission is granted for 1 year only. You may obtain a license for future website posting, All content posted to the web site must maintain the copyright information line on the bottom of each image,

A hyper-text must be included to the Homepage of the journal from which you are licensing at http//www. sciencedirect.com/science/journal/xxxxx or the Elsevier homepage for books at http://www. elsevier.com, and

Central Storage: This license does not include permission for a scanned version of the material to be stored in a central repository such as that provided by Heron/XanEdu.

17. Author website for journals with the following additional clauses:

All content posted to the web site must maintain the copyright information line on the bottom of each image, and the permission granted is limited to the personal version of your paper. You are not allowed to download and post the published electronic version of your article (whether PDF or HTML, proof or final version), nor may you scan the printed edition to create an electronic version A hyper-text must be included to the Homepage of the journal from which you are licensing at http:/www.sciencedirect.com/science/journal/xxxxx . As part of our normal production process, you will receive an e-mail notice when your article appears on Elsevier's online service ScienceDirect (www. sciencedirect.com). That e-mail will include the article's Digital Object Identifier (DOI). This number provides the electronic link to the published article and should be included in the posting of your personal version. We ask that you wait until you receive this e-mail and have the DOI to do any posting.

Central Storage: This license does not include permission for a scanned version of the material to be stored in a central repository such as that provided by Heron/XanEdu.

18. Author website for books with the following additional clauses

Authors are permitted to place a brief summary of their work online only.

A hyper-text must be included to the Elsevier homepage at http://www.elsevier.com. All content posted to the web site must maintain the copyright information line on the bottom of each image. You are not allowed to download and post the published electronic version of your chapter, nor may you scan the printed edition to create an electronic version.

Central Storage: This license does not include permission for a scanned version of the material to be stored in a central repository such as that provided by Heron/XanEdu.

19. Website (regular and for author): A hyper-text must be included to the Homepage of the journal from which you are licensing at $\underline{\mathrm{htt}} \mathrm{f} / \mathrm{www}$. sciencedirect.com/science/journal $/ \mathrm{xxxxx}$. or for books to the Elsevier homepage at http $/ /$ www. elsevier.com

20. Thesis/Dissertation: If your license is for use in a thesis/dissertation your thesis may be submitted to your institution in either print or electronic form. Should your thesis be published commercially, please reapply for permission. These requirements include permission for the Library and Archives of Canada to supply single copies, on demand, of the complete thesis and include permission for UMI to supply single copies, on demand, of the complete thesis. Should your thesis 
be published commercially, please reapply for permission.

21. Other Conditions:

v1.6

If you would like to pay for this license now, please remit this license along with your payment made payable to "COPYRIGHT CLEARANCE CENTER" otherwise you will be invoiced within 48 hours of the license date. Payment should be in the form of a check or money order referencing your account number and this invoice number RLNK501118119. Once you receive your invoice for this order, you may pay your invoice by credit card.

Please follow instructions provided at that time.

Make Payment To:

Copyright Clearance Center

Dept 001

P.O. Box 843006

Boston, MA 02284-3006

For suggestions or comments regarding this order, contact RightsLink Customer Support: customercare@copyright.com or +1-877-622-5543 (toll free in the US) or +1-978-6462777.

Gratis licenses (referencing $\$ 0$ in the Total field) are free. Please retain this printable license for your reference. No payment is required. 
For Figures 2.3, 2.6, 2.7, 2.8, 2.10, 2.11, and 2.2 (In Order)

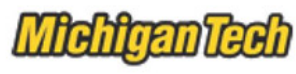

Ryan Foley <rkfoley@mtu.edu>

\section{Permission to Use Copyrighted Material}

Terri Kelly <terri@sae.org>

Thu, Oct 17, 2013 at 4:37 PM

To: Ryan Foley <rkfoley@mtu.edu>

Dear Ryan,

Thank you for your correspondence requesting permission to reprint the figures noted below from various SAE papers in your Master's Thesis for Michigan Technological University.

Permission is hereby granted and subject to the following conditions:

- Permission is for this one time single use only. New requests are required for further use or distribution of the SAE material.

- The following credit statement must appear below the figures: "Reprinted with permission from SAE Paper No. $X X X X X^{*} \odot X^{\circ} X X^{*}$ SAE International." *please insert the paper number and **year of publication

- We also request that you credit the original source (author/s, paper number and SAE) in the reference section of your publication.

- Permission does not cover any third-party copyrighted work which may appear in the material requested.

Again, thank you for contacting SAE for this permission.

Best regards,

Terri Kelly

Intellectual Property Rights Administrator

SAEINTERNATIONAL

400 Commonwealth Drive

Warrendale, PA 15096

e terri@sae.org

www.sae.org 
From: Ryan Foley [mailto:rkfoley@mtu.edu]

Sent: Saturday, September 21, 2013 11:42 AM

To: copyright

Subject: Permission to Use Copyrighted Material

$\mathrm{Hi}$,

I am working on my Masters Thesis at Michigan Technological University. The working title is "Experimental Investigation into Particulate Matter Distribution in Catalyzed Particulate Filters using a 3D Terahertz Wave Scanner", although it may change slightly. The expected publication date is December 2013. I am guessing that 4-6 print copies will be distributed between my 3 committee members, myself, and possibly my family. There will likely be a hard-copy available in Michigan Technological University's library. An electronic version will be available through ProQuest, and a subscription to ProQuest will be required to view it. I am requesting permission to use the figures listed from the works listed below. I would greatly appreciate permission to use these figures in my thesis. If you require additional information, feel free to contact me. Please leave my original text in your reply.

1). Opris, Cornelius N., and Johnson, John H., 1998, "A 2-D Computational Model Describing the Flow and Filtration Characteristics of a Ceramic Diesel Particulate Trap," 980545, SAE International.

Figure 22

2). Piscaglia, F., Rutland, C. J., and Foster, D. E., 2005, "Development of a CFD Model to Study the Hydrodynamic Characteristics and the Soot Deposition Mechanism on the Porous Wall of a Diesel Particulate Filter," 2005-01-0963, SAE International.

Figure 11

3). Yi, Yong, 2006, "Simulating the Soot Loading in Wall-flow DPF Using a Three-Dimensional Macroscopic Model," 2006-010264, SAE International.

Figures 7 and 9

4). Pinturaud, D., Charlet, A., Caillol, C., Higelin, P., Girot, P., and Briot, A., 2007, "Experimental Study of DPF Loading and Incomplete Regeneration," 2007-24-0094, Consiglio Nazionale delle Ricerche. SAE Naples Section. SAE International

Figures 16 and 18

5). Ohara, E., Mizuno, Y., Miyairi, Y., Mizutani, T., Yuuki, K., Noguchi, Y., Hiramatsu, T., Makino, M., Takahashi, A., Sakai, H., Tanaka, M., Martin, A., Fujii, S., Busch, P., Toyoshima, T., Ito, T., Lappas, I., and Vogt, C. D., 2007, "Filtration Behavior of Diesel Particulate Filters (1)," 2007-01-0921, SAE International.

Figure 4 
Thank you very much for considering my request and spending time on the matter. I look forward to your reply.

Regards,

Ryan Foley

\section{Ryan Foley}

Graduate Research Assistant

Mechanical Engineering

Michigan Technological University

Office: MEEM Room B009

Nothing in this message is intended to constitute an electronic signature unless a specific statement to the contrary is included in this message. Confidentiality Note: This message is intended only for the person or entity to which it is addressed. It may contain confidential and/or proprietary material. Any review, transmission, dissemination or other use, or taking of any action in reliance upon this message by persons or entities other than the intended recipient is prohibited. If you received this message in error, please contact the sender and delete it from your computer. 